DRAFt VERSION July 16, 2019

Typeset using IATEX twocolumn style in AASTeX61

\title{
ANALYSIS OF HELIUM-RICH WHITE DWARFS POLLUTED BY HEAVY ELEMENTS IN THE GAIA ERA
}

\author{
S. Coutu,${ }^{1}$ P. Dufour,${ }^{1}$ P. Bergeron,${ }^{1}$ S. Blouin,${ }^{1}$ E. Loranger,${ }^{1}$ N.F. Allard,${ }^{2,3}$ And B. H. Dunlap ${ }^{4,5}, 6$ \\ ${ }^{1}$ Département de Physique, Université de Montréal, Montréal, QC H3C 3J\%, Canada; coutu@astro.umontreal.ca, \\ dufourpa@astro.umontreal.ca.,bergeron@astro.umontreal.ca,sblouin@astro.umontreal.ca \\ ${ }^{2}$ GEPI, Observatoire de Paris, Université PSL, CNRS, UMR 8111, 61 avenue de l'Observatoire, F-75014 Paris, France \\ ${ }^{3}$ Sorbonne Université, CNRS, UMR 7095, Institut d'Astrophysique de Paris, 98bis boulevard Arago, F-75014 Paris, France \\ ${ }^{4}$ Department of Physics and Astronomy, University of North Carolina at Chapel Hill, Chapel Hill, NC 27599, USA \\ ${ }^{5}$ Department of Astronomy and McDonald Observatory, University of Texas at Austin, Austin, TX 78712, USA \\ ${ }^{6}$ McDonald Observatory, Fort Davis, TX-79734, USA
}

We present a homogeneous analysis of 1023 DBZ/DZ(A) and 319 DQ white dwarf stars taken from the Montreal White Dwarf Database. This represents a significant increase over the previous comprehensive studies on these types of objects. We use new trigonometric parallax measurements from the Gaia second data release, together with photometry from the Sloan Digital Sky Survey, Pan-STARRS, Gaia, or BVRI from the literature, which allow the determination of the mass for the majority of the objects in our sample. We use the photometric and spectroscopic techniques with the most recent model atmospheres available, which include high-density effects, to accurately determine the effective temperature, surface gravity, and heavy element abundances for each object. We study the abundance of hydrogen in DBZ/DZ white dwarfs and the properties of the accreted planetesimals. We explore the nature of the second sequence of DQ stars using proper motions from Gaia, and highlight evidence of crystallization in massive DQ stars. We also present mass distributions for both spectral types. Finally, we discuss the implications of our findings in the context of the spectral evolution of white dwarfs, and provide the atmospheric parameters for each star.

Keywords: stars: abundances - stars: atmospheres - stars: evolution - white dwarfs 


\section{INTRODUCTION}

White dwarfs represent the final evolutionary phase of main sequence stars with initial mass below $\sim 8 M_{\odot}$ and are characterized by their high surface gravity, typically $g=10^{8} \mathrm{~cm} \mathrm{~s}^{-2}$. Because of this, elements heavier than helium will sink below the photosphere in characteristic timescales that are many orders of magnitude smaller than the cooling age of the star (Paquette et al. 1986). This gravitational separation also explains why most white dwarfs - about $80 \%$ - have pure hydrogen atmospheres. The only absorption lines present in their spectra are those of hydrogen, and they are collectively known as DA stars. For a smaller fraction, practically no hydrogen survives the late phases of stellar evolution, and a thin opaque helium layer - the heaviest element remaining - will float on top and form the atmosphere. Depending on the effective temperature, they are classified as DO white dwarfs if they show ionized helium lines, and DB stars if only neutral helium lines can be observed. Below $T_{\text {eff }} \sim 12,000 \mathrm{~K}$, spectra of pure helium atmosphere white dwarfs become featureless as there is not enough energy to populate the lower energy levels of He I line transitions. Such objects with continuous optical spectra are classified as DC white dwarfs. Note that a similar phenomenon also happens for hydrogen-rich white dwarfs cooler than about $5000 \mathrm{~K}$ as the electrons are mostly found in the ground state, preventing Balmer line transitions. In the absence of physical mechanisms competing with gravitational settling, the optical spectra of all white dwarfs should thus show only hydrogen lines, helium lines, or pure continuum. Nevertheless, white dwarfs with traces of heavy elements do exist, indicating that gravitational settling is not acting alone. These "contaminated" white dwarfs are found mainly in two categories: i) those with traces of heavy elements (other than carbon) in their optical spectra, and ii) those with primarily carbon absorption lines, either molecular or atomic.

\subsection{Heavy Element Pollution}

White dwarfs showing absorption lines from elements such as calcium, magnesium, or iron are collectively known as DZ stars if their spectra show only heavy element lines, DBZ stars if they show helium and heavy elements, and DAZ stars if they display hydrogen and metal features. Model atmospheres show that both the DZ and DBZ stars are helium dominated while the DAZs are hydrogen dominated. The presence of heavy elements in these objects is now understood as external accretion of matter from a disk of debris resulting from the destruction of a rocky object (asteroid or small planets) by the white dwarf's tidal forces (see Jura \& Young
2014, and references therein). These objects are thus temporarily imprinted (diffusion timescales range from a few hundred thousands to a few million years ${ }^{1}$, much shorter than the cooling age) with the chemical composition of the polluting body, providing a unique opportunity to study the chemical composition of extra-solar bodies (Zuckerman et al. 2007; Klein et al. 2010, 2011; Dufour et al. 2012; Jura et al. 2012; Xu et al. 2014; Koester et al. 2014; Xu et al. 2016, 2017; Blouin et al. 2019b). High-resolution or UV spectroscopic observations of some samples have shown that 25 to $50 \%$ of all white dwarfs are contaminated by heavy elements at some level (Zuckerman et al. 2003, 2010; Koester et al. 2014).

Moreover, since observational evidence indicates that these white dwarfs had (or still have) at least some sort of planetary system around them, they can provide information about the correlation between stellar mass and planet occurrence, which can give insight into the planet formation process (Johnson et al. 2010). Indeed, most observational exoplanet surveys are biased towards lower mass stars. The Kepler mission prioritized G-type stars (Batalha et al. 2010), which have masses around 1 $M_{\odot}$. More massive stars of type $\mathrm{O}$ and $\mathrm{B}$ are observed much less frequently by Kepler, mostly because of their scarcity (they spend a very short time on the main sequence), and the Kepler field of view was chosen to avoid young stellar populations (Batalha et al. 2010). Doppler surveys also favor Sun-like stars, as their spectral properties make their detection easier (Johnson et al. 2010). Thus, very little is known about the relation between planet occurrence and stellar mass above $M=2-3 M_{\odot}$. While massive white dwarfs - which had massive main sequence progenitors - are fainter, the selection bias is much less important, especially with Gaia DR2, which is expected to be volume-complete within $\sim 70$ parsecs (Gentile Fusillo et al. 2019). Inferences of planetary systems around white dwarfs are thus not subjected to the same limitations.

\subsection{Carbon Pollution}

White dwarfs showing mainly carbon features are collectively known as DQ stars. They represent $9 \%$ of the white dwarfs in the local sample $(D<20 \mathrm{pc}$, Giammichele et al. 2012). They show only atomic carbon lines when $T_{\text {eff }} \gtrsim 10,000 \mathrm{~K}$, and molecular $\mathrm{C}_{2}$ bands at lower effective temperatures, with a smooth transition around that temperature where both molec-

\footnotetext{
${ }^{1}$ Based on calculations by G. Fontaine, included in the MWDD and available at http://www.montrealwhitedwarfdatabase.org/ evolution.html
} 
ular bands and atomic carbon lines are simultaneously present. Model atmosphere analyses have shown that they are helium-dominated with carbon abundances ranging from $\log (\mathrm{C} / \mathrm{He})=-7$ to -2 (Weidemann \& Koester 1995; Dufour et al. 2005; Koester \& Knist 2006), and effective temperatures between $\sim 5000 \mathrm{~K}$ and 12,000 $\mathrm{K}$. A model where the deep helium convection zone catches up with the settling carbon in the core, and dredges it up to the surface, can successfully account for the observed abundances in most objects (Fontaine \& Brassard 2005; Dufour et al. 2005). Dufour et al. (2005) showed, however, that several DQ stars had larger than average carbon abundances, forming a distinct sequence about 1 dex above the bulk of the sample in a $\log (\mathrm{C} / \mathrm{He})$ vs $T_{\text {eff }}$ diagram (see also Koester \& Knist 2006). Since the only object with a measured trigonometric parallax belonging to this second sequence was massive, Dufour et al. proposed that they could represent the high-mass tail of the white dwarf mass distribution. However, Brassard et al. (2007) showed that an evolutionary sequence at $1 M_{\odot}$ does not correctly predict the carbon abundance pattern that is empirically observed, indicating that another explanation to account for these stars must be sought.

While the cooler end of the DQ sequence is consistent with the expectations from the dredge-up model, very few stars were known at that time to test the theory on the hotter side $\left(T_{\text {eff }} \geq 13,000 \mathrm{~K}\right)$. Many hot objects showing mainly ionized or neutral carbon lines (and also oxygen in a few cases) had been identified in the Sloan Digital Sky Survey (SDSS) by Liebert et al. (2003). The interpretation then was that these objects were a hotter version of the cool DQ white dwarfs, which had helium-dominated atmospheres with traces of carbon. However, when the hottest objects were analyzed using state-of-the-art model atmospheres, it was found in fact that the main atmospheric constituent was carbon, not helium (Dufour et al. 2007a, 2008). Dufour et al. (2013) then showed that, in a $u-g$ vs $g-r$ colorcolor diagram, the hot carbon-dominated atmosphere DQ stars $\left(T_{\text {eff }} \sim 18,000-24,000 \mathrm{~K}\right.$ with mainly ionized carbon lines) seem to form a sequence that connects with warm DQ white dwarfs with neutral atomic lines $\left(T_{\text {eff }} \sim 10,000-16,000 \mathrm{~K}\right)$, followed by cooler DQ stars with strong molecular bands $\left(T_{\text {eff }}<10,000 \mathrm{~K}\right.$ on the second sequence mentioned above). Unfortunately, very few trigonometric parallax measurements were available for these objects at that time, and thus the massive nature of the elusive sequence was somewhat speculative.

Since the studies of Dufour et al. (2005, 2007b) the latest comprehensive analyses of large samples of DQ and DZ stars, respectively - there have been sev- eral improvements in stellar atmosphere modeling (for example, Blouin et al. 2017, Blouin et al. 2018a). Simultaneously, several surveys have enlarged the sample of spectroscopically confirmed white dwarfs considerably, and consequently, the number of known DQ/DZ/DBZ white dwarfs has increased by more than a factor of five. Also, thanks to the second Gaia data release in April 2018 , distances are now available, for the first time, for most of these objects, a quantity required to obtain precise measurements of their stellar masses.

The availability of improved model atmospheres and new data motivated us to perform an updated homogeneous analysis of all these metal-polluted white dwarfs. We describe the model atmospheres in Section 2, the observational data in Section 3, and the methodology in Section 4. We present the analysis for the DBZ/DZ stars in Section 5, and for the DQ stars in Section 6. We then discuss the implications of our results on our understanding of the spectral evolution of white dwarfs in Section 7. Our conclusions follow in Section 8.

\section{MODEL ATMOSPHERES}

Our DZ/DBZ/DQ model atmosphere code is similar to that outlined in Dufour et al. (2005, 2007b), but with several physical improvements described at length in Blouin et al. (2018a,b). Of particular importance for the study of DZ/DBZ stars are the new line profile calculations following the unified line shape theory of Allard et al. (1999) for strong transitions, the most important being Ca II H\&K, Mg I $\lambda 2852$ and $\mathrm{Mg}$ II $\lambda 2795 / 2802$, the Mgb triplet, and Ca I $\lambda 4226$. Less important transitions use Lorentzian or quasistatic van der Waals broadening profiles (Walkup et al. 1984, D. Koester, private communication). For the DQ model atmospheres, one of the main improvements over Dufour et al. (2005) is the replacement of the "just overlapping line approximation" (Zeidler-K.T. \& Koester 1982), to describe the $\mathrm{C}_{2}$ Swan band opacity, with a complete linelist provided by J. O. Hornkohl (private communication; see Parigger et al. 2015 for details of the methodology). We find that the use of this new linelist provides a much better representation of the shape of the observed Swan bands in DQ white dwarfs, particularly in the region around $4300 \AA$, which was poorly fitted in Dufour et al. (2005), compared to the prescription of Zeidler-K.T. \& Koester (1982), Brooke et al. (2013), or Kurucz linelists ${ }^{2}$. The atomic linelist of Kurucz has also been replaced by the compilation from the Vienna Atomic Line Database (VALD, Piskunov et al. 1995).

\footnotetext{
${ }^{2}$ http://kurucz.harvard.edu/linelists.html
} 
For the DZ/DBZ(A) white dwarfs, we generated a 4dimensional grid of model atmospheres and synthetic spectra with $T_{\text {eff }}$ varying from $4000 \mathrm{~K}$ to $16,000 \mathrm{~K}$ by steps of $500 \mathrm{~K}, \log g$ from 7.0 to 9.0 by steps of 0.5 dex, $\log \mathrm{Ca} / \mathrm{He}$ from -12 to -7 by steps of $0.5 \mathrm{dex}$, and $\log \mathrm{H} / \mathrm{He}$ from -7 to -3 by steps of 1 dex. We also generated a grid with no hydrogen. Metal-to-metal ratios have been fixed to that of chondrites (Lodders 2003) with respect to calcium. Calcium thus serves as a proxy for the abundances of all other heavy elements. While metal-to-metal ratios certainly differ from that of chondrites in many objects, our assumption provides a good first order approximation of the contribution of free electrons and opacity from heavy elements (see Dufour et al. 2007b, and also discussion below).

For the DQ model grid, since no DQ white dwarfs are found at high effective temperature and low carbon abundance, or at low effective temperature and high carbon abundance, we generated two separate 3dimensional grids. The first grid is generated with $T_{\text {eff }}$ from $8000 \mathrm{~K}$ to $16,000 \mathrm{~K}$ by steps of $500 \mathrm{~K}, \log g$ from 7 to 9 by steps of $0.5 \mathrm{dex}$, and $\log \mathrm{C} / \mathrm{He}$ from -5 to -1 by steps of 0.5 dex. The second grid covers $T_{\text {eff }}$ from 6000 $\mathrm{K}$ to $10,000 \mathrm{~K}$ by steps of $500 \mathrm{~K}, \log g$ from 7 to 9 by steps of $0.5 \mathrm{dex}$, and $\log \mathrm{C} / \mathrm{He}$ from -8 to -4 by steps of 0.5 dex. No hydrogen is included in these models, but some smaller grids with hydrogen were generated to test its effects, as discussed in Section 6.3.

\section{OBSERVATIONS}

\subsection{Note on Naming Convention}

Some white dwarfs can have up to 20 different names. Here we decided to adopt names based on ICRS coordinates at epoch and equinox 2000 instead of mixing names from different catalogs. Stars will be named $\mathrm{JHHMM} \pm \mathrm{DDMM}$, where the first four digits correspond to the right ascension in hours and minutes, and the last four digits to the declination in degrees and minutes in sexagesimal notation. In some cases, a second relevant name will be written in parentheses. Table 5 provides cross-references for the names of the objects in our sample with Gaia source id, MWDD id, and the full coordinates.

\subsection{Sample Selection}

We first selected from the Montreal White Dwarf Database $^{3}$ (Dufour et al. 2017, hereafter MWDD) all objects with either a $\mathrm{Q}$ or a $\mathrm{Z}$ in their spectral classification. This includes DZ, DZA, DBZ, DBZA, DQ,

\footnotetext{
3 www.montrealwhitedwarf database.org
}

etc. We did not include DAZ white dwarfs because they have hydrogen-rich atmospheres, and we are only interested here in helium-rich atmospheres. Most objects in our sample have previously been identified in various white dwarf catalogs from the Sloan Digital Sky Survey (SDSS; Kepler et al. 2015, 2016; Kleinman et al. 2004, 2013; Eisenstein et al. 2006). Many objects have also been classified as uncertain, such as DQ: or DC-DQ. We thus visually inspected every spectrum and rejected those that did not show the appropriate spectral lines, or were too noisy to make a reliable classification (spectra with better signal-to-noise will eventually confirm their spectral types). We rejected known unresolved binary systems as well as magnetic white dwarfs. In order to ensure a homogeneous analysis based exclusively on optical features, we also rejected some objects classified as DQ white dwarfs but with carbon features detected only in the ultraviolet. We also excluded stars that had carbon-dominated atmospheres, the so-called Hot DQ stars $\left(T_{\text {eff }} \gtrsim 18,000 \mathrm{~K}\right)$, or any object for which our preliminary analysis gave atmospheric parameters outside of our model grid. Finally, DQpec white dwarfs with strong distorted Swan bands are also left out of our analysis because there are still large uncertainties regarding their modeling (see Blouin et al. 2019c).

All objects in our sample were then cross-matched with Gaia DR2 to retrieve photometric and astrometric data. For one object, J0739+0513 (Procyon B), we use the parallax from Hipparcos $(284.56 \pm 1.26$, van Leeuwen 2007). A few objects with negative parallaxes or very large uncertainties $\left(\sigma_{\pi} / \pi>1\right)$ were rejected; these are unlikely to be white dwarfs, and if they are, the data are too imprecise to allow any satisfactory analysis. BailerJones (2015) demonstrated that inverting the parallax to estimate the distance might lead to unreliable distances when the uncertainty on the parallax is larger than $20 \%$, and that it can lead to incorrect error estimates. Fortunately, $87 \%$ of our parallax sample have smaller uncertainties. In fact, the difference between a probabilistic analysis distance and the inverse of the parallax is less than $1 \%$ for the majority of objects when $\sigma_{\pi} / \pi<0.1$

With the availability of trigonometric parallaxes and broadband colors from Gaia for the majority of the objects in our sample, it is now possible to place them accurately in an observational Hertzsprung-Russell (HR) diagram (Gaia Collaboration et al. 2018). This is illustrated in Figure 1, where stars that have been erroneously classified as white dwarfs can easily be identified. Examples of such stars are the giant star SDSS J174618.94+262217.0 (Green 2013) and SDSS J192013.71+383917.7, two objects that have been er- 


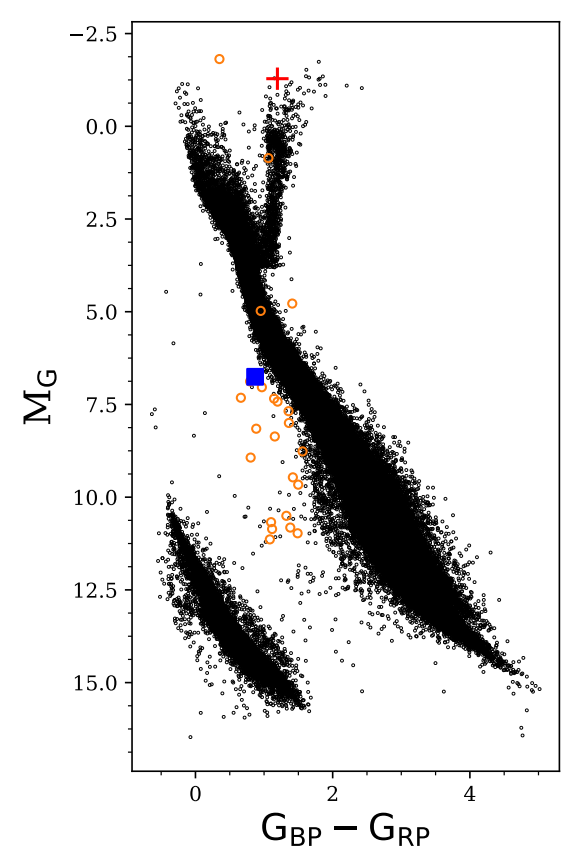

Figure 1. Gaia HR diagram for objects misclassified as white dwarfs (orange circles). SDSS J174618.94+262217.0 and SDSS J192013.71+383917.7, the two examples discussed in the text and displayed in Figure 2, are represented by a red cross and a blue square, respectively. Black dots are Gaia objects within $100 \mathrm{pc}$ selected using the cuts proposed in Gaia Collaboration et al. (2018, Appendix B). White dwarf candidates are located in the bottom left portion of the diagram.

roneously classified as a DQ and a DZ white dwarf, respectively (Kleinman et al. 2013). The similarity of the spectra of these non-degenerate stars, displayed in Figure 2, with genuine DQ and DZ white dwarfs stresses the importance of using photometric and parallax measurements in combination to spectroscopic data during the classification process. Overall, 25 objects, listed in Table 1, previously classified as white dwarfs in various catalogs were rejected this way.

To constitute our final sample, we removed 63 stars that had parameters outside our model grids (these will be analyzed elsewhere) and 63 white dwarfs for which no optical spectroscopy was available. In the end, we are left with a sample of 1023 DZ (679 with parallax measurements) and 317 DQ (303 with parallax measurements) white dwarfs, which we analyze in a homogeneous fashion in the next sections.

\subsection{Photometric and Spectroscopic Data}

We cross-matched our sample with Pan-STARRS DR1 and SDSS DR14 to retrieve grizy and ugriz PSF magnitudes, respectively. Since SDSS photometry is available for $95 \%$ of our sample, while only $80 \%$ of our objects

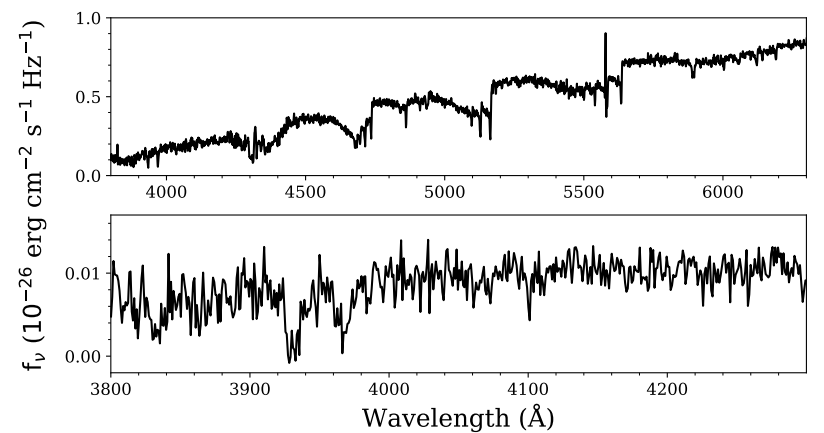

Figure 2. Optical spectra of SDSS J174618.94+262217.0 (top) and SDSS J192013.71+383917.7 (bottom), two objects that were previously misclassified as DQ and DZ white dwarfs, respectively.

have Pan-STARRS photometry, we decided to rely primarily on SDSS photometry for the sake of homogeneity. Note that although Pan-STARRS covers a much larger part of the sky than SDSS, most spectroscopically identified white dwarfs were discovered in the SDSS. Furthermore, SDSS has proven to be reliable many times (see Genest-Beaulieu \& Bergeron 2019), while possible Pan-STARRS biases have not been fully explored yet. When SDSS ugriz is not available for an object, we rely on Pan-STARRS data, previously published $B V R I$ photometry, or simply Gaia broadband photometry, in that order of priority. For J0739+0513 (Procyon B), we rely on HST photometry (Provencal et al. 1997).

The majority of the spectra used in this study are drawn from SDSS $^{4}$. The spectra of 51 objects without SDSS spectroscopy are taken from Limoges et al. (2013, 2015), Subasavage et al. (2007, 2009), Bergeron et al. $(1997,2001)$, or archival data secured by the Montreal group in the last few decades. Finally, the spectroscopic data for Procyon B are from HST (Provencal et al. 2002).

\section{ATMOSPHERIC PARAMETER DETERMINATION}

Our fitting method is similar to that described in Dufour et al. (2005, 2007b) for DQ and DZ stars, respectively. Briefly, we first convert the observed magnitudes into average fluxes following the method described in Holberg \& Bergeron (2006). The magnitudes on the AB system (SDSS, Pan-STARRS) are converted using the relation $m=-2.5 \log f_{\nu}^{m}-48.60$, where $f_{\nu}^{m}$ is the stellar flux averaged over the bandpass response; the corrections from Eisenstein et al. (2006) are applied to put the SDSS photometry on the AB system. The magni-

\footnotetext{
${ }^{4}$ https://www.sdss.org/dr14/spectro/spectro_basics/
} 
Table 1. Objects rejected due to their location in the Gaia HR diagram

\begin{tabular}{ccc}
\hline J Name & Gaia source id & MWDD id \\
\hline J0205+0058 & 2508108382280944768 & 2MASS J02053812+0058354 \\
J2112+0912 & 1743430270303257728 & 2MASS J21120843+0912019 \\
J1642+3617 & 1328051961501737088 & Cl* NGC 6205 KAD 606 \\
J0239-0003 & 2498734800840646400 & SDSS J023955.79-000312.0 \\
J0814+3508 & 902942352007933568 & SDSS J081424.51+350822.9 \\
J0815+1537 & 655412730925191808 & SDSS J081502.24+153709.7 \\
J0914-0022 & 3842424562164246656 & SDSS J091455.49-002219.2 \\
J1136+4702 & 787028812551381248 & SDSS J113612.16+470206.8 \\
J1208-0008 & 3698751446481826432 & SDSS J120853.36-000847.3 \\
J1228+2057 & 3952475922233611776 & SDSS J122829.50+205747.0 \\
J1336+1535 & 3742286441879709952 & SDSS J133644.21+153554.2 \\
J1357+1252 & 3727838511198756224 & SDSS J135713.37+125230.9 \\
J1507+1824 & 1211873229279489536 & SDSS J150741.36+182406.8 \\
J1549+0436 & 4426232766560567296 & SDSS J154918.87+043651.0 \\
J1610+0247 & 4412254439017190400 & SDSS J161041.62+024706.2 \\
J1714+2324 & 4568671714800327680 & SDSS J171432.25+232423.0 \\
J1746+2622 & 4582265290590354304 & SDSS J174618.94+262217.0 \\
J1920+3839 & 2052876071205883520 & SDSS J192013.71+383917.7 \\
J2105+0828 & 1744023628624108288 & SDSS J210511.38+082853.7 \\
J2106+0642 & 1736767710874927872 & SDSS J210632.52+064233.5 \\
J2111+0915 & 1743431683347518464 & SDSS J211157.26+091554.2 \\
J2153+2750 & 1800030173963458048 & SDSS J215306.03+275057.3 \\
J2259-1001 & 2606526522781415168 & SDSS J225931.60-100146.9 \\
J0124+0541 & 2563653437678125568 & USNO-B1.0 0956-00013686 \\
\hline 0111 & 339405495907200 & WD 0255+009.1 \\
\hline
\end{tabular}

tudes on the Vega system (BVRI, Gaia) are converted using the relation $m=-2.5 \log f_{\lambda}^{m}+c_{m}$, where $c_{m}$ is the zero point in the corresponding bandpass. These zero points are calculated using the observed fluxes and magnitudes for Vega. For the BVRI bandpass response, we use Cohen et al. (2003), and for Gaia, we use the revised bandpasses ${ }^{5}$, while for the Vega flux, we use alpha_lyr_stis_008.fits from the CALSPEC Calibration Database ${ }^{6}$. Our results are summarized in Table 2.

Photometric data are also dereddened following the procedure described in Harris et al. (2006) using the extinction maps of Schlafly \& Finkbeiner (2011). We

\footnotetext{
5 https://www.cosmos.esa.int/web/gaia/iow_20180316

6 http://www.stsci.edu/hst/observatory/crds/calspec. html
}

Table 2. Zero points derived for different bandpasses.

\begin{tabular}{ccc}
\hline Bandpass & Zero point & Vega mag \\
\hline$G$ & -21.51572 & 0.00 \\
$G_{\mathrm{BP}}$ & -20.99456 & 0.00 \\
$G_{\mathrm{RP}}$ & -22.22677 & 0.00 \\
$B$ & -20.46331 & $0.024^{1}$ \\
$V$ & -21.06662 & $0.026^{1}$ \\
$R$ & -21.64524 & $0.033^{1}$ \\
$I$ & -22.37877 & $0.029^{1}$ \\
\hline
\end{tabular}

(1)Holberg \& Bergeron (2006)

thus consider the extinction to be negligible for stars with $D<100 \mathrm{pc}$, to be maximum for those located at $|z|>250 \mathrm{pc}$ from the galactic plane $(z$ is the distance from the galactic), and to vary linearly between these 
two regimes. Genest-Beaulieu \& Bergeron (2019) compared this method to that used by Gentile Fusillo et al. (2019) and came to the conclusion that they lead to similar results for white dwarfs in the SDSS. When no parallax measurement is available, we first obtain the photometric distance assuming $\log \mathrm{g}=8.0$, and then apply a dereddening correction using this distance, and repeat the process until the distance (and the reddening) converge to a single value.

Next we transform the monochromatic Eddington fluxes from our model grids into fluxes averaged over each bandpass. These synthetic average fluxes $H_{\lambda}^{m}$ (or $\left.H_{\nu}^{m}\right)$ are related to the observed fluxes by the equation:

$$
f_{\lambda}^{m}=4 \pi\left(\frac{R}{D}\right)^{2} H_{\lambda}^{m}
$$

where $R$ is the radius of the star, and $D$ its distance from Earth. In the above equation, the average model fluxes depend on $T_{\text {eff }}, \log g$, and the atmospheric composition, which refers to the carbon abundance for DQ stars, and to the hydrogen and calcium abundances for $\mathrm{DBZ} / \mathrm{DZ}$ stars. The best fit between observed photometry and synthetic fluxes is obtained using the nonlinear least square steepest decent method of Levenberg-Marquardt (Press et al. 1986), with the values of $T_{\text {eff }}$ and the solid angle $\pi(R / D)^{2}$ left as free parameters, while $\log g$ and the atmospheric composition are kept fixed during the first iteration.

From the derived value of the solid angle and the distance given by the inverse of the parallax, we determine the radius $R$ of the star. The values of $\log g$ and mass are then derived by interpolating in evolutionary models similar to those described in Fontaine et al. (2001) but with $\mathrm{C} / \mathrm{O}$ cores, $\log q(\mathrm{He}) \equiv \log M_{\mathrm{He}} / M_{\star}=-2$ (where $M_{\star}$ is the mass of the star) and $\log q(\mathrm{H})=$ -10 , which are representative of helium-rich atmosphere white dwarfs (Dufour et al. 2005). We repeat the process this time using the newly determined surface gravity until convergence is reached. If the parallax is unknown, we simply assume $\log g=8$ and derive a radius $R$ from the same evolutionary models, which yields a photometric distance when combined with the solid angle. The uncertainties on the effective temperature and the solid angle are obtained directly from the covariance matrix of the fitting procedure.

We next determine the chemical composition by fitting the spectroscopic data, again using the LevenbergMarquardt method, keeping $T_{\text {eff }}$ and $\log g$ fixed to the values obtained from the photometric fit. For cool DQ stars, we fit the Swan bands between 4000 and $6500 \AA$, while for hotter DQs, we use the atomic absorption lines between 4500 and $5500 \AA$. The carbon abundance, the solid angle, and a first or second degree polynomial to account for uncertainties in the flux calibration (see Dufour et al. 2005) — are considered free parameters during the fitting procedure. For DBZ/DZ stars, we begin by fixing the hydrogen abundance, either by fitting the $\mathrm{H} \alpha$ spectral line if it is visible, or by fixing $\log \mathrm{H} / \mathrm{He}$ at the detection limit (we also fit stars not showing $\mathrm{H} \alpha$ with our hydrogen-free grid; see Section 5 for a discussion of this matter). This detection limit was estimated by calculating, at each temperature and $\log g$ value in our grid, the amount of hydrogen required to reach a threshold of $500 \mathrm{~m} \AA$ for the equivalent width of the $\mathrm{H} \alpha$ line. We then fit the $\mathrm{Ca}$ II H\&K absorption lines to determine $\log \mathrm{Ca} / \mathrm{He}$. Then we repeat the photometric fit, but this time using the values of $\log g$, calcium abundance (or carbon abundance in the case of DQ white dwarfs), and hydrogen abundance obtained in this last iteration. We repeat the procedure, typically 3 to 5 iterations (or more in some cases), until the parameters have converged to a stable solution.

To obtain the uncertainty on the abundances measured from the spectroscopic fit, we rely on the same method described in Bergeron et al. (1992), where we first assume an arbitrary standard deviation $\sigma=1$ for each data point, then calculate the root-mean-square deviation of the observed spectrum from the best-fit model spectrum. This is then propagated into the covariance matrix, from which the formal uncertainties of the fitted atmospheric parameters are obtained. This gives an uncertainty estimation that depends mostly on the signal-to-noise ratio of the observed spectra.

\section{DZ/DBZ(A) WHITE DWARFS}

\subsection{Photometric and Spectroscopic Fits}

Following the method described in Section 4, we determined the atmospheric parameters for all DZ/DBZ(A) white dwarfs in our sample. Figure 3 shows typical fits of the energy distribution and the Ca II H\&K lines region (all our fits are available in Appendix I), while our final parameters are given in Table 6 . The fit to the $\mathrm{H} \alpha$ line, when present, is shown as an inset. Note that for stars that do not show hydrogen, we report a solution with the hydrogen abundance fixed at the detection limit (see explanation below).

We note that other lines, mostly Mg and Fe, are sometimes observed in addition to Ca II H\&K. Since in our model grids, the abundances of these elements relative to calcium are fixed to the chondrite values, a visual inspection of the quality of the "fit" to these lines provides a quick assessment of the validity of this approximation, which appears to be adequate (or close enough) for most stars that show such lines in our sample. How- 

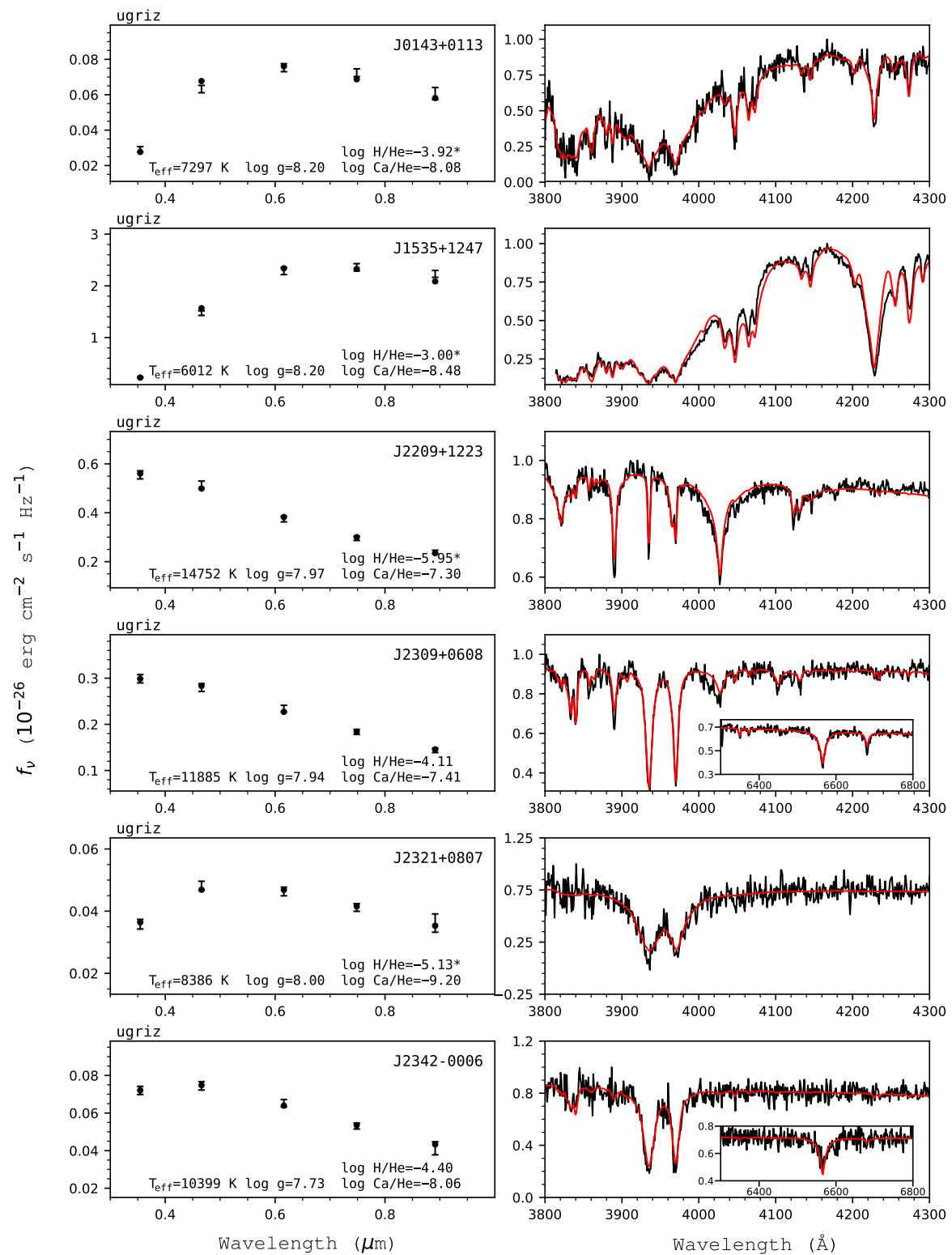

Figure 3. Examples of fits to our sample of DBZ/DZ(A) white dwarfs. Left panels: Photometric fits where error bars represent the observed data, while filled circles correspond to average model fluxes. A dagger symbol indicates that log $g$ is fixed at 8.0 (no parallax measurement available), while a star symbol indicates a value of $\log \mathrm{H} / \mathrm{He}$ fixed at the visibility limit. Right panels: Spectroscopic fits (red) to the normalized observed spectra (black). The inset shows the fit to H $\alpha$ when present. A version of this figure with all our fits is available in Appendix I.

ever, it is certainly possible that some elements depart significantly from the chondrite values. Since what is most important, as far as the mass and effective temperature determinations are concerned, is the amount of free electrons in the photosphere (effects due to the redistribution of the flux absorbed in the UV can also play a role in some cases), small deviations should only have a minimal impact on those parameters, even more so when hydrogen is present, because electrons from ionized hydrogen will dominate the free electron budget.

Recently, Hollands et al. $(2017,2018)$ analyzed a large sample of 230 cool DZ white dwarfs and determined abundances of individual elements. Their results indicate that to first order, objects are found with chemical compositions not too far from that of bulk Earth ratios, at least for the most visible elements, which are calcium, magnesium, and iron. Although their param- 
eters were obtained using a different model atmosphere code that do not include all the improvements included in our code, a comparison of their results for 197 objects in common with our sample suggests that the exact metal-to-metal ratios used in their analysis has only a modest impact on the effective temperature determination. We find that the effective temperatures obtained with our approach are, on average, only $11 \mathrm{~K}$ lower than theirs, but with a somewhat large $330 \mathrm{~K}$ standard deviation. We find no correlation between the differences in effective temperature and the deviation to the chondrite abundances (relative to $\mathrm{Ca}$ ) that they report. Instead, we find that these differences are correlated with $\log g$ values. Since the Gaia second data release was not available at the time the analyses of Hollands et al. (2017, 2018) were published, the authors assumed $\log g=8$ for their whole sample, while our analysis makes use of the newly available parallax measurements to determine the surface gravities. This distinction seems to be the main explanation for the different atmospheric parameters derived in both studies. Indeed, we find a strong correlation between the differences in effective temperature and the departure from $\log g=8$, our temperatures being higher (lower) for larger (smaller) $\log g$ values. Similarly, there is also a correlation between the differences in $\log \mathrm{Ca} / \mathrm{He}$ and $\log g$. This result is not surprising given that the difference in the strength of the calcium absorption features resulting from a change in surface gravity will need to be compensated by an appropriate change in calcium abundance, which in turn will affect the number of free electrons, and thus the effective temperature determination. We thus conclude that the atmospheric parameters derived using our approach should be reliable, and not significantly affected by our assumption of the metal-to-metal ratios.

Nevertheless, when better spectroscopic data (highresolution or UV observations) for a given object indicate some departure from our approximation, atmospheric parameters should always be re-derived in a self-consistent way for better precision. For example, for the DZA white dwarf Ross 640 analyzed by Blouin et al. (2018a), changes in the abundances of $\mathrm{Mg}$ and $\mathrm{Fe}$ relative to $\mathrm{Ca}$ affected the UV flux level sufficiently to warrant the calculations of a specific grid with modified abundances in order to obtain atmospheric parameters in a self-consistent way. As a result, their final effective temperature was $250 \mathrm{~K}$ cooler, and the corresponding mass $0.04 M_{\odot}$ lower, than what we obtain here with our approach. Unfortunately, since high-resolution or ultraviolet spectroscopic data are not likely to become available for every object in our sample in a foreseeable future, we are forced to adopt this approximation, in particular given the fact that most objects in our sample show only calcium lines. To conclude on this topic, while our approach provides the best atmospheric parameters that are possible to infer with the available data, the solutions for individual objects will always suffer from small intrinsic uncertainties related to our adopted metal abundances.

\subsection{Hydrogen Abundance Measurements}

While for most objects in our sample, the metal-tometal ratio assumed in our analysis has only a modest impact on our atmospheric parameters, the abundance of hydrogen, on the other hand, has a deeper impact because it can be one of the main free electron donors, even when present below the visibility limit. For instance, Bergeron et al. (2019) recently showed that adding undetectable traces of hydrogen in the models had a nonnegligible effect on the mass determination of heliumrich white dwarfs (this is also discussed in Dufour et al. 2005 in a similar context, but with carbon as the main electron donor).

Only 105 of the 1023 (10\%) white dwarfs in our sample show $\mathrm{H} \alpha$ in their spectrum. This is much less than the $25 \%$ containing hydrogen reported by Dufour et al. (2007b) for two main reasons. The first one is that the presence of hydrogen for $7 \%$ of their objects was determined indirectly from the shape of the Ca II $\mathrm{H} \& \mathrm{~K}$ absorption features, in the sense that much better fits to these lines could be achieved when hydrogen was included. However, the study of Dufour et al. was based on Lorentzian profiles, with $\log g$ fixed at 8.0, while here we use the unified line shape theory of Allard et al. (1999), with surface gravities constrained by parallax measurements. As a consequence, we no longer find objects with spectroscopic fits that are significantly improved by adding hydrogen, indicating that the need to add hydrogen in the Dufour et al. analysis was probably only a way to compensate sub-optimal line profiles and/or incorrect surface gravities. The second reason is that since Dufour et al., the proportion of DZ white dwarfs too cool to show hydrogen has increased substantially, thanks to the thorough search for metal-polluted white dwarfs near the main-sequence color space (Koester et al. 2011; Hollands et al. 2017, 2018).

Figure 4 shows the abundance of hydrogen as a function of effective temperature for all the DBZ/DZ(A) white dwarfs in our sample. For stars that do not show $\mathrm{H} \alpha$, we determine the maximum amount of hydrogen that can be added without being detected (the scatter is explained by variations in $\log g$ for each object). While the hydrogen content, and thus its impact on the free electron budget, is well constrained for $T_{\text {eff }} \gtrsim 9000 \mathrm{~K}$, 


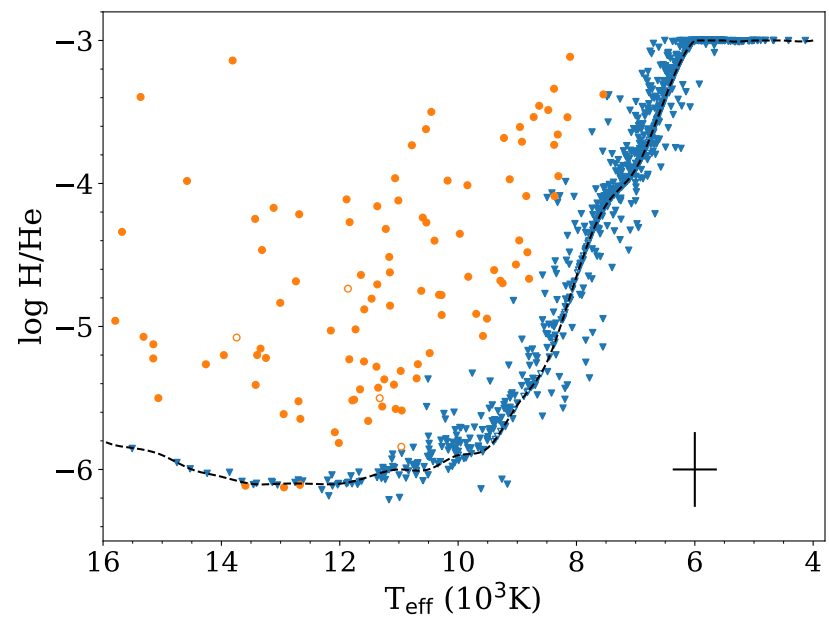

Figure 4. Hydrogen abundance as a function of effective temperature for our sample of $\mathrm{DBZ} / \mathrm{DZ}(\mathrm{A})$ white dwarfs. Objects with parallax measurements are represented with filled symbols, while open symbols are used for stars for which we assumed $\log \mathrm{g}=8$. Orange circles are objects with hydrogen abundances determined from fitting $\mathrm{H} \alpha$, while blue triangles correspond to upper limits. The dashed line represents the visibility limit, defined as an equivalent width of $0.5 \AA$ for $\mathrm{H} \alpha$ at $\log g=8$. The black error bars represent the average uncertainties.

increasingly large quantities of hydrogen can be hidden as the effective temperature decreases. To examine the impact of the unknown amount of hydrogen on our atmospheric parameter determinations, we fit each star in our sample with no detectable $\mathrm{H} \alpha$ with both a hydrogenfree model grid, and with the abundance of hydrogen fixed at the detection limit. The results of this experiment are displayed in Figure 5.

Clearly, masses and effective temperatures are significantly reduced for the coolest stars when hydrogen is included. This reduction in both effective temperature and mass was recently explained by Bergeron et al. (2019, see their Figures 10 and 11). Briefly, adding hydrogen in the model increases the number of free electrons, which in turn increases the $\mathrm{He}^{-}$free-free opacity. This has a quite dramatic effect on the continuum, as can be appreciated from Figure 6, where the energy distribution of two models that differ only by their hydrogen content are shown. As a result of the increased $\mathrm{He}^{-}$free-free opacity, a lower temperature and a larger solid angle are required to match the observed fluxes, which translate into a larger radius, and thus a smaller mass. Note that this effect practically disappears for effective temperatures above $\sim 11,000 \mathrm{~K}$, as the contribution from ionized helium starts to dominate the free electron budget.

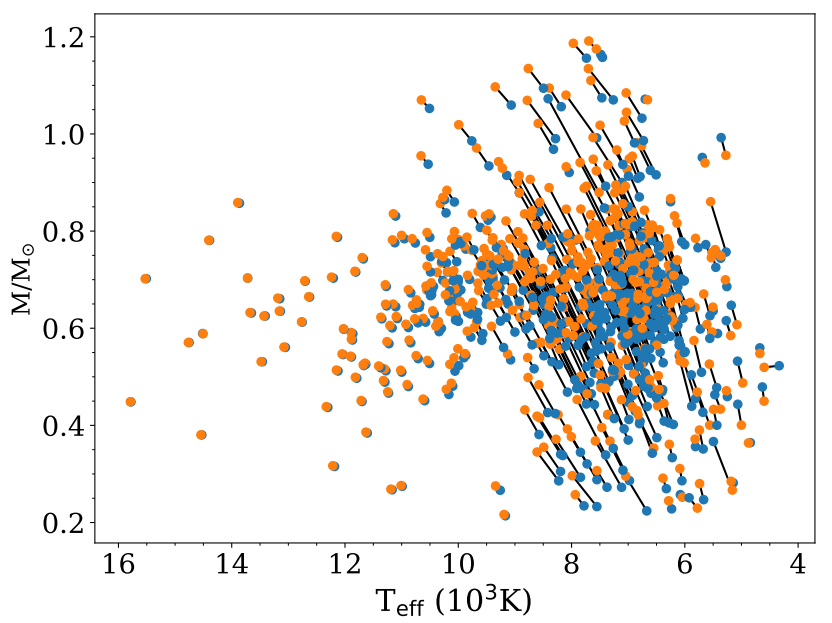

Figure 5. Comparison of masses and effective temperatures obtained with hydrogen-free models (orange dots) and with models where the hydrogen abundance was set at the visibility limit (blue dots). A black line connects the two solutions for each object.

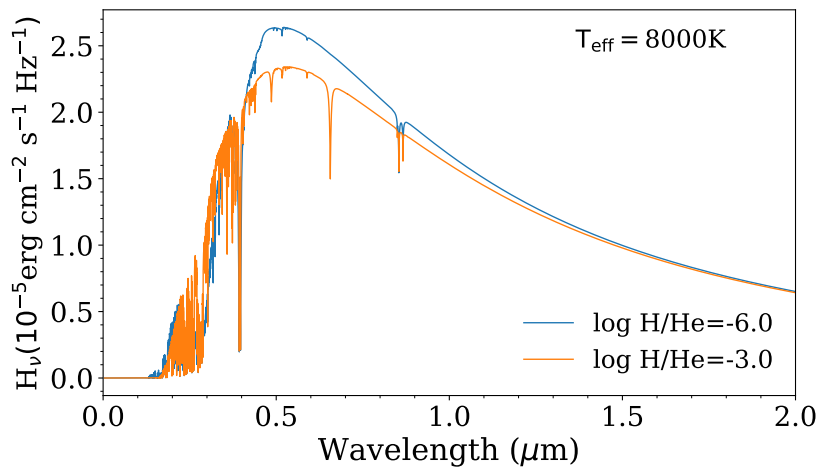

Figure 6. Synthetic spectra (Eddington fluxes) for models at $T_{\text {eff }}=8000 \mathrm{~K}, \log \mathrm{Ca} / \mathrm{He}=-9$, and $\log g=8$, with two different hydrogen abundances indicated in the figure.

\subsection{Mass Distributions}

As discussed above, the parameters we derive depend intimately on the assumed amount of invisible hydrogen present in the star. It is certainly reasonable to expect at least some amount of hydrogen to be present in each object. After all, not only have these white dwarfs been traveling through the interstellar medium for billions of years, but potentially, they also have accreted bodies that may have contained large amounts of water and ice (Klein et al. 2010; Farihi 2011; Farihi et al. 2013; Raddi et al. 2015). While it is impossible to determine exactly the hydrogen abundance in each object, we know this abundance must lie within the two limiting cases explored here. We thus, in what follows, take a deeper look at the consequences this parameter has on the derived 


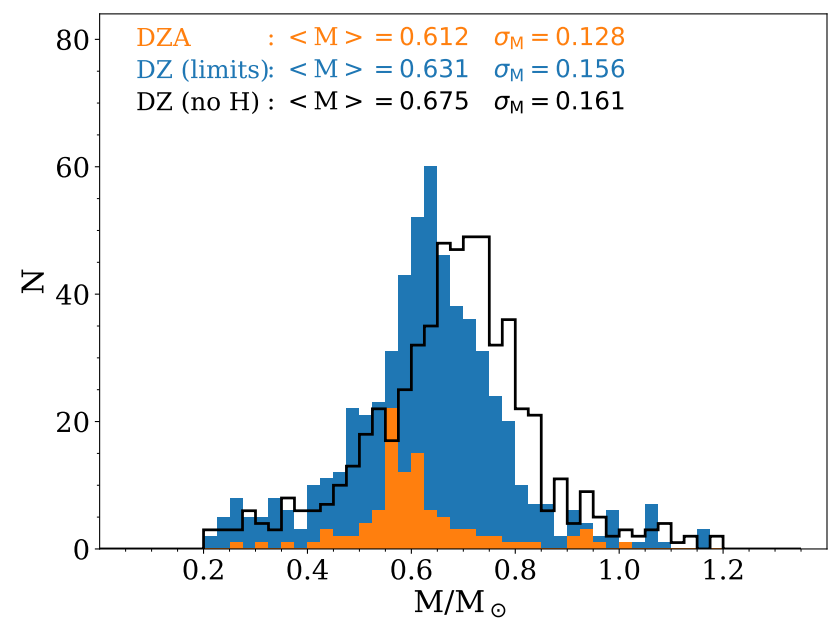

Figure 7. Mass distributions for DZ and DZA white dwarfs in our parallax sample. The DZA stars are shown in orange, while the DZ stars fitted with hydrogen-free models or with the hydrogen abundance set to the visibility limit are shown in black and blue, respectively.

global properties of the sample, and most importantly on the mass distribution.

Figure 7 compares the mass distributions of our parallax sample for stars with hydrogen abundances measured directly from $\mathrm{H} \alpha$ (105 objects) with those with no detectable $\mathrm{H} \alpha$ feature (918 objects) analyzed both with hydrogen-free models and with hydrogen abundances set to the visibility limit. We find that the mean mass for the DBZA/DZA stars is $0.612 M_{\odot}$, very close to the value recently reported by Genest-Beaulieu \& Bergeron (2019) for DA $\left(0.617 M_{\odot}\right)$ and DB $\left(0.620 M_{\odot}\right)$ white dwarfs. However, the mean mass is significantly higher $\left(0.675 M_{\odot}\right)$ for stars without $\mathrm{H} \alpha$ if no hydrogen is included in our models. This indicates that the effective temperatures and masses obtained from hydrogen-free models are probably overestimated, most likely due to the presence of invisible traces of hydrogen in these stars. Adding the maximum amount of hydrogen pushes the mass distribution towards values that now become consistent (mean mass of $0.631 M_{\odot}$ ) with the mean mass of DA and DB white dwarfs, although the peak is still slightly shifted towards higher masses with respect to the DBZA/DZA stars.

Since there is no reason to believe that these objects represent a distinct massive population, this probably means that our effective temperatures and masses are still slightly overestimated for many objects using our approach, most likely due to small deviations of the abundances of some elements with respect to the chondrite values. For example, if the accretion episode responsible for the metal pollution in some of these stars has stopped a long time ago, elements with different masses will start to settle with different timescales (photospheric abundances will be very close to that of the polluting body only during the early phase and steady state phase; Dupuis et al. 1993; Koester 2009), leading to abundances that can depart significantly from our assumed ratios. This will also affect, to a lesser extent, the relative strength of the absorption in the UV. Consequently, the uncertainties on the atmospheric parameters for these stars will be intrinsically larger than the statistical values reported here. Spectroscopic observations in the ultraviolet for every object in our sample would be required for a more accurate analysis, but unfortunately, such data will not become available anytime soon. Note that DZA white dwarfs are less affected by these uncertainties because when hydrogen is present in sufficient quantities to be detectable, it will also be the main free electron donor at the photosphere, diminishing the impact of small variations of heavy element abundance ratios with respect to chondrites.

Even though it is not possible to derive the exact photospheric hydrogen abundance for each object, the fact that the peak of the mass distribution appears more realistic when traces of hydrogen are included suggests that most of these objects probably have abundances close to the detection limit. As will be discussed in Section 7 , this corresponds also approximately to the amount of hydrogen expected if such DZ stars are the results of convectively mixed DA white dwarfs. Consequently, our adopted atmospheric parameters reported in Table 6 are obtained with the hydrogen abundance set to the visibility limit, keeping in mind that the true solution for individual objects may be off by a few hundred degrees and a few hundredth solar mass, depending on their real hydrogen (and also metal) content.

Note finally that objects in Table 6 with $M \lesssim 0.47$ $M_{\odot}$ are most likely unresolved double degenerate binaries, because such low mass white dwarfs would have low mass progenitors on the main sequence, with lifetimes longer than the age of the Milky Way. Such binaries are more luminous, resulting in a larger solid angle for a given distance, and thus a larger radius (lower mass) is inferred when analyzed under the assumption of a single object.

\subsection{Accreted Material}

Figure 8 shows the abundance of calcium as a function of effective temperature for the $1023 \mathrm{DBZ} / \mathrm{DZ}(\mathrm{A})$ white dwarfs in our sample. The two gaps in the distribution reported by Dufour et al. (2007b), namely between 5000 and $6000 \mathrm{~K}$ as well as in the top right corner of the diagram, have been mostly filled, thanks to the re- 


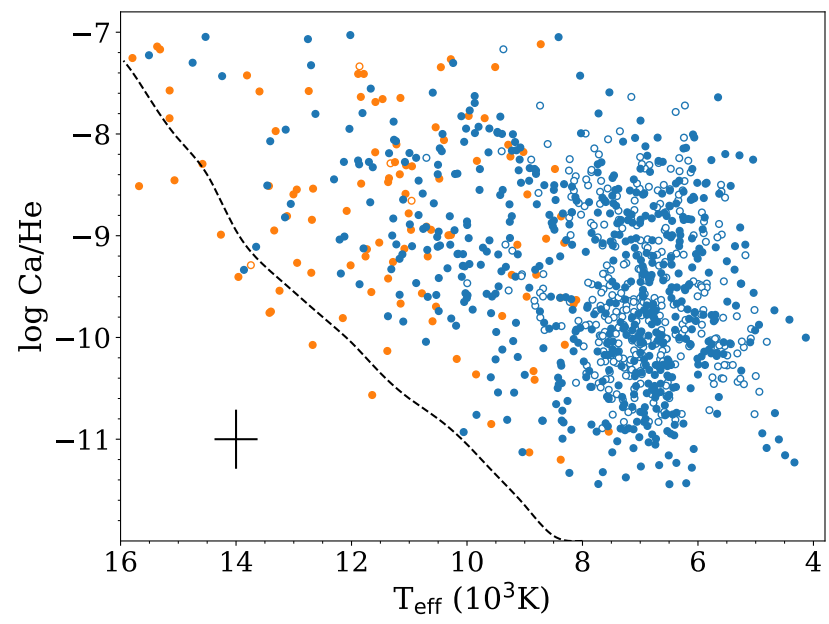

Figure 8. Calcium abundance as a function of effective temperature for all DZ white dwarfs in our sample. The black cross indicates the mean error bar. White dwarfs with a parallax measurement are shown with filled symbols, while $\log g=8$ was assumed for objects with open symbols. Orange circles represent DZA stars with hydrogen abundances determined by fitting $\mathrm{H} \alpha$, and blue circles represent DZ stars with the hydrogen abundance fixed at the detection limit. The dashed line indicates the detection limit of calcium, defined as an equivalent width of $0.5 \AA$ for the Ca II $\mathrm{H}$ line.

cent discovery of many new cool polluted white dwarfs (Kepler et al. 2015, 2016; Kleinman et al. 2013; Koester et al. 2011; Hollands et al. 2017, 2018). The absence of objects at high effective temperatures and low calcium abundances is still present, and is due to the detection limit of $\mathrm{Ca}$ II $\mathrm{H} \& \mathrm{~K}$ lines, as indicated in the figure. At the typical resolution of our spectroscopic observations, stars with such parameters would simply appear as DC white dwarfs below $T_{\text {eff }} \sim 12,000 \mathrm{~K}$, and as DB stars above this temperature.

More interesting than the calcium-to-helium abundance ratio is the total mass of calcium contained in the convection zone of these white dwarfs. Using the derived effective temperature and surface gravity of each object, we can calculate the total mass of the helium convection zone using envelope models similar to those described in Fontaine et al. (2001, see Dufour et al. 2010 for further details) ranging from $T_{\text {eff }}=7000 \mathrm{~K}$ to 30,000 $\mathrm{K}, \log g=7.5$ to 9.0 , and with the ML2 $/ \alpha=1.0$ version of the mixing-length theory (the effect of changing the mixing length is negligible below $T_{\text {eff }} \sim 16,000 \mathrm{~K}$; see Figures 9 and 10 of Rolland et al. 2018). Our results are presented in Figure 9. Note that these values represent only lower limits to the total mass accreted since we do not know how much mass has already diffused at the bottom of the convection zone. Also shown for comparison in Figure 9 is the estimated mass of calcium present

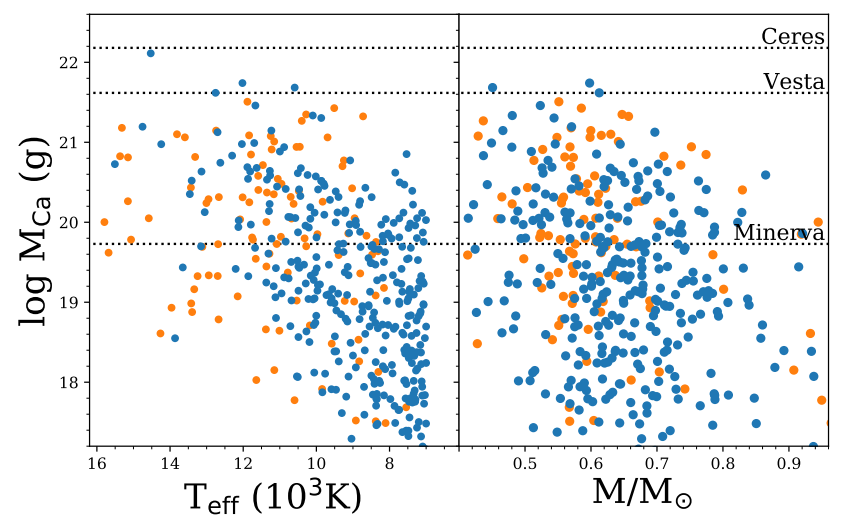

Figure 9. Total mass of calcium in the convection zone as a function of effective temperature (left) and mass (right). Orange circles are objects with hydrogen abundances determined by fitting $\mathrm{H} \alpha$ and blue circles are objects with the hydrogen abundance fixed at the detection limit. We also show the estimated mass of calcium in Ceres, Vesta, and Minerva, assuming that calcium constitutes $1.6 \%$ of their total mass as in bulk Earth (Farihi 2011).

in well-known asteroids in the solar system, assuming that calcium accounts for $1.6 \%$ of the mass of a typical planetesimal (see Farihi 2011). Our results are similar to those presented in Farihi (2011), with masses ranging from $10^{18} \mathrm{~g}$ to $10^{22} \mathrm{~g}$, with possibly smaller mass values at lower effective temperatures (the lack of low calcium mass values at high effective temperatures is simply a visibility limit effect; see Figure 8). Surprisingly, the total mass of calcium remains constant as a function of the white dwarf mass between $0.5 M_{\odot}$ and $0.75 M_{\odot}$, with a possible decrease in more massive white dwarfs, although we are probably dealing here with small number statistics.

\subsection{Main Sequence Progenitors}

It is also possible to estimate the mass of the white dwarf progenitors using the empirical initial-final mass relation (IFMR). While there are many different IFMR published (see Figure 8 of Williams et al. 2009 for a comparison), most of them rely on the observations of star clusters. El-Badry et al. (2018) propose an alternative way of calculating the relation using the Gaia colormagnitude diagram. Their results agree remarkably well with those of Williams et al. (2009), which we decided to use for simplicity. One must keep in mind that this relation includes many systematic errors, one being the possible dependence on initial metallicity (Marigo \& Girardi 2007), information that is lost when evolving to the white dwarf phase. Because of this, it is difficult to determine accurate initial masses of individual objects, but we can obtain a good idea of the distribution. The 


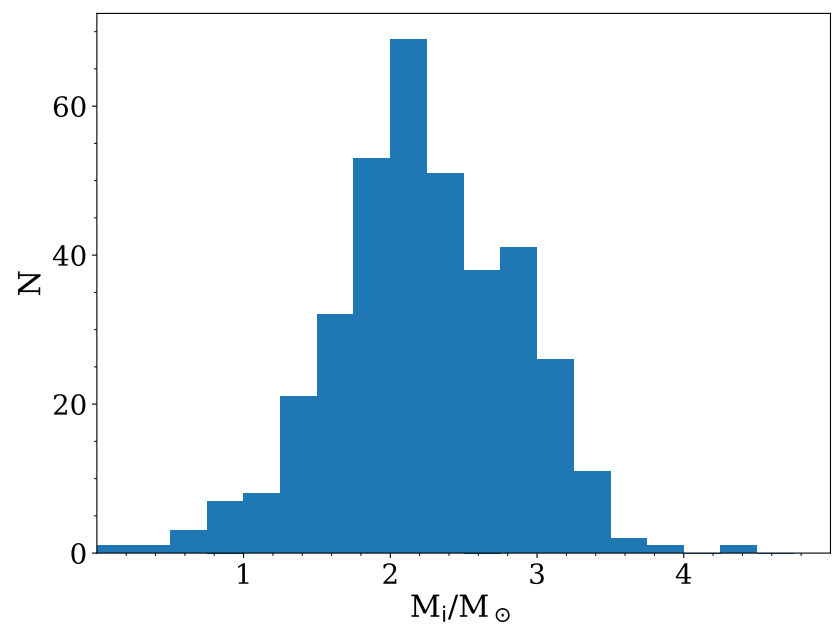

Figure 10. Mass distribution of white dwarf progenitors based on the IMFR of Williams et al. (2009) for the $\mathrm{DBZ} / \mathrm{DZ}(\mathrm{A})$ stars in our sample with $\sigma_{\pi} / \pi<0.1$.

result is shown in Figure 10 for stars with $\sigma_{\pi} / \pi<0.1$. Note that below $M_{i}=1 M_{\odot}$, the results are meaningless, since the white dwarfs in these bins have masses below $0.47 M_{\odot}$, and are most probably unresolved double degenerate binaries (single white dwarfs with such low mass have long main sequence lifetime, and are not expected to have evolved into white dwarfs within the age of the galactic disk yet).

On the basis of the results displayed in Figure 10, we find that 41 white dwarfs had main sequence progenitors with masses above $3 M_{\odot}$, indicating that the formation of rocky objects around massive stars may not be exceptional at all. Note that the Extrasolar Planets Encyclopaedia ${ }^{7}$ reports only 9 exoplanets (out of 3802 exoplanets with the mass of the host star determined) around 8 different stars with masses above $3 M_{\odot}$. The very small number of known planets around massive stars is mostly due to various selection effects, as the method used to find them (transit, radial velocity, direct imaging) are all less efficient around large, bright, and massive main sequence stars. Nevertheless, theoretical models of planet formation predict that planet occurrence around massive stars should be higher, and the presence of rocky material in the photosphere of many polluted white dwarfs with massive main sequence progenitors seems to confirm this hypothesis. However, to properly study planet occurrence and its correlation with stellar mass, an analysis of a large sample of DC and DB white dwarfs would be necessary, preferably for

\footnotetext{
${ }^{7}$ http://exoplanet.eu/
}

a complete-volume sample, something that is outside the scope of this work.

\subsection{Discussion of Individual Objects}

$J 0005+7313$ - The helium lines at 3889 and $4026 \AA$ are deeper than those predicted by the model, indicating that the temperature determined from photometry, $T_{\text {eff }}$ $=12,673 \mathrm{~K}$, is probably underestimated. Using Gaia photometry, we find $T_{\text {eff }}=13,152 \mathrm{~K}$, which is still too cool for a good spectroscopic fit. Bergeron et al. (2011) found $T_{\text {eff }}=14,410 \mathrm{~K}, \log \mathrm{H} / \mathrm{He}=-5.97$, and no metals, using only spectroscopy. The absence of metals is not the explanation, since we obtain $12,715 \mathrm{~K}$ fitting photometry with our metal-free models. Because their technique is independent of photometry, one possible explanation is the reddening due to interstellar absorption. In this work, we use the procedure of Harris et al. (2006), and since the star is only at 34.7 parsecs, no correction for reddening is applied. If we use the procedure of Gentile Fusillo et al. (2019) instead, we need to apply $3.2 \%$ of the maximum absorption along the line of sight, and we now obtain $T_{\text {eff }}=13,622 \mathrm{~K}$, which provides a much better agreement with the spectra. Three dimensional reddening maps made with Gaia should eventually allow better correction for reddening.

$J 0152+2418$ - The Mg I lines at $\sim 3830 \AA$ and the red wing of the $\mathrm{Ca}$ II $\mathrm{H} \& \mathrm{~K}$ lines are not well reproduced by our model. One possible explanation is that the abundance ratios differ significantly from that of chondrites. A deficiency in magnesium (and possibly $\mathrm{Fe}$ and other elements) may explain this discrepancy. More specific adjustments of the various abundances would be necessary to obtain a good fit.

$J 0209+2914, J 1242+0829$, and J1424+5657 - All three white dwarfs have "flattened" Ca II lines, similar to those observed in $\mathrm{J} 1249+6514$, not analyzed here, which has been identified as magnetic by Hollands et al. (2017). The spectra do not show other lines that could be used to detect line splitting. We can only consider the values we found to be approximate, but if the presence of magnetism is confirmed, J1242+0829 would be the hottest known magnetic DZ star with $T_{\text {eff }} \sim 8123 \mathrm{~K}$.

J0302-0108 (GD 40) - Our effective temperature of $T_{\text {eff }}=13,594 \mathrm{~K}$ is much lower than that reported by Voss et al. (2007) $-T_{\text {eff }}=15,316 \mathrm{~K}$ (with $\log g$ fixed at 8 ) - obtained on the basis of metal-free models. They also relied on optical spectra rather than photometry to derive the temperature. Part of this large difference is probably attributable to the lack of heavy elements in their model atmosphere calculations, but since our predicted helium lines are a tiny bit too shallow compared to the observations, it is also possible that our effective 
temperature is slightly underestimated, possibly due to our neglect of any reddening correction given that the distance is only $64 \mathrm{pc}$.

J0555-0410 (LP 658-2) and J2201+0219 - While these objects do not show a clear $\mathrm{H} \alpha$ absorption line, setting the hydrogen abundance at the detection limit leads to completely spurious fits, so we decided to adopt hydrogen-free models for these stars. J0555-0410 was also analyzed in Blouin et al. (2018a) who also found a lower hydrogen abundance of $\log \mathrm{H} / \mathrm{He}<-5$. The difference of $61 \mathrm{~K}$ in $T_{\text {eff }}$ between both temperature estimates can be explained by the different sets of photometry used ( $B V R I+J H K$ instead of Pan-STARRS grizy). Blouin et al. were also able to constrain $\log \mathrm{Mg} / \mathrm{He}$ to -8.66 , a value much higher than the -9.92 we used here on the basis of the chondrite ratio.

$J 0801+5329, J 0842-1347$, and J1428+4403 - These are objects similar to $J 0152+2418$ where the magnesium lines and the shape of the Ca II H\&K lines are not well reproduced. Reducing $\log \mathrm{H} / \mathrm{He}$ improves the fit in one case $(\mathrm{J} 1428+4403)$, but not to the point where the fit is satisfactory. Additional adjustments of individual metal abundances are most probably needed for these objects.

$J 0846+3538$ and $J 1356+4047$ - Both stars seem to have an overabundance of magnesium and sharper than predicted Ca II H\&K lines, as opposed to J0152+2418 and other similar objects discussed above. Again, a fit using a tailor-made grid with different abundance ratios would probably allow a better fit for these objects.

J1214+7822 - It was impossible to obtain a good spectroscopic fit for both the Ca II H\&K lines and the Ca I line at $4226 \AA$ simultaneously. We found the best agreement with $H \& \mathrm{~K}$ by using hydrogen-free models and ignoring the $\mathrm{Ca}$ I line. However, with a mass of only 0.31 $M_{\odot}$, this star is most likely a double degenerate system, and a DZ + DC system could explain the unusually narrow $H \& K$ spectral lines at this temperature. Alternatively, Limoges et al. (2015) suggested that this object could have a hydrogen-rich atmosphere, with a lower atmospheric pressure and thus narrower absorption lines.

J1234+5606 — The SDSS magnitudes for this object are $\sim 0.3$ mag fainter than Pan-STARRS and Gaia, which leads to two different possible solutions. We could not reach a conclusion regarding this discrepancy, but the SDSS colors lead to a much better spectroscopic fit of the helium lines at 5876 and $6678 \AA$, indicating a good estimate of the effective temperature. We thus decided to adopt the SDSS photometric data set.

J2253-0646 (WD 2251-070) - Blouin et al. (2019a) found for this object $T_{\text {eff }}=4170 \pm 90 \mathrm{~K}, \log g=$ $8.06 \pm 0.08$, and $\log \mathrm{Ca} / \mathrm{He}=-9.8 \pm 0.2$ from fitting $B V R I+J H K$ and Pan-STARRS photometry, while we find $T_{\text {eff }}=4132 \pm 53 \mathrm{~K}, \log g=8.03 \pm 0.07$, and $\log \mathrm{Ca} / \mathrm{He}=-10.00 \pm 0.05$. The values are very close, but Blouin et al. (2019a) show a much better fit in their Figure 9. This is not surprising because they relied on improved line profile calculations for the Ca I line at 4226 $\AA$. These improved calculations are important only for objects with $T_{\text {eff }} \lesssim 4500 \mathrm{~K}$, and thus they have little impact on our analysis. Only 4 objects in our sample are in that temperature range; J1636+1619 and J0555-0410 do not show Ca I line, and J1214+7822 has already been discussed above.

\section{DQ WHITE DWARFS}

\subsection{Carbon Abundances}

Following the method described in Section 4, we obtained the atmospheric parameters for all 317 DQ stars in our sample by fitting simultaneously the spectral energy distribution and the carbon features (atomic and/or molecular). Figure 11 shows examples of spectroscopic and photometric fits for typical DQ white dwarfs (all our fits are available in Appendix I, and our final parameters are given in Table 7). The figures show the spectral region used for the fit, i.e. either the Swan bands between 4000 and $6500 \AA$, or the carbon lines between 4500 and $5500 \AA$ for the hotter objects. Note that for some objects with weaker molecular absorption features, we used a smaller region centered on the observed bands to achieve a good fit.

Figure 12 shows the carbon abundance as a function of effective temperature, using a color scale to indicate the mass of each object. Two distinct populations are clearly present: one sequence with "normal" mass $(\sim 0.6$ $\left.M_{\odot}\right)$ DQ white dwarfs (bluish circles) at low effective temperature, and a second sequence (reddish circles) of massive white dwarfs $\left(M \geq 0.8 M_{\odot}\right)$, with carbon abundances increasing with effective temperature. The first sequence follows nicely the expected evolutionary path for $0.6 M_{\odot}$ DQ stars with $\log q(\mathrm{He})=-2.0$ (Dantona \& Mazzitelli 1979, Iben \& MacDonald 1985, Fontaine \& Brassard 2005). Note that in Dufour et al. (2005), the bulk of the stars was following the evolutionary sequence for $\log q(\mathrm{He})$ closer to -3.0 . This is mainly due to the fact that in our improved models, the band strengths with our new line list are slightly higher than with the Zeidler-K.T. \& Koester (1982) prescription, resulting in a systematic downward shift in carbon abundances compared to the values published in Dufour et al. (2005). Note also that the abrupt disappearance of normal mass DQ stars above $\sim 9500 \mathrm{~K}$ is simply due to the visibility limit of carbon at these temperatures; in order to see carbon features in the optical at higher effective temperatures, the abundance must be at least $\log (\mathrm{C} / \mathrm{He})=$ 

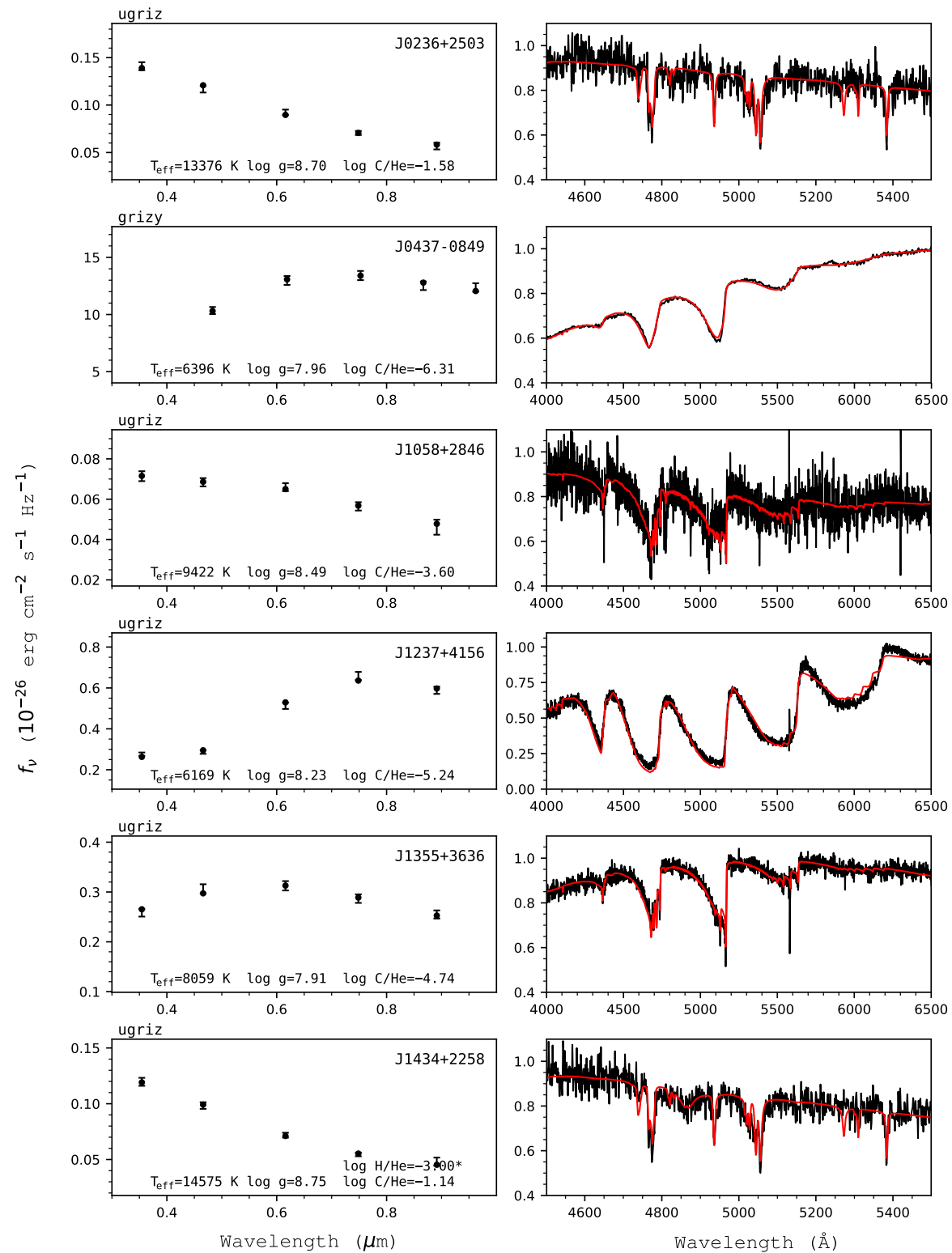

Figure 11. Examples of fits to DQ white dwarfs in our sample. In the left panels, error bars represent the observed data, while filled circles correspond to our best fit model, with the atmospheric parameters given in each panel. The photometry used in the fit is indicated at the top left of each panel. A dagger symbol indicates that the $\log g$ value has been fixed at 8.0, when no trigonometric parallax is available. A star symbol indicates that the value of $\log \mathrm{H} / \mathrm{He}$ has been fixed rather than fitted. The right panels show our spectroscopic fits (red) to the normalized observed spectra (black). The complete version of this figure is available in Appendix I.

-4.5. Unless an ultraviolet spectrum is available, objects following the predicted sequence in that range of effective temperature will simply be classified as DB white dwarfs. Also, the absence of stars below $T_{\text {eff }} \sim 6500 \mathrm{~K}$ is artificial since these were not included in our sample due to the uncertainties in modeling the pressure-shifted Swan bands (the so-called peculiar DQ, or DQpec; see Kowalski 2010, Blouin et al. 2019c).
More importantly, our analysis confirms the presence of a second sequence (Dufour et al. 2005; Koester \& Knist 2006) with an abundance about one dex higher than the bulk of DQ white dwarfs at $M \sim 0.6 M_{\odot}$. It appears that this second sequence is indeed composed of massive white dwarfs, as suggested by Dufour et al. (2005), at least for the hottest ones $\left(T_{\text {eff }} \gtrsim 9000 \mathrm{~K}\right)$. In fact, almost all DQ white dwarfs with an effective 


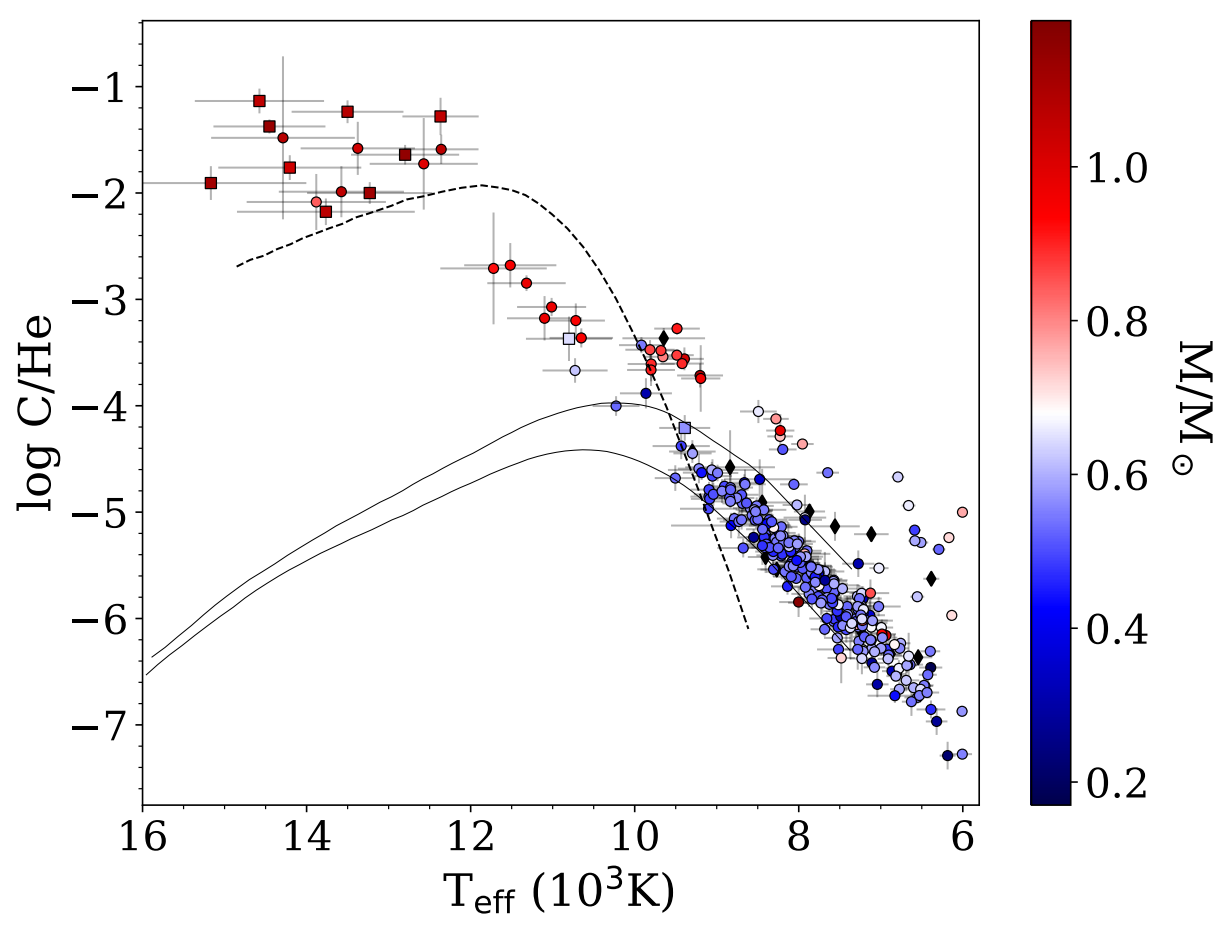

Figure 12. Carbon abundances as a function of effective temperature for DQ white dwarfs using a color scale for the mass of each object (objects without parallax measurements are shown as black diamonds). A square indicates an object fitted with models including a trace of hydrogen $(\log \mathrm{H} / \mathrm{He}=-3$, see text). Solid lines represent evolutionary models from Fontaine \& Brassard (2005) at $0.6 M_{\odot}$ with, from top to bottom, $\log q(\mathrm{He})=-3$ and $\log q(\mathrm{He})=-2$, while the dashed line is for $1.0 M_{\odot}$ and $\log q(\mathrm{He})=-5$. The standard dredge-up scenario struggles to explain the observed abundance pattern for massive objects.

temperature above $10,000 \mathrm{~K}$ have masses higher than $0.8 M_{\odot}$. The coolest objects on the second sequence, however, do not appear to have larger masses. These massive DQ stars will be discussed in greater detail in Section 6.4.

\subsection{Mass Distribution}

The mass distribution of the DQ white dwarfs in our sample, displayed in Figure 13, reveals the two distinct populations very clearly, with the bulk of our sample centered around $0.55 M_{\odot}$, and a second bump centered around $1 M_{\odot}$ where all the hottest DQ stars in our sample are found. As stated before in the case of $\mathrm{DBZ} / \mathrm{DZ}(\mathrm{A})$ white dwarfs, DQ stars with derived masses below $\sim 0.45 M_{\odot}$ are probably unresolved double degenerate binaries. Such low mass stars, if isolated, could not have been formed from single star evolution within the lifetime of the Galaxy. Moreover, these objects are usually interpreted as helium-core white dwarfs whose core mass was truncated by mass transfer with a companion. The presence of carbon in these apparently low-mass DQ stars indicates that at least one component has a carbon core. These binary are thus probably composed of two non-DA stars (e.g., DQ + DC), which

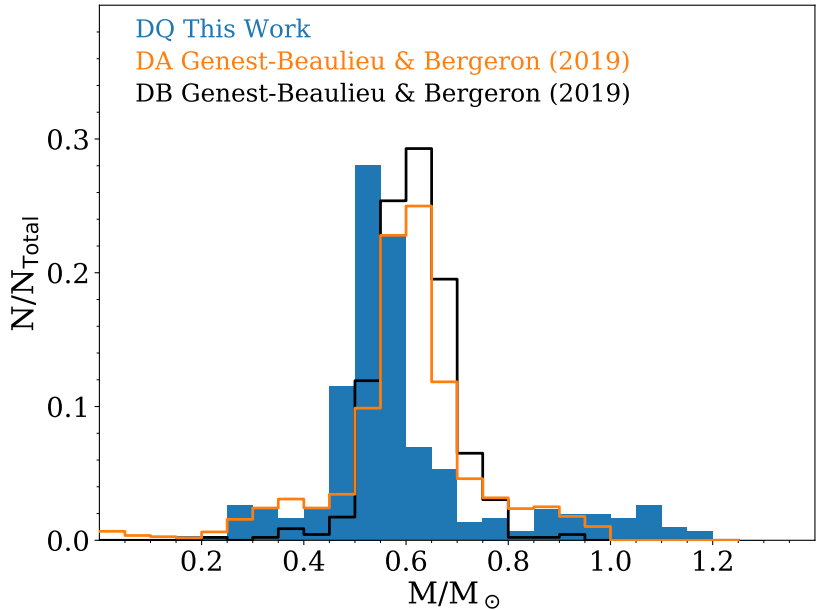

Figure 13. Mass distribution of DQ white dwarfs (blue), compared to the photometric mass distributions of DA and DB white dwarfs (orange and black lines, respectively) taken from Figure 21 of Genest-Beaulieu \& Bergeron (2019).

means that the masses reported here are most likely underestimated.

Surprisingly, the main peak of the mass distribution for the DQ white dwarfs in our sample is shifted by $\sim 0.05 M_{\odot}$ relative to that obtained for the DA and 
DB white dwarfs analyzed by Genest-Beaulieu \& Bergeron (2019, see their Figure 21), raising suspicions about our mass determinations of DQ stars. A way to test the accuracy of our method for the mass determination is to look at the well-known DQ white dwarf Procyon B (J0739+0513), which has a very accurate dynamical mass determination of $0.592 \pm 0.006 M_{\odot}$ (Bond et al. 2015). Using our standard hybrid photometric/spectroscopic approach, we obtain a slightly lower mass of $0.554 \pm 0.013 M_{\odot}$ using HST photometry. This indicates we are probably dealing with a systematic shift in the mass determinations of DQ white dwarfs using our models ${ }^{8}$. Uncertainties related to our dereddening procedure for our $T_{\text {eff }}$ and mass determinations should be irrelevant here given the proximity of Procyon B. In fact, if we take only objects within $100 \mathrm{pc}$, where reddening should be minimal along the line of sight for most stars, we still find that the peak of the mass distribution is too low. In order to identify the origin of this shift in mass, we performed several tests using various model grids where we replaced our treatment of the molecular band opacity with the old just overlapping line approximation (Zeidler-K.T. \& Koester 1982). We also tried various treatments for the line broadening, and included undetectable traces of hydrogen or oxygen as well. In the end, similar mass distributions were always obtained, and we could not pinpoint the exact cause of this discrepancy.

We note, however, that several lines in the ultraviolet have wings that extend several hundreds of angstroms from the line center. This is probably not physically realistic, and the use of the impact approximation is certainly not appropriate for these lines. Unfortunately, the data required to compute line profiles with the unified line shape theory of Allard et al. (1999) are not available for these lines. For stars with available spectroscopic observations in the UV (Holberg et al. 2003) ${ }^{9}$, the use of the quasistatic van der Waals broadening (Walkup et al. 1984, D. Koester, private communication) provides a good fit to the asymmetric C I $1930 \AA$ line, although the carbon abundance needed to reproduce the observations is different than that determined from the Swan bands. This problem is not new (see Dufour 2011, for a detailed discussion), and the origin of this discrepancy still remains mysterious to this day, although it is most likely related to the uncertainties of the ultraviolet opacities. In fact, for DQ stars in the intermediate temperature regime where both atomic lines and molecular

\footnotetext{
8 Note that a recent study by Koester \& Kepler (2019) also finds a DQ mass distribution peaking near $0.55 M_{\odot}$.

${ }^{9}$ http://vega.lpl.arizona.edu/newsips/low/
}

bands are present ( 9500 to $11,000 \mathrm{~K})$, the models also have difficulties reproducing both types of absorption features simultaneously. Examples of this problem are illustrated in Figure 14. While it is possible to obtain a good spectroscopic fit by increasing the effective temperature and carbon abundance for these objects, doing so would then produce an energy distribution completely at odds with the photometric observations (see right panel of Figure 14). A similar problem, based on an analysis using D. Koester's model atmosphere code, was also reported by Gänsicke et al. (2010) for two DQ stars showing traces of oxygen. For these problematic DQ stars in our sample, we compare in Table 3 the results of our standard approach with the parameters obtained by fitting only the optical spectra, thus ignoring all photometric information. We note that even though the exact atmospheric parameters are somewhat uncertain because of this problem, the massive nature $(M>0.8$ $M_{\odot}$ ) of these objects remains unquestionable, even when allowing for a conservative uncertainty of $0.15 M_{\odot}$.

While the problems mentioned above become apparent only for stars that show both atomic and molecular lines, it is likely, however, that the whole temperature, abundance, and mass scales for DQ white dwarfs are affected, which could perhaps also explain the $\sim 0.05 M_{\odot}$ shift of the peak of the DQ mass distribution relative to DA and DB white dwarfs. Until this problem is solved, the absolute values of the atmospheric parameters for all DQ white dwarfs should be considered uncertain, although the relative values between objects in the sample are probably reliable.

\subsection{The Effects of Hydrogen}

The presence of hydrogen is a rare phenomenon in DQ white dwarfs (see Dufour 2011). Hydrogen abundance measurements are reported only for two relatively hot and massive DQ stars in the literature, namely G3526 (Thejll et al. 1990) and G227-5 (Wegner \& Koester 1985), while its presence is also inferred from the detection of a $\mathrm{CH}$ molecular band in two other objects - G9937 and GJ 841B, both magnetic white dwarfs (Blouin et al. 2019c; Vornanen et al. 2010). Our sample of cool DQ white dwarfs with molecular carbon bands contains only six objects showing $\mathrm{H} \alpha$, three of which have masses below $0.47 M_{\odot}$, indicating they are most probably unresolved double degenerate binaries composed of a DQ and a DA white dwarf; these are listed in Table 4. Leggett et al. (2018) also identified two such DQ+DA unresolved binaries (see their Figure 7). Except for the hotter DQ $\mathrm{J} 1243+1651$, the other objects have an $\mathrm{H} \alpha$ line that is too narrow to be reproduced by helium-rich models, and we believe they are also DQ + DA binaries. Hence, of 


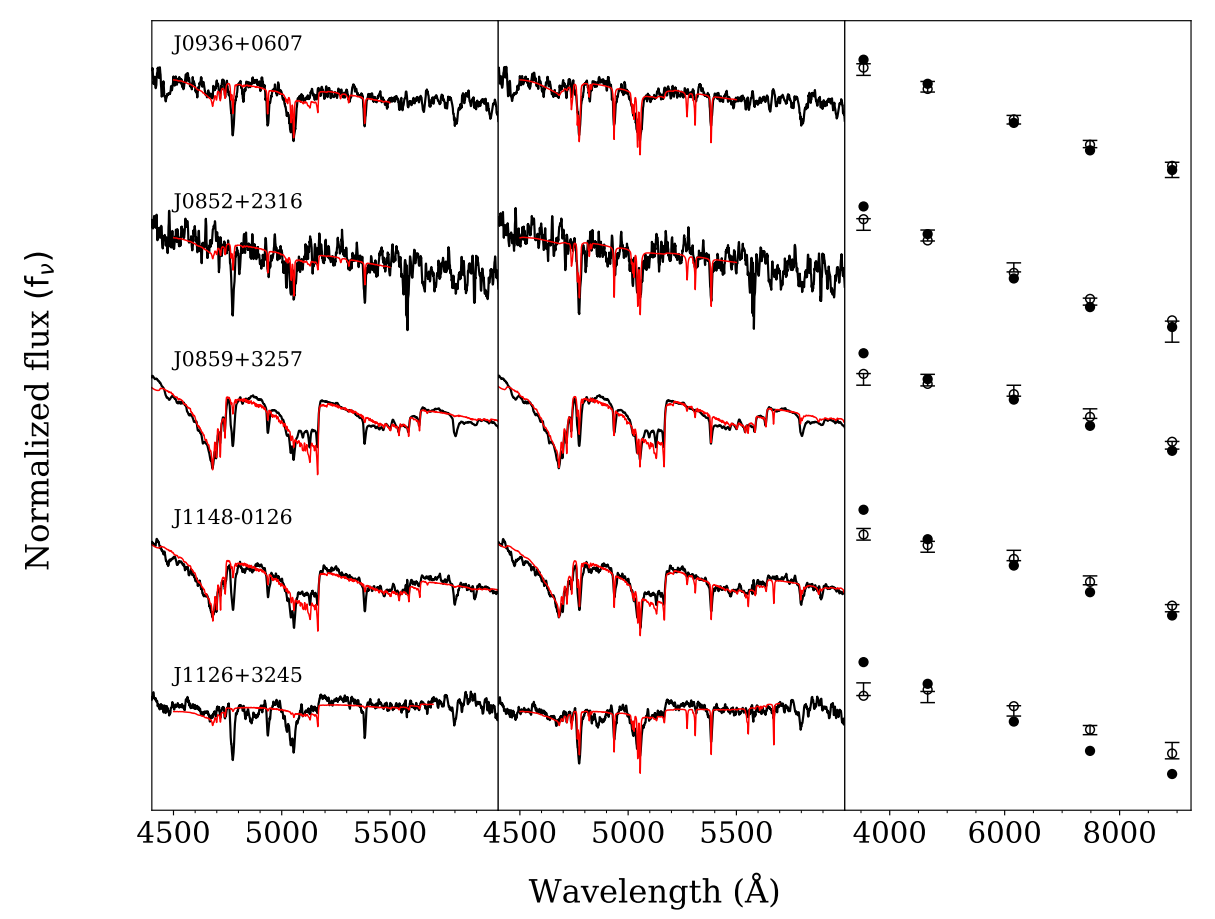

Figure 14. Left panel: best solutions when the effective temperature is obtained from fits to photometric data. Middle panel: best solutions when fitting only the optical spectra. Right panel: corresponding energy distributions for the two solutions. Open circles represent the best photometric/spectroscopic solutions (our standard approach), while filled circles correspond to the solutions with the effective temperature and carbon abundance determined solely from the optical spectra.

Table 3. Parameters of DQ white dwarfs with bad spectroscopic fits when the effective temperature is derived from photometry

\begin{tabular}{c|cccccc}
\hline & \multicolumn{3}{|c}{ Photometric fits } & \multicolumn{3}{c}{ Spectroscopic fits } \\
Name & $T_{\text {eff }}(\mathrm{K})$ & $\log \mathrm{C} / \mathrm{He}$ & $M / M_{\odot}$ & $T_{\text {eff }}(\mathrm{K})$ & $\log \mathrm{C} / \mathrm{He}$ & $M / M_{\odot}$ \\
\hline $\mathrm{J} 0852+2316$ & 11099 & -3.18 & 0.97 & 12733 & -1.99 & 1.12 \\
$\mathrm{~J} 0859+3257$ & 9486 & -3.52 & 0.87 & 10407 & -2.87 & 0.97 \\
$\mathrm{~J} 0936+0607$ & 11013 & -3.07 & 0.97 & 12109 & -1.99 & 1.09 \\
$\mathrm{~J} 1000+1005$ & 7869 & -4.99 & & 10395 & -2.87 & \\
$\mathrm{~J} 1126+3245$ & 9297 & -4.43 & & 11369 & -2.73 & \\
$\mathrm{~J} 1140+0735$ & 10651 & -3.36 & 0.94 & 12395 & -1.60 & 1.12 \\
$\mathrm{~J} 1140+1824$ & 9656 & -3.54 & 0.81 & 10921 & -2.62 & 0.94 \\
$\mathrm{~J} 1148-0126$ & 9680 & -3.48 & 0.88 & 10868 & -2.62 & 1.00 \\
$\mathrm{~J} 2248+2826$ & 9390 & -4.21 & 0.57 & 11920 & -2.02 & 0.90 \\
\hline
\end{tabular}

the 293 DQ white dwarfs with temperatures between $\sim 6500 \mathrm{~K}$ and $10,500 \mathrm{~K}$ in our sample, there is compelling evidence for the presence of hydrogen in only two objects, both hotter than $9000 \mathrm{~K}$.

As mentioned in Section 5.1, undetectable traces of hydrogen can still have an impact on the atmospheric parameter determination of helium-atmosphere white dwarfs, although in the case of DQ white dwarfs, the contribution of free electrons from carbon is usually much more important than that of heavy elements in DZ stars. To test the effect of hydrogen on the determination of the atmospheric parameters of cool DQ white dwarfs, we generated a small grid of models with $\log \mathrm{H} / \mathrm{He}=-3$, and refitted every object in our sample 
Table 4. DQ white dwarfs showing hydrogen lines and Swan bands

\begin{tabular}{c|cc}
\hline Name & $T_{\text {eff }}(\mathrm{K})$ & $\mathrm{M} / M_{\odot}$ \\
\hline $\mathrm{J} 0836+0437$ & 8420 & 0.46 \\
$\mathrm{~J} 0928+2638$ & 7108 & 0.28 \\
$\mathrm{~J} 0950+3238$ & 8268 & \\
$\mathrm{~J} 1243+1651$ & 10227 & 0.53 \\
$\mathrm{~J} 1406+3402$ & 7042 & 0.30 \\
$\mathrm{~J} 2310-0057$ & 7647 & 0.54 \\
\hline
\end{tabular}

with $T_{\text {eff }}<10,000 \mathrm{~K}$. We find that by assuming such a hydrogen abundance, we could produce $\mathrm{H} \alpha$ and $\mathrm{H} \beta$ absorption features that would be easily detected above $8000 \mathrm{~K}$, as well as a $\mathrm{CH}$ molecular band near $4300 \AA$ for objects cooler than $\sim 8500 \mathrm{~K}$. Even by assuming an abundance that is clearly ruled out by the spectroscopic observations, we find that the presence of hydrogen has only a marginal impact on our atmospheric parameters, increasing the derived effective temperatures by $150 \mathrm{~K}$ and masses by $0.035 M_{\odot}$, on average. We can thus safely consider that the presence of undetectable traces of hydrogen does not affect significantly the parameters of cool DQ stars.

In the atomic lines regime $\left(T_{\text {eff }} \gtrsim 10,000 \mathrm{~K}\right)$, however, spectroscopic observations tell a different story. Although $\mathrm{H} \alpha$ is blended with the carbon lines near 6588 $\AA, \mathrm{H} \beta$ can be detected very clearly. These lines are observed in $40 \%$ of the objects $(10 / 25)$. We thus generated additional grids with $\log \mathrm{H} / \mathrm{He}=-2,-3$, and -4 , with $T_{\text {eff }}$ ranging from $10,000 \mathrm{~K}$ to $16,000 \mathrm{~K}$, We found that, surprisingly, the $\log \mathrm{H} / \mathrm{He}=-3$ grid best reproduces the observations for almost every star, suggesting that the hydrogen abundance appears relatively constant in the objects where hydrogen is detected. Therefore, the solutions with $\log \mathrm{H} / \mathrm{He}=-3$ are presented in Table 7 when the Balmer lines are observed. Note that it is possible that DQ stars with no detectable hydrogen features also contain some traces of hydrogen, since the Balmer lines are barely predicted for some objects with higher carbon abundance and lower effective temperature. The presence of hydrogen in these objects has a marginal impact on the measured effective temperature $(265 \mathrm{~K}$ on average) and mass $\left(0.032 M_{\odot}\right.$ on average), but the effect can be quite significant for the carbon abundance (around 0.5 dex in some cases). Since we do not know the exact hydrogen abundance for these objects, we provide the solutions without hydrogen in Table 7.

\subsection{Massive DQ White Dwarfs}

We now turn our attention to the population of massive $\left(M>0.8 M_{\odot}\right)$ DQ white dwarfs clearly distinguishable in Figures 12 and 13. First, the carbon abundance pattern observed for these objects indicates that they followed a different evolutionary path than their cooler normal mass counterparts, since the standard dredgeup prediction for massive white dwarfs completely fails to explain their chemical composition (see also Brassard et al. 2007). Second, such massive DQ stars could not have DB white dwarfs as progenitor because practically no massive helium-rich white dwarfs exist at higher effective temperatures (Bergeron et al. 2011; GenestBeaulieu \& Bergeron 2019; Beauchamp et al. 1996). Instead, the massive sequence connects nicely with the carbon-dominated atmosphere white dwarfs at higher effective temperature, the so-called Hot DQ stars $\left(T_{\text {eff }}\right.$ $\geq 18,000 \mathrm{~K}$ and $\log (\mathrm{C} / \mathrm{He}) \geq 0$, see Dufour et al. 2007a, 2008, 2013). Note that Gaia trigonometric parallax measurements confirm that the carbon-dominated atmosphere white dwarfs are massive as well (Dunlap et al., submitted). We thus believe that our massive DQ stars represent cooler versions of the carbon-atmosphere white dwarfs.

The many unusual properties of Hot DQ white dwarfs - unique chemical composition, high mass, and high incidence of magnetism - recently prompted Dunlap \& Clemens (2015) to propose that these hot carbonatmosphere white dwarfs represent a population of merged white dwarfs (failed type Ia supernovae). The key piece of evidence for this proposed scenario comes from the kinematic properties of the sample, which provide an independent age indicator (Dunlap \& Clemens 2015). Indeed, one interesting characteristic that stands out about the Hot DQ population is their very high tangential velocity, as a group, compared to other white dwarfs with the same age and mass. Over time, a population of stars is kinematically heated through gravitational interactions (Wegg \& Phinney 2012). Hence, as a population gets older, its velocity dispersion increases. In particular, Dunlap \& Clemens showed that there is a discrepancy between the young age inferred from the derived atmospheric parameters of carbon-atmosphere white dwarfs ${ }^{10}$, and the old age derived from the velocity dispersion. The merger scenario provides an elegant solution to this dilemma because the reheating induced by the merging event resets the cooling age clock, and consequently, the cooling age derived from the effective temperature and mass thus becomes meaningless. If the

\footnotetext{
10 Such hot massive white dwarfs should descend from massive, short-live main-sequence progenitors, and they should thus have a small total age if they evolved as single stars.
} 


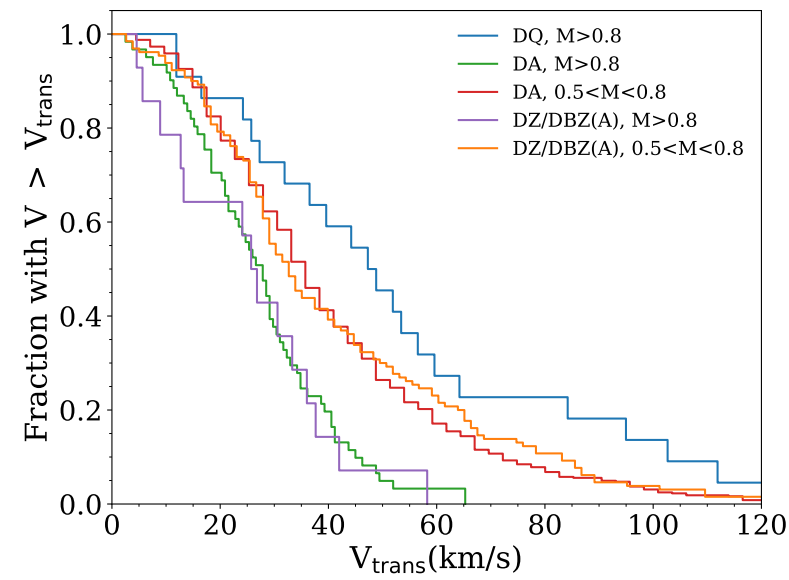

Figure 15. Cumulative distribution of transverse velocities for massive DQ, DBZ/DZ(A) (this paper) and DA (GenestBeaulieu \& Bergeron 2019) white dwarfs with $T_{\text {eff }}$ between 10,000 and $16,000 \mathrm{~K}$.

massive DQ white dwarfs observed in Figures 12 and 13 are indeed cooled down versions of the Hot DQ stars, a kinematic analysis of the sample should then show the same discrepancy between the two age indicators.

We show in Figure 15 the cumulative distribution of transverse velocities, $V_{\text {trans }}$, for our sample of massive DQ white dwarfs, calculated using Gaia distances and proper motions. We selected DQ white dwarfs with $T_{\text {eff }}>10,000 \mathrm{~K}$ and $M>0.8 M_{\odot}$, a point where there appears to be a clean separation between the two DQ populations. Since there is no DQ white dwarf with "normal" masses in this temperature range, we compare the properties of the massive DQ stars with those of our sample of DBZ/DZ(A) as well as DA white dwarfs with signal-to-noise ratio higher than 25 taken from GenestBeaulieu \& Bergeron (2019), a large and clean sample perfectly suited for this comparison. Also, in order to make a meaningful comparison, we selected only $\mathrm{DBZ} / \mathrm{DZ}(\mathrm{A})$ and DA white dwarfs in the same range of effective temperature where the massive DQ stars are found, i.e. between $10,000 \mathrm{~K}$ and 16,000 K, and we compare the distributions of transverse velocities for stars with masses greater than $0.8 M_{\odot}$ and in the $0.5-0.8$ $M_{\odot}$ mass range.

First, we notice that the transverse velocity distribution for $\mathrm{DBZ} / \mathrm{DZ}(\mathrm{A})$ and $\mathrm{DA}$ white dwarfs are very similar, and that those with $M>0.8 M_{\odot}$ are much less dispersed than those with $0.5<M<0.8 M_{\odot}$. This is exactly what we expect, since massive white dwarfs have massive progenitors with shorter main sequence lifetimes, corresponding to a shorter total age, and thus a smaller velocity dispersion (Wegg \& Phinney
2012). However, the velocity dispersion for the massive DQ stars displayed in Figure 15 is extremely broad, in sharp contrast with what is expected for a population of massive white dwarfs. We find indeed that $45 \%$ (10 out of 22) of the massive $\left(M>0.8 M_{\odot}\right)$ DQ population has $V_{\text {trans }}>50 \mathrm{~km} \mathrm{~s}^{-1}$, while the fractions are only $5 \%$ (3 out of 61 ) and $7 \%$ (1 out of 14) for DA and DBZ/DZ(A) with similar atmospheric parameters, respectively. The fact that the distribution is even larger than that of stars in the $0.5-0.8 M_{\odot}$ mass range strongly supports the idea that these objects are the descendants of the carbon-dominated atmosphere white dwarfs (the Hot DQ stars), and consequently, that they represent the outcome of the merging of two white dwarfs.

It is interesting to note, however, that very few massive DQ stars are found with a large magnetic field $(\mathrm{J} 1040+0635$ and $\mathrm{J} 1036+6522$ are the only two examples, Williams et al. 2013), in contrast with the very large fraction of magnetic Hot DQ white dwarfs (at least $70 \%$ are magnetic at some level, Dufour et al. 2013). Magnetic fields of a few MG would be easily detectable through line splitting in our spectra. It thus seems that as helium finds its way to the top of the photosphere, the strength of the magnetic field is also reduced significantly, as these stars cool down. If our hypothesis that the Hot DQ stars represent the progenitors of the massive DQ in our sample is correct, then the latter are probably still magnetic at some level. Unfortunately, the SDSS spectra used in our analysis lack the signal-tonoise ratio and spectral resolution required to properly identify weaker magnetic fields of a few $100 \mathrm{kG}$. We predict that a large fraction of these objects will eventually show magnetism when high-resolution spectroscopic observations become available. Also, at least one third of the Hot DQ stars are variable (Montgomery et al. 2008; Barlow et al. 2008; Dunlap et al. 2010; Lawrie et al. 2013; Dufour et al. 2011), presumably due to the presence of magnetic spots at the surface of a rapidly rotating star. If magnetism is indeed still present at some level, a large fraction of the massive DQ stars should also be variable.

Finally, we notice that there appears to be a correlation between the mass and the effective temperature, the hottest objects near 16,000 K being significantly more massive $\left(\sim 0.3 M_{\odot}\right)$ than their cooler counterparts near $10,000 \mathrm{~K}$. As it is expected that white dwarfs should evolve at constant masses, we first believed that some missing ingredient in our models was responsible for either an overestimation of the masses of the hottest stars, or an underestimation for the cooler ones. We experimented with many test grids incorporating traces of oxygen, hydrogen, as well as different treatments of line broadening, but none of these experiments affected the 
relative masses of our object in a significant way. As mentioned in Section 6.2, we know there are some uncertainties concerning the absolute values of the atmospheric parameters of DQ white dwarfs that may explain the shifted position of the peak of the mass distribution and the difficulties in reproducing simultaneously the atomic and molecular features. However, it is unlikely that the shortcomings of our models translate into uncertainties on the masses much larger than $0.1 M_{\odot}$ (the radius of the star is tightly constrained from the measured energy distribution and distance), not enough to explain the clear correlation with effective temperature that we observe in our sample. We thus believe that the observed correlation is real and that it is most probably the manifestation of an observational bias due to the crystallization of the core of these white dwarfs (see next section).

\subsection{The Crystallization Sequence}

As a white dwarf cools off, thermal energy is gradually lost from the star in the form of radiation, until the kinetic motions of the ions lose amplitude and eventually become correlated. The ionic state then evolves from a gas to a fluid to a solid, a process referred to as crystallization (Fontaine et al. 2001). This liquid-tosolid transition, which begins in the center of the stellar core and slowly moves outwards, represents a first-order phase transition, and is thus accompanied by a release of latent heat, which contributes to slowing down the cooling process significantly. Over 50 years ago, van Horn (1968) predicted that this crystallization process would cause a decrease of the cooling rate during the transition, and would cause a pile-up of objects that could be detected in HR diagrams. The first direct observational evidence of this crystallization process was reported by Tremblay et al. (2019), in the form of a characteristic pile-up of white dwarfs forming a tight sequence in the Gaia $\mathrm{M}_{G}$ versus $G_{\mathrm{BP}}-G_{\mathrm{RP}} \mathrm{HR}$ diagram, the so-called "Q branch" (see their Figure 2).

Our second, more massive sequence of DQ white dwarfs is particularly interesting in the context of crystallization, because for $\sim 0.6 M_{\odot}$ white dwarfs, crystallization occurs at the same time as another physical process referred to as convective coupling, which takes place when the base of the superficial convection zone reaches into the degenerate interior (Fontaine et al. 2001). Convective coupling also decreases the cooling process temporarily, and in $\sim 0.6 M_{\odot}$ white dwarfs, the effects of crystallization and convective coupling cannot be disentangled. Fortunately, crystallization occurs at much higher effective temperature for more massive white dwarfs, making the massive sequence of DQ white

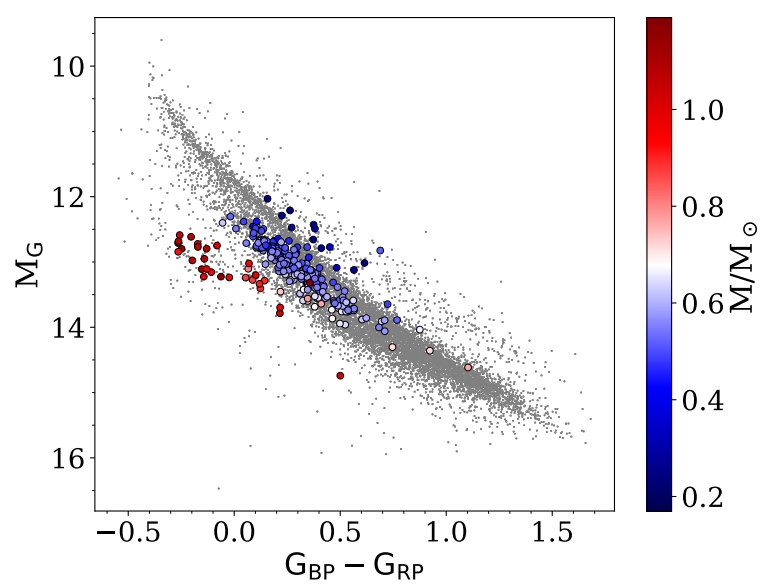

Figure 16. Absolute $G$ magnitudes as a function of $G_{\mathrm{BP}}-$ $G_{\mathrm{RP}}$. Filled circles represent DQ stars with $\sigma_{\pi} / \pi<0.1$, using a color scale for the mass of each object. Black dots are white dwarf candidates within 100 pc selected from Gaia DR2 using the cuts proposed in Appendix B of Gaia Collaboration et al. (2018).

dwarfs ideal objects to examine the crystallization process.

Figure 16 shows the color-magnitude diagram for our sample of DQ white dwarfs with measured parallaxes and $\sigma_{\pi} / \pi<0.1$. The lowest red point in this diagram is $\mathrm{J} 0841+3329$, for which the Gaia phot_bp_rp_excess_factor value is 1.34 , indicating possibly a bad quality of magnitudes according to Gaia Collaboration et al. (2018). Our best fit based on SDSS photometry predicts a lower magnitude in the $G$ bandpass. By removing this object, all our massive DQ stars fall in the region of the crystallization sequence reported by Tremblay et al. (2019).

Figure 17 shows the masses as a function of effective temperature for the same sample of DQ stars, together with the sample of DA white dwarfs analyzed by Bergeron et al. (2019, see their Figure 14). The black solid lines are also described at length in Bergeron et al. Briefly, the left line corresponds to the onset of crystallization at the center of an evolving model (the same as in Tremblay et al. 2019), while the right line indicates the location where $80 \%$ of the total mass of the star has solidified. Upon crystallization, latent heat is slowly released, and the white dwarf cooling is slowed down, a process that is well illustrated by the tightening of the isochrones (shown in red) between the two black lines. Figure 17 reveals that most of our massive DQ stars fall within these lines, indicating a possible pile-up due to crystallization. 


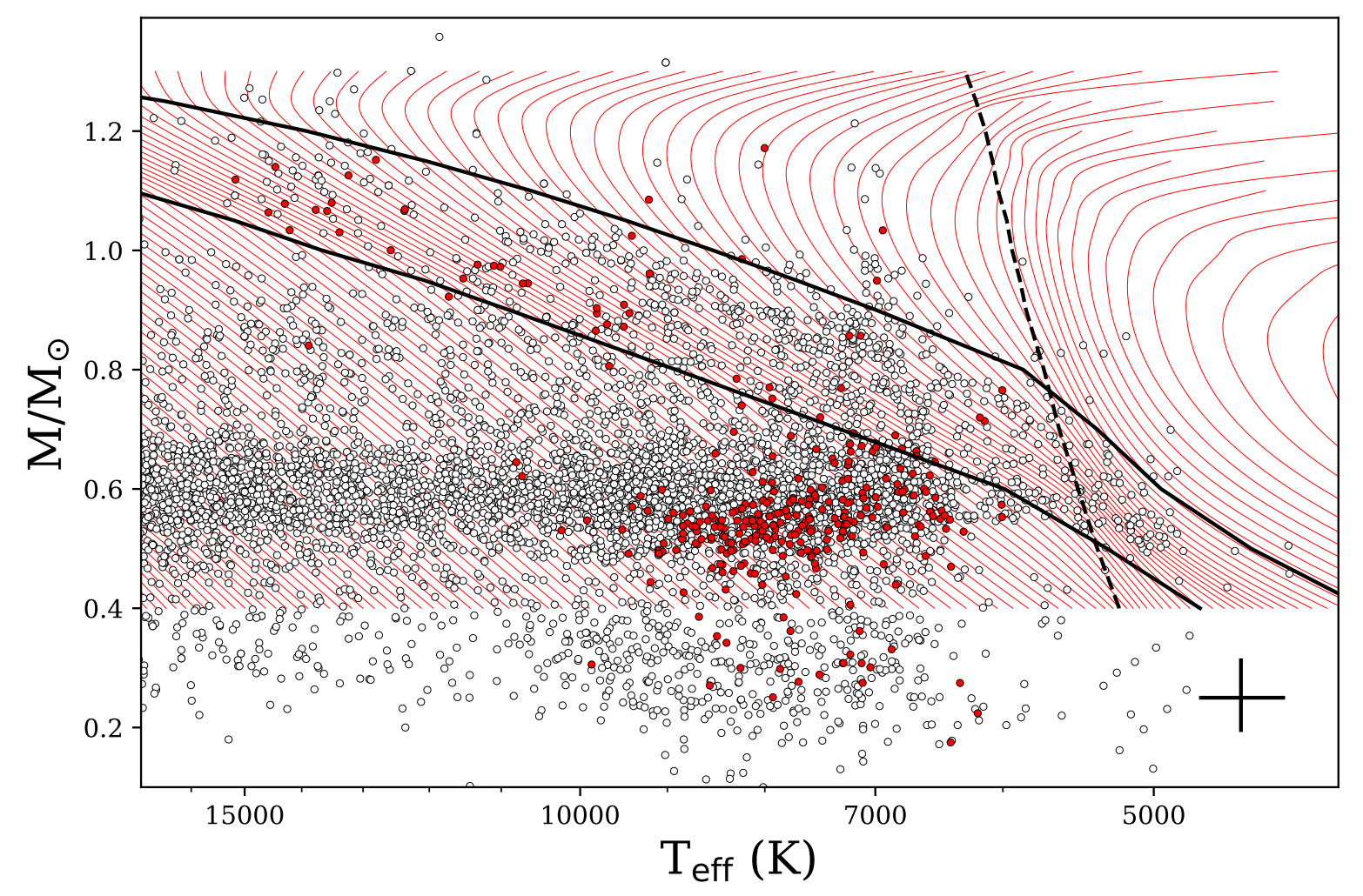

Figure 17. Mass as a function of $T_{\text {eff }}$ for the DQ white dwarfs in our sample (red dots), and DA white dwarfs spectroscopically identified in the MWDD with $\sigma_{\pi} / \pi<0.1$ (white dots), taken from Figure 14 of Bergeron et al. (2019). Also reproduced are their theoretical isochrones obtained from cooling sequences with $\mathrm{C} / \mathrm{O}$-core compositions, $q(\mathrm{He})=10^{-2}$, and $q(\mathrm{H})=10^{-4}$, equally spaced by $\Delta \log \tau_{\text {cool }}=0.02$ (in years). The lower black solid curve indicates the onset of crystallization at the center of evolving models, while the upper one indicates the location where $80 \%$ of the total mass has solidified. The dashed curve indicates the onset of convective coupling. The black cross corresponds to the mean errors of each fitted parameter.

Figure 18 shows the masses of $\mathrm{DBZ} / \mathrm{DZ}(\mathrm{A})$ white dwarfs in our sample as a function of effective temperature together with the same isochrones and crystallization sequences as before. The signature of crystallization cannot be seen, but this could be due to the fact that there are much less massive stars above $T_{\text {eff }}=12,000$ $\mathrm{K}$ which is consistent with the idea that $\mathrm{DBZ}(\mathrm{A})$ white dwarfs belong to the same population as $\mathrm{DB}(\mathrm{A})$ stars. At high temperatures, it is also more difficult to see metal absorption lines (see the detection limit in Figure 8) and the diagram is thus much less populated in that range.

\subsection{Discussion of Individual Objects}

$J 0901+5751, J 0922+2928$, and J1423+5729 - These stars show many O I lines, especially at 6156 and $7772 \AA$, and at 5330 and $5435 \AA$ in the case of J1423+5729. For J0922 +2928 , Gänsicke et al. (2010) found $T_{\text {eff }}=8270 \mathrm{~K}$ and $\log \mathrm{C} / \mathrm{He}=-2.6$ using oxygen-rich models, while we found $T_{\text {eff }}=8022 \mathrm{~K}$ and $\log \mathrm{C} / \mathrm{He}=-4.93$. They also found $\log \mathrm{H} / \mathrm{He}<-5.0$ and $\log \mathrm{O} / \mathrm{He}=-2.0$, which implies that oxygen is more abundant than carbon. White dwarfs with such a high amount of oxygen in their atmosphere must have followed a different evolutionary path, and the authors suggest that they could be $\mathrm{O} / \mathrm{Ne}$-core white dwarfs surrounded by a layer of carbon and oxygen. Such white dwarfs would be massive. According to our results, only one of these can be qualified as massive, but we determined the mass using evolutionary models with $\mathrm{C} / \mathrm{O}$ cores and did not take atmospheric oxygen into account in our analysis. However, if we take the values of Gänsicke et al. (2010) for J0922+2928, all these objects fall above the first sequence in Figure 12, suggesting indeed a different evolutionary path. We also note that these objects are rare and have little impact on the conclusions of this work.

$J 1040+0635$ - This star shows magnetic splitting, suggesting that our solution may be uncertain. To evaluate the influence of the magnetic field on our result, 


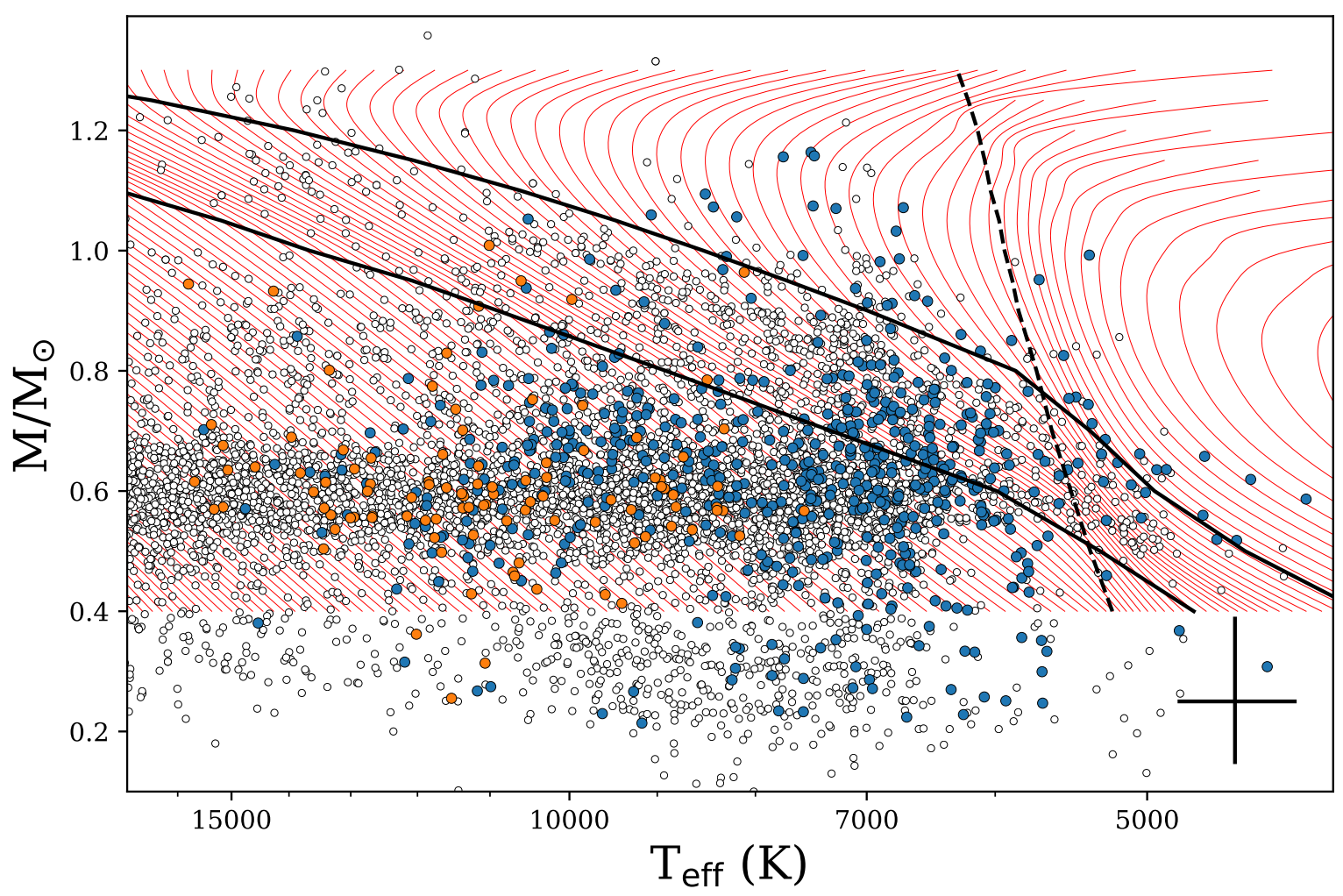

Figure 18. Same as Figure 17 but for DZ/DBZ white dwarfs. Blue circles correspond to DZ/DBZ stars, while orange circles represent DZA/DBZA stars.

we fitted the magnetic white dwarf J1036+6522 (not included in this analysis), which shows similar line splitting. We found $T_{\text {eff }}=15,637 \mathrm{~K}, \log g=8.8$, and $\log \mathrm{C} / \mathrm{He}=-1.87$, the carbon abundance being very approximate since we do not include splitting in this exercise. Williams et al. (2013) used magnetic synthetic spectra to fit this object and estimated $T_{\text {eff }} \sim 15,500 \mathrm{~K}$, $\log g \sim 9$, and $\log \mathrm{C} / \mathrm{He}=-1$, which is consistent with our solution.

J2101+3148 - This star shows a discrepancy for the Swan band at $4700 \AA$ A. Dufour et al. (2005) also reported this problem without further explanation. With a larger sample, we can now compare this object with other stars with similar properties (for example $\mathrm{J} 1424+0833$ and J1118-0314), and this discrepancy is not observed anywhere else. A change in temperature of $\pm 500 \mathrm{~K}$ does not improve the situation. However, the finding chart on SIMBAD clearly shows a red object very near the white dwarf (potentially a companion or an object along the line of sight) that probably contaminates the spectra.

\section{ON THE SPECTRAL EVOLUTION OF WHITE DWARFS}

Thanks to the advent of Gaia, we now have, for the first time, detailed mass distributions for a large sample of DQ and DBZ/DZ(A) white dwarfs. Although there are still uncertainties regarding the individual mass determinations (see Sections 5.1 and 6.2), a lot of information can be extracted from the overall shape of these mass distributions, and in particular with respect to the spectral evolution of these white dwarfs.

The mass distribution of DQ white dwarfs shows two distinct peaks (see Figure 13), one centered around 0.55 $M_{\odot}$ and another one centered around $1 M_{\odot}$. As discussed above, the location of the first peak is slightly shifted relative to that of DA and DB white dwarfs (see Figure 21 of Genest-Beaulieu \& Bergeron 2019), most probably indicating a systematic error due to some unknown opacity source in the ultraviolet. Putting aside this problem, it is interesting to compare the overall shape of these mass distributions. The main difference between the mass distribution of DB stars and that of DA stars is the absence of a high-mass tail in the former. This difference was first noted by Beauchamp et al. (1996), and later confirmed by Bergeron et al. (2011) and Genest-Beaulieu \& Bergeron (2019). The mass dis- 


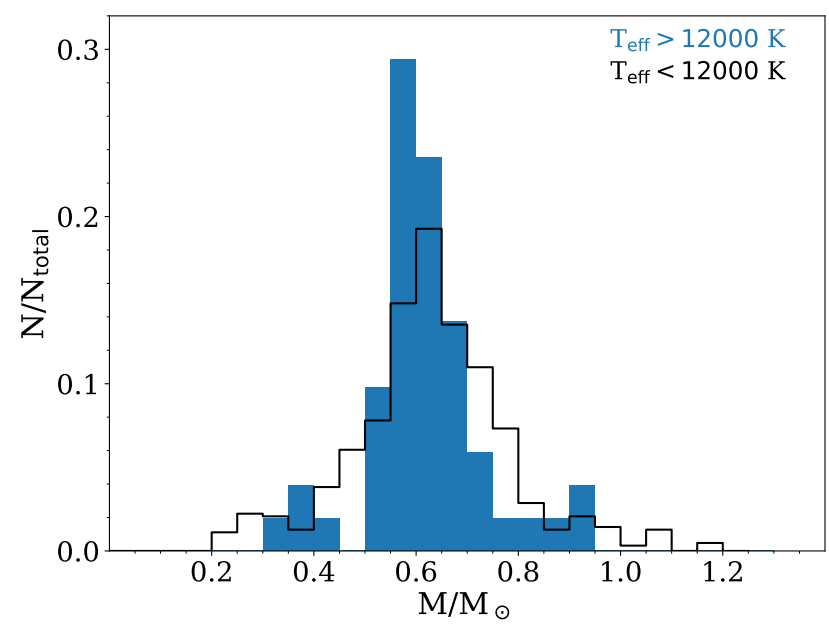

Figure 19. Comparison of the mass distributions of DBZ/DZ(A) white dwarfs for effective temperatures above and below $12,000 \mathrm{~K}$.

tribution of our DQ sample does not show an extended tail extending to masses above $1 M_{\odot}$, but rather exhibits two distinct peaks with a clear separation near $0.8 M_{\odot}$. As discussed in Section 6.4, there is compelling evidence that the second peak represents a population of merged white dwarfs. Hence, the mass distribution of DQ stars with $T_{\text {eff }}<10,000 \mathrm{~K}$ - effectively removing practically all the massive objects - resemble much more that of DB white dwarfs than that of DA stars, reinforcing the belief that the progenitor of $\mathrm{DQ}$ white dwarfs are helium-rich DB stars.

In contrast, the mass distribution of $\mathrm{DBZ} / \mathrm{DZ}(\mathrm{A})$ white dwarfs (see Figure 7) reveals a fair number of massive objects forming some sort of a tail similar to that observed in DA white dwarfs. The similitude is even more striking in Figure 19 where we show the mass distributions of our sample for effective temperatures above and below 12,000 K. We see that at low effective temperatures, the mass distribution - composed essentially of DZ(A) stars - clearly shows a high mass tail, which is barely present in the mass distribution of hotter white dwarfs, containing essentially only DBZ(A) white dwarfs.

The most logical explanation for this phenomenon is that the progenitors of a significant fraction of $\mathrm{DZ}(\mathrm{A})$ white dwarfs are DA stars that were convectively mixed, and transformed into helium-atmosphere white dwarfs. Indeed, it is well known that the ratio of non-DA to DA white dwarfs increases considerably with decreasing effective temperatures. This has been interpreted as the result of convective mixing, a process which becomes important below 12,000 K, as discussed at length in Rolland et al. (2018) and references therein. This is a process where the thin hydrogen layer is mixed with the deeper and more massive helium layer through convection, effectively turning a hydrogen-rich white dwarf into a helium-rich one. Our results thus suggest that many $\mathrm{DZ}(\mathrm{A})$ stars, and certainly most of the massive ones, represent the outcome of mixed DA white dwarfs that have also accreted planetary material. In fact, Rolland et al. (2018) also demonstrated that most DZA stars below $T_{\text {eff }} \sim 12,000 \mathrm{~K}$ could not be the descendants of DBA (or DBZA) stars, and that these objects must be the result of hydrogen-rich stars that turned helium-rich as a result of convective mixing. For the DBZ(A) white dwarfs, it is reasonable to consider these objects as being simply the metal-polluted version of $\mathrm{DB}(\mathrm{A})$ stars. These polluted DBZ(A) stars, when they cool off, will eventually become helium-rich white dwarfs, or DZ(A) stars if they also accrete material (they would not become DQ stars, however, see below).

Finally, the DQ white dwarfs with masses near the peak of the mass distribution - which rarely show any trace of hydrogen (see Section 6.3) - are most likely the descendants of the so-called "pure helium" DB stars that show no hydrogen feature. In these nearly hydrogenfree DB stars, hydrogen has probably been largely depleted during the earlier born-again post-AGB evolutionary phase (Rolland et al. 2018). This interpretation could simultaneously explain the rarity of DQZ objects ${ }^{11}$ (i.e. DQ stars showing traces of metals), if the surroundings of the progenitor have been cleared of rocky debris during the active earlier post-AGB phases.

\section{SUMMARY AND CONCLUSIONS}

We presented a homogeneous analysis of 1023 DBZ/DZ(A) and 319 DQ white dwarfs based on state-of-the-art model atmospheres using new parallaxes, proper motions, and photometry from Gaia DR2, as well as photometry from SDSS, Pan-STARRS, previously published $B V R I$, and spectroscopy from various sources. This represents a significant increase over the previous comprehensive studies on these types of objects, namely those of Dufour et al. (2007b, 159 DZs) and Dufour et al. (2005, 56 DQs). Calcium abundance measurements for our large sample of DBZ/DZ(A) white dwarfs indicate that the rocky objects that polluted their photosphere had masses similar to those of large asteroids found in our solar system. We found several polluted white dwarfs with progenitor masses well above $3 M_{\odot}$, confirming that the formation of rocky material is also

\footnotetext{
11 There are only 4 stars with Ca II H\&K absorption lines in our sample: J0739+0513 (Procyon B), J0900+0331, J1332+2740, and $\mathrm{J} 1534+4145$.
} 
common for early type stars. The availability of parallax measurements for nearly three quarters of our sample allowed us to determine, for the first time, meaningful mass distributions for these types of objects (the mass distributions for DQ and DZ stars of Dufour et al. 2005, 2007b contained only 11 and 16 objects, respectively). These mass distributions revealed several interesting aspects about the properties of our samples that we summarize here:

1. The mean mass for the DBZ/DZ white dwarfs (i.e. objects not showing $\mathrm{H} \alpha$ ) is significantly higher than that of DBZA/DZA stars. The two distributions are in much better agreement when undetectable traces of hydrogen are included in the model fits.

2. The mass distribution of $\mathrm{DZ}(\mathrm{A})$ white dwarfs cooler than $T_{\text {eff }}=12,000 \mathrm{~K}$ shows a high-mass tail similar to that observed for DA stars. This high-mass tail is absent for objects in our sample hotter than $12,000 \mathrm{~K}$. We interpret this as a signature that a significant fraction of the DZ(A) stars are convectively mixed DA white dwarfs that have accreted rocky material.

3. The mass distribution of DQ white dwarfs shows two distinct peaks, one centered at $0.55 M_{\odot}$, whose carbon abundances are well explained by the standard carbon dredge-up scenario, and another one centered at $\sim 1 M_{\odot}$, whose high kinematic properties are consistent with the idea that they represent a population of merged white dwarfs. We note that the location of the $0.55 M_{\odot}$ peak is slightly shifted towards smaller masses relative to that of DB white dwarfs, most probably due to some unknown opacity source in the ultraviolet in our DQ models.

While traces of hydrogen are detected (or needed) in nearly all DBZ/DZ(A) white dwarfs, its presence is extremely rare in cool DQ stars. This indicates that the nature of these two populations of helium-atmosphere white dwarfs are clearly distinct. The most logical way to explain the abundance pattern and mass distributions of these objects is to interpret hydrogen-free DB white dwarfs as progenitors of cool DQ stars, while the other types of cool helium-atmosphere white dwarfs, namely the DC and DZ(A) stars, would originate from both convectively mixed DA and cooled down $\mathrm{DB}(\mathrm{A})$ white dwarfs. Within this scenario, the rarity of both hydrogen and heavy elements in DQ white dwarfs is also naturally explained by invoking particularly active post-AGB phases that would eliminate practically all the remaining hydrogen, as well as most nearby rocky objects in orbit.

The presence of hydrogen in $40 \%$ of the hotter DQ is, however, somewhat mysterious. We showed that this population of massive stars have much larger space velocities than what is expected for a relatively young star population. As originally proposed by Dunlap \& Clemens (2015) in the case of Hot DQ white dwarfs, this indicates that the massive DQ stars in our sample, which are most likely cooled-down version of the Hot DQs, would also be the result of the merging of two $\mathrm{C} / \mathrm{O}$ white dwarfs. Dufour et al. (2007a, 2008) proposed that the Hot DQ white dwarfs would disguise themselves as massive DB white dwarfs until the underlying convection zone completely dilutes the thin helium layer, effectively transforming the object into a $\mathrm{C} / \mathrm{O}$-dominated atmosphere when the star cools to about 24,000 K. However, such massive, and most probably magnetic, DB white dwarfs are very scarce. Another possibility is that these merger remnants could instead hide as DA stars with very thin hydrogen layers, or possibly as some of the massive magnetic white dwarfs that are often found at higher effective temperatures. We predict that spectropolarimetric or high-resolution spectroscopic observations of massive DQ white dwarfs should reveal that most of them are magnetic at some level.

Interestingly, we find there is a correlation between the stellar mass and the effective temperature for these massive DQ white dwarfs. Despite the uncertainties associated with the temperature, abundance, and mass scales for these objects (due most probably to some missing opacity in the ultraviolet), we believe the observed trend to be real, and that it represents a manifestation of an accumulation of stars at certain effective temperatures due to the slowing of the cooling process, when the stellar core eventually crystallizes. Future work should address the shortcomings in the modeling of DQ white dwarfs in order to reduce the uncertainties on the atmospheric parameters of these objects.

This work was supported in part by NSERC (Canada) and the Fund FRQNT (Québec). BHD acknowledges support from the Wootton Center for Astrophysical Plasma Properties under the United States Department of Energy collaborative agreement DE-FOA-0001634, from the United States Department of Energy grant under DE-SC0010623. This work has made use of the Montreal White Dwarf Database (Dufour et al. 2017), and also the VALD database, operated at Uppsala University, the Institute of Astronomy RAS in Moscow, and the University of Vienna, the SIMBAD database, 
operated at CDS, Strasbourg, France (Wenger et al. 2000), data from the European Space Agency (ESA) mission Gaia (https://www.cosmos.esa.int/gaia), processed by the Gaia Data Processing and Analysis Consortium (DPAC, https://www.cosmos.esa.int/ web/gaia/dpac/consortium). Funding for the DPAC has been provided by national institutions, in particular the institutions participating in the Gaia Multilateral Agreement (Gaia Collaboration et al. 2016, 2018). Funding for the Sloan Digital Sky Survey IV has been provided by the Alfred P. Sloan Foundation, the U.S. Department of Energy Office of Science, and the Participating Institutions. SDSS-IV acknowledges support and resources from the Center for High-Performance Computing at the University of Utah. The SDSS web site is www.sdss.org. SDSS-IV is managed by the Astrophysical Research Consortium for the Participating Institutions of the SDSS Collaboration including the Brazilian Participation Group, the Carnegie Institution for Science, Carnegie Mellon University, the Chilean Participation Group, the French Participation Group, Harvard-Smithsonian Center for Astrophysics, Instituto de Astrofísica de Canarias, The Johns Hopkins University, Kavli Institute for the Physics and Mathematics of the Universe (IPMU) / University of Tokyo, the Korean Participation Group, Lawrence Berkeley National Laboratory, Leibniz Institut für Astrophysik Potsdam (AIP), Max-Planck-Institut für Astronomie (MPIA Heidelberg), Max-Planck-Institut für Astrophysik (MPA Garching), Max-Planck-Institut für Extraterrestrische Physik (MPE), National Astronomical Observatories of China, New Mexico State University, New York University, University of Notre Dame, Observatário Nacional /
MCTI, The Ohio State University, Pennsylvania State University, Shanghai Astronomical Observatory, United Kingdom Participation Group, Universidad Nacional Autónoma de México, University of Arizona, University of Colorado Boulder, University of Oxford, University of Portsmouth, University of Utah, University of Virginia, University of Washington, University of Wisconsin, Vanderbilt University, and Yale University (Abolfathi et al. 2018). The Pan-STARRS1 Surveys (PS1) and the PS1 public science archive have been made possible through contributions by the Institute for Astronomy, the University of Hawaii, the Pan-STARRS Project Office, the Max-Planck Society and its participating institutes, the Max Planck Institute for Astronomy, Heidelberg and the Max Planck Institute for Extraterrestrial Physics, Garching, The Johns Hopkins University, Durham University, the University of Edinburgh, the Queen's University Belfast, the Harvard-Smithsonian Center for Astrophysics, the Las Cumbres Observatory Global Telescope Network Incorporated, the National Central University of Taiwan, the Space Telescope Science Institute, the National Aeronautics and Space Administration under Grant No. NNX08AR22G issued through the Planetary Science Division of the NASA Science Mission Directorate, the National Science Foundation Grant No. AST-1238877, the University of Maryland, Eotvos Lorand University (ELTE), the Los Alamos National Laboratory, and the Gordon and Betty Moore Foundation (Chambers et al. 2016).

Softwares: Astropy (Astropy Collaboration et al. 2013, 2018), Matplotlib (Hunter 2007), NumPy (Oliphant 2006; van der Walt et al. 2011), PySpecKit (Ginsburg \& Mirocha 2011).

\section{REFERENCES}

Abolfathi, B., Aguado, D. S., Aguilar, G., et al. 2018, ApJS, 235, 42

Allard, N. F., Royer, A., Kielkopf, J. F., \& Feautrier, N. 1999, PhRvA, 60, 1021

Astropy Collaboration, Robitaille, T. P., Tollerud, E. J., et al. 2013, A\&A, 558, A33

Astropy Collaboration, Price-Whelan, A. M., Sipőcz, B. M., et al. 2018, AJ, 156, 123

Bailer-Jones, C. A. L. 2015, PASP, 127, 994

Barlow, B. N., Dunlap, B. H., Rosen, R., \& Clemens, J. C. 2008, ApJL, 688, L95

Batalha, N. M., Borucki, W. J., Koch, D. G., et al. 2010, ApJL, 713, L109
Beauchamp, A., Wesemael, F., Bergeron, P., Liebert, J., \& Saffer, R. A. 1996, in Astronomical Society of the Pacific Conference Series, Vol. 96, Hydrogen Deficient Stars, ed. C. S. Jeffery \& U. Heber, 295

Bergeron, P., Dufour, P., Fontaine, G., et al. 2019, ApJ, 876,67

Bergeron, P., Leggett, S. K., \& Ruiz, M. T. 2001, ApJS, 133,413

Bergeron, P., Ruiz, M. T., \& Leggett, S. K. 1997, ApJS, 108, 339

Bergeron, P., Saffer, R. A., \& Liebert, J. 1992, ApJ, 394, 228

Bergeron, P., Wesemael, F., Dufour, P., et al. 2011, ApJ, 737, 28 
Blouin, S., Allard, N. F., Leininger, T., Gadéa, F. X., \& Dufour, P. 2019a, ApJ, 875, 137

Blouin, S., Dufour, P., \& Allard, N. F. 2018a, ApJ, 863, 184

Blouin, S., Dufour, P., Allard, N. F., \& Kilic, M. 2018b, ApJ, 867, 161

Blouin, S., Dufour, P., Allard, N. F., et al. 2019b, ApJ, 872, 188

Blouin, S., Dufour, P., Thibeault, C., \& Allard, N. F. 2019c, ApJ, 878, 63

Blouin, S., Kowalski, P. M., \& Dufour, P. 2017, ApJ, 848, 36

Bond, H. E., Gilliland, R. L., Schaefer, G. H., et al. 2015, ApJ, 813, 106

Brassard, P., Fontaine, G., Dufour, P., \& Bergeron, P. 2007, in Astronomical Society of the Pacific Conference Series, Vol. 372, 15th European Workshop on White Dwarfs, ed. R. Napiwotzki \& M. R. Burleigh, 19

Brooke, J. S., Bernath, P. F., Schmidt, T. W., \& Bacskay, G. B. 2013, Journal of Quantitative Spectroscopy and Radiative Transfer, 124, 11 .

http://www.sciencedirect.com/science/article/pii/ S0022407313000800

Chambers, K. C., Magnier, E. A., Metcalfe, N., et al. 2016, arXiv e-prints, arXiv:1612.05560

Cohen, M., Megeath, S. T., Hammersley, P. L., Martín-Luis, F., \& Stauffer, J. 2003, AJ, 125, 2645

Dantona, F., \& Mazzitelli, I. 1979, A\&A, 74, 161

Dufour, P. 2011, Stars with Unusual Compositions: Carbon and Oxygen in Cool White Dwarfs, ed. D. W. Hoard, $53-88$

Dufour, P., Béland, S., Fontaine, G., Chayer, P., \& Bergeron, P. 2011, ApJL, 733, L19

Dufour, P., Bergeron, P., \& Fontaine, G. 2005, ApJ, 627, 404

Dufour, P., Blouin, S., Coutu, S., et al. 2017, in Astronomical Society of the Pacific Conference Series, Vol. 509, 20th European White Dwarf Workshop, ed. P.-E. Tremblay, B. Gaensicke, \& T. Marsh, 3

Dufour, P., Fontaine, G., Liebert, J., Schmidt, G. D., \& Behara, N. 2008, ApJ, 683, 978

Dufour, P., Kilic, M., Fontaine, G., et al. 2010, ApJ, 719, 803

—. 2012, ApJ, 749, 6

Dufour, P., Liebert, J., Fontaine, G., \& Behara, N. 2007a, Nature, 450, 522

Dufour, P., Vornanen, T., Bergeron, P., \& Fontaine, A., B. 2013, in Astronomical Society of the Pacific Conference Series, Vol. 469, 18th European White Dwarf Workshop., 167

Dufour, P., Bergeron, P., Liebert, J., et al. 2007b, ApJ, 663, 1291
Dunlap, B. H., Barlow, B. N., \& Clemens, J. C. 2010, ApJL, 720, L159

Dunlap, B. H., \& Clemens, J. C. 2015, in Astronomical Society of the Pacific Conference Series, Vol. 493, 19th European Workshop on White Dwarfs, ed. P. Dufour, P. Bergeron, \& G. Fontaine, 547

Dupuis, J., Fontaine, G., \& Wesemael, F. 1993, ApJS, 87, 345

Eisenstein, D. J., Liebert, J., Koester, D., et al. 2006, AJ, 132,676

El-Badry, K., Rix, H.-W., \& Weisz, D. R. 2018, ApJL, 860, L17

Farihi, J. 2011, in American Institute of Physics Conference Series, Vol. 1331, American Institute of Physics

Conference Series, ed. S. Schuh, H. Drechsel, \& U. Heber, 193-210

Farihi, J., Gänsicke, B. T., \& Koester, D. 2013, Science, 342,218

Fontaine, G., \& Brassard, P. 2005, in Astronomical Society of the Pacific Conference Series, Vol. 334, 14th European Workshop on White Dwarfs, ed. D. Koester \&

S. Moehler, 49

Fontaine, G., Brassard, P., \& Bergeron, P. 2001, PASP, 113,409

Gaia Collaboration, Prusti, T., de Bruijne, J. H. J., et al. 2016, A\&A, 595, A1

Gaia Collaboration, Babusiaux, C., van Leeuwen, F., et al. 2018, A\&A, 616, A10

Gänsicke, B. T., Koester, D., Girven, J., Marsh, T. R., \& Steeghs, D. 2010, Science, 327, 188

Genest-Beaulieu, C., \& Bergeron, P. 2019, ApJ, 871, 169

Gentile Fusillo, N. P., Tremblay, P.-E., Gänsicke, B. T., et al. 2019, MNRAS, 482, 4570

Giammichele, N., Bergeron, P., \& Dufour, P. 2012, ApJS, 199, 29

Ginsburg, A., \& Mirocha, J. 2011, PySpecKit: Python Spectroscopic Toolkit, Astrophysics Source Code Library, , , ascl:1109.001

Green, P. 2013, ApJ, 765, 12

Harris, H. C., Munn, J. A., Kilic, M., et al. 2006, AJ, 131, 571

Holberg, J. B., Barstow, M. A., \& Burleigh, M. R. 2003, ApJS, 147, 145

Holberg, J. B., \& Bergeron, P. 2006, AJ, 132, 1221

Hollands, M. A., Gänsicke, B. T., \& Koester, D. 2018, MNRAS, 477, 93

Hollands, M. A., Koester, D., Alekseev, V., Herbert, E. L., \& Gänsicke, B. T. 2017, MNRAS, 467, 4970

Hunter, J. D. 2007, Computing In Science \& Engineering, 9, 90 
Iben, I., J., \& MacDonald, J. 1985, ApJ, 296, 540

Johnson, J. A., Aller, K. M., Howard, A. W., \& Crepp, J. R. 2010, PASP, 122, 905

Jura, M., Xu, S., Klein, B., Koester, D., \& Zuckerman, B. 2012, ApJ, 750, 69

Jura, M., \& Young, E. D. 2014, Annual Review of Earth and Planetary Sciences, 42, 45

Kepler, S. O., Pelisoli, I., Koester, D., et al. 2015, MNRAS, 446, 4078

一. 2016, MNRAS, 455, 3413

Klein, B., Jura, M., Koester, D., \& Zuckerman, B. 2011, ApJ, 741, 64

Klein, B., Jura, M., Koester, D., Zuckerman, B., \& Melis, C. 2010, ApJ, 709, 950

Kleinman, S. J., Harris, H. C., Eisenstein, D. J., et al. 2004, ApJ, 607, 426

Kleinman, S. J., Kepler, S. O., Koester, D., et al. 2013, ApJS, 204, 5

Koester, D. 2009, A\&A, 498, 517

Koester, D., Gänsicke, B. T., \& Farihi, J. 2014, A\&A, 566, A34

Koester, D., Girven, J., Gänsicke, B. T., \& Dufour, P. 2011, A\&A, 530, A114

Koester, D., \& Kepler, S. O. 2019, arXiv e-prints, arXiv:1905.11174

Koester, D., \& Knist, S. 2006, A\&A, 454, 951

Kowalski, P. M. 2010, A\&A, 519, L8

Lawrie, K. A., Burleigh, M. R., Dufour, P., \& Hodgkin, S. T. 2013, MNRAS, 433, 1599

Leggett, S. K., Bergeron, P., Subasavage, J. P., et al. 2018, ApJS, 239, 26

Liebert, J., Harris, H. C., Dahn, C. C., et al. 2003, AJ, 126, 2521

Limoges, M.-M., Bergeron, P., \& Lépine, S. 2015, ApJS, 219, 19

Limoges, M.-M., Lépine, S., \& Bergeron, P. 2013, AJ, 145, 136

Lodders, K. 2003, ApJ, 591, 1220

Marigo, P., \& Girardi, L. 2007, A\&A, 469, 239

Montgomery, M. H., Williams, K. A., Winget, D. E., et al. 2008, ApJL, 678, L51

Oliphant, T. 2006, NumPy: A guide to NumPy, USA: Trelgol Publishing, , . http://www .numpy.org/

Paquette, C., Pelletier, C., Fontaine, G., \& Michaud, G. 1986, ApJS, 61, 197

Parigger, C. G., Woods, A. C., Surmick, D. M., et al. 2015, Spectrochimica Acta Part B: Atomic Spectroscopy, 107, 132. http://www.sciencedirect.com/science/ article/pii/S058485471500066X
Piskunov, N. E., Kupka, F., Ryabchikova, T. A., Weiss, W. W., \& Jeffery, C. S. 1995, A\&AS, 112, 525

Press, W. H., Flannery, B. P., \& Teukolsky, S. A. 1986, Numerical recipes. The art of scientific computing (Cambridge University Press)

Provencal, J. L., Shipman, H. L., Koester, D., Wesemael, F., \& Bergeron, P. 2002, ApJ, 568, 324

Provencal, J. L., Shipman, H. L., Wesemael, F., et al. 1997, ApJ, 480, 777

Raddi, R., Gänsicke, B. T., Koester, D., et al. 2015, MNRAS, 450, 2083

Rolland, B., Bergeron, P., \& Fontaine, G. 2018, ApJ, 857, 56

Schlafly, E. F., \& Finkbeiner, D. P. 2011, ApJ, 737, 103

Subasavage, J. P., Henry, T. J., Bergeron, P., et al. 2007, AJ, 134, 252

Subasavage, J. P., Jao, W.-C., Henry, T. J., et al. 2009, AJ, 137,4547

Thejll, P., Shipman, H. L., MacDonald, J., \& Macfarland, W. M. 1990, ApJ, 361, 197

Tremblay, P.-E., Fontaine, G., Fusillo, N. P. G., et al. 2019, Nature, 565, 202

van der Walt, S., Colbert, S. C., \& Varoquaux, G. 2011,

Computing in Science Engineering, 13, 22

van Horn, H. M. 1968, ApJ, 151, 227

van Leeuwen, F. 2007, A\&A, 474, 653

Vornanen, T., Berdyugina, S. V., Berdyugin, A. V., \&

Piirola, V. 2010, ApJ, 720, L52

Voss, B., Koester, D., Napiwotzki, R., Christlieb, N., \&

Reimers, D. 2007, A\&A, 470, 1079

Walkup, R., Stewart, B., \& Pritchard, D. E. 1984, Phys. Rev. A, 29, 169.

https://link.aps.org/doi/10.1103/PhysRevA.29.169

Wegg, C., \& Phinney, E. S. 2012, MNRAS, 426, 427

Wegner, G., \& Koester, D. 1985, ApJ, 288, 746

Weidemann, V., \& Koester, D. 1995, A\&A, 297, 216

Wenger, M., Ochsenbein, F., Egret, D., et al. 2000, A\&AS, 143,9

Williams, K. A., Bolte, M., \& Koester, D. 2009, ApJ, 693, 355

Williams, K. A., Winget, D. E., Montgomery, M. H., et al. 2013, ApJ, 769, 123

Xu, S., Jura, M., Dufour, P., \& Zuckerman, B. 2016, ApJL, 816, L22

Xu, S., Jura, M., Koester, D., Klein, B., \& Zuckerman, B. 2014, ApJ, 783, 79

Xu, S., Zuckerman, B., Dufour, P., et al. 2017, ApJL, 836, L7

Zeidler-K.T., E. M., \& Koester, D. 1982, A\&A, 113, 173 
Zuckerman, B., Koester, D., Melis, C., Hansen, B. M., \& Jura, M. 2007, ApJ, 671, 872
Zuckerman, B., Koester, D., Reid, I. N., \& Hünsch, M. 2003, ApJ, 596, 477

Zuckerman, B., Melis, C., Klein, B., Koester, D., \& Jura, M. 2010, ApJ, 722, 725 
Table 5. Object names, spectral types, and coordinates

\begin{tabular}{|c|c|c|c|c|c|}
\hline J Name & Gaia source id & MWDD id & Sp. type & R.A & Dec \\
\hline J0000-0026 & 2449884942327523456 & SDSS J000052.44-002610.5 & DQ & 0.21853 & -0.43626 \\
\hline J0000-0850 & 2441060055844546816 & WD 2357-091 & DQ & 0.04817 & -8.83566 \\
\hline J0002+3209 & 2873769975034246272 & SDSS J000215.65+320914.1 & $\mathrm{DZ}$ & 0.56522 & 32.15387 \\
\hline J0002-0627 & & SDSS J000232.35-062746.0 & $\mathrm{DZ}$ & 0.63479 & -6.46278 \\
\hline J0003+2240 & & SDSS J000341.93+224043.7 & $\mathrm{DZ}$ & 0.92471 & 22.67881 \\
\hline J0004+0819 & & SDSS J000418.68+081929.9 & $\mathrm{DZ}$ & 1.07787 & 8.32499 \\
\hline J0004+2432 & 2849976676552398976 & SDSS J000426.95+243258.9 & DBZ & 1.11226 & 24.54971 \\
\hline J0004+2531 & 2850199499454835968 & SDSS J000439.38+253150.6 & $\mathrm{DZ}$ & 1.16411 & 25.53071 \\
\hline $\mathrm{J} 0005+0018$ & 2546096749538393600 & WD $0003+000$ & $\mathrm{DZ}$ & 1.48845 & 0.30924 \\
\hline $\mathrm{J} 0005+4003$ & 2881925666956510848 & GD 1 & DZA & 1.25425 & 40.05996 \\
\hline $\mathrm{J} 0005+7313$ & 537563127588521600 & GD 408 & DBZA & 1.27842 & 73.21927 \\
\hline J0006+0520 & 2742043289409950336 & SDSS J000614.53+052039.0 & $\mathrm{DZ}$ & 1.56054 & 5.34418 \\
\hline J0006+1800 & 2773073470346643200 & WD $0003+177$ & $\mathrm{DQ}$ & 1.59557 & 18.00373 \\
\hline J0007+2821 & 2860138053779074560 & SDSS J000705.02+282104.2 & $\mathrm{DQ}$ & 1.77087 & 28.35123 \\
\hline J0008+2507 & 2849930668862492544 & GALEX 2667408659217122775 & DQA & 2.04310 & 25.13154 \\
\hline J0008-1034 & 2427847052815180544 & WD $0005-108$ & $\mathrm{DQ}$ & 2.03141 & -10.56821 \\
\hline J0009+0152 & 2546743262376424064 & SDSS J000950.09+015219.6 & $\mathrm{DZ}$ & 2.45871 & 1.87211 \\
\hline J0010-0430 & 2444911924250219648 & SDSS J001052.56-043014.3 & $\mathrm{DZ}$ & 2.71886 & -4.50390 \\
\hline J0010-0628 & & SDSS J001037.06-062857.7 & $\mathrm{DZ}$ & 2.65442 & -6.48272 \\
\hline J0013+1109 & & SDSS J001309.43+110949.0 & $\mathrm{DZ}$ & 3.28929 & 11.16361 \\
\hline J0015+0309 & 2548437124462728704 & SDSS J001523.91+030909.0 & $\mathrm{DQ}$ & 3.84950 & 3.15249 \\
\hline J0015+1830 & 2797320927258478464 & SDSS J001557.38+183034.6 & $\mathrm{DZ}$ & 3.98905 & 18.50957 \\
\hline J0017+0230 & & SDSS J001703.79+023008.7 & $\mathrm{DZ}$ & 4.26579 & 2.50242 \\
\hline J0018-0012 & 2545263281069744512 & PB 5858 & $\mathrm{DZ}$ & 4.70597 & -0.20132 \\
\hline $\mathrm{J} 0019+1848$ & 2797345597551222656 & SDSS J001910.66+184824.0 & $\mathrm{DZ}$ & 4.79449 & 18.80673 \\
\hline $\mathrm{J} 0019+2209$ & 2800362726177065984 & SDSS J001949.26+220926.5 & $\mathrm{DZ}$ & 4.95519 & 22.15746 \\
\hline J0024-1107 & 2424780514884907648 & WD $0022-114$ & DQ & 6.22903 & -11.12868 \\
\hline J0026-0006 & & SDSS J002639.47-000630.7 & DBZ & 6.66444 & -0.10851 \\
\hline J0027+0102 & 2543967197379493120 & SDSS J002758.56+010250.4 & $\mathrm{DZ}$ & 6.99400 & 1.04736 \\
\hline J0027+3350 & 2863148619694318208 & SDSS J002755.88+335018.5 & $\mathrm{DZ}$ & 6.98276 & 33.83842 \\
\hline J0030-0254 & & SDSS J003058.58-025436.5 & $\mathrm{DZ}$ & 7.74408 & -2.91014 \\
\hline J0031+2040 & 2796487909762143232 & SDSS J003104.21+204033.6 & $\mathrm{DZ}$ & 7.76759 & 20.67601 \\
\hline J0031+3252 & & SDSS J003137.63+325215.5 & $\mathrm{DZ}$ & 7.90679 & 32.87099 \\
\hline J0032+0827 & 2749781068130558464 & SDSS J003244.25+082720.1 & $\mathrm{DZ}$ & 8.18420 & 8.45562 \\
\hline J0033+0418 & 2554073392865462272 & SDSS J003328.58+041834.6 & DQ & 8.36893 & 4.30958 \\
\hline J0033+2845 & 2857867974583987200 & SDSS J003300.59+284504.8 & $\mathrm{DZ}$ & 8.25228 & 28.75131 \\
\hline J0036-1112 & 2425347347488993408 & WD $0033-114$ & $\mathrm{DZ}$ & 9.00572 & -11.20389 \\
\hline J0038+1645 & 2782417115863764224 & SDSS J003803.63+164504.4 & $\mathrm{DZ}$ & 9.51504 & 16.75136 \\
\hline
\end{tabular}


Table 5. Object names, spectral types, and coordinates - continued

\begin{tabular}{|c|c|c|c|c|c|}
\hline J Name & Gaia source id & MWDD id & Sp. type & R.A & Dec \\
\hline $\mathrm{J} 0039+1416$ & & SDSS J003916.55+141623.4 & DZ: & 9.81896 & 14.27318 \\
\hline J0040+2349 & 2806346096656318208 & SDSS J004038.99+234904.5 & $\mathrm{DZ}$ & 10.16250 & 23.81796 \\
\hline $\mathrm{J} 0040+2723$ & 2809357354062968704 & SDSS J004011.38+272353.0 & $\mathrm{DZ}$ & 10.04719 & 27.39809 \\
\hline J0040+3016 & 2858528884154261120 & SDSS J004015.15+301619.5 & $\mathrm{DZ}$ & 10.06312 & 30.27209 \\
\hline J0041+1511 & 2779758050071090688 & WD $0038+149$ & $\mathrm{DZ}$ & 10.34829 & 15.18586 \\
\hline J0041+7321 & 536979286914485760 & WD $0038+730$ & $\mathrm{DQ}$ & 10.44775 & 73.35407 \\
\hline $\mathrm{J} 0042+2255$ & 2803222521560799104 & SDSS J004235.36+225536.5 & $\mathrm{DZ}$ & 10.64740 & 22.92686 \\
\hline J0044+0418 & 2551258990991085184 & LSPM J0044+0418 & $\mathrm{DZ}$ & 11.21537 & 4.30535 \\
\hline J0044+1259 & 2776033626230867712 & SDSS J004440.31+125923.3 & DQ & 11.16782 & 12.98991 \\
\hline J0046+0024 & 2549074978645621632 & SDSS J004646.15+002430.9 & $\mathrm{DZ}$ & 11.69232 & 0.40859 \\
\hline J0046+0635 & 2556270869932443648 & SDSS J004605.61+063513.6 & $\mathrm{DZ}$ & 11.52333 & 6.58730 \\
\hline J0046+2717 & 2808923867308433152 & SDSS J004634.22+271737.6 & $\mathrm{DZ}$ & 11.64256 & 27.29386 \\
\hline J0049+0523 & 2552928187080872832 & Wolf 28 & $\mathrm{DZ}$ & 12.29124 & 5.38861 \\
\hline $\mathrm{J} 0050+1828$ & & SDSS J005029.89+182816.2 & $\mathrm{DZ}$ & 12.62454 & 18.47117 \\
\hline J0050-0621 & 2523124962338002432 & SDSS J005004.40-062104.1 & $\mathrm{DZ}$ & 12.51833 & -6.35115 \\
\hline J0052+1846 & & SDSS J005247.16+184649.5 & $\mathrm{DZ}$ & 13.19650 & 18.78042 \\
\hline J0053+3115 & & SDSS J005304.15+311555.8 & $\mathrm{DZ}$ & 13.26729 & 31.26553 \\
\hline $\mathrm{J} 0055+0850$ & 2581058706745690752 & SDSS J005505.42+085030.5 & DQ & 13.77256 & 8.84182 \\
\hline$J 0055+2413$ & 2804037057813910272 & SDSS J005537.21+241347.1 & $\mathrm{DZ}$ & 13.90504 & 24.22978 \\
\hline J0056+0005 & & SDSS J005651.25+000559.8 & $\mathrm{DZ}$ & 14.21354 & 0.09997 \\
\hline $\mathrm{J} 0056+2453$ & & SDSS J005649.27+245335.4 & $\mathrm{DZ}$ & 14.20529 & 24.89317 \\
\hline J0058+0507 & 2552263669740622720 & SDSS J005818.82+050711.0 & $\mathrm{DZ}$ & 14.57842 & 5.11975 \\
\hline J0058+1102 & 2582516968401861632 & LSPM J0058+1102 & $\mathrm{DZ}$ & 14.58887 & 11.04418 \\
\hline J0059+0017 & 2536491205505048960 & WD $0056+000$ & $\mathrm{DZ}$ & 14.77823 & 0.29035 \\
\hline $\mathrm{J} 0100+0814$ & & SDSS J010039.50+081401.0 & DQ & 15.16458 & 8.23364 \\
\hline J0102+1817 & & SDSS J010258.13+181733.3 & $\mathrm{DZ}$ & 15.74221 & 18.29261 \\
\hline $\mathrm{J} 0102+3112$ & & SDSS J010245.58+311216.5 & $\mathrm{DZ}$ & 15.68992 & 31.20461 \\
\hline J0103-0429 & 2525099577846697856 & SDSS J010351.91-042913.7 & $\mathrm{DQ}$ & 15.96612 & -4.48721 \\
\hline J0104-0030 & & SDSS J010425.85-003004.8 & $\mathrm{DZ}$ & 16.10771 & -0.50136 \\
\hline J0106+2412 & & SDSS J010651.88+241258.5 & $\mathrm{DZ}$ & 16.71617 & 24.21625 \\
\hline J0106-0103 & 2533057533770551680 & WD $0103-013$ & DZA & 16.62438 & -1.06233 \\
\hline J0107+0102 & 2537913801752406400 & LSPM J0107+0102 & $\mathrm{DQ}$ & 16.95082 & 1.04461 \\
\hline J0107+2650 & 306779618349361920 & LSPM J0107+2650 & $\mathrm{DZ}$ & 16.86880 & 26.83933 \\
\hline J0107+2905 & 308384183771218048 & SDSS J010726.83+290557.1 & $\mathrm{DZ}$ & 16.86179 & 29.09931 \\
\hline J0108-0537 & 2476852079906496384 & SDSS J010825.79-053755.6 & $\mathrm{DZ}$ & 17.10747 & -5.63215 \\
\hline J0111+1501 & 2591195654198397696 & GALEX 2418435336417843954 & $\mathrm{DZ}$ & 17.99707 & 15.02440 \\
\hline$J 0111+2848$ & & SDSS J011100.82+284800.2 & $\mathrm{DZ}$ & 17.75342 & 28.80008 \\
\hline J0113-0006 & 2534630488233088256 & SDSS J011338.36-000632.9 & $\mathrm{DZ}$ & 18.40984 & -0.10913 \\
\hline
\end{tabular}


Table 5. Object names, spectral types, and coordinates - continued

\begin{tabular}{|c|c|c|c|c|c|}
\hline J Name & Gaia source id & MWDD id & Sp. type & R.A & Dec \\
\hline J0113-0959 & 2471465301860389120 & WD $0111-102$ & DZA & 18.49577 & -9.98704 \\
\hline J0114+3505 & 320947998199092992 & SDSS J011421.17+350547.1 & $\mathrm{DZ}$ & 18.58831 & 35.09644 \\
\hline J0115-0542 & 2481890145264070400 & SDSS J011512.11-054217.7 & $\mathrm{DZ}$ & 18.80047 & -5.70483 \\
\hline J0116+0328 & 2562882748746769280 & SDSS J011636.75+032823.9 & $\mathrm{DZ}$ & 19.15313 & 3.47331 \\
\hline J0116+0345 & 2562897214197044224 & SDSS J011646.62+034504.0 & $\mathrm{DZ}$ & 19.19425 & 3.75111 \\
\hline J0116+1744 & 2785761314839472768 & SDSS J011614.28+174425.6 & $\mathrm{DZ}$ & 19.05950 & 17.74047 \\
\hline $\mathrm{J} 0116+2050$ & 2787507098786135552 & SDSS J011646.10+205001.9 & $\mathrm{DZ}$ & 19.19208 & 20.83386 \\
\hline J0116+2229 & & SDSS J011636.01+222935.2 & $\mathrm{DZ}$ & 19.15004 & 22.49311 \\
\hline $\mathrm{J} 0116+2346$ & 293138458619500800 & SDSS J011639.84+234644.4 & DQ & 19.16600 & 23.77902 \\
\hline $\mathrm{J} 0116+2402$ & 293251570878605696 & SDSS J011642.68+240223.8 & DQ & 19.17784 & 24.04001 \\
\hline J0117+0021 & 2534988314843345536 & SDSS J011759.81+002138.1 & $\mathrm{DZ}$ & 19.49921 & 0.36057 \\
\hline J0117+0039 & & SDSS J011749.68+003900.5 & $\mathrm{DZ}$ & 19.45700 & 0.65014 \\
\hline J0118+1610 & 2591754107321120896 & Wolf 1516 & $\mathrm{DQ}$ & 19.50031 & 16.17238 \\
\hline $\mathrm{J} 0119+1758$ & & SDSS J011908.15+175832.8 & $\mathrm{DZ}$ & 19.78396 & 17.97581 \\
\hline J0120-0041 & 2533741365578975744 & [GMS97] NGC 450121 & DBZ & 20.18653 & -0.69975 \\
\hline J0121+1504 & & SDSS J012110.32+150433.3 & $\mathrm{DZ}$ & 20.29300 & 15.07594 \\
\hline J0121+3440 & 320029150076023808 & 2MASS J01213782+3440431 & $\mathrm{DZ}$ & 20.40748 & 34.67861 \\
\hline $\mathrm{J} 0123+1324$ & 2590150327877551616 & WD $0120+131$ & $\mathrm{DZ}$ & 20.91567 & 13.40932 \\
\hline $\mathrm{J} 0125+2346$ & & SDSS J012513.92+234638.8 & $\mathrm{DZ}$ & 21.30800 & 23.77744 \\
\hline J0126+2534 & 295286835620380032 & SDSS J012620.48+253433.6 & $\mathrm{DZ}$ & 21.58533 & 25.57601 \\
\hline J0127+1545 & & SDSS J012755.15+154541.1 & $\mathrm{DZ}$ & 21.97979 & 15.76142 \\
\hline $\mathrm{J} 0130+2218$ & & SDSS J013013.30+221814.1 & $\mathrm{DZ}$ & 22.55542 & 22.30394 \\
\hline $\mathrm{J} 0131+0250$ & & SDSS J013121.28+025007.6 & $\mathrm{DZ}$ & 22.83867 & 2.83544 \\
\hline J0134+1857 & 95799314257405696 & SDSS J013422.70+185704.6 & $\mathrm{DZ}$ & 23.59458 & 18.95131 \\
\hline $\mathrm{J} 0135+1302$ & 2586182156053386240 & WD $0132+127$ & $\mathrm{DZ}$ & 23.76715 & 13.04457 \\
\hline J0135-0027 & 2509739915804015232 & SDSS J013557.23-002705.1 & $\mathrm{DZ}$ & 23.98846 & -0.45144 \\
\hline J0136+0229 & & SDSS J013647.58+022953.7 & $\mathrm{DZ}$ & 24.19825 & 2.49828 \\
\hline J0136+2004 & 96167513213751936 & SDSS J013655.67+200429.8 & $\mathrm{DQ}$ & 24.23200 & 20.07496 \\
\hline J0137+0047 & 2510118903716498816 & SDSS J013719.01+004743.5 & $\mathrm{DQ}$ & 24.32920 & 0.79541 \\
\hline J0138+0031 & 2509910992940012416 & SDSS J013831.12+003101.6 & $\mathrm{DZ}$ & 24.62970 & 0.51713 \\
\hline J0140+3125 & & SDSS J014031.53+312502.0 & $\mathrm{DZ}$ & 25.13137 & 31.41723 \\
\hline J0140+3357 & 317634168937635200 & SDSS J014043.78+335723.7 & $\mathrm{DZ}$ & 25.18254 & 33.95644 \\
\hline J0141+2921 & 302222623688124800 & SDSS J014135.06+292153.5 & $\mathrm{DZ}$ & 25.39614 & 29.36479 \\
\hline J0142+0118 & 2510436112821077760 & SDSS J014213.26+011834.8 & $\mathrm{DZ}$ & 25.55525 & 1.30969 \\
\hline J0143+0113 & 2510420204262205312 & SDSS J014300.52+011356.8 & $\mathrm{DZ}$ & 25.75218 & 1.23249 \\
\hline J0143+0424 & & SDSS J014331.92+042441.4 & $\mathrm{DZ}$ & 25.88300 & 4.41153 \\
\hline J0144+0305 & & SDSS J014441.64+030536.2 & $\mathrm{DZ}$ & 26.17350 & 3.09339 \\
\hline J0144+1920 & & SDSS J014415.12+192021.4 & $\mathrm{DZ}$ & 26.06300 & 19.33928 \\
\hline
\end{tabular}


Table 5. Object names, spectral types, and coordinates - continued

\begin{tabular}{|c|c|c|c|c|c|}
\hline J Name & Gaia source id & MWDD id & Sp. type & R.A & Dec \\
\hline J0144-0757 & 2465432384637068672 & SDSS J014405.93-075758.1 & DQ & 26.02473 & -7.96612 \\
\hline $\mathrm{J} 0145+2317$ & 290678477446232960 & G $34-49$ & DQ & 26.33124 & 23.29825 \\
\hline J0145-0822 & 2465343629137442816 & WD $0143-086$ & DQ & 26.47522 & -8.37268 \\
\hline J0147+1541 & & SDSS J014732.99+154104.6 & $\mathrm{DZ}$ & 26.88746 & 15.68461 \\
\hline J0147-0623 & & SDSS J014709.40-062349.9 & $\mathrm{DZ}$ & 26.78917 & -6.39721 \\
\hline J0148-0112 & 2506055761575043456 & SDSS J014834.00-011235.9 & $\mathrm{DZ}$ & 27.14167 & -1.20997 \\
\hline $\mathrm{J} 0150+1354$ & 2576012498130773376 & [VV2006] J015008.6+135433 & $\mathrm{DZ}$ & 27.53562 & 13.90944 \\
\hline $\mathrm{J} 0150+1710$ & 91408178348709376 & SDSS J015048.48+171039.0 & $\mathrm{DZ}$ & 27.70200 & 17.17750 \\
\hline $\mathrm{J} 0152+2418$ & 99064833727036160 & SDSS J015217.94+241850.6 & $\mathrm{DZ}$ & 28.07480 & 24.31413 \\
\hline $\mathrm{J} 0153+0101$ & & SDSS J015330.76+010135.9 & $\mathrm{DZ}$ & 28.37817 & 1.02664 \\
\hline J0154+1403 & 77986298173874176 & WD $0151+138$ & DQ & 28.67395 & 14.05221 \\
\hline J0154-0040 & 2506157672559170944 & SDSS J015433.59-004047.1 & $\mathrm{DQ}$ & 28.63993 & -0.67976 \\
\hline J0158-0942 & 2462572103921051648 & SDSS J015849.02-094225.3 & $\mathrm{DZ}$ & 29.70411 & -9.70698 \\
\hline J0200+0040 & 2507853291287991808 & WD $0157+004$ & $\mathrm{DZ}$ & 30.00837 & 0.67178 \\
\hline $\mathrm{J} 0200+0715$ & 2567842095943560448 & LSPM J0200+0715 & DQ & 30.09463 & 7.25006 \\
\hline J0201+2015 & 94296969056387328 & SDSS J020128.66+201521.8 & $\mathrm{DZ}$ & 30.36942 & 20.25606 \\
\hline J0201-0039 & 2506764706057099520 & WD 0158-009 & $\mathrm{DZ}$ & 30.38433 & -0.65903 \\
\hline $\mathrm{J} 0202+2459$ & & SDSS J020253.24+245921.4 & $\mathrm{DZ}$ & 30.72183 & 24.98928 \\
\hline J0208-0315 & & SDSS J020844.85-031535.4 & DQ & 32.18681 & -3.25986 \\
\hline J0209+0558 & 2520139096777929344 & SDSS J020942.37+055854.7 & $\mathrm{DZ}$ & 32.42649 & 5.98187 \\
\hline $\mathrm{J} 0209+1425$ & 77451939817925888 & WD $0206+141$ & DQ & 32.27529 & 14.42246 \\
\hline J0209+2914 & 300086341315084544 & SDSS J020930.15+291423.8 & $\mathrm{DZ}$ & 32.37561 & 29.24007 \\
\hline J0211-0046 & 2494849302842058624 & SDSS J021154.61-004607.2 & DZA & 32.97755 & -0.76867 \\
\hline J0213+2848 & 107870405742373248 & SDSS J021353.94+284855.5 & $\mathrm{DZ}$ & 33.47457 & 28.81548 \\
\hline J0214+0536 & 2519877790967868672 & SDSS J021457.89+053653.6 & $\mathrm{DZ}$ & 33.74121 & 5.61489 \\
\hline J0218-0919 & 5176159517007960576 & PHL 1255 & $\mathrm{DZ}$ & 34.65289 & -9.32915 \\
\hline J0221-0445 & & SDSS J022141.04-044507.3 & $\mathrm{DZ}$ & 35.42100 & -4.75203 \\
\hline J0225-0018 & 2500048030040892800 & 2SLAQ J022509.10-001854.6 & $\mathrm{DZ}$ & 36.28790 & -0.31516 \\
\hline J0226-0444 & & VIPERS 116178525 & $\mathrm{DZ}$ & 36.72992 & -4.73941 \\
\hline J0227+0018 & & SDSS J022751.36+001813.8 & $\mathrm{DQ}$ & 36.96400 & 0.30386 \\
\hline J0228-0009 & 2500373657281469056 & SDSS J022851.97-000938.7 & $\mathrm{DZ}$ & 37.21655 & -0.16077 \\
\hline J0231-0142 & & SDSS J023150.30-014204.5 & $\mathrm{DZ}$ & 37.95958 & -1.70125 \\
\hline J0234-0510 & & SDSS J023407.46-051028.1 & $\mathrm{DZ}$ & 38.53108 & -5.17448 \\
\hline J0236+2503 & 126173494772886144 & SDSS J023633.74+250348.9 & $\mathrm{DQ}$ & 39.14059 & 25.06357 \\
\hline J0239+0027 & 2498791288251035520 & 2SLAQ J023945.02+002745.0 & $\mathrm{DQ}$ & 39.93753 & 0.46252 \\
\hline J0241-0533 & 5184841501339099008 & SDSS J024142.65-053351.5 & DZA & 40.42773 & -5.56418 \\
\hline J0243+0101 & 2498928486686282368 & WD $0240+008$ & $\mathrm{DQ}$ & 40.88657 & 1.01978 \\
\hline J0248+3408 & 139937559287208192 & SDSS J024802.27+340802.4 & DQ & 42.00951 & 34.13424 \\
\hline
\end{tabular}


Table 5. Object names, spectral types, and coordinates - continued

\begin{tabular}{|c|c|c|c|c|c|}
\hline J Name & Gaia source id & MWDD id & Sp. type & R.A & Dec \\
\hline J0251+7341 & 547501815051141248 & EGGR 474 & $\mathrm{DZ}$ & 42.96365 & 73.69309 \\
\hline $\mathrm{J} 0252+0054$ & 2499087366116251392 & SDSS J025253.20+005439.4 & $\mathrm{DZ}$ & 43.22167 & 0.91095 \\
\hline J0252-0401 & 5185475919548087808 & SDSS J025206.06-040130.3 & $\mathrm{DZ}$ & 43.02506 & -4.02508 \\
\hline J0253+3414 & 139799845455078912 & SDSS J025357.12+341436.8 & $\mathrm{DQ}$ & 43.48792 & 34.24362 \\
\hline J0302-0108 & 5187830356195791488 & GD 40 & DBZA & 45.72127 & -1.14272 \\
\hline J0305+0557 & 3940546595098240 & SDSS J030538.53+055734.4 & DQ & 46.41053 & 5.95946 \\
\hline J0308-0657 & 5179967194494422912 & WD $0305-071$ & $\mathrm{DZ}$ & 47.00165 & -6.95002 \\
\hline J0314+0524 & 4081563256565120 & GALEX 2691967329460291916 & $\mathrm{DZ}$ & 48.54539 & 5.41018 \\
\hline J0314-0827 & 5167512029854029184 & SDSS J031448.24-082755.2 & $\mathrm{DZ}$ & 48.70109 & -8.46535 \\
\hline $\mathrm{J} 0319+3630$ & 234469931207274112 & PM J03196+3630 & $\mathrm{DZ}$ & 49.90945 & 36.50824 \\
\hline J0320-0716 & 5169190159475821312 & WD $0318-074$ & DQ & 50.22548 & -7.27374 \\
\hline J0332-0037 & 3263359238612842880 & WD 0329-007 & $\mathrm{DQ}$ & 53.07595 & -0.62287 \\
\hline J0352-0605 & 3245199640146267264 & SDSS J035222.85-060506.3 & $\mathrm{DQ}$ & 58.09523 & -6.08509 \\
\hline J0411-0548 & 3197201731345817856 & WD 0409-059 & $\mathrm{DZ}$ & 62.94123 & -5.81348 \\
\hline J0416+0713 & 3297444373952016640 & SDSS J041601.25+071308.9 & DQ & 64.00522 & 7.21911 \\
\hline J0437-0849 & 3186021141200137472 & GJ 3306 & $\mathrm{DQ}$ & 69.44746 & -8.81915 \\
\hline J0438+4109 & 203931163247581184 & GD 61 & DBZA & 69.66406 & 41.15899 \\
\hline $\mathrm{J} 0447+1124$ & 3295565239861125888 & SDSS J044751.21+112403.8 & $\mathrm{DZ}$ & 71.96337 & 11.40109 \\
\hline J0536+6154 & 286154360759650048 & SDSS J053607.02+615409.8 & DBZA & 84.02918 & 61.90271 \\
\hline J0555-0410 & 3022956969731332096 & NAME HL 4 & $\mathrm{DZ}$ & 88.78954 & -4.16786 \\
\hline J0618+6413 & 1008292775881784192 & SDSS J061844.92+641337.3 & $\mathrm{Z}$ & 94.68737 & 64.22709 \\
\hline J0627+1002 & 3327488430402704000 & WD $0625+10$ & $\mathrm{DZ}$ & 96.90657 & 10.03718 \\
\hline J0649+3726 & 943713544541017088 & SDSS J064912.36+372638.6 & $\mathrm{DZ}$ & 102.30144 & 37.44418 \\
\hline J0707+3825 & 946311759236089984 & SDSS J070731.45+382531.0 & $\mathrm{DQ}$ & 106.88101 & 38.42534 \\
\hline $\mathrm{J} 0710+3740$ & 946030529073021440 & GJ $3431 \mathrm{~B}$ & DQ & 107.55900 & 37.67200 \\
\hline J0721+3928 & & SDSS J072144.23+392843.3 & $\mathrm{DZ}$ & 110.43433 & 39.47872 \\
\hline J0721+3955 & 948173369860748160 & SDSS J072109.48+395539.6 & $\mathrm{DZ}$ & 110.28926 & 39.92783 \\
\hline J0723+3908 & 900040942684340608 & SDSS J072339.09+390839.5 & $\mathrm{DQ}$ & 110.91285 & 39.14440 \\
\hline J0730+3456 & 893881581625207680 & SDSS J073029.07+345613.4 & DBZA & 112.62115 & 34.93706 \\
\hline J0732+2746 & 872428494859607680 & SDSS J073217.01+274641.5 & DBZA & 113.07087 & 27.77822 \\
\hline J0733+3727 & 898724140071904640 & SDSS J073334.19+372735.8 & $\mathrm{DZ}$ & 113.39249 & 37.45998 \\
\hline J0734+4448 & 973522301201644416 & SDSS J073416.91+444801.3 & DBZ & 113.57047 & 44.80037 \\
\hline J0736+4118 & 924478859922238720 & SDSS J073635.22+411828.2 & $\mathrm{DZ}$ & 114.14675 & 41.30783 \\
\hline J0737+6455 & 1089540191183664768 & SDSS J073703.83+645524.6 & $\mathrm{DQ}$ & 114.26592 & 64.92357 \\
\hline J0738+3844 & 899277915974764032 & WD $0735+388$ & DZA & 114.64991 & 38.74394 \\
\hline J0739+0513 & & * alf CMi B & DQZ & 114.82449 & 5.22410 \\
\hline J0739+3024 & & SDSS J073957.64+302439.8 & $\mathrm{DZ}$ & 114.99017 & 30.41108 \\
\hline $\mathrm{J} 0740+1810$ & 671366580221327616 & SDSS J074008.45+181047.2 & DQ & 115.03524 & 18.17983 \\
\hline
\end{tabular}


Table 5. Object names, spectral types, and coordinates - continued

\begin{tabular}{|c|c|c|c|c|c|}
\hline J Name & Gaia source id & MWDD id & Sp. type & R.A & Dec \\
\hline J0740-1724 & 5717278911884258176 & GJ $283 \mathrm{~A}$ & DZA & 115.08662 & -17.41365 \\
\hline J0741+3146 & 880328657704678016 & SDSS J074153.45+314620.4 & $\mathrm{DZ}$ & 115.47275 & 31.77235 \\
\hline J0741+4829 & 981804273754602496 & SDSS J074122.94+482925.9 & $\mathrm{DZ}$ & 115.34558 & 48.49053 \\
\hline J0742+2422 & & SDSS J074226.82+242229.0 & $\mathrm{DZ}$ & 115.61175 & 24.37472 \\
\hline $\mathrm{J} 0742+4348$ & 925185708460455936 & SDSS J074204.79+434835.7 & $\mathrm{DQ}$ & 115.51993 & 43.80994 \\
\hline J0743+4240 & 924315311863541248 & GALEX 2419490867580507837 & DBZ & 115.94924 & 42.67311 \\
\hline $\mathrm{J} 0744+1640$ & 667952729758500224 & SDSS J074456.21+164041.8 & $\mathrm{DZ}$ & 116.23425 & 16.67829 \\
\hline J0744+2701 & 875121993405936640 & SDSS J074450.24+270132.6 & $\mathrm{DZ}$ & 116.20933 & 27.02572 \\
\hline J0744+4408 & & SDSS J074444.03+440845.6 & $\mathrm{DZ}$ & 116.18350 & 44.14603 \\
\hline J0744+4649 & 927618687178189056 & SDSS J074414.66+464912.5 & $\mathrm{DZ}$ & 116.06108 & 46.82017 \\
\hline $\mathrm{J} 0747+3732$ & 919872455957849984 & WD $0744+376$ & $\mathrm{DZ}$ & 116.96413 & 37.53805 \\
\hline $\mathrm{J} 0747+4001$ & 920780618202557312 & WD $0744+401$ & $\mathrm{DZ}$ & 116.93149 & 40.01945 \\
\hline J0747+4825 & 933721354665822720 & SDSS J074729.50+482539.7 & $\mathrm{DZ}$ & 116.87292 & 48.42772 \\
\hline J0748+2544 & & SDSS J074847.45+254431.7 & $\mathrm{DZ}$ & 117.19771 & 25.74214 \\
\hline J0748+3506 & 894591148878550144 & WD $0745+352$ & $\mathrm{DZ}$ & 117.09114 & 35.11352 \\
\hline J0749+3124 & 880840789603989504 & SDSS J074942.87+312424.6 & $\mathrm{DZ}$ & 117.42865 & 31.40686 \\
\hline $\mathrm{J} 0749+4343$ & 925919460672962816 & WD $0746+438$ & $\mathrm{DZ}$ & 117.49277 & 43.71835 \\
\hline $\mathrm{J} 0750+1328$ & 3152307739573943040 & SDSS J075059.15+132855.4 & DQ & 117.74646 & 13.48206 \\
\hline $\mathrm{J} 0750+2329$ & 675367939256072704 & SDSS J075000.55+232945.8 & DQ & 117.50230 & 23.49610 \\
\hline$J 0751+1310$ & 3152283103641386112 & SDSS J075156.89+131018.3 & DBZ & 117.98704 & 13.17175 \\
\hline $\mathrm{J} 0753+2332$ & 675220948295250560 & SDSS J075333.61+233200.1 & DBZA & 118.39009 & 23.53339 \\
\hline$J 0753+2741$ & & SDSS J075306.34+274146.7 & $\mathrm{DZ}$ & 118.27642 & 27.69633 \\
\hline $\mathrm{J} 0753+6613$ & 1094930963554291968 & SDSS J075307.34+661354.0 & $\mathrm{DZ}$ & 118.28067 & 66.23172 \\
\hline$J 0755+2112$ & 673563468875449344 & SDSS J075543.97+211219.6 & $\mathrm{DZ}$ & 118.93321 & 21.20547 \\
\hline J0756+3353 & 881567910387854592 & SDSS J075647.77+335314.6 & $\mathrm{DZ}$ & 119.19904 & 33.88742 \\
\hline J0758+1013 & & SDSS J075853.46+101347.4 & $\mathrm{DZ}$ & 119.72275 & 10.22985 \\
\hline$J 0758+3225$ & 881133160914293888 & SDSS J075846.91+322523.4 & $\mathrm{DZ}$ & 119.69545 & 32.42317 \\
\hline $\mathrm{J} 0800+2242$ & 674322750375093376 & SDSS J080003.90+224210.1 & $\mathrm{DZ}$ & 120.01625 & 22.70281 \\
\hline J0801+1344 & 653532394243172736 & SDSS J080132.37+134410.9 & $\mathrm{DZ}$ & 120.38487 & 13.73638 \\
\hline J0801+1414 & 654305389572962944 & SDSS J080127.10+141455.0 & DBZA & 120.36291 & 14.24867 \\
\hline J0801+5329 & 936641833642799744 & SDSS J080131.16+532900.9 & $\mathrm{DZ}$ & 120.37972 & 53.48352 \\
\hline J0802+3012 & 877285278237322496 & SDSS J080211.41+301256.8 & $\mathrm{DZ}$ & 120.54762 & 30.21581 \\
\hline J0802+4050 & 921482450218605440 & SDSS J080234.18+405015.2 & DBZA & 120.64244 & 40.83756 \\
\hline J0802+4257 & 922800562797653248 & SDSS J080211.74+425747.8 & $\mathrm{DZ}$ & 120.54892 & 42.96328 \\
\hline J0803+4502 & 929208615347675776 & WD $0759+451$ & DZA & 120.88109 & 45.04934 \\
\hline J0804+0750 & 3145823678987592576 & SDSS J080405.76+075055.1 & $\mathrm{DQ}$ & 121.02394 & 7.84869 \\
\hline J0805+3832 & 908957092928687104 & EGGR 346 & $\mathrm{DZ}$ & 121.40687 & 38.53688 \\
\hline J0805+5406 & 1032748903780398592 & SDSS J080513.13+540615.6 & DBZA & 121.30480 & 54.10434 \\
\hline
\end{tabular}


Table 5. Object names, spectral types, and coordinates - continued

\begin{tabular}{|c|c|c|c|c|c|}
\hline J Name & Gaia source id & MWDD id & Sp. type & R.A & Dec \\
\hline J0806+3055 & & SDSS J080626.70+305555.7 & $\mathrm{DZ}$ & 121.61125 & 30.93216 \\
\hline J0806+3640 & 907059606440721024 & SDSS J080615.26+364018.1 & $\mathrm{DZ}$ & 121.56359 & 36.67170 \\
\hline J0806+3702 & 907085926000534400 & SDSS J080607.45+370209.1 & $\mathrm{DZ}$ & 121.53104 & 37.03589 \\
\hline J0806+3747 & 907379121944136832 & SDSS J080602.91+374720.6 & $\mathrm{DZ}$ & 121.51218 & 37.78910 \\
\hline J0807+1949 & 669750259471847680 & SDSS J080708.48+194950.7 & DQA & 121.78535 & 19.83062 \\
\hline J0807+4930 & 934631788948274304 & SDSS J080740.69+493059.7 & $\mathrm{DZ}$ & 121.91954 & 49.51649 \\
\hline $\mathrm{J} 0808+2118$ & & SDSS J080858.40+211827.1 & $\mathrm{DZ}$ & 122.24333 & 21.30756 \\
\hline J0808+2809 & 683943477117809024 & SDSS J080830.98+280915.8 & $\mathrm{DZ}$ & 122.12908 & 28.15439 \\
\hline $\mathrm{J} 0809+1034$ & 3146882852282366208 & SDSS J080910.28+103449.2 & $\mathrm{DZ}$ & 122.29283 & 10.58033 \\
\hline $\mathrm{J} 0809+3106$ & 877057614905717760 & SDSS J080949.93+310642.0 & DBZA & 122.45810 & 31.11170 \\
\hline $\mathrm{J} 0812+0942$ & 3146529359293906816 & SDSS J081252.84+094228.2 & $\mathrm{DZ}$ & 123.22021 & 9.70787 \\
\hline J0812+3614 & & SDSS J081240.79+361427.8 & $\mathrm{DZ}$ & 123.16996 & 36.24108 \\
\hline J0813+3047 & 900818263045818880 & SDSS J081323.31+304744.0 & $\mathrm{DQ}$ & 123.34715 & 30.79562 \\
\hline J0813+3453 & & SDSS J081341.18+345327.7 & $\mathrm{DZ}$ & 123.42158 & 34.89103 \\
\hline J0813+3506 & 902929806407158400 & SDSS J081335.75+350620.2 & $\mathrm{DZ}$ & 123.39896 & 35.10564 \\
\hline J0814+2455 & 682315757527779584 & WD $0811+250$ & $\mathrm{DQ}$ & 123.70203 & 24.92825 \\
\hline $\mathrm{J} 0816+2330$ & & SDSS J081606.19+233030.1 & $\mathrm{DZ}$ & 124.02583 & 23.50839 \\
\hline$J 0818+1247$ & & SDSS J081828.12+124717.2 & $\mathrm{DZ}$ & 124.61721 & 12.78812 \\
\hline J0818-3110 & 5548080118369905408 & WD $0816-310$ & $\mathrm{DZ}$ & 124.66775 & -31.17231 \\
\hline J0819+0027 & 3077580153347317248 & SDSS J081937.67+002716.7 & DZA & 124.90696 & 0.45463 \\
\hline J0819+5732 & 1035126803834023296 & NLTT 19272 & $\mathrm{Z}$ & 124.81237 & 57.54963 \\
\hline J0822+3910 & 908497870729287424 & SDSS J082224.01+391028.9 & $\mathrm{DZ}$ & 125.60009 & 39.17472 \\
\hline $\mathrm{J} 0822+5202$ & 1028460327395772416 & SDSS J082221.21+520248.1 & $\mathrm{DZ}$ & 125.58838 & 52.04672 \\
\hline J0822+5203 & 1028460670993157504 & SDSS J082233.41+520308.4 & $\mathrm{DZ}$ & 125.63922 & 52.05234 \\
\hline $\mathrm{J} 0823+2015$ & 664002940394103808 & SDSS J082346.00+201557.1 & $\mathrm{DZ}$ & 125.94176 & 20.26594 \\
\hline $\mathrm{J} 0825+2009$ & 663815301863336448 & SDSS J082502.20+200921.1 & $\mathrm{DZ}$ & 126.25918 & 20.15586 \\
\hline $\mathrm{J} 0825+2148$ & 664271633551603328 & SDSS J082535.44+214823.9 & $\mathrm{DZ}$ & 126.39767 & 21.80667 \\
\hline J0827+1731 & 662082918214070144 & SDSS J082708.67+173120.6 & DZA & 126.78614 & 17.52237 \\
\hline J0827+2857 & 707499173712737408 & SDSS J082723.78+285729.9 & $\mathrm{DZ}$ & 126.84908 & 28.95833 \\
\hline J0827+3304 & 902982754763873408 & SDSS J082720.59+330438.0 & $\mathrm{DZ}$ & 126.83579 & 33.07725 \\
\hline J0827+5504 & & SDSS J082709.96+550413.5 & $\mathrm{DZ}$ & 126.79150 & 55.07042 \\
\hline J0827+6017 & 1041818603399415040 & SDSS J082748.76+601738.3 & DQ & 126.95321 & 60.29391 \\
\hline $\mathrm{J} 0829+0759$ & 599123782896409984 & SDSS J082927.85+075911.4 & $\mathrm{DZ}$ & 127.36615 & 7.98653 \\
\hline J0829+3551 & & SDSS J082903.99+355125.7 & $\mathrm{DZ}$ & 127.26662 & 35.85717 \\
\hline J0830-0319 & 3066599197577336960 & SDSS J083033.66-031911.0 & $\mathrm{DZ}$ & 127.64013 & -3.31969 \\
\hline J0831+1801 & 659207660948780160 & SDSS J083151.29+180124.1 & $\mathrm{DZ}$ & 127.96371 & 18.02336 \\
\hline J0832-0408 & 3065744460430556032 & SDSS J083231.57-040807.8 & $\mathrm{DQ}$ & 128.13145 & -4.13527 \\
\hline J0833+0512 & 3092743556860766208 & SDSS J083317.64+051201.4 & DBZ & 128.32353 & 5.20040 \\
\hline
\end{tabular}


Table 5. Object names, spectral types, and coordinates - continued

\begin{tabular}{|c|c|c|c|c|c|}
\hline J Name & Gaia source id & MWDD id & Sp. type & R.A & Dec \\
\hline J0833+3638 & 904025332305851904 & SDSS J083310.19+363846.9 & DQ & 128.29246 & 36.64636 \\
\hline $\mathrm{J} 0834+2422$ & 678517387234326528 & SDSS J083421.14+242212.9 & DBZA & 128.58807 & 24.37026 \\
\hline J0834+3927 & & SDSS J083430.67+392721.0 & $\mathrm{DZ}$ & 128.62779 & 39.45583 \\
\hline J0834+4641 & 918504427269976192 & WD $0831+468$ & $\mathrm{DZ}$ & 128.64445 & 46.69187 \\
\hline J0835+0906 & 596684963026743936 & SDSS J083556.32+090619.4 & $\mathrm{DZ}$ & 128.98463 & 9.10545 \\
\hline J0836+0437 & 3092463658136334208 & SDSS J083608.54+043757.6 & $\mathrm{DQ}+\mathrm{DA}$ & 129.03557 & 4.63272 \\
\hline J0836+4817 & 1014805114533282432 & WD $0833+484$ & DQ & 129.15747 & 48.29796 \\
\hline J0837+0321 & 3080032407874946560 & SDSS J083728.83+032129.0 & $\mathrm{DQ}$ & 129.37012 & 3.35806 \\
\hline J0838+1121 & 601707227200568448 & SDSS J083815.75+112118.8 & DQ & 129.56563 & 11.35533 \\
\hline $\mathrm{J} 0838+2322$ & 666207736086203648 & SDSS J083858.56+232252.9 & $\mathrm{DZ}$ & 129.74400 & 23.38137 \\
\hline J0838+5455 & 1030661240436915072 & SDSS J083844.36+545556.2 & $\mathrm{DZ}$ & 129.68483 & 54.93231 \\
\hline J0839+2158 & 665206291446423040 & SDSS J083935.88+215844.7 & $\mathrm{DZ}$ & 129.89953 & 21.97904 \\
\hline J0839+2618 & 702830819140107904 & SDSS J083930.08+261804.9 & DBZA & 129.87535 & 26.30137 \\
\hline J0840+0237 & 3079168604051593856 & SDSS J084020.79+023733.4 & $\mathrm{DZ}$ & 130.08663 & 2.62597 \\
\hline $\mathrm{J} 0840+1306$ & 603090206670150400 & SDSS J084023.21+130624.9 & $\mathrm{DZ}$ & 130.09671 & 13.10692 \\
\hline J0840+3202 & & SDSS J084036.32+320209.0 & $\mathrm{DZ}$ & 130.15133 & 32.03586 \\
\hline $\mathrm{J} 0840+3812$ & & SDSS J084043.88+381231.7 & $\mathrm{DZ}$ & 130.18283 & 38.20881 \\
\hline $\mathrm{J} 0840+4529$ & 917539532801552640 & US 1431 & DQ & 130.00509 & 45.49275 \\
\hline J0841+3329 & 710392886455231360 & SDSS J084131.55+332915.6 & DQ & 130.38147 & 33.48763 \\
\hline J0841+3723 & 909933248799545344 & CBS 78 & DBZ & 130.47928 & 37.38666 \\
\hline J0841+5414 & 1030373477628554624 & SDSS J084110.08+541453.6 & $\mathrm{DZ}$ & 130.29194 & 54.24822 \\
\hline J0842+1406 & 609236712891803264 & SDSS J084223.14+140615.9 & $\mathrm{DZ}$ & 130.59642 & 14.10443 \\
\hline J0842+1536 & & SDSS J084239.85+153628.8 & $\mathrm{DZ}$ & 130.66604 & 15.60801 \\
\hline J0842+3625 & 717705287317984000 & SDSS J084200.24+362540.0 & DZA & 130.50102 & 36.42781 \\
\hline J0842-1347 & 5734737438536674432 & WD $0840-136$ & $\mathrm{DZ}$ & 130.70194 & -13.78700 \\
\hline J0843+1247 & 602283130775405056 & SDSS J084353.36+124732.0 & $\mathrm{DZ}$ & 130.97233 & 12.79222 \\
\hline J0843+5614 & 1031206323326386432 & SDSS J084300.23+561452.8 & $\mathrm{DZ}$ & 130.75102 & 56.24794 \\
\hline J0844+5446 & 1030783114428872704 & SDSS J084415.68+544632.4 & $\mathrm{DZ}$ & 131.06533 & 54.77569 \\
\hline J0845+1258 & 608294087828806016 & SDSS J084551.48+125810.4 & $\mathrm{DZ}$ & 131.46459 & 12.96954 \\
\hline J0845+4115 & 913170417190431488 & SDSS J084502.72+411547.5 & $\mathrm{DZ}$ & 131.26134 & 41.26322 \\
\hline J0845+4802 & & SDSS J084500.85+480223.0 & $\mathrm{DZ}$ & 131.25354 & 48.03975 \\
\hline J0845+5352 & 1029607839578658560 & WD $0841+540$ & $\mathrm{DZ}$ & 131.35417 & 53.86910 \\
\hline J0845+6143 & 1042192506072573312 & SDSS J084531.38+614336.4 & $\mathrm{DQ}$ & 131.38057 & 61.72684 \\
\hline J0846+1024 & 598707239788040448 & SDSS J084624.72+102406.0 & $\mathrm{DQ}$ & 131.60308 & 10.40176 \\
\hline J0846+3538 & 717393648787722624 & Ton 949 & $\mathrm{DZ}$ & 131.68506 & 35.64266 \\
\hline J0847+1830 & 660222170879118208 & LB 8684 & $\mathrm{DQ}$ & 131.95512 & 18.50487 \\
\hline J0847+1919 & 661085768542888064 & SDSS J084719.85+191906.2 & $\mathrm{DQ}$ & 131.83268 & 19.31836 \\
\hline J0847+4507 & 917238713292494080 & SDSS J084709.12+450734.9 & $\mathrm{DZ}$ & 131.78802 & 45.12637 \\
\hline
\end{tabular}


Table 5. Object names, spectral types, and coordinates - continued

\begin{tabular}{|c|c|c|c|c|c|}
\hline J Name & Gaia source id & MWDD id & Sp. type & R.A & Dec \\
\hline J0848+0028 & 3075425797751507840 & WD $0846+006$ & $\mathrm{DZ}$ & 132.24116 & 0.47636 \\
\hline J0848+3548 & 717762977319125632 & SDSS J084849.42+354858.0 & $\mathrm{DZ}$ & 132.20596 & 35.81613 \\
\hline J0848+5214 & 1029230844528601472 & WD $0844+524$ & $\mathrm{DZ}$ & 132.11670 & 52.23955 \\
\hline J0849+0710 & 584716401001115392 & SDSS J084906.69+071030.0 & DZA & 132.27788 & 7.17501 \\
\hline $\mathrm{J} 0849+1827$ & 660212752014880256 & WD $0846+186$ & $\mathrm{DZ}$ & 132.27167 & 18.45876 \\
\hline $\mathrm{J} 0849+1836$ & 660236219716267392 & SDSS J084953.98+183633.9 & $\mathrm{DZ}$ & 132.47489 & 18.60945 \\
\hline $\mathrm{J} 0849+4036$ & 912415155781038464 & SDSS J084911.86+403649.6 & $\mathrm{DZ}$ & 132.29947 & 40.61382 \\
\hline J0850+0709 & 584707506124191616 & SDSS J085030.31+070937.0 & $\mathrm{DQ}$ & 132.62629 & 7.16028 \\
\hline J0851+0538 & 582802361711105152 & SDSS J085141.72+053852.1 & $\mathrm{DZ}$ & 132.92387 & 5.64781 \\
\hline$J 0851+1543$ & 609898313949732608 & WD $0848+159$ & $\mathrm{DZ}$ & 132.75101 & 15.71723 \\
\hline J0852+0428 & 581820218658061952 & SDSS J085239.65+042804.4 & DQ & 133.16526 & 4.46791 \\
\hline J0852+1124 & & SDSS J085214.39+112403.7 & $\mathrm{DZ}$ & 133.05996 & 11.40103 \\
\hline J0852+2316 & 689246765295367808 & SDSS J085235.43+231644.3 & $\mathrm{DQ}$ & 133.14759 & 23.27900 \\
\hline $\mathrm{J} 0852+3402$ & 716230640363477760 & SDSS J085217.60+340211.3 & $\mathrm{DZ}$ & 133.07333 & 34.03649 \\
\hline $\mathrm{J} 0852+5124$ & & SDSS J085242.75+512435.3 & $\mathrm{DZ}$ & 133.17812 & 51.40981 \\
\hline J0852+5223 & 1017236379556868352 & SDSS J085212.53+522336.2 & $\mathrm{DQ}$ & 133.05217 & 52.39337 \\
\hline $\mathrm{J} 0853+1603$ & 611387460714600064 & SDSS J085329.09+160342.1 & $\mathrm{DZ}$ & 133.37121 & 16.06169 \\
\hline $\mathrm{J} 0854+0603$ & 583593696549710080 & SDSS J085454.99+060345.7 & $\mathrm{DZ}$ & 133.72913 & 6.06272 \\
\hline $\mathrm{J} 0854+1847$ & 660375033059366400 & SDSS J085423.47+184733.8 & $\mathrm{DZ}$ & 133.59777 & 18.79277 \\
\hline J0855+0639 & 584402318633098624 & LSPM J0855+0639 & $\mathrm{DQ}$ & 133.77770 & 6.65127 \\
\hline $\mathrm{J} 0855+5438$ & & SDSS J085515.78+543848.3 & $\mathrm{Z}$ & 133.81575 & 54.64675 \\
\hline J0856+4513 & 1010184519997258240 & SDSS J085626.94+451336.9 & $\mathrm{DQ}$ & 134.11228 & 45.22695 \\
\hline J0857+0603 & 583604897824754688 & SDSS J085709.01+060357.4 & $\mathrm{DQ}$ & 134.28755 & 6.06599 \\
\hline J0857+1438 & 608798110831521280 & SDSS J085750.55+143812.9 & $\mathrm{DZ}$ & 134.46061 & 14.63697 \\
\hline $\mathrm{J} 0857+2630$ & 691813197233587712 & SDSS J085749.95+263026.5 & $\mathrm{DZ}$ & 134.45812 & 26.50736 \\
\hline J0859+3257 & 712888090655562624 & EGGR 182 & $\mathrm{DQ}$ & 134.81131 & 32.95338 \\
\hline $\mathrm{J} 0859+5732$ & 1037518722660955392 & WD $0856+577$ & DBZ & 134.98828 & 57.54723 \\
\hline J0859+6016 & 1038076351151065088 & G $234-51$ & $\mathrm{DQ}$ & 134.89893 & 60.27066 \\
\hline J0900+0331 & 578379365734698752 & SDSS J090051.91+033149.3 & DQZ & 135.21635 & 3.53037 \\
\hline J0900+1020 & 603543888359619968 & SDSS J090041.82+102047.5 & $\mathrm{DZ}$ & 135.17425 & 10.34653 \\
\hline J0901+0752 & 584968773279756032 & SDSS J090146.87+075206.8 & $\mathrm{DZ}$ & 135.44529 & 7.86858 \\
\hline J0901+1113 & & SDSS J090159.01+111314.3 & $\mathrm{DZ}$ & 135.49587 & 11.22064 \\
\hline J0901+5337 & 1017732705977200640 & SDSS J090108.41+533749.0 & $\mathrm{DZ}$ & 135.28504 & 53.63028 \\
\hline J0901+5751 & 1037553185478639360 & WD $0858+580$ & $\mathrm{DQ}$ & 135.49130 & 57.85997 \\
\hline J0902+5037 & 1016048043709449088 & WD $0858+508$ & $\mathrm{DQ}$ & 135.50152 & 50.62307 \\
\hline J0903-0120 & & SDSS J090322.57-012014.1 & $\mathrm{DZ}$ & 135.84404 & -1.33728 \\
\hline J0904+3954 & 719439423313644672 & SDSS J090449.72+395416.4 & $\mathrm{DQ}$ & 136.20724 & 39.90461 \\
\hline J0904-0029 & 3842783861947967232 & 2SLAQ J090451.98-002951.6 & $\mathrm{DZ}$ & 136.21636 & -0.49764 \\
\hline
\end{tabular}


Table 5. Object names, spectral types, and coordinates - continued

\begin{tabular}{|c|c|c|c|c|c|}
\hline J Name & Gaia source id & MWDD id & Sp. type & R.A & Dec \\
\hline J0905+0133 & 577045589410219904 & WD $0902+017$ & $\mathrm{DZ}$ & 136.32380 & 1.55211 \\
\hline J0905+0846 & 591125419920174976 & SDSS J090522.38+084657.9 & $\mathrm{DZ}$ & 136.34325 & 8.78278 \\
\hline J0905+0904 & 591207406550605312 & EGGR 532 & DQ & 136.31164 & 9.07396 \\
\hline J0905+5235 & 1017429511350062464 & WD $0902+527$ & $\mathrm{DZ}$ & 136.48424 & 52.59251 \\
\hline J0906+1141 & & SDSS J090652.19+114149.9 & $\mathrm{DZ}$ & 136.71750 & 11.69722 \\
\hline J0906+1628 & 610909070077557632 & SDSS J090605.37+162835.5 & $\mathrm{DZ}$ & 136.52237 & 16.47653 \\
\hline J0906+5956 & 1039309620944640256 & SDSS J090655.64+595654.1 & $\mathrm{DZ}$ & 136.73189 & 59.94849 \\
\hline J0907+2533 & 688417016269720704 & SDSS J090707.34+253313.4 & $\mathrm{DZ}$ & 136.78049 & 25.55389 \\
\hline J0908+4119 & & SDSS J090814.52+411918.3 & $\mathrm{DZ}$ & 137.06050 & 41.32177 \\
\hline J0908+5136 & 1016558973019185664 & SDSS J090803.35+513633.1 & $\mathrm{DZ}$ & 137.01403 & 51.60914 \\
\hline J0909-0045 & 3842065090580902144 & 2SLAQ J090945.07-004559.3 & $\mathrm{DZ}$ & 137.43779 & -0.76648 \\
\hline J0910+1045 & 591988265964810112 & SDSS J091056.79+104541.8 & $\mathrm{DZ}$ & 137.73662 & 10.76164 \\
\hline J0911+2433 & & SDSS J091142.33+243306.6 & $\mathrm{DZ}$ & 137.92637 & 24.55183 \\
\hline J0912-0128 & & SDSS J091211.80-012813.0 & $\mathrm{DZ}$ & 138.04917 & -1.47028 \\
\hline J0913+2627 & 688954024621015040 & SDSS J091322.38+262752.0 & $\mathrm{DZ}$ & 138.34321 & 26.46444 \\
\hline J0913+3116 & 699920381797443712 & LP $313-27$ & $\mathrm{DZ}$ & 138.42393 & 31.26954 \\
\hline J0913+4127 & & SDSS J091356.05+412728.6 & $\mathrm{DZ}$ & 138.48358 & 41.45797 \\
\hline $\mathrm{J} 0915+2019$ & 636095892172507264 & SDSS J091500.58+201903.0 & DQ & 138.75242 & 20.31753 \\
\hline J0916+0110 & & WD $0914+013$ & $\mathrm{DZ}$ & 139.16886 & 1.16844 \\
\hline J0916+1011 & 591040864898749312 & PG 0913+104 & $\mathrm{DQ}$ & 139.01120 & 10.18630 \\
\hline J0916+2540 & 687914432080614528 & SDSS J091621.36+254028.4 & $\mathrm{DZ}$ & 139.08878 & 25.67475 \\
\hline J0916-0212 & 3838621076566036736 & SDSS J091633.07-021256.1 & $\mathrm{DZ}$ & 139.13779 & -2.21558 \\
\hline J0917+2146 & & SDSS J091703.40+214602.4 & $\mathrm{DZ}$ & 139.26417 & 21.76733 \\
\hline J0917+2224 & & SDSS J091722.79+222437.2 & $\mathrm{DZ}$ & 139.34496 & 22.41033 \\
\hline J0918+4843 & 1012116705524378112 & SDSS J091830.27+484323.0 & DQ & 139.62616 & 48.72318 \\
\hline $\mathrm{J} 0919+0236$ & 3845201035182583424 & WD $0916+028$ & DQ & 139.84244 & 2.60141 \\
\hline J0920+3603 & 714642425880664448 & SDSS J092046.95+360353.5 & DQ & 140.19553 & 36.06497 \\
\hline J0921+1204 & 593551801563915520 & SDSS J092110.80+120411.1 & DBZA & 140.29503 & 12.06977 \\
\hline J0921+3421 & 702332431134751360 & SDSS J092153.46+342136.9 & DQ & 140.47275 & 34.36030 \\
\hline J0922+2007 & 637318274224686720 & SDSS J092249.95+200747.1 & DZA & 140.70826 & 20.12975 \\
\hline J0922+2928 & 695775871499726208 & WD J0922+2928 & DQ & 140.53417 & 29.46971 \\
\hline J0923+1842 & & [VV2010] J092356.5+184242 & $\mathrm{DQ}$ & 140.98545 & 18.71184 \\
\hline J0923+4823 & & SDSS J092326.28+482318.1 & $\mathrm{DZ}$ & 140.85950 & 48.38836 \\
\hline J0924+4301 & & SDSS J092450.03+430136.4 & $\mathrm{DZ}$ & 141.20846 & 43.02680 \\
\hline J0925+2256 & & SDSS J092515.41+225634.8 & $\mathrm{DZ}$ & 141.31421 & 22.94300 \\
\hline J0925+3130 & 700557819367554432 & SDSS J092523.10+313019.0 & $\mathrm{DZ}$ & 141.34627 & 31.50545 \\
\hline J0925+5256 & 1019891219101344256 & SDSS J092525.52+525651.9 & $\mathrm{DQ}$ & 141.35638 & 52.94780 \\
\hline J0926+0605 & & SDSS J092638.40+060532.3 & $\mathrm{DQ}$ & 141.66008 & 6.09208 \\
\hline
\end{tabular}


Table 5. Object names, spectral types, and coordinates - continued

\begin{tabular}{|c|c|c|c|c|c|}
\hline J Name & Gaia source id & MWDD id & Sp. type & R.A & Dec \\
\hline J0926+4725 & 819574013135499776 & SDSS J092613.45+472521.1 & DQ & 141.55608 & 47.42256 \\
\hline J0926+6212 & 1039978089654509952 & SDSS J092627.59+621201.7 & DQ & 141.61507 & 62.20044 \\
\hline J0928+1801 & 631623697346239744 & SDSS J092832.62+180149.0 & $\mathrm{DZ}$ & 142.13595 & 18.03035 \\
\hline J0928+2638 & 694176429973819520 & SDSS J092825.16+263856.3 & $\mathrm{DQ}+\mathrm{DA}$ & 142.10482 & 26.64904 \\
\hline J0928+6124 & 1039177168448595328 & WD $0924+616$ & $\mathrm{DZ}$ & 142.00745 & 61.40946 \\
\hline $\mathrm{J} 0929+3310$ & 701307621874580864 & SDSS J092909.03+331011.7 & $\mathrm{DQ}$ & 142.28768 & 33.16993 \\
\hline $\mathrm{J} 0929+4247$ & 814515018401000064 & SDSS J092932.50+424757.9 & $\mathrm{DZ}$ & 142.38542 & 42.79942 \\
\hline $\mathrm{J} 0930+2601$ & 646091930337657472 & SDSS J093046.01+260136.2 & DZA & 142.69171 & 26.02675 \\
\hline J0930+2959 & 697155586793611648 & SDSS J093017.14+295940.7 & DQ & 142.57157 & 29.99471 \\
\hline $\mathrm{J} 0930+3013$ & 697184758211607680 & GALEX 2736932956989820904 & DZA & 142.62391 & 30.22338 \\
\hline J0931+0730 & 586292933172097408 & SDSS J093130.31+073054.6 & DBZ & 142.87629 & 7.51517 \\
\hline $\mathrm{J} 0931+1210$ & & SDSS J093152.85+121022.1 & $\mathrm{DZ}$ & 142.97021 & 12.17281 \\
\hline J0931+1230 & & SDSS J093107.94+123020.4 & $\mathrm{DQ}$ & 142.78308 & 12.50567 \\
\hline J0932+4856 & 825789513712009216 & SDSS J093210.54+485601.7 & $\mathrm{DZ}$ & 143.04397 & 48.93381 \\
\hline J0933+6334 & & SDSS J093320.01+633441.2 & $\mathrm{DZ}$ & 143.33350 & 63.57805 \\
\hline J0934+0822 & 587953535032442624 & SDSS J093423.17+082225.3 & $\mathrm{DZ}$ & 143.59663 & 8.37373 \\
\hline J0934+1158 & 613987187238694144 & SDSS J093448.86+115854.3 & DQ & 143.70375 & 11.98174 \\
\hline $\mathrm{J} 0934+2626$ & 646155255335699584 & SDSS J093414.16+262641.2 & $\mathrm{DZ}$ & 143.55898 & 26.44475 \\
\hline $\mathrm{J} 0934+5632$ & 1025033665052512000 & SDSS J093412.30+563232.8 & $\mathrm{DZ}$ & 143.55131 & 56.54237 \\
\hline J0935+0037 & 3841058384606465664 & SDSS J093545.45+003750.8 & 4 & 143.93937 & 0.63080 \\
\hline $\mathrm{J} 0935+2417$ & 644881986510775680 & SDSS J093537.57+241708.7 & DQ & 143.90640 & 24.28569 \\
\hline J0935+2420 & 644883773217189632 & SDSS J093552.63+242042.8 & $\mathrm{DZ}$ & 143.96935 & 24.34527 \\
\hline $\mathrm{J} 0935+4450$ & 818151103353879808 & US 739 & $\mathrm{DZ}$ & 143.78716 & 44.84049 \\
\hline J0936+0607 & 3852516395279467264 & SDSS J093638.07+060710.0 & $\mathrm{DQ}$ & 144.15867 & 6.11948 \\
\hline J0936+1815 & 633002244409170688 & SDSS J093635.96+181511.9 & DBZA & 144.14983 & 18.25333 \\
\hline $\mathrm{J} 0937+2842$ & 696007387416777856 & SDSS J093708.92+284205.7 & DBZA & 144.28714 & 28.70152 \\
\hline $\mathrm{J} 0937+3646$ & 798980568368200320 & SDSS J093704.99+364647.2 & DZA & 144.27087 & 36.77975 \\
\hline $\mathrm{J} 0937+5228$ & 1020417502917748096 & WD $0933+526$ & $\mathrm{DZ}$ & 144.32982 & 52.46731 \\
\hline J0938+6343 & 1064557259776082944 & SDSS J093855.08+634314.4 & $\mathrm{DZ}$ & 144.72962 & 63.72057 \\
\hline J0939+4136 & 813599709331004288 & SDSS J093916.04+413612.9 & $\mathrm{DZ}$ & 144.81683 & 41.60358 \\
\hline J0939+5019 & 826349066346658304 & SDSS J093944.58+501917.6 & $\mathrm{DZ}$ & 144.93579 & 50.32156 \\
\hline $\mathrm{J} 0939+5201$ & 1020158400426591872 & [VV98b] J093937.9+520146 & $\mathrm{DQ}$ & 144.90700 & 52.02873 \\
\hline J0939+5550 & 1022128244227188224 & WD $0936+560$ & DZA & 144.92632 & 55.84687 \\
\hline J0940+0210 & 3847322577227869952 & WD $0937+023$ & $\mathrm{DQ}$ & 145.01933 & 2.17295 \\
\hline J0940+6136 & 1063014099502601088 & GALEX 2485743040223777830 & $\mathrm{DZ}$ & 145.10506 & 61.61359 \\
\hline J0940+6422 & 1064649481314008832 & SDSS J094024.00+642201.9 & $\mathrm{DZ}$ & 145.10025 & 64.36723 \\
\hline J0941+0901 & 588332900903805824 & 2MASS J09411519+0901549 & $\mathrm{DQ}$ & 145.31326 & 9.03189 \\
\hline J0941+4414 & 820840165198320640 & US 846 & DQ & 145.40866 & 44.24954 \\
\hline
\end{tabular}


Table 5. Object names, spectral types, and coordinates - continued

\begin{tabular}{|c|c|c|c|c|c|}
\hline J Name & Gaia source id & MWDD id & Sp. type & R.A & Dec \\
\hline J0941+5022 & 826323257887507584 & SDSS J094148.75+502214.5 & $\mathrm{DZ}$ & 145.45318 & 50.37072 \\
\hline J0942+0743 & 3854236409423135488 & SDSS J094210.50+074354.8 & $\mathrm{DZ}$ & 145.54380 & 7.73189 \\
\hline J0942+5755 & 1025583077264885248 & WD $0938+581$ & $\mathrm{DZ}$ & 145.52589 & 57.93218 \\
\hline J0944+3939 & 801200997741011840 & SDSS J094415.33+393943.0 & $\mathrm{DZ}$ & 146.06391 & 39.66195 \\
\hline J0944+4408 & 820167229722388864 & SDSS J094451.59+440856.7 & $\mathrm{DZ}$ & 146.21500 & 44.14909 \\
\hline J0944-0039 & 3827999107046766720 & WD 0941-004 & DBZ & 146.13033 & -0.65938 \\
\hline J0945+0846 & 3854957448532256000 & SDSS J094530.20+084624.8 & $\mathrm{DZ}$ & 146.37589 & 8.77356 \\
\hline J0945+5558 & 1022098175160994560 & WD $0941+562$ & $\mathrm{DQ}$ & 146.33656 & 55.97721 \\
\hline J0946+2024 & 639674321484637312 & SDSS J094648.94+202423.2 & $\mathrm{DZ}$ & 146.70392 & 20.40647 \\
\hline J0947+1916 & 627435932433330048 & SDSS J094757.96+191611.6 & $\mathrm{DZ}$ & 146.99150 & 19.26984 \\
\hline $\mathrm{J} 0947+4238$ & 819704575845347328 & SDSS J094743.09+423841.3 & $\mathrm{DZ}$ & 146.92959 & 42.64483 \\
\hline J0948+1232 & 613553056239392768 & LSPM J0948+1232 & $\mathrm{DQ}$ & 147.24135 & 12.54527 \\
\hline J0948+3008 & 744609512219878400 & SDSS J094813.74+300851.2 & $\mathrm{DZ}$ & 147.05731 & 30.14761 \\
\hline $\mathrm{J} 0950+3238$ & 793334404360693632 & CSO 20 & DQA & 147.51397 & 32.64153 \\
\hline $\mathrm{J} 0950+4716$ & 821800829123228544 & SDSS J095023.70+471603.7 & $\mathrm{DZ}$ & 147.59875 & 47.26772 \\
\hline $\mathrm{J} 0950+4848$ & 826488184631550208 & SDSS J095053.05+484811.8 & $\mathrm{DZ}$ & 147.72104 & 48.80328 \\
\hline $\mathrm{J} 0950+5315$ & 1020653077580086784 & EGGR 251 & DQ & 147.57142 & 53.25412 \\
\hline J0951+4033 & 807280785942808320 & SDSS J095119.85+403322.4 & DZA & 147.83278 & 40.55627 \\
\hline $\mathrm{J} 0951+6243$ & 1063462764670566016 & WD $0947+629$ & DQ & 147.90664 & 62.73020 \\
\hline J0953+1510 & 616712429888284288 & SDSS J095339.13+151017.7 & $\mathrm{DZ}$ & 148.41304 & 15.17161 \\
\hline J0953+1719 & 620070750715749504 & SDSS J095354.97+171953.1 & $\mathrm{Z}$ & 148.47904 & 17.33142 \\
\hline J0954+1814 & 626343705070034432 & SDSS J095449.30+181454.7 & DBZA & 148.70542 & 18.24853 \\
\hline J0954+3347 & 795177254568857600 & SDSS J095406.43+334716.5 & $\mathrm{DZ}$ & 148.52687 & 33.78802 \\
\hline J0954+5635 & 1046044748139868544 & SDSS J095435.86+563518.2 & $\mathrm{DZ}$ & 148.64941 & 56.58836 \\
\hline $\mathrm{J} 0955+5233$ & 828417999336648320 & SDSS J095523.49+523350.3 & $\mathrm{DZ}$ & 148.84791 & 52.56391 \\
\hline J0956+5912 & 1049598202217308160 & WD $0953+594$ & DZA & 149.18811 & 59.21129 \\
\hline $\mathrm{J} 0957+2822$ & 743306350418730112 & SDSS J095731.02+282232.4 & $\mathrm{DZ}$ & 149.37924 & 28.37571 \\
\hline J0958+0550 & 3850187217335099904 & SDSS J095854.96+055021.3 & DBAZ & 149.72906 & 5.83906 \\
\hline J0959+2556 & 643650361688870656 & SDSS J095934.77+255618.8 & $\mathrm{DZ}$ & 149.89489 & 25.93849 \\
\hline J0959+4537 & 821981423908534656 & SDSS J095934.95+453725.4 & $\mathrm{DQ}$ & 149.89563 & 45.62374 \\
\hline $\mathrm{J} 1000+1005$ & & SDSS J100059.82+100531.7 & $\mathrm{DQ}$ & 150.24942 & 10.09192 \\
\hline $\mathrm{J} 1000+3518$ & 795577030124098304 & GALEX 2684261956267803251 & DBZA & 150.15443 & 35.30611 \\
\hline $\mathrm{J} 1000+4420$ & 808316555959581568 & US 1170 & DBZA & 150.00505 & 44.33787 \\
\hline J1000-0230 & & SDSS J100031.86-023050.0 & $\mathrm{DZ}$ & 150.13275 & -2.51389 \\
\hline $\mathrm{J} 1002+0313$ & 3836479227914477184 & SDSS J100237.36+031325.6 & $\mathrm{DZ}$ & 150.65568 & 3.22378 \\
\hline $\mathrm{J} 1003+1421$ & & SDSS J100309.89+142117.4 & $\mathrm{DZ}$ & 150.79121 & 14.35483 \\
\hline $\mathrm{J} 1004+0451$ & 3849136565255477760 & SDSS J100421.25+045117.2 & $\mathrm{DZ}$ & 151.08856 & 4.85480 \\
\hline $\mathrm{J} 1004+4231$ & 807172995146946816 & SDSS J100406.63+423151.5 & $\mathrm{DZ}$ & 151.02772 & 42.53097 \\
\hline
\end{tabular}


Table 5. Object names, spectral types, and coordinates - continued

\begin{tabular}{|c|c|c|c|c|c|}
\hline J Name & Gaia source id & MWDD id & Sp. type & R.A & Dec \\
\hline $\mathrm{J} 1005+1746$ & & SDSS J100516.78+174635.3 & $\mathrm{DZ}$ & 151.31992 & 17.77647 \\
\hline $\mathrm{J} 1005+2032$ & 628573377212774656 & SDSS J100553.03+203242.5 & $\mathrm{DZ}$ & 151.47096 & 20.54517 \\
\hline $\mathrm{J} 1005+2244$ & & SDSS J100537.43+224403.1 & $\mathrm{DZ}$ & 151.40596 & 22.73421 \\
\hline $\mathrm{J} 1005+2655$ & 739870788898835584 & SDSS J100509.60+265524.0 & $\mathrm{DZ}$ & 151.29010 & 26.92341 \\
\hline J1005-0114 & 3830084537007178880 & 2QZ J100523.5-011429 & $\mathrm{DQ}$ & 151.34833 & -1.24131 \\
\hline $\mathrm{J} 1006+1752$ & 623291048475264768 & SDSS J100609.16+175221.3 & $\mathrm{DZ}$ & 151.53817 & 17.87261 \\
\hline $\mathrm{J} 1006+6116$ & 1050383902059149184 & SDSS J100617.24+611613.3 & $\mathrm{DZ}$ & 151.57190 & 61.27027 \\
\hline $\mathrm{J} 1008+0248$ & 3836544614496629248 & WD $1005+030$ & $\mathrm{DZ}$ & 152.09894 & 2.81114 \\
\hline $\mathrm{J} 1008+4349$ & 808795706807426688 & SDSS J100817.03+434931.7 & DBZA & 152.07106 & 43.82549 \\
\hline $\mathrm{J} 1009+3337$ & 747179998603216640 & SDSS J100953.25+333754.4 & $\mathrm{DZ}$ & 152.47187 & 33.63181 \\
\hline $\mathrm{J} 1010+2119$ & & SDSS J101048.43+211944.2 & $\mathrm{DZ}$ & 152.70179 & 21.32897 \\
\hline $\mathrm{J} 1010+2300$ & 630102488648801024 & SDSS J101009.54+230017.5 & $\mathrm{DQ}$ & 152.53967 & 23.00494 \\
\hline J1010+3948 & 803490735004698496 & SDSS J101007.84+394852.2 & DZA & 152.53271 & 39.81453 \\
\hline $\mathrm{J} 1012+0040$ & 3831946697387407232 & SDSS J101219.90+004019.7 & $\mathrm{DQ}$ & 153.08290 & 0.67214 \\
\hline $\mathrm{J} 1012+4435$ & 808936302560071040 & SDSS J101217.24+443529.1 & $\mathrm{DZ}$ & 153.07190 & 44.59143 \\
\hline J1012-1843 & 5669427512997660800 & WD 1009-184 & $\mathrm{DZ}$ & 153.00780 & -18.72591 \\
\hline $\mathrm{J} 1013+4350$ & 808657370204219904 & SDSS J101336.56+435054.7 & DQ & 153.40239 & 43.84851 \\
\hline J1013-0251 & & SDSS J101309.20-025105.4 & $\mathrm{DZ}$ & 153.28833 & -2.85153 \\
\hline $\mathrm{J} 1014+2827$ & & SDSS J101451.15+282701.6 & $\mathrm{DZ}$ & 153.71312 & 28.45046 \\
\hline $\mathrm{J} 1015+3518$ & 753390452593714688 & SDSS J101509.57+351813.8 & $\mathrm{DQ}$ & 153.78987 & 35.30393 \\
\hline $\mathrm{J} 1015+4141$ & 805192602906283520 & SDSS J101558.21+414131.7 & DZA & 153.99258 & 41.69215 \\
\hline $\mathrm{J} 1015+6327$ & & SDSS J101506.59+632722.2 & $\mathrm{DZ}$ & 153.77746 & 63.45618 \\
\hline $\mathrm{J} 1017+1948$ & 625133520725582208 & SDSS J101704.38+194848.3 & DBZA & 154.26827 & 19.81342 \\
\hline $\mathrm{J} 1017+2419$ & 725630326616623872 & SDSS J101750.24+241911.6 & $\mathrm{DZ}$ & 154.45935 & 24.31999 \\
\hline $\mathrm{J} 1017+3447$ & 752599079099409408 & SDSS J101711.54+344710.5 & $\mathrm{DZ}$ & 154.29818 & 34.78637 \\
\hline $\mathrm{J} 1017+3736$ & 754237557583214336 & SDSS J101750.38+373637.5 & $\mathrm{DQ}$ & 154.45969 & 37.61052 \\
\hline $\mathrm{J} 1018+0344$ & 3860024719667494912 & SDSS J101805.16+034435.6 & $\mathrm{DZ}$ & 154.52157 & 3.74324 \\
\hline J1018+0838 & 3875789001991057536 & 2MASS J10180002+0838206 & $\mathrm{DQ}$ & 154.50015 & 8.63904 \\
\hline J1018+3708 & 754117577672241920 & SDSS J101800.81+370840.6 & DBZ & 154.50350 & 37.14464 \\
\hline J1018+3726 & 754220107131130624 & CBS 127 & DZA & 154.66160 & 37.44956 \\
\hline J1019+2045 & & SDSS J101959.51+204553.4 & $\mathrm{DZ}$ & 154.99800 & 20.76486 \\
\hline $\mathrm{J} 1019+3535$ & & SDSS J101924.73+353527.6 & $\mathrm{DZ}$ & 154.85308 & 35.59103 \\
\hline $\mathrm{J} 1019+3752$ & 754266832080416512 & SDSS J101929.78+375218.9 & DBZA & 154.87410 & 37.87193 \\
\hline $\mathrm{J} 1022+2845$ & 741044173964155136 & USNO-B1.0 1187-00180823 & $\mathrm{DQ}$ & 155.54874 & 28.75244 \\
\hline J1023-0014 & & SDSS J102306.10-001434.8 & $\mathrm{DZ}$ & 155.77542 & -0.24300 \\
\hline $\mathrm{J} 1024+1014$ & & SDSS J102438.05+101410.5 & $\mathrm{DZ}$ & 156.15854 & 10.23625 \\
\hline $\mathrm{J} 1024+2227$ & & SDSS J102421.89+222747.1 & $\mathrm{DZ}$ & 156.09121 & 22.46310 \\
\hline J1024+4531 & 833191803946601344 & SDSS J102414.83+453109.9 & $\mathrm{DZ}$ & 156.06179 & 45.51942 \\
\hline
\end{tabular}


Table 5. Object names, spectral types, and coordinates - continued

\begin{tabular}{|c|c|c|c|c|c|}
\hline J Name & Gaia source id & MWDD id & Sp. type & R.A & Dec \\
\hline J1024-0017 & & 2SLAQ J102456.50-001732.4 & $\mathrm{DZ}$ & 156.23541 & -0.29232 \\
\hline $\mathrm{J} 1025+2016$ & & SDSS J102540.75+201613.8 & $\mathrm{DZ}$ & 156.41979 & 20.27050 \\
\hline $\mathrm{J} 1026+1948$ & 624581428448988288 & SDSS J102607.59+194808.0 & $\mathrm{DZ}$ & 156.53155 & 19.80217 \\
\hline $\mathrm{J} 1026+2646$ & & SDSS J102620.95+264611.6 & $\mathrm{DZ}$ & 156.58729 & 26.76991 \\
\hline $\mathrm{J} 1026+2725$ & 728685628908570240 & SDSS J102635.07+272507.6 & DQ & 156.64610 & 27.41882 \\
\hline $\mathrm{J} 1026+5807$ & 1047132925349510784 & LSPM J1026+5807 & DQ & 156.64922 & 58.12079 \\
\hline $\mathrm{J} 1027+1218$ & 3883495444630204672 & SDSS J102705.07+121836.0 & DQ & 156.77130 & 12.31006 \\
\hline $\mathrm{J} 1027+4532$ & 830203606220330240 & SDSS J102756.87+453223.3 & $\mathrm{DZ}$ & 156.98707 & 45.53980 \\
\hline $\mathrm{J} 1028+2507$ & & SDSS J102834.08+250724.6 & $\mathrm{DZ}$ & 157.14200 & 25.12352 \\
\hline $\mathrm{J} 1028+2537$ & 727648003464905728 & SDSS J102836.21+253732.8 & DQ & 157.15092 & 25.62570 \\
\hline J1028-0135 & 3782563919332193792 & 2QZ J102807.8-013553 & DBZA & 157.03270 & -1.59797 \\
\hline $\mathrm{J} 1029+1227$ & & SDSS J102956.06+122729.6 & $\mathrm{DZ}$ & 157.48358 & 12.45825 \\
\hline $\mathrm{J} 1029+2013$ & & SDSS J102930.40+201318.8 & $\mathrm{DZ}$ & 157.37667 & 20.22192 \\
\hline $\mathrm{J} 1029+3103$ & 747729754417122304 & SDSS J102955.65+310353.6 & $\mathrm{DZ}$ & 157.48187 & 31.06489 \\
\hline $\mathrm{J} 1030+0026$ & 3855110692965549312 & 2QZ J103024.3+002613 & $\mathrm{DZ}$ & 157.60118 & 0.43729 \\
\hline $\mathrm{J} 1031+0936$ & 3870096319192789120 & SDSS J103123.91+093657.8 & $\mathrm{DZ}$ & 157.84967 & 9.61602 \\
\hline $\mathrm{J} 1031+1203$ & 3883044266905679488 & SDSS J103126.19+120340.4 & $\mathrm{DZ}$ & 157.85916 & 12.06126 \\
\hline $\mathrm{J} 1031+2217$ & 722275854075503616 & SDSS J103102.52+221714.7 & DQ & 157.76050 & 22.28742 \\
\hline $\mathrm{J} 1032+1338$ & & SDSS J103205.15+133833.4 & $\mathrm{DZ}$ & 158.02150 & 13.64262 \\
\hline $\mathrm{J} 1032+2101$ & 720841884754600960 & SDSS J103205.85+210131.1 & $\mathrm{DQ}$ & 158.02437 & 21.02531 \\
\hline J1032+3509 & 751262111614979328 & SDSS J103242.42+350937.3 & $\mathrm{DQ}$ & 158.17683 & 35.16045 \\
\hline $\mathrm{J} 1032+4519$ & 830034079569393536 & SDSS J103210.68+451929.9 & $\mathrm{DQ}$ & 158.04467 & 45.32518 \\
\hline $\mathrm{J} 1032-0240$ & 3781631086795267328 & 2QZ J103257.4-024012 & DBZA & 158.23946 & -2.66978 \\
\hline $\mathrm{J} 1033+1809$ & 3890742944243942528 & SDSS J103352.89+180935.3 & $\mathrm{DZ}$ & 158.47037 & 18.15980 \\
\hline $\mathrm{J} 1033+2837$ & 729218617173430784 & SDSS J103310.69+283707.5 & $\mathrm{DZ}$ & 158.29454 & 28.61867 \\
\hline $\mathrm{J} 1033+6247$ & 1052295059426254208 & SDSS J103300.12+624747.9 & $\mathrm{DZ}$ & 158.25054 & 62.79664 \\
\hline $\mathrm{J} 1034+2245$ & 721648380828681984 & WD $1032+230$ & $\mathrm{DZ}$ & 158.68095 & 22.76358 \\
\hline $\mathrm{J} 1036+4837$ & 834176141731355392 & SDSS J103651.09+483754.0 & DZA & 159.21293 & 48.63169 \\
\hline J1037+0709 & 3862624171313960960 & SDSS J103729.82+070902.7 & DBZA & 159.37429 & 7.15077 \\
\hline $\mathrm{J} 1037+4341$ & 828871861427262464 & PB 424 & $\mathrm{DZ}$ & 159.48588 & 43.68460 \\
\hline J1038+0432 & 3858626690632865664 & SDSS J103839.01+043223.8 & $\mathrm{DZ}$ & 159.66254 & 4.53997 \\
\hline $\mathrm{J} 1038+1342$ & 3884773386379596416 & SDSS J103806.75+134225.3 & $\mathrm{DZ}$ & 159.52812 & 13.70706 \\
\hline J1038-0036 & & WD 1035-003 & $\mathrm{DZ}$ & 159.53837 & -0.60617 \\
\hline $\mathrm{J} 1039+2648$ & 725041881733642112 & SDSS J103923.87+264822.9 & DBZA & 159.84949 & 26.80636 \\
\hline $\mathrm{J} 1039+4612$ & 830469417454250496 & SDSS J103941.85+461224.3 & $\mathrm{DZ}$ & 159.92458 & 46.20681 \\
\hline $\mathrm{J} 1040+0635$ & 3859527160591518464 & SDSS J104052.40+063519.7 & $\mathrm{DQ}$ & 160.21833 & 6.58881 \\
\hline $\mathrm{J} 1040+1349$ & 3884858083134254848 & SDSS J104028.33+134935.5 & $\mathrm{DZ}$ & 160.11804 & 13.82656 \\
\hline $\mathrm{J} 1040+2408$ & 723361209490895744 & SDSS J104046.48+240759.5 & $\mathrm{DZ}$ & 160.19382 & 24.13338 \\
\hline
\end{tabular}


Table 5. Object names, spectral types, and coordinates - continued

\begin{tabular}{|c|c|c|c|c|c|}
\hline J Name & Gaia source id & MWDD id & Sp. type & R.A & Dec \\
\hline $\mathrm{J} 1041+2200$ & 3989437719293787776 & SDSS J104137.38+220032.4 & DQ & 160.40575 & 22.00897 \\
\hline $\mathrm{J} 1041+2746$ & 734116705248896128 & SDSS J104119.39+274617.6 & $\mathrm{DZ}$ & 160.33073 & 27.77157 \\
\hline $\mathrm{J} 1041+3432$ & 750285088159627392 & SDSS J104130.65+343240.7 & $\mathrm{DZ}$ & 160.37767 & 34.54469 \\
\hline $\mathrm{J} 1041+4110$ & 779606383212607104 & SDSS J104156.42+411013.1 & $\mathrm{DZ}$ & 160.48508 & 41.17031 \\
\hline $\mathrm{J} 1042+5833$ & 855241272691342336 & SDSS J104204.27+583347.7 & DQ & 160.51788 & 58.56326 \\
\hline $\mathrm{J} 1043+0303$ & 3857475197016034432 & WD $1041+033$ & $\mathrm{DQ}$ & 160.94469 & 3.05513 \\
\hline $\mathrm{J} 1043+3516$ & 750713313579071232 & SDSS J104319.84+351641.6 & $\mathrm{DZ}$ & 160.83279 & 35.27836 \\
\hline $\mathrm{J} 1044+2023$ & 3987528623509801984 & SDSS J104433.42+202313.0 & $\mathrm{DZ}$ & 161.13942 & 20.38692 \\
\hline $\mathrm{J} 1044+2143$ & 3989359653967394816 & SDSS J104414.35+214337.3 & $\mathrm{DZ}$ & 161.05979 & 21.72706 \\
\hline $\mathrm{J} 1045+2134$ & 3989166483518724480 & SDSS J104505.66+213447.2 & DQ & 161.27358 & 21.57978 \\
\hline $\mathrm{J} 1045+5254$ & 849175610638319616 & SDSS J104504.99+525443.3 & DBZ & 161.27079 & 52.91203 \\
\hline $\mathrm{J} 1045+6009$ & 855674583352060544 & SDSS J104552.51+600921.5 & $\mathrm{DZ}$ & 161.46886 & 60.15590 \\
\hline $\mathrm{J} 1045+6254$ & 1055070364214118272 & SDSS J104511.21+625442.3 & $\mathrm{DZ}$ & 161.29676 & 62.91173 \\
\hline $\mathrm{J} 1046+1329$ & 3884925157638345856 & SDSS J104658.12+132911.3 & $\mathrm{DZ}$ & 161.74217 & 13.48649 \\
\hline $\mathrm{J} 1046+2424$ & 723524383888419840 & SDSS J104652.44+242438.6 & $\mathrm{DZ}$ & 161.71861 & 24.41086 \\
\hline $\mathrm{J} 1047+5219$ & 837084590505236864 & SDSS J104708.84+521935.4 & $\mathrm{DZ}$ & 161.78688 & 52.32647 \\
\hline $\mathrm{J} 1047+5912$ & 855387061061127168 & PSO J161.8956+59.2131 & DQ & 161.89590 & 59.21366 \\
\hline $\mathrm{J} 1048+2623$ & 729995971891636992 & SDSS J104849.77+262333.6 & $\mathrm{DZ}$ & 162.20732 & 26.39267 \\
\hline $\mathrm{J} 1049+1659$ & 3982534641696914688 & SDSS J104906.61+165923.6 & DQA & 162.27753 & 16.98992 \\
\hline $\mathrm{J} 1049+2128$ & & SDSS J104955.29+212832.6 & $\mathrm{DZ}$ & 162.48038 & 21.47574 \\
\hline $\mathrm{J} 1049+5154$ & 836823151552466304 & USNO-B1.0 1419-00233520 & $\mathrm{Z}$ & 162.29808 & 51.90662 \\
\hline J1049-0007 & 3806278083041021312 & [VV2006] J104915.1-000707 & DZA & 162.31276 & -0.11840 \\
\hline $\mathrm{J} 1050+3138$ & 736735600211990144 & SDSS J105019.09+313820.1 & $\mathrm{DZ}$ & 162.57952 & 31.63895 \\
\hline $\mathrm{J} 1051+2751$ & & SDSS J105152.70+275118.7 & $\mathrm{DZ}$ & 162.96958 & 27.85519 \\
\hline $\mathrm{J} 1051+5947$ & & SDSS J105111.87+594735.2 & $\mathrm{DZ}$ & 162.79925 & 59.79287 \\
\hline J1051-0303 & & SDSS J105145.62-030359.3 & $\mathrm{DZ}$ & 162.94008 & -3.06647 \\
\hline $\mathrm{J} 1052+0659$ & 3864644627009594368 & SDSS J105221.56+065915.4 & $\mathrm{DZ}$ & 163.08985 & 6.98763 \\
\hline $\mathrm{J} 1052+5911$ & 861320712999661568 & SBSS $1049+594$ & $\mathrm{DQ}$ & 163.20250 & 59.19730 \\
\hline J1055+3509 & 762519594559623168 & GALEX 2684543414064644977 & $\mathrm{DZ}$ & 163.93342 & 35.15739 \\
\hline $\mathrm{J} 1055+3725$ & 774872607698171392 & SDSS J105533.73+372542.7 & $\mathrm{DZ}$ & 163.89058 & 37.42855 \\
\hline J1055+4115 & 778285113833405056 & SDSS J105523.29+411550.1 & $\mathrm{DZ}$ & 163.84710 & 41.26391 \\
\hline $\mathrm{J} 1056+0128$ & 3808050667584060672 & SDSS J105601.50+012825.0 & DZA & 164.00628 & 1.47358 \\
\hline J1056+5714 & 857165662854257792 & PSO J164.1738+57.2466 & $\mathrm{DZ}$ & 164.17387 & 57.24697 \\
\hline J1056-0004 & 3804750723950984448 & SDSS J105616.89-000449.5 & DZA & 164.07032 & -0.08043 \\
\hline $\mathrm{J} 1057+1053$ & & SDSS J105741.62+105305.3 & $\mathrm{DZ}$ & 164.42342 & 10.88483 \\
\hline J1057-0413 & 3789156870225942656 & G $163-28$ & $\mathrm{DZ}$ & 164.44838 & -4.22504 \\
\hline J1058+1124 & 3871463179649436544 & SDSS J105855.32+112440.2 & $\mathrm{DZ}$ & 164.73050 & 11.41117 \\
\hline $\mathrm{J} 1058+2846$ & 732964137889540992 & SDSS J105817.66+284609.3 & DQ & 164.57346 & 28.76942 \\
\hline
\end{tabular}


Table 5. Object names, spectral types, and coordinates - continued

\begin{tabular}{|c|c|c|c|c|c|}
\hline J Name & Gaia source id & MWDD id & Sp. type & R.A & Dec \\
\hline $\mathrm{J} 1058+3022$ & 733397830802440704 & SDSS J105815.65+302225.8 & DZ & 164.56530 & 30.37378 \\
\hline J1058+3143 & 736915954478557824 & SDSS J105826.60+314358.3 & $\mathrm{DZ}$ & 164.61084 & 31.73290 \\
\hline $\mathrm{J} 1058+3440$ & 762250454729037440 & SDSS J105854.34+344018.0 & DQ & 164.72642 & 34.67169 \\
\hline J1058+6041 & 861745571164680064 & SDSS J105853.70+604136.8 & $\mathrm{DZ}$ & 164.72379 & 60.69358 \\
\hline J1059+5038 & & SDSS J105910.24+503827.5 & $\mathrm{DZ}$ & 164.79267 & 50.64099 \\
\hline $\mathrm{J} 1100+1439$ & & SDSS J110049.52+143915.4 & DZ & 165.20633 & 14.65428 \\
\hline $\mathrm{J} 1100+1758$ & 3983742627018262656 & GALEX 2698265319179290502 & DQA & 165.24184 & 17.96865 \\
\hline $\mathrm{J} 1101+2730$ & & SDSS J110141.48+273017.9 & $\mathrm{DZ}$ & 165.42283 & 27.50499 \\
\hline $\mathrm{J} 1101+5218$ & 836517723541888000 & SDSS J110140.41+521806.6 & DQ & 165.41809 & 52.30202 \\
\hline $\mathrm{J} 1102+0214$ & 3808536101967194368 & SDSS J110234.21+021459.2 & DZ & 165.64254 & 2.24979 \\
\hline $\mathrm{J} 1102+1755$ & 3983562478910025728 & SDSS J110251.37+175552.2 & $\mathrm{DZ}$ & 165.71404 & 17.93117 \\
\hline $\mathrm{J} 1102+2653$ & 730298028350540288 & SDSS J110202.02+265335.5 & $\mathrm{DZ}$ & 165.50843 & 26.89324 \\
\hline $\mathrm{J} 1102+2827$ & & SDSS J110216.09+282730.7 & $\mathrm{DZ}$ & 165.56708 & 28.45854 \\
\hline J1103+4144 & 778183305928430592 & SDSS J110304.15+414434.9 & $\mathrm{DZ}$ & 165.76735 & 41.74307 \\
\hline J1104+0711 & 3818299730867320704 & SDSS J110438.38+071129.8 & $\mathrm{DZ}$ & 166.15996 & 7.19165 \\
\hline J1104+1657 & & SDSS J110426.37+165723.0 & $\mathrm{DZ}$ & 166.10987 & 16.95642 \\
\hline J1104+2439 & 3995629412866477696 & SDSS J110446.03+243933.3 & DZA & 166.19186 & 24.65930 \\
\hline $\mathrm{J} 1104+6426$ & 1055640907669798272 & SDSS J110456.77+642606.2 & DZ & 166.23663 & 64.43498 \\
\hline J1104-1607 & 3562462135495771648 & SDSS J110424.47-160717.0 & $\mathrm{DZ}$ & 166.10201 & -16.12136 \\
\hline J1105+0228 & & SDSS J110556.17+022849.0 & 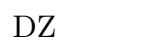 & 166.48408 & 2.48029 \\
\hline J1105+1949 & & SDSS J110550.98+194938.7 & $\mathrm{DZ}$ & 166.46242 & 19.82744 \\
\hline J1105+3801 & 763282281967678848 & SDSS J110502.99+380111.3 & $\mathrm{DZ}$ & 166.26249 & 38.01979 \\
\hline $\mathrm{J} 1106+0104$ & 3805152217493874304 & SDSS J110608.27+010451.7 & $\mathrm{DZ}$ & 166.53446 & 1.08103 \\
\hline J1106-0039 & 3803570123340582400 & 2QZ J110644.2-003928 & $\mathrm{DZ}$ & 166.68450 & -0.65767 \\
\hline $\mathrm{J} 1107+4059$ & 777512951728187392 & SDSS J110759.46+405910.8 & DQ & 166.99806 & 40.98633 \\
\hline J1108+1349 & 3968318334306923264 & SDSS J110831.47+134950.7 & DQ & 167.13112 & 13.83078 \\
\hline $\mathrm{J} 1108+3033$ & 732899545876664832 & SDSS J110836.29+303304.6 & $\mathrm{DZ}$ & 167.15122 & 30.55134 \\
\hline $\mathrm{J} 1109+2112$ & & SDSS J110930.19+211241.0 & $\mathrm{DZ}$ & 167.37563 & 21.21136 \\
\hline J1109+4249 & 778707154499553792 & SDSS J110912.21+424956.0 & DQ & 167.30078 & 42.83225 \\
\hline $\mathrm{J} 1112+0700$ & 3818095805815429760 & SDSS J111215.06+070052.4 & $\mathrm{DZ}$ & 168.06275 & 7.01460 \\
\hline $\mathrm{J} 1113+2228$ & 3991951069730422784 & SDSS J111349.33+222842.9 & $\mathrm{DZ}$ & 168.45558 & 22.47854 \\
\hline$J 1113+4455$ & 785117341649573248 & SDSS J111336.00+445505.1 & DQ & 168.40016 & 44.91819 \\
\hline $\mathrm{J} 1113+4550$ & 788216246452663040 & SDSS J111350.59+455051.2 & $\mathrm{DZ}$ & 168.46073 & 45.84758 \\
\hline J1114+2957 & & SDSS J111409.30+295730.3 & $\mathrm{DZ}$ & 168.53871 & 29.95822 \\
\hline $\mathrm{J} 1115+1720$ & & SDSS J111549.85+172056.8 & DZ & 168.95771 & 17.34914 \\
\hline J1116-1228 & 3565086394873693184 & SDSS J111639.67-122859.0 & DQ & 169.16537 & -12.48307 \\
\hline J1117+0826 & & SDSS J111733.31+082605.3 & $\mathrm{DZ}$ & 169.38879 & 8.43483 \\
\hline $\mathrm{J} 1117+3311$ & 757581378601957376 & SDSS J111741.76+331121.1 & $\mathrm{DZ}$ & 169.42405 & 33.18922 \\
\hline
\end{tabular}


Table 5. Object names, spectral types, and coordinates - continued

\begin{tabular}{|c|c|c|c|c|c|}
\hline J Name & Gaia source id & MWDD id & Sp. type & R.A & Dec \\
\hline $\mathrm{J} 1118+0838$ & & SDSS J111817.14+083801.9 & $\mathrm{DZ}$ & 169.57142 & 8.63386 \\
\hline J1118-0314 & 3790040465258127616 & LAWD 35 & DQ & 169.56277 & -3.23488 \\
\hline $\mathrm{J} 1120+1200$ & 3964058791901412608 & SDSS J112014.78+120053.3 & DQ & 170.06158 & 12.01481 \\
\hline $\mathrm{J} 1120+4252$ & 783773326123820928 & SDSS J112023.61+425200.7 & $\mathrm{DQ}$ & 170.09848 & 42.86685 \\
\hline J1120-1102 & 3565714697049767936 & SDSS J112010.61-110259.5 & DQ & 170.04430 & -11.04992 \\
\hline $\mathrm{J} 1121+1417$ & 3966668139152301568 & SDSS J112125.75+141713.6 & DZA & 170.35727 & 14.28716 \\
\hline $\mathrm{J} 1122+5041$ & 838344081075034240 & WD $1120+509$ & DZA & 170.74312 & 50.69632 \\
\hline $\mathrm{J} 1123+3026$ & 4023114386062671872 & SDSS J112320.76+302634.3 & $\mathrm{DZ}$ & 170.83654 & 30.44270 \\
\hline $\mathrm{J} 1123+3347$ & 759151210623548672 & SDSS J112348.02+334713.7 & DQ & 170.95008 & 33.78717 \\
\hline $\mathrm{J} 1125+2853$ & 4022064867854157696 & SDSS J112505.92+285311.0 & $\mathrm{DZ}$ & 171.27473 & 28.88637 \\
\hline $\mathrm{J} 1125+3823$ & 761097999039664896 & SDSS J112544.76+382316.0 & $\mathrm{DZ}$ & 171.43667 & 38.38782 \\
\hline $\mathrm{J} 1126+3245$ & & SDSS J112634.19+324530.2 & $\mathrm{DQ}$ & 171.64240 & 32.75822 \\
\hline $\mathrm{J} 1126+4419$ & 784290543265061504 & SDSS J112604.28+441938.6 & DQ & 171.51805 & 44.32738 \\
\hline $\mathrm{J} 1126+5241$ & 839578798273255040 & SDSS J112617.16+524155.1 & DZA & 171.57158 & 52.69865 \\
\hline J1127-0138 & 3796601418644353536 & 2QZ J112730.3-013801 & $\mathrm{DZ}$ & 171.87686 & -1.63386 \\
\hline $\mathrm{J} 1129+2917$ & 4021995632981461760 & SDSS J112909.12+291705.0 & $\mathrm{DZ}$ & 172.28806 & 29.28471 \\
\hline J1129-0152 & 3793667612383707904 & SDSS J112956.98-015229.5 & DZA & 172.48745 & -1.87490 \\
\hline $\mathrm{J} 1130+1036$ & 3915497520830530688 & SDSS J113001.60+103614.8 & $\mathrm{DZ}$ & 172.50671 & 10.60415 \\
\hline J1130-0734 & 3592850369145726976 & SDSS J113027.20-073451.8 & DQ & 172.61330 & -7.58097 \\
\hline $\mathrm{J} 1131+0736$ & 3910703753212366080 & SDSS J113127.45+073627.8 & 2 & 172.86443 & 7.60775 \\
\hline $\mathrm{J} 1131+1845$ & 3977321925788597504 & SDSS J113104.23+184558.8 & DQ & 172.76778 & 18.76641 \\
\hline $\mathrm{J} 1131+2315$ & 3980618845764418432 & SDSS J113146.45+231542.4 & $\mathrm{DZ}$ & 172.94345 & 23.26180 \\
\hline $\mathrm{J} 1132+1002$ & & SDSS J113238.95+100207.9 & $\mathrm{DZ}$ & 173.16229 & 10.03556 \\
\hline $\mathrm{J} 1132+3323$ & & SDSS J113209.59+332353.0 & $\mathrm{DZ}$ & 173.03996 & 33.39806 \\
\hline $\mathrm{J} 1132+4705$ & 785774510304049280 & SDSS J113208.77+470523.3 & $\mathrm{DZ}$ & 173.03657 & 47.08982 \\
\hline J1132-0106 & 3794102366153078016 & SDSS J113256.01-010622.7 & $\mathrm{DZ}$ & 173.23338 & -1.10633 \\
\hline $\mathrm{J} 1133+0610$ & 3909508996390412032 & SDSS J113300.18+061037.9 & $\mathrm{DZ}$ & 173.25102 & 6.17723 \\
\hline $\mathrm{J} 1133+1804$ & 3974222196351837056 & SDSS J113319.76+180443.4 & $\mathrm{DZ}$ & 173.33233 & 18.07875 \\
\hline J1133+1900 & 3977309075246562944 & SDSS J113300.58+190056.4 & $\mathrm{DQ}$ & 173.25242 & 19.01569 \\
\hline $\mathrm{J} 1133+6331$ & 863792964894515712 & WD $1131+637$ & $\mathrm{DQ}$ & 173.49982 & 63.52039 \\
\hline J1134+1236 & 3917474786334558976 & SDSS J113426.79+123652.5 & $\mathrm{DZ}$ & 173.61162 & 12.61461 \\
\hline $\mathrm{J} 1134+1542$ & & SDSS J113410.85+154245.9 & $\mathrm{DZ}$ & 173.54525 & 15.71276 \\
\hline J1134+5928 & 858433945220510848 & SDSS J113408.45+592846.1 & $\mathrm{DZ}$ & 173.53535 & 59.47945 \\
\hline $\mathrm{J} 1137+0343$ & 3800902265750001664 & USNO-B1.0 0937-00210798 & $\mathrm{DZ}$ & 174.29715 & 3.72396 \\
\hline J1137-0023 & 3794254579793954688 & 2QZ J113728.3-002343 & $\mathrm{DZ}$ & 174.36813 & -0.39533 \\
\hline J1138-0130 & & SDSS J113818.90-013044.4 & $\mathrm{DZ}$ & 174.57875 & -1.51233 \\
\hline J1139+6239 & 862907411357721088 & SDSS J113912.67+623908.9 & $\mathrm{DZ}$ & 174.80285 & 62.65242 \\
\hline J1139+6737 & 1058091311066785280 & SDSS J113939.48+673737.4 & $\mathrm{DZ}$ & 174.91458 & 67.62708 \\
\hline
\end{tabular}


Table 5. Object names, spectral types, and coordinates - continued

\begin{tabular}{|c|c|c|c|c|c|}
\hline J Name & Gaia source id & MWDD id & Sp. type & R.A & Dec \\
\hline J1139-0132 & & SDSS J113953.58-013240.8 & $\mathrm{DZ}$ & 174.97325 & -1.54467 \\
\hline $\mathrm{J} 1140+0735$ & 3910091810567523456 & SDSS J114059.85+073530.1 & DQ & 175.24938 & 7.59174 \\
\hline $\mathrm{J} 1140+1540$ & 3972370343892609280 & SDSS J114056.78+154014.3 & DQ & 175.23668 & 15.67069 \\
\hline $\mathrm{J} 1140+1824$ & 3974116235214146432 & SDSS J114006.35+182402.3 & $\mathrm{DQ}$ & 175.02664 & 18.40067 \\
\hline J1140+5328 & 840377937068042752 & SDSS J114054.87+532827.4 & DZA & 175.22870 & 53.47426 \\
\hline $\mathrm{J} 1141+3836$ & 766499483055284864 & SDSS J114136.53+383611.9 & $\mathrm{DQ}$ & 175.40208 & 38.60329 \\
\hline $\mathrm{J} 1142+0352$ & 3896760021627103360 & SDSS J114256.66+035207.9 & DQ & 175.73608 & 3.86889 \\
\hline $\mathrm{J} 1142+1104$ & 3916465060998691328 & SDSS J114209.56+110438.6 & $\mathrm{DZ}$ & 175.53983 & 11.07742 \\
\hline $\mathrm{J} 1143+1928$ & 3975786629599506816 & SDSS J114339.16+192855.3 & $\mathrm{DZ}$ & 175.91311 & 19.48209 \\
\hline J1143-0145 & 3793121807939483776 & 2QZ J114310.2-014529 & $\mathrm{DZ}$ & 175.79281 & -1.75797 \\
\hline $\mathrm{J} 1144+1218$ & 3916712206301454720 & SDSS J114441.92+121829.2 & $\mathrm{DZ}$ & 176.17454 & 12.30815 \\
\hline J1144+3259 & 4024772621396103808 & SDSS J114402.34+325911.7 & $\mathrm{DZ}$ & 176.00984 & 32.98665 \\
\hline J1144+3720 & 4032793627440382592 & SDSS J114408.05+372007.8 & $\mathrm{DZ}$ & 176.03354 & 37.33550 \\
\hline $\mathrm{J} 1145+6619$ & 1056978016888546048 & SDSS J114551.29+661923.5 & $\mathrm{DZ}$ & 176.46369 & 66.32313 \\
\hline J1145-6450 & 5332606522595645952 & GJ 440 & DQ & 176.42882 & -64.84152 \\
\hline $\mathrm{J} 1147+4928$ & 787923780658695680 & USNO-B1.0 1394-00221747 & $\mathrm{DZ}$ & 176.80098 & 49.46696 \\
\hline $\mathrm{J} 1147+5429$ & & SDSS J114709.09+542940.8 & $\mathrm{DZ}$ & 176.78792 & 54.49463 \\
\hline $\mathrm{J} 1148+4109$ & 768373570560162176 & SDSS J114804.59+410928.5 & $\mathrm{DZ}$ & 177.01898 & 41.15797 \\
\hline $\mathrm{J} 1148+4124$ & 768406070577604864 & SDSS J114850.37+412429.0 & $\mathrm{DZ}$ & 177.20990 & 41.40807 \\
\hline $\mathrm{J} 1148+5708$ & 845306291781647360 & SDSS J114830.05+570840.6 & $\mathrm{Z}$ & 177.12539 & 57.14472 \\
\hline J1148-0126 & 3794415245931016320 & WD $1146-011$ & DQ & 177.21536 & -1.43686 \\
\hline $\mathrm{J} 1149+0519$ & 3897357571836998784 & SDSS J114944.95+051947.7 & $\mathrm{DZ}$ & 177.43729 & 5.32994 \\
\hline $\mathrm{J} 1149+4943$ & 787933057786712960 & SDSS J114901.99+494328.2 & $\mathrm{DZ}$ & 177.25829 & 49.72440 \\
\hline $\mathrm{J} 1150+4928$ & 787888523268272000 & SDSS J115015.74+492843.7 & $\mathrm{DZ}$ & 177.56554 & 49.47872 \\
\hline $\mathrm{J} 1151+4527$ & 773341881353587968 & SDSS J115149.92+452729.8 & DQ & 177.95822 & 45.45820 \\
\hline $\mathrm{J} 1151-2732$ & 3487220772397809536 & WD $1149-272$ & DQ & 177.90044 & -27.53920 \\
\hline $\mathrm{J} 1152+2834$ & 4019646766906456448 & SDSS J115243.92+283419.8 & $\mathrm{DZ}$ & 178.18297 & 28.57224 \\
\hline $\mathrm{J} 1152+5101$ & & SDSS J115207.15+510126.2 & $\mathrm{DZ}$ & 178.02983 & 51.02389 \\
\hline $\mathrm{J} 1155+4327$ & 769790390076725376 & CSO 1241 & DZ & 178.94766 & 43.46428 \\
\hline $\mathrm{J} 1156+2212$ & 4000563990987070592 & LSPM J1156+2212 & $\mathrm{DQ}$ & 179.01444 & 22.20827 \\
\hline $\mathrm{J} 1157+6138$ & & SDSS J115748.35+613845.9 & $\mathrm{DZ}$ & 179.45146 & 61.64610 \\
\hline $\mathrm{J} 1158+0454$ & 3896383782491848192 & SDSS J115818.76+045447.5 & $\mathrm{DZ}$ & 179.57817 & 4.91321 \\
\hline $\mathrm{J} 1158+1845$ & 3927086888783407616 & SDSS J115809.38+184557.3 & $\mathrm{DZ}$ & 179.53908 & 18.76592 \\
\hline $\mathrm{J} 1158+4712$ & 774517602881392256 & SDSS J115822.32+471214.9 & $\mathrm{DZ}$ & 179.59309 & 47.20416 \\
\hline $\mathrm{J} 1158+5942$ & 846585951516974976 & SDSS J115809.88+594210.3 & $\mathrm{DZ}$ & 179.54125 & 59.70279 \\
\hline $\mathrm{J} 1159+2059$ & & SDSS J115934.82+205903.1 & $\mathrm{DZ}$ & 179.89508 & 20.98421 \\
\hline $\mathrm{J} 1159+4045$ & 4035112669261814912 & SDSS J115958.93+404541.7 & $\mathrm{DZ}$ & 179.99557 & 40.76163 \\
\hline J1159-4629 & 5377861592235273856 & WD $1157-462$ & DQ & 179.99246 & -46.48483 \\
\hline
\end{tabular}


Table 5. Object names, spectral types, and coordinates - continued

\begin{tabular}{|c|c|c|c|c|c|}
\hline J Name & Gaia source id & MWDD id & Sp. type & R.A & Dec \\
\hline $\mathrm{J} 1201+3400$ & 4028120776036373760 & SDSS J120154.70+340055.9 & $\mathrm{DQ}$ & 180.47795 & 34.01537 \\
\hline $\mathrm{J} 1203+0834$ & 3899809757644549632 & WD $1200+088$ & $\mathrm{DZ}$ & 180.85067 & 8.56937 \\
\hline $\mathrm{J} 1203+2323$ & & SDSS J120324.42+232350.7 & $\mathrm{DZ}$ & 180.85175 & 23.39742 \\
\hline $\mathrm{J} 1203+2439$ & 4002914643768684288 & SDSS J120319.78+243955.7 & DBZA & 180.83255 & 24.66546 \\
\hline $\mathrm{J} 1203+6451$ & 1585063422960992256 & WD $1200+651$ & DQ & 180.88292 & 64.85039 \\
\hline $\mathrm{J} 1204+1030$ & & SDSS J120435.35+103044.8 & $\mathrm{DZ}$ & 181.14729 & 10.51244 \\
\hline $\mathrm{J} 1204+5007$ & 1546867312429206656 & SDSS J120436.40+500730.9 & $\mathrm{DZ}$ & 181.15162 & 50.12517 \\
\hline $\mathrm{J} 1205+3536$ & 4029735271423173248 & SDSS J120548.97+353642.4 & $\mathrm{DZ}$ & 181.45408 & 35.61180 \\
\hline $\mathrm{J} 1205+4312$ & 1537735902720638720 & SDSS J120543.74+431226.6 & $\mathrm{DZ}$ & 181.43224 & 43.20739 \\
\hline $\mathrm{J} 1206+1310$ & & SDSS J120646.49+131020.1 & $\mathrm{DZ}$ & 181.69371 & 13.17225 \\
\hline $\mathrm{J} 1206+1454$ & 3921972269928228096 & SDSS J120630.63+145435.6 & $\mathrm{DZ}$ & 181.62762 & 14.90992 \\
\hline $\mathrm{J} 1206+2650$ & 4006630542098974464 & SDSS J120603.74+265026.6 & $\mathrm{DZ}$ & 181.51558 & 26.84075 \\
\hline $\mathrm{J} 1208+1907$ & 3950937048336547840 & SDSS J120840.86+190710.2 & $\mathrm{DZ}$ & 182.17028 & 19.11951 \\
\hline $\mathrm{J} 1209+3026$ & 4014467830917382912 & SDSS J120943.03+302629.1 & $\mathrm{DZ}$ & 182.42933 & 30.44134 \\
\hline $\mathrm{J} 1209+5355$ & 15730020853464858888 & SDSS J120936.50+535525.7 & DQ & 182.40215 & 53.92382 \\
\hline $\mathrm{J} 1210+0553$ & 3895376870362295680 & SDSS J121048.44+055348.2 & DBZA & 182.70194 & 5.89676 \\
\hline $\mathrm{J} 1210+3136$ & 4014824794238339840 & SDSS J121051.66+313659.9 & $\mathrm{DZ}$ & 182.71535 & 31.61664 \\
\hline $\mathrm{J} 1210+4332$ & & SDSS J121050.42+433214.1 & $\mathrm{DZ}$ & 182.71008 & 43.53725 \\
\hline $\mathrm{J} 1210+4751$ & 1546026289113665792 & SDSS J121011.79+475132.8 & $\mathrm{Z}$ & 182.54916 & 47.85908 \\
\hline $\mathrm{J} 1211+2326$ & 4001872474248647936 & SDSS J121106.43+232623.0 & $\mathrm{DZ}$ & 182.77694 & 23.43964 \\
\hline $\mathrm{J} 1212+5409$ & 1573053006478894464 & SDSS J121218.68+540938.7 & $\mathrm{DZ}$ & 183.07791 & 54.16074 \\
\hline $\mathrm{J} 1212+5452$ & 1573476112296657408 & SDSS J121211.03+545222.6 & $\mathrm{DQ}$ & 183.04591 & 54.87302 \\
\hline $\mathrm{J} 1212+6351$ & 1584414053970166784 & SDSS J121231.65+635157.4 & $\mathrm{DZ}$ & 183.13194 & 63.86588 \\
\hline $\mathrm{J} 1214+2216$ & 3953431908939316096 & SDSS J121437.07+221628.3 & $\mathrm{DZ}$ & 183.65461 & 22.27447 \\
\hline $\mathrm{J} 1214+7822$ & 1717341818608187648 & LP $20-214$ & $\mathrm{DZ}$ & 183.63264 & 78.38237 \\
\hline $\mathrm{J} 1215+3953$ & 1532901560546248320 & SDSS J121543.40+395348.8 & $\mathrm{DZ}$ & 183.93088 & 39.89687 \\
\hline $\mathrm{J} 1215+4700$ & 1545134482103819136 & SDSS J121510.64+470011.0 & DQA & 183.79437 & 47.00313 \\
\hline $\mathrm{J} 1216+6208$ & & SDSS J121632.42+620838.3 & $\mathrm{DZ}$ & 184.13508 & 62.14400 \\
\hline J1217+1157 & 3908228546380210432 & SDSS J121731.31+115715.9 & $\mathrm{DZ}$ & 184.38050 & 11.95443 \\
\hline $\mathrm{J} 1217+6420$ & 1584767615678182272 & SDSS J121701.53+642000.1 & $\mathrm{DZ}$ & 184.25635 & 64.33336 \\
\hline J1218+0023 & 3698477839885075200 & SDSS J121837.12+002304.0 & $\mathrm{DZ}$ & 184.65466 & 0.38441 \\
\hline $\mathrm{J} 1218+6023$ & & SDSS J121843.28+602304.2 & $\mathrm{DZ}$ & 184.68033 & 60.38451 \\
\hline $\mathrm{J} 1219+0848$ & 3902219234298271488 & SDSS J121905.53+084840.1 & $\mathrm{DZ}$ & 184.77304 & 8.81117 \\
\hline $\mathrm{J} 1219+3018$ & & SDSS J121927.94+301842.2 & $\mathrm{DZ}$ & 184.86642 & 30.31174 \\
\hline $\mathrm{J} 1219+5720$ & & SDSS J121952.80+572004.1 & $\mathrm{DZ}$ & 184.97000 & 57.33449 \\
\hline $\mathrm{J} 1220+0929$ & & SDSS J122035.76+092948.1 & $\mathrm{DZ}$ & 185.14900 & 9.49672 \\
\hline $\mathrm{J} 1220+2700$ & 4009486184250297344 & SDSS J122021.63+270007.8 & $\mathrm{DQ}$ & 185.09023 & 27.00212 \\
\hline $\mathrm{J} 1222+6343$ & 1583984114858734720 & SDSS J122204.47+634354.6 & $\mathrm{DZ}$ & 185.51878 & 63.73189 \\
\hline
\end{tabular}


Table 5. Object names, spectral types, and coordinates - continued

\begin{tabular}{|c|c|c|c|c|c|}
\hline J Name & Gaia source id & MWDD id & Sp. type & R.A & Dec \\
\hline $\mathrm{J} 1223+1600$ & & SDSS J122303.79+160036.5 & $\mathrm{DZ}$ & 185.76579 & 16.01017 \\
\hline $\mathrm{J} 1223+1935$ & & SDSS J122335.34+193531.9 & $\mathrm{DZ}$ & 185.89725 & 19.59219 \\
\hline $\mathrm{J} 1224+2838$ & 4010119017615780608 & SDSS J122437.07+283853.0 & $\mathrm{DZ}$ & 186.15446 & 28.64805 \\
\hline $\mathrm{J} 1225+4706$ & 1542337839923130624 & PSO J186.4406+47.1036 & $\mathrm{DQ}$ & 186.44143 & 47.10363 \\
\hline J1225-0245 & 3693806156714162816 & 2QZ J122555.3-024547 & $\mathrm{DZ}$ & 186.48067 & -2.76261 \\
\hline $\mathrm{J} 1226+4445$ & 1541711569263906944 & GALEX 2686197079552822523 & DBZ & 186.70841 & 44.75394 \\
\hline $\mathrm{J} 1227+4434$ & & SDSS J122713.08+443431.8 & $\mathrm{DZ}$ & 186.80450 & 44.57552 \\
\hline $\mathrm{J} 1227+6330$ & 1583921850718408960 & WD $1225+637$ & $\mathrm{DZ}$ & 186.88939 & 63.50818 \\
\hline $\mathrm{J} 1229+0743$ & 3901145183236527872 & SDSS J122943.92+074311.8 & $\mathrm{DZ}$ & 187.43300 & 7.71994 \\
\hline $\mathrm{J} 1229+1606$ & & SDSS J122948.48+160640.4 & $\mathrm{DZ}$ & 187.45200 & 16.11125 \\
\hline $\mathrm{J} 1229+3021$ & 4012015915692131456 & SDSS J122906.01+302108.8 & $\mathrm{DZ}$ & 187.27511 & 30.35263 \\
\hline $\mathrm{J} 1229+4254$ & 1535265643690386816 & SDSS J122929.04+425414.4 & $\mathrm{DZ}$ & 187.37100 & 42.90402 \\
\hline $\mathrm{J} 1229+5129$ & 1568771851797540736 & SDSS J122953.17+512925.2 & $\mathrm{DZ}$ & 187.47158 & 51.49034 \\
\hline $\mathrm{J} 1230+0813$ & & SDSS J123013.19+081317.8 & $\mathrm{DZ}$ & 187.55496 & 8.22161 \\
\hline $\mathrm{J} 1230+3143$ & 4012552339927419520 & SDSS J123024.04+314339.7 & $\mathrm{DZ}$ & 187.60017 & 31.72773 \\
\hline $\mathrm{J} 1232+3232$ & & SDSS J123216.13+323240.4 & $\mathrm{DZ}$ & 188.06721 & 32.54456 \\
\hline $\mathrm{J} 1232+3729$ & & SDSS J123229.31+372954.0 & $\mathrm{DZ}$ & 188.12212 & 37.49836 \\
\hline $\mathrm{J} 1233+1253$ & 3931756484602030848 & 2MASS J12334759+1253454 & DQ & 188.44831 & 12.89606 \\
\hline $\mathrm{J} 1234+3051$ & 4012252894807339264 & SDSS J123412.27+305123.1 & $\mathrm{DZ}$ & 188.55117 & 30.85644 \\
\hline $\mathrm{J} 1234+5208$ & 1568852322304497280 & SDSS J123415.21+520808.1 & $\mathrm{DZ}$ & 188.56333 & 52.13564 \\
\hline $\mathrm{J} 1234+5606$ & 15715845399805885544 & SBSS $1232+563$ & DBZA & 188.63615 & 56.11195 \\
\hline J1234-0330 & 3681368652978824960 & WD $1232-032$ & $\mathrm{DZ}$ & 188.73315 & -3.51310 \\
\hline $\mathrm{J} 1235+3918$ & 1533514911941019008 & SDSS J123534.86+391820.4 & DQ & 188.89516 & 39.30573 \\
\hline $\mathrm{J} 1236+2144$ & & SDSS J123645.05+214419.8 & $\mathrm{DZ}$ & 189.18771 & 21.73883 \\
\hline $\mathrm{J} 1236+3502$ & 1518558259563900416 & SDSS J123646.98+350246.8 & DQ & 189.19582 & 35.04632 \\
\hline $\mathrm{J} 1237+4156$ & 1534384148897669248 & WD $1235+422$ & $\mathrm{DQ}$ & 189.46763 & 41.94019 \\
\hline $\mathrm{J} 1238+1328$ & 3932223532230002944 & SDSS J123838.90+132815.3 & $\mathrm{DZ}$ & 189.66208 & 13.47094 \\
\hline $\mathrm{J} 1238+2149$ & 3955605402909063552 & SDSS J123826.93+214937.7 & $\mathrm{DZ}$ & 189.61221 & 21.82714 \\
\hline $\mathrm{J} 1239+0756$ & 3710371635120188288 & SDSS J123927.94+075611.1 & $\mathrm{DZ}$ & 189.86642 & 7.93644 \\
\hline $\mathrm{J} 1239+3522$ & & SDSS J123941.79+352210.2 & $\mathrm{DZ}$ & 189.92412 & 35.36950 \\
\hline $\mathrm{J} 1240+1603$ & 3935035773736441216 & SDSS J124006.86+160357.9 & $\mathrm{DZ}$ & 190.02858 & 16.06608 \\
\hline $\mathrm{J} 1240+1911$ & 3948086603096305664 & SDSS J124020.22+191157.9 & $\mathrm{DZ}$ & 190.08425 & 19.19944 \\
\hline $\mathrm{J} 1240+2748$ & 3962505117546749696 & SDSS J124018.47+274835.3 & $\mathrm{DQ}$ & 190.07693 & 27.80989 \\
\hline J1240-0037 & 3695821316713228032 & SDSS J124006.35-003700.8 & $\mathrm{DZ}$ & 190.02647 & -0.61693 \\
\hline J1240-0144 & 3683297604395370240 & [VV2003b] J124035.0-014500 & DQ & 190.14537 & -1.74991 \\
\hline $\mathrm{J} 1241+3010$ & 4011269965771874688 & SDSS J124159.86+301024.4 & $\mathrm{DZ}$ & 190.49942 & 30.17344 \\
\hline J1242+0829 & 3710792163957803648 & SDSS J124201.80+082945.7 & $\mathrm{DZ}$ & 190.50750 & 8.49603 \\
\hline $\mathrm{J} 1243+1651$ & 3935214272577795712 & WD $1240+171$ & DQA & 190.78028 & 16.85953 \\
\hline
\end{tabular}


Table 5. Object names, spectral types, and coordinates - continued

\begin{tabular}{|c|c|c|c|c|c|}
\hline J Name & Gaia source id & MWDD id & Sp. type & R.A & Dec \\
\hline $\mathrm{J} 1243+3607$ & 1520035689658571264 & SDSS J124338.97+360729.0 & DQ & 190.91237 & 36.12468 \\
\hline $\mathrm{J} 1244+2709$ & 3961476455699563136 & SDSS J124433.81+270923.9 & DQ & 191.14082 & 27.15663 \\
\hline $\mathrm{J} 1244+3724$ & 1520393997305175424 & SDSS J124456.83+372415.5 & $\mathrm{DZ}$ & 191.23679 & 37.40431 \\
\hline $\mathrm{J} 1244+5732$ & 1577634018596346624 & SDSS J124420.37+573239.6 & $\mathrm{DZ}$ & 191.08483 & 57.54425 \\
\hline $\mathrm{J} 1244-0118$ & 3683519637024466176 & SDSS J124437.69-011838.4 & $\mathrm{DZ}$ & 191.15702 & -1.31066 \\
\hline $\mathrm{J} 1245+0822$ & 3710635414832030976 & SDSS J124547.11+082231.4 & $\mathrm{DZ}$ & 191.44629 & 8.37542 \\
\hline $\mathrm{J} 1245+6700$ & 1682132737571666560 & SDSS J124522.69+670036.2 & $\mathrm{DZ}$ & 191.34458 & 67.00999 \\
\hline $\mathrm{J} 1246+1155$ & 3928060510626899200 & WD $1243+121$ & $\mathrm{DZ}$ & 191.62548 & 11.92240 \\
\hline J1246-0236 & & SDSS J124644.88-023619.1 & $\mathrm{DZ}$ & 191.68700 & -2.60531 \\
\hline $\mathrm{J} 1247+4113$ & 1522140502806435456 & LSPM J1247+4113 & DQ & 191.75815 & 41.22808 \\
\hline $\mathrm{J} 1247+4934$ & 1567391625404274304 & WD $1244+498$ & DBZA & 191.76365 & 49.57320 \\
\hline $\mathrm{J} 1248+1411$ & 3930813172345263872 & SDSS J124805.39+141140.4 & DBZ & 192.02248 & 14.19454 \\
\hline J1249+0806 & 3709828029699575808 & USNO-B1.0 0981-00259963 & $\mathrm{DZ}$ & 192.42920 & 8.10377 \\
\hline $\mathrm{J} 1249+2800$ & 3961998375830065152 & SDSS J124926.17+280058.3 & $\mathrm{DQ}$ & 192.35904 & 28.01617 \\
\hline $\mathrm{J} 1249+3407$ & 1515081328918440064 & WIRED J124911.87+340705.5 & DQ & 192.29950 & 34.11820 \\
\hline $\mathrm{J} 1250+0205$ & 3702648596727152768 & 2QZ J125004.5+020517 & $\mathrm{DQ}$ & 192.51896 & 2.08844 \\
\hline $\mathrm{J} 1250+5249$ & 1569926755619671040 & SDSS J125014.58+524931.8 & $\mathrm{DZ}$ & 192.56065 & 52.82546 \\
\hline $\mathrm{J} 1251+4646$ & 1531053247143615488 & PB 10 & DQ & 192.96487 & 46.77426 \\
\hline $\mathrm{J} 1252+1943$ & 3942041105914664832 & LSPM J1252+1943 & DQ & 193.18958 & 19.71999 \\
\hline $\mathrm{J} 1252+3327$ & & SDSS J125228.96+332756.4 & $\mathrm{Z}$ & 193.12067 & 33.46567 \\
\hline $\mathrm{J} 1252+3330$ & & SDSS J125238.85+333008.3 & $\mathrm{Z}$ & 193.16188 & 33.50231 \\
\hline $\mathrm{J} 1252+6401$ & & SDSS J125200.11+640104.9 & $\mathrm{DZ}$ & 193.00046 & 64.01804 \\
\hline $\mathrm{J} 1253+0139$ & 3690553556505229312 & WD $1251+019$ & DQ & 193.49839 & 1.65712 \\
\hline $\mathrm{J} 1253+1808$ & 3940985407247934848 & SDSS J125353.84+180837.9 & DBZ & 193.47446 & 18.14384 \\
\hline $\mathrm{J} 1253+3842$ & & SDSS J125350.98+384225.7 & $\mathrm{DZ}$ & 193.46242 & 38.70714 \\
\hline $\mathrm{J} 1254+2233$ & 3956500645892257408 & GALEX 2695907992019142607 & DBZA & 193.53623 & 22.56331 \\
\hline $\mathrm{J} 1254+3551$ & 1516793058069558784 & SDSS J125454.93+355145.7 & $\mathrm{DZ}$ & 193.72888 & 35.86269 \\
\hline J1254-0236 & 3682412703692940032 & WD $1251-023$ & $\mathrm{DZ}$ & 193.55745 & -2.60237 \\
\hline $\mathrm{J} 1255+4001$ & & SDSS J125515.83+400133.2 & $\mathrm{DZ}$ & 193.81596 & 40.02592 \\
\hline $\mathrm{J} 1257+3238$ & & SDSS J125710.13+323848.5 & $\mathrm{DZ}$ & 194.29221 & 32.64682 \\
\hline J1257+5938 & 1578968688278446080 & SDSS J125724.77+593818.7 & $\mathrm{DQ}$ & 194.35333 & 59.63848 \\
\hline J1257-0310 & & SDSS J125720.87-031025.1 & $\mathrm{DZ}$ & 194.33696 & -3.17365 \\
\hline $\mathrm{J} 1259+3112$ & 1465529324856790656 & SDSS J125922.03+311215.2 & $\mathrm{DZ}$ & 194.84179 & 31.20424 \\
\hline $\mathrm{J} 1259+4729$ & 1554975145532543744 & SDSS J125945.32+472953.6 & $\mathrm{DZ}$ & 194.93887 & 47.49828 \\
\hline J1302+0923 & 3734180047591687936 & SDSS J130201.30+092351.6 & $\mathrm{DQ}$ & 195.50536 & 9.39777 \\
\hline $\mathrm{J} 1302+2215$ & 3944459138142121728 & SDSS J130247.76+221551.0 & $\mathrm{DZ}$ & 195.69900 & 22.26419 \\
\hline J1303+4055 & 1527325967146769664 & SDSS J130328.07+405545.5 & $\mathrm{DZ}$ & 195.86696 & 40.92933 \\
\hline $\mathrm{J} 1303+4418$ & 1529446512759983616 & SDSS J130355.87+441815.8 & $\mathrm{DZ}$ & 195.98277 & 44.30442 \\
\hline
\end{tabular}


Table 5. Object names, spectral types, and coordinates - continued

\begin{tabular}{|c|c|c|c|c|c|}
\hline J Name & Gaia source id & MWDD id & Sp. type & R.A & Dec \\
\hline $\mathrm{J} 1307+0307$ & 3692497733580887040 & WD $1305+033$ & DZA & 196.94308 & 3.12832 \\
\hline $\mathrm{J} 1308+0258$ & & SDSS J130830.03+025844.5 & $\mathrm{DZ}$ & 197.12517 & 2.97903 \\
\hline $\mathrm{J} 1308+0957$ & 3735751868182285312 & SDSS J130826.36+095724.0 & $\mathrm{DZ}$ & 197.10983 & 9.95669 \\
\hline $\mathrm{J} 1309+3812$ & & SDSS J130902.65+381210.3 & $\mathrm{DZ}$ & 197.26104 & 38.20289 \\
\hline $\mathrm{J} 1309+4445$ & 1529705447748085376 & SDSS J130945.62+444540.9 & DQ & 197.44023 & 44.76139 \\
\hline $\mathrm{J} 1309+4913$ & 1554843655110340992 & PB 85 & $\mathrm{DZ}$ & 197.27188 & 49.23329 \\
\hline $\mathrm{J} 1310+0645$ & & SDSS J131021.96+064556.5 & $\mathrm{DZ}$ & 197.59150 & 6.76572 \\
\hline $\mathrm{J} 1310+1323$ & & SDSS J131049.65+132354.3 & $\mathrm{DZ}$ & 197.70687 & 13.39842 \\
\hline $\mathrm{J} 1310+3149$ & 1466195014723444480 & SDSS J131028.90+314901.5 & $\mathrm{DZ}$ & 197.62041 & 31.81710 \\
\hline $\mathrm{J} 1311+1019$ & 3732881936675288192 & SDSS J131116.29+101903.1 & $\mathrm{DZ}$ & 197.81788 & 10.31753 \\
\hline $\mathrm{J} 1313+3811$ & 1522894419890933504 & SDSS J131352.32+381111.3 & DQ & 198.46807 & 38.18644 \\
\hline $\mathrm{J} 1313+5738$ & 1566553007268519168 & LB 2597 & DZA & 198.40429 & 57.63378 \\
\hline $\mathrm{J} 1313+5813$ & 1566610796052681728 & SDSS J131308.79+581321.9 & $\mathrm{DZ}$ & 198.28671 & 58.22271 \\
\hline $\mathrm{J} 1314+3748$ & 1522822577973910016 & SDSS J131420.49+374806.5 & $\mathrm{DZ}$ & 198.58542 & 37.80181 \\
\hline $\mathrm{J} 1314+5223$ & 1557096756297688704 & SBSS $1312+526$ & DBZA & 198.52814 & 52.39741 \\
\hline $\mathrm{J} 1315+4711$ & 1551346520939849728 & NLTT 33455 & $\mathrm{DQ}$ & 198.89459 & 47.18593 \\
\hline $\mathrm{J} 1316+0810$ & 3731530843043705216 & SDSS J131640.71+081058.6 & DQ & 199.16970 & 8.18298 \\
\hline $\mathrm{J} 1316+1916$ & 3939691969258025216 & SDSS J131606.82+191602.9 & DZA & 199.02843 & 19.26748 \\
\hline $\mathrm{J} 1316+1918$ & 3939694335783773696 & SDSS J131612.87+191806.5 & $\mathrm{DZ}$ & 199.05362 & 19.30183 \\
\hline J1316+5117 & 1556792019777662208 & SDSS J131655.06+511747.3 & $\mathrm{Z}$ & 199.22924 & 51.29641 \\
\hline J1316-2007 & 3506567328028533120 & LHS 2710 & $\mathrm{Z}$ & 199.08156 & -20.12557 \\
\hline $\mathrm{J} 1318+2022$ & 3940212274480243072 & SDSS J131826.64+202234.9 & $\mathrm{DZ}$ & 199.61100 & 20.37639 \\
\hline $\mathrm{J} 1319+0844$ & 3731667388643923840 & SDSS J131953.50+084422.8 & $\mathrm{DZ}$ & 199.97292 & 8.73972 \\
\hline $\mathrm{J} 1319+1401$ & 3742980950976788352 & SDSS J131930.66+140137.1 & $\mathrm{DQ}$ & 199.87764 & 14.02705 \\
\hline $\mathrm{J} 1319+3025$ & 1463122974939958528 & OMHR 10489 & $\mathrm{DZ}$ & 199.90127 & 30.42227 \\
\hline $\mathrm{J} 1319+3641$ & 1474478765391306368 & SDSS J131900.19+364149.8 & $\mathrm{DZ}$ & 199.75079 & 36.69717 \\
\hline $\mathrm{J} 1320+0204$ & 3688377657353004032 & SDSS J132005.53+020419.0 & $\mathrm{DZ}$ & 200.02304 & 2.07197 \\
\hline $\mathrm{J} 1320+4332$ & 1526350322380366720 & SDSS J132020.95+433201.1 & $\mathrm{DZ}$ & 200.08729 & 43.53366 \\
\hline J1321+1614 & 3744460000274429312 & SDSS J132147.12+161446.4 & $\mathrm{DZ}$ & 200.44642 & 16.24627 \\
\hline $\mathrm{J} 1321+2019$ & 3940245740865078656 & SDSS J132108.28+201957.6 & $\mathrm{DZ}$ & 200.28450 & 20.33267 \\
\hline J1321-0237 & 3638091080740030336 & SDSS J132144.04-023751.4 & $\mathrm{DZ}$ & 200.43346 & -2.63099 \\
\hline $\mathrm{J} 1322+1224$ & & SDSS J132251.72+122459.0 & $\mathrm{DZ}$ & 200.71550 & 12.41639 \\
\hline J1322+3730 & 1474623595985489664 & [VV2010] J132232.1+373033 & $\mathrm{DQ}$ & 200.63362 & 37.50919 \\
\hline $\mathrm{J} 1322+6707$ & 1684435806410554368 & SDSS J132236.35+670704.9 & DZA & 200.65149 & 67.11804 \\
\hline J1323+2112 & & SDSS J132351.98+211225.7 & $\mathrm{DZ}$ & 200.96658 & 21.20714 \\
\hline J1323+3849 & 1476388823247556608 & SDSS J132330.45+384929.1 & DBZA & 200.87690 & 38.82489 \\
\hline J1325+6521 & 1678166558611938944 & WD $1323+656$ & DZA & 201.27895 & 65.35893 \\
\hline $\mathrm{J} 1326+1854$ & 3939012569855670144 & SDSS J132615.67+185416.6 & $\mathrm{DZ}$ & 201.56535 & 18.90458 \\
\hline
\end{tabular}


Table 5. Object names, spectral types, and coordinates - continued

\begin{tabular}{|c|c|c|c|c|c|}
\hline J Name & Gaia source id & MWDD id & Sp. type & R.A & Dec \\
\hline $\mathrm{J} 1326+4235$ & & SDSS J132649.53+423513.1 & $\mathrm{DZ}$ & 201.70638 & 42.58699 \\
\hline $\mathrm{J} 1327+4508$ & 1550148770522625024 & SDSS J132751.98+450805.4 & $\mathrm{DZ}$ & 201.96674 & 45.13487 \\
\hline $\mathrm{J} 1327+5519$ & & SDSS J132720.10+551927.7 & $\mathrm{DZ}$ & 201.83375 & 55.32438 \\
\hline $\mathrm{J} 1328+3640$ & 1475033331568894080 & SDSS J132825.33+364016.9 & $\mathrm{DQ}$ & 202.10570 & 36.67143 \\
\hline $\mathrm{J} 1329+0746$ & 3719493011785709056 & USNO-B1.0 0977-00289412 & DQ & 202.37961 & 7.78118 \\
\hline $\mathrm{J} 1329+1301$ & 3739809925082831104 & SDSS J132941.79+130131.9 & $\mathrm{DZ}$ & 202.42412 & 13.02553 \\
\hline $\mathrm{J} 1329+6459$ & & SDSS J132933.78+645917.7 & $\mathrm{DZ}$ & 202.39075 & 64.98826 \\
\hline $\mathrm{J} 1331+6704$ & 1684391409332966400 & WD $1329+673$ & $\mathrm{DQ}$ & 202.86259 & 67.07209 \\
\hline $\mathrm{J} 1332+1522$ & 3744903481417287552 & SDSS J133205.52+152248.3 & $\mathrm{DZ}$ & 203.02317 & 15.38020 \\
\hline $\mathrm{J} 1332+2355$ & 1443309397450725376 & SDSS J133221.56+235502.1 & DQA & 203.08983 & 23.91728 \\
\hline $\mathrm{J} 1332+2740$ & 1448881864114808448 & SDSS J133205.63+274003.9 & DQZ & 203.02355 & 27.66770 \\
\hline $\mathrm{J} 1332+5446$ & & SDSS J133242.19+544644.3 & $\mathrm{DZ}$ & 203.17579 & 54.77897 \\
\hline J1333+2357 & 1444622768385439104 & SDSS J133313.73+235721.9 & $\mathrm{DQ}$ & 203.30730 & 23.95598 \\
\hline $\mathrm{J} 1333+3254$ & 1469124246843402624 & GALEX 2699672702652776758 & DBZ & 203.27224 & 32.90003 \\
\hline $\mathrm{J} 1333+6349$ & 1665473315344805760 & WD $1331+640$ & DBZA & 203.27912 & 63.82677 \\
\hline $\mathrm{J} 1334+1622$ & 3745402968934425216 & SDSS J133420.09+162235.8 & $\mathrm{DQ}$ & 203.58353 & 16.37695 \\
\hline $\mathrm{J} 1335+2254$ & & SDSS J133555.81+225422.2 & $\mathrm{DZ}$ & 203.98254 & 22.90617 \\
\hline $\mathrm{J} 1336+3012$ & & SDSS J133620.04+301200.7 & $\mathrm{DZ}$ & 204.08350 & 30.20020 \\
\hline $\mathrm{J} 1336+3547$ & 1471788161655374080 & SDSS J133624.26+354751.2 & $\mathrm{DZ}$ & 204.10113 & 35.79770 \\
\hline J1338+1334 & 3740641224592907904 & SDSS J133836.18+133400.0 & DDLA & 204.65076 & 13.56667 \\
\hline $\mathrm{J} 1338+3255$ & 1468984471427930496 & SDSS J133826.43+325540.8 & $\mathrm{Z}$ & 204.61012 & 32.92800 \\
\hline J1338-0130 & 3661716738057880320 & WD $1335-012$ & $\mathrm{DZ}$ & 204.60410 & -1.50473 \\
\hline $\mathrm{J} 1339+2643$ & 1445611018884630912 & SDSS J133905.98+264322.9 & $\mathrm{DZ}$ & 204.77492 & 26.72303 \\
\hline $\mathrm{J} 1339+6401$ & 1665809972061825408 & SDSS J133938.04+640128.1 & $\mathrm{DZ}$ & 204.90842 & 64.02442 \\
\hline $\mathrm{J} 1340+0835$ & 3724917864718061056 & SDSS J134019.55+083520.1 & $\mathrm{DZ}$ & 205.08152 & 8.58892 \\
\hline $\mathrm{J} 1340+2702$ & 1445645279839722368 & SDSS J134050.31+270219.0 & $\mathrm{DZ}$ & 205.20962 & 27.03864 \\
\hline $\mathrm{J} 1341+0346$ & 3713647252057777536 & SDSS J134124.28+034628.7 & DQA & 205.35117 & 3.77464 \\
\hline $\mathrm{J} 1341+1338$ & 3740678745426551296 & SDSS J134156.63+133845.3 & $\mathrm{DZ}$ & 205.48594 & 13.64595 \\
\hline $\mathrm{J} 1341+2216$ & 1443411828126496512 & SDSS J134107.38+221644.4 & $\mathrm{DZ}$ & 205.28075 & 22.27903 \\
\hline $\mathrm{J} 1341+3024$ & 1456378364457038208 & SDSS J134116.56+302448.8 & $\mathrm{DZ}$ & 205.31897 & 30.41355 \\
\hline J1341-0112 & 3661660211993375360 & WD 1339-009 & $\mathrm{DZ}$ & 205.43375 & -1.21064 \\
\hline J1341-3124 & 6174669289396761088 & WD $1338-311$ & $\mathrm{DZ}$ & 205.35804 & -31.41408 \\
\hline $\mathrm{J} 1342+0522$ & 3714962577202183168 & SDSS J134226.93+052248.6 & $\mathrm{DZ}$ & 205.61222 & 5.38018 \\
\hline $\mathrm{J} 1342+1813$ & & SDSS J134203.60+181332.8 & $\mathrm{DZ}$ & 205.51500 & 18.22578 \\
\hline J1343+2941 & 1455527965227944064 & SDSS J134353.14+294114.1 & $\mathrm{DZ}$ & 205.97140 & 29.68723 \\
\hline $\mathrm{J} 1343+4641$ & 1503980964467235584 & SDSS J134317.31+464104.7 & $\mathrm{DZ}$ & 205.82221 & 46.68464 \\
\hline J1344+1849 & 1248152852388496896 & PB 4069 & $\mathrm{DQ}$ & 206.11017 & 18.82532 \\
\hline $\mathrm{J} 1344+6505$ & 1671965931506508288 & WD $1343+653$ & $\mathrm{DZ}$ & 206.24730 & 65.08681 \\
\hline
\end{tabular}


Table 5. Object names, spectral types, and coordinates - continued

\begin{tabular}{|c|c|c|c|c|c|}
\hline J Name & Gaia source id & MWDD id & Sp. type & R.A & Dec \\
\hline $\mathrm{J} 1345+1153$ & 3728178127148086784 & SDSS J134520.99+115357.6 & $\mathrm{DZ}$ & 206.33746 & 11.89935 \\
\hline $\mathrm{J} 1345+5854$ & 1659379924983087488 & SDSS J134508.12+585421.3 & $\mathrm{DZ}$ & 206.28376 & 58.90588 \\
\hline $\mathrm{J} 1347+0720$ & 3721524466892038656 & SDSS J134721.36+072017.6 & $\mathrm{DZ}$ & 206.83900 & 7.33825 \\
\hline $\mathrm{J} 1347+1415$ & 3740936580903900160 & SDSS J134711.47+141528.0 & $\mathrm{DZ}$ & 206.79779 & 14.25781 \\
\hline $\mathrm{J} 1347+1528$ & 3741346355143254272 & SDSS J134747.94+152851.0 & DQ & 206.94975 & 15.48083 \\
\hline $\mathrm{J} 1347+1733$ & 1244597066144718336 & SDSS J134751.54+173343.0 & $\mathrm{DZ}$ & 206.96475 & 17.56197 \\
\hline $\mathrm{J} 1347+3817$ & 1496440964697320576 & SDSS J134707.57+381749.7 & DQ & 206.78154 & 38.29714 \\
\hline $\mathrm{J} 1347+5019$ & 1558850305545116928 & SDSS J134716.07+501941.9 & $\mathrm{DQ}$ & 206.81686 & 50.32829 \\
\hline $\mathrm{J} 1349+0209$ & & SDSS J134955.76+020931.2 & $\mathrm{DZ}$ & 207.48233 & 2.15867 \\
\hline $\mathrm{J} 1349+0243$ & 3665065605662377088 & WD $1347+029$ & DBZ & 207.48426 & 2.71927 \\
\hline $\mathrm{J} 1350+1058$ & 3727077099396496000 & SDSS J135054.02+105808.0 & $\mathrm{DZ}$ & 207.72508 & 10.96892 \\
\hline $\mathrm{J} 1350+2148$ & & SDSS J135024.97+214814.8 & $\mathrm{DZ}$ & 207.60404 & 21.80414 \\
\hline J1351+1900 & 1245205027355144064 & SDSS J135116.60+190006.3 & DBZ & 207.81914 & 19.00180 \\
\hline $\mathrm{J} 1351+2645$ & 1450680729561299840 & SDSS J135123.86+264546.5 & $\mathrm{DZ}$ & 207.84942 & 26.76292 \\
\hline $\mathrm{J} 1351+4253$ & 1502063317405058304 & LSPM J1351+4253 & $\mathrm{DZ}$ & 207.82669 & 42.88803 \\
\hline $\mathrm{J} 1351+6136$ & 1664233581625001856 & SDSS J135137.07+613607.1 & $\mathrm{DZ}$ & 207.90446 & 61.60199 \\
\hline $\mathrm{J} 1351+6623$ & 1672147149062665472 & WD $1350+666$ & DQ & 207.89347 & 66.38730 \\
\hline $\mathrm{J} 1352+2218$ & 1251457881262355456 & SDSS J135201.98+221811.4 & DQ & 208.00825 & 22.30317 \\
\hline $\mathrm{J} 1352+2658$ & 1450701860800499712 & [CCH92] 1350.7+2713 & DQ & 208.24451 & 26.98175 \\
\hline $\mathrm{J} 1353+1347$ & 3728893389526098432 & SDSS J135320.79+134720.7 & DL & 208.33663 & 13.78908 \\
\hline $\mathrm{J} 1353+3239$ & 1457834740625970560 & SDSS J135343.13+323925.0 & $\mathrm{DZ}$ & 208.42970 & 32.65701 \\
\hline $\mathrm{J} 1354+1217$ & 3727701007821059072 & SDSS J135409.79+121732.1 & $\mathrm{DQ}$ & 208.54079 & 12.29228 \\
\hline J1355+0810 & 3721831501224246272 & SDSS J135543.54+081029.5 & $\mathrm{DZ}$ & 208.93142 & 8.17486 \\
\hline $\mathrm{J} 1355+3636$ & 1495340933377121792 & CSO 1025 & $\mathrm{DQ}$ & 208.81924 & 36.60353 \\
\hline $\mathrm{J} 1356+0236$ & 3664591647431946240 & SDSS J135654.01+023641.5 & $\mathrm{DZ}$ & 209.22504 & 2.61153 \\
\hline $\mathrm{J} 1356+2416$ & 1257923215792065152 & [VV2010] J135632.7+241605 & $\mathrm{DZ}$ & 209.13583 & 24.26841 \\
\hline $\mathrm{J} 1356+4047$ & 1498645171976598144 & SDSS J135637.78+404703.4 & $\mathrm{DZ}$ & 209.15752 & 40.78429 \\
\hline $\mathrm{J} 1356+4342$ & & SDSS J135609.74+434220.8 & $\mathrm{DZ}$ & 209.04058 & 43.70579 \\
\hline J1356-0009 & 3660622479174640640 & WD $1353+000$ & $\mathrm{DQ}$ & 209.11767 & -0.16148 \\
\hline J1357+2949 & 1453674429141463936 & SDSS J135739.52+294921.5 & $\mathrm{DQ}$ & 209.41475 & 29.82261 \\
\hline J1357+5727 & & SDSS J135702.22+572709.1 & $\mathrm{DZ}$ & 209.25925 & 57.45254 \\
\hline $\mathrm{J} 1358+0552$ & 3672120793821311744 & SDSS J135810.42+055237.6 & $\mathrm{DQ}$ & 209.54342 & 5.87711 \\
\hline $\mathrm{J} 1359+3810$ & & SDSS J135953.17+381057.6 & $\mathrm{DZ}$ & 209.97154 & 38.18267 \\
\hline $\mathrm{J} 1400+4507$ & 1505711149093085184 & SDSS J140048.98+450758.7 & $\mathrm{DZ}$ & 210.20404 & 45.13299 \\
\hline J1400-0154 & 3657765432570156160 & 2QZ J140051.6-015413 & $\mathrm{DQ}$ & 210.21494 & -1.90396 \\
\hline $\mathrm{J} 1401+2840$ & 1452751492209135616 & SDSS J140115.38+284048.8 & $\mathrm{DZ}$ & 210.31407 & 28.68019 \\
\hline J1401+3009 & 1453786643751444352 & SDSS J140101.13+300943.3 & $\mathrm{DZ}$ & 210.25469 & 30.16204 \\
\hline J1401+3659 & 1483683911099065088 & SDSS J140137.31+365909.9 & $\mathrm{DZ}$ & 210.40546 & 36.98607 \\
\hline
\end{tabular}


Table 5. Object names, spectral types, and coordinates - continued

\begin{tabular}{|c|c|c|c|c|c|}
\hline J Name & Gaia source id & MWDD id & Sp. type & R.A & Dec \\
\hline $\mathrm{J} 1402+1113$ & 3724362336470991488 & SDSS J140256.39+111332.3 & DQ & 210.73492 & 11.22577 \\
\hline $\mathrm{J} 1402+2506$ & 1258268604177744256 & SDSS J140216.29+250643.0 & DZ & 210.56791 & 25.11192 \\
\hline $\mathrm{J} 1402+2518$ & 1258284336642384768 & SDSS J140207.62+251827.4 & $\mathrm{DZ}$ & 210.53175 & 25.30761 \\
\hline $\mathrm{J} 1402+4742$ & 1509394551702059392 & SDSS J140210.98+474245.3 & $\mathrm{DZ}$ & 210.54573 & 47.71263 \\
\hline J1403+1954 & 1246965487205372672 & SDSS J140336.35+195455.2 & DBZA & 210.90153 & 19.91530 \\
\hline $\mathrm{J} 1404+3620$ & 1483427385588999936 & SDSS J140410.72+362056.8 & $\mathrm{DZ}$ & 211.04486 & 36.34920 \\
\hline J1404-0232 & 3647147315636540032 & SDSS J140445.10-023237.2 & $\mathrm{DZ}$ & 211.18797 & -2.54369 \\
\hline J1405+1549 & 1231399834234447488 & SDSS J140557.10+154940.5 & $\mathrm{DZ}$ & 211.48805 & 15.82794 \\
\hline $\mathrm{J} 1405+2542$ & & SDSS J140525.20+254212.4 & $\mathrm{DZ}$ & 211.35500 & 25.70344 \\
\hline $\mathrm{J} 1406+0148$ & 3667337544578212736 & WD $1403+020$ & DQ & 211.63511 & 1.81065 \\
\hline $\mathrm{J} 1406+0204$ & 3667357915607541632 & WD $1403+023$ & DQ & 211.60707 & 2.07972 \\
\hline J1406+1814 & 1245445167566647936 & SDSS J140653.67+181444.8 & $\mathrm{DZ}$ & 211.72362 & 18.24578 \\
\hline $\mathrm{J} 1406+3402$ & 1481854770426696704 & SDSS J140641.03+340200.3 & $\mathrm{DQ}+\mathrm{DA}$ & 211.67090 & 34.03353 \\
\hline J1406-0037 & 36596435358686664832 & 2QZ J140615.9-003715 & $\mathrm{DZ}$ & 211.56660 & -0.62081 \\
\hline $\mathrm{J} 1407+2039$ & 1247128489804218368 & SDSS J140723.04+203918.6 & DQ & 211.84613 & 20.65518 \\
\hline $\mathrm{J} 1407+3218$ & 1478409656899499392 & SDSS J140730.28+321812.9 & $\mathrm{DZ}$ & 211.87617 & 32.30361 \\
\hline J1408+1535 & 1231351421362931584 & SDSS J140820.04+153507.3 & $\mathrm{DZ}$ & 212.08357 & 15.58531 \\
\hline $\mathrm{J} 1408+1750$ & & SDSS J140838.48+175058.5 & $\mathrm{DZ}$ & 212.16033 & 17.84961 \\
\hline $\mathrm{J} 1409+1254$ & & SDSS J140935.97+125415.3 & $\mathrm{DZ}$ & 212.39988 & 12.90428 \\
\hline $\mathrm{J} 1409+1511$ & 1230575131794474752 & SDSS J140918.87+151136.3 & $\mathrm{D}$ & 212.32875 & 15.19360 \\
\hline$J 1410+1445$ & 1230479444218162432 & SDSS J141037.54+144544.7 & DBZ & 212.65644 & 14.76234 \\
\hline $\mathrm{J} 1411+3410$ & 1479231236900790144 & SDSS J141140.27+341039.4 & $\mathrm{DZ}$ & 212.91779 & 34.17764 \\
\hline $\mathrm{J} 1412+1343$ & 1227084358469891328 & SDSS J141248.87+134324.1 & $\mathrm{DZ}$ & 213.20362 & 13.72339 \\
\hline $\mathrm{J} 1412+4011$ & & SDSS J141215.36+401138.2 & $\mathrm{DZ}$ & 213.06400 & 40.19397 \\
\hline $\mathrm{J} 1413+1659$ & & SDSS J141334.88+165941.1 & $\mathrm{DZ}$ & 213.39533 & 16.99478 \\
\hline $\mathrm{J} 1413+1820$ & & SDSS J141333.27+182030.1 & $\mathrm{DZ}$ & 213.38863 & 18.34172 \\
\hline J1414-0113 & 3647680024725171968 & WD 1411-009 & $\mathrm{DZ}$ & 213.61061 & -1.23184 \\
\hline $\mathrm{J} 1416+0230$ & 3666876265090396672 & SDSS J141615.25+023050.6 & $\mathrm{DZ}$ & 214.06354 & 2.51406 \\
\hline J1416+3016 & 1285060846393119360 & SDSS J141648.86+301653.9 & DQ & 214.20359 & 30.28167 \\
\hline $\mathrm{J} 1417+2412$ & 1255667705126644224 & SDSS J141745.42+241222.6 & DQ & 214.43935 & 24.20630 \\
\hline $\mathrm{J} 1417+2931$ & & SDSS J141706.52+293105.2 & $\mathrm{DZ}$ & 214.27717 & 29.51813 \\
\hline $\mathrm{J} 1419+4400$ & 1504806251022988160 & SDSS J141936.11+440019.9 & $\mathrm{DZ}$ & 214.90046 & 44.00553 \\
\hline $\mathrm{J} 1420+4405$ & & SDSS J142055.12+440507.0 & $\mathrm{DZ}$ & 215.22967 & 44.08528 \\
\hline J1420+4759 & 1507764869311437568 & SDSS J142055.87+475917.5 & $\mathrm{DZ}$ & 215.23278 & 47.98817 \\
\hline $\mathrm{J} 1421+1843$ & 1239668986308981632 & SDSS J142120.11+184351.6 & $\mathrm{DZ}$ & 215.33387 & 18.73104 \\
\hline $\mathrm{J} 1421+5703$ & 1611374216521821952 & SDSS J142119.93+570329.9 & $\mathrm{DZ}$ & 215.33307 & 57.05834 \\
\hline J1422+5229 & 1604959219009055360 & SDSS J142258.01+522925.8 & DBZA & 215.74177 & 52.49048 \\
\hline $\mathrm{J} 1423+5729$ & 1611364359572790016 & WD $1422+577$ & DQ & 215.92766 & 57.49703 \\
\hline
\end{tabular}


Table 5. Object names, spectral types, and coordinates - continued

\begin{tabular}{|c|c|c|c|c|c|}
\hline J Name & Gaia source id & MWDD id & Sp. type & R.A & Dec \\
\hline $\mathrm{J} 1424+0833$ & 1175788425887673728 & SDSS J142413.37+083316.4 & $\mathrm{DQ}$ & 216.05573 & 8.55448 \\
\hline $\mathrm{J} 1424+5657$ & 1611313365425136384 & SDSS J142456.64+565720.8 & $\mathrm{DZ}$ & 216.23598 & 56.95570 \\
\hline $\mathrm{J} 1425+1801$ & 1238621701484033664 & SDSS J142531.98+180116.2 & DQ & 216.38327 & 18.02127 \\
\hline $\mathrm{J} 1425+2302$ & 1254382616552890496 & SDSS J142525.77+230245.3 & DBZA & 216.35740 & 23.04602 \\
\hline $\mathrm{J} 1425-0050$ & 3652865390281317120 & WD $1422-006$ & $\mathrm{DZ}$ & 216.31844 & -0.84690 \\
\hline $\mathrm{J} 1427+4825$ & 1507446659477664512 & SDSS J142744.98+482527.3 & $\mathrm{DZ}$ & 216.93736 & 48.42424 \\
\hline $\mathrm{J} 1427+6110$ & 1666378698746614528 & EGGR 362 & DQ & 216.86790 & 61.17402 \\
\hline J1428+3238 & 1287258636998635008 & SDSS J142812.54+323817.7 & $\mathrm{DQ}$ & 217.05217 & 32.63829 \\
\hline $\mathrm{J} 1428+4210$ & 1491662379787040896 & SDSS J142820.31+421051.5 & $\mathrm{DZ}$ & 217.08463 & 42.18097 \\
\hline $\mathrm{J} 1428+4403$ & 1494157691363079168 & WD $1426+442$ & $\mathrm{DZ}$ & 217.14056 & 44.06304 \\
\hline $\mathrm{J} 1429+3841$ & 1487653182435218048 & SDSS J142939.38+384113.2 & $\mathrm{DZ}$ & 217.41413 & 38.68701 \\
\hline $\mathrm{J} 1429+5839$ & 1611891639821969408 & SDSS J142931.17+583927.9 & $\mathrm{DZ}$ & 217.37998 & 58.65776 \\
\hline $\mathrm{J} 1430+3245$ & & SDSS J143022.06+324521.7 & $\mathrm{DZ}$ & 217.59192 & 32.75603 \\
\hline J1430-0151 & 36495539704955866432 & SDSS J143007.15-015129.5 & $\mathrm{DZ}$ & 217.52983 & -1.85824 \\
\hline $\mathrm{J} 1431+3750$ & 1487348067958833536 & SDSS J143144.83+375011.8 & DQ & 217.93686 & 37.83661 \\
\hline $\mathrm{J} 1432+0354$ & 3668760342689186048 & WD $1430+041$ & DZA & 218.14925 & 3.90645 \\
\hline $\mathrm{J} 1432+1522$ & 1228332918347939968 & SDSS J143253.37+152222.1 & $\mathrm{DZ}$ & 218.22235 & 15.37280 \\
\hline $\mathrm{J} 1432+3542$ & 1480227871175119616 & SDSS J143247.94+354202.0 & $\mathrm{DZ}$ & 218.19975 & 35.70058 \\
\hline $\mathrm{J} 1433+0714$ & 1172289608089050496 & SDSS J143350.83+071452.2 & DBZA & 218.46183 & 7.24785 \\
\hline J1433+6139 & & SDSS J143351.85+613931.7 & DZ & 218.46604 & 61.65881 \\
\hline $\mathrm{J} 1434+2258$ & 1242732500581829632 & SDSS J143437.82+225859.5 & DQA & 218.65765 & 22.98324 \\
\hline $\mathrm{J} 1435+2120$ & 1241254207198151168 & SDSS J143550.13+212014.5 & $\mathrm{DQ}$ & 218.95887 & 21.33736 \\
\hline $\mathrm{J} 1435+4554$ & 1494738469724112512 & SDSS J143548.73+455419.1 & DQ & 218.95313 & 45.90526 \\
\hline $\mathrm{J} 1435+5318$ & 1605233036060102144 & SDSS J143534.01+531815.0 & DQA & 218.89174 & 53.30418 \\
\hline $\mathrm{J} 1436+2152$ & & SDSS J143637.94+215231.3 & $\mathrm{DZ}$ & 219.15808 & 21.87539 \\
\hline $\mathrm{J} 1438+1046$ & & SDSS J143810.86+104639.6 & $\mathrm{DZ}$ & 219.54525 & 10.77767 \\
\hline $\mathrm{J} 1438+2234$ & 1242472569161161472 & SDSS J143859.38+223402.4 & DQ & 219.74742 & 22.56733 \\
\hline $\mathrm{J} 1440+0958$ & 1174728432253334784 & SDSS J144006.80+095835.4 & $\mathrm{DQ}$ & 220.02836 & 9.97659 \\
\hline $\mathrm{J} 1440+1227$ & & SDSS J144026.02+122720.9 & $\mathrm{DZ}$ & 220.10842 & 12.45581 \\
\hline J1440-0232 & 3648790157806712448 & SDSS J144022.52-023222.2 & $\mathrm{DZ}$ & 220.09384 & -2.53951 \\
\hline J1441+0831 & 1172831907840206848 & SDSS J144124.34+083104.6 & $\mathrm{DZ}$ & 220.35151 & 8.51797 \\
\hline $\mathrm{J} 1441+2347$ & 1266711131199577216 & SDSS J144152.14+234704.2 & $\mathrm{DQ}$ & 220.46734 & 23.78444 \\
\hline $\mathrm{J} 1441+4714$ & 1590927320335127424 & SDSS J144129.56+471452.6 & $\mathrm{DZ}$ & 220.37300 & 47.24799 \\
\hline $\mathrm{J} 1441+5215$ & 1605820896823841536 & SDSS J144147.78+521531.4 & $\mathrm{DZ}$ & 220.44881 & 52.25872 \\
\hline $\mathrm{J} 1442+4202$ & 1490005484484145280 & SDSS J144221.53+420250.1 & $\mathrm{DQ}$ & 220.58971 & 42.04728 \\
\hline $\mathrm{J} 1442+5833$ & 1616918538265393536 & SDSS J144237.91+583321.4 & $\mathrm{DZ}$ & 220.65798 & 58.55590 \\
\hline $\mathrm{J} 1443+2054$ & & SDSS J144326.39+205422.1 & $\mathrm{DZ}$ & 220.85996 & 20.90614 \\
\hline $\mathrm{J} 1443+3014$ & & SDSS J144354.13+301413.3 & $\mathrm{DZ}$ & 220.97554 & 30.23706 \\
\hline
\end{tabular}


Table 5. Object names, spectral types, and coordinates - continued

\begin{tabular}{|c|c|c|c|c|c|}
\hline J Name & Gaia source id & MWDD id & Sp. type & R.A & Dec \\
\hline $\mathrm{J} 1443+3810$ & 1486866348722547072 & SDSS J144337.01+381025.7 & DQ & 220.90413 & 38.17387 \\
\hline $\mathrm{J} 1443+5833$ & & SDSS J144301.55+583301.6 & $\mathrm{DZ}$ & 220.75646 & 58.55046 \\
\hline $\mathrm{J} 1444+0434$ & 1158766577140118144 & WD $1441+047$ & DQ & 221.03024 & 4.57966 \\
\hline $\mathrm{J} 1444+1151$ & & SDSS J144449.31+115124.7 & $\mathrm{DZ}$ & 221.20546 & 11.85689 \\
\hline J1444+2059 & & SDSS J144416.52+205946.7 & $\mathrm{DZ}$ & 221.06883 & 20.99633 \\
\hline $\mathrm{J} 1444+4741$ & & SDSS J144416.62+474104.3 & $\mathrm{DZ}$ & 221.06925 & 47.68455 \\
\hline $\mathrm{J} 1444+5741$ & 1616801852593079040 & SDSS J144453.67+574147.3 & $\mathrm{DZ}$ & 221.22369 & 57.69648 \\
\hline $\mathrm{J} 1445+0913$ & & SDSS J144535.03+091340.4 & $\mathrm{DZ}$ & 221.39600 & 9.22791 \\
\hline $\mathrm{J} 1445+5850$ & 1617010931601618432 & WD $1443+590$ & DBZ & 221.32512 & 58.84227 \\
\hline $\mathrm{J} 1445-0208$ & 3648744214542134912 & SDSS J144516.24-020849.6 & $\mathrm{DZ}$ & 221.31766 & -2.14710 \\
\hline $\mathrm{J} 1446+0111$ & & SDSS J144612.30+011139.1 & $\mathrm{DZ}$ & 221.55125 & 1.19422 \\
\hline $\mathrm{J} 1446+0112$ & & SDSS J144604.69+011209.7 & $\mathrm{DZ}$ & 221.51954 & 1.20272 \\
\hline $\mathrm{J} 1447+1340$ & & SDSS J144722.94+134038.3 & $\mathrm{DZ}$ & 221.84558 & 13.67731 \\
\hline $\mathrm{J} 1448+1047$ & 1175204104176381696 & SDSS J144804.49+104709.1 & $\mathrm{DZ}$ & 222.01871 & 10.78586 \\
\hline $\mathrm{J} 1448+2946$ & & SDSS J144820.62+294629.5 & $\mathrm{DZ}$ & 222.08592 & 29.77486 \\
\hline J1448+2958 & 1282587151389251968 & SDSS J144830.61+295817.2 & $\mathrm{DZ}$ & 222.12754 & 29.97147 \\
\hline J1448-0047 & 3650615686411758080 & WD $1445-005$ & DQ & 222.03361 & -0.79886 \\
\hline $\mathrm{J} 1449+2916$ & 1281751453832480128 & SDSS J144954.84+291633.7 & DQ & 222.47850 & 29.27603 \\
\hline $\mathrm{J} 1452+1346$ & 1185098132573773184 & SDSS J145213.98+134633.5 & DBZ & 223.05827 & 13.77596 \\
\hline $\mathrm{J} 1452+4544$ & & SDSS J145216.86+454448.6 & $\mathrm{DZ}$ & 223.07025 & 45.74684 \\
\hline $\mathrm{J} 1452+6020$ & 1617584636152648448 & WD $1451+605$ & DQ & 223.15241 & 60.34342 \\
\hline $\mathrm{J} 1453+2452$ & & SDSS J145351.49+245244.0 & $\mathrm{DZ}$ & 223.46454 & 24.87889 \\
\hline $\mathrm{J} 1453+3003$ & & SDSS J145304.75+300311.5 & $\mathrm{DZ}$ & 223.26979 & 30.05322 \\
\hline J1453+3241 & 1289479302593197056 & SDSS J145309.32+324145.1 & $\mathrm{DZ}$ & 223.28883 & 32.69589 \\
\hline $\mathrm{J} 1453+4719$ & 1590415360233819776 & SDSS J145312.96+471910.5 & DQ & 223.30403 & 47.31958 \\
\hline $\mathrm{J} 1455+2010$ & 1237285592992374784 & SDSS J145558.75+201057.6 & $\mathrm{DZ}$ & 223.99479 & 20.18267 \\
\hline $\mathrm{J} 1455+4209$ & 1489435937460963712 & SDSS J145524.89+420910.8 & DQ & 223.85373 & 42.15300 \\
\hline J1458+4719 & 1587462866571138048 & SDSS J145832.11+471955.8 & DBZA & 224.63386 & 47.33219 \\
\hline $\mathrm{J} 1500+2315$ & & SDSS J150028.02+231554.0 & $\mathrm{DZ}$ & 225.11679 & 23.26501 \\
\hline $\mathrm{J} 1501+0627$ & 1160138114816313472 & SDSS J150136.90+062720.5 & $\mathrm{DQ}$ & 225.40373 & 6.45582 \\
\hline $\mathrm{J} 1501+3807$ & 1295272393132844288 & SDSS J150134.65+380747.2 & $\mathrm{DZ}$ & 225.39437 & 38.12978 \\
\hline $\mathrm{J} 1501+5609$ & 1612720366646956928 & SDSS J150119.76+560914.5 & $\mathrm{DZ}$ & 225.33233 & 56.15403 \\
\hline $\mathrm{J} 1502+3744$ & 1295063619067459712 & SDSS J150228.71+374452.9 & $\mathrm{DZ}$ & 225.61962 & 37.74803 \\
\hline $\mathrm{J} 1503+4414$ & & SDSS J150302.85+441423.2 & $\mathrm{DZ}$ & 225.76187 & 44.23978 \\
\hline $\mathrm{J} 1503+4642$ & & SDSS J150351.94+464243.6 & $\mathrm{DZ}$ & 225.96642 & 46.71214 \\
\hline $\mathrm{J} 1506+4152$ & 1392973720770987392 & BSO 24 & $\mathrm{DZ}$ & 226.60932 & 41.87949 \\
\hline J1506+5844 & 1614158871454061696 & SDSS J150603.62+584449.2 & $\mathrm{DZ}$ & 226.51518 & 58.74693 \\
\hline $\mathrm{J} 1507+2633$ & 1268369057294713472 & SDSS J150710.20+263312.5 & $\mathrm{DZ}$ & 226.79250 & 26.55350 \\
\hline
\end{tabular}


Table 5. Object names, spectral types, and coordinates - continued

\begin{tabular}{|c|c|c|c|c|c|}
\hline J Name & Gaia source id & MWDD id & Sp. type & R.A & Dec \\
\hline $\mathrm{J} 1507+4034$ & & SDSS J150739.03+403408.9 & $\mathrm{DZ}$ & 226.91267 & 40.56916 \\
\hline $\mathrm{J} 1508+0622$ & 1157277288640874496 & SDSS J150822.60+062242.1 & $\mathrm{DZ}$ & 227.09417 & 6.37836 \\
\hline $\mathrm{J} 1509+1620$ & 1184399079402015616 & SDSS J150901.11+162012.3 & DQ & 227.25481 & 16.33653 \\
\hline $\mathrm{J} 1509+1838$ & 1211965588257187840 & SDSS J150926.04+183825.4 & $\mathrm{DZ}$ & 227.35850 & 18.64039 \\
\hline $\mathrm{J} 1510+3814$ & 1292517802972365952 & SDSS J151054.85+381450.2 & DZA & 227.72857 & 38.24729 \\
\hline $\mathrm{J} 1511+2654$ & 1265489814296750592 & SDSS J151133.87+265437.9 & $\mathrm{DZ}$ & 227.89112 & 26.91053 \\
\hline $\mathrm{J} 1511+5008$ & 1589607700223131264 & SDSS J151118.19+500800.7 & $\mathrm{DQ}$ & 227.82576 & 50.13355 \\
\hline $\mathrm{J} 1512+5304$ & & SDSS J151221.62+530426.3 & $\mathrm{DZ}$ & 228.09008 & 53.07398 \\
\hline $\mathrm{J} 1514+1423$ & 1183073068378187776 & SDSS J151417.00+142354.1 & $\mathrm{DQ}$ & 228.57084 & 14.39839 \\
\hline $\mathrm{J} 1514+5012$ & 1589669582111773312 & SDSS J151441.86+501209.7 & $\mathrm{DZ}$ & 228.67448 & 50.20273 \\
\hline $\mathrm{J} 1515+1348$ & 1182836742097801600 & SDSS J151522.04+134836.5 & $\mathrm{DZ}$ & 228.84183 & 13.81014 \\
\hline $\mathrm{J} 1515+4532$ & 1395405256376599552 & SDSS J151514.54+453211.1 & $\mathrm{DZ}$ & 228.81058 & 45.53642 \\
\hline J1516+2118 & 1214926813587984768 & SDSS J151610.57+211824.4 & $\mathrm{DZ}$ & 229.04421 & 21.30685 \\
\hline J1516-0040 & 4418628372344562048 & SDSS J151642.97-004042.7 & DBZA & 229.17903 & -0.67847 \\
\hline $\mathrm{J} 1517+2256$ & 1215384313505335424 & SDSS J151734.42+225618.7 & $\mathrm{DQ}$ & 229.39359 & 22.93847 \\
\hline J1518+0506 & 1156006287558141056 & SDSS J151835.63+050627.5 & $\mathrm{DZ}$ & 229.64845 & 5.10763 \\
\hline J1518+5619 & 1600924702825302784 & SDSS J151806.55+561935.1 & $\mathrm{DZ}$ & 229.52720 & 56.32644 \\
\hline J1519+3856 & 1388487923784151168 & SDSS J151942.66+385658.7 & DQ & 229.92776 & 38.94961 \\
\hline $\mathrm{J} 1520+2703$ & 1271298912183099520 & SDSS J152030.30+270321.5 & DQ & 230.12625 & 27.05597 \\
\hline $\mathrm{J} 1520+2718$ & 1271338327098920704 & SDSS J152033.66+271839.7 & $\mathrm{DZ}$ & 230.14025 & 27.31103 \\
\hline $\mathrm{J} 1523+5601$ & & SDSS J152345.85+560135.0 & $\mathrm{DZ}$ & 230.94104 & 56.02641 \\
\hline J1524+4049 & 1389075921987168512 & SDSS J152449.58+404938.1 & $\mathrm{DZ}$ & 231.20658 & 40.82724 \\
\hline $\mathrm{J} 1524+4052$ & & SDSS J152418.19+405252.1 & $\mathrm{DZ}$ & 231.07579 & 40.88114 \\
\hline J1525+1625 & 1207623754277319040 & SDSS J152554.24+162547.0 & $\mathrm{DZ}$ & 231.47600 & 16.42972 \\
\hline $\mathrm{J} 1525+4258$ & 1394025712881244928 & SDSS J152521.94+425805.8 & $\mathrm{DZ}$ & 231.34123 & 42.96834 \\
\hline $\mathrm{J} 1526+1308$ & & SDSS J152615.36+130846.8 & $\mathrm{DZ}$ & 231.56400 & 13.14633 \\
\hline $\mathrm{J} 1527+2752$ & 1271077944705755904 & SDSS J152702.59+275213.6 & $\mathrm{DQ}$ & 231.76079 & 27.87050 \\
\hline $\mathrm{J} 1528+0442$ & 4428393925389673344 & SDSS J152854.10+044226.6 & $\mathrm{DQ}$ & 232.22541 & 4.70746 \\
\hline $\mathrm{J} 1528+0753$ & 1163021962017933312 & SDSS J152819.04+075346.2 & $\mathrm{DZ}$ & 232.07933 & 7.89617 \\
\hline J1528+4003 & 1388228984500678016 & SDSS J152840.08+400336.0 & DQ & 232.16704 & 40.06006 \\
\hline $\mathrm{J} 1528+5134$ & 1595970711451752192 & SDSS J152812.03+513445.2 & $\mathrm{DQ}$ & 232.05018 & 51.57925 \\
\hline $\mathrm{J} 1531+2505$ & & SDSS J153100.63+250546.2 & $\mathrm{DZ}$ & 232.75262 & 25.09617 \\
\hline $\mathrm{J} 1531+4240$ & 1391331222197901696 & SDSS J153129.26+424015.7 & $\mathrm{DZ}$ & 232.87198 & 42.67105 \\
\hline $\mathrm{J} 1532+1006$ & & SDSS J153226.52+100629.4 & $\mathrm{DZ}$ & 233.11050 & 10.10817 \\
\hline J1532+1611 & & SDSS J153233.08+161140.5 & $\mathrm{DZ}$ & 233.13783 & 16.19458 \\
\hline $\mathrm{J} 1532+2748$ & & SDSS J153240.34+274818.3 & $\mathrm{DZ}$ & 233.16808 & 27.80508 \\
\hline J1533+5301 & 1596218239007316608 & SDSS J153333.95+530107.8 & $\mathrm{DZ}$ & 233.39151 & 53.01877 \\
\hline $\mathrm{J} 1534+1242$ & & SDSS J153407.58+124254.4 & $\mathrm{DZ}$ & 233.53162 & 12.71511 \\
\hline
\end{tabular}


Table 5. Object names, spectral types, and coordinates - continued

\begin{tabular}{|c|c|c|c|c|c|}
\hline J Name & Gaia source id & MWDD id & Sp. type & R.A & Dec \\
\hline $\mathrm{J} 1534+1345$ & 1193929989788210944 & SDSS J153417.60+134515.6 & DBZ & 233.57340 & 13.75431 \\
\hline $\mathrm{J} 1534+4145$ & 1390295486540598656 & LSPM J1534+4145 & DQZ & 233.69828 & 41.76643 \\
\hline $\mathrm{J} 1535+1247$ & 1193520666521113344 & WD $1532+12$ & $\mathrm{DZ}$ & 233.77421 & 12.79589 \\
\hline $\mathrm{J} 1537+1337$ & 1194088628700386432 & SDSS J153746.05+133733.5 & $\mathrm{DQ}$ & 234.44184 & 13.62610 \\
\hline $\mathrm{J} 1537+3608$ & & SDSS J153745.52+360818.6 & $\mathrm{DZ}$ & 234.43967 & 36.13851 \\
\hline $\mathrm{J} 1537+4625$ & 1400755548676988544 & SDSS J153724.24+462521.1 & $\mathrm{DZ}$ & 234.35103 & 46.42251 \\
\hline $\mathrm{J} 1539+1930$ & 1210014401793691264 & SDSS J153946.30+193055.4 & $\mathrm{DZ}$ & 234.94292 & 19.51539 \\
\hline $\mathrm{J} 1539+5248$ & & SDSS J153930.32+524819.3 & $\mathrm{DZ}$ & 234.87633 & 52.80537 \\
\hline $\mathrm{J} 1540+2109$ & 1217134048821424384 & SDSS J154057.43+210944.6 & $\mathrm{DZ}$ & 235.23929 & 21.16239 \\
\hline $\mathrm{J} 1540+3737$ & 1377011285837278080 & SDSS J154045.28+373747.8 & $\mathrm{DZ}$ & 235.18868 & 37.62994 \\
\hline $\mathrm{J} 1540+5149$ & 1596598601310586368 & SDSS J154025.18+514921.6 & $\mathrm{DZ}$ & 235.10491 & 51.82271 \\
\hline $\mathrm{J} 1540+5352$ & & SDSS J154022.80+535239.8 & $\mathrm{DZ}$ & 235.09519 & 53.87753 \\
\hline J1541+0715 & & SDSS J154152.58+071549.2 & $\mathrm{DZ}$ & 235.46908 & 7.26367 \\
\hline $\mathrm{J} 1542+1314$ & 1190978660061347584 & SDSS J154238.98+131455.7 & $\mathrm{DQ}$ & 235.66258 & 13.24876 \\
\hline $\mathrm{J} 1542+2544$ & 1223037571566004992 & SDSS J154234.73+254438.7 & DQ & 235.64474 & 25.74419 \\
\hline $\mathrm{J} 1542+4329$ & 1396483602405444352 & SDSS J154248.67+432902.4 & $\mathrm{DQ}$ & 235.70274 & 43.48404 \\
\hline $\mathrm{J} 1542+4650$ & 1400829525199398912 & SDSS J154201.75+465020.2 & $\mathrm{DZ}$ & 235.50733 & 46.83897 \\
\hline $\mathrm{J} 1543+2024$ & & SDSS J154349.79+202442.9 & $\mathrm{DZ}$ & 235.95746 & 20.41192 \\
\hline $\mathrm{J} 1544+2345$ & 1218155666922071552 & SDSS J154403.30+234516.6 & $\mathrm{DZ}$ & 236.01364 & 23.75466 \\
\hline $\mathrm{J} 1545+2854$ & 1224851624015623680 & SDSS J154518.44+285412.9 & $\mathrm{DQ}$ & 236.32697 & 28.90363 \\
\hline $\mathrm{J} 1545+4921$ & 1401734938659475456 & SDSS J154540.21+492145.1 & $\mathrm{DZ}$ & 236.41756 & 49.36258 \\
\hline $\mathrm{J} 1545+5236$ & 1596740644469446656 & SDSS J154510.31+523618.4 & $\mathrm{DZ}$ & 236.29299 & 52.60505 \\
\hline $\mathrm{J} 1546+1750$ & & SDSS J154630.17+175034.5 & $\mathrm{DZ}$ & 236.62571 & 17.84294 \\
\hline $\mathrm{J} 1546+3927$ & 1377594233157992704 & SDSS J154623.07+392723.6 & $\mathrm{DZ}$ & 236.59614 & 39.45656 \\
\hline $\mathrm{J} 1547+0659$ & 4430165650933432576 & SDSS J154729.96+065909.5 & $\mathrm{DZ}$ & 236.87495 & 6.98600 \\
\hline $\mathrm{J} 1548+0222$ & 4423437704923843968 & SDSS J154807.93+022257.1 & $\mathrm{DZ}$ & 237.03304 & 2.38256 \\
\hline $\mathrm{J} 1548+2135$ & 1216820413129873280 & SDSS J154815.23+213528.4 & DBZA & 237.06350 & 21.59122 \\
\hline J1548+5626 & 1598666993137041280 & WD $1547+565$ & $\mathrm{DQ}$ & 237.04443 & 56.44659 \\
\hline J1549+0239 & 4423539654567531136 & WD $1547+028$ & $\mathrm{DZ}$ & 237.47203 & 2.65824 \\
\hline $\mathrm{J} 1549+1906$ & & SDSS J154933.23+190646.7 & $\mathrm{DZ}$ & 237.38846 & 19.11299 \\
\hline $\mathrm{J} 1549+2020$ & 1204133766931561344 & SDSS J154941.55+202023.8 & $\mathrm{DZ}$ & 237.42312 & 20.33997 \\
\hline $\mathrm{J} 1549+2633$ & 1223368966945806848 & SDSS J154913.46+263301.1 & $\mathrm{DZ}$ & 237.30608 & 26.55033 \\
\hline $\mathrm{J} 1549+4422$ & 1396603002496338432 & SDSS J154907.11+442257.9 & $\mathrm{DZ}$ & 237.27962 & 44.38275 \\
\hline $\mathrm{J} 1550+2013$ & & SDSS J155030.78+201309.6 & $\mathrm{DZ}$ & 237.62825 & 20.21933 \\
\hline $\mathrm{J} 1550+5214$ & & SDSS J155033.47+521438.4 & $\mathrm{DZ}$ & 237.63946 & 52.24401 \\
\hline $\mathrm{J} 1551+0824$ & 4454432667131057792 & SDSS J155155.26+082431.7 & $\mathrm{DQ}$ & 237.98022 & 8.40887 \\
\hline J1551+1529 & & SDSS J155106.77+152955.3 & $\mathrm{DZ}$ & 237.77821 & 15.49872 \\
\hline $\mathrm{J} 1551+2547$ & & SDSS J155113.05+254752.1 & $\mathrm{DZ}$ & 237.80437 & 25.79783 \\
\hline
\end{tabular}


Table 5. Object names, spectral types, and coordinates - continued

\begin{tabular}{|c|c|c|c|c|c|}
\hline J Name & Gaia source id & MWDD id & Sp. type & R.A & Dec \\
\hline J1552+0815 & 4454420740003558528 & SDSS J155240.07+081516.8 & $\mathrm{DZ}$ & 238.16698 & 8.25469 \\
\hline $\mathrm{J} 1552+1148$ & 1189726007080211200 & SDSS J155202.39+114829.1 & $\mathrm{DQ}$ & 238.00999 & 11.80812 \\
\hline J1552+3910 & 1376870960665371136 & SDSS J155206.42+391017.6 & DQ & 238.02713 & 39.17135 \\
\hline J1554+0336 & 4425127624234553984 & WD $1551+037$ & $\mathrm{DQ}$ & 238.55633 & 3.60965 \\
\hline J1554+1735 & 1202552914026910976 & SDSS J155429.01+173545.9 & $\mathrm{DZ}$ & 238.62085 & 17.59626 \\
\hline J1555+3123 & 1321574115723174144 & SDSS J155530.18+312310.4 & $\mathrm{DQ}$ & 238.87579 & 31.38624 \\
\hline J1555+3219 & 1321789143262245248 & SDSS J155539.51+321914.1 & DQ & 238.91469 & 32.32054 \\
\hline $\mathrm{J} 1557+2157$ & 1206198821567293056 & SDSS J155724.87+215746.4 & DZA & 239.35360 & 21.96288 \\
\hline J1558+0312 & 4424935480278206976 & WD $1556+033$ & $\mathrm{DZ}$ & 239.71916 & 3.21193 \\
\hline J1558+1507 & 1192938917495381504 & SDSS J155847.89+150735.4 & $\mathrm{DZ}$ & 239.69962 & 15.12641 \\
\hline J1558+2512 & 1219957873855946240 & USNO-B1.0 1152-00234632 & $\mathrm{DZ}$ & 239.57211 & 25.21415 \\
\hline $\mathrm{J} 1559+0445$ & 4425689367298237312 & SDSS J155917.21+044549.4 & $\mathrm{DZ}$ & 239.82173 & 4.76371 \\
\hline $\mathrm{J} 1600+1833$ & 1203094762803715712 & SDSS J160020.65+183325.6 & $\mathrm{DZ}$ & 240.08604 & 18.55711 \\
\hline $\mathrm{J} 1600+3819$ & 1379356410995780480 & SDSS J160032.88+381904.5 & $\mathrm{DZ}$ & 240.13700 & 38.31794 \\
\hline $\mathrm{J} 1600+4120$ & & SDSS J160000.12+412033.4 & $\mathrm{DZ}$ & 240.00050 & 41.34264 \\
\hline J1600-0009 & & WD $1557-000 \mathrm{~B}$ & $\mathrm{DZ}$ & 240.05225 & -0.15147 \\
\hline J1601+1824 & & SDSS J160143.58+182437.9 & $\mathrm{DZ}$ & 240.43158 & 18.41053 \\
\hline J1602+1923 & & SDSS J160221.46+192351.6 & $\mathrm{DZ}$ & 240.58942 & 19.39769 \\
\hline $\mathrm{J} 1603+4140$ & & SDSS J160359.58+414047.8 & $\mathrm{DZ}$ & 240.99825 & 41.67997 \\
\hline $\mathrm{J} 1604+0831$ & 4451614618828537216 & SDSS J160401.31+083109.0 & DBZA & 241.00544 & 8.51917 \\
\hline J1604+1830 & 1200053822878445056 & SDSS J160429.80+183035.4 & $\mathrm{DZ}$ & 241.12421 & 18.50983 \\
\hline J1606+1908 & & SDSS J160612.76+190801.3 & $\mathrm{DZ}$ & 241.55317 & 19.13369 \\
\hline $\mathrm{J} 1606+4712$ & 1399018870061627520 & SDSS J160646.32+471207.7 & $\mathrm{Z}$ & 241.69300 & 47.20213 \\
\hline J1607+3950 & 1379917024487277056 & SDSS J160702.05+395058.6 & $\mathrm{DZ}$ & 241.75854 & 39.84964 \\
\hline J1607+5321 & 1428807010879180672 & GALEX 2432438716509192504 & $\mathrm{DZ}$ & 241.79941 & 53.36601 \\
\hline J1608+1342 & 4458463884776394880 & SDSS J160819.44+134204.3 & $\mathrm{DZ}$ & 242.08103 & 13.70116 \\
\hline J1608+3831 & 1379023018454640256 & SDSS J160859.59+383146.3 & $\mathrm{DZ}$ & 242.24829 & 38.52953 \\
\hline $\mathrm{J} 1609+0655$ & 4450425359563998720 & SDSS J160935.86+065511.9 & $\mathrm{DQ}$ & 242.39945 & 6.91999 \\
\hline J1610+3036 & 1318849697708771584 & SDSS J161018.94+303618.9 & $\mathrm{DQ}$ & 242.57900 & 30.60522 \\
\hline J1610+3714 & 1378301463949155200 & SDSS J161038.92+371403.0 & DBZA & 242.66219 & 37.23415 \\
\hline J1610+4006 & & SDSS J161026.10+400619.7 & $\mathrm{DZ}$ & 242.60879 & 40.10547 \\
\hline J1611+1117 & 4456951094215638016 & SDSS J161114.32+111708.3 & $\mathrm{DZ}$ & 242.80963 & 11.28563 \\
\hline J1612+1845 & & SDSS J161216.54+184512.3 & $\mathrm{DZ}$ & 243.06892 & 18.75342 \\
\hline J1612+3534 & & SDSS J161248.17+353434.8 & $\mathrm{DZ}$ & 243.20071 & 35.57635 \\
\hline J1613+5116 & 1424602959450847872 & WD $1611+513$ & $\mathrm{DQ}$ & 243.31400 & 51.26899 \\
\hline J1615+1746 & 1200496999080913920 & SDSS J161544.48+174627.4 & $\mathrm{DZ}$ & 243.93539 & 17.77426 \\
\hline J1616+3303 & 1322623152896019328 & SDSS J161603.00+330301.2 & $\mathrm{DZ}$ & 244.01254 & 33.05031 \\
\hline J1616+3924 & 1380443823700583552 & SDSS J161653.36+392444.4 & $\mathrm{DQ}$ & 244.22240 & 39.41232 \\
\hline
\end{tabular}


Table 5. Object names, spectral types, and coordinates - continued

\begin{tabular}{|c|c|c|c|c|c|}
\hline J Name & Gaia source id & MWDD id & Sp. type & R.A & Dec \\
\hline $\mathrm{J} 1618+4452$ & 1385558957951613952 & SDSS J161801.33+445220.7 & $\mathrm{DZ}$ & 244.50558 & 44.87243 \\
\hline $\mathrm{J} 1619+4745$ & & SDSS J161922.43+474553.7 & $\mathrm{DZ}$ & 244.84346 & 47.76493 \\
\hline $\mathrm{J} 1620+1809$ & 1200428928142394496 & SDSS J162004.03+180912.6 & DQ & 245.01692 & 18.15366 \\
\hline $\mathrm{J} 1621+2253$ & 1301685290406051072 & SDSS J162153.82+225309.5 & $\mathrm{DQ}$ & 245.47431 & 22.88597 \\
\hline $\mathrm{J} 1622+4731$ & & SDSS J162247.25+473146.8 & $\mathrm{DZ}$ & 245.69688 & 47.52968 \\
\hline $\mathrm{J} 1622+6319$ & 1628970289513198976 & SDSS J162236.76+631908.9 & DZA & 245.65316 & 63.31918 \\
\hline $\mathrm{J} 1624+3310$ & & SDSS J162408.57+331019.0 & $\mathrm{DZ}$ & 246.03571 & 33.17194 \\
\hline $\mathrm{J} 1626+3303$ & & SDSS J162612.73+330308.2 & $\mathrm{DZ}$ & 246.55308 & 33.05228 \\
\hline $\mathrm{J} 1627+1443$ & 4463884614540773632 & SDSS J162702.24+144324.7 & $\mathrm{DZ}$ & 246.75938 & 14.72350 \\
\hline $\mathrm{J} 1627+4646$ & 1410054324670763904 & SDSS J162703.34+464658.2 & $\mathrm{DZ}$ & 246.76392 & 46.78286 \\
\hline $\mathrm{J} 1628+3646$ & 1331106782752978688 & Ross 640 & DZA & 247.10418 & 36.77107 \\
\hline $\mathrm{J} 1629+1758$ & 4467141230543287808 & SDSS J162946.94+175805.2 & $\mathrm{DZ}$ & 247.44559 & 17.96811 \\
\hline J1633+1840 & 4467283273702508800 & SDSS J163310.02+184011.7 & $\mathrm{DZ}$ & 248.29185 & 18.66986 \\
\hline $\mathrm{J} 1634+5710$ & 1431176943768691328 & WD $1633+572$ & $\mathrm{DQ}$ & 248.58980 & 57.16913 \\
\hline $\mathrm{J} 1636+1619$ & 4465939601772584448 & SDSS J163601.33+161907.1 & $\mathrm{DZ}$ & 249.00558 & 16.31866 \\
\hline J1638+1244 & 4460911603818614272 & SDSS J163857.94+124420.6 & $\mathrm{DQ}$ & 249.74131 & 12.73905 \\
\hline J1638+3837 & 1331552115026136448 & SDSS J163827.68+383726.5 & $\mathrm{DZ}$ & 249.61533 & 38.62403 \\
\hline $\mathrm{J} 1640+3154$ & 1312731877573336448 & SDSS J164026.65+315453.9 & $\mathrm{DZ}$ & 250.11106 & 31.91493 \\
\hline $\mathrm{J} 1640+7310$ & 1654560662439820416 & WD $1641+732$ & DQ & 250.10153 & 73.17614 \\
\hline $\mathrm{J} 1641+1856$ & 4562675699936729216 & SDSS J164104.95+185602.1 & DL & 250.27062 & 18.93392 \\
\hline $\mathrm{J} 1641+4833$ & 1410448259071414528 & SDSS J164143.31+483301.6 & Q & 250.43058 & 48.55041 \\
\hline $\mathrm{J} 1642+3211$ & 1312718893887366784 & SDSS J164208.47+321136.6 & $\mathrm{DZ}$ & 250.53529 & 32.19350 \\
\hline $\mathrm{J} 1642+3749$ & & SDSS J164211.83+374954.9 & $\mathrm{DZ}$ & 250.54929 & 37.83192 \\
\hline $\mathrm{J} 1642+4529$ & & SDSS J164224.39+452911.8 & $\mathrm{DZ}$ & 250.60163 & 45.48664 \\
\hline $\mathrm{J} 1643+1422$ & 4461739604794187520 & SDSS J164336.31+142250.2 & $\mathrm{DZ}$ & 250.90126 & 14.38067 \\
\hline $\mathrm{J} 1643+4002$ & 1355756420402482688 & WD $1641+401$ & DQ & 250.86892 & 40.03450 \\
\hline $\mathrm{J} 1643+4129$ & 1356453338975443840 & SDSS J164317.39+412959.2 & DQ & 250.82246 & 41.49978 \\
\hline $\mathrm{J} 1644+3853$ & 1352596698862254208 & SDSS J164427.54+385357.7 & $\mathrm{DZ}$ & 251.11475 & 38.89936 \\
\hline $\mathrm{J} 1646+2742$ & 1307617155638441344 & GALEX 2680180581990401605 & DBZA & 251.59259 & 27.71011 \\
\hline $\mathrm{J} 1647+2636$ & 1307226283551364224 & SDSS J164744.70+263646.7 & $\mathrm{DZ}$ & 251.93592 & 26.61258 \\
\hline $\mathrm{J} 1647+4156$ & & SDSS J164723.80+415644.5 & $\mathrm{DZ}$ & 251.84917 & 41.94569 \\
\hline $\mathrm{J} 1647+4350$ & 1357729871975804160 & SDSS J164701.52+435047.9 & $\mathrm{DQ}$ & 251.75643 & 43.84661 \\
\hline J1647+5119 & 1413228614740924032 & SDSS J164700.05+511922.3 & DQ: & 251.75009 & 51.32270 \\
\hline J1648+5241 & 1413937593584775168 & SDSS J164842.83+524131.5 & $\mathrm{DZ}$ & 252.17858 & 52.69192 \\
\hline $\mathrm{J} 1649+2238$ & 4565805356705582976 & SDSS J164939.23+223807.2 & $\mathrm{DZ}$ & 252.41346 & 22.63536 \\
\hline $\mathrm{J} 1649+4152$ & & SDSS J164912.04+415241.8 & $\mathrm{DZ}$ & 252.30017 & 41.87828 \\
\hline $\mathrm{J} 1650+4055$ & & SDSS J165020.80+405550.2 & $\mathrm{DZ}$ & 252.58667 & 40.93061 \\
\hline $\mathrm{J} 1653+1113$ & 4447744196162611200 & SDSS J165309.19+111345.1 & DQ: & 253.28830 & 11.22931 \\
\hline
\end{tabular}


Table 5. Object names, spectral types, and coordinates - continued

\begin{tabular}{|c|c|c|c|c|c|}
\hline J Name & Gaia source id & MWDD id & Sp. type & R.A & Dec \\
\hline $\mathrm{J} 1654+3157$ & 1313265900922425856 & SDSS J165436.86+315754.4 & $\mathrm{DQ}$ & 253.65361 & 31.96510 \\
\hline $\mathrm{J} 1655+2134$ & 4565249351715525888 & SDSS J165518.49+213428.2 & $\mathrm{DZ}$ & 253.82704 & 21.57450 \\
\hline $\mathrm{J} 1655+3722$ & 1351330100122743040 & WD $1653+374$ & DQ & 253.91046 & 37.37976 \\
\hline $\mathrm{J} 1657+3738$ & 1351667753271611264 & SDSS J165741.58+373824.2 & $\mathrm{DZ}$ & 254.42331 & 37.64005 \\
\hline $\mathrm{J} 1657+4759$ & 1408298645123645824 & SDSS J165751.23+475911.6 & $\mathrm{DZ}$ & 254.46337 & 47.98644 \\
\hline $\mathrm{J} 1700+2047$ & & SDSS J170029.59+204704.3 & $\mathrm{DZ}$ & 255.12329 & 20.78456 \\
\hline $\mathrm{J} 1703+2541$ & 4572969916208600704 & SDSS J170326.57+254103.8 & DZA & 255.86069 & 25.68437 \\
\hline $\mathrm{J} 1706+2541$ & & SDSS J170638.11+254111.7 & $\mathrm{DZ}$ & 256.65879 & 25.68661 \\
\hline $\mathrm{J} 1707+4250$ & 1355088432729232384 & SDSS J170715.38+425043.1 & $\mathrm{DZ}$ & 256.81408 & 42.84533 \\
\hline $\mathrm{J} 1708+0257$ & 4388138816124225792 & GJ 1211 & $\mathrm{DZ}$ & 257.03319 & 2.96030 \\
\hline $\mathrm{J} 1711+2201$ & 4567999535238106880 & SDSS J171139.82+220152.9 & $\mathrm{DZ}$ & 257.91594 & 22.03137 \\
\hline $\mathrm{J} 1712+3406$ & 1335069349644072064 & SDSS J171221.94+340609.8 & DQ & 258.09142 & 34.10275 \\
\hline $\mathrm{J} 1713+3240$ & 1334374153353733888 & LSPM J1713+3240 & DQ & 258.42396 & 32.66922 \\
\hline J1714+3658 & 1340140572149710336 & SDSS J171403.75+365810.5 & DBZA & 258.51553 & 36.96963 \\
\hline $\mathrm{J} 1714+5501$ & 1420411964723649408 & WD $1713+550$ & $\mathrm{DZ}$ & 258.65175 & 55.02010 \\
\hline $\mathrm{J} 1715+2804$ & 4575202298472648576 & SDSS J171513.63+280403.9 & $\mathrm{DZ}$ & 258.80683 & 28.06774 \\
\hline $\mathrm{J} 1719+5623$ & 1420738111655252992 & SDSS J171900.63+562350.5 & $\mathrm{DZ}$ & 259.75263 & 56.39736 \\
\hline $\mathrm{J} 1722+2723$ & 4574208168163608832 & SDSS J172223.31+272326.2 & DZA & 260.59709 & 27.39064 \\
\hline $\mathrm{J} 1724+5333$ & 1416339274870578176 & WD $1723+536$ & DQ & 261.23076 & 53.56494 \\
\hline $\mathrm{J} 1728+0815$ & 4490308833894507008 & SDSS J172822.61+081549.3 & DBZA & 262.09425 & 8.26367 \\
\hline $\mathrm{J} 1728+3250$ & 4601940290956951424 & GALEX 2680391675338031787 & DBZ & 262.23419 & 32.84605 \\
\hline J1728+5558 & 1422012892308493568 & GD 524 & DQA & 262.23425 & 55.97295 \\
\hline J1833+1945 & 4525569007873380736 & WD $1831+19$ & DQ & 278.49312 & 19.76472 \\
\hline J1838+4046 & 2110571565187233152 & SDSS J183806.18+404629.8 & $\mathrm{DQ}$ & 279.52583 & 40.77487 \\
\hline J2007-1208 & 6880697900670837632 & SDSS J200757.10-120832.0 & DBZA & 301.98789 & -12.14222 \\
\hline J2046-0715 & 6907027901322999552 & WD $2043-074$ & DQ & 311.60188 & -7.25526 \\
\hline J2050-0110 & 4226851932184135552 & SDSS J205059.11-011021.9 & $\mathrm{DZ}$ & 312.74631 & -1.17276 \\
\hline J2053-0702 & 6909994246262080000 & WD $2050-072$ & DQ & 313.31806 & -7.03449 \\
\hline J2101+3148 & 1864760695541016832 & EGGR 262 & DQ & 315.43652 & 31.81073 \\
\hline J2103-0108 & 6917166017006522368 & SDSS J210303.47-010842.5 & $\mathrm{DZ}$ & 315.76450 & -1.14510 \\
\hline J2107-0055 & 2689055590685508864 & SDSS J210733.93-005557.7 & $\mathrm{DZ}$ & 316.89132 & -0.93269 \\
\hline J2109-0039 & 2689160181727792512 & SDSS J210916.51-003921.6 & $\mathrm{DZ}$ & 317.31883 & -0.65602 \\
\hline $\mathrm{J} 2110+0512$ & & SDSS J211045.34+051214.8 & $\mathrm{DZ}$ & 317.68892 & 5.20411 \\
\hline J2110-0001 & 2689964337045144704 & SDSS J211003.74-000152.9 & DBZA & 317.51554 & -0.03130 \\
\hline J2111-0036 & 2689129601561746560 & SDSS J211130.04-003628.7 & DQ & 317.87516 & -0.60796 \\
\hline J2114-0051 & 2688921828223018496 & SDSS J211412.77-005125.5 & $\mathrm{DZ}$ & 318.55321 & -0.85708 \\
\hline J2123+0016 & 2690911738111175936 & SDSS J212312.20+001653.5 & $\mathrm{DZ}$ & 320.80083 & 0.28154 \\
\hline J2124-0114 & 2686055710647408128 & 2SLAQ J212424.69-011452.5 & $\mathrm{DZ}$ & 321.10293 & -1.24790 \\
\hline
\end{tabular}


Table 5. Object names, spectral types, and coordinates - continued

\begin{tabular}{|c|c|c|c|c|c|}
\hline J Name & Gaia source id & MWDD id & Sp. type & R.A & Dec \\
\hline $\mathrm{J} 2127+0319$ & 2692018980680713856 & SDSS J212757.26+031939.6 & $\mathrm{DZ}$ & 321.98858 & 3.32767 \\
\hline J2130-0243 & 2684869956075600640 & SDSS J213024.38-024302.2 & $\mathrm{DZ}$ & 322.60158 & -2.71728 \\
\hline J2132-0203 & & SDSS J213254.64-020309.0 & $\mathrm{DZ}$ & 323.22767 & -2.05253 \\
\hline $\mathrm{J} 2135+0003$ & 2687339493552038400 & SDSS J213503.32+000318.4 & $\mathrm{DQ}$ & 323.76378 & 0.05515 \\
\hline $\mathrm{J} 2136+1137$ & 1766826194114406656 & SDSS J213621.56+113726.8 & DZA & 324.08984 & 11.62413 \\
\hline $\mathrm{J} 2139+0354$ & & SDSS J213954.44+035403.7 & $\mathrm{DZ}$ & 324.97683 & 3.90103 \\
\hline J2140-0045 & 2686998713666980992 & 2SLAQ J214058.24-004528.8 & DQ & 325.24271 & -0.75800 \\
\hline $\mathrm{J} 2141+0225$ & & SDSS J214105.26+022543.7 & $\mathrm{DZ}$ & 325.27192 & 2.42883 \\
\hline J2141-3300 & 6592315723192176896 & WD $2138-332$ & $\mathrm{DZ}$ & 325.48985 & -33.00828 \\
\hline $\mathrm{J} 2142+0908$ & 1741347932717756928 & SDSS J214247.87+090858.8 & DQ & 325.69946 & 9.14967 \\
\hline $\mathrm{J} 2142+2059$ & 1792830060723673472 & GJ 836.5 & DQ & 325.67086 & 20.99948 \\
\hline $\mathrm{J} 2142+2252$ & 1794409921493969792 & 2MASS J21420390+2252290 & $\mathrm{DZ}$ & 325.51622 & 22.87484 \\
\hline J2143-0019 & & SDSS J214323.03-001934.6 & $\mathrm{DZ}$ & 325.84596 & -0.32631 \\
\hline $\mathrm{J} 2149+2039$ & 1792618572237988608 & SDSS J214944.06+203921.7 & $\mathrm{DQ}$ & 327.43353 & 20.65606 \\
\hline J2150-0113 & 2674012072593383168 & 6dFGS gJ215027.8-011348 & DQ & 327.61591 & -1.23055 \\
\hline J2151-0112 & 2680013864187927168 & 2SLAQ J215144.77-011247.4 & $\mathrm{DZ}$ & 327.93641 & -1.21320 \\
\hline $\mathrm{J} 2155+4103$ & 1959573541696236032 & 2MASS J21550637+4103067 & $\mathrm{DZ}$ & 328.77654 & 41.05186 \\
\hline $\mathrm{J} 2156+0559$ & 2697327113581223936 & SDSS J215652.94+055925.1 & DQ & 329.22050 & 5.99043 \\
\hline J2157+1137 & 2726854738863780992 & SDSS J215759.09+113730.1 & DQ & 329.49622 & 11.62510 \\
\hline $\mathrm{J} 2157+1206$ & 2728434462194932352 & SDSS J215752.30+120603.1 & DL & 329.46792 & 12.10089 \\
\hline $\mathrm{J} 2201+0219$ & 2683345934175922176 & LSPM J2201+0219 & $\mathrm{DZ}$ & 330.34661 & 2.32685 \\
\hline $\mathrm{J} 2202+0013$ & & SDSS J220242.32+001336.1 & $\mathrm{DZ}$ & 330.67633 & 0.22672 \\
\hline $\mathrm{J} 2202+0320$ & & SDSS J220251.46+032039.3 & $\mathrm{DZ}$ & 330.71442 & 3.34425 \\
\hline J2206+1007 & 2727009632564240384 & SDSS J220651.57+100744.2 & $\mathrm{DZ}$ & 331.71488 & 10.12897 \\
\hline $\mathrm{J} 2207+0135$ & 2682344450881423360 & VIPERS 403166921 & DQ & 331.94253 & 1.58604 \\
\hline $\mathrm{J} 2207+2319$ & & SDSS J220750.08+231955.6 & $\mathrm{DZ}$ & 331.95867 & 23.33211 \\
\hline $\mathrm{J} 2209+1223$ & 2727904257071365760 & WD J2209+1223 & DBZ & 332.39521 & 12.39349 \\
\hline$J 2211+2145$ & 1782054361439384960 & SDSS J221132.37+214526.8 & $\mathrm{DZ}$ & 332.88488 & 21.75747 \\
\hline $\mathrm{J} 2213+0103$ & & SDSS J221353.85+010334.1 & $\mathrm{DZ}$ & 333.47437 & 1.05947 \\
\hline $\mathrm{J} 2218+2123$ & 1778839767397091200 & SDSS J221850.23+212303.7 & $\mathrm{DQ}$ & 334.70948 & 21.38478 \\
\hline J2218+3908 & 1956838712683591296 & GD 401 & $\mathrm{DZ}$ & 334.50476 & 39.14552 \\
\hline $\mathrm{J} 2220+0140$ & & SDSS J222057.35+014001.0 & $\mathrm{DZ}$ & 335.23896 & 1.66697 \\
\hline $\mathrm{J} 2220+1420$ & 2734603650138728448 & SDSS J222032.10+142033.4 & DQ & 335.13379 & 14.34263 \\
\hline $\mathrm{J} 2225+1251$ & 2730798480916011648 & SDSS J222552.64+125116.8 & $\mathrm{DQ}$ & 336.46933 & 12.85467 \\
\hline $\mathrm{J} 2225+2338$ & 1878579387730218112 & SDSS J222503.71+233855.1 & $\mathrm{DZ}$ & 336.26546 & 23.64864 \\
\hline $\mathrm{J} 2228+1207$ & 2730508416002618752 & 2MASS J22280206+1207334 & $\mathrm{DZ}$ & 337.00857 & 12.12594 \\
\hline J2230+1905 & & SDSS J223014.70+190514.4 & $\mathrm{DZ}$ & 337.56125 & 19.08736 \\
\hline $\mathrm{J} 2230+3052$ & 1900628306721576064 & SDSS J223010.83+305240.9 & $\mathrm{DZ}$ & 337.54512 & 30.87805 \\
\hline
\end{tabular}


Table 5. Object names, spectral types, and coordinates - continued

\begin{tabular}{|c|c|c|c|c|c|}
\hline J Name & Gaia source id & MWDD id & Sp. type & R.A & Dec \\
\hline $\mathrm{J} 2231+0040$ & 2702211693987574400 & SDSS J223159.94+004046.4 & $\mathrm{DZ}$ & 337.99974 & 0.67956 \\
\hline J2231+0906 & 2711092621203766912 & LSPM J2231+0906 & $\mathrm{DZ}$ & 337.75415 & 9.11086 \\
\hline $\mathrm{J} 2232+0109$ & 2702382156944616832 & SDSS J223222.32+010920.7 & $\mathrm{DZ}$ & 338.09301 & 1.15577 \\
\hline J2235-0056 & & SDSS J223507.65-005607.7 & $\mathrm{DZ}$ & 338.78188 & -0.93549 \\
\hline $\mathrm{J} 2236+0109$ & 2654669468332355584 & USNO-A2.0 0900-20178217 & DBAZ & 339.22070 & 1.16064 \\
\hline J2236+0413 & & SDSS J223601.38+041324.7 & $\mathrm{DZ}$ & 339.00575 & 4.22356 \\
\hline $\mathrm{J} 2238+0101$ & 2654620329611594496 & SDSS J223841.06+010150.1 & $\mathrm{DZ}$ & 339.67106 & 1.03068 \\
\hline J2238+0213 & & SDSS J223811.10+021352.9 & $\mathrm{DZ}$ & 339.54629 & 2.23137 \\
\hline J2238-0113 & 2652983573409260800 & SDSS J223815.97-011336.9 & $\mathrm{DZ}$ & 339.56661 & -1.22690 \\
\hline $\mathrm{J} 2239+2303$ & 1876067076676460800 & SDSS J223937.63+230328.9 & DQ & 339.90665 & 23.05808 \\
\hline $\mathrm{J} 2239+2932$ & & SDSS J223956.24+293225.7 & $\mathrm{DZ}$ & 339.98433 & 29.54048 \\
\hline J $2243+3210$ & 1890668861674532224 & SDSS J224346.29+321027.1 & DBZ & 340.94300 & 32.17422 \\
\hline $\mathrm{J} 2245+2748$ & 1884129791148258304 & SDSS J224524.71+274827.8 & $\mathrm{DZ}$ & 341.35301 & 27.80781 \\
\hline $\mathrm{J} 2248+1318$ & 2719990109813842688 & SDSS J224828.65+131838.5 & DBZ & 342.11940 & 13.31071 \\
\hline $\mathrm{J} 2248+2826$ & 1884548739436672640 & SDSS J224827.82+282637.2 & DQA & 342.11606 & 28.44385 \\
\hline J2248-0153 & 2649709841962009088 & SDSS J224847.11-015301.8 & $\mathrm{DZ}$ & 342.19629 & -1.88386 \\
\hline $\mathrm{J} 2250+1240$ & 2719705542461133568 & SDSS J225000.22+124019.8 & DQ & 342.50088 & 12.67219 \\
\hline J2253-0646 & 2611561706216413696 & GJ 1276 & $\mathrm{DZ}$ & 343.47244 & -6.78180 \\
\hline J2258-0006 & & SDSS J225833.44-000614.8 & $\mathrm{DZ}$ & 344.63933 & -0.10411 \\
\hline J2258-0829 & 2607019447587739008 & SDSS J225809.37-082920.1 & DBZA & 344.53907 & -8.48893 \\
\hline $\mathrm{J} 2259+1251$ & 2718973478170263808 & SDSS J225950.66+125119.3 & $\mathrm{DZ}$ & 344.96096 & 12.85542 \\
\hline $\mathrm{J} 2259+1748$ & & SDSS J225935.37+174825.6 & $\mathrm{DZ}$ & 344.89738 & 17.80713 \\
\hline $\mathrm{J} 2300+2204$ & 2835805586577740288 & LP $401-31$ & $\mathrm{DZ}$ & 345.09152 & 22.07111 \\
\hline J2302+1626 & 2816958617248201984 & SDSS J230227.96+162602.6 & $\mathrm{DZ}$ & 345.61651 & 16.43408 \\
\hline $\mathrm{J} 2302+2430$ & 2843077206728009472 & SDSS J230249.37+243027.9 & DQ & 345.70583 & 24.50797 \\
\hline $\mathrm{J} 2304+2415$ & 2842312874347797632 & SDSS J230414.49+241554.0 & $\mathrm{DZ}$ & 346.06044 & 24.26513 \\
\hline $\mathrm{J} 2305+2307$ & 2841985288602442752 & GALEX 2665262369570030960 & DZA & 346.40428 & 23.13154 \\
\hline J2306+2111 & 2832478464391764480 & SDSS J230620.01+211118.5 & DBZA & 346.58340 & 21.18850 \\
\hline $\mathrm{J} 2309+0608$ & 2665008240592600704 & PB 5274 & DBZA & 347.47097 & 6.13894 \\
\hline $\mathrm{J} 2310+2203$ & 2838626486737671040 & SDSS J231019.68+220323.3 & $\mathrm{DZ}$ & 347.58191 & 22.05649 \\
\hline J2310-0057 & 2638932738224859776 & WD $2307-012$ & DQA & 347.62609 & -0.96271 \\
\hline J2311+1042 & 2810602344823007872 & SDSS J231106.56+104251.7 & $\mathrm{DZ}$ & 347.77733 & 10.71447 \\
\hline $\mathrm{J} 2311+1510$ & & SDSS J231129.04+151022.1 & $\mathrm{DZ}$ & 347.87100 & 15.17281 \\
\hline J2311+3343 & & SDSS J231143.15+334334.2 & $\mathrm{DZ}$ & 347.92979 & 33.72618 \\
\hline J2311-0041 & 2650975899537545728 & USNO-A2.0 0825-19880842 & DBZ & 347.92326 & -0.68351 \\
\hline J2314-0632 & 2631876970245863552 & GJ 893.1 & DQ & 348.60494 & -6.54663 \\
\hline J2315-0209 & 2638553754605793408 & EGGR 554 & $\mathrm{DZ}$ & 348.82836 & -2.16128 \\
\hline $\mathrm{J} 2316+0041$ & & 2SLAQ J231601.88+004131.2 & $\mathrm{DZ}$ & 349.00788 & 0.69201 \\
\hline
\end{tabular}


Table 5. Object names, spectral types, and coordinates - continued

\begin{tabular}{|c|c|c|c|c|c|}
\hline J Name & Gaia source id & MWDD id & Sp. type & R.A & Dec \\
\hline J2318+2916 & & SDSS J231820.78+291617.3 & $\mathrm{DZ}$ & 349.58658 & 29.27149 \\
\hline J2318-0320 & 2637115142424897536 & SDSS J231847.23-032013.0 & $\mathrm{DZ}$ & 349.69679 & -3.33697 \\
\hline $\mathrm{J} 2319+3018$ & 2870363305758068480 & SDSS J231937.39+301848.4 & $\mathrm{DZ}$ & 349.90564 & 30.31348 \\
\hline $\mathrm{J} 2321+0807$ & 2664985631884840704 & SDSS J232112.68+080715.3 & $\mathrm{DZ}$ & 350.30287 & 8.12096 \\
\hline $\mathrm{J} 2324+1135$ & 2762920717413900416 & SDSS J232423.50+113542.6 & $\mathrm{DZ}$ & 351.09797 & 11.59513 \\
\hline $\mathrm{J} 2324+1207$ & 2811012462660275968 & PG $2322+119$ & DZA & 351.24155 & 12.12760 \\
\hline $\mathrm{J} 2328+0711$ & & SDSS J232828.78+071119.4 & $\mathrm{DZ}$ & 352.11992 & 7.18873 \\
\hline $\mathrm{J} 2328+0830$ & & SDSS J232833.31+083028.4 & $\mathrm{DZ}$ & 352.13883 & 8.50790 \\
\hline $\mathrm{J} 2330+1015$ & 2761789865409701760 & SDSS J233011.79+101541.4 & $\mathrm{DZ}$ & 352.54921 & 10.26146 \\
\hline $\mathrm{J} 2330+2620$ & 2865440826559975296 & SDSS J233056.20+262048.4 & $\mathrm{DZ}$ & 352.73416 & 26.34677 \\
\hline $\mathrm{J} 2330+2805$ & 2865936671944556160 & SDSS J233054.31+280517.4 & $\mathrm{DZ}$ & 352.72617 & 28.08821 \\
\hline $\mathrm{J} 2333+1058$ & & SDSS J233320.38+105830.2 & $\mathrm{DZ}$ & 353.33492 & 10.97505 \\
\hline J2336+1657 & & SDSS J233625.13+165751.4 & $\mathrm{DZ}$ & 354.10471 & 16.96428 \\
\hline $\mathrm{J} 2336+2021$ & 2826113047981744896 & SDSS J233610.58+202126.1 & $\mathrm{DZ}$ & 354.04411 & 20.35727 \\
\hline $\mathrm{J} 2337+2607$ & & SDSS J233719.10+260708.6 & $\mathrm{DZ}$ & 354.32958 & 26.11906 \\
\hline $\mathrm{J} 2339+4631$ & 1939136468100337536 & SDSS J233911.63+463142.4 & DZA & 354.79837 & 46.52845 \\
\hline $\mathrm{J} 2340+0124$ & 2646118355949741440 & SDSS J234034.61+012416.6 & $\mathrm{DZ}$ & 355.14421 & 1.40461 \\
\hline $\mathrm{J} 2340+0817$ & 2758086538448464512 & SDSS J234048.74+081753.3 & $\mathrm{DZ}$ & 355.20284 & 8.29811 \\
\hline $\mathrm{J} 2340+1621$ & & SDSS J234000.97+162127.0 & $\mathrm{DZ}$ & 355.00404 & 16.35751 \\
\hline $\mathrm{J} 2341+0059$ & 2646042317848350464 & SDSS J234131.41+005957.9 & $\mathrm{DZ}$ & 355.38088 & 0.99942 \\
\hline $\mathrm{J} 2341+2448$ & 2852254177449825152 & SDSS J234141.51+244854.3 & $\mathrm{Z}$ & 355.42284 & 24.81501 \\
\hline J2341-0101 & 2641006004837594752 & SDSS J234132.82-010104.5 & $\mathrm{DQ}$ & 355.38677 & -1.01794 \\
\hline J2342-0006 & 2642625482386020224 & SDSS J234245.90-000632.4 & DZA & 355.69129 & -0.10902 \\
\hline $\mathrm{J} 2343+2242$ & 2827053439660604544 & SDSS J234355.80+224223.2 & $\mathrm{DZ}$ & 355.98244 & 22.70644 \\
\hline $\mathrm{J} 2343+3155$ & & SDSS J234315.21+315556.7 & $\mathrm{DZ}$ & 355.81338 & 31.93244 \\
\hline $\mathrm{J} 2343-0010$ & 2642621084339810560 & SDSS J234307.67-001016.3 & $\mathrm{DZ}$ & 355.78196 & -0.17120 \\
\hline $\mathrm{J} 2344+1343$ & 27708787377626588816 & SDSS J234457.13+134342.1 & $\mathrm{DZ}$ & 356.23805 & 13.72838 \\
\hline $\mathrm{J} 2344+1813$ & & SDSS J234444.70+181343.5 & $\mathrm{DZ}$ & 356.18625 & 18.22876 \\
\hline $\mathrm{J} 2345+2857$ & & SDSS J234530.32+285739.8 & $\mathrm{DZ}$ & 356.37633 & 28.96108 \\
\hline J2346+2816 & & SDSS J234644.52+281601.0 & $\mathrm{DZ}$ & 356.68550 & 28.26696 \\
\hline J2348+1341 & 2770165738831638784 & GALEX 2665121662146448800 & DBZA & 357.21916 & 13.69466 \\
\hline J2348-4428 & 6531195177474061824 & WD $2345-447$ & $\mathrm{DZ}$ & 357.06986 & -44.46993 \\
\hline $\mathrm{J} 2349+0325$ & & SDSS J234909.76+032535.2 & $\mathrm{DZ}$ & 357.29067 & 3.42647 \\
\hline J2351+0633 & 2744849968934530304 & SDSS J235125.70+063305.3 & $\mathrm{DZ}$ & 357.85711 & 6.55154 \\
\hline $\mathrm{J} 2351+3612$ & 2878778418002027648 & SDSS J235139.39+361203.1 & DBZ & 357.91413 & 36.20087 \\
\hline $\mathrm{J} 2352+1922$ & & SDSS J235224.26+192247.3 & $\mathrm{DZ}$ & 358.10108 & 19.37982 \\
\hline J2352+3344 & & SDSS J235249.13+334439.2 & $\mathrm{DZ}$ & 358.20471 & 33.74423 \\
\hline J2352-0146 & 2449345460075810048 & SDSS J235254.92-014651.9 & $\mathrm{DZ}$ & 358.22883 & -1.78111 \\
\hline
\end{tabular}


Table 5. Object names, spectral types, and coordinates - continued

\begin{tabular}{ccclcc}
\hline \hline J Name & Gaia source id & \multicolumn{1}{c}{ MWDD id } & Sp. type & R.A & Dec \\
\hline J2353+0415 & 2743352635960001792 & SDSS J235313.49+041525.4 & DZ & 358.30621 & 4.25706 \\
J2353+3720 & 2879694208111191168 & SDSS J235336.08+372055.3 & DBZA & 358.40036 & 37.34868 \\
J2354+4027 & 1921351390779081600 & EGGR 507 & DQ & 358.73449 & 40.45821 \\
J2355+1431 & 2770512570327553408 & SDSS J235516.61+143136.1 & DZ & 358.81910 & 14.52674 \\
J2356-0320 & & SDSS J235625.20-032020.5 & DZ & 359.10500 & -3.33906 \\
J2357+2348 & & SDSS J235715.03+234848.9 & DZ & 359.31263 & 23.81360 \\
J2357-0324 & 2448097411298706560 & SDSS J235737.37-032408.7 & DZ & 359.40571 & -3.40244 \\
J2358+0445 & & SDSS J235810.49+044517.8 & DZ & 359.54371 & 4.75497 \\
J2359+0243 & 2739899276095504640 & SDSS J235937.33+024357.0 & DZ & 359.90554 & 2.73250 \\
J2359+0347 & 2740318086946217472 & SDSS J235926.15+034758.5 & DZ & 359.85896 & 3.79961 \\
\hline
\end{tabular}


Table 6. Atmospheric parameters for DZ stars. A dagger indicates that the distance is a photometric distance based on the value of $\log g=8$ in the absence of a parallax.

\begin{tabular}{|c|c|c|c|c|c|c|}
\hline $\mathrm{J}$ Name & eff $(K)$ & $\log g$ & $M / M_{\odot}$ & $\log \mathrm{Ca} / \mathrm{He}$ & $\log \mathrm{H} / \mathrm{He}$ & $D(\mathrm{pc})$ \\
\hline J0002+3209 & $6447(121)$ & $8.30\left({ }_{-0.22}^{+0.23}\right)$ & $0.77\left({ }_{-0.14}^{+0.16}\right)$ & -9.00 & $<-3.08$ & 28.7) \\
\hline J0002-0627 & $7306(428)$ & 8.00 & & -9.6 & 5 & 3) \\
\hline $\mathrm{J} 0003+2240$ & $5493(269)$ & 8.00 & & $-10.27(0.22)$ & 00 & 229. \\
\hline J0004+0819 & $5896(234)$ & 8.00 & & $-8.86(0.16)$ & $<-3.00$ & $337.4(27.7)$ \\
\hline $\mathrm{J} 0004+2432$ & $10490(614)$ & $8.26\left({ }_{-0.25}^{+0.28}\right)$ & $0.75\left({ }_{-0.16}^{+0.18}\right)$ & $-9.74(0.18)$ & $<-5.82$ & $287.9(49.8)$ \\
\hline $\mathrm{J} 0004+2531$ & 12132(1141) & $8.32\left({ }_{-0.62}^{+0.81}\right)$ & $0.79\left({ }_{-0.36}^{+0.45}\right)$ & $-8.27(0.35)$ & $<-6.04$ & $431.5(214.5)$ \\
\hline $\mathrm{J} 0005+0018$ & $8073(208)$ & $7.81\left({ }_{-0.14}^{+0.14}\right)$ & $0.47\left({ }_{-0.07}^{+0.08}\right)$ & $-10.05(0.09)$ & $<-5.01$ & $156.4(11.6)$ \\
\hline $\mathrm{J} 0005+4003$ & $8829(427)$ & $8.03\left({ }_{-0.09}^{+0.09}\right)$ & $0.59\left({ }_{-0.05}^{+0.06}\right)$ & $-10.42(0.04)$ & $-4.48(0.21)$ & $64.3(0.3)$ \\
\hline $\mathrm{J} 0005+7313$ & $12673(759)$ & $7.95\left({ }_{-0.08}^{+0.08}\right)$ & $0.56\left({ }_{-0.04}^{+0.05}\right)$ & $-10.07(0.36)$ & $-6.11(0.00)$ & $34.7(0.0)$ \\
\hline $\mathrm{J} 0006+0520$ & $5906(160)$ & $7.97\left({ }_{-0.25}^{+0.27}\right)$ & $0.55\left({ }_{-0.14}^{+0.17}\right)$ & $-9.78(0.10)$ & $<-3.00$ & $178.3(27.0)$ \\
\hline $\mathrm{J} 0009+0152$ & $7159(212)$ & $8.25\left({ }_{-0.30}^{+0.32}\right)$ & $0.73\left({ }_{-0.19}^{+0.22}\right)$ & $-9.45(0.12)$ & $<-3.81$ & $180.4(38.1)$ \\
\hline J0010-0430 & $7089(160)$ & $8.25\left(_{-0.27}^{+0.29}\right)$ & $0.73\left({ }_{-0.17}^{+0.19}\right)$ & $-8.04(0.09)$ & $<-3.77$ & $204.1(38.4)$ \\
\hline J0010-0628 & $7883(1189)$ & 8.00 & & $-9.87(0.29)$ & $<-4.51$ & $488.1(105.7)$ \\
\hline J0013+1109 & $5939(283)$ & 8.00 & & $-9.96(0.67)$ & $<-3.00$ & $275.2(24.9)$ \\
\hline J0015+1830 & $7346(417)$ & 8.00 & & $-8.86(0.30)$ & $<-4.07$ & $320.0(26.3)$ \\
\hline $\mathrm{J} 0017+0230$ & $7477(495)$ & 8.00 & & $-9.06(0.68)$ & $<-4.14$ & $448.5(42.1)$ \\
\hline J0018-0012 & $9958(3$ & $8.16\left({ }_{-0.08}^{+0.08}\right)$ & $0.68\left({ }_{-0.05}^{+0.05}\right)$ & $-9.28(0.04)$ & $<-5.81$ & ) \\
\hline $\mathrm{J} 0019+1848$ & 6977 & $7.39\left({ }_{-0.51}^{+0.63}\right)$ & $0.29\left({ }_{-0.14}^{+0.30}\right)$ & $-10.34(0.12)$ & $<-4.28$ & ) \\
\hline $\mathrm{J} 0019+2209$ & 567 & $7.58\left({ }_{-0.30}^{+0.34}\right)$ & $0.35\left({ }_{-0.11}^{+0.17}\right)$ & $-10.18(0.16)$ & $<-3.00$ & $37.7(31.5)$ \\
\hline J0026-0006 & $5020(1185)$ & 8.00 & & $-10.75(0.11)$ & $<-3.00$ & $324.9(154.2)$ \\
\hline J0027+0102 & $8573(374)$ & $8.23\left({ }_{-0.31}^{+0.34}\right)$ & $0.72\left({ }_{-0.19}^{+0.22}\right)$ & $-9.25(0.14)$ & $<-4.95$ & $246.8(52.9)$ \\
\hline $\mathrm{J} 0027+3350$ & $7114(384)$ & 8.00 & & $-9.62(0.21)$ & $<-3.97$ & $297.0(22.3)$ \\
\hline J0030-0254 & 7011(904) & 8.00 & & $-9.99(0.61)$ & $<-3.91$ & $451.8(87.1)$ \\
\hline $\mathrm{J} 0031+2040$ & $7853(584)$ & 8.00 & & $-8.87(0.42)$ & $<-4.47$ & $373.8(40.4)$ \\
\hline J0031+3252 & $6537(332)$ & 8.00 & & $-10.01(0.23)$ & $<-3.44$ & $287.6(23.8)$ \\
\hline J0032+0827 & $5771(320)$ & $7.84\left({ }_{-0.20}^{+0.20}\right)$ & $0.48\left({ }_{-0.10}^{+0.12}\right)$ & $-10.16(0.10)$ & $<-3.00$ & $133.6(8.5)$ \\
\hline J0033+2845 & $7117(353)$ & $7.35\left(_{-1.47}^{+1.24}\right)$ & $0.27\left({ }_{-0.25}^{+0.69}\right)$ & $-11.09(0.18)$ & $<-4.47$ & $401.2(218.5)$ \\
\hline J0036-1112 & $7513(108)$ & $8.20\left({ }_{-0.04}^{+0.04}\right)$ & $0.70\left({ }_{-0.02}^{+0.02}\right)$ & $-9.06(0.08)$ & $<-4.02$ & $52.3(0.3)$ \\
\hline $\mathrm{J} 0038+1645$ & $6609(313)$ & $8.54\left({ }_{-0.40}^{+0.46}\right)$ & $0.93\left({ }_{-0.26}^{+0.26}\right)$ & $-10.06(0.19)$ & $<-3.05$ & $170.3(55.3)$ \\
\hline J0039+1416 & $6557(327)$ & 8.00 & & $-8.77(0.24)$ & $<-3.46$ & $327.0(29.2)$ \\
\hline $\mathrm{J} 0040+2349$ & $8170(231)$ & $8.22\left({ }_{-0.11}^{+0.11}\right)$ & $0.72\left({ }_{-0.07}^{+0.07}\right)$ & $-9.73(0.06)$ & $<-4.54$ & $158.6(10.0)$ \\
\hline $\mathrm{J} 0040+2723$ & $6448(375)$ & $7.71\left({ }_{-0.86}^{+1.14}\right)$ & $0.42\left({ }_{-0.28}^{+0.70}\right)$ & $-10.39(0.22)$ & $<-3.61$ & $280.0(154.9)$ \\
\hline J0040+3016 & $7411(658)$ & 8.00 & & $-10.25(0.12)$ & $<-4.10$ & $302.8(39.2)$ \\
\hline J0041+1511 & $8259(278)$ & $8.02\left({ }_{-0.16}^{+0.16}\right)$ & $0.59\left({ }_{-0.09}^{+0.10}\right)$ & $-10.62(0.08)$ & $<-4.95$ & $187.7(16.5)$ \\
\hline J0042+2255 & $7726(495)$ & $7.48\left({ }_{-0.77}^{+1.05}\right)$ & $0.32\left({ }_{-0.20}^{+0.60}\right)$ & $-11.44(0.23)$ & $<-5.04$ & $373.8(180.4)$ \\
\hline J0044+0418 & $6058(54)$ & $8.20\left({ }_{-0.04}^{+0.04}\right)$ & $0.70\left({ }_{-0.03}^{+0.03}\right)$ & $-9.76(0.05)$ & $<-3.00$ & $74.1(1.3)$ \\
\hline J0046+0024 & $9866(266)$ & $8.29\left({ }_{-0.24}^{+0.26}\right)$ & $0.76\left(_{-0.15}^{+0.17}\right)$ & $-7.70(0.18)$ & $<-5.72$ & $230.9(39.8)$ \\
\hline
\end{tabular}


Table 6. Atmospheric parameters for DZ stars - continued.

\begin{tabular}{|c|c|c|c|c|c|c|}
\hline J Name & $T_{\text {eff }}(\mathrm{K})$ & $\log g$ & $M / M_{\odot}$ & $\log \mathrm{Ca} / \mathrm{He}$ & $\log \mathrm{H} / \mathrm{He}$ & $D(\mathrm{pc})$ \\
\hline J0046+0635 & $6738(134)$ & $3.04\left({ }_{-0.07}^{+0.07}\right)$ & $0.60\left({ }_{-0.04}^{+0.05}\right)$ & $-11.05(0.05)$ & $<-3.63$ & $101.9(3.0)$ \\
\hline $\mathrm{J} 0046+2717$ & $8041(262)$ & $8.53\left({ }_{-0.24}^{+0.26}\right)$ & $0.92\left({ }_{-0.16}^{+0.16}\right)$ & $-7.43(0.23)$ & $<-4.09$ & $219.9(42.3)$ \\
\hline $\mathrm{J} 0049+0523$ & $6106(211)$ & $.20\left({ }_{-0.08}^{+0.08}\right)$ & $0.70\left({ }_{-0.05}^{+0.06}\right)$ & $-9.92(0.03)$ & $<-3.01$ & $4.3(0.0)$ \\
\hline $\mathrm{J} 0050+1828$ & $7029(372)$ & 8.00 & & $-9.33(0.26)$ & $<-3.92$ & $323.6(25.2)$ \\
\hline J0050-0621 & $6466(234)$ & $8.29\left({ }_{-0.31}^{+0.34}\right)$ & $0.76\left({ }_{-0.20}^{+0.22}\right)$ & $-9.21(0.16)$ & $<-3.10$ & $194.4(43.0)$ \\
\hline $\mathrm{J} 0052+1846$ & $5021(138)$ & 8.00 & & $-9.73(0.37)$ & $<-3.00$ & $198.3(13.6)$ \\
\hline $\mathrm{J} 0053+3115$ & $6290(374)$ & 8.00 & & $-9.71(0.71)$ & $<-3.19$ & $282.8(28.2)$ \\
\hline $\mathrm{J} 0055+2413$ & $7652(412)$ & 8.00 & & $-9.10(0.86)$ & $<-4.27$ & $353.4(26.7)$ \\
\hline $\mathrm{J} 0056+0005$ & $6567(306)$ & 8.00 & & $-9.85(0.39)$ & $<-3.47$ & $346.3(26.4)$ \\
\hline $\mathrm{J} 0056+2453$ & $5206(153)$ & 8.00 & & $-10.10(0.31)$ & $<-3.01$ & 203.1(13.3) \\
\hline $\mathrm{J} 0058+0507$ & $8817(759)$ & 8.00 & & $-9.69(0.27)$ & $<-5.45$ & $426.1(50.8)$ \\
\hline $\mathrm{J} 0058+1102$ & 6366(93) & $8.04\left({ }_{-0.09}^{+0.09}\right)$ & $0.60\left({ }_{-0.05}^{+0.06}\right)$ & $-9.67(0.09)$ & $<-3.23$ & $113.3(5.5)$ \\
\hline $\mathrm{J} 0059+0017$ & $10839(415)$ & $8.15\left({ }_{-0.18}^{+0.19}\right)$ & $0.67\left({ }_{-0.11}^{+0.13}\right)$ & $-8.80(0.12)$ & $<-5.94$ & $242.6(29.0)$ \\
\hline $\mathrm{J} 0102+1817$ & $6374(245)$ & 8.00 & & $-9.18(0.25)$ & $<-3.27$ & 293.1(19.6) \\
\hline $\mathrm{J} 0102+3112$ & $7001(566)$ & 8.00 & & $-9.61(0.34)$ & $<-3.90$ & $331.6(39.0)$ \\
\hline J0104-0030 & $7615(931)$ & 8.00 & & $-9.37(0.77)$ & $<-4.23$ & $592.6(105.9)$ \\
\hline $\mathrm{J} 0106+2412$ & $5590(4814)$ & 8.00 & & $-10.40(0.07)$ & $<-3.00$ & $349.3(419.7)$ \\
\hline J0106-0103 & $11222(493)$ & $7.90\left({ }_{-0.14}^{+0.15}\right)$ & $0.53\left({ }_{-0.07}^{+0.09}\right)$ & $-8.10(0.09)$ & $-4.32(0.12)$ & $226.1(17.8)$ \\
\hline $\mathrm{J} 0107+2650$ & $5258(80)$ & $8.18\left({ }_{-0.06}^{+0.06}\right)$ & $0.69\left({ }_{-0.04}^{+0.04}\right)$ & $-9.47(0.17)$ & $<-3.00$ & $68.3(1.6)$ \\
\hline $\mathrm{J} 0107+2905$ & $6904(1$ & $7.88\left({ }_{-0.18}^{+0.19}\right)$ & $0.51\left({ }_{-0.09}^{+0.11}\right)$ & $-9.38(0$ & $<-0.90$ & $171.9(18.5)$ \\
\hline J0108-0537 & 632 & $7.35\left({ }_{-0.42}^{+0.48}\right)$ & $0.27\left({ }_{-0.11}^{+0.21}\right)$ & $-9.10(0.14)$ & & $60.3(60.4)$ \\
\hline $\mathrm{J} 0111+1501$ & $10838(4$ & $7.98\left({ }_{-0.17}^{+0.18}\right)$ & $0.57\left({ }_{-0.09}^{+0.11}\right)$ & $-8.46(0.12)$ & $<-6.01$ & $75.7(28.3)$ \\
\hline $\mathrm{J} 0111+2848$ & $7217(964)$ & 8.00 & & $-9.47(0.48)$ & $<-4.02$ & $399.8(75.4)$ \\
\hline J0113-0006 & $7617(202)$ & $7.92\left({ }_{-0.15}^{+0.15}\right)$ & $0.53\left({ }_{-0.08}^{+0.09}\right)$ & $-10.17(0.08)$ & $<-4.32$ & $176.9(14.6)$ \\
\hline J0113-0959 & $10480(384)$ & $8.00\left({ }_{-0.07}^{+0.07}\right)$ & $0.58\left({ }_{-0.04}^{+0.04}\right)$ & $-8.44(0.09)$ & $-5.19(0.13)$ & $129.9(2.3)$ \\
\hline $\mathrm{J} 0114+3505$ & $6233(165)$ & 8.00 & & $-8.46(0.15)$ & $<-3.14$ & $242.1(12.8)$ \\
\hline J0115-0542 & $7739(417)$ & $7.76\left({ }_{-0.62}^{+0.81}\right)$ & $0.44\left({ }_{-0.23}^{+0.50}\right)$ & $-10.14(0.12)$ & $<-4.67$ & $324.7(135.3)$ \\
\hline $\mathrm{J} 0116+0328$ & $7204(368)$ & 8.00 & & $-9.64(0.22)$ & $<-4.01$ & $281.5(21.5)$ \\
\hline $\mathrm{J} 0116+0345$ & $7377(500)$ & 8.00 & & $-9.61(0.29)$ & $<-4.08$ & $301.5(30.6)$ \\
\hline $\mathrm{J} 0116+1744$ & $7122(215)$ & $8.20\left({ }_{-0.20}^{+0.21}\right)$ & $0.70\left({ }_{-0.13}^{+0.14}\right)$ & $-10.02(0.15)$ & $<-3.83$ & $193.2(25.7)$ \\
\hline $\mathrm{J} 0116+2050$ & 6258( & $8.02\left({ }_{-0.03}^{+0.03}\right)$ & $0.58\left({ }_{-0.02}^{+0.02}\right)$ & $-8.81(0.05)$ & $<-3.15$ & $73.6(1.0)$ \\
\hline $\mathrm{J} 0116+2229$ & $8603(394)$ & 8.00 & & $-8.10(0.83)$ & $<-5.33$ & $444.2(30.4)$ \\
\hline $\mathrm{J} 0117+0021$ & $7068(86)$ & $8.08\left({ }_{-0.08}^{+0.09}\right)$ & $0.63\left({ }_{-0.05}^{+0.06}\right)$ & $-8.57(0.06)$ & $<-3.89$ & $141.3(7.0)$ \\
\hline $\mathrm{J} 0117+0039$ & $6135(510)$ & 8.00 & & $-10.06(0.35)$ & $<-3.07$ & $388.3(57.7)$ \\
\hline $\mathrm{J} 0119+1758$ & $5327(143)$ & 8.00 & & $-9.79(0.25)$ & $<-3.01$ & $238.5(17.1)$ \\
\hline J0120-0041 & $11792(423)$ & $7.84\left({ }_{-0.11}^{+0.12}\right)$ & $0.50\left({ }_{-0.06}^{+0.07}\right)$ & $-9.12(0.24)$ & $<-6.10$ & $227.4(13.9)$ \\
\hline $\mathrm{J} 0121+1504$ & $8149(836)$ & 8.00 & & $-8.56(0.52)$ & $<-4.84$ & $579.2(92.6)$ \\
\hline $\mathrm{J} 0121+3440$ & $6906(134)$ & $8.13\left({ }_{-0.05}^{+0.05}\right)$ & $0.65\left(\begin{array}{l}+0.03 \\
-0.03\end{array}\right)$ & $-10.80(0.06)$ & $<-3.72$ & $38.9(0.2)$ \\
\hline
\end{tabular}


Table 6. Atmospheric parameters for DZ stars - continued.

\begin{tabular}{|c|c|c|c|c|c|c|}
\hline J Name & $T_{\text {eff }}(\mathrm{K})$ & $\log g$ & $M / M_{\odot}$ & $\log \mathrm{Ca} / \mathrm{He}$ & $\log \mathrm{H} / \mathrm{He}$ & $D(\mathrm{pc})$ \\
\hline $\mathrm{J} 0123+1324$ & $7341(239)$ & $.33\left(\begin{array}{l}+0.19 \\
-0.18\end{array}\right)$ & $0.79\left({ }_{-0.12}^{+0.12}\right)$ & $-9.85(0.25)$ & $<-3.86$ & $178.8(22.0)$ \\
\hline $\mathrm{J} 0125+2346$ & $7009(477)$ & 8.00 & & $-10.19(0.27)$ & $<-3.91$ & $347.8(36.1)$ \\
\hline $\mathrm{J} 0126+2534$ & $5692(162)$ & $8.58\left({ }_{-0.18}^{+0.19}\right)$ & $0.95\left({ }_{-0.12}^{+0.12}\right)$ & $-9.11(0.20)$ & $<-3.01$ & $113.0(15.5)$ \\
\hline $\mathrm{J} 0127+1545$ & $6576(307)$ & 8.00 & & $-8.95(0.29)$ & $<-3.48$ & $329.7(26.2)$ \\
\hline $\mathrm{J} 0130+2218$ & $6568(677)$ & 8.00 & & $-9.26(($ & $<-3.48$ & $385.9(59.4)$ \\
\hline $\mathrm{J} 0131+0250$ & $4808(139)$ & 8.00 & & $-10.04(0.21)$ & $<-3.00$ & $164.1(11.8)$ \\
\hline $\mathrm{J} 0134+1857$ & $6525(253)$ & $8.15\left({ }_{-0.29}^{+0.32}\right)$ & $0.67\left({ }_{-0.18}^{+0.21}\right)$ & $-10.22(0.13)$ & $<-3.28$ & $210.5(40.8)$ \\
\hline $\mathrm{J} 0135+1302$ & $6005(63)$ & $8.11\left({ }_{-0.07}^{+0.07}\right)$ & $0.64\left({ }_{-0.04}^{+0.05}\right)$ & $-9.23(0.14)$ & $<-3.00$ & $98.9(4.0)$ \\
\hline J0135-0027 & $4811(127)$ & $7.62\left({ }_{-0.22}^{+0.23}\right)$ & $0.37\left({ }_{-0.09}^{+0.11}\right)$ & $-11.09(0.11)$ & $<-3.00$ & $108.5(11.7)$ \\
\hline $\mathrm{J} 0136+0229$ & $7032(422)$ & 8.00 & & $-9.19(0.47)$ & $<-3.92$ & $408.2(36.1)$ \\
\hline $\mathrm{J} 0138+0031$ & $9494(329)$ & $8.13\left({ }_{-0.24}^{+0.26}\right)$ & $0.66\left(_{-0.14}^{+0.17}\right)$ & $-9.95(0.14)$ & $<-5.77$ & $216.2(34.4)$ \\
\hline $\mathrm{J} 0140+3125$ & $6741(674)$ & 8.00 & & $-9.67(0.24)$ & $<-3.67$ & $343.3(54.6)$ \\
\hline $\mathrm{J} 0140+3357$ & $6473(133)$ & $8.09\left({ }_{-0.08}^{+0.08}\right)$ & $0.63\left({ }_{-0.05}^{+0.05}\right)$ & $-10.95(0.10)$ & $<-3.28$ & $101.5(4.1)$ \\
\hline $\mathrm{J} 0141+2921$ & $6647(189)$ & $7.87\left({ }_{-0.19}^{+0.21}\right)$ & $0.50\left({ }_{-0.10}^{+0.12}\right)$ & $-10.54(0.07)$ & $<-3.69$ & $184.2(20.3)$ \\
\hline $\mathrm{J} 0142+0118$ & $6727(244)$ & $7.84\left({ }_{-0.26}^{+0.28}\right)$ & $0.48\left({ }_{-0.13}^{+0.17}\right)$ & $-11.00(0.11)$ & $<-3.79$ & $219.4(32.8)$ \\
\hline $\mathrm{J} 0143+0113$ & $7297(95)$ & $8.20\left({ }_{-0.09}^{+0.09}\right)$ & $0.70\left({ }_{-0.06}^{+0.06}\right)$ & $-8.08(0.05)$ & $<-3.92$ & $145.1(8.0)$ \\
\hline $\mathrm{J} 0143+0424$ & $7403(393)$ & 8.00 & & $-8.61(0.34)$ & $<-4.10$ & $359.5(29.3)$ \\
\hline $\mathrm{J} 0144+0305$ & $6272(280)$ & 8.00 & & $-9.96(0.37)$ & $<-3.17$ & $288.8(22.6)$ \\
\hline $\mathrm{J} 0144+1920$ & $5863(198)$ & 8.00 & & $-9.06(0.11$ & $<-3.00$ & 190.4(12.6) \\
\hline $\mathrm{J} 0147+1541$ & 8762 & 8.00 & & $-8.58(1.43$ & $<-5.42$ & 7.1) \\
\hline J0147-06 & 73 & 8.00 & & $-9.56(0$. & $<-4.06$ & 5) \\
\hline J0148-0112 & $6791(166)$ & $7.69\left({ }_{-0.87}^{+1.17}\right)$ & $0.41\left({ }_{-0.27}^{+0.71}\right)$ & $-9.04(0.17)$ & $<-3.94$ & $312.5(177.7)$ \\
\hline $\mathrm{J} 0150+1354$ & $6996(124)$ & $8.32\left({ }_{-0.14}^{+0.15}\right)$ & $0.78\left({ }_{-0.09}^{+0.10}\right)$ & $-8.34(0.15)$ & $<-3.63$ & $170.0(16.8)$ \\
\hline $\mathrm{J} 0150+1710$ & $8422(598)$ & $7.72\left({ }_{-0.52}^{+0.66}\right)$ & $0.43\left({ }_{-0.19}^{+0.40}\right)$ & $-10.39(0.16)$ & $<-5.47$ & $374.5(126.0)$ \\
\hline $\mathrm{J} 0152+2418$ & $7465(115)$ & $8.08\left({ }_{-0.04}^{+0.04}\right)$ & $0.62\left(_{-0.03}^{+0.03}\right)$ & $-9.24(0.07)$ & $<-4.07$ & $52.9(0.3)$ \\
\hline $\mathrm{J} 0153+0101$ & $7480(774)$ & 8.00 & & $-9.95(0.35)$ & $<-4.14$ & $478.2(73.6)$ \\
\hline J0158-0942 & $6126(177)$ & $8.06\left({ }_{-0.28}^{+0.30}\right)$ & $0.61\left({ }_{-0.16}^{+0.20}\right)$ & $-10.07(0.13)$ & $<-3.04$ & $176.8(31.9)$ \\
\hline $\mathrm{J} 0200+0040$ & 10467(359) & $8.16\left({ }_{-0.10}^{+0.10}\right)$ & $0.68\left({ }_{-0.06}^{+0.06}\right)$ & $-9.10(0.14)$ & $<-5.90$ & $169.4(8.4)$ \\
\hline $\mathrm{J} 0201+2015$ & $6306(194)$ & $8.16\left({ }_{-0.21}^{+0.23}\right)$ & $0.67\left({ }_{-0.13}^{+0.15}\right)$ & $-8.99(0.13)$ & $<-3.10$ & $170.2(23.2)$ \\
\hline J0201-0039 & $9863(261)$ & $8.15\left({ }_{-0.06}^{+0.06}\right)$ & $0.67\left({ }_{-0.04}^{+0.04}\right)$ & $-7.99(0.05)$ & $<-5.81$ & $111.2(2.0)$ \\
\hline $\mathrm{J} 0202+2459$ & $6248(353)$ & 8.00 & & $-9.19(0.47)$ & $<-3.15$ & $359.4(40.1)$ \\
\hline $\mathrm{J} 0209+0558$ & $8575(464)$ & $7.62\left({ }_{-0.57}^{+0.79}\right)$ & $0.38\left({ }_{-0.18}^{+0.46}\right)$ & $-9.91(0.22)$ & $<-5.63$ & $382.1(150.4)$ \\
\hline $\mathrm{J} 0209+2914$ & $6790(226)$ & $8.52\left({ }_{-0.15}^{+0.16}\right)$ & $0.91\left({ }_{-0.10}^{+0.10}\right)$ & $-9.27(0.10)$ & $<-3.22$ & $146.3(16.3)$ \\
\hline J0211-0046 & $10701(454)$ & $7.79\left({ }_{-0.31}^{+0.37}\right)$ & $0.46\left({ }_{-0.14}^{+0.21}\right)$ & $-8.91(0.50)$ & $-5.36(0.57)$ & $354.5(71.9)$ \\
\hline $\mathrm{J} 0213+2848$ & $6920(316)$ & $7.80\left({ }_{-0.23}^{+0.25}\right)$ & $0.46\left({ }_{-0.11}^{+0.14}\right)$ & $-10.69(0.08)$ & $<-3.96$ & $207.7(25.1)$ \\
\hline $\mathrm{J} 0214+0536$ & $7492(355)$ & $8.01\left({ }_{-0.36}^{+0.40}\right)$ & $0.58\left({ }_{-0.19}^{+0.26}\right)$ & $-10.16(0.13)$ & $<-4.13$ & $269.1(63.0)$ \\
\hline J0218-0919 & $10273(321)$ & $8.19\left({ }_{-0.06}^{+0.06}\right)$ & $0.70\left(_{-0.04}^{+0.04}\right)$ & $-9.81(0.04)$ & $<-5.84$ & $96.2(1.1)$ \\
\hline J0221-0445 & $7719(873)$ & 8.00 & & $-10.45(0.30)$ & $<-4.33$ & $423.8(70.4)$ \\
\hline
\end{tabular}


Table 6. Atmospheric parameters for DZ stars - continued.

\begin{tabular}{|c|c|c|c|c|c|c|}
\hline J Name & $T_{\text {eff }}(\mathrm{K})$ & $\log g$ & $M / M_{\odot}$ & $\log \mathrm{Ca} / \mathrm{He}$ & $\log \mathrm{H} / \mathrm{He}$ & $D(\mathrm{pc})$ \\
\hline J0225-0018 & $6207(101)$ & $24(+0.15)$ & $73\left(\begin{array}{l}+0.10 \\
-0.09\end{array}\right)$ & $-10.01(0.12)$ & $<-3.02$ & $135.4(13.1)$ \\
\hline J0226-0444 & $7247(757)$ & 8.00 & & $-10.37(0.20)$ & $<-4.03$ & $405.8(64.3)$ \\
\hline J0228-0009 & $8573(312)$ & $3.41\left({ }_{-0.27}^{+0.30}\right)$ & $0.84\left({ }_{-0.18}^{+0.19}\right)$ & $-8.79(0.79)$ & $<-4.64$ & $227.1(47.1)$ \\
\hline J0231-0142 & $7037(736)$ & 8.00 & & $-10.17(0.23)$ & $<-3.92$ & $397.6(65.2)$ \\
\hline J0234-0510 & $6696(245)$ & 8.00 & & $-8.55(0$ & $<-3.62$ & $303.9(19.3)$ \\
\hline J0241-0533 & $8379(226)$ & $7.99\left({ }_{-0.09}^{+0.09}\right)$ & $0.57\left({ }_{-0.05}^{+0.05}\right)$ & $-9.61(0.07)$ & $-3.34(0.11)$ & $143.3(5.6)$ \\
\hline $\mathrm{J} 0251+7341$ & 7531 & $8.07\left({ }_{-0.05}^{+0.05}\right)$ & $0.62\left({ }_{-0.03}^{+0.03}\right)$ & $-9.97(0$ & $<-4.12$ & $61.4(0.3)$ \\
\hline $\mathrm{J} 0252+0054$ & 7433( & $8.01\left({ }_{-0.17}^{+0.18}\right)$ & $0.58\left({ }_{-0.10}^{+0.11}\right)$ & $-8.55(0.21)$ & $<-4.10$ & 205. \\
\hline J0252-0401 & $6936(150)$ & $8.03\left({ }_{-0.27}^{+0.29}\right)$ & $0.59\left(_{-0}^{+0}\right.$ & $-8.59(($ & $<-3.83$ & $220.4(37.9)$ \\
\hline J0302-0108 & $13594(1735)$ & $8.02\left({ }_{-0.18}^{+0.19}\right)$ & $0.60\left({ }_{-0.10}^{+0.12}\right)$ & $-7.58(0.10)$ & $-6.11(0$ & $64.4(0.3)$ \\
\hline J0308-0657 & $10049(487)$ & $8.30\left({ }_{-0.26}^{+0.28}\right)$ & $0.77\left({ }_{-0.16}^{+0.18}\right)$ & $-8.08(0.54)$ & $<-5.72$ & $289.0(52.3)$ \\
\hline $\mathrm{J} 0314+0524$ & 14241 & $8.09\left({ }_{-0.21}^{+0.23}\right)$ & & -7.43 & $<-6$ & 1.4) \\
\hline J0314-0827 & $11810(625)$ & $8.21\left(_{-0}^{+0}\right.$ & $0.72\left(_{-0}^{+0}\right.$ & $-7.79(0.78)$ & $<-6.05$ & $353.2(126.4)$ \\
\hline $\mathrm{J} 0319+3630$ & $6335(307)$ & $8.12\left({ }_{-0.11}^{+0.12}\right)$ & $0.64\left({ }_{-0.07}^{+0.08}\right)$ & $-9.37(0.14)$ & $<-3.15$ & $32.0(0.1)$ \\
\hline J0411-0548 & $10069(652)$ & $8.43\left({ }_{-0.30}^{+0.33}\right)$ & $0.86\left({ }_{-0.20}^{+0.21}\right)$ & $-9.18(0.43)$ & $<-5.60$ & $291.7(65.6)$ \\
\hline $\mathrm{J} 0438+4109$ & $15680(1633)$ & $8.04\left({ }_{-0.15}^{+0.16}\right)$ & $0.62\left({ }_{-0.09}^{+0.10}\right)$ & $-8.51(0.56)$ & $-4.34(0$ & $53.7(0.2)$ \\
\hline $\mathrm{J} 0447+1124$ & $6598(141)$ & $7.81\left({ }_{-0.27}^{+0.29}\right)$ & $0.47\left({ }_{-0.13}^{+0.17}\right)$ & $-9.28(0$ & $<-3.69$ & 206.6(33.6) \\
\hline $\mathrm{J} 0536+6154$ & 10953(398) & $8.02\left({ }_{-0.06}^{+0.07}\right)$ & $0.59\left(_{-0}^{+0}\right.$ & $-8.32(0.06)$ & $-5.59(0$ & $132.7(2.1)$ \\
\hline J0555-0410 & $4491(67)$ & $7.92\left({ }_{-0.06}^{+0.06}\right)$ & & -11.16 & $<-30$ & 6.4 \\
\hline $\mathrm{J} 0618+641$ & 90 & $8.02\left({ }_{-0.11}^{+0.11}\right)$ & $0.59\left(_{+}^{+}\right.$ & -10.37( & --0.04 & $100.1(9$. \\
\hline $\mathrm{J} 0627+1002$ & 8 & & & $-10.50(0$ & & \\
\hline $\mathrm{J} 0649+3726$ & 66 & $8.25\left({ }_{-0.13}^{+0.14}\right)$ & $0.74\left({ }_{-0.09}^{+0.09}\right)$ & $-9.98(0$ & & B) \\
\hline J0721+3928 & $5997(1$ & 8.00 & & $-9.47(0$ & $<-3.00$ & 189.1(9.9) \\
\hline $\mathrm{J} 0721+3955$ & $6731(90)$ & $7.92\left({ }_{-0.05}^{+0.05}\right)$ & $0.53\left({ }_{-0.03}^{+0.03}\right)$ & $-9.73(0$ & $<-3.72$ & $96.1(1.9)$ \\
\hline $\mathrm{J} 0730+3456$ & $10970(420)$ & $8.04\left({ }_{-0.07}^{+0.07}\right)$ & $0.61\left({ }_{-0.04}^{+0.04}\right)$ & $-8.94(0.06)$ & $-5.31(0.09)$ & $98.0(1.2)$ \\
\hline $\mathrm{J} 0732+2746$ & $12084(553)$ & $8.01\left({ }_{-0.15}^{+0.16}\right)$ & $0.59\left({ }_{-0.08}^{+0.10}\right)$ & $-8.76(0.15)$ & $-5.74(0.33)$ & $238.0(21.3)$ \\
\hline $\mathrm{J} 0733+3727$ & $7599(291)$ & $7.88\left({ }_{-0.37}^{+0.42}\right)$ & $0.51\left({ }_{-0.18}^{+0.26}\right)$ & $-10.84(0.21)$ & $<-4.35$ & $252.0(59.2)$ \\
\hline $\mathrm{J} 0734+4448$ & $13863(8$ & $8.42\left({ }_{-0.19}^{+0.20}\right)$ & $0.86\left({ }_{-0.12}^{+0.13}\right)$ & $-9.34(0.56)$ & $<-6.02$ & $288.5(38.4)$ \\
\hline J0736+4118 & $4676(70)$ & $7.99\left(_{-0.11}^{+0.11}\right)$ & $0.56\left(_{-0.06}^{+0.07}\right)$ & $-9.73(0.24)$ & $<-3.00$ & $80.3(3.9)$ \\
\hline J0738+3844 & $10280(279)$ & $8.11\left({ }_{-0.22}^{+0.24}\right)$ & $0.65\left({ }_{-0.13}^{+0.16}\right)$ & $-7.27(0.21)$ & $-4.78(0.51)$ & $300.5(45.4)$ \\
\hline $\mathrm{J} 0739+3024$ & $7106(624)$ & 8.00 & & $-9.75(0.3$ & $<-3.96$ & $427.7(56.1)$ \\
\hline J0740-1724 & $7545(58$ & $7.99\left({ }_{-0.16}^{+0.16}\right)$ & $0.57\left({ }_{-0.09}^{+0.10}\right)$ & $-10.93(0.05)$ & $-3.38(0.04)$ & $9.2(0.0)$ \\
\hline $\mathrm{J} 0741+3146$ & $6065(113)$ & $8.29\left({ }_{-0.19}^{+0.20}\right)$ & $0.76\left({ }_{-0.12}^{+0.13}\right)$ & $-8.63(0.12)$ & $<-3.00$ & $54.5(20.5)$ \\
\hline $\mathrm{J} 0741+4829$ & $6741(2$ & $8.34\left({ }_{-0.19}^{+0.20}\right)$ & $0.80\left({ }_{-0.13}^{+0.14}\right)$ & -10.59( & $<-3.32$ & $174.9(23.7)$ \\
\hline $\mathrm{J} 0742+2422$ & $7106(469)$ & 8.00 & & $-10.13(0.27)$ & $<-3.96$ & $32.4(33.3)$ \\
\hline $\mathrm{J} 0743+4240$ & 1230 & $7.72\left({ }_{-0.47}^{+0.63}\right)$ & $0.44\left({ }_{-0.17}^{+0.37}\right)$ & $-8.45(0.86)$ & $<-6.14$ & $511.7(172.1)$ \\
\hline $\mathrm{J} 0744+1640$ & 494 & $8.11\left({ }_{-0.23}^{+0.24}\right)$ & $0.64\left({ }_{-0.14}^{+0.16}\right)$ & $-9.88(0$ & $<-3.00$ & $118.5(16.8)$ \\
\hline $\mathrm{J} 0744+2701$ & $7735(189)$ & $8.20\left({ }_{-0.30}^{+0.33}\right)$ & $0.70\left({ }_{-0.19}^{+0.22}\right)$ & $-8.30(0.19)$ & $<-4.17$ & $246.0(52.1)$ \\
\hline
\end{tabular}


Table 6. Atmospheric parameters for DZ stars - continued.

\begin{tabular}{|c|c|c|c|c|c|c|}
\hline J Name & $T_{\text {eff }}(\mathrm{K})$ & $\log g$ & $M / M_{\odot}$ & $\log \mathrm{Ca} / \mathrm{He}$ & $\log \mathrm{H} / \mathrm{He}$ & $D(\mathrm{pc})$ \\
\hline $\mathrm{J} 0744+4408$ & $6655(246)$ & 8.00 & & $-8.69(0.24)$ & $<-3.57$ & $310.2(21.0)$ \\
\hline $\mathrm{J} 0744+4649$ & $5045(61)$ & 15( & $0.66 \stackrel{+}{+}^{+}$ & $-8.25(0.03)$ & $<-3.00$ & $56.0(0.9)$ \\
\hline $\mathrm{J} 0747+3732$ & $10165(373)$ & $7.79\left({ }_{-0.24}^{+0.27}\right)$ & $0.46\left({ }_{-0.11}^{+0.15}\right)$ & $-9.18(0.24)$ & $<-6.00$ & $322.4(47.3)$ \\
\hline $\mathrm{J} 0747+4001$ & $8688(268)$ & $8.14\left({ }_{-0.09}^{+0.09}\right)$ & $0.66\left({ }_{-0.06}^{+0.06}\right)$ & $-10.82(0.18)$ & $<-5.21$ & $150.6(5.9)$ \\
\hline $\mathrm{J} 0747+4825$ & $6647(185)$ & $7.83\left({ }_{-0.31}^{+0.34}\right)$ & $0.48\left({ }_{-0.14}^{+0.20}\right)$ & $-10.45(0.08)$ & $<-3.72$ & $198.1(36.5)$ \\
\hline $\mathrm{J} 0748+2544$ & 6497( & 8.00 & & -10.60 & $<-3.40$ & 883.1 \\
\hline $\mathrm{J} 0748+3506$ & 11168 & $7.27\left({ }_{-0.71}^{+1.26}\right)$ & $0.27\left({ }_{-0.14}^{+0.65}\right)$ & $-9.23(\mathrm{C}$ & $<-6.21$ & 600.7 \\
\hline $\mathrm{J} 0749+3124$ & 6678 & $8.12\left(_{-}^{+}\right.$ & $0.65\left(_{-}^{+}\right.$ & $-9.97(0.04)$ & $<-3.47$ & $91.7(2.1)$ \\
\hline $\mathrm{J} 0749+4343$ & $9459(477)$ & $55\left(_{-}^{+}\right.$ & $0.93\left(_{-}^{+}\right.$ & $-9.18(0.25)$ & $<-5.38$ & $253.3(54.6)$ \\
\hline $\mathrm{J} 0751+1310$ & $11886(458)$ & $7.92\left({ }_{-0.10}^{+0.10}\right)$ & & $-8.26(0.19)$ & $<-6.10$ & $227.9(10.7)$ \\
\hline $\mathrm{J} 0753+2332$ & 11150 & $8.50\left({ }_{-0.61}^{+0.82}\right)$ & $0.91\left(_{-}^{+}\right.$ & $-9.67(0.34)$ & $-4.85(C$ & $340.3(178.2)$ \\
\hline $\mathrm{J} 0753+2741$ & 6548 & 8.00 & & $-9.41(0.47)$ & $<-3.45$ & 388. \\
\hline $\mathrm{J} 0753+6613$ & $7485(179)$ & 8.03( & $0.59\left(_{-c}^{+c}\right.$ & $-9.45(0.17)$ & $<-4.12$ & $198.5(1$ \\
\hline $\mathrm{J} 0755+2112$ & 6843 & $8.25\left(_{-c}^{+c}\right.$ & $0.73\left(_{-c}^{+c}\right.$ & $-9.46(0$ & $<-3.53$ & $148.3(10.9)$ \\
\hline $\mathrm{J} 0756+3353$ & 7909 & $7.82\left({ }_{-0.24}^{+0.26}\right)$ & $0.48\left({ }_{-0.12}^{+0.15}\right)$ & $-9.69(0$ & $<-4.78$ & $262.8(3$ \\
\hline $\mathrm{J} 0758+1013$ & $5600(144)$ & 8.00 & & $-8.50(0.14)$ & $<-3.00$ & $219.3(13.0)$ \\
\hline $\mathrm{J} 0758+3225$ & $10532(467)$ & $8.55\left({ }_{-0.24}^{+0.26}\right)$ & $0.94\left(_{-c}^{+c}\right.$ & $-9.58(0$ & $<-5.56$ & $236.2(44.7)$ \\
\hline $\mathrm{J} 0800+2242$ & $6153(172)$ & $8.05\left({ }_{-0.51}^{+0.58}\right)$ & $0.60\left(_{-0}^{+0}\right.$ & $-9.82(0.17)$ & $<-3.06$ & $210.6(71.8)$ \\
\hline $\mathrm{J} 0801+13$ & 100 & $8.12\left({ }_{-0.47}^{+0.56}\right)$ & $0.66\left(_{-0}^{+0}\right.$ & $-9.30(0$ & $<-5.85$ & $4.7)$ \\
\hline $\mathrm{J} 0801+141$ & 150 & $8.08\left({ }_{-0.11}^{+0.11}\right)$ & $0.64\left(_{-}^{+}\right.$ & $-8.46(0$ & -5 & 11 \\
\hline $\mathrm{J} 0801+5329$ & 88 & & & $-8.36(0$ & & $2.1(0)$. \\
\hline $\mathrm{J} 0802+3012$ & 1047 & $8.14\left(_{+}^{+}\right.$ & $0.67\left({ }_{-0.35}^{+0.56}\right)$ & $-8.14(0$ & $<-5.91$ & T. \\
\hline $\mathrm{J} 0802+4050$ & 132 & $7.91\left(_{-}^{+}\right.$ & $0.54\left({ }_{-0.06}^{+0.07}\right)$ & $-9.54(0.26)$ & $-5.22(0$. & 196.7( \\
\hline $\mathrm{J} 0802+4257$ & $6705(230)$ & $7.83\left({ }_{-0.55}^{+0.66}\right)$ & $0.48\left({ }_{-0.23}^{+0.42}\right)$ & $-9.85(0.13)$ & $<-3.77$ & $250.8(88.9)$ \\
\hline $\mathrm{J} 0803+4502$ & $9225(327)$ & $8.18\left({ }_{-0.21}^{+0.22}\right)$ & $0.69\left({ }_{-0.13}^{+0.15}\right)$ & $-9.38(0.23)$ & $-3.68(0.37)$ & $238.5(33.2)$ \\
\hline $\mathrm{J} 0805+3832$ & $10680(354)$ & $8.11\left({ }_{-0.06}^{+0.06}\right)$ & $0.65\left({ }_{-0.04}^{+0.04}\right)$ & $-9.60(0.02)$ & $<-5.95$ & $45.3(0.1)$ \\
\hline $\mathrm{J} 0805+5406$ & $12942(535)$ & $8.08\left({ }_{-0.07}^{+0.07}\right)$ & $0.64\left({ }_{-0.04}^{+0.05}\right)$ & $-9.27(0.19)$ & $-6.13(0.37)$ & $154.6(3.7)$ \\
\hline $\mathrm{J} 0806+3055$ & $6708(153)$ & 8.00 & & $-8.72(0.14)$ & $<-3.63$ & $255.0(10.0)$ \\
\hline $\mathrm{J} 0806+3640$ & 10881 & $7.81\left({ }_{-0.55}^{+0.73}\right)$ & $0.48\left({ }_{-0.21}^{+0.46}\right)$ & $-8.23(0.47)$ & $<-6.06$ & $459.9(182.2)$ \\
\hline $\mathrm{J} 0806+3702$ & $6751(229)$ & $8.02\left(_{-0}^{+0}\right.$ & $0.59\left(_{-}^{+}\right.$ & $-10.48(0.13)$ & $<-3.66$ & 209.4(37.6) \\
\hline $\mathrm{J} 0806+3747$ & 9800 & $0.101-$ & $0.70\left(_{-}^{+}\right.$ & $-7.93(0.1$ & $<-5.78$ & 0.3) \\
\hline $\mathrm{J} 0807+4930$ & 5 & $8.15\left({ }_{-0.27}^{+0.29}\right)$ & $0.66\left(_{-0.16}^{+0.19}\right)$ & $-8.21(0.1$ & $<-3.00$ & (0.1. \\
\hline J0808+2118 & $9048(1059)$ & 8.00 & & $-9.38(0.40)$ & $<-5.58$ & $577.7(99.0)$ \\
\hline $\mathrm{J} 0808+2809$ & $6878(1$ & $8.24\left({ }_{-0.25}^{+0.27}\right)$ & $0.73\left({ }_{-0.16}^{+0.18}\right)$ & $-8.21(0.13)$ & $<-3.58$ & $214.5(37.6)$ \\
\hline $\mathrm{J} 0809+1034$ & $6842(138)$ & $8.27\left({ }_{-0.07}^{+0.07}\right)$ & $0.75\left({ }_{-0.05}^{+0.05}\right)$ & $-10.18(0.06)$ & $<-3.51$ & $109.0(4.1)$ \\
\hline $\mathrm{J} 0809+3106$ & $11657(4$ & $7.85\left({ }_{-0.10}^{+0.11}\right)$ & $0.50\left({ }_{-0.05}^{+0.06}\right)$ & $-9.55(0.24)$ & $-5.44(0.26)$ & $230.0(11.8)$ \\
\hline J0812+0942 & 10593 & $7.76\left(_{-0.15}^{+0.17}\right)$ & $0.45\left(_{-0.07}^{+0.09}\right)$ & $-7.59(0$ & $<-6.08$ & $293.0(26.5)$ \\
\hline $\mathrm{J} 0812+$ & $7612(388)$ & 8.00 & & $-9.79(0.23)$ & $<-4.23$ & $360.7(27.3)$ \\
\hline
\end{tabular}


Table 6. Atmospheric parameters for DZ stars - continued.

\begin{tabular}{|c|c|c|c|c|c|c|}
\hline J Name & $T_{\text {eff }}(\mathrm{K})$ & $\log g$ & $M / M_{\odot}$ & $\log \mathrm{Ca} / \mathrm{He}$ & $\log \mathrm{H} / \mathrm{He}$ & $D(\mathrm{pc})$ \\
\hline $\mathrm{J} 0813+3453$ & $6753(300)$ & 8.00 & & $-9.61(0.35)$ & $<-3.68$ & $331.4(22.3)$ \\
\hline $\mathrm{J} 0813+3506$ & $7094(172)$ & $8.06\left({ }_{-0.40}^{+0.45}\right)$ & $0.61\left(_{-0}^{+0}\right.$ & $-9.12(0.25)$ & $<-3.91$ & $247.4(67.6)$ \\
\hline $\mathrm{J} 0816+2330$ & $7143(415)$ & 8.00 & & $-9.81(0.35)$ & $<-3.98$ & $310.9(25.6)$ \\
\hline $\mathrm{J} 0818+1247$ & $6872(264)$ & 8.00 & & $-9.03(0.36)$ & $<-3.80$ & $310.6(19.3)$ \\
\hline J0818-3110 & $6535(645)$ & $8.28\left(\begin{array}{c}+0.19 \\
-0.18\end{array}\right)$ & $0.75\left(\begin{array}{l}+0.13 \\
-0.12\end{array}\right)$ & $-8.99(0.04)$ & $<-3.17$ & $19.4(0.0)$ \\
\hline $\mathrm{J} 0819+0027$ & $11366(567)$ & $8.19\left({ }_{-0.21}^{+0.23}\right)$ & $0.70\left({ }_{-0.13}^{+0.15}\right)$ & $-8.47(0.21)$ & $-4.16(0$ & $250.3(34.8)$ \\
\hline $\mathrm{J} 0819+5732$ & $6074(8$ & $7.99\left({ }_{-0.04}^{+0.04}\right)$ & $0.56\left({ }_{-0.03}^{+0.03}\right)$ & $-11.10(0.10)$ & $<-3.04$ & $54.5(0.3)$ \\
\hline $\mathrm{J} 0822+3910$ & $11277(765)$ & $7.87\left({ }_{-0.59}^{+0.81}\right)$ & $0.51\left({ }_{-0.24}^{+0.51}\right)$ & $-8.83(0.37)$ & $<-6.05$ & $440.1(191.2)$ \\
\hline $\mathrm{J} 0822+5202$ & $6639(346)$ & 8.00 & & $-10.28(0.13)$ & $<-3.55$ & $262.3(22.8)$ \\
\hline $\mathrm{J} 0822+5203$ & 10039(369) & $8.07\left({ }_{-0.19}^{+0.20}\right)$ & $0.62\left({ }_{-0.11}^{+0.13}\right)$ & $-9.30(0.09)$ & $<-5.87$ & $237.6(27.7)$ \\
\hline $\mathrm{J} 0823+2015$ & $10575(374)$ & $8.05\left({ }_{-0.12}^{+0.12}\right)$ & $0.61\left({ }_{-0.07}^{+0.08}\right)$ & $-8.68(0.07)$ & $<-5.98$ & $220.3(14.4)$ \\
\hline $\mathrm{J} 0825+2009$ & $8457(397)$ & $8.00\left({ }_{-0.26}^{+0.28}\right)$ & $0.58\left(_{-0}^{+0}\right.$ & $-9.89(0.24)$ & $<-5.21$ & $271.6(43.7)$ \\
\hline $\mathrm{J} 0825+2148$ & $6411(147)$ & $8.22\left(\begin{array}{l}+0.10 \\
-0.10\end{array}\right)$ & $0.71\left(_{-0}^{+0}\right.$ & $-10.96(0.19)$ & $<-3.12$ & $126.7(7.2)$ \\
\hline $\mathrm{J} 0827+1731$ & $10537(382)$ & $8.06\left({ }_{-0.08}^{+0.08}\right)$ & $0.62\left({ }_{-0.05}^{+0.05}\right)$ & $-9.70(0.04)$ & $-4.27(0.07)$ & $129.1(2.7)$ \\
\hline $\mathrm{J} 0827+2857$ & $7465(277)$ & $8.78\left({ }_{-0.30}^{+0.35}\right)$ & $1.07\left({ }_{-0.19}^{+0.17}\right)$ & $-8.68(0.57)$ & $<-3.57$ & $183.4(51.3)$ \\
\hline $\mathrm{J} 0827+3304$ & $8489(203)$ & $7.94\left({ }_{-0.21}^{+0.23}\right)$ & $0.54\left({ }_{-0.11}^{+0.14}\right)$ & $-8.50(0.36)$ & $<-5.32$ & 259.1(33.9) \\
\hline $\mathrm{J} 0827+5504$ & $7371(511)$ & 8.00 & & $-10.14(0.30)$ & $<-4.08$ & $341.2(35.5)$ \\
\hline $\mathrm{J} 0829+0759$ & $10016(287)$ & $8.15\left({ }_{-0.09}^{+0.09}\right)$ & $0.67\left({ }_{-0.06}^{+0.06}\right)$ & $-7.95(0.10)$ & $<-5.83$ & $176.1(8.7)$ \\
\hline $\mathrm{J} 0829+3551$ & 6682( & 8.00 & & $-10.28(0$ & $<-3.60$ & $.5)$ \\
\hline J0830-0319 & 6365( & $7.70\left({ }_{-0.12}^{+0.13}\right)$ & $0.41\left({ }_{-0.06}^{+0.06}\right)$ & -9.51 & $<-3.52$ & 17 \\
\hline $\mathrm{J} 0831+1801$ & 710 & 8.00 & & $-10.50(0.17)$ & $<-3.96$ & 6) \\
\hline $\mathrm{J} 0833+0512$ & 12185( & $7.42\left({ }_{-0.30}^{+0.41}\right)$ & & $-9.37(0.30)$ & $<-6.18$ & $424.0(91.5)$ \\
\hline $\mathrm{J} 0834+2422$ & 1159 & $8.04\left({ }_{-0.11}^{+0.12}\right)$ & $0.61\left({ }_{-0.06}^{+0.07}\right)$ & $-8.18(0.15)$ & $-5.25(0$ & $214.2(13.0)$ \\
\hline $\mathrm{J} 0834+3927$ & $8117(673)$ & 8.00 & & $-8.42(0.37)$ & $<-4.80$ & $532.6(66.0)$ \\
\hline $\mathrm{J} 0834+4641$ & $7065(84)$ & $8.04\left({ }_{-0.07}^{+0.07}\right)$ & $0.60\left({ }_{-0.04}^{+0.04}\right)$ & $-9.20(0.13)$ & $<-3.91$ & $123.1(4.4)$ \\
\hline $\mathrm{J} 0835+0906$ & $8546(315)$ & $8.18\left({ }_{-0.13}^{+0.13}\right)$ & $0.69\left({ }_{-0.08}^{+0.09}\right)$ & $-9.60(0.11)$ & $<-5.00$ & $155.7(11.3)$ \\
\hline $\mathrm{J} 0838+2322$ & $6037(61)$ & $8.25\left({ }_{-0.05}^{+0.05}\right)$ & $0.73\left({ }_{-0.03}^{+0.03}\right)$ & $-9.84(0.13)$ & $<-3.00$ & $84.4(2.2)$ \\
\hline $\mathrm{J} 0838+5455$ & $6795(315)$ & $8.25\left({ }_{-0.47}^{+0.53}\right)$ & $0.73\left({ }_{-0.28}^{+0.34}\right)$ & $-10.51(0.27)$ & $<-3.47$ & $205.8(69.3)$ \\
\hline $\mathrm{J} 0839+2158$ & $8340(265)$ & $8.26\left({ }_{-0.14}^{+0.14}\right)$ & $0.74\left({ }_{-0.09}^{+0.09}\right)$ & $-8.78(0.31)$ & $<-4.63$ & $162.3(14.0)$ \\
\hline $\mathrm{J} 0839+2618$ & $14581(1486)$ & $8.09\left({ }_{-0.24}^{+0.27}\right)$ & $0.64\left({ }_{-0.14}^{+0.17}\right)$ & $-8.29(0.67)$ & $-3.98(0.28)$ & $315.8(39.2)$ \\
\hline $\mathrm{J} 0840+0237$ & $7398(302)$ & $7.53\left({ }_{-0.41}^{+0.51}\right)$ & $0.34\left(_{-c}^{+c}\right.$ & $-11.02(0.10)$ & $<-4.59$ & 18.1(80.7) \\
\hline $\mathrm{J} 0840+1306$ & $6854(21$ & $7.96\left({ }_{-0.32}^{+0.35}\right)$ & $0.55\left({ }_{-0.17}^{+0.22}\right)$ & $-11.03(0.16)$ & $<-3.81$ & $198.5(39.7)$ \\
\hline $\mathrm{J} 0840+3202$ & $7535(886)$ & 8.00 & & -10 & -4.17 & 3.8) \\
\hline $\mathrm{J} 0840+3812$ & $7421(760)$ & 8.00 & & $-9.96(0.26)$ & $<-4.11$ & $478.6(68.9)$ \\
\hline $\mathrm{J} 0841+3723$ & $12036(493)$ & $7.93\left({ }_{-0.08}^{+0.08}\right)$ & $0.55\left({ }_{-0.04}^{+0.05}\right)$ & $-7.95(0.08)$ & $<-6.11$ & $171.3(5.2)$ \\
\hline $\mathrm{J} 0841+5414$ & $7884(304)$ & $7.83\left({ }_{-0.20}^{+0.21}\right)$ & $0.48\left({ }_{-0.10}^{+0.12}\right)$ & $-10.33(0.07)$ & $<-4.73$ & $220.5(24.1)$ \\
\hline $\mathrm{J} 0842+1406$ & $7111(55)$ & $7.99\left({ }_{-0.04}^{+0.04}\right)$ & $0.57\left({ }_{-0.03}^{+0.03}\right)$ & $-8.45(0.06)$ & $<-3.97$ & $99.1(2.1)$ \\
\hline $\mathrm{J} 0842+1536$ & $5932(137)$ & 8.00 & & $-9.88(0.23)$ & $<-3.00$ & $198.7(9.1)$ \\
\hline
\end{tabular}


Table 6. Atmospheric parameters for DZ stars - continued.

\begin{tabular}{|c|c|c|c|c|c|c|}
\hline J Name & $T_{\text {eff }}(\mathrm{K})$ & $\log g$ & $M / M_{\odot}$ & $\log \mathrm{Ca} / \mathrm{He}$ & $\log \mathrm{H} / \mathrm{He}$ & $D(\mathrm{pc})$ \\
\hline $\mathrm{J} 0842+3625$ & $10322(424)$ & $3.02\left({ }_{-0.13}^{+0.13}\right)$ & $0.59\left({ }_{-0.07}^{+0.08}\right)$ & $-9.00(0.15)$ & $-4.78(0.21)$ & $213.7(14.2)$ \\
\hline J0842-1347 & $5010(89)$ & $8.05\left({ }_{-0.06}^{+0.06}\right)$ & $0.60\left({ }_{-0.04}^{+0.04}\right)$ & $-9.93(0.13)$ & $<-3.00$ & $14.8(0.0)$ \\
\hline $\mathrm{J} 0843+1247$ & $6507(163)$ & $8.52\left({ }_{-0.15}^{+0.16}\right)$ & $0.92\left({ }_{-0.10}^{+0.10}\right)$ & $-10.23(0.13)$ & $<-3.00$ & $134.9(15.4)$ \\
\hline $\mathrm{J} 0843+5614$ & $6581(183)$ & $8.32\left(\begin{array}{l}+0.25 \\
-0.24\end{array}\right)$ & $0.78\left(_{-0.16}^{+0.17}\right)$ & $-8.78(0.15)$ & $<-3.17$ & $183.9(31.4)$ \\
\hline $\mathrm{J} 0844+5446$ & $7033(412)$ & $8.36\left({ }_{-0.35}^{+0.39}\right)$ & $0.81\left(\begin{array}{l}+0.25 \\
-0.23\end{array}\right)$ & $-9.77(0.16)$ & $<-3.62$ & $216.9(55.3)$ \\
\hline $\mathrm{J} 0845+1258$ & $9473(329)$ & $8.38\left({ }_{-0.37}^{+0.42}\right)$ & $0.82\left({ }_{-0.24}^{+0.26}\right)$ & $-7.98(0.37)$ & $<-5.57$ & $275.4(79.2)$ \\
\hline $\mathrm{J} 0845+4115$ & $8194(2$ & $7.43\left({ }_{-0.26}^{+0.32}\right)$ & $0.31\left({ }_{-0.08}^{+0.13}\right)$ & $-9.62(0.25)$ & $<-5.51$ & $297.3(47.2)$ \\
\hline $\mathrm{J} 0845+4802$ & $8145(871)$ & 8.00 & & $-8.46(0.50)$ & $<-4.84$ & 608.1 \\
\hline $\mathrm{J} 0845+5352$ & $7138(167)$ & $8.21\left({ }_{-0.09}^{+0.09}\right)$ & $0.71\left({ }_{-0.06}^{+0.06}\right)$ & $-10.68(0.14)$ & $<-3.83$ & 125.4 \\
\hline $\mathrm{J} 0846+3538$ & $8791(204)$ & $8.16\left({ }_{-0.05}^{+0.05}\right)$ & $0.68\left({ }_{-0.03}^{+0.03}\right)$ & $-8.69(0.10)$ & $<-5.27$ & $24.6(0.0)$ \\
\hline $\mathrm{J} 0847+4507$ & $8921(394)$ & $8.46\left({ }_{-0.17}^{+0.18}\right)$ & $0.88\left({ }_{-0.12}^{+0.12}\right)$ & $-9.51(0.22)$ & $<-5.09$ & $210.6(26.0)$ \\
\hline $\mathrm{J} 0848+0028$ & $11667(380)$ & $7.89\left({ }_{-0.10}^{+0.11}\right)$ & $0.52\left({ }_{-0.05}^{+0.06}\right)$ & $-7.55(0.08)$ & $<-6.09$ & $236.4(12.9)$ \\
\hline $\mathrm{J} 0848+3548$ & $7864(189)$ & $8.17\left({ }_{-0.06}^{+0.06}\right)$ & $0.68\left({ }_{-0.04}^{+0.04}\right)$ & $-10.49(0.04)$ & $<-4.30$ & 89.3( \\
\hline $\mathrm{J} 0848+5214$ & $8349(221)$ & $8.07\left({ }_{-0.07}^{+0.07}\right)$ & $0.62\left(_{-0}^{+0}\right.$ & $-10.72(0.06)$ & $<-4.98$ & $106.1(2.4)$ \\
\hline $\mathrm{J} 0849+0710$ & $8477(218)$ & $8.32\left({ }_{-0.17}^{+0.18}\right)$ & $0.78\left({ }_{-0.11}^{+0.12}\right)$ & $-8.34(0.29)$ & $-3.49(0.55)$ & $198.6(24.3)$ \\
\hline $\mathrm{J} 0849+1827$ & $6127(67)$ & $8.06\left({ }_{-0.05}^{+0.05}\right)$ & $0.61\left({ }_{-0.03}^{+0.03}\right)$ & $-10.50(0.09)$ & $<-3.05$ & $88.4(2.0)$ \\
\hline $\mathrm{J} 0849+1836$ & $8805(287)$ & $8.03\left({ }_{-0.37}^{+0.41}\right)$ & $0.60\left({ }_{-0.20}^{+0.27}\right)$ & $-8.39(0.38)$ & $<-5.41$ & $309.6(76.2)$ \\
\hline $\mathrm{J} 0849+4036$ & $9388(295)$ & $8.20\left({ }_{-0.08}^{+0.08}\right)$ & $0.70\left({ }_{-0.05}^{+0.05}\right)$ & $-10.12(0.09)$ & $<-5.69$ & $123.2(3.5)$ \\
\hline $\mathrm{J} 0851+0538$ & $7415(183)$ & $.09\left({ }_{-0.18}^{+0.19}\right)$ & $0.63\left({ }_{-0.11}^{+0.12}\right)$ & $-9.50(0.31)$ & $<-4.04$ & $195.0(22.6)$ \\
\hline $\mathrm{J} 0851+1543$ & 649 & $3.21\left({ }_{-0.05}^{+0.05}\right)$ & $0.71\left({ }_{-0.03}^{+0.03}\right)$ & -0.1 & 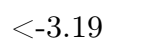 & $96.1(2.4)$ \\
\hline $\mathrm{J} 0852+1124$ & 66 & 8.00 & & $-10.21(0.30)$ & & 3) \\
\hline $\mathrm{J} 0852+3402$ & 5036 & $7.98\left({ }_{-0.29}^{+0.31}\right)$ & $0.56\left(_{-0.16}^{+0.20}\right)$ & $-9.56(0.25)$ & $<-3.00$ & 4) \\
\hline $\mathrm{J} 0852+5124$ & $7827(721)$ & 8.00 & & $-9.63(0.42)$ & $<-4.44$ & $432.7(60.4)$ \\
\hline $\mathrm{J} 0853+1603$ & $7538(526)$ & $8.11\left({ }_{-1.02}^{+1.88}\right)$ & $0.64\left({ }_{-0.44}^{+1.05}\right)$ & $-10.32(0.18)$ & $<-4.09$ & $307.6(248.6)$ \\
\hline $\mathrm{J} 0854+0603$ & $7340(345)$ & $7.85\left({ }_{-0.38}^{+0.43}\right)$ & $0.49\left(_{-0.17}^{+0.26}\right)$ & $-10.90(0.17)$ & $<-4.19$ & $282.9(65.2)$ \\
\hline $\mathrm{J} 0854+1847$ & $8119(243)$ & $8.23\left({ }_{-0.13}^{+0.14}\right)$ & $0.72\left({ }_{-0.09}^{+0.09}\right)$ & $-9.94(0.09)$ & $<-4.48$ & $170.7(14.5)$ \\
\hline $\mathrm{J} 0855+5438$ & $7903(936)$ & 8.00 & & $-8.50(1.25)$ & $<-4.53$ & $615.5(111.4)$ \\
\hline $\mathrm{J} 0857+1438$ & $8180(201)$ & $8.11\left({ }_{-0.14}^{+0.15}\right)$ & $0.64\left({ }_{-0.09}^{+0.10}\right)$ & $-9.09(0.42)$ & $<-4.72$ & $184.7(16.2)$ \\
\hline $\mathrm{J} 0857+2630$ & $6402(127)$ & $7.82\left({ }_{-0.22}^{+0.24}\right)$ & $0.47\left({ }_{-0.11}^{+0.14}\right)$ & $-10.86(0.11)$ & $<-3.46$ & $144.0(18.9)$ \\
\hline $\mathrm{J} 0859+5732$ & $13140(9$ & $8.08\left({ }_{-0.20}^{+0.21}\right)$ & $0.63\left({ }_{-0.11}^{+0.13}\right)$ & $-7.96(0.37)$ & $<-6.09$ & $301.8(36.1)$ \\
\hline $\mathrm{J} 0900+1020$ & $7301(378)$ & 8.00 & & $-10.61(0.18)$ & $<-4.05$ & $316.7(24.7)$ \\
\hline $\mathrm{J} 0901+0752$ & $6995(117)$ & $7.91\left({ }_{-0.29}^{+0.31}\right)$ & $0.52\left({ }_{-0.15}^{+0.19}\right)$ & $-7.87(0.08)$ & $<-3.95$ & $216.7(38.8)$ \\
\hline $\mathrm{J} 0901+1113$ & $7545(465)$ & 8.00 & & $-10.51(0.23)$ & $<-4.18$ & $378.7(35.8)$ \\
\hline J0901+5337 & $5802(284)$ & 8.00 & & $-10.71(0.65)$ & $<-3.00$ & $253.0(23.9)$ \\
\hline J0903-0120 & $8731(1234)$ & 8.00 & & $-9.80(0.26)$ & $<-5.41$ & $536.4(107.7)$ \\
\hline J0904-0029 & $7933(421)$ & 8.00 & & $-10.35(0.12)$ & $<-4.57$ & $329.5(25.2)$ \\
\hline $\mathrm{J} 0905+0133$ & $7977(227)$ & $8.25\left({ }_{-0.12}^{+0.13}\right)$ & $0.73\left(_{-0.08}^{+0.08}\right)$ & $-10.24(0.12)$ & $<-4.33$ & $157.9(11.9)$ \\
\hline $\mathrm{J} 0905+0846$ & $6152(201)$ & 8.00 & & $-10.09(0.28)$ & $<-3.08$ & $242.2(14.0)$ \\
\hline
\end{tabular}


Table 6. Atmospheric parameters for DZ stars - continued.

\begin{tabular}{|c|c|c|c|c|c|c|}
\hline J Name & $T_{\text {eff }}(\mathrm{K})$ & $\log g$ & $M / M_{\odot}$ & $\log \mathrm{Ca} / \mathrm{He}$ & $\log \mathrm{H} / \mathrm{He}$ & $D(\mathrm{pc})$ \\
\hline $\mathrm{J} 0905+5235$ & $7650(126)$ & $8.15\left({ }_{-0.09}^{+0.09}\right)$ & $0.67\left({ }_{-0.06}^{+0.06}\right)$ & $-9.07(0.22)$ & $<-4.14$ & $143.4(7.8)$ \\
\hline J0906+1141 & $6592(161)$ & 8.00 & & $-8.50(0.19)$ & $<-3.50$ & $282.9(12.5)$ \\
\hline J0906+1628 & $7099(340)$ & 8.00 & & $-10.60(0.22)$ & $<-3.96$ & $270.7(19.7)$ \\
\hline J0906+5956 & $9953(277)$ & $8.31\left({ }_{-0.11}^{+0.12}\right)$ & $0.77\left({ }_{-0.07}^{+0.08}\right)$ & $-7.77(0.16)$ & $<-5.71$ & $214.3(15.5)$ \\
\hline $\mathrm{J} 0907+2533$ & $8745(286)$ & $8.22\left(\begin{array}{l}+0.15 \\
-0.15\end{array}\right)$ & $0.71\left({ }_{-0.09}^{+0.10}\right)$ & $-9.30(0.09)$ & $<-5.16$ & $184.2(17.0)$ \\
\hline J0908+4119 & $6268(206)$ & 8.00 & & $-9.87(0.46)$ & $<-3.17$ & $268.7(15.3)$ \\
\hline $\mathrm{J} 0908+5136$ & $5811(62)$ & $7.80\left(\begin{array}{l}+0.09 \\
-0.09\end{array}\right)$ & $0.46\left(_{-0.04}^{+0.05}\right)$ & $-10.12(0.10)$ & $<-3.00$ & $125.2(5.6)$ \\
\hline J0909-0045 & $9160(293)$ & $8.13\left({ }_{-0.13}^{+0.13}\right)$ & $0.66\left(_{-0.08}^{+0.09}\right)$ & $-9.40(0.08)$ & $<-5.59$ & $196.4(14.3)$ \\
\hline J0910+1045 & $9425(270)$ & $8.26\left(_{-0.11}^{+0.11}\right)$ & $0.74\left({ }_{-0.07}^{+0.08}\right)$ & $-8.60(0.10)$ & $<-5.66$ & $202.0(13.7)$ \\
\hline $\mathrm{J} 0911+2433$ & $6731(655)$ & 8.00 & & $-10.06(0.44)$ & $<-3.66$ & $433.2(70.9)$ \\
\hline J0912-0128 & $7509(409)$ & 8.00 & & $-9.33(0.28)$ & $<-4.16$ & $323.5(26.3)$ \\
\hline $\mathrm{J} 0913+2627$ & $5342(96)$ & $8.22\left({ }_{-0.13}^{+0.14}\right)$ & $0.71\left({ }_{-0.09}^{+0.09}\right)$ & $-9.69(0.18)$ & $<-3.00$ & $106.8(9.0)$ \\
\hline J0913+3116 & $5446(45)$ & $8.29\left(_{-0.05}^{+0.05}\right)$ & $0.76\left(_{-0.03}^{+0.03}\right)$ & $-10.06(0.06)$ & $<-3.00$ & $55.0(1.3)$ \\
\hline $\mathrm{J} 0913+4127$ & $5932(126)$ & 8.00 & & $-8.77(0.32)$ & $<-3.00$ & $229.2(10.0)$ \\
\hline $\mathrm{J} 0916+0110$ & $7527(339)$ & 8.00 & & $-10.34(0.08)$ & $<-4.17$ & $238.8(15.5)$ \\
\hline $\mathrm{J} 0916+2540$ & $5652(57)$ & $8.13\left({ }_{-0.04}^{+0.04}\right)$ & $0.65\left(_{-0.03}^{+0.03}\right)$ & $-7.64(0.04)$ & $<-3.00$ & $46.3(0.4)$ \\
\hline J0916-0212 & $6672(300)$ & $7.19\left({ }_{-0.59}^{+0.57}\right)$ & $0.22\left({ }_{-0.13}^{+0.22}\right)$ & $-11.25(0.09)$ & $<-4.21$ & $326.1(84.9)$ \\
\hline $\mathrm{J} 0917+2146$ & $6674(319)$ & 8.00 & & $-9.29(0.33)$ & $<-3.59$ & $357.8(28.1)$ \\
\hline $\mathrm{J} 0917+2224$ & $6771(432)$ & 8.00 & & $-10.64(0.27)$ & $<-3.70$ & $311.6(31.9)$ \\
\hline J0921+1204 & $13314(898)$ & $7.95\left({ }_{-0.30}^{+0.34}\right)$ & $0.56\left(_{-0.15}^{+0.21}\right)$ & $-7.97(0.55)$ & $-4.47(0.44)$ & $406.3(79.9)$ \\
\hline $\mathrm{J} 0922+2007$ & $8377(218)$ & $7.98\left({ }_{-0.08}^{+0.08}\right)$ & $0.57\left({ }_{-0.04}^{+0.05}\right)$ & $-11.20(0.20)$ & $-3.73(0.16)$ & $120.0(3.7)$ \\
\hline $\mathrm{J} 0923+4823$ & $9334(1365)$ & 8.00 & & $-9.09(0.60)$ & $<-5.77$ & $705.1(138.0)$ \\
\hline $\mathrm{J} 0924+4301$ & $5537(117)$ & 8.00 & & $-10.08(0.20)$ & $<-3.00$ & $209.6(9.1)$ \\
\hline $\mathrm{J} 0925+2256$ & $6748(297)$ & 8.00 & & $-9.74(0.35)$ & $<-3.67$ & $303.5(21.0)$ \\
\hline $\mathrm{J} 0925+3130$ & $5901(58)$ & 8.00 & & $-8.97(0.12)$ & $<-3.00$ & $88.9(2.0)$ \\
\hline J0928+1801 & $101 \S$ & $8.22\left({ }_{-0.14}^{+0.15}\right)$ & $0.72\left({ }_{-0.09}^{+0.10}\right)$ & $-9.88(0.11)$ & $<-5.81$ & $210.8(18.7)$ \\
\hline $\mathrm{J} 0928+6124$ & $9167(303)$ & $7.08\left({ }_{-1.46}^{+1.55}\right)$ & $0.21\left({ }_{-0.19}^{+0.77}\right)$ & $-8.08(0.40)$ & $<-6.10$ & $582.6(370.6)$ \\
\hline $\mathrm{J} 0929+4247$ & $6683(88)$ & $7.86\left({ }_{-0.22}^{+0.24}\right)$ & $0.50\left({ }_{-0.11}^{+0.14}\right)$ & $-8.26(0.15)$ & $<-3.72$ & $217.7(29.1)$ \\
\hline J0930+2601 & $9843(636)$ & $8.26\left(_{-0.30}^{+0.33}\right)$ & $0.74\left(\begin{array}{l}+0.22 \\
-0.19\end{array}\right)$ & $-10.36(0.29)$ & $-4.01(0.46)$ & $259.5(54.2)$ \\
\hline J0930+3013 & 11366(508) & $7.98\left({ }_{-0.17}^{+0.19}\right)$ & $0.57\left(\begin{array}{l}+0.11 \\
-0.09\end{array}\right)$ & $-9.42(0.09)$ & $-4.71(0.12)$ & $242.7(24.7)$ \\
\hline J0931+0730 & 11699(537) & $7.75\left(_{-0.50}^{+0.68}\right)$ & $0.45\left(_{-0.19}^{+0.41}\right)$ & $-8.28(0.27)$ & $<-6.10$ & $445.6(162.6)$ \\
\hline $\mathrm{J} 0931+1210$ & $7431(424)$ & 8.00 & & $-9.11(0.34)$ & $<-4.11$ & $378.1(31.0)$ \\
\hline J0932+4856 & $8831(257)$ & $8.08\left({ }_{-0.28}^{+0.30}\right)$ & $0.63\left(\begin{array}{l}+0.20 \\
-0.16\end{array}\right)$ & $-8.34(0.47)$ & $<-5.39$ & $288.8(53.7)$ \\
\hline $\mathrm{J} 0933+6334$ & $6265(165)$ & 8.00 & & $-8.55(0.33)$ & $<-3.17$ & $265.1(14.6)$ \\
\hline J0934+0822 & $9453(291)$ & $8.19\left({ }_{-0.07}^{+0.08}\right)$ & $0.70\left({ }_{-0.05}^{+0.05}\right)$ & $-9.50(0.03)$ & $<-5.72$ & $110.6(3.2)$ \\
\hline J0934+2626 & $8367(354)$ & $8.30\left({ }_{-0.33}^{+0.37}\right)$ & $0.77\left({ }_{-0.21}^{+0.24}\right)$ & $-10.28(0.16)$ & $<-4.60$ & $236.4(56.9)$ \\
\hline J0934+5632 & $6494(92)$ & $8.23\left({ }_{-0.06}^{+0.06}\right)$ & $0.72\left(\begin{array}{l}+0.04 \\
-0.04\end{array}\right)$ & $-9.55(0.41)$ & $<-3.18$ & $108.7(3.3)$ \\
\hline $\mathrm{J} 0935+0037$ & $10098(490)$ & $7.82\left(\begin{array}{l}+0.85 \\
-0.61\end{array}\right)$ & $0.48\left(\begin{array}{l}+0.53 \\
-0.23\end{array}\right)$ & $-7.83(0.44)$ & $<-5.98$ & $492.7(222.6)$ \\
\hline
\end{tabular}


Table 6. Atmospheric parameters for DZ stars - continued.

\begin{tabular}{|c|c|c|c|c|c|c|}
\hline J Name & $T_{\text {eff }}(\mathrm{K})$ & $\log g$ & $M / M_{\odot}$ & $\log \mathrm{Ca} / \mathrm{He}$ & $\log \mathrm{H} / \mathrm{He}$ & $D(\mathrm{pc})$ \\
\hline $\mathrm{J} 0935+2420$ & $8289(284)$ & $7.72\left({ }_{-0.38}^{+0.45}\right)$ & $0.42\left({ }_{-0.15}^{+0.26}\right)$ & $-9.60(0.33)$ & $<-5.35$ & $294.0(71.2)$ \\
\hline $\mathrm{J} 0935+4450$ & $7484(170)$ & $8.02\left(\begin{array}{l}+0.07 \\
-0.07\end{array}\right)$ & $0.59\left(\begin{array}{l}+0.04 \\
-0.04\end{array}\right)$ & $-11.13(0.14)$ & $<-4.12$ & $109.6(2.9)$ \\
\hline J0936+1815 & $11520(641)$ & $7.21\left({ }_{-0.44}^{+0.70}\right)$ & $0.26\left(_{-0.09}^{+0.28}\right)$ & $-9.07(0.82)$ & $-5.66(0.61)$ & $548.1(190.5)$ \\
\hline $\mathrm{J} 0937+2842$ & $13743(865)$ & 8.00 & & $-9.29(0.61)$ & $-5.08(0.31)$ & $365.1(25.5)$ \\
\hline $\mathrm{J} 0937+3646$ & $11733(565)$ & $7.95\left({ }_{-0.15}^{+0.16}\right)$ & $0.55\left(_{-0.08}^{+0.10}\right)$ & $-9.13(0.37)$ & $-5.02(0.32)$ & $286.0(23.4)$ \\
\hline $\mathrm{J} 0937+5228$ & $6621(80)$ & $7.97\left({ }_{-0.09}^{+0.09}\right)$ & $0.56\left(_{-0.05}^{+0.06}\right)$ & $-8.59(0.07)$ & $<-3.56$ & $142.2(7.3)$ \\
\hline $\mathrm{J} 0938+6343$ & $7980(743)$ & 8.00 & & $-10.09(0.22)$ & $<-4.63$ & $392.4(52.7)$ \\
\hline $\mathrm{J} 0939+4136$ & $6251(124)$ & $8.44\left(\begin{array}{l}+0.25 \\
-0.23\end{array}\right)$ & $0.86\left(_{-0.16}^{+0.16}\right)$ & $-8.14(0.16)$ & $<-3.00$ & $152.0(27.3)$ \\
\hline J0939+5019 & $6086(97)$ & $7.98\left({ }_{-0.11}^{+0.11}\right)$ & $0.56\left(_{-0.06}^{+0.07}\right)$ & $-8.01(0.26)$ & $<-3.04$ & $145.6(8.8)$ \\
\hline $\mathrm{J} 0939+5550$ & $9023(186)$ & $8.07\left(\begin{array}{l}+0.05 \\
-0.05\end{array}\right)$ & $0.62\left({ }_{-0.03}^{+0.03}\right)$ & $-8.18(0.05)$ & $-4.57(0.06)$ & $68.4(0.4)$ \\
\hline $\mathrm{J} 0940+6136$ & $9205(202)$ & $8.29\left({ }_{-0.05}^{+0.05}\right)$ & $0.76\left(_{-0.03}^{+0.03}\right)$ & $-8.00(0.05)$ & $<-5.53$ & $113.9(1.7)$ \\
\hline $\mathrm{J} 0940+6422$ & $6875(177)$ & $7.74\left({ }_{-0.11}^{+0.11}\right)$ & $0.43\left({ }_{-0.05}^{+0.06}\right)$ & $-10.87(0.08)$ & $<-3.97$ & $155.2(7.6)$ \\
\hline J0941+5022 & $11230(437)$ & $7.79\left(\begin{array}{l}+0.20 \\
-0.19\end{array}\right)$ & $0.47\left(\begin{array}{l}+0.11 \\
-0.09\end{array}\right)$ & $-8.07(0.18)$ & $<-6.07$ & $354.3(39.6)$ \\
\hline $\mathrm{J} 0942+0743$ & $9777(299)$ & $8.00\left({ }_{-0.19}^{+0.20}\right)$ & $0.58\left({ }_{-0.11}^{+0.13}\right)$ & $-8.35(0.16)$ & $<-5.89$ & $214.9(25.9)$ \\
\hline $\mathrm{J} 0942+5755$ & $11264(454)$ & $8.10\left({ }_{-0.17}^{+0.18}\right)$ & $0.65\left({ }_{-0.10}^{+0.11}\right)$ & $-8.06(0.44)$ & & 29 \\
\hline J0944+3939 & $11106(862)$ & $8.39\left({ }_{-0.21}^{+0.23}\right)$ & $0.83\left({ }_{-0.14}^{+0.15}\right)$ & $-8.64(0.24)$ & $<-5.89$ & $235.3(34.1)$ \\
\hline $\mathrm{J} 0944+4408$ & $10997(425)$ & $7.87\left(\begin{array}{l}+0.25 \\
-0.23\end{array}\right)$ & $0.51\left({ }_{-0.11}^{+0.15}\right)$ & $-8.19(0.34)$ & $<-6.04$ & $346.6(50.0)$ \\
\hline J0944-0039 & $12625(604)$ & $8.13\left({ }_{-0.07}^{+0.08}\right)$ & $0.66\left(_{-0.05}^{+0.05}\right)$ & $-7.80(0.08)$ & $<-6.08$ & $160.3(4.5)$ \\
\hline $\mathrm{J} 0945+0846$ & $10880(409)$ & $8.03\left({ }_{-0.15}^{+0.16}\right)$ & $0.60\left({ }_{-0.09}^{+0.10}\right)$ & $-9.47(0.21)$ & $<-5.99$ & $227.0(20.5)$ \\
\hline J0946+2024 & $7557(191)$ & 8.00 & & $-8.05(0.22)$ & $<-4.19$ & $338.2(14.2)$ \\
\hline $\mathrm{J} 0947+1916$ & $9996(359)$ & 8.00 & & $-9.47(0.27)$ & $<-5.90$ & $297.5(14.1)$ \\
\hline $\mathrm{J} 0947+4238$ & $9611(335)$ & & $0.81\left({ }_{-0.14}^{+0.15}\right)$ & $-9.05(0.25)$ & $<-5.62$ & $216.2(34.2)$ \\
\hline $\mathrm{J} 0948+3008$ & $6233(137)$ & $7.22\left({ }_{-0.38}^{+0.39}\right)$ & $0.23\left({ }_{-0.09}^{+0.14}\right)$ & $-9.52(0.15)$ & $<-3.75$ & $278.4(52.1)$ \\
\hline $\mathrm{J} 0950+4716$ & $6803(172)$ & $8.45\left({ }_{-0.22}^{+0.24}\right)$ & $0.87\left(\begin{array}{l}+0.15 \\
-0.15\end{array}\right)$ & $-9.39(0.18)$ & $<-3.28$ & $164.6(28.0)$ \\
\hline $\mathrm{J} 0950+4848$ & $6995(230)$ & $8.52\left({ }_{-0.21}^{+0.23}\right)$ & $0.91\left(_{-0.14}^{+0.14}\right)$ & $-8.84(0.26)$ & $<-3.43$ & $174.3(28.9)$ \\
\hline $\mathrm{J} 0951+4033$ & 83 & $8.20\left({ }_{-0.05}^{+0.06}\right)$ & $0.70\left({ }_{-0.04}^{+0.04}\right)$ & $-10.07(0.03)$ & $-3.95(0.09)$ & $72.3(0.6)$ \\
\hline $\mathrm{J} 0953+1510$ & $6933(220)$ & $8.11\left({ }_{-0.26}^{+0.28}\right)$ & $0.65\left(_{-0.16}^{+0.19}\right)$ & $-10.83(0.20)$ & $<-3.76$ & $181.7(3$ \\
\hline J0953+1719 & $7072(305)$ & $7.66\left({ }_{-0.72}^{+0.95}\right)$ & $0.39\left(_{-0.23}^{+0.58}\right)$ & $-10.35(0.15)$ & $<-4.14$ & $315.2(148.3)$ \\
\hline $\mathrm{J} 0954+1814$ & $11325(445)$ & 8.00 & & $-8.29(0.12)$ & $-5.50(0.19)$ & $217.1(9.4)$ \\
\hline $\mathrm{J} 0954+3347$ & $10290(345)$ & $8.25\left({ }_{-0.07}^{+0.07}\right)$ & $0.74\left({ }_{-0.05}^{+0.05}\right)$ & $-9.09(0.08)$ & $<-5.80$ & $143.4(4.1)$ \\
\hline $\mathrm{J} 0954+5635$ & $9404(314)$ & $8.16\left(_{-0.15}^{+0.16}\right)$ & $0.68\left(\begin{array}{l}+0.10 \\
-0.09\end{array}\right)$ & $-9.35(0.22)$ & $<-5.72$ & $216.1(20.2)$ \\
\hline $\mathrm{J} 0955+5233$ & $7620(175)$ & $7.92\left(\begin{array}{l}+0.23 \\
-0.22\end{array}\right)$ & $0.53\left(\begin{array}{l}+0.14 \\
-0.11\end{array}\right)$ & $-8.65(0.15)$ & $<-4.32$ & $245.8(32.9)$ \\
\hline J0956+5912 & $8723(105)$ & $8.13\left({ }_{-0.05}^{+0.05}\right)$ & $0.66\left(_{-0.03}^{+0.03}\right)$ & $-7.12(0.09)$ & $-3.54(0.18)$ & $138.8(3.1)$ \\
\hline $\mathrm{J} 0957+2822$ & $6494(218)$ & $7.62\left(\begin{array}{l}+0.37 \\
-0.32\end{array}\right)$ & $0.37\left(\begin{array}{l}+0.19 \\
-0.12\end{array}\right)$ & $-11.44(0.33)$ & $<-3.75$ & $240.4(44.6)$ \\
\hline $\mathrm{J} 0958+0550$ & $10960(402)$ & 8.00 & & $-8.66(0.09)$ & $-5.84(0.25)$ & $179.9(7.2)$ \\
\hline J0959+2556 & $9304(284)$ & $8.17\left({ }_{-0.07}^{+0.07}\right)$ & $0.68\left({ }_{-0.04}^{+0.04}\right)$ & $-10.81(0.04)$ & $<-5.66$ & $57.1(0.5)$ \\
\hline $\mathrm{J} 1000+3518$ & $12666(640)$ & $7.95\left(_{-0.11}^{+0.11}\right)$ & $0.56\left(_{-0.06}^{+0.06}\right)$ & $-8.54(0.15)$ & $-5.65(0.16)$ & $212.4(10.0)$ \\
\hline $\mathrm{J} 1000+4420$ & $13430(909)$ & $7.85\left(\begin{array}{l}+0.20 \\
-0.18\end{array}\right)$ & $0.50\left(\begin{array}{l}+0.11 \\
-0.09\end{array}\right)$ & $-8.51(0.24)$ & $-4.25(0.14)$ & $255.2(23.4)$ \\
\hline
\end{tabular}


Table 6. Atmospheric parameters for DZ stars - continued.

\begin{tabular}{|c|c|c|c|c|c|c|}
\hline J Name & $T_{\text {eff }}(\mathrm{K})$ & $\log g$ & $M / M_{\odot}$ & $\log \mathrm{Ca} / \mathrm{He}$ & $\log \mathrm{H} / \mathrm{He}$ & $D(\mathrm{pc})$ \\
\hline $\mathrm{J} 1000-0230$ & $5318(203)$ & 8.00 & & $-10.16(0.24)$ & $<-3.01$ & $188.4(15.1)$ \\
\hline $\mathrm{J} 1002+0313$ & 9997(333) & $8.06\left({ }_{-0.14}^{+0.15}\right)$ & $0.61\left({ }_{-0.08}^{+0.09}\right)$ & $-8.89(0.11)$ & $<-5.87$ & $214.0(18.3)$ \\
\hline $\mathrm{J} 1003+1421$ & $5725(312)$ & 8.00 & & $-10.46(0.73)$ & $<-3.00$ & $298.2(30.5)$ \\
\hline $\mathrm{J} 1004+0451$ & $7797(194)$ & $7.82\left({ }_{-0.22}^{+0.24}\right)$ & $0.47\left({ }_{-0.11}^{+0.14}\right)$ & $-9.77(0.19)$ & $<-4.65$ & $211.5(28.0)$ \\
\hline $\mathrm{J} 1004+4231$ & $8152(356)$ & $8.33\left({ }_{-0.18}^{+0.19}\right)$ & $0.79\left({ }_{-0.12}^{+0.12}\right)$ & $-10.01(0.15)$ & $<-4.36$ & $189.9(22.2)$ \\
\hline $\mathrm{J} 1005+1746$ & $5749(214)$ & 8.00 & & $-10.39(0.47)$ & $<-3.00$ & $229.9(16.3)$ \\
\hline $\mathrm{J} 1005+2032$ & $6522(281)$ & 8.00 & & $-10.44(0$ & $<-3.43$ & $266.1(19.0)$ \\
\hline $\mathrm{J} 1005+2244$ & $6006(224)$ & 8.00 & & $-9.60(0$ & $<-3.00$ & 267.1(19.1) \\
\hline $\mathrm{J} 1005+2655$ & $8038(206)$ & $8.01\left({ }_{-0.10}^{+0.10}\right)$ & $0.58\left({ }_{-0.06}^{+0.06}\right)$ & $-9.90(0.04)$ & $<-4.69$ & $141.5(6.8)$ \\
\hline $\mathrm{J} 1006+1752$ & $5883(127)$ & $8.43\left({ }_{-0.16}^{+0.17}\right)$ & $0.85\left({ }_{-0.11}^{+0.11}\right)$ & $-8.82(0.24)$ & $<-3.00$ & $135.8(15.6)$ \\
\hline $\mathrm{J} 1006+6116$ & $6935(229)$ & $8.10\left({ }_{-0.18}^{+0.19}\right)$ & $0.63\left({ }_{-0.11}^{+0.12}\right)$ & $-10.74(0.12)$ & $<-3.77$ & $183.0(19.6)$ \\
\hline $\mathrm{J} 1008+0248$ & $7648(184)$ & $7.95\left({ }_{-0.10}^{+0.10}\right)$ & $0.55\left({ }_{-0.06}^{+0.06}\right)$ & $-11.32(0.13)$ & $<-4.31$ & $118.2(6.0)$ \\
\hline $\mathrm{J} 1008+4349$ & $11380(444)$ & $8.02\left({ }_{-0.09}^{+0.09}\right)$ & $0.60\left({ }_{-0.05}^{+0.06}\right)$ & $-10.13(0.09)$ & $-5.28(0.10)$ & $146.1(5.4)$ \\
\hline $\mathrm{J} 1009+3337$ & $6543(244)$ & 8.00 & & $-10.11(0.30)$ & $<-3.45$ & $238.8(14.7)$ \\
\hline $\mathrm{J} 1010+2119$ & $6968(408)$ & 8.00 & & $-9.64(0.37)$ & $<-3.88$ & $401.8(35.2)$ \\
\hline $\mathrm{J} 1010+3948$ & $8371(170)$ & $8.05\left({ }_{-0.06}^{+0.06}\right)$ & $0.61\left({ }_{-0.04}^{+0.04}\right)$ & $-8.81(0.06)$ & $-4.09(0.12)$ & $122.8(2.9)$ \\
\hline $\mathrm{J} 1012+4435$ & $9065(483)$ & $8.75\left({ }_{-0.74}^{+1.43}\right)$ & $1.06\left({ }_{-0.47}^{+0.86}\right)$ & $-8.48(0.73)$ & $<-4.82$ & $219.4(161.8)$ \\
\hline $\mathrm{J} 1012-1843$ & $5938(213)$ & $8.05\left({ }_{-0.08}^{+0.09}\right)$ & $0.60\left({ }_{-0.05}^{+0.05}\right)$ & $-10.19(0.05)$ & $<-3.00$ & $18.1(0.0)$ \\
\hline $\mathrm{J} 1013-0251$ & $7065(631)$ & 8.00 & & $-9.84(0.33)$ & $<-3.9$ & $385.0(53.1)$ \\
\hline $\mathrm{J} 1014+2827$ & $6243(196)$ & 8.00 & & $-8.04(0.41)$ & $<-3.15$ & $64.1(18.0)$ \\
\hline $\mathrm{J} 1015+4141$ & 969 & $7.95\left({ }_{-0.14}^{+0.15}\right)$ & $0.55\left({ }_{-0.08}^{+0.09}\right)$ & $-7.84(0.09)$ & $-4.91(0.20)$ & 3) \\
\hline $\mathrm{J} 1015+6327$ & $8230(595)$ & 8.00 & & $-8.82(0.48)$ & $<-4.95$ & $494.5(55.2)$ \\
\hline $\mathrm{J} 1017+1948$ & 15794(1718) & $8.56\left({ }_{-0.32}^{+0.36}\right)$ & $0.94\left({ }_{-0.21}^{+0.21}\right)$ & $-7.25(0.62)$ & $-4.96(0.71)$ & $291.1(74.6)$ \\
\hline $\mathrm{J} 1017+2419$ & $6831(97)$ & $8.51\left({ }_{-0.25}^{+0.27}\right)$ & $0.91\left({ }_{-0.17}^{+0.17}\right)$ & $-8.37(0.08)$ & $<-3.26$ & $132.4(27.1)$ \\
\hline $\mathrm{J} 1017+3447$ & $5995(143)$ & $7.97\left({ }_{-0.22}^{+0.23}\right)$ & $0.55\left({ }_{-0.12}^{+0.14}\right)$ & $-10.27(0.23)$ & $<-3.00$ & $182.8(23.8)$ \\
\hline $\mathrm{J} 1018+0344$ & $9264(370)$ & $8.17\left({ }_{-0.36}^{+0.40}\right)$ & $0.68\left({ }_{-0.21}^{+0.26}\right)$ & $-8.69(0.44)$ & $<-5.64$ & $275.4(68.7)$ \\
\hline $\mathrm{J} 1018+3708$ & $15508(1643)$ & $8.18\left({ }_{-0.17}^{+0.18}\right)$ & $0.70\left({ }_{-0.10}^{+0.11}\right)$ & $-7.23(0.28)$ & $<-5.85$ & $272.3(20.3)$ \\
\hline $\mathrm{J} 1018+3726$ & $10543(361)$ & $7.98\left({ }_{-0.07}^{+0.07}\right)$ & $0.57\left({ }_{-0.04}^{+0.04}\right)$ & $-7.93(0.22)$ & $-3.62(1.83)$ & $137.7(2.9)$ \\
\hline $\mathrm{J} 1019+2045$ & $5552(133)$ & 8.00 & & $-9.45(0.44)$ & $<-3.00$ & $191.7(9.6)$ \\
\hline $\mathrm{J} 1019+3535$ & $6079(227)$ & 8.00 & & $-9.45(0.50)$ & $<-3.04$ & $261.2(17.6)$ \\
\hline $\mathrm{J} 1019+3752$ & $12697(593)$ & $8.04\left({ }_{-0.17}^{+0.18}\right)$ & $0.61\left({ }_{-0.10}^{+0.11}\right)$ & $-9.36(0.19)$ & $-5.52(0.19)$ & $43.9(25.7)$ \\
\hline $\mathrm{J} 1023-0014$ & $7549(603)$ & 8.00 & & $-9.42(0.45)$ & $<-4.18$ & $397.9(46.7)$ \\
\hline $\mathrm{J} 1024+1014$ & $6226(129)$ & 8.00 & & $-7.72(0.10)$ & $<-3.13$ & $195.1(9.4)$ \\
\hline $\mathrm{J} 1024+2227$ & $7517(434)$ & 8.00 & & $-10.41(0.17)$ & $<-4.16$ & $367.2(30.9)$ \\
\hline $\mathrm{J} 1024+4531$ & $6375(134)$ & $8.38\left({ }_{-0.16}^{+0.16}\right)$ & $0.82\left({ }_{-0.10}^{+0.11}\right)$ & $-8.68(0.16)$ & $<-3.01$ & $148.7(16.5)$ \\
\hline $\mathrm{J} 1024-0017$ & $7370(390)$ & 8.00 & & $-8.84(0.29)$ & $<-4.08$ & $355.4(27.5)$ \\
\hline $\mathrm{J} 1025+2016$ & $7612(680)$ & 8.00 & & $-9.95(0.58)$ & $<-4.23$ & $461.7(59.5)$ \\
\hline $\mathrm{J} 1026+1948$ & 10703(387) & 8.00 & & $-8.23(0.09)$ & $<-6.01$ & $246.9(10.3)$ \\
\hline
\end{tabular}


Table 6. Atmospheric parameters for DZ stars - continued.

\begin{tabular}{|c|c|c|c|c|c|c|}
\hline J Name & $T_{\text {eff }}(\mathrm{K})$ & $\log g$ & $M / M_{\odot}$ & $\log \mathrm{Ca} / \mathrm{He}$ & $\log \mathrm{H} / \mathrm{He}$ & $D(\mathrm{pc})$ \\
\hline $\mathrm{J} 1026+2646$ & $6803(371)$ & 8.00 & & $-10.56(0.24)$ & $<-3.73$ & $305.3(26.8)$ \\
\hline $\mathrm{J} 1027+4532$ & $9076(202)$ & $7.92\left({ }_{-0.21}^{+0.23}\right)$ & $0.53\left({ }_{-0.11}^{+0.14}\right)$ & $-8.13(0.07)$ & $<-5.65$ & $195.8(25.9)$ \\
\hline $\mathrm{J} 1028+2507$ & $5847(214)$ & 8.00 & & $-9.47(0.41)$ & $<-3.00$ & $273.8(20.0)$ \\
\hline J1028-0135 & $12948(776)$ & $7.95\left({ }_{-0.19}^{+0.21}\right)$ & $0.56\left(_{-0.10}^{+0.12}\right)$ & $-8.55(0.27)$ & $-5.61(0.23)$ & $304.3(34.1)$ \\
\hline $\mathrm{J} 1029+1227$ & $7158(401)$ & 8.00 & & $-10.33(0.24)$ & $<-3.99$ & $304.9(26.4)$ \\
\hline $\mathrm{J} 1029+2013$ & $6281(232)$ & 8.00 & & $-10.75(C$ & $<-3.18$ & $226.8(14.0)$ \\
\hline $\mathrm{J} 1029+3103$ & $9423(3$ & $8.39\left({ }_{-0.38}^{+0.43}\right)$ & $0.83\left({ }_{-0.25}^{+0.27}\right)$ & $-7.83(0$ & $<-5.55$ & $268.3(79.9)$ \\
\hline $\mathrm{J} 1030+0026$ & 6747( & $8.02\left({ }_{-0.12}^{+0.12}\right)$ & $0.58\left({ }_{-0.07}^{+0.08}\right)$ & $-10.37(0.08)$ & $<-3.66$ & $148.5(9.8)$ \\
\hline $\mathrm{J} 1031+0936$ & $11104(423)$ & $8.10\left({ }_{-0.12}^{+0.13}\right)$ & $0.64\left({ }_{-0.07}^{+0.08}\right)$ & $-9.84(0.14)$ & $<-5.98$ & $200.9(14.0)$ \\
\hline $\mathrm{J} 1031+1203$ & $8486(177)$ & $8.12\left({ }_{-0.06}^{+0.06}\right)$ & $0.65\left({ }_{-0.04}^{+0.04}\right)$ & $-8.94(0.12)$ & $<-5.05$ & $100.5(2.6)$ \\
\hline $\mathrm{J} 1032+1338$ & $5588(131)$ & 8.00 & & $-8.99(0$ & $<-3.00$ & $208.8(11.5)$ \\
\hline $\mathrm{J} 1032-0240$ & $11010(518)$ & $8.66\left({ }_{-0.31}^{+0.36}\right)$ & & $-8.78(0$ & -4.12( & $173.9(47.1)$ \\
\hline $\mathrm{J} 1033+1809$ & $6324(94)$ & $8.35\left({ }_{-0.09}^{+0.10}\right)$ & $0.80\left({ }_{-0.06}^{+0.06}\right)$ & $-8.46(0.36)$ & $<-3.01$ & $117.1(7.3)$ \\
\hline $\mathrm{J} 1033+2837$ & $6681(240)$ & 8.00 & & $-9.05(0.27)$ & $<-3.60$ & $291.2(17.5)$ \\
\hline $\mathrm{J} 1033+6247$ & $7474(171)$ & $8.09\left({ }_{-0.07}^{+0.07}\right)$ & $0.63\left({ }_{-0.05}^{+0.05}\right)$ & $-10.04(0.09)$ & $<-4.07$ & $127.5(4.1)$ \\
\hline $\mathrm{J} 1034+2245$ & $7214(75)$ & $8.21\left({ }_{-0.03}^{+0.03}\right)$ & $0.71\left({ }_{-0.02}^{+0.02}\right)$ & $-8.85(0.04)$ & $<-3.88$ & $40.8(0.2)$ \\
\hline $\mathrm{J} 1036+4837$ & $11247(470)$ & $7.71\left({ }_{-0.21}^{+0.24}\right)$ & $0.43\left({ }_{-0.09}^{+0.12}\right)$ & $-8.28(0.19)$ & $-5.37(0.30)$ & $346.0(44.4)$ \\
\hline $\mathrm{J} 1037+0709$ & $11153(462)$ & $8.10\left({ }_{-0.17}^{+0.18}\right)$ & $0.64\left({ }_{-0.10}^{+0.12}\right)$ & $-7.65(0.11)$ & $-4.62(0.32)$ & $258.3(28.4)$ \\
\hline $\mathrm{J} 1037+4341$ & $11075(425)$ & $8.06\left({ }_{-0.12}^{+0.13}\right)$ & $0.62\left({ }_{-0.07}^{+0.08}\right)$ & $-8.28(0.11)$ & $<-5.99$ & $242.9(17.1)$ \\
\hline $\mathrm{J} 1038+0432$ & 6319 & 8.00 & & $-8.37(0$ & 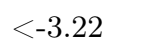 & $216.2(9.0)$ \\
\hline $\mathrm{J} 1038+1342$ & 908 & $8.25\left({ }_{-0.11}^{+0.12}\right)$ & $0.74\left({ }_{-0.07}^{+0.08}\right)$ & $-8.25(0$ & & $72.7(12.7)$ \\
\hline J1038-0036 & $7795(82)$ & 8.00 & & $-8.14(0.06)$ & $<-4.41$ & $65.0(1.4)$ \\
\hline $\mathrm{J} 1039+2648$ & $13421(637)$ & $7.97\left({ }_{-0.11}^{+0.11}\right)$ & $0.57\left({ }_{-0.06}^{+0.07}\right)$ & $-9.76(0.32)$ & $-5.41(0.14)$ & $183.8(9.4)$ \\
\hline $\mathrm{J} 1039+4612$ & $6994(91)$ & $7.77\left({ }_{-0.13}^{+0.14}\right)$ & $0.45\left(_{-0.06}^{+0.07}\right)$ & $-9.45(0.14)$ & $<-4.02$ & $134.7(10.1)$ \\
\hline $\mathrm{J} 1040+1349$ & $7605(339)$ & 8.00 & & $-10.24(0.14)$ & $<-4.23$ & $293.2(19.0)$ \\
\hline $\mathrm{J} 1040+2408$ & $5899(67)$ & $8.12\left({ }_{-0.06}^{+0.06}\right)$ & $0.64\left({ }_{-0.04}^{+0.04}\right)$ & $-8.63(0.14)$ & $<-3.00$ & $93.3(2.5)$ \\
\hline $\mathrm{J} 1041+2746$ & $7480(222)$ & $7.84\left({ }_{-0.35}^{+0.40}\right)$ & $0.49\left({ }_{-0.16}^{+0.24}\right)$ & $-10.12(0.08)$ & $<-4.30$ & $220.9(49.0)$ \\
\hline $\mathrm{J} 1041+3432$ & $7672(249)$ & $8.35\left({ }_{-0.33}^{+0.36}\right)$ & $0.80\left({ }_{-0.21}^{+0.24}\right)$ & $-8.28(0.21)$ & $<-4.04$ & $254.8(62.7)$ \\
\hline $\mathrm{J} 1041+4110$ & $7204(150)$ & $8.34\left({ }_{-0.30}^{+0.33}\right)$ & $0.79\left(_{-0.20}^{+0.22}\right)$ & $-8.84(0.25)$ & $<-3.77$ & $204.4(46.5)$ \\
\hline $\mathrm{J} 1043+3516$ & $6115(58)$ & $8.15\left({ }_{-0.05}^{+0.05}\right)$ & $0.67\left({ }_{-0.03}^{+0.03}\right)$ & $-8.84(0.07)$ & $<-3.02$ & $85.5(2.3)$ \\
\hline $\mathrm{J} 1044+2023$ & $8411(293$ & $8.11\left({ }_{-0.08}^{+0.08}\right)$ & $0.64\left({ }_{-0.05}^{+0.05}\right)$ & $-10.46(0.03)$ & $<-4.99$ & $89.2(1.6)$ \\
\hline $\mathrm{J} 1044+2143$ & $10000(401)$ & $8.19\left({ }_{-0.15}^{+0.16}\right)$ & $0.70\left({ }_{-0.10}^{+0.11}\right)$ & $-8.86(0.15)$ & $<-5.80$ & $210.1(20.2)$ \\
\hline $\mathrm{J} 1045+5254$ & $10990(641)$ & $7.30\left({ }_{-0.90}^{+1.61}\right)$ & $0.27\left({ }_{-0.18}^{+0.87}\right)$ & $-8.89(0.55)$ & $<-6.20$ & $84.9(464.3)$ \\
\hline $\mathrm{J} 1045+6009$ & $7651(387)$ & $7.80\left({ }_{-0.39}^{+0.45}\right)$ & $0.47\left({ }_{-0.17}^{+0.27}\right)$ & $-10.43(0.13)$ & $<-4.51$ & $313.2(74.3)$ \\
\hline $\mathrm{J} 1045+6254$ & $9566(574)$ & $8.02\left({ }_{-0.36}^{+0.41}\right)$ & $0.59\left({ }_{-0.19}^{+0.26}\right)$ & $-9.60(0.38)$ & $<-5.86$ & $378.3(87.9)$ \\
\hline $\mathrm{J} 1046+1329$ & 5251 & $7.97\left({ }_{-0.20}^{+0.21}\right)$ & $0.55\left({ }_{-0.11}^{+0.13}\right)$ & $-9.47(0.21)$ & $<-3.01$ & $140.2(16.6)$ \\
\hline $\mathrm{J} 1046+2424$ & 6252 & $8.09\left({ }_{-0.05}^{+0.05}\right)$ & $0.63\left({ }_{-0.03}^{+0.03}\right)$ & $-10.85(0.06)$ & $<-3.11$ & $59.1(0.7)$ \\
\hline $\mathrm{J} 1047+5219$ & $7095(254)$ & $8.56\left({ }_{-0.22}^{+0.24}\right)$ & $0.94\left(\begin{array}{l}+0.15 \\
-0.15\end{array}\right)$ & $-9.87(0.15)$ & $<-3.49$ & $165.2(28.8)$ \\
\hline
\end{tabular}


Table 6. Atmospheric parameters for DZ stars - continued.

\begin{tabular}{|c|c|c|c|c|c|c|}
\hline J Name & $T_{\text {eff }}(\mathrm{K})$ & $\log g$ & $M / M_{\odot}$ & $\log \mathrm{Ca} / \mathrm{He}$ & $\log \mathrm{H} / \mathrm{He}$ & $D(\mathrm{pc})$ \\
\hline $\mathrm{J} 1048+2623$ & $6911(182)$ & 8.00 & & $-9.10(0.32)$ & $<-3.83$ & $274.7(12.2)$ \\
\hline $\mathrm{J} 1049+2128$ & $6850(452)$ & 8.00 & & $-9.83(0.31)$ & $<-3.78$ & $368.7(39.1)$ \\
\hline $\mathrm{J} 1049+5154$ & $7224(73)$ & $8.10\left({ }_{-0.04}^{+0.04}\right)$ & $0.64\left({ }_{-0.02}^{+0.02}\right)$ & $-8.77(0.05)$ & $<-3.95$ & $79.7(1.0)$ \\
\hline J1049-0007 & $9286(221)$ & $7.98\left({ }_{-0.13}^{+0.14}\right)$ & $0.57\left({ }_{-0.07}^{+0.08}\right)$ & $-8.11(0.08)$ & $-4.68(0.16)$ & $197.9(15.1)$ \\
\hline $\mathrm{J} 1050+3138$ & $9394(324)$ & $8.22\left({ }_{-0.11}^{+0.12}\right)$ & $0.72\left(_{-0.07}^{+0.08}\right)$ & $-8.00(0.21)$ & $<-5.67$ & $187.5(12.5)$ \\
\hline $\mathrm{J} 1051+2751$ & $8735(591)$ & 8.00 & & $-7.72(0.32)$ & $<-5.41$ & $633.4(66.7)$ \\
\hline $\mathrm{J} 1051+5947$ & $6995(521)$ & 8.00 & & $-10.33(0.33)$ & $<-3.90$ & $332.2(39.0)$ \\
\hline $\mathrm{J} 1051-0303$ & $7241(539)$ & 8.00 & & $-9.27(1$ & $<-4.03$ & 349.6( \\
\hline $\mathrm{J} 1052+0659$ & $11224(469)$ & $7.97\left({ }_{-0.20}^{+0.22}\right)$ & $0.57\left({ }_{-0.11}^{+0.13}\right)$ & $-9.09(0.50)$ & $<-6.02$ & $320.3(40.3)$ \\
\hline $\mathrm{J} 1055+3509$ & $10767(397)$ & $8.10\left({ }_{-0.10}^{+0.11}\right)$ & $0.64\left({ }_{-0.06}^{+0.07}\right)$ & $-8.59(0.07)$ & $<-5.96$ & $197.4(11.1)$ \\
\hline $\mathrm{J} 1055+3725$ & $5869(104)$ & $7.76\left({ }_{-0.19}^{+0.20}\right)$ & $0.44\left(\begin{array}{l}+0.11 \\
-0.09\end{array}\right)$ & $-8.49(0.11)$ & $<-3.00$ & $177.0(19.0)$ \\
\hline $\mathrm{J} 1055+4115$ & $10058(395)$ & $7.88\left({ }_{-0.16}^{+0.17}\right)$ & $0.51\left(_{-0}^{+0}\right.$ & $-10.93(0.24)$ & $<-5.95$ & $245.2(22.3)$ \\
\hline $\mathrm{J} 1056+0128$ & $10278(378)$ & $8.07\left({ }_{-0.07}^{+0.07}\right)$ & $0.62\left({ }_{-0.04}^{+0.05}\right)$ & $-8.99(0.06)$ & $-4.92(0$ & $118.7(2.0)$ \\
\hline $\mathrm{J} 1056+5714$ & $7300(181)$ & $8.05\left({ }_{-0.07}^{+0.07}\right)$ & $0.60\left({ }_{-0.04}^{+0.04}\right)$ & $-10.69(0.09)$ & $<-4.02$ & $94.3(1.2)$ \\
\hline $\mathrm{J} 1056-0004$ & $11862(494)$ & 8.00 & & $-7.34(0.35)$ & $-4.74(0.79)$ & 402.1(18.4) \\
\hline $\mathrm{J} 1057+1053$ & $5947(252)$ & 8.00 & & $-9.49(0.28)$ & $<-3.00$ & $280.0(23.8)$ \\
\hline $\mathrm{J} 1057-0413$ & $6529(375)$ & $8.05\left({ }_{-0.14}^{+0.14}\right)$ & $0.60\left({ }_{-0.08}^{+0.09}\right)$ & $-10.28(0.02)$ & $<-3.38$ & $36.3(0.1)$ \\
\hline $\mathrm{J} 1058+1124$ & $10153(358)$ & $8.16\left({ }_{-0.13}^{+0.14}\right)$ & $0.68\left({ }_{-0.08}^{+0.09}\right)$ & $-9.18(0.11)$ & $<-5.84$ & $229.5(18.5)$ \\
\hline $\mathrm{J} 1058+3022$ & 10216 & $8.40\left({ }_{-0.28}^{+0.31}\right)$ & $0.84\left(_{-0}^{+0}\right.$ & $-8.95(0.24)$ & $<-5.65$ & $238.0(51.8)$ \\
\hline $\mathrm{J} 1058+3143$ & 713 & $8.14\left({ }_{-0.07}^{+0.07}\right)$ & $0.66\left(_{-0}^{+0}\right.$ & $-8.87(0$ & $<-3.00$ & $125.4(5.2)$ \\
\hline $\mathrm{J} 1058+6041$ & 93 & $8.29\left({ }_{-0.27}^{+0.29}\right)$ & $0.77\left({ }_{-0.17}^{+0.19}\right)$ & $-9.31(0$ & $<-5.59$ & $.9(53.7)$ \\
\hline $\mathrm{J} 1059+5038$ & $6922(288)$ & 8.00 & & $-9.57(0.29)$ & $<-3.84$ & $302.5(18.9)$ \\
\hline $\mathrm{J} 1100+1439$ & $7146(478)$ & 8.00 & & $-10.01(0.31)$ & $<-3.98$ & $359.8(37.3)$ \\
\hline $\mathrm{J} 1101+2730$ & $7735(629)$ & 8.00 & & $-9.02(0.59)$ & $<-4.35$ & $470.1(55.0)$ \\
\hline $\mathrm{J} 1102+0214$ & $5699(58)$ & $8.14\left({ }_{-0.08}^{+0.08}\right)$ & $0.66\left({ }_{-0.05}^{+0.06}\right)$ & $-9.78(0.14)$ & $<-3.00$ & $96.5(4.8)$ \\
\hline $\mathrm{J} 1102+1755$ & $6949(159)$ & $8.30\left({ }_{-0.18}^{+0.19}\right)$ & $0.77\left({ }_{-0.12}^{+0.13}\right)$ & $-9.60(0.15)$ & $<-3.60$ & $166.8(20.8)$ \\
\hline $\mathrm{J} 1102+2653$ & $6938(140)$ & $8.27\left({ }_{-0.18}^{+0.19}\right)$ & $0.75\left({ }_{-0.12}^{+0.13}\right)$ & $-9.36(0.12)$ & $<-3.61$ & $177.5(22.0)$ \\
\hline $\mathrm{J} 1102+2827$ & $6324(222)$ & 8.00 & & $-8.24(0.44)$ & $<-3.22$ & $278.6(19.5)$ \\
\hline $\mathrm{J} 1103+4144$ & $5812(92)$ & $7.59\left({ }_{-0.18}^{+0.20}\right)$ & $0.36\left({ }_{-0.07}^{+0.09}\right)$ & $-9.36(0.08)$ & $<-3.03$ & $153.7(15.2)$ \\
\hline $\mathrm{J} 1104+0711$ & 11167(399) & $8.03\left({ }_{-0.08}^{+0.08}\right)$ & $0.60\left({ }_{-0.04}^{+0.05}\right)$ & $-9.58(0.09)$ & $<-6.00$ & $166.6(4.7)$ \\
\hline $\mathrm{J} 1104+1657$ & $6959(339)$ & 8.00 & & $-9.40(0.56)$ & $<-3.87$ & $303.0(22.1)$ \\
\hline $\mathrm{J} 1104+2439$ & $10453(305)$ & $8.27\left({ }_{-0.10}^{+0.11}\right)$ & $0.75\left({ }_{-0.07}^{+0.07}\right)$ & $-7.34(0.09)$ & $-3.50(0.26)$ & $164.6(11.0)$ \\
\hline $\mathrm{J} 1104+6426$ & $5668(148)$ & $7.29\left({ }_{-0.61}^{+0.62}\right)$ & $0.25\left({ }_{-0.15}^{+0.27}\right)$ & $-10.75(0.26)$ & $<-3.09$ & $272.2(77.1)$ \\
\hline J1104-1607 & $8182(559)$ & $8.75\left({ }_{-0.29}^{+0.34}\right)$ & $1.06\left({ }_{-0.18}^{+0.17}\right)$ & $-8.52(0.52)$ & $<-3.99$ & $189.2(48.6)$ \\
\hline $\mathrm{J} 1105+0228$ & $5848(143)$ & 8.00 & & $-9.51(0.40)$ & $<-3.00$ & $170.9(8.0)$ \\
\hline $\mathrm{J} 1105+1949$ & $6616(498)$ & 8.00 & & $-10.13(0.43)$ & $<-3.53$ & $370.0(46.1)$ \\
\hline $\mathrm{J} 1105+3801$ & $7843(280)$ & $7.54\left({ }_{-1.46}^{+1.83}\right)$ & $0.34\left({ }_{-0.31}^{+0.97}\right)$ & $-8.97(0.41)$ & $<-5.10$ & $411.4(312.9)$ \\
\hline $\mathrm{J} 1106+0104$ & $6989(218)$ & $8.15\left({ }_{-0.16}^{+0.16}\right)$ & $0.67\left({ }_{-0.10}^{+0.11}\right)$ & $-11.27(0.29)$ & $<-3.77$ & $172.1(16.5)$ \\
\hline
\end{tabular}


Table 6. Atmospheric parameters for DZ stars - continued.

\begin{tabular}{|c|c|c|c|c|c|c|}
\hline J Name & $T_{\text {eff }}(\mathrm{K})$ & $\log g$ & $M / M_{\odot}$ & $\log \mathrm{Ca} / \mathrm{He}$ & $\log \mathrm{H} / \mathrm{He}$ & $D(\mathrm{pc})$ \\
\hline J1106-0039 & $11350(457)$ & $.06\left(\begin{array}{l}+0.27 \\
-0.25\end{array}\right)$ & $0.62\left({ }_{-0.14}^{+0.18}\right)$ & $-8.19(0.13)$ & $<-6.02$ & $285.8(48.1)$ \\
\hline $\mathrm{J} 1108+3033$ & $6530(207)$ & $8.00\left({ }_{-0.25}^{+0.27}\right)$ & $0.57\left({ }_{-0.14}^{+0.17}\right)$ & $-10.37(0.09)$ & $<-3.44$ & $188.7(28.8)$ \\
\hline $\mathrm{J} 1109+2112$ & $6684(268)$ & 8.00 & & $-10.83(0.20)$ & $<-3.60$ & $228.9(15.2)$ \\
\hline $\mathrm{J} 1112+0700$ & $6664(120)$ & $7.76\left({ }_{-0.15}^{+0.15}\right)$ & $0.44\left(_{-}^{+}\right.$ & $-10.00(0.17)$ & $<-3.80$ & $156.3(12.7)$ \\
\hline $\mathrm{J} 1113+2228$ & $6631(196)$ & 8.00 & & $-10.76(0$ & $<-3.55$ & $199.9(9.9)$ \\
\hline $\mathrm{J} 1113+4550$ & $9836(315)$ & $8.09\left({ }_{-0.09}^{+0.09}\right)$ & $0.63\left({ }_{-0.05}^{+0.06}\right)$ & $-10.76(0.18)$ & $<-5.85$ & $138.4(5.1)$ \\
\hline $\mathrm{J} 1114+2957$ & $6689(167)$ & 8.00 & & $-9.95(0$ & $<-3.61$ & $179.8(7$ \\
\hline $\mathrm{J} 1115+1720$ & $8112(7$ & 8.00 & & -10.45( & $<-4.80$ & 433. \\
\hline $\mathrm{J} 1117+0826$ & $8657(667)$ & 8.00 & & $-9.93(0.24)$ & $<-5.37$ & $412.0(45.5)$ \\
\hline $\mathrm{J} 1117+3311$ & $7918(432)$ & $8.32\left({ }_{-0.39}^{+0.44}\right)$ & $0.78\left({ }_{-0.25}^{+0.28}\right)$ & $-9.71(0$ & $<-4.21$ & $253.3(72.5)$ \\
\hline $\mathrm{J} 1118+0838$ & $9058(876)$ & 8.00 & & $-9.40(0.32)$ & $<-5.59$ & $468.7(67.1)$ \\
\hline $\mathrm{J} 1121+1417$ & $8921(352)$ & $8.04\left({ }_{-0.13}^{+0.13}\right)$ & $0.60\left({ }_{-0.07}^{+0.08}\right)$ & $-11.13(0.19)$ & $-3.71(($ & $164.5(8.4)$ \\
\hline $\mathrm{J} 1122+5041$ & $9512(181)$ & $8.01\left({ }_{-0.17}^{+0.18}\right)$ & $0.59\left({ }_{-0.09}^{+0.11}\right)$ & $-7.34(0.23)$ & $-4.95(0$ & $257.8(27.4)$ \\
\hline $\mathrm{J} 1123+3026$ & $9215(377)$ & 8.00 & & $-9.15(0.20)$ & $<-5.69$ & $270.9(15.0)$ \\
\hline $\mathrm{J} 1125+2853$ & $10952(431)$ & $8.32\left({ }_{-0.14}^{+0.15}\right)$ & $0.78\left({ }_{-0.09}^{+0.10}\right)$ & $-9.10(0.14)$ & $<-5.89$ & $222.8(20.8)$ \\
\hline $\mathrm{J} 1125+3823$ & $10437(330)$ & $8.17\left({ }_{-0.07}^{+0.07}\right)$ & $0.69\left({ }_{-0.04}^{+0.05}\right)$ & $-8.17(0.07)$ & $<-5.88$ & $131.2(3.8)$ \\
\hline $\mathrm{J} 1126+5241$ & $8967(266)$ & $8.07\left({ }_{-0.08}^{+0.08}\right)$ & $0.62\left({ }_{-0.05}^{+0.05}\right)$ & $-9.60(0.04)$ & $-4.40(0.17)$ & $140.1(3.8)$ \\
\hline $\mathrm{J} 1127-0138$ & $7655(95)$ & $8.11\left({ }_{-0.05}^{+0.05}\right)$ & $0.64\left({ }_{-0.03}^{+0.03}\right)$ & $-8.63(0.22)$ & $<-4.17$ & $95.0(2.0)$ \\
\hline $\mathrm{J} 1129+2917$ & $8346(440)$ & $7.92\left({ }_{-0.40}^{+0.46}\right)$ & $0.53\left(_{-0}^{+0}\right.$ & $-10.99(0$ & $<-5.20$ & $256.8(65.0)$ \\
\hline J1129-0152 & 89 & $8.05\left({ }_{-0.14}^{+0.14}\right)$ & $0.61\left({ }_{-0.08}^{+0.09}\right)$ & $-0.05(0)$ & -3 & 5) \\
\hline $\mathrm{J} 1130+1036$ & 91 & $8.52\left({ }_{-0.08}^{+0.09}\right)$ & $0.91\left({ }_{-0.05}^{+0.06}\right)$ & & & 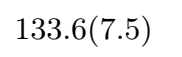 \\
\hline $\mathrm{J} 1131+2315$ & $7656(529)$ & 8.00 & & $-10.89(0.18)$ & $<-4.27$ & $353.4(37.0)$ \\
\hline $\mathrm{J} 1132+1002$ & $7239(479)$ & 8.00 & & $-9.69(0$ & $<-4.02$ & $314.4(30.5)$ \\
\hline $\mathrm{J} 1132+3323$ & $6168(170)$ & 8.00 & & $-8.26(0$. & $<-3.09$ & $256.1(14.1)$ \\
\hline $\mathrm{J} 1132+4705$ & $7553(372)$ & $7.21\left({ }_{-1.47}^{+1.39}\right)$ & $0.23\left({ }_{-0.21}^{+0.73}\right)$ & $-10.17(0.23)$ & $<-5.14$ & $434.0(252.9)$ \\
\hline $\mathrm{J} 1132-0106$ & $7755(417)$ & $8.01\left({ }_{-0.48}^{+0.55}\right)$ & $0.58\left({ }_{-0.24}^{+0.36}\right)$ & $-10.93(0.19)$ & $<-4.35$ & $272.2(85.9)$ \\
\hline $\mathrm{J} 1133+0610$ & $10711(364)$ & $8.05\left({ }_{-0.08}^{+0.09}\right)$ & $0.61\left({ }_{-0.05}^{+0.05}\right)$ & $-10.04(0.07)$ & $<-5.99$ & $158.6(5.7)$ \\
\hline $\mathrm{J} 1133+1804$ & $7780(356)$ & $7.21\left({ }_{-1.47}^{+1.38}\right)$ & $0.23\left({ }_{-0.21}^{+0.72}\right)$ & $-9.04(0.20)$ & $<-5.36$ & $422.8(246.6)$ \\
\hline $\mathrm{J} 1134+1236$ & $6960(172)$ & $8.36\left(_{-0.12}^{+0.13}\right)$ & $0.81\left({ }_{-0.08}^{+0.09}\right)$ & $-9.58(0.10)$ & $<-3.54$ & $144.7(12.2)$ \\
\hline $\mathrm{J} 1134+1542$ & $6850(223)$ & 8.00 & & $-8.52(0.20)$ & $<-3.78$ & $317.2(18.7)$ \\
\hline $\mathrm{J} 1134+5928$ & $8378(274)$ & $8.08\left({ }_{-0.11}^{+0.11}\right)$ & $0.63\left({ }_{-0.07}^{+0.07}\right)$ & $-10.25(0.14)$ & $<-5.00$ & $195.1(10.8)$ \\
\hline $\mathrm{J} 1137+0343$ & $6793(126)$ & $8.09\left({ }_{-0.05}^{+0.05}\right)$ & $0.63\left({ }_{-0.03}^{+0.03}\right)$ & $-10.95(0.10)$ & $<-3.64$ & $65.0(0.9)$ \\
\hline $\mathrm{J} 1137-0023$ & $10003(359)$ & $8.18\left({ }_{-0.15}^{+0.16}\right)$ & $0.69\left({ }_{-0.09}^{+0.10}\right)$ & $-9.61(0.0$ & $<-5.80$ & $.4(20.2)$ \\
\hline $\mathrm{J} 1138-0130$ & $5493(231)$ & 8.00 & & $-10.18(0.18)$ & $<-3.00$ & $229.4(19.2)$ \\
\hline $\mathrm{J} 1139+6239$ & 7252 & $7.87\left({ }_{-0.29}^{+0.32}\right)$ & $0.50\left({ }_{-0.14}^{+0.19}\right)$ & $-9.06(0.28)$ & $<-4.12$ & $270.7(48.6)$ \\
\hline $\mathrm{J} 1139+6737$ & $6600(196)$ & $7.94\left({ }_{-0.21}^{+0.22}\right)$ & $0.54\left({ }_{-0.11}^{+0.14}\right)$ & $-10.05(0$. & $<-3.57$ & $206.6(25.6)$ \\
\hline J1139-0132 & $5204(152)$ & 8.00 & & $-10.46(0.20)$ & $<-3.01$ & $200.8(12.5)$ \\
\hline $\mathrm{J} 1140+5328$ & $9829(323)$ & $8.14\left({ }_{-0.12}^{+0.12}\right)$ & $0.67\left({ }_{-0.07}^{+0.08}\right)$ & $-8.26(0.12)$ & $-4.65(0.39)$ & $219.4(15.0)$ \\
\hline
\end{tabular}


Table 6. Atmospheric parameters for DZ stars - continued.

\begin{tabular}{|c|c|c|c|c|c|c|}
\hline J Name & $T_{\text {eff }}(\mathrm{K})$ & $\log g$ & $M / M_{\odot}$ & $\log \mathrm{Ca} / \mathrm{He}$ & $\log \mathrm{H} / \mathrm{He}$ & $D(\mathrm{pc})$ \\
\hline $\mathrm{J} 1142+1104$ & $10424(341)$ & $8.08\left({ }_{-0.18}^{+0.19}\right)$ & $0.63\left({ }_{-0.11}^{+0.12}\right)$ & $-8.00(0.10)$ & $<-5.94$ & $242.1(28.2)$ \\
\hline $\mathrm{J} 1143+1928$ & $7845(198)$ & $8.11\left({ }_{-0.10}^{+0.11}\right)$ & $0.64\left(_{-}^{+}\right.$ & $-10.86(0.06)$ & $<-4.34$ & $115.7(6.7)$ \\
\hline $\mathrm{J} 1143-0145$ & $9583(342)$ & $7.99\left({ }_{-0.15}^{+0.16}\right)$ & $0.58\left({ }_{-0.09}^{+0.10}\right)$ & $-9.76(0.06)$ & $<-5.88$ & $208.1(18.1)$ \\
\hline $\mathrm{J} 1144+1218$ & $5500(47)$ & $8.11\left({ }_{-0.04}^{+0.04}\right)$ & $0.64\left({ }_{-0.02}^{+0.02}\right)$ & $-9.25(0.09)$ & $<-3.00$ & $50.5(0.6)$ \\
\hline $\mathrm{J} 1144+3259$ & $7324(289)$ & $7.98\left({ }_{-0.12}^{+0.13}\right)$ & $0.56\left(_{-0.07}^{+0.08}\right)$ & $-10.28(0.08)$ & $<-4.08$ & $142.5(8.1)$ \\
\hline $\mathrm{J} 1144+3720$ & $7379(165)$ & $7.84\left({ }_{-0.32}^{+0.35}\right)$ & $0.49\left({ }_{-0.15}^{+0.21}\right)$ & $-8.54(0.17)$ & $<-4.22$ & $279.6(55.5)$ \\
\hline $\mathrm{J} 1145+6619$ & $7556(480)$ & $8.64\left({ }_{-0.46}^{+0.57}\right)$ & $0.99\left({ }_{-0.30}^{+0.28}\right)$ & $-10.04(0$ & $<-3.78$ & $220.9(88.9)$ \\
\hline $\mathrm{J} 1147+4928$ & $7573(174)$ & $3.07\left({ }_{-0.06}^{+0.06}\right)$ & $0.62\left({ }_{-0.04}^{+0.04}\right)$ & $-10.06(0.05)$ & $<-4.14$ & 102.0 \\
\hline $\mathrm{J} 1147+5429$ & $5178(117)$ & 8.00 & & $-9.19(0.64)$ & $<-3.01$ & $204.4(12.3)$ \\
\hline $\mathrm{J} 1148+4109$ & $6698(98)$ & $7.89\left({ }_{-0.07}^{+0.07}\right)$ & $0.51\left({ }_{-0.04}^{+0.04}\right)$ & $-10.03(0.05)$ & $<-3.72$ & $117.9(3.4)$ \\
\hline $\mathrm{J} 1148+4124$ & $11119(433)$ & $8.31\left({ }_{-0.15}^{+0.15}\right)$ & $0.78\left({ }_{-0.10}^{+0.10}\right)$ & $-9.18(0.29)$ & $<-5.93$ & $249.4(24.6)$ \\
\hline $\mathrm{J} 1148+5708$ & $10207(337)$ & $8.25\left({ }_{-0.07}^{+0.08}\right)$ & $0.73\left(_{-0}^{+0}\right.$ & $-8.39(0.09)$ & $<-5.79$ & $170.7(5.4)$ \\
\hline $\mathrm{J} 1149+0519$ & $7004(94)$ & $7.84\left({ }_{-0.19}^{+0.20}\right)$ & $0.48\left({ }_{-0.10}^{+0.12}\right)$ & $-8.88(0.15)$ & $<-3.99$ & $189.4(21.9)$ \\
\hline $\mathrm{J} 1149+4943$ & $7597(464)$ & 8.00 & & $-10.41(0.19)$ & $<-4.22$ & $372.0(33.8)$ \\
\hline $\mathrm{J} 1150+4928$ & $7261(227)$ & $8.11\left({ }_{-0.16}^{+0.17}\right)$ & $0.64\left({ }_{-0.10}^{+0.11}\right)$ & $-9.51(0.17)$ & $<-3.96$ & $192.0(19.1)$ \\
\hline $\mathrm{J} 1152+2834$ & $6844(232)$ & $8.02\left({ }_{-0.25}^{+0.26}\right)$ & $0.58\left({ }_{-0.14}^{+0.17}\right)$ & $-10.32(0.10)$ & $<-3.76$ & $187.5(28.4)$ \\
\hline $\mathrm{J} 1152+5101$ & $5080(132)$ & 8.00 & & $-9.92(0.32)$ & $<-3.00$ & $178.5(11.0)$ \\
\hline $\mathrm{J} 1155+4327$ & $9902(339)$ & $7.93\left({ }_{-0.10}^{+0.10}\right)$ & $0.54\left({ }_{-0.05}^{+0.06}\right)$ & $-9.73(0.09)$ & $<-5.92$ & $174.9(8.0)$ \\
\hline $\mathrm{J} 1157+6138$ & $7008(4$ & 8.00 & & $-8.54(0$ & $<-3.91$ & $378.0(42.8)$ \\
\hline $\mathrm{J} 1158+0454$ & 543 & $8.12{ }_{+}^{+}$ & $0.65\left(_{-0}^{+0}\right.$ & $-8.51(C$ & $<-3$. & 0) \\
\hline $\mathrm{J} 1158+1845$ & 69 & & $0.67\left({ }_{-0.13}^{+0.14}\right)$ & -8.1 & . & 5) \\
\hline $\mathrm{J} 1158+4712$ & 8026 & $8.32\left({ }_{-0.41}^{+0.47}\right)$ & $0.78\left({ }_{-0.26}^{+0.30}\right)$ & $-7.99(0.17)$ & $<-4.28$ & 2) \\
\hline $\mathrm{J} 1158+5942$ & 581 & $7.87\left({ }_{-0.19}^{+0.20}\right)$ & $0.50\left({ }_{-0.10}^{+0.12}\right)$ & $-10.04(0$ & $<-3.00$ & 0) \\
\hline $\mathrm{J} 1159+2059$ & $7407(659)$ & 8.00 & & $-10.56(0.23)$ & $<-4.10$ & $398.3(54.3)$ \\
\hline $\mathrm{J} 1159+4045$ & $7839(186)$ & $8.00\left({ }_{-0.08}^{+0.08}\right)$ & $0.58\left({ }_{-0.05}^{+0.05}\right)$ & $-9.92(0.09)$ & $<-4.46$ & $137.7(4.7)$ \\
\hline $\mathrm{J} 1203+0834$ & $6009(54)$ & $7.96\left({ }_{-0.05}^{+0.05}\right)$ & $0.55\left({ }_{-0.03}^{+0.03}\right)$ & $-10.21(0.05)$ & $<-3.01$ & $62.7(1.2)$ \\
\hline $\mathrm{J} 1203+2323$ & $6559(316)$ & 8.00 & & $-8.92(0.27)$ & $<-3.47$ & $312.6(25.5)$ \\
\hline $\mathrm{J} 1203+2439$ & $13959(1027)$ & $8.17\left({ }_{-0.12}^{+0.13}\right)$ & $0.69\left({ }_{-0.08}^{+0.08}\right)$ & $-9.40(0.17)$ & $-5.20(0.07)$ & $99.2(1.1)$ \\
\hline $\mathrm{J} 1204+1030$ & $5630(212)$ & 8.00 & & $-10.08(0.35)$ & $<-3.00$ & $242.1(17.6)$ \\
\hline $\mathrm{J} 1204+5007$ & $8496(498)$ & $8.81\left({ }_{-0.26}^{+0.30}\right)$ & $1.09\left({ }_{-0.16}^{+0.14}\right)$ & $-8.55(0.44)$ & $<-4.10$ & $206.8(49.6)$ \\
\hline $\mathrm{J} 1205+3536$ & $5918(1$ & $8.14\left({ }_{-0.18}^{+0.18}\right)$ & $0.66\left(_{-0.11}^{+0.12}\right)$ & $-8.94(0.06)$ & $<-3.00$ & $157.7(17.2)$ \\
\hline $\mathrm{J} 1205+4312$ & $7000(3$ & $1\left({ }_{-0.49}^{+0.61}\right)$ & $0.37\left({ }_{-0.16}^{+0.34}\right)$ & $-10.73(0.16)$ & $<-4.12$ & (101.7) \\
\hline $\mathrm{J} 1206+1310$ & $6644(519)$ & 8.00 & & $-10.17(0.34)$ & $<-3.56$ & $332.6(42.0)$ \\
\hline $\mathrm{J} 1206+1454$ & $6774(226)$ & $8.38\left({ }_{-0.54}^{+0.65}\right)$ & $0.82\left({ }_{-0.34}^{+0.38}\right)$ & $-9.80(0.16)$ & $<-3.32$ & $216.3(92.2)$ \\
\hline $\mathrm{J} 1206+2650$ & $6322(169)$ & 8.00 & & $-10.02(0.20)$ & $<-3.22$ & $203.5(9.9)$ \\
\hline $\mathrm{J} 1208+1907$ & $7375(1$ & $8.14\left({ }_{-0.09}^{+0.09}\right)$ & $0.66\left({ }_{-0.06}^{+0.06}\right)$ & $-10.47(0.07)$ & $<-3.99$ & $120.6(5.5)$ \\
\hline $\mathrm{J} 1209+3026$ & $6850(97)$ & $8.01\left({ }_{-0.12}^{+0.12}\right)$ & $0.58\left(_{-0.07}^{+0.08}\right)$ & $-9.42(0.17)$ & $<-3.77$ & $161.7(11.5)$ \\
\hline $\mathrm{J} 1210+0553$ & 9391(558) & $7.69\left({ }_{-0.15}^{+0.16}\right)$ & $0.41\left({ }_{-0.06}^{+0.08}\right)$ & $-9.79(0.06)$ & $-4.61(0.16)$ & $194.9(11.7)$ \\
\hline
\end{tabular}


Table 6. Atmospheric parameters for DZ stars - continued.

\begin{tabular}{|c|c|c|c|c|c|c|}
\hline J Name & $T_{\text {eff }}(\mathrm{K})$ & $\log g$ & $M / M_{\odot}$ & $\log \mathrm{Ca} / \mathrm{He}$ & $\log \mathrm{H} / \mathrm{He}$ & $D(\mathrm{pc})$ \\
\hline $\mathrm{J} 1210+3136$ & $9121(275)$ & $8.12\left(\begin{array}{l}+0.10 \\
-0.10\end{array}\right)$ & $0.65\left({ }_{-0.06}^{+0.06}\right)$ & $-9.18(0.13)$ & $<-5.57$ & $175.0(8.3)$ \\
\hline $\mathrm{J} 1210+4332$ & $7926(783)$ & 8.00 & & $-10.28(0.25)$ & $<-4.56$ & $474.1(67.2)$ \\
\hline $\mathrm{J} 1210+4751$ & $7263(348)$ & $8.77\left({ }_{-0.38}^{+0.46}\right)$ & $1.07\left({ }_{-0.24}^{+0.20}\right)$ & $-9.90(0.22)$ & $<-3.41$ & $177.8(62.8)$ \\
\hline $\mathrm{J} 1211+2326$ & 6699(199) & $8.77\left({ }_{-0.24}^{+0.27}\right)$ & $1.07\left({ }_{-0.15}^{+0.13}\right)$ & $-8.04(0.16)$ & $<-3.02$ & $149.8(31.6)$ \\
\hline $\mathrm{J} 1212+5409$ & $8388(241)$ & $8.14\left({ }_{-0.07}^{+0.07}\right)$ & $0.67\left({ }_{-0.04}^{+0.04}\right)$ & $-9.61(0.04)$ & $<-4.90$ & $123.7(2.5)$ \\
\hline $\mathrm{J} 1212+6351$ & $7596(140)$ & $7.76\left({ }_{-0.15}^{+0.16}\right)$ & $0.44\left({ }_{-0.07}^{+0.09}\right)$ & $-8.67(0.09)$ & $<-4.51$ & $241.1(20.8)$ \\
\hline $\mathrm{J} 1214+2216$ & $7575(194)$ & $7.99\left({ }_{-0.11}^{+0.11}\right)$ & $0.57\left({ }_{-0.06}^{+0.07}\right)$ & $-10.58(0.10)$ & $<-4.21$ & $146.1(8.4)$ \\
\hline $\mathrm{J} 1214+7822$ & $4329(61)$ & $7.48\left({ }_{-0.07}^{+0.07}\right)$ & $0.31\left({ }_{-0.02}^{+0.03}\right)$ & $-11.23(0.13)$ & $<-30.00$ & $32.7(0.1)$ \\
\hline $\mathrm{J} 1215+3953$ & $9971(436)$ & $8.31\left({ }_{-0.27}^{+0.29}\right)$ & $0.78\left({ }_{-0.17}^{+0.19}\right)$ & $-9.02(0.35)$ & $<-5.71$ & $257.6(49.6)$ \\
\hline $\mathrm{J} 1216+6208$ & $7624(439)$ & 8.00 & & $-8.89(0.52)$ & $<-4.24$ & $421.8(35.5)$ \\
\hline $\mathrm{J} 1217+1157$ & $6149(155)$ & $7.53\left({ }_{-0.29}^{+0.33}\right)$ & $0.33\left({ }_{-0.10}^{+0.16}\right)$ & $-9.48(0.07)$ & $<-3.36$ & $230.0(37.7)$ \\
\hline $\mathrm{J} 1217+6420$ & $10688(402)$ & $8.10\left({ }_{-0.13}^{+0.13}\right)$ & $0.64\left(_{-0}^{+0}\right.$ & $-9.14(0.10)$ & $<-5.96$ & $245.2(18.1)$ \\
\hline $\mathrm{J} 1218+0023$ & $6456(77)$ & $8.31\left({ }_{-0.07}^{+0.08}\right)$ & $0.77\left({ }_{-0.05}^{+0.05}\right)$ & $-9.51(0.13)$ & $<-3.09$ & $112.3(5.3)$ \\
\hline $\mathrm{J} 1218+6023$ & $6700(416)$ & 8.00 & & $-9.70(0.41)$ & $<-3.62$ & $337.9(32.6)$ \\
\hline $\mathrm{J} 1219+0848$ & $7964(520)$ & 8.00 & & $-8.71(0.16)$ & $<-4.61$ & $390.2(35.9)$ \\
\hline $\mathrm{J} 1219+3018$ & $8193(383)$ & 8.00 & & $-8.62(0.34)$ & $<-4.90$ & $415.6(29.9)$ \\
\hline $\mathrm{J} 1219+5720$ & $7249(510)$ & 8.00 & & $-10.26(0.24)$ & $<-4.03$ & $341.8(36.6)$ \\
\hline $\mathrm{J} 1220+0929$ & $6842(239)$ & 8.00 & & $-8.31(0.10)$ & $<-3.77$ & $285.7(17.5)$ \\
\hline $\mathrm{J} 1222+6343$ & 10027 & $8.25\left({ }_{-0.15}^{+0.16}\right)$ & $0.74\left({ }_{-0.10}^{+0.10}\right)$ & $-9.56(0$ & $<-5.76$ & $.9)$ \\
\hline $\mathrm{J} 1223+1600$ & 76 & 8.00 & & $-8.57(0.56)$ & $<-4.31$ & .2) \\
\hline $\mathrm{J} 1223+1935$ & $5313(207)$ & 8.00 & & $-10.44(0.30)$ & $<-3.01$ & $232.5(18.1)$ \\
\hline $\mathrm{J} 1224+2838$ & $5096(84)$ & $8.07\left({ }_{-0.07}^{+0.08}\right)$ & $0.61\left({ }_{-0.05}^{+0.05}\right)$ & $-10.05(0.13)$ & $<-3.00$ & $81.7(2.4)$ \\
\hline $\mathrm{J} 1225-0245$ & $6934(245)$ & $8.16\left({ }_{-0.41}^{+0.46}\right)$ & $0.67\left({ }_{-0.24}^{+0.30}\right)$ & $-10.22(0.12)$ & $<-3.72$ & $209.5(59.1)$ \\
\hline $\mathrm{J} 1226+4445$ & $11861(1068)$ & $8.01\left({ }_{-0.20}^{+0.21}\right)$ & $0.59\left(_{-0.11}^{+0.13}\right)$ & $-9.48(0.18)$ & $<-6.09$ & $222.7(9.3)$ \\
\hline $\mathrm{J} 1227+4434$ & $6231(218)$ & 8.00 & & $-9.92(0.30)$ & $<-3.14$ & $277.4(16.6)$ \\
\hline $\mathrm{J} 1227+6330$ & $7418(82)$ & $8.07\left({ }_{-0.04}^{+0.04}\right)$ & $0.62\left({ }_{-0.02}^{+0.02}\right)$ & $-8.71(0.09)$ & $<-4.05$ & $96.8(1.3)$ \\
\hline $\mathrm{J} 1229+0743$ & $6079(141)$ & $7.32\left({ }_{-0.49}^{+0.54}\right)$ & $0.26\left({ }_{-0.13}^{+0.23}\right)$ & $-8.62(0.12)$ & $<-3.47$ & $263.5(67.2)$ \\
\hline $\mathrm{J} 1229+1606$ & $8025(562)$ & 8.00 & & $-8.79(0.71)$ & $<-4.68$ & $498.9(51.4)$ \\
\hline $\mathrm{J} 1229+3021$ & $6000(138)$ & $8.31\left(\left(_{-0.17}^{+0.18}\right)\right.$ & $0.77\left({ }_{-0.11}^{+0.12}\right)$ & $-10.36(0.21)$ & $<-3.00$ & $142.0(16.7)$ \\
\hline $\mathrm{J} 1229+4254$ & $11868(692)$ & $7.99\left({ }_{-0.51}^{+0.64}\right)$ & $0.58\left({ }_{-0.24}^{+0.41}\right)$ & $-8.30(0.55)$ & $<-6.09$ & $470.0(172.3)$ \\
\hline $\mathrm{J} 1229+5129$ & $9660(388)$ & $8.06\left({ }_{-0.13}^{+0.13}\right)$ & $0.62\left({ }_{-0.08}^{+0.08}\right)$ & $-9.57(0.12)$ & $<-5.85$ & $227.6(15.1)$ \\
\hline $\mathrm{J} 1230+0813$ & $7133(941)$ & 8.00 & & $-10.71(0.30)$ & $<-3.98$ & $449.5(93.7)$ \\
\hline $\mathrm{J} 1230+3143$ & $6282(149)$ & $7.69\left({ }_{-0.31}^{+0.35}\right)$ & $0.41\left({ }_{-0.13}^{+0.19}\right)$ & $-9.64(0.17)$ & $<-3.41$ & $28.6(42.2)$ \\
\hline $\mathrm{J} 1232+3232$ & $7682(862)$ & 8.00 & & $-10.24(0.22)$ & $<-4.29$ & $468.1(77.8)$ \\
\hline $\mathrm{J} 1232+3729$ & $7000(535)$ & 8.00 & & $-9.33(1.54)$ & $<-3.90$ & $478.0(55.8)$ \\
\hline $\mathrm{J} 1234+3051$ & $7552(380)$ & $7.39\left({ }_{-0.56}^{+0.71}\right)$ & $0.29\left({ }_{-0.14}^{+0.35}\right)$ & $-10.58(0.23)$ & $<-4.95$ & $371.0(125.0)$ \\
\hline $\mathrm{J} 1234+5208$ & $7534(63)$ & $8.05\left({ }_{-0.04}^{+0.04}\right)$ & $0.61\left({ }_{-0.02}^{+0.03}\right)$ & $-7.59(0.07)$ & $<-4.13$ & $115.1(2.1)$ \\
\hline $\mathrm{J} 1234+5606$ & $11787(423)$ & $8.30\left({ }_{-0.06}^{+0.06}\right)$ & $0.77\left({ }_{-0.04}^{+0.04}\right)$ & $-7.41(0.06)$ & $-5.52(0.14)$ & $173.8(3.6)$ \\
\hline
\end{tabular}


Table 6. Atmospheric parameters for DZ stars - continued.

\begin{tabular}{|c|c|c|c|c|c|c|}
\hline J Name & $T_{\text {eff }}(\mathrm{K})$ & $\log g$ & $M / M_{\odot}$ & $\log \mathrm{Ca} / \mathrm{He}$ & $\log \mathrm{H} / \mathrm{He}$ & $D(\mathrm{pc})$ \\
\hline J1234-0330 & $9184(287)$ & $19\left({ }_{-0.07}^{+0.08}\right)$ & $0.70\left(\begin{array}{l}+0.05 \\
-0.05\end{array}\right)$ & $-10.54(0.07)$ & $<-5.57$ & $107.1(2.6)$ \\
\hline $\mathrm{J} 1236+2144$ & $6660(612)$ & 8.00 & & $-9.79(0.40)$ & $<-3.58$ & $405.5(60.7)$ \\
\hline $\mathrm{J} 1238+1328$ & $6785(147)$ & $8.23\left({ }_{-0.15}^{+0.15}\right)$ & $0.72\left({ }_{-0.09}^{+0.10}\right)$ & $-9.69(0.22)$ & $<-3.49$ & $141.6(13.6)$ \\
\hline $\mathrm{J} 1238+2149$ & $5768(128)$ & $8.30\left({ }_{-0.19}^{+0.20}\right)$ & $0.77\left({ }_{-0.12}^{+0.13}\right)$ & $-8.26(0.32)$ & $<-3.00$ & $150.6(19.1)$ \\
\hline $\mathrm{J} 1239+0756$ & $6962(182)$ & $8.29\left({ }_{-0.23}^{+0.24}\right)$ & $0.76\left({ }_{-0.15}^{+0.16}\right)$ & $-9.38(0.41)$ & $<-3.62$ & $204.0(33.3)$ \\
\hline $\mathrm{J} 1239+3522$ & $7717(719)$ & 8.00 & & $-10.05(0$ & $<-4.33$ & $470.6(63.2)$ \\
\hline $\mathrm{J} 1240+1603$ & 6499(1 & $8.19\left({ }_{-0.18}^{+0.18}\right)$ & $0.70\left({ }_{-0.11}^{+0.12}\right)$ & $-10.56(0.12)$ & $<-3.21$ & $150.8(17.3)$ \\
\hline $\mathrm{J} 1240+1911$ & $7137(437)$ & 8.00 & & $-10.03(0.32)$ & $<-3.98$ & $340.6(30.7)$ \\
\hline $\mathrm{J} 1240-0037$ & $8514(177)$ & $8.13\left({ }_{-0.06}^{+0.06}\right)$ & $0.66\left({ }_{-0.04}^{+0.04}\right)$ & $-8.91(0.20)$ & $<-5.05$ & $114.5(2.6)$ \\
\hline $\mathrm{J} 1241+3010$ & $7525(233)$ & $8.08\left({ }_{-0.22}^{+0.23}\right)$ & $0.63\left({ }_{-0.13}^{+0.15}\right)$ & $-9.66(0.22)$ & $<-4.10$ & $217.7(30.3)$ \\
\hline $\mathrm{J} 1242+0829$ & 8123( & $8.17\left({ }_{-0.13}^{+0.14}\right)$ & $0.68\left({ }_{-0.08}^{+0.09}\right)$ & $-8.62(0.12)$ & $<-4.57$ & $183.5(15.5)$ \\
\hline $\mathrm{J} 1244+3724$ & $6637(87)$ & $8.14\left({ }_{-0.10}^{+0.10}\right)$ & $0.66\left({ }_{-0.06}^{+0.07}\right)$ & $-9.37(0.10)$ & $<-3.41$ & $142.2(8.7)$ \\
\hline $\mathrm{J} 1244+5732$ & $6973(496)$ & 8.00 & & $-10.36(0.17)$ & $<-3.88$ & $310.6(35.5)$ \\
\hline $\mathrm{J} 1244-0118$ & $8466(185)$ & $8.14\left({ }_{-0.10}^{+0.10}\right)$ & $0.67\left({ }_{-0.06}^{+0.07}\right)$ & $-8.65(0.17)$ & $<-4.98$ & $164.7(9.5)$ \\
\hline $\mathrm{J} 1245+0822$ & $5924(159)$ & $7.30\left({ }_{-1.25}^{+0.96}\right)$ & $0.25\left({ }_{-0.22}^{+0.48}\right)$ & $-9.16(0.19)$ & $<-3.31$ & $308.1(131.7)$ \\
\hline $\mathrm{J} 1245+6700$ & $7842(338)$ & $7.40\left({ }_{-0.39}^{+0.50}\right)$ & $0.29\left({ }_{-0.11}^{+0.23}\right)$ & $-10.49(0.14)$ & $<-5.25$ & $363.6(89.1)$ \\
\hline $\mathrm{J} 1246+1155$ & $8182(142)$ & $8.05\left({ }_{-0.05}^{+0.05}\right)$ & $0.61\left({ }_{-0.03}^{+0.03}\right)$ & $-8.77(0.10)$ & $<-4.81$ & $79.9(0.8)$ \\
\hline $\mathrm{J} 1246-0236$ & $5540(1292)$ & 8.00 & & $-10.27(0.21)$ & $<-3.00$ & $459.2(210.8)$ \\
\hline $\mathrm{J} 1247+4934$ & 15150 & $7.97\left({ }_{-0.12}^{+0.13}\right)$ & $0.57\left({ }_{-0.07}^{+0.07}\right)$ & $-7.57(0.14)$ & -5.1 & 120 \\
\hline $\mathrm{J} 1248+1411$ & 12755 & $8.05\left({ }_{-0.24}^{+0.27}\right)$ & $0.61\left({ }_{-0.13}^{+0.17}\right)$ & $-7.07(0.40)$ & -0.03 & 7.9) \\
\hline $\mathrm{J} 1249+0806$ & $7016(166)$ & & $0.67\left({ }_{-0.05}^{+0.05}\right)$ & $-10.98(0.14)$ & $<-3.79$ & $16.2(4.1)$ \\
\hline $\mathrm{J} 1250+5249$ & $6889(270)$ & $8.62\left({ }_{-0.50}^{+0.64}\right)$ & $0.98\left({ }_{-0.33}^{+0.30}\right)$ & $-9.17(0.24)$ & $<-3.23$ & $197.6(88.4)$ \\
\hline $\mathrm{J} 1252+3327$ & $7283(461$ & 8.00 & & $-10.83(0.19)$ & $<-4.04$ & $337.1(32.6)$ \\
\hline $\mathrm{J} 1252+3330$ & $6922(699)$ & 8.00 & & $-9.77(0.42)$ & $<-3.84$ & $457.7(69.4)$ \\
\hline $\mathrm{J} 1252+6401$ & $6394(414)$ & 8.00 & & $-10.26(0.27)$ & $<-3.29$ & $327.9(37.3)$ \\
\hline $\mathrm{J} 1253+1808$ & $13459(741)$ & $7.90\left({ }_{-0.30}^{+0.35}\right)$ & $0.53\left({ }_{-0.15}^{+0.21}\right)$ & $-8.51(0.31)$ & $<-6.11$ & $312.5(63.1)$ \\
\hline $\mathrm{J} 1253+3842$ & $7367(540)$ & 8.00 & & $-10.19(0.26)$ & $<-4.08$ & $435.9(48.7)$ \\
\hline $\mathrm{J} 1254+2233$ & $15366(1585)$ & $8.20\left({ }_{-0.19}^{+0.21}\right)$ & $0.71\left({ }_{-0.12}^{+0.13}\right)$ & $-7.14(0.22)$ & $-3.40(0.13)$ & $277.5(26.5)$ \\
\hline $\mathrm{J} 1254+3551$ & $6685(174)$ & $8.20\left({ }_{-0.28}^{+0.30}\right)$ & $0.70\left({ }_{-0.17}^{+0.20}\right)$ & $-8.79(0.15)$ & $<-3.40$ & $213.8(40.7)$ \\
\hline $\mathrm{J} 1254-0236$ & $6903(123)$ & $8.23\left({ }_{-0.05}^{+0.05}\right)$ & $0.72\left({ }_{-0.03}^{+0.03}\right)$ & $-10.27(0.08)$ & $<-3.62$ & $78.7(1.2)$ \\
\hline $\mathrm{J} 1255+4001$ & $6297(326)$ & 8.00 & & $-10.19(0.33)$ & $<-3.20$ & $372.1(34.4)$ \\
\hline $\mathrm{J} 1257+3238$ & $5531(209)$ & 8.00 & & $-8.63(0.17)$ & $<-3.00$ & $230.5(21.9)$ \\
\hline $\mathrm{J} 1257-0310$ & $6471(166)$ & 8.00 & & $-8.34(0.10)$ & $<-3.37$ & $226.9(10.7)$ \\
\hline $\mathrm{J} 1259+3112$ & $5976(82)$ & $8.20\left({ }_{-0.08}^{+0.08}\right)$ & $0.70\left({ }_{-0.05}^{+0.05}\right)$ & $-9.13(0.24)$ & $<-3.00$ & $115.3(5.5)$ \\
\hline $\mathrm{J} 1259+4729$ & $5706(207)$ & $7.90\left(\begin{array}{l}+0.38 \\
-0.35\end{array}\right)$ & $0.51\left({ }_{-0.17}^{+0.24}\right)$ & $-9.83(0.37)$ & $<-3.00$ & $210.7(42.9)$ \\
\hline $\mathrm{J} 1302+2215$ & $7092(310)$ & $7.45\left({ }_{-0.67}^{+0.84}\right)$ & $0.31\left({ }_{-0.18}^{+0.46}\right)$ & $-10.38(0.24)$ & $<-4.33$ & $365.8(145.7)$ \\
\hline $\mathrm{J} 1303+4055$ & $6542(115)$ & $8.09\left({ }_{-0.09}^{+0.09}\right)$ & $0.63\left(_{-0.05}^{+0.06}\right)$ & $-8.81(0.08)$ & $<-3.36$ & $147.0(7.0)$ \\
\hline $\mathrm{J} 1303+4418$ & $6849(449)$ & 8.00 & & $-10.83(0.26)$ & $<-3.77$ & $294.2(31.2)$ \\
\hline
\end{tabular}


Table 6. Atmospheric parameters for DZ stars - continued.

\begin{tabular}{|c|c|c|c|c|c|c|}
\hline J Name & $T_{\text {eff }}(\mathrm{K})$ & $\log g$ & $M / M_{\odot}$ & $\log \mathrm{Ca} / \mathrm{He}$ & $\log \mathrm{H} / \mathrm{He}$ & $D(\mathrm{pc})$ \\
\hline $\mathrm{J} 1307+0307$ & $8152(208)$ & $91\left(\begin{array}{l}+0.08 \\
-0.08\end{array}\right)$ & $0.53\left({ }_{-0.04}^{+0.05}\right)$ & $-9.67(0.07)$ & $-3.54(0.11)$ & $134.5(4.7)$ \\
\hline $\mathrm{J} 1308+0258$ & $6075(160)$ & 8.00 & & $-9.00(0.11)$ & $<-3.03$ & $214.0(11.1)$ \\
\hline $\mathrm{J} 1308+0957$ & $7946(373)$ & 8.00 & & $-8.00(0.28)$ & $<-4.58$ & $417.2(30.2)$ \\
\hline $\mathrm{J} 1309+3812$ & $8234(650)$ & 8.00 & & $-8.36(0.44)$ & $<-4.95$ & $545.0(65.9)$ \\
\hline $\mathrm{J} 1309+4913$ & $8677(243)$ & $8.10\left({ }_{-0.06}^{+0.06}\right)$ & $0.64\left({ }_{-0.04}^{+0.04}\right)$ & $-10.11(0.03)$ & $<-5.26$ & $78.9(0.5)$ \\
\hline $\mathrm{J} 1310+0645$ & $7935(662)$ & 8.00 & & $-8.45(0.25)$ & $<-4.57$ & $469.5(59.8)$ \\
\hline $\mathrm{J} 1310+1323$ & $7701(675)$ & 8.00 & & $-9.98(0.33)$ & $<-4.31$ & $394.5(50.4)$ \\
\hline $\mathrm{J} 1310+3149$ & $6925(449)$ & 8.00 & & -10.04( & $<-3.84$ & 7.5) \\
\hline $\mathrm{J} 1311+1019$ & $7359(152)$ & $7.72\left({ }_{-0.24}^{+0.26}\right)$ & $0.42\left(\begin{array}{l}+0.14 \\
-0.10\end{array}\right)$ & $-8.99(C$ & $<-4.33$ & $251.6(35.0)$ \\
\hline $\mathrm{J} 1313+5738$ & $8801(227)$ & $7.99\left({ }_{-0.06}^{+0.06}\right)$ & $0.57\left({ }_{-0.03}^{+0.04}\right)$ & $-9.38(0.03)$ & $-4.67(0.17)$ & $68.0(0.3)$ \\
\hline $\mathrm{J} 1313+5813$ & $6808(136)$ & $7.91\left({ }_{-0.12}^{+0.12}\right)$ & $0.52\left({ }_{-0.06}^{+0.07}\right)$ & $-9.75(0.20)$ & $<-3.81$ & $189.9(12.6)$ \\
\hline $\mathrm{J} 1314+3748$ & $6107(147)$ & $8.40\left({ }_{-0.25}^{+0.27}\right)$ & $0.83\left({ }_{-0.17}^{+0.18}\right)$ & $-8.64(C$ & $<-3.00$ & $168.3(31.6)$ \\
\hline $\mathrm{J} 1314+5223$ & 11351(434) & $8.01\left(_{-0}^{+0}\right.$ & $0.59\left(_{-0}^{+0}\right.$ & $-8.43(0.08)$ & $-5.43(0.15)$ & $143.6(2.6)$ \\
\hline $\mathrm{J} 1316+1916$ & $12686(674)$ & $8.11\left({ }_{-0.12}^{+0.13}\right)$ & $0.65\left({ }_{-0.07}^{+0.08}\right)$ & $-8.84(0.16)$ & $-4.22(0.15)$ & $230.0(13.4)$ \\
\hline $\mathrm{J} 1316+1918$ & $5250(129)$ & $7.81\left({ }_{-0.35}^{+0.38}\right)$ & $0.46\left({ }_{-0.16}^{+0.23}\right)$ & $-10.33(0.30)$ & $<-3.01$ & $185.0(37.4)$ \\
\hline $\mathrm{J} 1316+5117$ & $6465(195)$ & 8.00 & & $-9.20(0.36)$ & $<-3.36$ & $282.7(14.6)$ \\
\hline J1316-2007 & $4667(103)$ & $8.14\left({ }_{-0.06}^{+0.06}\right)$ & $0.66\left({ }_{-0.04}^{+0.04}\right)$ & $-10.74(0.09)$ & $<-3.00$ & $23.2(0.1)$ \\
\hline $\mathrm{J} 1318+2022$ & $6167(166)$ & $8.24\left({ }_{-0.23}^{+0.24}\right)$ & $0.72\left({ }_{-0.15}^{+0.16}\right)$ & $-9.49(0.29)$ & $<-3.01$ & $181.8(28.2)$ \\
\hline $\mathrm{J} 1319+0844$ & $9040(273)$ & $8.16\left({ }_{-0.07}^{+0.07}\right)$ & $0.68\left(_{-0}^{+0}\right.$ & $-11.13(0$ & $<-5.48$ & 108.5( \\
\hline $\mathrm{J} 1319+3025$ & 95 & & $0.65\left(_{-}^{+}\right.$ & -9.57 & $<-5.79$ & $160.6(5$. \\
\hline $\mathrm{J} 1319+3641$ & 73 & & & $-8.85(0$ & & 8) \\
\hline $\mathrm{J} 1320+0204$ & 7070 & $8.22\left({ }_{-0.73}^{+0.95}\right)$ & $0.71\left({ }_{-0.39}^{+0.54}\right)$ & $-9.02(0.3$ & $<-3.78$ & $282.0(1$ \\
\hline $\mathrm{J} 1320+4332$ & $6649(262)$ & $7.83\left({ }_{-0.24}^{+0.26}\right)$ & $0.48\left({ }_{-0.12}^{+0.15}\right)$ & $-10.32(0.13)$ & $<-3.72$ & $215.6(28.2)$ \\
\hline $\mathrm{J} 1321+1614$ & $6659(101)$ & $8.18\left({ }_{-0.06}^{+0.06}\right)$ & $0.69\left(_{-0.04}^{+0.04}\right)$ & $-9.98(0.08)$ & $<-3.39$ & $103.3(3.2)$ \\
\hline $\mathrm{J} 1321+2019$ & $7484(273)$ & $8.95\left({ }_{-0.23}^{+0.26}\right)$ & $1.16\left(_{-0.12}^{+0.10}\right)$ & $-8.61(0.32)$ & $<-3.39$ & $156.3(34.9)$ \\
\hline $\mathrm{J} 1321-0237$ & $5632(117)$ & $7.92\left({ }_{-0.29}^{+0.31}\right)$ & $0.53\left({ }_{-0.15}^{+0.19}\right)$ & $-8.59(0.27)$ & $<-3.00$ & $171.2(29.7)$ \\
\hline $\mathrm{J} 1322+1224$ & $6824(372)$ & 8.00 & & $-9.42(0.44)$ & $<-3.75$ & $367.7(31.2)$ \\
\hline $\mathrm{J} 1322+6707$ & $9971(435)$ & $8.52\left({ }_{-0.27}^{+0.29}\right)$ & $0.92\left({ }_{-0.18}^{+0.18}\right)$ & $-7.82(0.27)$ & $-4.35(0.46)$ & $287.1(61.4)$ \\
\hline $\mathrm{J} 1323+2112$ & $6871(525)$ & 8.00 & & $-10.03(0.31)$ & $<-3.80$ & $417.3(50.0)$ \\
\hline $\mathrm{J} 1323+3849$ & $10623(454)$ & $7.82\left({ }_{-0.11}^{+0.11}\right)$ & $0.48\left({ }_{-0.05}^{+0.06}\right)$ & $-8.94(0.09)$ & $-4.75(0.19)$ & $229.2(10.3)$ \\
\hline $\mathrm{J} 1325+6521$ & $11833(601)$ & $8.04\left({ }_{-0.13}^{+0.14}\right)$ & $0.61\left({ }_{-0.08}^{+0.09}\right)$ & $-8.49(0$ & $-4.27(0.27)$ & 17.2) \\
\hline $\mathrm{J} 1326+1854$ & $9259(423)$ & $7.29\left({ }_{-0.56}^{+0.82}\right)$ & $0.27\left({ }_{-0.12}^{+0.38}\right)$ & $-8.86(0.48)$ & $<-6.07$ & 13.5) \\
\hline $\mathrm{J} 1326+4235$ & $6948(418)$ & 8.00 & & $-10.09(0.20)$ & $<-3.86$ & $360.9(34.4)$ \\
\hline $\mathrm{J} 1327+4508$ & $7073(2$ & $8.37\left({ }_{-0.18}^{+0.19}\right)$ & $0.81\left({ }_{-0.12}^{+0.12}\right)$ & -10.48 & $<-3.64$ & $185.3(22.1)$ \\
\hline $\mathrm{J} 1327+5519$ & $6941(450)$ & 8.00 & & $-9.33(0.59)$ & $<-3.86$ & $387.3(38.3)$ \\
\hline $\mathrm{J} 1329+1301$ & $6800(129)$ & $7.68\left({ }_{-0.22}^{+0.24}\right)$ & $0.40\left({ }_{-0.09}^{+0.12}\right)$ & $-8.83(0.10)$ & $<-3.95$ & $228.0(28.9)$ \\
\hline $\mathrm{J} 1329+6459$ & $6904(499)$ & 8.00 & & $-9.44(0.58)$ & $<-3.82$ & $399.0(44.0)$ \\
\hline $\mathrm{J} 1332+1522$ & $7313(161)$ & $8.03\left({ }_{-0.07}^{+0.07}\right)$ & $0.59\left({ }_{-0.04}^{+0.05}\right)$ & $-10.58(0.08)$ & $<-4.03$ & $116.4(3.4)$ \\
\hline
\end{tabular}


Table 6. Atmospheric parameters for DZ stars - continued.

\begin{tabular}{|c|c|c|c|c|c|c|}
\hline J Name & $T_{\text {eff }}(\mathrm{K})$ & $\log g$ & $M / M_{\odot}$ & $\log \mathrm{Ca} / \mathrm{He}$ & $\log \mathrm{H} / \mathrm{He}$ & $D(\mathrm{pc})$ \\
\hline $\mathrm{J} 1332+5446$ & 9279(1339) & 8.00 & & $-9.54(0.32)$ & $<-5.73$ & 632.1(134.6) \\
\hline $\mathrm{J} 1333+3254$ & $12021(492)$ & $8.02\left({ }_{-0.13}^{+0.14}\right)$ & $0.60{ }_{-}^{+}$ & $-7.03(0.09)$ & $<-6.10$ & $296.8(22.5)$ \\
\hline $\mathrm{J} 1333+6349$ & $15316(1559)$ & $7.96\left({ }_{-0.16}^{+0.17}\right)$ & $0.57\left({ }_{-0.08}^{+0.10}\right)$ & $-7.17(0.19)$ & $-5.07(0.18)$ & $292.7(17.4)$ \\
\hline $\mathrm{J} 1335+2254$ & $6388(332)$ & 8.00 & & $-9.97(0.43)$ & $<-3.28$ & $346.3(31.4)$ \\
\hline $\mathrm{J} 1336+3012$ & $9400(786)$ & 8.00 & & $-8.17(0.39)$ & $<-5.81$ & $560.1(63.3)$ \\
\hline $\mathrm{J} 1336+3547$ & $6247(31)$ & $8.08\left({ }_{-0.03}^{+0.03}\right)$ & $0.62\left({ }_{-0.02}^{+0.02}\right)$ & $-8.85(0.03)$ & $<-3.11$ & $59.7(0.4)$ \\
\hline $\mathrm{J} 1338+1334$ & $13396(634)$ & $8.05\left({ }_{-0.13}^{+0.13}\right)$ & $0.61\left({ }_{-0.07}^{+0.08}\right)$ & $-9.75(C$ & $-5.20(0.21)$ & $252.4(17.4)$ \\
\hline $\mathrm{J} 1338+3255$ & $7104(286)$ & $8.19\left({ }_{-0.29}^{+0.31}\right)$ & $0.69\left({ }_{-0.18}^{+0.21}\right)$ & $-10.23(0.12)$ & $<-3.83$ & $230.5(45$ \\
\hline $\mathrm{J} 1338-0130$ & $10338(322)$ & $8.02\left({ }_{-0.07}^{+0.07}\right)$ & $0.59\left({ }_{-0.04}^{+0.04}\right)$ & $-9.32(0.08)$ & $<-5.96$ & $138.6(3.5)$ \\
\hline $\mathrm{J} 1339+2643$ & $6456(53)$ & $8.07\left({ }_{-0.06}^{+0.06}\right)$ & $0.62\left({ }_{-0.04}^{+0.04}\right)$ & $-9.28(0.09)$ & $<-3.29$ & $103.8(3.3)$ \\
\hline $\mathrm{J} 1339+6401$ & $8070(616)$ & 8.00 & & $-9.84(C$ & $<-4.74$ & $387.7(42.4)$ \\
\hline $\mathrm{J} 1340+0835$ & $7546(180)$ & $8.23\left(_{-0 .}^{+0 .}\right.$ & & $-10.60(0.14)$ & $<-4.03$ & $112.4(4.9)$ \\
\hline $\mathrm{J} 1340+2702$ & $8414(277)$ & $8.78\left(_{-0}^{+0}\right.$ & $1.07\left(_{-C}^{+C}\right.$ & $-7.05(0.22)$ & $<-4.07$ & $246.9(91.1)$ \\
\hline $\mathrm{J} 1341+1338$ & $10507(368)$ & $7.91\left(_{-}^{+}\right.$ & $0.53\left({ }_{-0.09}^{+0.11}\right)$ & $-8.40(0.16)$ & $<-6.04$ & $271.9(27.7)$ \\
\hline $\mathrm{J} 1341+2216$ & $8102(214)$ & $7.98\left({ }_{-0.14}^{+0.15}\right)$ & $0.56\left({ }_{-0.08}^{+0.09}\right)$ & $-8.66(0.15)$ & $<-4.81$ & $214.6(17.3)$ \\
\hline $\mathrm{J} 1341+3024$ & $6436(183)$ & $7.96\left({ }_{-0.20}^{+0.21}\right)$ & $0.55\left({ }_{-0.11}^{+0.13}\right)$ & $-10.76(0.13)$ & $<-3.37$ & $166.5(19.5)$ \\
\hline $\mathrm{J} 1341-0112$ & $8533(242)$ & $8.30\left({ }_{-0.16}^{+0.17}\right)$ & $0.77\left(_{-0}^{+0}\right.$ & $-8.87(0.41)$ & $<-4.77$ & $196.4(21.3)$ \\
\hline J1341-3124 & $7935(463)$ & $8.01\left({ }_{-0.12}^{+0.12}\right)$ & $0.58\left(_{-0}^{+0}\right.$ & $-10.39(0.05)$ & $<-4.56$ & $67.8(0.9)$ \\
\hline $\mathrm{J} 1342+0522$ & $8189(150)$ & $8.02\left({ }_{-0.12}^{+0.12}\right)$ & $0.59\left({ }_{-0.07}^{+0.08}\right)$ & $-8.77(0.25)$ & $<-4.86$ & $154.8(10.9)$ \\
\hline $\mathrm{J} 1342+1813$ & $5635(1$ & 8.00 & & $-8.88(0$ & $<-3.00$ & $01.4(10.7)$ \\
\hline $\mathrm{J} 1343+294$ & 11 & & $0.69\left(_{-0.10}^{+0.11}\right)$ & $-7.88(0$ & & 0) \\
\hline $\mathrm{J} 1343+4641$ & 6531 & $8.05\left({ }_{-0.16}^{+0.17}\right)$ & $0.60\left({ }_{-0.10}^{+0.11}\right)$ & $-10.92(0.16)$ & $<-3.39$ & 1) \\
\hline $\mathrm{J} 1344+6505$ & 105 & $8.15\left({ }_{-0.19}^{+0.21}\right)$ & $0.68\left(_{-0.12}^{+0.13}\right)$ & $-9.01(0.39)$ & $<-5.91$ & 1) \\
\hline $\mathrm{J} 1345+1153$ & $6064(102)$ & $8.22\left({ }_{-0.11}^{+0.11}\right)$ & $0.71\left({ }_{-0.07}^{+0.07}\right)$ & $-8.03(0.10)$ & $<-3.00$ & 120 \\
\hline $\mathrm{J} 1345+5854$ & $7258(270)$ & $8.22\left({ }_{-0.20}^{+0.21}\right)$ & $0.71\left({ }_{-0.13}^{+0.14}\right)$ & $-9.63(0.18)$ & $<-3.89$ & $219.0(28.2)$ \\
\hline $\mathrm{J} 1347+0720$ & $6787(232)$ & 8.00 & & $-9.76(0.23)$ & $<-3.71$ & $237.6(12.2)$ \\
\hline $\mathrm{J} 1347+1415$ & $6489(76)$ & $8.18\left({ }_{-0.10}^{+0.10}\right)$ & $0.69\left({ }_{-0.06}^{+0.07}\right)$ & $-8.56(0.07)$ & $<-3.21$ & $136.8(8.9)$ \\
\hline $\mathrm{J} 1347+1733$ & $6953(208)$ & $8.22\left({ }_{-0.25}^{+0.27}\right)$ & $0.71\left({ }_{-0.16}^{+0.18}\right)$ & $-9.53(0.13)$ & $<-3.68$ & $183.3(32.0)$ \\
\hline $\mathrm{J} 1349+0209$ & $5935(164)$ & 8.00 & & $-9.51(0.22)$ & $<-3.00$ & $198.8(10.4)$ \\
\hline $\mathrm{J} 1349+0243$ & $13408(863)$ & $8.06\left({ }_{-0.12}^{+0.12}\right)$ & $0.63\left({ }_{-0.07}^{+0.08}\right)$ & $-8.07(0.20)$ & $<-6.10$ & $228.0(10.9)$ \\
\hline $\mathrm{J} 1350+1058$ & 535 & $8.64\left({ }_{-0.17}^{+0.18}\right)$ & $0.99\left(_{-0.11}^{+0.11}\right)$ & $-9.10(0.23)$ & $<-3.00$ & $05.3(14.6)$ \\
\hline $\mathrm{J} 1350+2148$ & $6997(725)$ & 8.00 & & $-9.70(0.45)$ & $<-3.90$ & $427.5(66.9)$ \\
\hline $\mathrm{J} 1351+1900$ & $14528(1593)$ & $7.58\left({ }_{-0.54}^{+0.88}\right)$ & $0.38\left({ }_{-0.15}^{+0.50}\right)$ & $-7.05(0.39)$ & $<-6.00$ & 709.1(311.6) \\
\hline $\mathrm{J} 1351+2645$ & $5850(109)$ & $7.86\left({ }_{-0.16}^{+0.16}\right)$ & $0.49\left({ }_{-0.08}^{+0.09}\right)$ & $-8.48(0.09)$ & $<-3.00$ & $171.2(14.7)$ \\
\hline $\mathrm{J} 1351+4253$ & $6506(104)$ & $8.12\left({ }_{-0.04}^{+0.05}\right)$ & $0.65\left({ }_{-0.03}^{+0.03}\right)$ & $-11.16(0.09)$ & $<-3.29$ & $39.4(0.1)$ \\
\hline $\mathrm{J} 1351+6136$ & 685 & $8.14\left({ }_{-0.08}^{+0.09}\right)$ & $0.66\left({ }_{-0.05}^{+0.06}\right)$ & $-10.45(0.14)$ & $<-3.66$ & $141.9(5.4)$ \\
\hline $\mathrm{J} 1353+1347$ & $8319(258)$ & $8.60\left({ }_{-0.25}^{+0.28}\right)$ & $0.97\left({ }_{-0.17}^{+0.16}\right)$ & $-8.49(0.22)$ & $<-4.13$ & $202.9(43.5)$ \\
\hline $\mathrm{J} 1353+3239$ & $8360(234)$ & $8.03\left({ }_{-0.07}^{+0.07}\right)$ & $0.60\left({ }_{-0.04}^{+0.05}\right)$ & $-10.82(0.04)$ & $<-5.06$ & $112.9(1.9)$ \\
\hline
\end{tabular}


Table 6. Atmospheric parameters for DZ stars - continued.

\begin{tabular}{|c|c|c|c|c|c|c|}
\hline J Name & $T_{\text {eff }}(\mathrm{K})$ & $\log g$ & $M / M_{\odot}$ & $\log \mathrm{Ca} / \mathrm{He}$ & $\log \mathrm{H} / \mathrm{He}$ & $D(\mathrm{pc})$ \\
\hline $\mathrm{J} 1355+0810$ & $7160(146)$ & $3.25\left({ }_{-0.14}^{+0.14}\right)$ & $0.74\left({ }_{-0.09}^{+0.09}\right)$ & $-9.04(0.17)$ & $<-3.81$ & $160.5(14.6)$ \\
\hline $\mathrm{J} 1356+0236$ & $7722(114)$ & $3.12\left({ }_{-0.26}^{+0.28}\right)$ & $0.65\left({ }_{-0.16}^{+0.19}\right)$ & $-7.80(0.09)$ & $<-4.22$ & $189.7(33.8)$ \\
\hline $\mathrm{J} 1356+2416$ & $6317(54)$ & $3.08\left({ }_{-0.05}^{+0.05}\right)$ & $0.62\left({ }_{-0.03}^{+0.03}\right)$ & $-8.95(0.13)$ & $<-3.16$ & $100.6(2.5)$ \\
\hline $\mathrm{J} 1356+4047$ & $6792(145)$ & $8.11\left({ }_{-0.06}^{+0.06}\right)$ & $0.64\left({ }_{-0.04}^{+0.04}\right)$ & $-10.08(0.07)$ & $<-3.62$ & $109.0(2.5)$ \\
\hline $\mathrm{J} 1356+4342$ & $6942(610)$ & 8.00 & & $-9.65(0$ & $<-3.86$ & $394.5(53.0)$ \\
\hline $\mathrm{J} 1357+5727$ & $6976(456)$ & 8.00 & & $-9.78(0$ & $<-3.88$ & $345.1(35.4)$ \\
\hline $\mathrm{J} 1359+3810$ & $6552(301)$ & 8.00 & & $-10.47(0$ & $<-3.46$ & $181.4(17.7)$ \\
\hline $\mathrm{J} 1400+4507$ & $7238(294)$ & $8.16\left({ }_{-0.22}^{+0.23}\right)$ & $0.67\left({ }_{-0.13}^{+0.15}\right)$ & $-9.97(0.09)$ & $<-3.92$ & $221.0(30.1)$ \\
\hline $\mathrm{J} 1401+2840$ & $9200(293)$ & $7.97\left({ }_{-0.10}^{+0.11}\right)$ & $0.56\left(_{-0.06}^{+0.06}\right)$ & $-10.04(0.05)$ & $<-5.70$ & $180.1(8.2)$ \\
\hline $\mathrm{J} 1401+3009$ & $8283(510)$ & $8.64\left({ }_{-0.27}^{+0.30}\right)$ & $0.99\left({ }_{-0.18}^{+0.17}\right)$ & $-9.04(0.28)$ & $<-4.09$ & $229.5(51.1)$ \\
\hline $\mathrm{J} 1401+3659$ & $5891(42)$ & $7.94\left({ }_{-0.04}^{+0.04}\right)$ & $0.54\left({ }_{-0.02}^{+0.03}\right)$ & $-10.14(0.09)$ & $<-3.00$ & $84.3(1.4)$ \\
\hline $\mathrm{J} 1402+2506$ & $9224(286)$ & $8.28\left({ }_{-0.07}^{+0.07}\right)$ & $0.75\left({ }_{-0.05}^{+0.05}\right)$ & $-8.81(0.08)$ & $<-5.55$ & $131.8(3.3)$ \\
\hline $\mathrm{J} 1402+2518$ & $6777(143)$ & $8.26\left({ }_{-0.09}^{+0.09}\right)$ & $0.74\left({ }_{-0.06}^{+0.06}\right)$ & $-9.67(0.09)$ & $<-3.44$ & $132.0(6.7)$ \\
\hline $\mathrm{J} 1402+4742$ & $7847(354)$ & 8.00 & & $-8.37(0.24)$ & $<-4.47$ & $407.1(29.0)$ \\
\hline $\mathrm{J} 1403+1954$ & $13117(867)$ & $8.14\left({ }_{-0.18}^{+0.19}\right)$ & $0.67\left({ }_{-0.11}^{+0.12}\right)$ & $-8.81(0.41)$ & $-4.17(0.30)$ & $274.6(27.3)$ \\
\hline $\mathrm{J} 1404+3620$ & $6448(45)$ & $8.27\left({ }_{-0.03}^{+0.03}\right)$ & $0.75\left({ }_{-0.02}^{+0.02}\right)$ & $-8.85(0.08)$ & $<-3.11$ & $81.7(1.2)$ \\
\hline $\mathrm{J} 1404-0232$ & $7597(164)$ & $8.17\left({ }_{-0.08}^{+0.08}\right)$ & $0.68\left({ }_{-0.05}^{+0.05}\right)$ & $-9.76(0.13)$ & $<-4.09$ & $128.1(4.8)$ \\
\hline $\mathrm{J} 1405+1549$ & $6952(61)$ & $8.02\left({ }_{-0.09}^{+0.09}\right)$ & $0.59\left({ }_{-0.05}^{+0.06}\right)$ & $-8.60(0.08)$ & $<-3.85$ & $124.7(6.7)$ \\
\hline $\mathrm{J} 1405+2542$ & $5653(150)$ & 8.00 & & $-10.28(0.35)$ & $<-3.00$ & $01.8(10.3)$ \\
\hline $\mathrm{J} 1406+1814$ & 8664 & $8.12\left({ }_{-0.11}^{+0.11}\right)$ & $0.65\left({ }_{-0.07}^{+0.07}\right)$ & $-9.86(0.06)$ & $<-5.22$ & $168.0(9.3)$ \\
\hline J1406-0037 & 7522 & $8.10\left({ }_{-0.45}^{+0.51}\right)$ & $0.64\left({ }_{-0.25}^{+0.34}\right)$ & $-8.78(0.24)$ & $<-4.09$ & $3.4(79.1)$ \\
\hline $\mathrm{J} 1407+3218$ & $6988(1$ & $7.70\left({ }_{-0.22}^{+0.24}\right)$ & $0.41\left({ }_{-0.09}^{+0.13}\right)$ & $-9.24(0.22)$ & $<-4.06$ & $244.2(30.9)$ \\
\hline $\mathrm{J} 1408+1535$ & 10502 & $8.32\left({ }_{-0.11}^{+0.11}\right)$ & $0.79\left({ }_{-0.07}^{+0.07}\right)$ & $-8.30(0.09)$ & $<-5.77$ & $184.3(12.1)$ \\
\hline $\mathrm{J} 1408+1750$ & $7484(472)$ & 8.00 & & $-9.58(0.49)$ & $<-4.14$ & $413.1(38.4)$ \\
\hline $\mathrm{J} 1409+1254$ & $6215(334)$ & 8.00 & & $-9.83(0.56)$ & $<-3.13$ & $316.2(30.3)$ \\
\hline $\mathrm{J} 1409+1511$ & $6542(112)$ & $8.14\left({ }_{-0.06}^{+0.06}\right)$ & $0.66\left({ }_{-0.04}^{+0.04}\right)$ & $-10.75(0.10)$ & $<-3.31$ & $87.2(1.8)$ \\
\hline $\mathrm{J} 1410+1445$ & $13152(674)$ & $8.12\left({ }_{-0.17}^{+0.18}\right)$ & $0.66\left({ }_{-0.10}^{+0.12}\right)$ & $-8.82(0.32)$ & $<-6.08$ & $321.4(35.2)$ \\
\hline $\mathrm{J} 1411+3410$ & $5527(93)$ & $8.39\left({ }_{-0.13}^{+0.14}\right)$ & $0.83\left({ }_{-0.09}^{+0.09}\right)$ & $-8.20(0.88)$ & $<-3.00$ & $120.1(11.1)$ \\
\hline $\mathrm{J} 1412+1343$ & $10166(382)$ & $8.12\left({ }_{-0.12}^{+0.13}\right)$ & $0.66\left(_{-0.08}^{+0.08}\right)$ & $-8.39(0.09)$ & $<-5.86$ & $232.5(16.4)$ \\
\hline $\mathrm{J} 1412+4011$ & $9370(797)$ & 8.00 & & $-7.17(0.48)$ & $<-5.79$ & $818.8(106.2)$ \\
\hline $\mathrm{J} 1413+1659$ & $7946(1078)$ & 8.00 & & $-9.88(0.36)$ & $<-4.58$ & $553.7(104.5)$ \\
\hline $\mathrm{J} 1413+1820$ & $7034(412)$ & 8.00 & & $-9.99(16.68)$ & $<-3.92$ & $303.4(27.1)$ \\
\hline $\mathrm{J} 1414-0113$ & $8697(238)$ & $7.98\left({ }_{-0.07}^{+0.07}\right)$ & $0.57\left({ }_{-0.04}^{+0.04}\right)$ & $-10.82(0.07)$ & $<-5.41$ & $108.4(1.9)$ \\
\hline $\mathrm{J} 1416+0230$ & $7003(401)$ & 8.00 & & $-10.07(0.24)$ & $<-3.90$ & $288.5(26.2)$ \\
\hline $\mathrm{J} 1417+2931$ & $5887(325)$ & 8.00 & & $-9.56(0.19)$ & $<-3.00$ & $328.5(36.0)$ \\
\hline $\mathrm{J} 1419+4400$ & $7292(273)$ & $8.33\left({ }_{-0.15}^{+0.15}\right)$ & $0.79\left({ }_{-0.10}^{+0.10}\right)$ & $-9.91(0.13)$ & $<-3.83$ & $197.9(18.0)$ \\
\hline $\mathrm{J} 1420+4405$ & $5780(227)$ & 8.00 & & $-10.03(0.41)$ & $<-3.00$ & $315.6(25.1)$ \\
\hline $\mathrm{J} 1420+4759$ & $11369(412)$ & $7.89\left({ }_{-0.11}^{+0.11}\right)$ & $0.52\left({ }_{-0.06}^{+0.06}\right)$ & $-9.79(0.17)$ & $<-6.06$ & 250.1(13.6) \\
\hline
\end{tabular}


Table 6. Atmospheric parameters for DZ stars - continued.

\begin{tabular}{|c|c|c|c|c|c|c|}
\hline J Name & $T_{\text {eff }}(\mathrm{K})$ & $\log g$ & $M / M_{\odot}$ & $\log \mathrm{Ca} / \mathrm{He}$ & $\log \mathrm{H} / \mathrm{He}$ & $D(\mathrm{pc})$ \\
\hline $\mathrm{J} 1421+1843$ & $6610(117)$ & $8.06\left({ }_{-0.26}^{+0.27}\right)$ & $0.61\left({ }_{-0.15}^{+0.18}\right)$ & $-8.27(0.11)$ & $<-3.46$ & $204.4(34.1)$ \\
\hline $\mathrm{J} 1421+5703$ & $6980(255)$ & $8.14\left({ }_{-0.19}^{+0.20}\right)$ & $0.66\left({ }_{-0.12}^{+0.13}\right)$ & $-9.94(0.12)$ & $<-3.78$ & $215.8(25.5)$ \\
\hline $\mathrm{J} 1422+5229$ & $12016(531)$ & $7.55\left({ }_{-0.20}^{+0.23}\right)$ & $0.36\left({ }_{-0.07}^{+0.10}\right)$ & $-9.29(0.32)$ & $-5.81(0.51)$ & $443.7(53.1)$ \\
\hline $\mathrm{J} 1424+5657$ & $6723(206)$ & $8.25\left({ }_{-0.10}^{+0.11}\right)$ & $0.73\left({ }_{-0.07}^{+0.07}\right)$ & $-9.95(0.11)$ & $<-3.40$ & $148.8(8.0)$ \\
\hline $\mathrm{J} 1425+2302$ & $11839(514)$ & $8.05\left({ }_{-0.14}^{+0.15}\right)$ & $0.62\left(_{-0.08}^{+0.09}\right)$ & $-7.64(0.14)$ & $-5.23(0.22)$ & $248.8(20.9)$ \\
\hline $\mathrm{J} 1425-0050$ & $7116(149)$ & $8.19\left({ }_{-0.05}^{+0.05}\right)$ & $0.70\left({ }_{-0.03}^{+0.04}\right)$ & $-10.90(0.07)$ & $<-3.83$ & $70.8(1.0)$ \\
\hline $\mathrm{J} 1427+4825$ & $7561(174)$ & $7.96\left({ }_{-0.06}^{+0.07}\right)$ & $0.55\left({ }_{-0.04}^{+0.04}\right)$ & $-10.87(0.08)$ & $<-4.23$ & $116.7(2.2)$ \\
\hline $\mathrm{J} 1428+4210$ & $7422(311)$ & $8.42\left({ }_{-0.35}^{+0.39}\right)$ & $0.85\left({ }_{-0.23}^{+0.24}\right)$ & $-9.66(0.21)$ & $<-3.85$ & $246.8(65.9)$ \\
\hline $\mathrm{J} 1428+4403$ & $6602(59)$ & $8.06\left({ }_{-0.03}^{+0.03}\right)$ & $0.61\left({ }_{-0.02}^{+0.02}\right)$ & $-9.44(0.11)$ & $<-3.45$ & $44.9(0.1)$ \\
\hline $\mathrm{J} 1429+3841$ & $5761(112)$ & $7.75\left({ }_{-0.23}^{+0.25}\right)$ & $0.43\left({ }_{-0.11}^{+0.14}\right)$ & $-10.07(0.22)$ & $<-3.00$ & $208.7(27.2)$ \\
\hline $\mathrm{J} 1429+5839$ & $9382(301)$ & $8.24\left({ }_{-0.11}^{+0.11}\right)$ & $0.73\left({ }_{-0.07}^{+0.07}\right)$ & $-8.55(0.33)$ & $<-5.65$ & $217.0(13.3)$ \\
\hline $\mathrm{J} 1430+3245$ & $7294(669)$ & 8.00 & & $-9.73(0.49)$ & $<-4.05$ & $491.2(66.8)$ \\
\hline $\mathrm{J} 1430-0151$ & $6098(88)$ & $7.95\left({ }_{-0.08}^{+0.08}\right)$ & $0.54\left(\begin{array}{l}+0.05 \\
-0.05\end{array}\right)$ & $-8.34(0.25)$ & $<-3.06$ & $118.9(4.8)$ \\
\hline $\mathrm{J} 1432+0354$ & $11164(532)$ & $8.05\left({ }_{-0.16}^{+0.17}\right)$ & $0.61\left({ }_{-0.09}^{+0.11}\right)$ & $-8.40(0.12)$ & $-4.51(0.17)$ & $238.2(22.5)$ \\
\hline $\mathrm{J} 1432+1522$ & $10489(406)$ & $8.14\left({ }_{-0.15}^{+0.16}\right)$ & $0.67\left({ }_{-0.09}^{+0.10}\right)$ & $-9.42(0.20)$ & $<-5.92$ & $244.1(23.0)$ \\
\hline $\mathrm{J} 1432+3542$ & $6800(193)$ & $7.86\left({ }_{-0.33}^{+0.37}\right)$ & $0.49\left({ }_{-0.16}^{+0.22}\right)$ & $-9.87(0.17)$ & $<-3.83$ & $260.4(53.3)$ \\
\hline $\mathrm{J} 1433+0714$ & $11282(418)$ & $7.98\left({ }_{-0.07}^{+0.07}\right)$ & $0.57\left({ }_{-0.04}^{+0.04}\right)$ & $-9.26(0.05)$ & $-5.56(0.09)$ & $97.6(1.3)$ \\
\hline $\mathrm{J} 1433+6139$ & $7843(464)$ & 8.00 & & $-8.35(0.36)$ & $<-4.46$ & $499.5(46.0)$ \\
\hline $\mathrm{J} 1436+2152$ & $7286(429)$ & 8.00 & & $-9.88(0.47)$ & $<-4.04$ & $338.2(28.8)$ \\
\hline $\mathrm{J} 1438+1046$ & $6971(70$ & 8.00 & & $-10.17(0.42)$ & $<-3.88$ & $461.0(74.3)$ \\
\hline $\mathrm{J} 1440+1227$ & 776 & 8.00 & & $-9.74(0.38$ & $<-4.37$ & $5.5)$ \\
\hline $\mathrm{J} 1440-0232$ & $6719(173)$ & $7.96\left({ }_{-0.09}^{+0.10}\right)$ & $0.55\left({ }_{-0.05}^{+0.06}\right)$ & $-10.56(0.09)$ & $<-3.68$ & $100.2(2.2)$ \\
\hline $\mathrm{J} 1441+0831$ & $7056(146)$ & $7.99\left({ }_{-0.08}^{+0.08}\right)$ & $0.57\left({ }_{-0.05}^{+0.05}\right)$ & $-10.83(0.04)$ & $<-3.94$ & $100.9(3.6)$ \\
\hline $\mathrm{J} 1441+4714$ & $7176(345)$ & 8.00 & & $-10.26(0.15)$ & $<-4.00$ & $308.5(22.2)$ \\
\hline $\mathrm{J} 1441+5215$ & $7839(414)$ & 8.00 & & $-9.48(0.37)$ & $<-4.46$ & $358.7(27.3)$ \\
\hline $\mathrm{J} 1442+5833$ & $6774(225)$ & $8.33\left({ }_{-0.24}^{+0.25}\right)$ & $0.79\left({ }_{-0.16}^{+0.17}\right)$ & $-9.20(0.19)$ & $<-3.37$ & $208.7(35.3)$ \\
\hline $\mathrm{J} 1443+2054$ & $6582(700)$ & 8.00 & & $-10.73(0.44)$ & $<-3.49$ & $380.6(67.6)$ \\
\hline $\mathrm{J} 1443+3014$ & $7001(302)$ & 8.00 & & $-8.23(0.29)$ & $<-30.00$ & $405.7(32.4)$ \\
\hline $\mathrm{J} 1443+5833$ & $7056(402)$ & 8.00 & & $-8.49(0.23)$ & $<-3.94$ & $398.7(38.7)$ \\
\hline $\mathrm{J} 1444+1151$ & $4937(185)$ & 8.00 & & $-10.53(0.23)$ & $<-3.00$ & $188.1(15.1)$ \\
\hline $\mathrm{J} 1444+2059$ & $7636(827)$ & 8.00 & & $-9.53(0.71)$ & $<-4.25$ & $539.1(87.2)$ \\
\hline $\mathrm{J} 1444+4741$ & $5431(167)$ & 8.00 & & $-10.07(0.29)$ & $<-3.00$ & $257.8(17.5)$ \\
\hline $\mathrm{J} 1444+5741$ & $9105(412)$ & $8.25\left({ }_{-0.27}^{+0.29}\right)$ & $0.74\left({ }_{-0.17}^{+0.19}\right)$ & $-8.36(1.00)$ & $<-5.48$ & $316.7(57.9)$ \\
\hline $\mathrm{J} 1445+0913$ & $7154(309)$ & 8.00 & & $-7.64(0.23)$ & $<-3.99$ & $380.4(29.1)$ \\
\hline $\mathrm{J} 1445+5850$ & $13052(673)$ & $7.96\left({ }_{-0.09}^{+0.09}\right)$ & $0.56\left({ }_{-0.05}^{+0.05}\right)$ & $-8.69(0.17)$ & $<-6.11$ & $179.0(3.9)$ \\
\hline $\mathrm{J} 1445-0208$ & $6467(86)$ & $8.14\left({ }_{-0.05}^{+0.05}\right)$ & $0.66\left({ }_{-0.03}^{+0.03}\right)$ & $-10.27(0.05)$ & $<-3.23$ & $87.3(1.6)$ \\
\hline $\mathrm{J} 1446+0111$ & $6391(632)$ & 8.00 & & $-10.81(0.54)$ & $<-3.29$ & $333.7(55.0)$ \\
\hline $\mathrm{J} 1446+0112$ & $7293(556)$ & 8.00 & & $-10.21(0.22)$ & $<-4.05$ & $326.5(36.2)$ \\
\hline
\end{tabular}


Table 6. Atmospheric parameters for DZ stars - continued.

\begin{tabular}{|c|c|c|c|c|c|c|}
\hline J Name & $T_{\text {eff }}(\mathrm{K})$ & $\log g$ & $M / M_{\odot}$ & $\log \mathrm{Ca} / \mathrm{He}$ & $\log \mathrm{H} / \mathrm{He}$ & $D(\mathrm{pc})$ \\
\hline $\mathrm{J} 1447+1340$ & $4999(175)$ & 8.00 & & $-10.38(0.30)$ & $<-3.00$ & $209.7(16.8)$ \\
\hline $\mathrm{J} 1448+1047$ & $6316(47)$ & $8.16\left({ }_{-0.04}^{+0.04}\right)$ & $0.67\left({ }_{-0.03}^{+0.03}\right)$ & $-9.06(0.06)$ & $<-3.10$ & $88.9(2.1)$ \\
\hline $\mathrm{J} 1448+2946$ & $7678(612)$ & 8.00 & & $-9.99(0.25)$ & $<-4.29$ & $409.9(48.2)$ \\
\hline $\mathrm{J} 1448+2958$ & $8819(268)$ & $8.28\left({ }_{-0.10}^{+0.10}\right)$ & $0.75\left({ }_{-0.06}^{+0.07}\right)$ & $-9.33(0.08)$ & $<-5.17$ & $162.3(9.1)$ \\
\hline $\mathrm{J} 1452+1346$ & $12206(546)$ & $8.19\left({ }_{-0.18}^{+0.19}\right)$ & $0.70\left(_{-0.11}^{+0.12}\right)$ & $-9.04(0.19)$ & $<-6.07$ & $256.4(29.9)$ \\
\hline $\mathrm{J} 1452+4544$ & $8678(650)$ & 8.00 & & $-9.38(0.27)$ & $<-5.38$ & $470.4(51.3)$ \\
\hline $\mathrm{J} 1453+2452$ & $5907(171)$ & 8.00 & & $-9.73(0.28)$ & $<-3.00$ & $240.4(14.1)$ \\
\hline $\mathrm{J} 1453+3003$ & $7644(539)$ & 8.00 & & $-9.37(40.72)$ & $<-4.26$ & $475.9(50.3)$ \\
\hline $\mathrm{J} 1453+3241$ & $6371(123)$ & $8.01\left({ }_{-0.09}^{+0.10}\right)$ & $0.58\left({ }_{-0.06}^{+0.06}\right)$ & $-10.74(0.11)$ & $<-3.26$ & $128.2(6.1)$ \\
\hline $\mathrm{J} 1455+2010$ & $7737(285)$ & $8.93\left({ }_{-0.37}^{+0.49}\right)$ & $1.16\left({ }_{-0.21}^{+0.17}\right)$ & $-8.38(0.24)$ & $<-3.64$ & $171.3(65.7)$ \\
\hline $\mathrm{J} 1458+4719$ & $12741(854)$ & $8.02\left(\begin{array}{l}+0.19 \\
-0.18\end{array}\right)$ & $0.60\left({ }_{-0.10}^{+0.12}\right)$ & $-7.58(0.29)$ & $-4.68(0.47)$ & $322.6(33.0)$ \\
\hline $\mathrm{J} 1500+2315$ & $6682(304)$ & 8.00 & & $-8.29(0.33)$ & $<-3.60$ & $371.4(31.9)$ \\
\hline $\mathrm{J} 1501+3807$ & $7206(483)$ & 8.00 & & $-10.73(0.24)$ & $<-4.01$ & $342.2(35.8)$ \\
\hline $\mathrm{J} 1501+5609$ & $9471(266)$ & $8.20\left({ }_{-0.07}^{+0.07}\right)$ & $0.70\left({ }_{-0.04}^{+0.05}\right)$ & $-7.99(0.06)$ & $<-5.72$ & $104.9(1.2)$ \\
\hline $\mathrm{J} 1502+3744$ & $5556(62)$ & $8.09\left({ }_{-0.06}^{+0.07}\right)$ & $0.63\left({ }_{-0.04}^{+0.04}\right)$ & $-10.05(0.15)$ & $<-3.00$ & $106.2(3.5)$ \\
\hline $\mathrm{J} 1503+4414$ & $8682(916)$ & 8.00 & & $-9.52(0.73)$ & $<-5.38$ & $570.7(85.4)$ \\
\hline $\mathrm{J} 1503+4642$ & $7089(724)$ & 8.00 & & $-9.91(0.88)$ & $<-3.95$ & $406.8(62.8)$ \\
\hline $\mathrm{J} 1506+4152$ & $9463(295)$ & $8.17\left({ }_{-0.07}^{+0.07}\right)$ & $0.68\left({ }_{-0.04}^{+0.04}\right)$ & $-10.54(0.04)$ & $<-5.74$ & $95.7(0.8)$ \\
\hline $\mathrm{J} 1506+5844$ & $7107(197)$ & $8.01\left({ }_{-0.10}^{+0.10}\right)$ & $0.58\left({ }_{-0.06}^{+0.06}\right)$ & $-10.56(0.08)$ & $<-3.95$ & $164.6(7.4)$ \\
\hline $\mathrm{J} 1507+2633$ & $7249(2$ & $7.89\left({ }_{-0.13}^{+0.13}\right)$ & $0.51\left({ }_{-0.07}^{+0.08}\right)$ & $-11.38(0.09)$ & $<-4.10$ & 14 \\
\hline $\mathrm{J} 1507+4034$ & 735 & 8.00 & & $-7.97(0.22)$ & $<-4.07$ & 7) \\
\hline $\mathrm{J} 1508+0622$ & 9991(352) & $7.90\left({ }_{-0.22}^{+0.23}\right)$ & $0.52\left({ }_{-0.11}^{+0.14}\right)$ & $-9.59(0.15)$ & $<-5.94$ & $261.0(34.7)$ \\
\hline $\mathrm{J} 1509+1838$ & $7943(282)$ & $7.86\left({ }_{-0.28}^{+0.31}\right)$ & $0.49\left({ }_{-0.14}^{+0.19}\right)$ & $-9.16(0.22)$ & $<-4.78$ & $262.8(45.5)$ \\
\hline $\mathrm{J} 1510+3814$ & $9248(230)$ & $7.89\left({ }_{-0.14}^{+0.15}\right)$ & $0.51\left({ }_{-0.07}^{+0.09}\right)$ & $-8.22(0.24)$ & $-4.70(0.37)$ & $259.5(21.4)$ \\
\hline $\mathrm{J} 1511+2654$ & $6381(172)$ & $8.10\left({ }_{-0.23}^{+0.24}\right)$ & $0.64\left({ }_{-0.14}^{+0.16}\right)$ & $-10.49(0.12)$ & $<-3.19$ & $176.3(25.9)$ \\
\hline $\mathrm{J} 1512+5304$ & $8221(593)$ & 8.00 & & $-8.25(0.28)$ & $<-4.94$ & $542.6(58.5)$ \\
\hline $\mathrm{J} 1514+5012$ & $10508(559)$ & $8.74\left({ }_{-0.23}^{+0.26}\right)$ & $1.05\left({ }_{-0.15}^{+0.14}\right)$ & $-9.11(0.43)$ & $<-5.37$ & $239.7(49.2)$ \\
\hline $\mathrm{J} 1515+1348$ & $6736(202)$ & $8.03\left({ }_{-0.22}^{+0.23}\right)$ & $0.59\left({ }_{-0.12}^{+0.15}\right)$ & $-10.53(0.12)$ & $<-3.63$ & $194.0(25.9)$ \\
\hline $\mathrm{J} 1515+4532$ & $7476(218)$ & $7.97\left({ }_{-0.17}^{+0.18}\right)$ & $0.56\left(_{-0.09}^{+0.11}\right)$ & $-9.10(0.21)$ & $<-4.16$ & $235.4(23.4)$ \\
\hline $\mathrm{J} 1516+2118$ & $9588(302)$ & $8.24\left({ }_{-0.07}^{+0.07}\right)$ & $0.73\left({ }_{-0.04}^{+0.04}\right)$ & $-10.39(0.06)$ & $<-5.72$ & $107.6(1.8)$ \\
\hline $\mathrm{J} 1516-0040$ & $13006(735)$ & $7.95\left({ }_{-0.10}^{+0.10}\right)$ & $0.56\left(_{-0.05}^{+0.06}\right)$ & $-8.59(0.14)$ & $-4.83(0.08)$ & $141.9(2.4)$ \\
\hline $\mathrm{J} 1518+0506$ & $5366(76)$ & $8.27\left({ }_{-0.07}^{+0.07}\right)$ & $0.74\left({ }_{-0.04}^{+0.04}\right)$ & $-9.33(0.22)$ & $<-3.00$ & $69.2(2.2)$ \\
\hline $\mathrm{J} 1518+5619$ & $7373(550)$ & 8.00 & & $-9.90(0.26)$ & $<-4.08$ & $369.1(39.5)$ \\
\hline $\mathrm{J} 1520+2718$ & $7414(568)$ & 8.00 & & $-9.75(0.17)$ & $<-4.10$ & $416.4(49.5)$ \\
\hline $\mathrm{J} 1523+5601$ & $7520(485)$ & 8.00 & & $-10.05(0.26)$ & $<-4.16$ & $386.1(36.3)$ \\
\hline $\mathrm{J} 1524+4049$ & $6250(93)$ & $8.22\left(\begin{array}{l}+0.09 \\
-0.09\end{array}\right)$ & $0.71\left({ }_{-0.06}^{+0.06}\right)$ & $-8.57(0.09)$ & $<-3.04$ & $146.4(7.7)$ \\
\hline $\mathrm{J} 1524+4052$ & $5777(485)$ & 8.00 & & $-10.11(0.44)$ & $<-3.00$ & $357.4(57.0)$ \\
\hline $\mathrm{J} 1525+1625$ & $6757(221)$ & $8.71\left({ }_{-0.19}^{+0.21}\right)$ & $1.03\left({ }_{-0.12}^{+0.11}\right)$ & $-9.81(0.14)$ & $<-3.08$ & $153.1(24.8)$ \\
\hline
\end{tabular}


Table 6. Atmospheric parameters for DZ stars - continued.

\begin{tabular}{|c|c|c|c|c|c|c|}
\hline J Name & $T_{\text {eff }}(\mathrm{K})$ & $\log g$ & $M / M_{\odot}$ & $\log \mathrm{Ca} / \mathrm{He}$ & $\log \mathrm{H} / \mathrm{He}$ & $D(\mathrm{pc})$ \\
\hline $\mathrm{J} 1525+4258$ & $10491(378)$ & $.22\left(\begin{array}{l}+0.11 \\
-0.10\end{array}\right)$ & $0.72\left({ }_{-0.07}^{+0.07}\right)$ & $-8.96(0.12)$ & $<-5.86$ & $226.7(13.1)$ \\
\hline $\mathrm{J} 1526+1308$ & $7373(630)$ & 8.00 & & $-10.20(0.33)$ & $<-4.08$ & $403.2(50.1)$ \\
\hline $\mathrm{J} 1528+0753$ & $7480(125)$ & $8.09\left({ }_{-0.14}^{+0.14}\right)$ & $0.63\left({ }_{-0.08}^{+0.09}\right)$ & $-8.43(0.11)$ & $<-4.07$ & $190.3(16.7)$ \\
\hline $\mathrm{J} 1531+2505$ & $7591(587)$ & 8.00 & & $-9.71(0.59)$ & $<-4.22$ & $410.5(46.2)$ \\
\hline $\mathrm{J} 1531+4240$ & $6998(126)$ & $8.00\left({ }_{-0.05}^{+0.05}\right)$ & $0.57\left({ }_{-0.03}^{+0.03}\right)$ & $-10.05(0.06)$ & $<-3.90$ & $86.7(1.0)$ \\
\hline $\mathrm{J} 1532+1006$ & $5085(178)$ & 8.00 & & $-10.01(0.30)$ & $<-3.00$ & $254.3(21.0)$ \\
\hline $\mathrm{J} 1532+1611$ & $7167(680)$ & 8.00 & & $-10.22(0.31)$ & $<-3.99$ & $449.2(63.8)$ \\
\hline $\mathrm{J} 1532+2748$ & $6871(452)$ & 8.00 & & $-9.12(0$. & $<-3.80$ & $450.5(55.6)$ \\
\hline $\mathrm{J} 1533+5301$ & $11632(459)$ & $7.90\left({ }_{-0.13}^{+0.14}\right)$ & $0.53\left({ }_{-0.07}^{+0.08}\right)$ & $-8.33(0.14)$ & $<-6.08$ & $321.4(24.1)$ \\
\hline $\mathrm{J} 1534+1242$ & $6386(265)$ & 8.00 & & $-7.78(0.18)$ & $<-3.28$ & $324.6(27.5)$ \\
\hline $\mathrm{J} 1534+1345$ & $13652(720)$ & $8.07\left({ }_{-0.18}^{+0.19}\right)$ & $0.63\left({ }_{-0.10}^{+0.12}\right)$ & $-9.11(1.04)$ & $<-6.08$ & $302.6(32.9)$ \\
\hline $\mathrm{J} 1535+1247$ & $6012(28)$ & $8.20\left({ }_{-0.02}^{+0.02}\right)$ & $0.70\left({ }_{-0.02}^{+0.02}\right)$ & $-8.48(0.03)$ & $<-3.00$ & $19.3(0.0)$ \\
\hline $\mathrm{J} 1537+3608$ & $5564(128)$ & 8.00 & & $-9.81(0.35)$ & $<-3.00$ & $198.8(9.5)$ \\
\hline $\mathrm{J} 1537+4625$ & $7445(173)$ & $8.16\left({ }_{-0.21}^{+0.22}\right)$ & $0.68\left({ }_{-0.13}^{+0.15}\right)$ & $-8.99(0.35)$ & $<-4.01$ & $230.7(32.4)$ \\
\hline $\mathrm{J} 1539+1930$ & $7140(203)$ & $8.15\left({ }_{-0.13}^{+0.13}\right)$ & $0.67\left({ }_{-0.08}^{+0.09}\right)$ & $-9.80(0.21)$ & $<-3.88$ & $166.2(12.9)$ \\
\hline $\mathrm{J} 1539+5248$ & $6995(422)$ & 8.00 & & $-10.45(0.20)$ & $<-3.90$ & $331.0(31.5)$ \\
\hline $\mathrm{J} 1540+2109$ & $7001(293)$ & $8.42\left({ }_{-0.37}^{+0.42}\right)$ & $0.85\left({ }_{-0.24}^{+0.26}\right)$ & $-9.96(0.15)$ & $<-3.52$ & $199.3(57.2)$ \\
\hline $\mathrm{J} 1540+3737$ & $8226(310)$ & $7.91\left({ }_{-0.27}^{+0.29}\right)$ & $0.52\left({ }_{-0.14}^{+0.18}\right)$ & $-8.89(1.01)$ & $<-5.07$ & $327.2(53.8)$ \\
\hline $\mathrm{J} 1540+5149$ & $8107(289)$ & $8.06\left({ }_{-0.19}^{+0.19}\right)$ & $0.61\left({ }_{-0.11}^{+0.13}\right)$ & $-9.92(0.20)$ & $<-4.70$ & $228.1(25.5)$ \\
\hline $\mathrm{J} 1540+5352$ & $6486(1$ & 8.00 & & $-8.71(0.28)$ & $<-3.39$ & 6) \\
\hline $\mathrm{J} 1541+0715$ & $7461(77$ & 8.00 & & $-10.24(0.29)$ & $<-4.13$ & $44.3(67.0)$ \\
\hline $\mathrm{J} 1542+4650$ & 6206 & $8.32\left({ }_{-0.18}^{+0.19}\right)$ & $0.78\left({ }_{-0.12}^{+0.13}\right)$ & $-8.14(0.24)$ & $<-3.00$ & $.3)$ \\
\hline $\mathrm{J} 1543+2024$ & $6341(255)$ & 8.00 & & $-8.65(0.19)$ & $<-3.24$ & $304.1(23.2)$ \\
\hline $\mathrm{J} 1544+2345$ & $6693(141)$ & $7.97\left({ }_{-0.13}^{+0.13}\right)$ & $0.56\left({ }_{-0.07}^{+0.08}\right)$ & $-10.27(0.08)$ & $<-3.64$ & $151.5(10.9)$ \\
\hline $\mathrm{J} 1545+4921$ & $9628(453)$ & $8.17\left({ }_{-0.29}^{+0.32}\right)$ & $0.68\left({ }_{-0.17}^{+0.21}\right)$ & $-8.18(0.70)$ & $<-5.78$ & $339.0(66.6)$ \\
\hline $\mathrm{J} 1545+5236$ & $6034(85)$ & $8.08\left({ }_{-0.10}^{+0.10}\right)$ & $0.62\left({ }_{-0.06}^{+0.06}\right)$ & $-9.18(0.12)$ & $<-3.00$ & $142.9(8.2)$ \\
\hline $\mathrm{J} 1546+1750$ & $8206(595)$ & 8.00 & & $-8.99(0.62)$ & $<-4.92$ & $491.0(52.4)$ \\
\hline $\mathrm{J} 1546+3927$ & $8924(216)$ & $8.13\left({ }_{-0.09}^{+0.09}\right)$ & $0.66\left({ }_{-0.06}^{+0.06}\right)$ & $-8.46(0.22)$ & $<-5.41$ & $191.6(9.4)$ \\
\hline $\mathrm{J} 1547+0659$ & $8737(244)$ & $8.04\left({ }_{-0.06}^{+0.06}\right)$ & $0.60\left({ }_{-0.04}^{+0.04}\right)$ & $-9.74(0.03)$ & $<-5.36$ & $87.8(0.9)$ \\
\hline $\mathrm{J} 1548+0222$ & $8359(277)$ & $8.32\left({ }_{-0.11}^{+0.11}\right)$ & $0.78\left({ }_{-0.07}^{+0.08}\right)$ & $-10.45(0.07)$ & $<-4.54$ & $156.0(10.1)$ \\
\hline $\mathrm{J} 1548+2135$ & $11084(442)$ & $7.99\left({ }_{-0.08}^{+0.09}\right)$ & $0.58\left({ }_{-0.05}^{+0.05}\right)$ & $-9.13(0.07)$ & $-5.41(0.11)$ & $167.1(4.9)$ \\
\hline $\mathrm{J} 1549+0239$ & $8684(487)$ & 8.00 & & $-9.60(0.50)$ & $<-5.38$ & $300.8(23.2)$ \\
\hline $\mathrm{J} 1549+1906$ & $6331(249)$ & 8.00 & & $-8.60(0.28)$ & $<-3.23$ & $286.6(21.2)$ \\
\hline $\mathrm{J} 1549+2020$ & $7150(251)$ & $8.34\left({ }_{-0.56}^{+0.68}\right)$ & $0.79\left({ }_{-0.34}^{+0.40}\right)$ & $-8.79(0.29)$ & $<-3.74$ & $240.5(104.6)$ \\
\hline $\mathrm{J} 1549+2633$ & $7178(184)$ & $8.28\left({ }_{-0.26}^{+0.28}\right)$ & $0.76\left({ }_{-0.17}^{+0.19}\right)$ & $-8.56(0.13)$ & $<-3.80$ & $225.9(41.7)$ \\
\hline $\mathrm{J} 1549+4422$ & $6428(158)$ & $8.01\left({ }_{-0.11}^{+0.12}\right)$ & $0.58\left({ }_{-0.07}^{+0.07}\right)$ & $-10.49(0.13)$ & $<-3.31$ & $163.7(9.4)$ \\
\hline $\mathrm{J} 1550+2013$ & $5944(334)$ & 8.00 & & $-9.77(0.56)$ & $<-3.00$ & $309.9(32.4)$ \\
\hline $\mathrm{J} 1550+5214$ & $7531(384)$ & 8.00 & & $-9.49(0.35)$ & $<-4.17$ & $388.1(29.9)$ \\
\hline
\end{tabular}


Table 6. Atmospheric parameters for DZ stars - continued.

\begin{tabular}{|c|c|c|c|c|c|c|}
\hline J Name & $T_{\text {eff }}(\mathrm{K})$ & $\log g$ & $M / M_{\odot}$ & $\log \mathrm{Ca} / \mathrm{He}$ & $\log \mathrm{H} / \mathrm{He}$ & $D(\mathrm{pc})$ \\
\hline $\mathrm{J} 1551+1529$ & $7501(1032)$ & 8.00 & & $-10.17(0.28)$ & $<-4.15$ & $520.9(106.1)$ \\
\hline $\mathrm{J} 1551+2547$ & $7677(671)$ & 8.00 & & $-8.46(0.47)$ & $<-4.29$ & $590.4(78.5)$ \\
\hline $\mathrm{J} 1552+0815$ & $10758(550)$ & $8.31\left({ }_{-0.46}^{+0.54}\right)$ & $0.78\left({ }_{-0.28}^{+0.34}\right)$ & $-8.93(0.49)$ & $<-5.85$ & $333.1(116.7)$ \\
\hline $\mathrm{J} 1554+1735$ & $6768(46)$ & $8.20\left({ }_{-0.03}^{+0.03}\right)$ & $0.70\left({ }_{-0.02}^{+0.02}\right)$ & $-8.71(0.05)$ & $<-3.50$ & $56.4(0.4)$ \\
\hline $\mathrm{J} 1557+2157$ & $8630(223)$ & $7.93\left({ }_{-0.09}^{+0.10}\right)$ & $0.54\left({ }_{-0.05}^{+0.06}\right)$ & $-9.03(0.11)$ & $-3.46(0.19)$ & $171.5(7.8)$ \\
\hline $\mathrm{J} 1558+0312$ & $7734(122)$ & $7.91\left({ }_{-0.10}^{+0.11}\right)$ & $0.52\left({ }_{-0.06}^{+0.06}\right)$ & $-8.77(0.17)$ & $<-4.46$ & $161.4(9.4)$ \\
\hline $\mathrm{J} 1558+1507$ & $10090(592)$ & $8.19\left({ }_{-0.12}^{+0.13}\right)$ & $0.70\left({ }_{-0.08}^{+0.08}\right)$ & $-9.40(0.10)$ & $<-5.81$ & $150.7(5.5)$ \\
\hline $\mathrm{J} 1558+2512$ & $7201(155)$ & $8.06\left({ }_{-0.05}^{+0.05}\right)$ & $0.61\left({ }_{-0.03}^{+0.03}\right)$ & $-10.89(0.05)$ & $<-3.97$ & $54.0(0.2)$ \\
\hline $\mathrm{J} 1559+0445$ & $7389(225)$ & $8.07\left(\left(_{-0.17}^{+0.18}\right)\right.$ & $0.62\left({ }_{-0.10}^{+0.11}\right)$ & $-10.14(0.12)$ & $<-4.04$ & $171.0(17.5)$ \\
\hline $\mathrm{J} 1600+1833$ & $8154(435)$ & $7.52\left({ }_{-0.58}^{+0.81}\right)$ & $0.34\left(_{-0}^{+0}\right.$ & $-10.14(0.27)$ & $<-5.41$ & $405.3(158.2)$ \\
\hline $\mathrm{J} 1600+3819$ & $7101(328)$ & 8.00 & & $-8.75(0.30)$ & $<-3.96$ & $365.2(26.3)$ \\
\hline $\mathrm{J} 1600+4120$ & $7765(639)$ & 8.00 & & $-9.88(0.36)$ & $<-4.38$ & 404. \\
\hline J1600-0009 & $6544(138)$ & 8.00 & & $-10.58(0.15)$ & $<-3.45$ & $117.0(4.4)$ \\
\hline $\mathrm{J} 1601+1824$ & $5240(196)$ & 8.00 & & $-10.04(C$ & $<-3.01$ & 219.1 \\
\hline $\mathrm{J} 1602+1923$ & $5267(324)$ & 8.00 & & $-10.41(0.28)$ & $<-3.01$ & $249.1(31.5)$ \\
\hline $\mathrm{J} 1603+4140$ & $7673(706)$ & 8.00 & & $-9.56(0.41)$ & $<-4.29$ & $453.7(63.7)$ \\
\hline $\mathrm{J} 1604+0831$ & $11757(452)$ & $7.89\left({ }_{-0.12}^{+0.13}\right)$ & $0.52\left({ }_{-0.06}^{+0.07}\right)$ & $-9.20(0$ & $-5.51(0.17)$ & $234.9(15.8)$ \\
\hline $\mathrm{J} 1604+1830$ & $6459(104)$ & $8.13\left({ }_{-0.10}^{+0.10}\right)$ & $0.65\left({ }_{-0.06}^{+0.07}\right)$ & $-9.61(0.12)$ & $<-3.24$ & $139.1(8.3)$ \\
\hline $\mathrm{J} 1606+1908$ & $7788(5$ & 8.00 & & -10.37( & $<-4.40$ & 384.3 \\
\hline $\mathrm{J} 1606+4712$ & 822 & & $0.29\left(_{-0.22}^{+0.72}\right)$ & $-11.33(0$ & -3.01 & 2) \\
\hline $\mathrm{J} 1607+3950$ & 739 & & & $-8.88(0$ & & ) \\
\hline $\mathrm{J} 1607+5321$ & $9657(44$ & $8.09\left({ }_{-0.20}^{+0.21}\right)$ & $0.63\left({ }_{-0.12}^{+0.14}\right)$ & $-9.16(0.17)$ & $<-5.84$ & $300.2(36.3)$ \\
\hline $\mathrm{J} 1608+1342$ & $9223(219)$ & $8.23\left({ }_{-0.09}^{+0.09}\right)$ & $0.73\left({ }_{-0.06}^{+0.06}\right)$ & $-8.16(0.25)$ & $<-5.57$ & $168.8(8.2)$ \\
\hline $\mathrm{J} 1608+3831$ & $6507(170)$ & $8.05\left({ }_{-0.11}^{+0.11}\right)$ & $0.61\left({ }_{-0.07}^{+0.07}\right)$ & $-10.39(0.08)$ & $<-3.35$ & $158.9(9.1)$ \\
\hline $\mathrm{J} 1610+3714$ & $11462(464)$ & $8.24\left({ }_{-0.12}^{+0.12}\right)$ & $0.74\left({ }_{-0.08}^{+0.08}\right)$ & $-7.66(0.11)$ & $-4.81(0.25)$ & $262.8(18.6)$ \\
\hline $\mathrm{J} 1610+4006$ & $6053(158)$ & 8.00 & & $-9.83(0.39)$ & $<-3.02$ & $238.7(11.6)$ \\
\hline $\mathrm{J} 1611+1117$ & $7676(169)$ & $7.85\left({ }_{-0.10}^{+0.10}\right)$ & $0.49\left(_{-0.05}^{+0.06}\right)$ & $-9.72(0.24)$ & $<-4.48$ & $168.2(8.0)$ \\
\hline $\mathrm{J} 1612+1845$ & $7376(531)$ & 8.00 & & $-10.06(0.18)$ & $<-4.08$ & $363.0(38.8)$ \\
\hline $\mathrm{J} 1612+3534$ & $7058(320)$ & 8.00 & & $-8.90(0.53)$ & $<-3.94$ & $379.1(27.0)$ \\
\hline $\mathrm{J} 1615+1746$ & $9613(456)$ & $7.14\left({ }_{-1.60}^{+2.49}\right)$ & $0.23\left({ }_{-0.21}^{+1.18}\right)$ & $-7.95(0.60)$ & $<-6.14$ & $740.2(633.9)$ \\
\hline $\mathrm{J} 1616+3303$ & $6382(72)$ & $7.93\left({ }_{-0.06}^{+0.06}\right)$ & $0.53\left({ }_{-0.03}^{+0.04}\right)$ & $-8.49(0.05)$ & $<-3.34$ & 127.3 \\
\hline $\mathrm{J} 1618+4452$ & 9867 & $7.94\left({ }_{-0.12}^{+0.13}\right)$ & $0.54\left({ }_{-0.07}^{+0.07}\right)$ & $-7.63(0.18)$ & $<-5.92$ & 266.0 \\
\hline $\mathrm{J} 1619+4745$ & 88 & 8.00 & & $-8.25(0.39)$ & $<-5.49$ & $555.0(53.8)$ \\
\hline $\mathrm{J} 1622+4731$ & $7716(279)$ & 8.00 & & $-8.20(0.22)$ & $<-4.33$ & $408.5(24.0)$ \\
\hline $\mathrm{J} 1622+6319$ & $11586(785)$ & $8.39\left({ }_{-0.19}^{+0.20}\right)$ & $0.83\left({ }_{-0.12}^{+0.13}\right)$ & $-7.69(0.33)$ & $-4.88(0.43)$ & $325.5(40.9)$ \\
\hline $\mathrm{J} 1624+3310$ & $6628(216)$ & 8.00 & & $-9.16(0.36)$ & $<-3.54$ & $300.0(16.4)$ \\
\hline $\mathrm{J} 1626+3303$ & $6918(261)$ & 8.00 & & $-8.34(0.21)$ & $<-3.84$ & $309.1(20.2)$ \\
\hline $\mathrm{J} 1627+1443$ & $9121(435)$ & $8.24\left({ }_{-0.24}^{+0.26}\right)$ & $0.73\left({ }_{-0.15}^{+0.17}\right)$ & $-10.19(0.20)$ & $<-5.50$ & $267.2(42.3)$ \\
\hline
\end{tabular}


Table 6. Atmospheric parameters for DZ stars - continued.

\begin{tabular}{|c|c|c|c|c|c|c|}
\hline J Name & $T_{\text {eff }}(\mathrm{K})$ & $\log g$ & $M / M_{\odot}$ & $\log \mathrm{Ca} / \mathrm{He}$ & $\log \mathrm{H} / \mathrm{He}$ & $D(\mathrm{pc})$ \\
\hline $\mathrm{J} 1627+4646$ & $5639(114)$ & $54\left({ }_{-0.19}^{+0.21}\right)$ & $0.33\left({ }_{-0.07}^{+0.10}\right)$ & $-10.59(0.36)$ & $<-3.01$ & $201.1(20.6)$ \\
\hline $\mathrm{J} 1628+3646$ & $8316(435)$ & $7.98\left({ }_{-0.09}^{+0.09}\right)$ & $0.57\left(_{-}^{+}\right.$ & $-9.07(0.08)$ & $-3.66(0.03)$ & $15.9(0.0)$ \\
\hline $\mathrm{J} 1629+1758$ & $10709(465)$ & $7.92\left({ }_{-0.23}^{+0.26}\right)$ & $0.54\left({ }_{-0.12}^{+0.15}\right)$ & $-8.91(0.37)$ & $<-6.04$ & $342.6(50.0)$ \\
\hline $\mathrm{J} 1633+1840$ & $10298(325)$ & $8.03\left({ }_{-0.07}^{+0.07}\right)$ & $0.60\left({ }_{-0.04}^{+0.04}\right)$ & $-8.81(0.06)$ & $<-5.95$ & $165.2(4.3)$ \\
\hline $\mathrm{J} 1636+1619$ & $4416(66)$ & $8.08\left({ }_{-0.09}^{+0.09}\right)$ & $0.62\left(_{-0.05}^{+0.06}\right)$ & $-9.83(0.20)$ & $<-3.00$ & $69.1(2.4)$ \\
\hline $\mathrm{J} 1638+3837$ & $7247(179)$ & $7.70\left({ }_{-0.25}^{+0.27}\right)$ & $0.41\left({ }_{-0.10}^{+0.15}\right)$ & $-9.19(0.25)$ & $<-4.25$ & $271.3(39.3)$ \\
\hline $\mathrm{J} 1640+3154$ & $6857(108)$ & $8.04\left({ }_{-0.06}^{+0.06}\right)$ & $0.60\left({ }_{-0.03}^{+0.04}\right)$ & $-9.98(0.06)$ & $<-3.75$ & 111.6 \\
\hline $\mathrm{J} 1641+1856$ & $5559(9$ & $8.01\left({ }_{-0.11}^{+0.11}\right)$ & $0.58\left({ }_{-0.06}^{+0.07}\right)$ & $-10.16(0.08)$ & $<-3.00$ & 131.2 \\
\hline $\mathrm{J} 1642+3211$ & $7278(341)$ & 8.00 & & $-10.30(0.22)$ & $<-4.04$ & $297.9(20.8)$ \\
\hline $\mathrm{J} 1642+3749$ & $6706(592)$ & 8.00 & & $-9.65(0.68)$ & $<-3.63$ & $473.6(66.6)$ \\
\hline $\mathrm{J} 1642+4529$ & $7649(431)$ & 8.00 & & $-9.98(0.20)$ & $<-4.26$ & $353.3(29.2)$ \\
\hline $\mathrm{J} 1643+1422$ & $6374(98)$ & 8.05( & $0.60\left(_{-c}^{+c}\right.$ & $-11.23(0.08)$ & $<-3.23$ & 75.7 \\
\hline $\mathrm{J} 1644+3853$ & $6054(158)$ & $8.32\left({ }_{-0.41}^{+0.47}\right)$ & $0.78\left({ }_{-0.26}^{+0.30}\right)$ & $-9.75(0.22)$ & $<-3.00$ & $182.3(56.4)$ \\
\hline $\mathrm{J} 1646+2742$ & $13810(1412)$ & $8.07\left({ }_{-0.25}^{+0.27}\right)$ & $0.63\left({ }_{-0.14}^{+0.17}\right)$ & $-7.42(0.31)$ & $-3.14(0$ & $378.3(50.1)$ \\
\hline $\mathrm{J} 1647+2636$ & $7034(1$ & $8.00\left({ }_{-0.05}^{+0.05}\right)$ & $0.57\left({ }_{-0.03}^{+0.03}\right)$ & $-9.99(0.05)$ & $<-3.93$ & $50.5(0.2)$ \\
\hline $\mathrm{J} 1647+4156$ & $6876(395)$ & 8.00 & & $-9.65(0.45)$ & $<-3.80$ & $359.7(32.0)$ \\
\hline $\mathrm{J} 1648+5241$ & $6934(225)$ & $8.29\left({ }_{-0.17}^{+0.17}\right)$ & $0.76\left({ }_{-0.11}^{+0.12}\right)$ & $-9.73(0.19)$ & $<-3.59$ & $185.5(20.5)$ \\
\hline $\mathrm{J} 1649+2238$ & $5180(86)$ & $8.12\left({ }_{-0.17}^{+0.17}\right)$ & $0.65\left({ }_{-0.10}^{+0.12}\right)$ & $-9.09(0.20)$ & $<-3.00$ & $137.1(14.2)$ \\
\hline $\mathrm{J} 1649+4152$ & $5678(2$ & 8.00 & & -10.01 & $<-3.00$ & 200.3 \\
\hline $\mathrm{J} 1650+4055$ & $7113(7$ & 8.00 & & -9.65( & م & 9) \\
\hline $\mathrm{J} 1655+2134$ & 70 & 8.00 & & $-10.05(0.18)$ & $<-3.93$ & 6) \\
\hline $\mathrm{J} 1657+3738$ & 7503 & $8.12\left({ }_{-0.09}^{+0.10}\right)$ & $\left.\begin{array}{l}.06 \\
.06\end{array}\right)$ & $-9.91(0.12)$ & $<-4.06$ & \\
\hline $\mathrm{J} 1657+4759$ & 7377 & 8.00 & & $-8.75(0.23)$ & $<-4.08$ & $359.1(22.3)$ \\
\hline $\mathrm{J} 1700+2047$ & $7156(473)$ & 8.00 & & $-8.84(0.74)$ & $<-3.99$ & $474.1(47.7)$ \\
\hline $\mathrm{J} 1703+2541$ & $8848(244)$ & $7.94\left({ }_{-0.06}^{+0.07}\right)$ & $0.54\left({ }_{-0.04}^{+0.04}\right)$ & $-10.33(0.03)$ & $-4.09(0.10)$ & $109.6(1.4)$ \\
\hline $\mathrm{J} 1706+2541$ & $5397(152)$ & 8.00 & & $-10.27(0.20)$ & $<-3.01$ & $193.0(12.4)$ \\
\hline $\mathrm{J} 1707+4250$ & $6574(335)$ & $7.55\left({ }_{-0.53}^{+0.66}\right)$ & $0.34\left({ }_{-0.17}^{+0.36}\right)$ & $-10.67(0.17)$ & $<-3.89$ & $340.0(109.1)$ \\
\hline $\mathrm{J} 1708+0257$ & $6396(262)$ & $8.11\left({ }_{-0.08}^{+0.09}\right)$ & $0.64\left({ }_{-0.05}^{+0.06}\right)$ & $-9.93(0.02)$ & $<-3.20$ & $17.9(0.0)$ \\
\hline $\mathrm{J} 1711+2201$ & $9256(340)$ & $8.06\left({ }_{-0.21}^{+0.23}\right)$ & $0.61\left({ }_{-0.12}^{+0.15}\right)$ & $-9.02(0.29)$ & $<-5.69$ & $286.4(38.8)$ \\
\hline $\mathrm{J} 1714+3658$ & $10680(429)$ & $7.77\left({ }_{-0.12}^{+0.12}\right)$ & $0.46\left({ }_{-0.06}^{+0.07}\right)$ & $-9.20(0.11)$ & $-5.26(0.19)$ & $244.6(13.6)$ \\
\hline $\mathrm{J} 1714+5501$ & $9761(589)$ & $8.63\left({ }_{-0.24}^{+0.26}\right)$ & $0.99\left(_{-0.16}^{+0.15}\right)$ & $-9.29(0.31)$ & $<-5.33$ & $6.0(50.3)$ \\
\hline $\mathrm{J} 1715+2804$ & $8328(238)$ & $8.16\left({ }_{-0.07}^{+0.07}\right)$ & $0.67\left({ }_{-0.05}^{+0.05}\right)$ & $-10.83(0.12)$ & $<-4.81$ & $127.0(2.6)$ \\
\hline $\mathrm{J} 1719+5623$ & $9783(309)$ & $8.08\left({ }_{-0.08}^{+0.09}\right)$ & $0.63\left({ }_{-0.05}^{+0.05}\right)$ & $-8.55(0.09)$ & $<-5.85$ & $203.4(7.6)$ \\
\hline $\mathrm{J} 1722+2723$ & $8108(367)$ & $8.60\left({ }_{-0.18}^{+0.19}\right)$ & $0.96\left({ }_{-0.12}^{+0.12}\right)$ & $-9.63(0.31)$ & $-3.11(0.27)$ & $208.8(29.2)$ \\
\hline $\mathrm{J} 1728+0815$ & $10597(628)$ & $8.57\left({ }_{-0.31}^{+0.35}\right)$ & $0.95\left({ }_{-0.20}^{+0.20}\right)$ & $-9.84(0.29)$ & $-4.24(0.39)$ & $255.0(64.1)$ \\
\hline $\mathrm{J} 1728+3250$ & $12123(496)$ & $7.87\left({ }_{-0.09}^{+0.09}\right)$ & $0.51\left({ }_{-0.04}^{+0.05}\right)$ & $-9.01(0.19)$ & $<-6.12$ & $230.8(8.0)$ \\
\hline $\mathrm{J} 2007-1208$ & $12151(506)$ & $7.95\left({ }_{-0.07}^{+0.08}\right)$ & $0.56\left(_{-0.04}^{+0.04}\right)$ & $-9.81(0.17)$ & $-5.03(0.07)$ & $111.2(1.2)$ \\
\hline $\mathrm{J} 2050-0110$ & $10240(306)$ & $8.44\left(\begin{array}{l}+0.22 \\
-0.20\end{array}\right)$ & $0.86\left({ }_{-0.14}^{+0.14}\right)$ & $-7.30(0.22)$ & $<-5.61$ & $249.4(39.2)$ \\
\hline
\end{tabular}


Table 6. Atmospheric parameters for DZ stars - continued.

\begin{tabular}{|c|c|c|c|c|c|c|}
\hline J Name & $T_{\text {eff }}(\mathrm{K})$ & $\log g$ & $M / M_{\odot}$ & $\log \mathrm{Ca} / \mathrm{He}$ & $\log \mathrm{H} / \mathrm{He}$ & $D(\mathrm{pc})$ \\
\hline $\mathrm{J} 2103-0108$ & 8496(195) & $8.07\left({ }_{-0.12}^{+0.13}\right)$ & $0.62\left({ }_{-0.07}^{+0.08}\right)$ & $-8.85(0.52)$ & $<-5.15$ & $188.8(13.6)$ \\
\hline $\mathrm{J} 2107-0055$ & $7276(167)$ & $7.90\left({ }_{-0.09}^{+0.10}\right)$ & $0.51\left({ }_{-0.05}^{+0.06}\right)$ & $-10.66(0.10)$ & $<-4.11$ & $125.3(5.5)$ \\
\hline $\mathrm{J} 2109-0039$ & $5764(92)$ & $7.82\left({ }_{-0.15}^{+0.16}\right)$ & $0.47\left({ }_{-0.08}^{+0.09}\right)$ & $-9.78(0.60)$ & $<-3.00$ & $153.0(12.9)$ \\
\hline $\mathrm{J} 2110+0512$ & $5704(197)$ & 8.00 & & $-10.01(0.48)$ & $<-3.00$ & $242.6(16.1)$ \\
\hline $\mathrm{J} 2110-0001$ & $10781(400)$ & $7.95\left({ }_{-0.09}^{+0.09}\right)$ & $0.55\left(\begin{array}{l}+0.05 \\
-0.05\end{array}\right)$ & $-9.57(0.07)$ & $-3.73(0.12)$ & $149.4(4.7)$ \\
\hline $\mathrm{J} 2114-0051$ & $6952(2067)$ & $7.35\left({ }_{-0.88}^{+0.96}\right)$ & $0.27\left({ }_{-0.20}^{+0.50}\right)$ & $-10.05(0.10)$ & $<-4.31$ & $238.7(33.5)$ \\
\hline $\mathrm{J} 2123+0016$ & $5492(90)$ & $8.29\left({ }_{-0.25}^{+0.27}\right)$ & $0.75\left(\begin{array}{l}+0.18 \\
-0.16\end{array}\right)$ & $-9.65(0.23)$ & $<-3.00$ & $112.1(20.0)$ \\
\hline $\mathrm{J} 2124-0114$ & $7732(153)$ & $8.05\left({ }_{-0.07}^{+0.07}\right)$ & $0.61\left({ }_{-0.04}^{+0.04}\right)$ & $-9.49(0.14)$ & $<-4.29$ & $121.1(3.4)$ \\
\hline $\mathrm{J} 2127+0319$ & $6957(132)$ & $8.09\left({ }_{-0.16}^{+0.17}\right)$ & $0.63\left({ }_{-0.10}^{+0.11}\right)$ & $-9.30(0.12)$ & $<-3.80$ & $159.7(16.7)$ \\
\hline $\mathrm{J} 2130-0243$ & $7234(402)$ & 8.00 & & $-9.96(0.23)$ & $<-4.02$ & $300.7(24.3)$ \\
\hline $\mathrm{J} 2132-0203$ & $5562(244)$ & 8.00 & & $-10.40(0.44)$ & $<-3.00$ & $239.2(20.8)$ \\
\hline $\mathrm{J} 2136+1137$ & $11642(523)$ & $8.13\left({ }_{-0.11}^{+0.11}\right)$ & $0.66\left({ }_{-0.06}^{+0.07}\right)$ & $-10.57(0$ & $-4.64(0$ & $175.0(7.8)$ \\
\hline $\mathrm{J} 2139+0354$ & $7688(889)$ & 8.00 & & $-9.69(0.47)$ & $<-4.30$ & $490.8(83.1)$ \\
\hline $\mathrm{J} 2141+0225$ & $6809(488)$ & 8.00 & & $-9.20(0.45)$ & $<-3.74$ & $446.6(51.6)$ \\
\hline $\mathrm{J} 2141-3300$ & $6908(760)$ & $8.05\left({ }_{-0.21}^{+0.23}\right)$ & $0.60\left({ }_{-0.12}^{+0.15}\right)$ & $-8.80(0.08)$ & $<-3.79$ & $16.1(0.0)$ \\
\hline $\mathrm{J} 2142+2252$ & $9505(297)$ & $8.09\left({ }_{-0.07}^{+0.07}\right)$ & $0.64\left({ }_{-0.04}^{+0.04}\right)$ & $-10.22(0.03)$ & $<-5.80$ & $46.6(0.2)$ \\
\hline $\mathrm{J} 2143-0019$ & $6638(381)$ & 8.00 & & $-9.63(0.44)$ & $<-3.55$ & $348.9(31.2)$ \\
\hline $\mathrm{J} 2151-0112$ & $6930(254)$ & 8.00 & & $-8.58(0.18)$ & $<-3.85$ & $286.6(17.3)$ \\
\hline $\mathrm{J} 2155+4103$ & $6106(317)$ & $8.17\left({ }_{-0.12}^{+0.13}\right)$ & $0.68\left({ }_{-0.08}^{+0.08}\right)$ & $-11.28(0.07)$ & $<-3.01$ & $59.0(0.5)$ \\
\hline $\mathrm{J} 2157+1206$ & $6103(8$ & $8.00\left({ }_{-0.13}^{+0.14}\right)$ & $0.57\left({ }_{-0.08}^{+0.08}\right)$ & $-9.03(0$ & $<-3.05$ & $0.4)$ \\
\hline $\mathrm{J} 2201+021$ & 45 & $7.92\left({ }_{-0.08}^{+0.08}\right)$ & $0.52\left({ }_{-0.04}^{+0.05}\right)$ & $-11.00(0$ & $<-3$ & $63.8(1.6)$ \\
\hline $\mathrm{J} 2202+0013$ & $7458(636)$ & 8.00 & & $-9.80(0.28)$ & $<-4.13$ & $431.7(54.3)$ \\
\hline $\mathrm{J} 2202+0320$ & $6421(407)$ & 8.00 & & $-9.68(0.29)$ & $<-3.32$ & $328.4(33.6)$ \\
\hline $\mathrm{J} 2206+1007$ & $5878(142)$ & $7.76\left({ }_{-0.41}^{+0.46}\right)$ & $0.44\left({ }_{-0.17}^{+0.28}\right)$ & $-10.27(0.19)$ & $<-3.00$ & $210.5(51.8)$ \\
\hline $\mathrm{J} 2207+2319$ & $7238(1143)$ & 8.00 & & $-9.73(0.65)$ & $<-4.02$ & $469.4(111.2)$ \\
\hline $\mathrm{J} 2209+1223$ & $14752(1192)$ & $7.97\left({ }_{-0.11}^{+0.12}\right)$ & $0.57\left({ }_{-0.06}^{+0.07}\right)$ & $-7.30(0.12)$ & $<-5.95$ & $165.8(3.8)$ \\
\hline $\mathrm{J} 2211+2145$ & $6731(239)$ & $8.63\left({ }_{-0.15}^{+0.16}\right)$ & $0.99\left({ }_{-0.10}^{+0.10}\right)$ & $-10.27(0.16)$ & $<-3.09$ & $131.2(15.6)$ \\
\hline $\mathrm{J} 2213+0103$ & $6706(256)$ & 8.00 & & $-9.19(0.25)$ & $<-3.63$ & $322.9(19.8)$ \\
\hline $\mathrm{J} 2218+3908$ & 8914(199) & $8.21\left({ }_{-0.05}^{+0.05}\right)$ & $0.71\left({ }_{-0.03}^{+0.03}\right)$ & $-8.34(0.15)$ & $<-5.34$ & $48.3(0.1)$ \\
\hline $\mathrm{J} 2220+0140$ & $6996(484)$ & 8.00 & & $-9.05(0.32)$ & $<-3.90$ & $376.7(39.1)$ \\
\hline $\mathrm{J} 2225+2338$ & $6100(114)$ & $7.99\left({ }_{-0.10}^{+0.11}\right)$ & $0.57\left({ }_{-0.06}^{+0.06}\right)$ & $-9.31(0.08)$ & $<-3.05$ & $123.9(6.8)$ \\
\hline $\mathrm{J} 2228+1207$ & $6887(92)$ & $8.09\left({ }_{-0.04}^{+0.04}\right)$ & $0.63\left({ }_{-0.02}^{+0.02}\right)$ & $-9.68(0.03)$ & $<-3.74$ & $33.9(0.1)$ \\
\hline $\mathrm{J} 2230+1905$ & $5593(134)$ & 8.00 & & $-8.57(0.48)$ & $<-3.00$ & $171.0(9.7)$ \\
\hline $\mathrm{J} 2230+3052$ & $6719(223)$ & $8.30\left({ }_{-0.17}^{+0.17}\right)$ & $0.76\left(_{-0.11}^{+0.12}\right)$ & $-10.70(0.24)$ & $<-3.34$ & $163.4(17.5)$ \\
\hline $\mathrm{J} 2231+0040$ & $8456(319)$ & $8.08\left({ }_{-0.24}^{+0.25}\right)$ & $0.63\left({ }_{-0.14}^{+0.17}\right)$ & $-10.66(0.26)$ & $<-5.08$ & $223.6(33.8)$ \\
\hline $\mathrm{J} 2231+0906$ & 5726 & $8.05\left({ }_{-0.08}^{+0.08}\right)$ & $0.60\left({ }_{-0.05}^{+0.05}\right)$ & $-9.84(0.07)$ & $<-3.00$ & $90.0(3.9)$ \\
\hline $\mathrm{J} 2232+0109$ & $6886(156)$ & $8.01\left({ }_{-0.11}^{+0.12}\right)$ & $0.58\left(_{-0.07}^{+0.07}\right)$ & $-10.55(0.15)$ & $<-3.80$ & $134.6(8.2)$ \\
\hline $\mathrm{J} 2235-0056$ & $6486(144)$ & 8.00 & & $-8.54(0.15)$ & $<-3.38$ & $214.3(8.5)$ \\
\hline
\end{tabular}


Table 6. Atmospheric parameters for DZ stars - continued.

\begin{tabular}{|c|c|c|c|c|c|c|}
\hline J Name & $T_{\text {eff }}(\mathrm{K})$ & $\log g$ & $M / M_{\odot}$ & $\log \mathrm{Ca} / \mathrm{He}$ & $\log \mathrm{H} / \mathrm{He}$ & $D(\mathrm{pc})$ \\
\hline $\mathrm{J} 2236+0109$ & 13339(708) & $3.34\left({ }_{-0.24}^{+0.26}\right)$ & $80\left(\begin{array}{l}+0.17 \\
-0.15\end{array}\right)$ & $-8.95(0.37)$ & $-5.16(0.25)$ & $253.6(44.4)$ \\
\hline $\mathrm{J} 2236+0413$ & $7145(577)$ & 8.00 & & $-10.32(0.22)$ & $<-3.98$ & $341.3(40.5)$ \\
\hline $\mathrm{J} 2238+0101$ & $7135(158)$ & $8.15\left({ }_{-0.10}^{+0.10}\right)$ & $0.67\left({ }_{-0.06}^{+0.07}\right)$ & $-9.93(0.11)$ & $<-3.87$ & $121.0(7.3)$ \\
\hline $\mathrm{J} 2238+0213$ & $7096(224)$ & 8.00 & & $-8.65(0.19)$ & $<-3.96$ & $268.5(13.1)$ \\
\hline $\mathrm{J} 2238-0113$ & $6222(198)$ & $7.53\left({ }_{-0.71}^{+0.87}\right)$ & $0.33\left({ }_{-0.20}^{+0.50}\right)$ & $-9.54(0.21)$ & $<-3.46$ & $290.8(121.1)$ \\
\hline $\mathrm{J} 2239+2932$ & $6595(451)$ & 8.00 & & $-9.41(0$ & $<-3.51$ & 335.1( \\
\hline $\mathrm{J} 2243+3210$ & $11295(447)$ & $7.83\left({ }_{-0.13}^{+0.14}\right)$ & $0.49\left(_{-0.06}^{+0.07}\right)$ & $-9.00(0.17)$ & $<-6.06$ & $252.6(17$ \\
\hline $\mathrm{J} 2245+2748$ & 6607( & $8.16\left({ }_{-0.18}^{+0.19}\right)$ & $0.68\left({ }_{-0.11}^{+0.13}\right)$ & $-10.27(0.08)$ & $<-3.35$ & $157.5(11.1)$ \\
\hline $\mathrm{J} 2248+1318$ & $11676(465)$ & $8.25\left({ }_{-0.13}^{+0.13}\right)$ & $0.74\left({ }_{-0.08}^{+0.09}\right)$ & $-8.67(0.13)$ & $<-6.02$ & $228.2(18.4)$ \\
\hline $\mathrm{J} 2248-0153$ & $7493(350)$ & 8.00 & & $-10.60(0.22)$ & $<-4.15$ & $262.9(18.1)$ \\
\hline $\mathrm{J} 2253-0646$ & $4132(53)$ & $8.03\left({ }_{-0.07}^{+0.07}\right)$ & $0.59\left({ }_{-0.04}^{+0.05}\right)$ & $-10.00(0.05)$ & $<-3.00$ & $8.5(0$ \\
\hline $\mathrm{J} 2258-0006$ & $8832(1006)$ & 8.00 & & $-9.64(0.33)$ & $<-5.46$ & 530 \\
\hline $\mathrm{J} 2258-0829$ & $14262(1391)$ & $8.54\left({ }_{-0.29}^{+0.33}\right)$ & $0.93\left({ }_{-0.19}^{+0.19}\right)$ & $-8.99(0.94)$ & $-5.26(0$ & $354.8(78.6)$ \\
\hline $\mathrm{J} 2259+1251$ & $6748(261)$ & $7.94\left({ }_{-0.31}^{+0.34}\right)$ & $0.54\left({ }_{-0.16}^{+0.22}\right)$ & $-10.53(0.11)$ & $<-3.72$ & $191.4(36.7)$ \\
\hline $\mathrm{J} 2259+1748$ & $7476(842)$ & 8.00 & & $-10.76(0.24)$ & $<-4.14$ & $317.9(54.5)$ \\
\hline $\mathrm{J} 2300+2204$ & $7000(122)$ & $8.06\left({ }_{-0.05}^{+0.05}\right)$ & $0.61\left({ }_{-0.03}^{+0.03}\right)$ & $-10.15(0.04)$ & $<-3.86$ & $60.8(0.4)$ \\
\hline $\mathrm{J} 2302+1626$ & $7311(299)$ & 8.00 & & $-8.93(0.39)$ & $<-4.05$ & $301.5(17.7)$ \\
\hline $\mathrm{J} 2304+2415$ & $5269(80)$ & $8.07\left({ }_{-0.07}^{+0.08}\right)$ & $0.62\left({ }_{-0.05}^{+0.05}\right)$ & $-8.92(0.16)$ & $<-3.01$ & $87.1(2.5)$ \\
\hline $\mathrm{J} 2305+2307$ & 1105 & $7.99\left({ }_{-0.10}^{+0.10}\right)$ & $0.58\left({ }_{-0.05}^{+0.06}\right)$ & -8.31( & -5 & 19 \\
\hline $\mathrm{J} 2306+2111$ & $9580(4$ & & $0.43\left({ }_{-0.10}^{+0.15}\right)$ & $-10.85(0$ & -5.07 & 8.8) \\
\hline $\mathrm{J} 2309+0608$ & 11885 & $7.94\left({ }_{-0.09}^{+0.09}\right)$ & $0.55\left({ }_{-0.05}^{+0.05}\right)$ & $-7.41(0.06)$ & & 年 \\
\hline $\mathrm{J} 2310+2203$ & 10049( & $8.06\left({ }_{-0.26}^{+0.29}\right)$ & $0.62\left(_{-0.15}^{+0.19}\right)$ & $-9.65(0$. & $<-5.88$ & $00.6(51.6)$ \\
\hline $\mathrm{J} 2311+1042$ & $6180(1$ & $8.25\left({ }_{-0.21}^{+0.22}\right)$ & $0.73\left({ }_{-0.13}^{+0.15}\right)$ & $-9.72(0.10)$ & $<-3.01$ & 17 \\
\hline $\mathrm{J} 2311+1510$ & $7073(472)$ & 8.00 & & $-10.17(0.16)$ & $<-3.95$ & $310.0(31.6)$ \\
\hline $\mathrm{J} 2311+3343$ & $6605(553)$ & 8.00 & & $-10.23(0.25)$ & $<-3.52$ & $353.2(48.5)$ \\
\hline $\mathrm{J} 2311-0041$ & $12701(746)$ & $8.18\left({ }_{-0.11}^{+0.12}\right)$ & $0.70\left({ }_{-0.07}^{+0.08}\right)$ & $-7.32(0.24)$ & $<-6.07$ & $234.4(13.7)$ \\
\hline $\mathrm{J} 2315-0209$ & $6389(60)$ & $8.10\left({ }_{-0.03}^{+0.03}\right)$ & $0.64\left({ }_{-0.02}^{+0.02}\right)$ & $-9.80(0.03)$ & $<-3.20$ & $29.7(0.1)$ \\
\hline $\mathrm{J} 2316+0041$ & $7696(565)$ & 8.00 & & $-8.69(0.41)$ & $<-4.31$ & $460.1(48.9)$ \\
\hline $\mathrm{J} 2318+2916$ & $6635(295)$ & 8.00 & & $-9.93(0.20)$ & $<-3.50$ & $298.8(21.3)$ \\
\hline $\mathrm{J} 2318-0320$ & $6935(542)$ & 8.00 & & $-10.27(0.15)$ & $<-3.85$ & $308.7(37.1)$ \\
\hline $\mathrm{J} 2319+3018$ & $7454(269)$ & $8.94\left({ }_{-0.34}^{+0.43}\right)$ & $1.16\left({ }_{-0.19}^{+0.16}\right)$ & $-8.41(0.34)$ & $<-3.38$ & $164.1(57.1)$ \\
\hline $\mathrm{J} 2321+0807$ & $8386(258)$ & $8.00\left({ }_{-0.25}^{+0.26}\right)$ & $0.58\left({ }_{-0.13}^{+0.17}\right)$ & $-9.20(0.15)$ & $<-5.13$ & $240.3(37.4)$ \\
\hline $\mathrm{J} 2324+1135$ & $7685(504)$ & 8.00 & & $-10.49(0.15)$ & $<-4.30$ & 304.0(29.3) \\
\hline $\mathrm{J} 2324+1207$ & $9128(246)$ & $7.91\left({ }_{-0.06}^{+0.06}\right)$ & $0.52\left({ }_{-0.03}^{+0.03}\right)$ & $-9.09(0.10)$ & $-3.97(0.18)$ & $50.3(0.2)$ \\
\hline $\mathrm{J} 2328+0711$ & $8735(758)$ & 8.00 & & $-9.56(0.29)$ & $<-5.41$ & $417.7(50.0)$ \\
\hline $\mathrm{J} 2328+0830$ & $5663(230)$ & 8.00 & & $-9.02(0.37)$ & $<-3.00$ & $242.0(22.0)$ \\
\hline $\mathrm{J} 2330+1015$ & $8661(447)$ & $7.89\left({ }_{-0.86}^{+1.34}\right)$ & $0.52\left({ }_{-0.32}^{+0.76}\right)$ & $-8.50(0.26)$ & $<-5.48$ & $360.3(237.9)$ \\
\hline $\mathrm{J} 2330+2620$ & $7263(384)$ & $7.57\left({ }_{-0.69}^{+0.91}\right)$ & $0.35\left({ }_{-0.20}^{+0.54}\right)$ & $-10.91(0.13)$ & $<-4.39$ & $324.4(142.3)$ \\
\hline
\end{tabular}


Table 6. Atmospheric parameters for DZ stars - continued.

\begin{tabular}{|c|c|c|c|c|c|c|}
\hline J Name & $T_{\text {eff }}(\mathrm{K})$ & $\log g$ & $M / M_{\odot}$ & $\log \mathrm{Ca} / \mathrm{He}$ & $\log \mathrm{H} / \mathrm{He}$ & $D(\mathrm{pc})$ \\
\hline $\mathrm{J} 2330+2805$ & $6470(228)$ & $8.16\left({ }_{-0.44}^{+0.50}\right)$ & $0.67\left(\begin{array}{l}+0.33 \\
-0.25\end{array}\right)$ & $-9.02(0.18)$ & $<-3.22$ & $215.8(65.8)$ \\
\hline $\mathrm{J} 2333+1058$ & $6971(343)$ & 8.00 & & $-8.30(0.28)$ & $<-3.88$ & $349.3(29.0)$ \\
\hline $\mathrm{J} 2336+1657$ & $7284(738)$ & 8.00 & & $-9.28(0.90)$ & $<-4.04$ & $455.2(65.6)$ \\
\hline $\mathrm{J} 2336+2021$ & $7311(534)$ & 8.00 & & $-9.80(0.25)$ & $<-4.05$ & $321.2(34.5)$ \\
\hline $\mathrm{J} 2337+2607$ & $7179(810)$ & 8.00 & & $-10.38(0.28)$ & $<-4.00$ & $393.2(69.5)$ \\
\hline $\mathrm{J} 2339+4631$ & $10178(372)$ & $7.95\left(_{-0.13}^{+0.14}\right)$ & $0.55\left(_{-0.07}^{+0.08}\right)$ & $-10.21(0.15)$ & $-3.98(0.22)$ & $226.3(15.8)$ \\
\hline $\mathrm{J} 2340+0124$ & $6167(63)$ & $8.06\left({ }_{-0.08}^{+0.09}\right)$ & $0.61\left({ }_{-0.05}^{+0.05}\right)$ & $-8.84(0.09)$ & $<-3.06$ & $115.0(5.7)$ \\
\hline $\mathrm{J} 2340+0817$ & $6123(146)$ & $8.18\left({ }_{-0.20}^{+0.21}\right)$ & $0.69\left({ }_{-0.12}^{+0.14}\right)$ & $-8.65(0.22)$ & $<-3.01$ & $146.0(19.0)$ \\
\hline $\mathrm{J} 2340+1621$ & $7637(773)$ & 8.00 & & $-10.14(0.08)$ & $<-4.25$ & $413.1(60.1)$ \\
\hline $\mathrm{J} 2341+0059$ & $6325(145)$ & $8.07\left({ }_{-0.24}^{+0.26}\right)$ & $0.62\left(\begin{array}{l}+0.17 \\
-0.14\end{array}\right)$ & $-10.00(0.16)$ & $<-3.17$ & $180.6(28.0)$ \\
\hline $\mathrm{J} 2341+2448$ & $6203(203)$ & $7.68\left({ }_{-0.19}^{+0.20}\right)$ & $0.40\left(\begin{array}{l}+0.10 \\
-0.08\end{array}\right)$ & $-11.43(0.18)$ & $<-3.31$ & $190.4(18.2)$ \\
\hline $\mathrm{J} 2342-0006$ & 10399(376) & $7.73\left({ }_{-0.21}^{+0.24}\right)$ & $0.44\left({ }_{-0.09}^{+0.13}\right)$ & $-8.06(0.09)$ & $-4.40(0.18)$ & $322.3(41.5)$ \\
\hline $\mathrm{J} 2343+2242$ & $8193(513)$ & $7.53\left({ }_{-1.10}^{+1.57}\right)$ & $0.34\left({ }_{-0.26}^{+0.89}\right)$ & $-10.91(0.18)$ & $<-5.43$ & $432.1(294.7)$ \\
\hline $\mathrm{J} 2343+3155$ & $7578(495)$ & 8.00 & & $-9.29(0.57)$ & $<-4.21$ & $436.6(42.9)$ \\
\hline $\mathrm{J} 2343-0010$ & $5672(112)$ & $7.45\left(_{-0.31}^{+0.35}\right)$ & $0.30\left({ }_{-0.10}^{+0.16}\right)$ & $-10.15(0.30)$ & $<-3.03$ & $210.6(3$ \\
\hline $\mathrm{J} 2344+1343$ & $7886(248)$ & $7.98\left({ }_{-0.48}^{+0.55}\right)$ & $0.56\left({ }_{-0.23}^{+0.36}\right)$ & $-8.67(0.40)$ & $<-4.53$ & $313.9(100.5)$ \\
\hline $\mathrm{J} 2344+1813$ & $7805(992)$ & 8.00 & & $-8.90(0.60)$ & $<-4.42$ & $539.3(95.9)$ \\
\hline $\mathrm{J} 2345+2857$ & $7548(458)$ & 8.00 & & $-9.83(0.40)$ & $<-4.18$ & $354.4(31.1)$ \\
\hline $\mathrm{J} 2346+2816$ & $8363(1646)$ & 8.00 & & $-9.89(0.26)$ & $<-5.11$ & $477.5(131.9)$ \\
\hline $\mathrm{J} 2348+1341$ & $11065(568)$ & $7.42\left({ }_{-0.21}^{+0.26}\right)$ & $0.31\left({ }_{-0.06}^{+0.10}\right)$ & $-8.59(0.17)$ & $-3.96(0.24)$ & $354.5(46.8)$ \\
\hline $\mathrm{J} 2348-4428$ & $4886(125)$ & $8.11\left({ }_{-0.08}^{+0.08}\right)$ & $0.64\left({ }_{-0.05}^{+0.05}\right)$ & $-10.94(0.39)$ & $<-3.00$ & $36.3(0.2)$ \\
\hline $\mathrm{J} 2349+0325$ & $7564(794)$ & 8.00 & & $-8.54(0.32)$ & $<-4.19$ & $557.2(85.6)$ \\
\hline $\mathrm{J} 2351+0633$ & $9724(328)$ & $8.29\left({ }_{-0.12}^{+0.12}\right)$ & $0.76\left({ }_{-0.08}^{+0.08}\right)$ & $-9.12(0.07)$ & $<-5.70$ & $174.3(12.3)$ \\
\hline $\mathrm{J} 2351+3612$ & $11311(803)$ & $7.94\left({ }_{-0.30}^{+0.33}\right)$ & $0.55\left({ }_{-0.15}^{+0.21}\right)$ & $-9.33(0.27)$ & $<-6.04$ & $344.7(65.5)$ \\
\hline $\mathrm{J} 2352+1922$ & $6126(209)$ & 8.00 & & $-8.38(0.28)$ & $<-3.06$ & $266.1(19.2)$ \\
\hline $\mathrm{J} 2352+3344$ & $6629(212)$ & 8.00 & & $-9.69(0.26)$ & $<-3.54$ & $270.0(13.7)$ \\
\hline $\mathrm{J} 2352-0146$ & $6568(182)$ & $8.05\left(_{-0.17}^{+0.17}\right)$ & $0.60\left({ }_{-0.10}^{+0.11}\right)$ & $-10.76(0.09)$ & $<-3.42$ & $154.0(15.2)$ \\
\hline $\mathrm{J} 2353+0415$ & $6551(330)$ & 8.00 & & $-10.01(0.23)$ & $<-3.46$ & $260.7(20.7)$ \\
\hline $\mathrm{J} 2353+3720$ & $15152(6173)$ & $8.14\left({ }_{-0.63}^{+0.84}\right)$ & $0.68\left({ }_{-0.32}^{+0.51}\right)$ & $-7.85(0.73)$ & $-5.22(0.47)$ & $422.2(94.8)$ \\
\hline $\mathrm{J} 2355+1431$ & $6523(113)$ & $8.10\left({ }_{-0.08}^{+0.08}\right)$ & $0.64\left({ }_{-0.05}^{+0.05}\right)$ & $-10.58(0.10)$ & $<-3.32$ & $111.3(4.6)$ \\
\hline $\mathrm{J} 2356-0320$ & $8219(854)$ & 8.00 & & $-9.65(0.39)$ & $<-4.94$ & $508.9(71.9)$ \\
\hline $\mathrm{J} 2357+2348$ & $6314(285)$ & 8.00 & & $-8.76(0.10)$ & $<-3.21$ & $270.0(21.8)$ \\
\hline $\mathrm{J} 2357-0324$ & $9152(358)$ & $8.07\left({ }_{-0.18}^{+0.19}\right)$ & $0.62\left({ }_{-0.11}^{+0.12}\right)$ & $-9.86(0.08)$ & $<-5.61$ & $199.2(21.7)$ \\
\hline $\mathrm{J} 2358+0445$ & $8065(724)$ & 8.00 & & $-8.68(0.48)$ & $<-4.73$ & $482.0(65.6)$ \\
\hline $\mathrm{J} 2359+0243$ & $7902(558)$ & 8.00 & & $-9.46(0.28)$ & $<-4.53$ & $387.4(38.8)$ \\
\hline $\mathrm{J} 2359+0347$ & $6886(203)$ & $8.36\left(_{-0.16}^{+0.16}\right)$ & $0.81\left(\left(_{-0.11}^{+0.11}\right)\right.$ & $-9.78(0.10)$ & $<-3.46$ & $153.9(16.7)$ \\
\hline
\end{tabular}


Table 7. Atmospheric parameters for DQ stars. A star indicate that the value of $\log \mathrm{H} / \mathrm{He}$ have been fixed rather than fitted. A dagger indicates that the distance is a photometric distance based on the value of $\log g$ $=8$ in the absence of a parallax.

\begin{tabular}{|c|c|c|c|c|c|c|}
\hline J Name & $T_{\text {eff }}(\mathrm{K})$ & $\log g$ & $M / M_{\odot}$ & $\log \mathrm{C} / \mathrm{He}$ & $\log \mathrm{H} / \mathrm{He}$ & $D(\mathrm{pc})$ \\
\hline J0000-0026 & $7420(163)$ & $7.87\left({ }_{-0.08}^{+0.09}\right)$ & $0.50\left({ }_{-0.04}^{+0.05}\right)$ & $-6.11(0.12)$ & & $125.9(4.5)$ \\
\hline J0000-0850 & $7853(226)$ & $7.42\left({ }_{-0.17}^{+0.19}\right)$ & $0.30\left({ }_{-0.05}^{+0.07}\right)$ & $-5.51(0.09)$ & & $207.3(18.4)$ \\
\hline $\mathrm{J} 0006+1800$ & $7422(155)$ & $7.90\left({ }_{-0.06}^{+0.06}\right)$ & $0.52\left({ }_{-0.03}^{+0.03}\right)$ & $-5.98(0.03)$ & & $57.9(0.4)$ \\
\hline $\mathrm{J} 0007+2821$ & $7409(195)$ & $7.92\left({ }_{-0.15}^{+0.15}\right)$ & $0.53\left({ }_{-0.08}^{+0.09}\right)$ & $-5.89(0.10)$ & & $184.5(15.1)$ \\
\hline $\mathrm{J} 0008+2507$ & $10801(525)$ & $8.10\left({ }_{-0.26}^{+0.28}\right)$ & $0.64\left(_{-0.15}^{+0.18}\right)$ & $-3.37(0.21)$ & $-3.00 *$ & $311.1(53.6)$ \\
\hline J0008-1034 & $7587(543)$ & $7.86\left({ }_{-0.24}^{+0.26}\right)$ & $0.50\left(\begin{array}{l}+0.15 \\
-0.12\end{array}\right)$ & $-5.85(0.14)$ & & $145.0(7.5)$ \\
\hline $\mathrm{J} 0015+0309$ & $7261(217)$ & $7.98\left({ }_{-0.20}^{+0.21}\right)$ & $0.56\left(_{-0.11}^{+0.13}\right)$ & $-6.03(0.12)$ & & $199.2(23.0)$ \\
\hline J0024-1107 & $8330(202)$ & $7.88\left(\begin{array}{l}+0.07 \\
-0.07\end{array}\right)$ & $0.51\left({ }_{-0.04}^{+0.04}\right)$ & $-5.09(0.06)$ & & $108.1(2.1)$ \\
\hline $\mathrm{J} 0033+0418$ & $7658(214)$ & $7.86\left(\begin{array}{l}+0.10 \\
-0.10\end{array}\right)$ & $0.49\left(\begin{array}{l}+0.06 \\
-0.05\end{array}\right)$ & $-6.00(0.07)$ & & $133.4(5.0)$ \\
\hline $\mathrm{J} 0041+7321$ & $9433(349)$ & $7.84\left(\begin{array}{l}+0.07 \\
-0.06\end{array}\right)$ & $0.49\left({ }_{-0.03}^{+0.04}\right)$ & $-4.38(0.12)$ & & $55.8(0.1)$ \\
\hline $\mathrm{J} 0044+1259$ & $7109(195)$ & $8.03\left(\begin{array}{l}+0.18 \\
-0.17\end{array}\right)$ & $0.59\left(\begin{array}{l}+0.12 \\
-0.10\end{array}\right)$ & $-6.28(0.10)$ & & $174.5(18.2)$ \\
\hline $\mathrm{J} 0055+0850$ & $6552(160)$ & $7.98\left(\begin{array}{l}+0.08 \\
-0.08\end{array}\right)$ & $0.56\left(_{-0.05}^{+0.05}\right)$ & $-6.74(0.06)$ & & $88.1(2.2)$ \\
\hline $\mathrm{J} 0100+0814$ & $6384(98)$ & 8.00 & & $-5.63(0.04)$ & & $165.9(5.2)$ \\
\hline J0103-0429 & $7215(227)$ & $7.49\left(_{-0.33}^{+0.40}\right)$ & $0.32\left(\begin{array}{l}+0.19 \\
-0.10\end{array}\right)$ & $-5.81(0.11)$ & & $269.7(53.3)$ \\
\hline $\mathrm{J} 0107+0102$ & $6600(108)$ & $8.04\left({ }_{-0.06}^{+0.06}\right)$ & $0.60\left({ }_{-0.04}^{+0.04}\right)$ & $-6.65(0.05)$ & & $93.3(2.2)$ \\
\hline $\mathrm{J} 0116+2346$ & $8047(189)$ & $7.93\left({ }_{-0.06}^{+0.06}\right)$ & $0.54\left(_{-0.03}^{+0.04}\right)$ & $-5.46(0.04)$ & & $89.4(1.1)$ \\
\hline $\mathrm{J} 0116+2402$ & $9217(258)$ & $7.97\left({ }_{-0.13}^{+0.14}\right)$ & $0.56\left(_{-0.07}^{+0.08}\right)$ & $-4.59(0.14)$ & & $175.8(13.5)$ \\
\hline $\mathrm{J} 0118+1610$ & $8833(218)$ & $7.96\left(_{-0.06}^{+0.06}\right)$ & $0.56\left(_{-0.03}^{+0.03}\right)$ & $-4.90(0.06)$ & & $16.8(0.0)$ \\
\hline $\mathrm{J} 0136+2004$ & $7115(211)$ & 8.00 & & $-5.21(0.07)$ & & $289.1(16.3)$ \\
\hline $\mathrm{J} 0137+0047$ & $8127(216)$ & $7.95\left({ }_{-0.23}^{+0.24}\right)$ & $0.55\left(\begin{array}{l}+0.15 \\
-0.12\end{array}\right)$ & $-5.28(0.19)$ & & $187.7(26.2)$ \\
\hline J0144-0757 & $7296(240)$ & $8.30\left({ }_{-0.17}^{+0.18}\right)$ & $0.77\left({ }_{-0.11}^{+0.12}\right)$ & $-6.06(0.11)$ & & $140.4(15.6)$ \\
\hline $\mathrm{J} 0145+2317$ & $7731(188)$ & $8.02\left({ }_{-0.06}^{+0.06}\right)$ & $0.58\left({ }_{-0.04}^{+0.04}\right)$ & $-5.55(0.02)$ & & $61.0(0.4)$ \\
\hline J0145-0822 & $8002(236)$ & $8.97\left({ }_{-0.05}^{+0.05}\right)$ & $1.17\left({ }_{-0.02}^{+0.02}\right)$ & $-5.85(0.14)$ & & $71.6(0.8)$ \\
\hline J0154+1403 & $6529(100)$ & $7.97\left({ }_{-0.05}^{+0.05}\right)$ & $0.55\left(_{-0.03}^{+0.03}\right)$ & $-6.72(0.05)$ & & $61.7(0.7)$ \\
\hline J0154-0040 & $7321(147)$ & $7.94\left({ }_{-0.07}^{+0.07}\right)$ & $0.54\left({ }_{-0.04}^{+0.04}\right)$ & $-6.01(0.09)$ & & $113.5(3.0)$ \\
\hline $\mathrm{J} 0200+0715$ & $6758(120)$ & $8.04\left({ }_{-0.07}^{+0.07}\right)$ & $0.60\left({ }_{-0.04}^{+0.04}\right)$ & $-6.23(0.04)$ & & $98.0(2.5)$ \\
\hline J0208-0315 & $7233(253)$ & 8.00 & & $-6.13(0.26)$ & & $235.2(13.5)$ \\
\hline $\mathrm{J} 0209+1425$ & $7117(192)$ & $7.45\left(\begin{array}{l}+0.23 \\
-0.21\end{array}\right)$ & $0.31\left({ }_{-0.07}^{+0.10}\right)$ & $-6.20(0.12)$ & & $184.3(21.0)$ \\
\hline $\mathrm{J} 0227+0018$ & $9646(504)$ & 8.00 & & $-3.37(0.10)$ & & $511.5(38.3)$ \\
\hline $\mathrm{J} 0236+2503$ & 13376(697) & $8.70\left({ }_{-0.11}^{+0.12}\right)$ & $1.03\left({ }_{-0.07}^{+0.07}\right)$ & $-1.58(0.25)$ & & $175.6(13.0)$ \\
\hline $\mathrm{J} 0239+0027$ & $7134(185)$ & $7.59\left({ }_{-0.20}^{+0.22}\right)$ & $0.36\left(_{-0.08}^{+0.11}\right)$ & $-5.97(0.14)$ & & $212.2(23.6)$ \\
\hline $\mathrm{J} 0243+0101$ & $8225(172)$ & $8.63\left({ }_{-0.16}^{+0.17}\right)$ & $0.99\left({ }_{-0.11}^{+0.10}\right)$ & $-4.23(0.15)$ & & $174.9(23.6)$ \\
\hline $\mathrm{J} 0248+3408$ & $6009(68)$ & $8.00\left(\begin{array}{l}+0.05 \\
-0.05\end{array}\right)$ & $0.57\left({ }_{-0.03}^{+0.03}\right)$ & $-6.87(0.03)$ & & $76.7(1.5)$ \\
\hline $\mathrm{J} 0253+3414$ & $7489(234)$ & $7.39\left(_{-0.28}^{+0.34}\right)$ & $0.29\left(_{-0.08}^{+0.14}\right)$ & $-5.98(0.20)$ & & $255.4(42.9)$ \\
\hline $\mathrm{J} 0305+0557$ & $8446(365)$ & 8.00 & & $-4.91(0.32)$ & & $305.7(20.3)$ \\
\hline J0320-0716 & $6510(89)$ & $8.02\left({ }_{-0.09}^{+0.09}\right)$ & $0.59\left(_{-0.05}^{+0.05}\right)$ & $-5.29(0.03)$ & & $125.4(5.9)$ \\
\hline
\end{tabular}


Table 7. Atmospheric parameters for DQ stars - continued.

\begin{tabular}{|c|c|c|c|c|c|c|}
\hline $\mathrm{J}$ Name & $T_{\text {eff }}(\mathrm{K})$ & $\log g$ & $M / M_{\odot}$ & $\log \mathrm{C} / \mathrm{He}$ & $\log \mathrm{H} / \mathrm{He}$ & $D(\mathrm{pc})$ \\
\hline J0332-0037 & $8017(190)$ & $8.01\left(\left(_{-0.07}^{+0.07}\right)\right.$ & $0.58\left({ }_{-0.04}^{+0.04}\right)$ & $-5.30(0.05)$ & & $110.6(2.5)$ \\
\hline J0352-0605 & $7519(179)$ & $7.81\left({ }_{-0.09}^{+0.09}\right)$ & $0.47\left({ }_{-0.05}^{+0.05}\right)$ & $-6.08(0.15)$ & & $132.8(4.8)$ \\
\hline $\mathrm{J} 0416+0713$ & $7266(213)$ & $7.96\left({ }_{-0.14}^{+0.14}\right)$ & $0.55\left(\begin{array}{l}+0.09 \\
-0.08\end{array}\right)$ & $-6.21(0.11)$ & & $153.5(11.0)$ \\
\hline J0437-0849 & $6396(121)$ & $7.96\left({ }_{-0.05}^{+0.05}\right)$ & $0.55\left({ }_{-0.03}^{+0.03}\right)$ & $-6.31(0.01)$ & & $9.4(0.0)$ \\
\hline $\mathrm{J} 0707+3825$ & $6929(191)$ & $7.82\left({ }_{-0.25}^{+0.27}\right)$ & $0.47\left({ }_{-0.12}^{+0.16}\right)$ & $-6.29(0.11)$ & & $219.8(31.8)$ \\
\hline $\mathrm{J} 0710+3740$ & $6643(159)$ & $7.94\left({ }_{-0.06}^{+0.06}\right)$ & $0.54\left({ }_{-0.03}^{+0.03}\right)$ & $-6.43(0.02)$ & & $24.4(0.0)$ \\
\hline J0723+3908 & $9086(230)$ & $7.84\left({ }_{-0.07}^{+0.07}\right)$ & $0.49\left({ }_{-0.03}^{+0.04}\right)$ & $-4.88(0.11)$ & & $118.0(2.3)$ \\
\hline $\mathrm{J} 0737+6455$ & $7215(176)$ & $7.69\left({ }_{-0.16}^{+0.17}\right)$ & $0.41\left({ }_{-0.07}^{+0.09}\right)$ & $-5.95(0.10)$ & & $196.2(16.8)$ \\
\hline $\mathrm{J} 0739+0513$ & $7585(33)$ & $7.96\left({ }_{-0.02}^{+0.02}\right)$ & $0.55\left(_{-0.01}^{+0.01}\right)$ & $-5.86(0.03)$ & & $3.5(0.0)$ \\
\hline $\mathrm{J} 0740+1810$ & 6993(144) & $8.15\left({ }_{-0.13}^{+0.13}\right)$ & $0.67\left({ }_{-0.08}^{+0.09}\right)$ & $-6.08(0.06)$ & & $138.1(10.9)$ \\
\hline $\mathrm{J} 0742+4348$ & $8042(208)$ & $7.95\left({ }_{-0.08}^{+0.09}\right)$ & $0.55\left(\begin{array}{l}+0.05 \\
-0.05\end{array}\right)$ & $-5.33(0.11)$ & & $139.6(4.5)$ \\
\hline $\mathrm{J} 0750+1328$ & $8085(210)$ & $7.91\left({ }_{-0.09}^{+0.10}\right)$ & $0.52\left({ }_{-0.05}^{+0.06}\right)$ & $-5.47(0.09)$ & & $162.4(6.6)$ \\
\hline $\mathrm{J} 0750+2329$ & $7194(218)$ & $8.19\left(\begin{array}{l}+0.19 \\
-0.19\end{array}\right)$ & $0.69\left({ }_{-0.12}^{+0.13}\right)$ & $-6.04(0.12)$ & & $187.0(23.0)$ \\
\hline $\mathrm{J} 0804+0750$ & $7828(236)$ & $7.84\left({ }_{-0.23}^{+0.25}\right)$ & $0.49\left(_{-0.11}^{+0.15}\right)$ & $-5.59(0.15)$ & & $214.2(29.2)$ \\
\hline J0807+1949 & $13501(682)$ & $8.78\left({ }_{-0.09}^{+0.10}\right)$ & $1.08\left({ }_{-0.05}^{+0.05}\right)$ & $-1.24(0.11)$ & $-3.00^{*}$ & $176.6(10.4)$ \\
\hline $\mathrm{J} 0813+3047$ & $7570(177)$ & $7.92\left({ }_{-0.09}^{+0.09}\right)$ & $0.53\left({ }_{-0.05}^{+0.05}\right)$ & $-5.75(0.09)$ & & $138.0(5.5)$ \\
\hline J0814+2455 & $7919(186)$ & $7.94\left({ }_{-0.06}^{+0.06}\right)$ & $0.54\left({ }_{-0.03}^{+0.04}\right)$ & $-5.71(0.05)$ & & $58.9(0.3)$ \\
\hline $\mathrm{J} 0827+6017$ & 6391(141) & $7.01\left({ }_{-0.43}^{+0.33}\right)$ & $0.18\left({ }_{-0.09}^{+0.09}\right)$ & $-6.46(0.05)$ & & $270.5(41.5)$ \\
\hline J0832-0408 & $6791(77)$ & $8.10\left({ }_{-0.04}^{+0.04}\right)$ & $0.63\left({ }_{-0.03}^{+0.03}\right)$ & $-4.67(0.01)$ & & $82.1(1.3)$ \\
\hline $\mathrm{J} 0833+3638$ & $7699(196)$ & $7.97\left({ }_{-0.10}^{+0.11}\right)$ & $0.56\left({ }_{-0.06}^{+0.06}\right)$ & $-5.65(0.12)$ & & $142.1(7.1)$ \\
\hline $\mathrm{J} 0836+0437$ & $8420(226)$ & $7.79\left({ }_{-0.11}^{+0.11}\right)$ & $0.46\left(_{-0.05}^{+0.06}\right)$ & $-5.28(0.10)$ & & $172.8(8.6)$ \\
\hline $\mathrm{J} 0836+4817$ & $7281(157)$ & $7.92\left({ }_{-0.08}^{+0.08}\right)$ & $0.53\left({ }_{-0.05}^{+0.05}\right)$ & $-6.29(0.19)$ & & $121.2(4.1)$ \\
\hline $\mathrm{J} 0837+0321$ & $8168(229)$ & $7.94\left({ }_{-0.12}^{+0.13}\right)$ & $0.54\left(\begin{array}{l}+0.08 \\
-0.07\end{array}\right)$ & $-5.56(0.12)$ & & $172.8(10.7)$ \\
\hline $\mathrm{J} 0838+1121$ & $7467(181)$ & $8.04\left({ }_{-0.11}^{+0.11}\right)$ & $0.60\left({ }_{-0.06}^{+0.07}\right)$ & $-5.72(0.06)$ & & $141.9(7.7)$ \\
\hline $\mathrm{J} 0840+4529$ & $7798(185)$ & $7.98\left({ }_{-0.07}^{+0.07}\right)$ & $0.56\left({ }_{-0.04}^{+0.04}\right)$ & $-5.66(0.05)$ & & $105.1(2.3)$ \\
\hline $\mathrm{J} 0841+3329$ & $6937(110)$ & $8.71\left({ }_{-0.06}^{+0.07}\right)$ & $1.03\left({ }_{-0.04}^{+0.04}\right)$ & $-6.16(0.07)$ & & $53.2(2.6)$ \\
\hline $\mathrm{J} 0845+6143$ & $7900(180)$ & $7.93\left({ }_{-0.06}^{+0.06}\right)$ & $0.53\left({ }_{-0.03}^{+0.04}\right)$ & $-5.36(0.03)$ & & $76.4(0.6)$ \\
\hline $\mathrm{J} 0846+1024$ & $8825(732)$ & $7.72\left({ }_{-0.26}^{+0.29}\right)$ & $0.43\left({ }_{-0.11}^{+0.15}\right)$ & $-5.13(0.12)$ & & $129.5(3.5)$ \\
\hline $\mathrm{J} 0847+1830$ & 8307(199) & $7.86\left({ }_{-0.06}^{+0.06}\right)$ & $0.50\left({ }_{-0.03}^{+0.04}\right)$ & $-5.54(0.07)$ & & $73.1(0.6)$ \\
\hline J0847+1919 & $7558(302)$ & 8.00 & & $-5.13(0.14)$ & & $361.8(26.2)$ \\
\hline $\mathrm{J} 0850+0709$ & $8379(230)$ & $7.53\left({ }_{-0.17}^{+0.19}\right)$ & $0.34\left({ }_{-0.06}^{+0.08}\right)$ & $-5.25(0.13)$ & & $240.3(22.1)$ \\
\hline $\mathrm{J} 0852+0428$ & $9918(273)$ & $7.94\left({ }_{-0.12}^{+0.13}\right)$ & $0.55\left(_{-0.07}^{+0.08}\right)$ & $-3.43(0.08)$ & & $209.8(14.7)$ \\
\hline $\mathrm{J} 0852+2316$ & $11099(458)$ & $8.61\left({ }_{-0.12}^{+0.13}\right)$ & $0.97\left(\begin{array}{l}+0.08 \\
-0.08\end{array}\right)$ & $-3.18(0.21)$ & & $190.7(17.4)$ \\
\hline $\mathrm{J} 0852+5223$ & $8837(547)$ & 8.00 & & $-4.58(0.35)$ & & $402.2(38.4)$ \\
\hline $\mathrm{J} 0855+0639$ & $7273(142)$ & $7.96\left({ }_{-0.06}^{+0.06}\right)$ & $0.55\left({ }_{-0.03}^{+0.03}\right)$ & $-6.08(0.05)$ & & $76.4(1.1)$ \\
\hline $\mathrm{J} 0856+4513$ & $9484(278)$ & $8.51\left({ }_{-0.17}^{+0.18}\right)$ & $0.91\left({ }_{-0.11}^{+0.11}\right)$ & $-3.27(0.06)$ & & $208.2(27.1)$ \\
\hline $\mathrm{J} 0857+0603$ & $8290(218)$ & $7.98\left({ }_{-0.08}^{+0.08}\right)$ & $0.56\left(_{-0.05}^{+0.05}\right)$ & $-5.25(0.13)$ & & $123.2(3.6)$ \\
\hline $\mathrm{J} 0859+3257$ & $9486(206)$ & $8.45\left({ }_{-0.04}^{+0.04}\right)$ & $0.87\left({ }_{-0.03}^{+0.03}\right)$ & $-3.52(0.02)$ & & $23.1(0.0)$ \\
\hline
\end{tabular}


Table 7. Atmospheric parameters for DQ stars - continued.

\begin{tabular}{|c|c|c|c|c|c|c|}
\hline $\mathrm{J}$ Name & $T_{\text {eff }}(\mathrm{K})$ & $\log g$ & $M / M_{\odot}$ & $\log \mathrm{C} / \mathrm{He}$ & $\log \mathrm{H} / \mathrm{He}$ & $D(\mathrm{pc})$ \\
\hline $\mathrm{J} 0859+6016$ & $7572(167)$ & $7.96\left({ }_{-0.06}^{+0.06}\right)$ & $0.55\left({ }_{-0.03}^{+0.03}\right)$ & $-5.64(0.05)$ & & $41.8(0.1)$ \\
\hline J0900+0331 & $7627(178)$ & $7.94\left({ }_{-0.08}^{+0.08}\right)$ & $0.54\left({ }_{-0.04}^{+0.05}\right)$ & $-5.87(0.07)$ & & $116.2(3.4)$ \\
\hline J0901+5751 & $13576(763)$ & $8.76\left({ }_{-0.09}^{+0.09}\right)$ & $1.07\left(\begin{array}{l}+0.05 \\
-0.05\end{array}\right)$ & $-1.99(0.24)$ & & $152.9(5.1)$ \\
\hline J0902+5037 & $7599(215)$ & $7.86\left({ }_{-0.13}^{+0.13}\right)$ & $0.50\left({ }_{-0.07}^{+0.08}\right)$ & $-5.81(0.19)$ & & $164.3(10.4)$ \\
\hline J0904+3954 & $7284(171)$ & $8.06\left({ }_{-0.14}^{+0.14}\right)$ & $0.61\left(\begin{array}{l}+0.09 \\
-0.08\end{array}\right)$ & $-5.79(0.11)$ & & $156.4(12.3)$ \\
\hline J0905+0904 & $8216(192)$ & $7.88\left({ }_{-0.06}^{+0.06}\right)$ & $0.51\left({ }_{-0.03}^{+0.03}\right)$ & $-5.41(0.04)$ & & $59.6(0.3)$ \\
\hline J0915+2019 & $8731(234)$ & $7.93\left({ }_{-0.16}^{+0.17}\right)$ & $0.54\left(\begin{array}{l}+0.10 \\
-0.09\end{array}\right)$ & $-5.09(0.14)$ & & $193.5(18.4)$ \\
\hline J0916+1011 & $8406(201)$ & $7.93\left({ }_{-0.06}^{+0.06}\right)$ & $0.53\left({ }_{-0.03}^{+0.03}\right)$ & $-5.21(0.03)$ & & $38.8(0.1)$ \\
\hline J0918+4843 & $9203(280)$ & $8.80\left({ }_{-0.14}^{+0.15}\right)$ & $1.09\left({ }_{-0.08}^{+0.08}\right)$ & $-3.72(0.11)$ & & $176.4(21.6)$ \\
\hline $\mathrm{J} 0919+0236$ & $11319(478)$ & $8.61\left({ }_{-0.10}^{+0.10}\right)$ & $0.98\left({ }_{-0.06}^{+0.06}\right)$ & $-2.85(0.07)$ & & $156.3(9.2)$ \\
\hline J0920+3603 & $7795(203)$ & $7.98\left({ }_{-0.11}^{+0.12}\right)$ & $0.56\left({ }_{-0.06}^{+0.07}\right)$ & $-5.72(0.10)$ & & $154.2(8.9)$ \\
\hline J0921+3421 & $8239(202)$ & $7.42\left({ }_{-0.11}^{+0.12}\right)$ & $0.30\left({ }_{-0.04}^{+0.05}\right)$ & $-5.20(0.06)$ & & $181.4(10.3)$ \\
\hline J0922+2928 & $8022(175)$ & $8.06\left({ }_{-0.09}^{+0.09}\right)$ & $0.61\left({ }_{-0.05}^{+0.06}\right)$ & $-4.93(0.04)$ & & $114.6(5.1)$ \\
\hline J0923+1842 & $6879(118)$ & 8.00 & & $-6.33(0.06)$ & & $89.9(2.8)$ \\
\hline J0925+5256 & $9505(251)$ & $7.92\left({ }_{-0.06}^{+0.07}\right)$ & $0.53\left({ }_{-0.03}^{+0.04}\right)$ & $-4.68(0.12)$ & & $126.0(2.2)$ \\
\hline J0926+0605 & $8404(217)$ & 8.00 & & $-5.42(0.15)$ & & $134.1(5.5)$ \\
\hline J0926+4725 & $7215(139)$ & $8.01\left({ }_{-0.06}^{+0.06}\right)$ & $0.58\left({ }_{-0.04}^{+0.04}\right)$ & $-6.31(0.09)$ & & $93.4(2.1)$ \\
\hline J0926+6212 & $7026(269)$ & $7.96\left({ }_{-0.53}^{+0.63}\right)$ & $0.55\left({ }_{-0.25}^{+0.41}\right)$ & $-5.89(0.09)$ & & $272.6(96.5)$ \\
\hline J0928+2638 & $7108(144)$ & $7.36\left({ }_{-0.07}^{+0.07}\right)$ & $0.28\left({ }_{-0.02}^{+0.03}\right)$ & $-6.42(0.07)$ & & $101.4(2.2)$ \\
\hline J0929+3310 & $6587(76)$ & $7.85\left({ }_{-0.06}^{+0.06}\right)$ & $0.49\left({ }_{-0.03}^{+0.04}\right)$ & $-5.17(0.02)$ & & $90.8(2.6)$ \\
\hline J0930+2959 & $8233(200)$ & $7.81\left({ }_{-0.14}^{+0.15}\right)$ & $0.47\left(_{-0.07}^{+0.08}\right)$ & $-5.28(0.07)$ & & $157.5(12.2)$ \\
\hline J0931+1230 & $6544(172)$ & 8.00 & & $-6.37(0.08)$ & & $177.2(8.3)$ \\
\hline J0934+1158 & $8421(217)$ & $7.81\left({ }_{-0.11}^{+0.11}\right)$ & $0.47\left({ }_{-0.05}^{+0.06}\right)$ & $-5.24(0.07)$ & & $159.7(8.7)$ \\
\hline $\mathrm{J} 0935+2417$ & $8697(224)$ & $7.93\left({ }_{-0.09}^{+0.09}\right)$ & $0.54\left({ }_{-0.05}^{+0.05}\right)$ & $-5.07(0.09)$ & & $126.6(4.7)$ \\
\hline J0936+0607 & $11013(421)$ & $8.61\left({ }_{-0.11}^{+0.11}\right)$ & $0.97\left({ }_{-0.07}^{+0.07}\right)$ & $-3.07(0.09)$ & & $162.0(12.1)$ \\
\hline J0939+5201 & $8527(201)$ & $7.96\left({ }_{-0.06}^{+0.06}\right)$ & $0.56\left(_{-0.03}^{+0.03}\right)$ & $-5.00(0.03)$ & & $73.6(0.6)$ \\
\hline $\mathrm{J} 0940+0210$ & $7229(134)$ & $8.12\left({ }_{-0.05}^{+0.05}\right)$ & $0.65\left({ }_{-0.03}^{+0.03}\right)$ & $-6.00(0.03)$ & & $52.0(0.3)$ \\
\hline J0941+0901 & $8550(200)$ & $7.32\left({ }_{-0.06}^{+0.06}\right)$ & $0.27\left({ }_{-0.02}^{+0.02}\right)$ & $-5.24(0.08)$ & & $89.5(1.0)$ \\
\hline J0941+4414 & $8222(203)$ & $7.98\left({ }_{-0.08}^{+0.08}\right)$ & $0.57\left({ }_{-0.05}^{+0.05}\right)$ & $-5.22(0.07)$ & & $134.5(4.6)$ \\
\hline J0945+5558 & $7371(212)$ & $8.12\left({ }_{-0.12}^{+0.12}\right)$ & $0.65\left({ }_{-0.07}^{+0.08}\right)$ & $-6.09(0.30)$ & & $168.2(10.4)$ \\
\hline J0948+1232 & $7218(146)$ & $8.16\left({ }_{-0.06}^{+0.06}\right)$ & $0.67\left({ }_{-0.04}^{+0.04}\right)$ & $-6.29(0.11)$ & & $80.5(1.3)$ \\
\hline $\mathrm{J} 0950+3238$ & 8268(199) & 8.00 & & $-5.54(0.06)$ & & $77.8(2.9)$ \\
\hline J0950+5315 & $8031(188)$ & $7.91\left({ }_{-0.06}^{+0.06}\right)$ & $0.52\left({ }_{-0.03}^{+0.04}\right)$ & $-5.62(0.04)$ & & $27.5(0.0)$ \\
\hline $\mathrm{J} 0951+6243$ & $9060(535)$ & $8.03\left({ }_{-0.18}^{+0.19}\right)$ & $0.60\left({ }_{-0.10}^{+0.12}\right)$ & $-4.60(0.08)$ & & $168.9(8.1)$ \\
\hline $\mathrm{J} 0959+4537$ & $7274(196)$ & $7.45\left({ }_{-0.29}^{+0.35}\right)$ & $0.31\left({ }_{-0.09}^{+0.15}\right)$ & $-5.48(0.12)$ & & $256.2(44.2)$ \\
\hline $\mathrm{J} 1000+1005$ & $7869(190)$ & 8.00 & & $-4.99(0.04)$ & & $155.4(6.4)$ \\
\hline $\mathrm{J} 1005-0114$ & $8376(233)$ & $7.85\left({ }_{-0.19}^{+0.20}\right)$ & $0.49\left(_{-0.09}^{+0.12}\right)$ & $-5.13(0.11)$ & & $196.5(21.4)$ \\
\hline $\mathrm{J} 1010+2300$ & $8534(204)$ & $7.89\left({ }_{-0.09}^{+0.09}\right)$ & $0.51\left({ }_{-0.05}^{+0.05}\right)$ & $-5.02(0.06)$ & & $137.3(5.4)$ \\
\hline
\end{tabular}


Table 7. Atmospheric parameters for DQ stars - continued.

\begin{tabular}{|c|c|c|c|c|c|c|}
\hline $\mathrm{J}$ Name & $T_{\text {eff }}(\mathrm{K})$ & $\log g$ & $M / M_{\odot}$ & $\log \mathrm{C} / \mathrm{He}$ & $\log \mathrm{H} / \mathrm{He}$ & $D(\mathrm{pc})$ \\
\hline $\mathrm{J} 1012+0040$ & $9184(229)$ & $7.75\left(\begin{array}{l}+0.07 \\
-0.07\end{array}\right)$ & $0.44\left({ }_{-0.03}^{+0.04}\right)$ & $-4.63(0.07)$ & & $119.2(3.3)$ \\
\hline $\mathrm{J} 1013+4350$ & $8569(263)$ & $7.95\left({ }_{-0.09}^{+0.10}\right)$ & $0.55\left({ }_{-0.05}^{+0.06}\right)$ & $-5.03(0.09)$ & & $144.1(5.5)$ \\
\hline $\mathrm{J} 1015+3518$ & $8097(208)$ & $7.79\left({ }_{-0.14}^{+0.15}\right)$ & $0.46\left(\begin{array}{l}+0.08 \\
-0.07\end{array}\right)$ & $-5.37(0.11)$ & & $173.0(12.7)$ \\
\hline $\mathrm{J} 1017+3736$ & $7184(137)$ & $7.95\left({ }_{-0.09}^{+0.09}\right)$ & $0.54\left({ }_{-0.05}^{+0.05}\right)$ & $-6.02(0.06)$ & & $124.0(5.3)$ \\
\hline $\mathrm{J} 1018+0838$ & $7576(164)$ & $7.93\left({ }_{-0.06}^{+0.06}\right)$ & $0.53\left({ }_{-0.03}^{+0.03}\right)$ & $-6.03(0.06)$ & & $43.8(0.2)$ \\
\hline $\mathrm{J} 1022+2845$ & $7244(162)$ & $7.94\left({ }_{-0.09}^{+0.10}\right)$ & $0.54\left({ }_{-0.05}^{+0.06}\right)$ & $-5.97(0.09)$ & & $139.7(6.1)$ \\
\hline $\mathrm{J} 1026+2725$ & $8270(223)$ & $7.95\left({ }_{-0.12}^{+0.12}\right)$ & $0.55\left(_{-0.07}^{+0.07}\right)$ & $-5.28(0.10)$ & & $150.9(9.5)$ \\
\hline $\mathrm{J} 1026+5807$ & $8133(189)$ & $7.90\left({ }_{-0.06}^{+0.06}\right)$ & $0.52\left({ }_{-0.03}^{+0.03}\right)$ & $-5.35(0.06)$ & & $69.1(0.5)$ \\
\hline $\mathrm{J} 1027+1218$ & $7230(146)$ & $8.11\left({ }_{-0.07}^{+0.07}\right)$ & $0.64\left(\begin{array}{l}+0.05 \\
-0.04\end{array}\right)$ & $-6.38(0.15)$ & & $97.1(3.0)$ \\
\hline $\mathrm{J} 1028+2537$ & $6672(115)$ & $7.91\left({ }_{-0.07}^{+0.07}\right)$ & $0.52\left({ }_{-0.04}^{+0.04}\right)$ & $-6.44(0.06)$ & & $109.7(2.8)$ \\
\hline $\mathrm{J} 1031+2217$ & $6986(172)$ & $8.57\left(\left(_{-0.17}^{+0.18}\right)\right.$ & $0.95\left(_{-0.11}^{+0.11}\right)$ & $-6.15(0.14)$ & & $135.9(18.2)$ \\
\hline $\mathrm{J} 1032+2101$ & $8193(212)$ & $8.00\left({ }_{-0.10}^{+0.11}\right)$ & $0.57\left({ }_{-0.06}^{+0.07}\right)$ & $-5.22(0.08)$ & & $166.9(8.7)$ \\
\hline $\mathrm{J} 1032+3509$ & $8332(223)$ & $7.86\left({ }_{-0.10}^{+0.10}\right)$ & $0.50\left({ }_{-0.05}^{+0.06}\right)$ & $-5.12(0.11)$ & & $146.8(6.0)$ \\
\hline $\mathrm{J} 1032+4519$ & $8905(227)$ & $7.86\left({ }_{-0.08}^{+0.08}\right)$ & $0.50\left({ }_{-0.04}^{+0.04}\right)$ & $-4.76(0.07)$ & & $126.1(3.6)$ \\
\hline $\mathrm{J} 1040+0635$ & $13882(849)$ & $8.40\left({ }_{-0.22}^{+0.24}\right)$ & $0.84\left(\begin{array}{l}+0.15 \\
-0.14\end{array}\right)$ & $-2.08(0.26)$ & & $277.3(42.8)$ \\
\hline $\mathrm{J} 1041+2200$ & $7608(347)$ & 8.00 & & $-5.69(0.19)$ & & $335.5(25.3)$ \\
\hline $\mathrm{J} 1042+5833$ & $7236(145)$ & $8.07\left({ }_{-0.08}^{+0.08}\right)$ & $0.62\left({ }_{-0.05}^{+0.05}\right)$ & $-5.88(0.07)$ & & $125.8(4.8)$ \\
\hline $\mathrm{J} 1043+0303$ & $6833(128)$ & $8.18\left({ }_{-0.11}^{+0.11}\right)$ & $0.69\left(_{-0.07}^{+0.07}\right)$ & $-6.25(0.10)$ & & $115.5(7.4)$ \\
\hline $\mathrm{J} 1045+2134$ & $7662(196)$ & $7.89\left({ }_{-0.11}^{+0.12}\right)$ & $0.51\left({ }_{-0.06}^{+0.07}\right)$ & $-5.72(0.10)$ & & $153.9(8.4)$ \\
\hline $\mathrm{J} 1047+5912$ & 8201(192) & $7.82\left({ }_{-0.06}^{+0.07}\right)$ & $0.48\left({ }_{-0.03}^{+0.04}\right)$ & $-5.39(0.08)$ & & $101.3(1.4)$ \\
\hline $\mathrm{J} 1049+1659$ & 12799(659) & $8.92\left({ }_{-0.11}^{+0.12}\right)$ & $1.15\left({ }_{-0.06}^{+0.05}\right)$ & $-1.64(0.09)$ & $-3.00^{*}$ & $173.6(14.9)$ \\
\hline $\mathrm{J} 1052+5911$ & $7903(204)$ & $7.90\left({ }_{-0.07}^{+0.08}\right)$ & $0.52\left({ }_{-0.04}^{+0.04}\right)$ & $-5.62(0.04)$ & & $113.8(2.0)$ \\
\hline $\mathrm{J} 1058+2846$ & $9422(265)$ & $8.49\left({ }_{-0.09}^{+0.09}\right)$ & $0.89\left({ }_{-0.06}^{+0.06}\right)$ & $-3.60(0.04)$ & & $154.3(8.9)$ \\
\hline $\mathrm{J} 1058+3440$ & $6779(161)$ & $8.15\left({ }_{-0.16}^{+0.17}\right)$ & $0.67\left({ }_{-0.10}^{+0.11}\right)$ & $-6.47(0.08)$ & & $165.7(16.9)$ \\
\hline $\mathrm{J} 1100+1758$ & $12367(465)$ & $8.76\left({ }_{-0.09}^{+0.10}\right)$ & $1.07\left({ }_{-0.05}^{+0.05}\right)$ & $-1.28(0.18)$ & $-3.00^{*}$ & $141.6(9.8)$ \\
\hline $\mathrm{J} 1101+5218$ & $8431(235)$ & $7.86\left({ }_{-0.12}^{+0.12}\right)$ & $0.50\left({ }_{-0.06}^{+0.07}\right)$ & $-5.11(0.10)$ & & $191.6(11.3)$ \\
\hline $\mathrm{J} 1107+4059$ & $7077(132)$ & $7.99\left({ }_{-0.05}^{+0.06}\right)$ & $0.57\left({ }_{-0.03}^{+0.03}\right)$ & $-6.46(0.04)$ & & $54.3(0.6)$ \\
\hline $\mathrm{J} 1108+1349$ & $7530(162)$ & $8.02\left({ }_{-0.06}^{+0.06}\right)$ & $0.59\left({ }_{-0.04}^{+0.04}\right)$ & $-6.18(0.07)$ & & $89.8(1.6)$ \\
\hline $\mathrm{J} 1109+4249$ & $8677(316)$ & $7.88\left({ }_{-0.09}^{+0.09}\right)$ & $0.51\left({ }_{-0.05}^{+0.05}\right)$ & $-5.34(0.09)$ & & $88.0(1.6)$ \\
\hline $\mathrm{J} 1113+4455$ & $8023(207)$ & $7.92\left({ }_{-0.12}^{+0.13}\right)$ & $0.53\left({ }_{-0.07}^{+0.08}\right)$ & $-5.52(0.09)$ & & $149.7(9.8)$ \\
\hline J1116-1228 & $8476(434)$ & $7.56\left({ }_{-0.62}^{+0.89}\right)$ & $0.35\left(_{-0.18}^{+0.51}\right)$ & $-4.69(0.19)$ & & $385.3(166.0)$ \\
\hline J1118-0314 & $9095(226)$ & $7.85\left({ }_{-0.06}^{+0.06}\right)$ & $0.50\left({ }_{-0.03}^{+0.03}\right)$ & $-4.85(0.05)$ & & $36.6(0.1)$ \\
\hline $\mathrm{J} 1120+1200$ & $8416(213)$ & $7.90\left({ }_{-0.09}^{+0.09}\right)$ & $0.52\left({ }_{-0.05}^{+0.05}\right)$ & $-5.33(0.12)$ & & $124.2(4.5)$ \\
\hline $\mathrm{J} 1120+4252$ & $7404(153)$ & $8.01\left({ }_{-0.08}^{+0.08}\right)$ & $0.58\left({ }_{-0.04}^{+0.05}\right)$ & $-5.96(0.10)$ & & $109.4(3.7)$ \\
\hline $\mathrm{J} 1120-1102$ & $9863(317)$ & $7.41\left({ }_{-0.36}^{+0.50}\right)$ & $0.31\left({ }_{-0.09}^{+0.22}\right)$ & $-3.88(0.14)$ & & $349.6(89.6)$ \\
\hline $\mathrm{J} 1123+3347$ & $7752(192)$ & $8.18\left({ }_{-0.13}^{+0.14}\right)$ & $0.69\left({ }_{-0.08}^{+0.09}\right)$ & $-5.69(0.09)$ & & $129.4(10.7)$ \\
\hline $\mathrm{J} 1126+3245$ & $9297(284)$ & 8.00 & & $-4.43(0.11)$ & & $165.1(7.1)$ \\
\hline $\mathrm{J} 1126+4419$ & $6997(134)$ & $8.02\left({ }_{-0.08}^{+0.08}\right)$ & $0.59\left({ }_{-0.05}^{+0.05}\right)$ & $-6.28(0.07)$ & & $121.4(4.5)$ \\
\hline
\end{tabular}


Table 7. Atmospheric parameters for DQ stars - continued.

\begin{tabular}{|c|c|c|c|c|c|c|}
\hline $\mathrm{J}$ Name & $T_{\text {eff }}(\mathrm{K})$ & $\log g$ & $M / M_{\odot}$ & $\log \mathrm{C} / \mathrm{He}$ & $\log \mathrm{H} / \mathrm{He}$ & $D(\mathrm{pc})$ \\
\hline J1130-0734 & $7925(260)$ & $8.13\left({ }_{-0.37}^{+0.42}\right)$ & $0.66\left({ }_{-0.21}^{+0.27}\right)$ & $-5.05(0.22)$ & & $234.9(60.1)$ \\
\hline $\mathrm{J} 1131+0736$ & $7530(170)$ & $7.82\left({ }_{-0.07}^{+0.08}\right)$ & $0.47\left({ }_{-0.04}^{+0.04}\right)$ & $-5.93(0.09)$ & & $116.5(2.7)$ \\
\hline $\mathrm{J} 1131+1845$ & $8396(252)$ & $7.90\left({ }_{-0.16}^{+0.16}\right)$ & $0.52\left(\begin{array}{l}+0.10 \\
-0.08\end{array}\right)$ & $-5.30(0.10)$ & & $153.9(13.0)$ \\
\hline $\mathrm{J} 1133+1900$ & $7821(196)$ & $7.64\left({ }_{-0.15}^{+0.16}\right)$ & $0.38\left({ }_{-0.06}^{+0.08}\right)$ & $-5.59(0.07)$ & & $184.1(14.8)$ \\
\hline $\mathrm{J} 1133+6331$ & $11517(562)$ & $8.57\left({ }_{-0.11}^{+0.11}\right)$ & $0.95\left(_{-0.07}^{+0.07}\right)$ & $-2.68(0.21)$ & & 193.6(11.0) \\
\hline $\mathrm{J} 1140+0735$ & $10651(387)$ & $8.56\left({ }_{-0.09}^{+0.09}\right)$ & $0.94\left({ }_{-0.06}^{+0.06}\right)$ & $-3.36(0.09)$ & & $160.9(9.6)$ \\
\hline $\mathrm{J} 1140+1540$ & $6472(116)$ & $7.96\left({ }_{-0.09}^{+0.09}\right)$ & $0.55\left(\begin{array}{l}+0.06 \\
-0.05\end{array}\right)$ & $-6.63(0.08)$ & & $115.9(5.1)$ \\
\hline $\mathrm{J} 1140+1824$ & $9656(228)$ & $8.35\left({ }_{-0.05}^{+0.05}\right)$ & $0.81\left({ }_{-0.03}^{+0.03}\right)$ & $-3.54(0.03)$ & & $94.8(1.4)$ \\
\hline $\mathrm{J} 1141+3836$ & $6004(48)$ & $8.30\left({ }_{-0.06}^{+0.06}\right)$ & $0.77\left({ }_{-0.04}^{+0.04}\right)$ & $-5.00(0.03)$ & & $79.1(2.6)$ \\
\hline $\mathrm{J} 1142+0352$ & $7702(234)$ & $7.72\left({ }_{-0.15}^{+0.16}\right)$ & $0.42\left({ }_{-0.07}^{+0.08}\right)$ & $-5.63(0.09)$ & & $182.4(14.2)$ \\
\hline $\mathrm{J} 1145-6450$ & $7951(351)$ & $8.01\left({ }_{-0.10}^{+0.10}\right)$ & $0.58\left({ }_{-0.06}^{+0.06}\right)$ & $-5.50(0.03)$ & & $4.6(0.0)$ \\
\hline $\mathrm{J} 1148-0126$ & $9680(222)$ & $8.46\left({ }_{-0.04}^{+0.04}\right)$ & $0.88\left({ }_{-0.03}^{+0.03}\right)$ & $-3.48(0.03)$ & & $68.1(0.7)$ \\
\hline $\mathrm{J} 1151+4527$ & $8698(208)$ & $7.88\left({ }_{-0.06}^{+0.06}\right)$ & $0.51\left({ }_{-0.03}^{+0.04}\right)$ & $-4.84(0.06)$ & & $106.4(1.6)$ \\
\hline $\mathrm{J} 1151-2732$ & $6436(148)$ & $7.97\left({ }_{-0.05}^{+0.06}\right)$ & $0.56\left(_{-0.03}^{+0.03}\right)$ & $-6.70(0.04)$ & & $25.2(0.0)$ \\
\hline $\mathrm{J} 1156+2212$ & $7022(117)$ & $8.14\left({ }_{-0.08}^{+0.08}\right)$ & $0.66\left({ }_{-0.05}^{+0.05}\right)$ & $-5.53(0.03)$ & & $110.0(4.9)$ \\
\hline J1159-4629 & $7101(249)$ & $8.02\left({ }_{-0.08}^{+0.09}\right)$ & $0.58\left({ }_{-0.05}^{+0.05}\right)$ & $-6.02(0.03)$ & & $63.1(0.5)$ \\
\hline $\mathrm{J} 1201+3400$ & $6134(62)$ & $8.22\left({ }_{-0.03}^{+0.03}\right)$ & $0.71\left({ }_{-0.02}^{+0.02}\right)$ & $-5.97(0.01)$ & & $40.5(0.2)$ \\
\hline $\mathrm{J} 1203+6451$ & $12359(455)$ & $8.77\left({ }_{-0.05}^{+0.05}\right)$ & $1.07\left({ }_{-0.03}^{+0.03}\right)$ & $-1.59(0.14)$ & & $87.3(0.8)$ \\
\hline $\mathrm{J} 1209+5355$ & $11721(649)$ & $8.53\left({ }_{-0.15}^{+0.16}\right)$ & $0.92\left({ }_{-0.10}^{+0.10}\right)$ & $-2.71(0.53)$ & & $225.9(20.8)$ \\
\hline $\mathrm{J} 1212+5452$ & $8186(195)$ & $7.95\left({ }_{-0.06}^{+0.06}\right)$ & $0.55\left(_{-0.03}^{+0.04}\right)$ & $-5.29(0.04)$ & & $94.5(1.0)$ \\
\hline $\mathrm{J} 1215+4700$ & $13230(764)$ & $8.87\left({ }_{-0.09}^{+0.09}\right)$ & $1.13\left({ }_{-0.05}^{+0.05}\right)$ & $-2.00(0.10)$ & $-3.00^{*}$ & $148.1(6.7)$ \\
\hline $\mathrm{J} 1220+2700$ & $6185(98)$ & $7.20\left({ }_{-0.09}^{+0.09}\right)$ & $0.22\left({ }_{-0.02}^{+0.03}\right)$ & $-7.29(0.13)$ & & $135.8(4.7)$ \\
\hline $\mathrm{J} 1225+4706$ & $6292(79)$ & $7.92\left({ }_{-0.07}^{+0.07}\right)$ & $0.53\left({ }_{-0.04}^{+0.04}\right)$ & $-5.35(0.05)$ & & $112.6(4.0)$ \\
\hline $\mathrm{J} 1233+1253$ & $6807(112)$ & $8.04\left({ }_{-0.05}^{+0.05}\right)$ & $0.60\left({ }_{-0.03}^{+0.03}\right)$ & $-6.52(0.04)$ & & $50.4(0.3)$ \\
\hline $\mathrm{J} 1235+3918$ & $9295(233)$ & $8.02\left({ }_{-0.05}^{+0.06}\right)$ & $0.59\left({ }_{-0.03}^{+0.03}\right)$ & $-4.45(0.05)$ & & $79.6(0.7)$ \\
\hline $\mathrm{J} 1236+3502$ & $9052(238)$ & $7.85\left({ }_{-0.08}^{+0.09}\right)$ & $0.49\left({ }_{-0.04}^{+0.05}\right)$ & $-4.66(0.16)$ & & $157.5(5.7)$ \\
\hline $\mathrm{J} 1237+4156$ & $6169(53)$ & $8.23\left({ }_{-0.03}^{+0.03}\right)$ & $0.72\left({ }_{-0.02}^{+0.02}\right)$ & $-5.24(0.01)$ & & $36.2(0.1)$ \\
\hline $\mathrm{J} 1240+2748$ & $8492(224)$ & $8.13\left({ }_{-0.38}^{+0.42}\right)$ & $0.66\left({ }_{-0.22}^{+0.28}\right)$ & $-4.05(0.11)$ & & $252.7(66.6)$ \\
\hline $\mathrm{J} 1240-0144$ & $8443(241)$ & $7.82\left(\begin{array}{l}+0.18 \\
-0.17\end{array}\right)$ & $0.47\left({ }_{-0.08}^{+0.10}\right)$ & $-5.32(0.18)$ & & $209.8(19.5)$ \\
\hline $\mathrm{J} 1243+1651$ & $10227(286)$ & $7.91\left({ }_{-0.07}^{+0.07}\right)$ & $0.53\left({ }_{-0.04}^{+0.04}\right)$ & $-4.00(0.09)$ & & $140.1(3.5)$ \\
\hline $\mathrm{J} 1243+3607$ & $8513(209)$ & $7.95\left({ }_{-0.07}^{+0.07}\right)$ & $0.55\left({ }_{-0.04}^{+0.04}\right)$ & $-4.94(0.08)$ & & $116.8(2.4)$ \\
\hline $\mathrm{J} 1244+2709$ & $7922(205)$ & $7.26\left({ }_{-0.08}^{+0.09}\right)$ & $0.25\left(_{-0.02}^{+0.03}\right)$ & $-5.07(0.05)$ & & $162.9(5.2)$ \\
\hline $\mathrm{J} 1247+4113$ & $8590(212)$ & $7.99\left({ }_{-0.06}^{+0.07}\right)$ & $0.57\left({ }_{-0.04}^{+0.04}\right)$ & $-4.96(0.06)$ & & $119.2(2.0)$ \\
\hline $\mathrm{J} 1249+2800$ & $7482(167)$ & $8.23\left({ }_{-0.07}^{+0.07}\right)$ & $0.72\left({ }_{-0.04}^{+0.05}\right)$ & $-6.37(0.24)$ & & $93.3(2.6)$ \\
\hline $\mathrm{J} 1249+3407$ & $8441(201)$ & $7.93\left({ }_{-0.06}^{+0.06}\right)$ & $0.54\left({ }_{-0.03}^{+0.04}\right)$ & $-5.16(0.04)$ & & $71.7(0.5)$ \\
\hline $\mathrm{J} 1250+0205$ & $7103(184)$ & $7.86\left({ }_{-0.16}^{+0.16}\right)$ & $0.49\left(_{-0.08}^{+0.10}\right)$ & $-6.04(0.07)$ & & $187.2(16.1)$ \\
\hline $\mathrm{J} 1251+4646$ & $8687(244)$ & $7.92\left({ }_{-0.07}^{+0.08}\right)$ & $0.53\left({ }_{-0.04}^{+0.04}\right)$ & $-4.92(0.09)$ & & $106.7(1.4)$ \\
\hline $\mathrm{J} 1252+1943$ & $6863(122)$ & $7.52\left({ }_{-0.08}^{+0.08}\right)$ & $0.33\left({ }_{-0.03}^{+0.03}\right)$ & $-6.49(0.04)$ & & $84.1(2.5)$ \\
\hline
\end{tabular}


Table 7. Atmospheric parameters for DQ stars - continued.

\begin{tabular}{|c|c|c|c|c|c|c|}
\hline $\mathrm{J}$ Name & $T_{\text {eff }}(\mathrm{K})$ & $\log g$ & $M / M_{\odot}$ & $\log \mathrm{C} / \mathrm{He}$ & $\log \mathrm{H} / \mathrm{He}$ & $D(\mathrm{pc})$ \\
\hline $\mathrm{J} 1253+0139$ & $8655(254)$ & $7.94\left({ }_{-0.12}^{+0.12}\right)$ & $0.55\left({ }_{-0.06}^{+0.07}\right)$ & $-4.74(0.14)$ & & $177.8(10.6)$ \\
\hline $\mathrm{J} 1257+5938$ & $7682(238)$ & $8.07\left({ }_{-0.15}^{+0.15}\right)$ & $0.62\left({ }_{-0.09}^{+0.10}\right)$ & $-5.56(0.18)$ & & $204.2(17.0)$ \\
\hline $\mathrm{J} 1302+0923$ & $7279(156)$ & $7.92\left({ }_{-0.07}^{+0.08}\right)$ & $0.53\left({ }_{-0.04}^{+0.04}\right)$ & $-5.91(0.05)$ & & $113.6(3.1)$ \\
\hline $\mathrm{J} 1309+4445$ & $7955(137)$ & $8.30\left({ }_{-0.05}^{+0.05}\right)$ & $0.77\left({ }_{-0.03}^{+0.03}\right)$ & $-4.36(0.03)$ & & $109.4(2.2)$ \\
\hline $\mathrm{J} 1313+3811$ & $6903(161)$ & $7.93\left({ }_{-0.18}^{+0.19}\right)$ & $0.54\left({ }_{-0.10}^{+0.12}\right)$ & $-6.34(0.15)$ & & $196.2(20.7)$ \\
\hline $\mathrm{J} 1315+4711$ & $7562(165)$ & $7.84\left({ }_{-0.06}^{+0.06}\right)$ & $0.49\left(_{-0.03}^{+0.03}\right)$ & $-6.01(0.05)$ & & $84.5(0.7)$ \\
\hline $\mathrm{J} 1316+0810$ & $6773(113)$ & $8.04\left({ }_{-0.06}^{+0.06}\right)$ & $0.60\left({ }_{-0.04}^{+0.04}\right)$ & $-6.66(0.07)$ & & $87.7(2.0)$ \\
\hline $\mathrm{J} 1319+1401$ & $7753(197)$ & $7.91\left({ }_{-0.09}^{+0.09}\right)$ & $0.52\left({ }_{-0.05}^{+0.05}\right)$ & $-5.57(0.09)$ & & $144.9(5.5)$ \\
\hline $\mathrm{J} 1322+3730$ & $7729(219)$ & $8.01\left({ }_{-0.09}^{+0.09}\right)$ & $0.58\left({ }_{-0.05}^{+0.06}\right)$ & $-5.85(0.12)$ & & $138.7(5.2)$ \\
\hline $\mathrm{J} 1328+3640$ & $8213(199)$ & $7.93\left({ }_{-0.07}^{+0.07}\right)$ & $0.54\left({ }_{-0.04}^{+0.04}\right)$ & $-5.14(0.03)$ & & $126.1(2.8)$ \\
\hline $\mathrm{J} 1329+0746$ & $8067(186)$ & $7.97\left({ }_{-0.06}^{+0.06}\right)$ & $0.56\left({ }_{-0.03}^{+0.04}\right)$ & $-5.51(0.05)$ & & $70.2(0.5)$ \\
\hline $\mathrm{J} 1331+6704$ & $8990(253)$ & $7.95\left({ }_{-0.08}^{+0.09}\right)$ & $0.55\left({ }_{-0.05}^{+0.05}\right)$ & $-4.63(0.08)$ & & $172.7(5.9)$ \\
\hline $\mathrm{J} 1332+2355$ & $14205(872)$ & $8.70\left({ }_{-0.13}^{+0.13}\right)$ & $1.03\left({ }_{-0.08}^{+0.08}\right)$ & $-1.76(0.12)$ & $-3.00^{*}$ & $226.8(19.4)$ \\
\hline $\mathrm{J} 1332+2740$ & $8316(200)$ & $7.97\left({ }_{-0.06}^{+0.06}\right)$ & $0.56\left(_{-0.03}^{+0.04}\right)$ & $-5.41(0.08)$ & & $71.8(0.6)$ \\
\hline $\mathrm{J} 1333+2357$ & $7847(199)$ & $7.97\left({ }_{-0.08}^{+0.09}\right)$ & $0.56\left({ }_{-0.05}^{+0.05}\right)$ & $-5.57(0.08)$ & & $131.7(4.4)$ \\
\hline $\mathrm{J} 1334+1622$ & $8757(216)$ & $7.97\left({ }_{-0.07}^{+0.07}\right)$ & $0.56\left(_{-0.04}^{+0.04}\right)$ & $-4.87(0.06)$ & & $118.4(2.4)$ \\
\hline $\mathrm{J} 1341+0346$ & $13765(1084)$ & $8.76\left({ }_{-0.20}^{+0.22}\right)$ & $1.07\left({ }_{-0.12}^{+0.11}\right)$ & $-2.18(0.13)$ & $-3.00^{*}$ & $215.5(33.9)$ \\
\hline $\mathrm{J} 1344+1849$ & $8137(201)$ & $7.79\left({ }_{-0.07}^{+0.08}\right)$ & $0.46\left(_{-0.04}^{+0.04}\right)$ & $-5.70(0.08)$ & & $115.3(2.2)$ \\
\hline $\mathrm{J} 1347+1528$ & $8306(228)$ & $8.19\left({ }_{-0.10}^{+0.10}\right)$ & $0.70\left({ }_{-0.06}^{+0.07}\right)$ & $-5.14(0.07)$ & & $147.3(7.7)$ \\
\hline $\mathrm{J} 1347+3817$ & $8118(213)$ & $8.08\left({ }_{-0.09}^{+0.09}\right)$ & $0.63\left({ }_{-0.06}^{+0.06}\right)$ & $-5.32(0.08)$ & & $156.3(6.7)$ \\
\hline $\mathrm{J} 1347+5019$ & $8195(169)$ & $7.89\left({ }_{-0.09}^{+0.09}\right)$ & $0.51\left({ }_{-0.05}^{+0.05}\right)$ & $-4.41(0.04)$ & & $154.2(6.2)$ \\
\hline $\mathrm{J} 1351+6623$ & $8935(219)$ & $7.97\left({ }_{-0.06}^{+0.06}\right)$ & $0.56\left({ }_{-0.03}^{+0.03}\right)$ & $-4.80(0.11)$ & & $100.2(0.9)$ \\
\hline $\mathrm{J} 1352+2218$ & $8000(208)$ & $7.94\left({ }_{-0.11}^{+0.11}\right)$ & $0.54\left({ }_{-0.06}^{+0.07}\right)$ & $-5.28(0.08)$ & & $169.6(9.2)$ \\
\hline $\mathrm{J} 1352+2658$ & $7849(265)$ & $7.94\left({ }_{-0.10}^{+0.10}\right)$ & $0.54\left({ }_{-0.06}^{+0.06}\right)$ & $-5.51(0.09)$ & & $154.0(6.6)$ \\
\hline $\mathrm{J} 1354+1217$ & $7756(219)$ & $7.58\left({ }_{-0.23}^{+0.26}\right)$ & $0.36\left(_{-0.09}^{+0.12}\right)$ & $-5.59(0.09)$ & & $215.9(28.5)$ \\
\hline $\mathrm{J} 1355+3636$ & $8059(169)$ & $7.91\left({ }_{-0.06}^{+0.06}\right)$ & $0.53\left({ }_{-0.03}^{+0.03}\right)$ & $-4.74(0.01)$ & & $85.7(0.8)$ \\
\hline J1356-0009 & $6555(85)$ & $8.08\left({ }_{-0.05}^{+0.05}\right)$ & $0.62\left({ }_{-0.03}^{+0.03}\right)$ & $-5.80(0.02)$ & & $78.1(1.3)$ \\
\hline $\mathrm{J} 1357+2949$ & $7581(185)$ & $7.96\left({ }_{-0.07}^{+0.08}\right)$ & $0.55\left({ }_{-0.04}^{+0.05}\right)$ & $-5.83(0.10)$ & & $119.1(2.7)$ \\
\hline $\mathrm{J} 1358+0552$ & $8360(223)$ & $7.88\left({ }_{-0.12}^{+0.12}\right)$ & $0.51\left({ }_{-0.06}^{+0.07}\right)$ & $-5.07(0.09)$ & & $170.6(10.3)$ \\
\hline J1400-0154 & $9394(237)$ & $8.69\left({ }_{-0.12}^{+0.12}\right)$ & $1.02\left({ }_{-0.07}^{+0.07}\right)$ & $-3.56(0.11)$ & & $146.0(13.6)$ \\
\hline $\mathrm{J} 1402+1113$ & $7238(250)$ & $8.00\left({ }_{-0.12}^{+0.12}\right)$ & $0.58\left({ }_{-0.07}^{+0.08}\right)$ & $-5.90(0.06)$ & & $114.7(3.4)$ \\
\hline $\mathrm{J} 1406+0148$ & $7713(186)$ & $7.91\left({ }_{-0.08}^{+0.08}\right)$ & $0.52\left({ }_{-0.04}^{+0.04}\right)$ & $-5.83(0.13)$ & & $112.9(2.8)$ \\
\hline $\mathrm{J} 1406+0204$ & $7680(186)$ & $7.35\left({ }_{-0.10}^{+0.11}\right)$ & $0.28\left({ }_{-0.03}^{+0.04}\right)$ & $-5.64(0.17)$ & & $177.1(7.8)$ \\
\hline $\mathrm{J} 1406+3402$ & $7042(137)$ & $7.43\left({ }_{-0.06}^{+0.07}\right)$ & $0.30\left({ }_{-0.02}^{+0.03}\right)$ & $-6.62(0.12)$ & & $99.5(1.3)$ \\
\hline $\mathrm{J} 1407+2039$ & $8387(209)$ & $7.73\left({ }_{-0.11}^{+0.11}\right)$ & $0.43\left({ }_{-0.05}^{+0.06}\right)$ & $-5.11(0.08)$ & & $157.9(8.3)$ \\
\hline $\mathrm{J} 1416+3016$ & $7518(253)$ & $8.15\left({ }_{-0.26}^{+0.28}\right)$ & $0.67\left({ }_{-0.16}^{+0.19}\right)$ & $-5.87(0.10)$ & & $224.4(38.6)$ \\
\hline $\mathrm{J} 1417+2412$ & $7930(194)$ & $8.06\left({ }_{-0.07}^{+0.07}\right)$ & $0.61\left({ }_{-0.04}^{+0.05}\right)$ & $-5.48(0.09)$ & & $119.9(3.2)$ \\
\hline $\mathrm{J} 1423+5729$ & $10727(397)$ & $8.07\left({ }_{-0.09}^{+0.09}\right)$ & $0.62\left({ }_{-0.05}^{+0.06}\right)$ & $-3.67(0.12)$ & & $174.7(5.9)$ \\
\hline
\end{tabular}


Table 7. Atmospheric parameters for DQ stars - continued.

\begin{tabular}{|c|c|c|c|c|c|c|}
\hline $\mathrm{J}$ Name & $T_{\text {eff }}(\mathrm{K})$ & $\log g$ & $M / M_{\odot}$ & $\log \mathrm{C} / \mathrm{He}$ & $\log \mathrm{H} / \mathrm{He}$ & $D(\mathrm{pc})$ \\
\hline $\mathrm{J} 1424+0833$ & $9094(226)$ & $7.85\left({ }_{-0.06}^{+0.07}\right)$ & $0.49\left({ }_{-0.03}^{+0.04}\right)$ & $-4.79(0.07)$ & & $112.8(2.1)$ \\
\hline $\mathrm{J} 1425+1801$ & $7924(204)$ & $7.86\left({ }_{-0.09}^{+0.09}\right)$ & $0.50\left({ }_{-0.05}^{+0.05}\right)$ & $-5.44(0.07)$ & & $132.0(4.5)$ \\
\hline $\mathrm{J} 1427+6110$ & $6461(96)$ & $7.98\left({ }_{-0.05}^{+0.05}\right)$ & $0.56\left({ }_{-0.03}^{+0.03}\right)$ & $-6.63(0.02)$ & & $43.0(0.1)$ \\
\hline $\mathrm{J} 1428+3238$ & $10718(355)$ & $8.56\left({ }_{-0.08}^{+0.08}\right)$ & $0.94\left({ }_{-0.05}^{+0.05}\right)$ & $-3.20(0.16)$ & & $159.0(7.3)$ \\
\hline $\mathrm{J} 1431+3750$ & $6319(132)$ & $7.37\left({ }_{-0.11}^{+0.12}\right)$ & $0.27\left({ }_{-0.04}^{+0.04}\right)$ & $-6.97(0.13)$ & & $158.2(7.9)$ \\
\hline $\mathrm{J} 1434+2258$ & $14575(788)$ & $8.75\left({ }_{-0.10}^{+0.11}\right)$ & $1.06\left({ }_{-0.06}^{+0.06}\right)$ & $-1.14(0.12)$ & $-3.00^{*}$ & $206.8(14.4)$ \\
\hline $\mathrm{J} 1435+2120$ & $8832(275)$ & $7.93\left({ }_{-0.14}^{+0.14}\right)$ & $0.54\left(\begin{array}{l}+0.09 \\
-0.07\end{array}\right)$ & $-4.77(0.13)$ & & $200.0(14.9)$ \\
\hline $\mathrm{J} 1435+4554$ & $6980(181)$ & $7.99\left({ }_{-0.13}^{+0.13}\right)$ & $0.57\left({ }_{-0.07}^{+0.08}\right)$ & $-6.18(0.12)$ & & $180.3(11.9)$ \\
\hline $\mathrm{J} 1435+5318$ & 15167(1164) & $8.85\left({ }_{-0.11}^{+0.12}\right)$ & $1.12\left({ }_{-0.06}^{+0.06}\right)$ & $-1.91(0.16)$ & $-3.00^{*}$ & $199.8(10.0)$ \\
\hline $\mathrm{J} 1438+2234$ & $8310(221)$ & $7.79\left({ }_{-0.14}^{+0.14}\right)$ & $0.46\left(_{-0.07}^{+0.08}\right)$ & $-5.37(0.14)$ & & $190.4(13.6)$ \\
\hline $\mathrm{J} 1440+0958$ & $8100(189)$ & $7.93\left({ }_{-0.06}^{+0.06}\right)$ & $0.54\left(\begin{array}{l}+0.04 \\
-0.03\end{array}\right)$ & $-5.49(0.06)$ & & $72.6(0.8)$ \\
\hline $\mathrm{J} 1441+2347$ & $7233(150)$ & $8.11\left({ }_{-0.11}^{+0.11}\right)$ & $0.64\left({ }_{-0.07}^{+0.07}\right)$ & $-5.76(0.07)$ & & $128.8(8.0)$ \\
\hline $\mathrm{J} 1442+4202$ & $8636(231)$ & $7.89\left({ }_{-0.12}^{+0.12}\right)$ & $0.52\left({ }_{-0.06}^{+0.07}\right)$ & $-4.91(0.09)$ & & $189.8(11.4)$ \\
\hline $\mathrm{J} 1443+3810$ & $8843(216)$ & $7.89\left({ }_{-0.06}^{+0.07}\right)$ & $0.52\left({ }_{-0.03}^{+0.04}\right)$ & $-4.86(0.09)$ & & $137.4(2.5)$ \\
\hline $\mathrm{J} 1444+0434$ & $9813(301)$ & $8.44\left({ }_{-0.18}^{+0.19}\right)$ & $0.87\left({ }_{-0.12}^{+0.12}\right)$ & $-3.47(0.09)$ & & $191.8(25.3)$ \\
\hline $\mathrm{J} 1448-0047$ & $7075(129)$ & $8.05\left({ }_{-0.06}^{+0.06}\right)$ & $0.60\left({ }_{-0.04}^{+0.04}\right)$ & $-6.31(0.08)$ & & $89.5(2.0)$ \\
\hline $\mathrm{J} 1449+2916$ & $7873(217)$ & $7.97\left({ }_{-0.12}^{+0.12}\right)$ & $0.56\left({ }_{-0.07}^{+0.07}\right)$ & $-5.77(0.12)$ & & $159.5(9.3)$ \\
\hline $\mathrm{J} 1452+6020$ & $12572(659)$ & $8.65\left({ }_{-0.12}^{+0.13}\right)$ & $1.00\left({ }_{-0.08}^{+0.08}\right)$ & $-1.73(0.43)$ & & $239.2(19.7)$ \\
\hline $\mathrm{J} 1453+4719$ & $7928(313)$ & $8.28\left({ }_{-0.18}^{+0.19}\right)$ & $0.75\left(_{-0.12}^{+0.13}\right)$ & $-5.39(0.16)$ & & $216.1(25.1)$ \\
\hline $\mathrm{J} 1455+4209$ & $14288(876)$ & $8.78\left({ }_{-0.13}^{+0.14}\right)$ & $1.08\left({ }_{-0.08}^{+0.07}\right)$ & $-1.48(0.77)$ & & $266.1(25.4)$ \\
\hline $\mathrm{J} 1501+0627$ & $7116(133)$ & $8.16\left({ }_{-0.07}^{+0.08}\right)$ & $0.67\left({ }_{-0.05}^{+0.05}\right)$ & $-6.08(0.07)$ & & $107.8(4.2)$ \\
\hline $\mathrm{J} 1509+1620$ & $8356(221)$ & $7.88\left({ }_{-0.14}^{+0.14}\right)$ & $0.51\left({ }_{-0.07}^{+0.08}\right)$ & $-5.33(0.12)$ & & $160.3(12.1)$ \\
\hline $\mathrm{J} 1511+5008$ & $7779(197)$ & $7.96\left({ }_{-0.08}^{+0.08}\right)$ & $0.55\left({ }_{-0.05}^{+0.05}\right)$ & $-5.62(0.15)$ & & $146.7(4.3)$ \\
\hline $\mathrm{J} 1514+1423$ & $6661(84)$ & $8.14\left({ }_{-0.07}^{+0.07}\right)$ & $0.66\left({ }_{-0.04}^{+0.05}\right)$ & $-4.94(0.05)$ & & $115.2(4.3)$ \\
\hline $\mathrm{J} 1517+2256$ & $7464(154)$ & $7.98\left({ }_{-0.06}^{+0.06}\right)$ & $0.57\left({ }_{-0.03}^{+0.03}\right)$ & $-6.07(0.06)$ & & $79.3(0.8)$ \\
\hline $\mathrm{J} 1519+3856$ & $8250(213)$ & $7.91\left({ }_{-0.09}^{+0.09}\right)$ & $0.53\left({ }_{-0.05}^{+0.05}\right)$ & $-5.34(0.10)$ & & $169.0(5.9)$ \\
\hline $\mathrm{J} 1520+2703$ & $7800(219)$ & $7.78\left({ }_{-0.12}^{+0.13}\right)$ & $0.45\left(_{-0.06}^{+0.07}\right)$ & $-5.61(0.07)$ & & $173.1(9.6)$ \\
\hline $\mathrm{J} 1527+2752$ & $8278(156)$ & $8.32\left({ }_{-0.07}^{+0.07}\right)$ & $0.78\left({ }_{-0.05}^{+0.05}\right)$ & $-4.12(0.04)$ & & $133.0(5.0)$ \\
\hline $\mathrm{J} 1528+0442$ & $7303(166)$ & $7.90\left({ }_{-0.12}^{+0.13}\right)$ & $0.52\left({ }_{-0.06}^{+0.07}\right)$ & $-6.03(0.17)$ & & $142.0(9.0)$ \\
\hline $\mathrm{J} 1528+4003$ & $7588(198)$ & $8.00\left({ }_{-0.08}^{+0.08}\right)$ & $0.57\left({ }_{-0.05}^{+0.05}\right)$ & $-5.83(0.09)$ & & $139.4(4.2)$ \\
\hline $\mathrm{J} 1528+5134$ & $7578(167)$ & $8.01\left({ }_{-0.06}^{+0.06}\right)$ & $0.58\left({ }_{-0.04}^{+0.04}\right)$ & $-5.65(0.04)$ & & $103.8(1.5)$ \\
\hline $\mathrm{J} 1534+4145$ & $7684(172)$ & $7.92\left({ }_{-0.06}^{+0.06}\right)$ & $0.53\left({ }_{-0.03}^{+0.04}\right)$ & $-6.10(0.07)$ & & $65.8(0.3)$ \\
\hline $\mathrm{J} 1537+1337$ & $7952(192)$ & $7.89\left({ }_{-0.08}^{+0.08}\right)$ & $0.52\left({ }_{-0.04}^{+0.05}\right)$ & $-5.53(0.08)$ & & $117.6(3.4)$ \\
\hline $\mathrm{J} 1542+1314$ & $7952(191)$ & $7.96\left({ }_{-0.07}^{+0.08}\right)$ & $0.55\left({ }_{-0.04}^{+0.05}\right)$ & $-5.44(0.04)$ & & $116.9(3.1)$ \\
\hline $\mathrm{J} 1542+2544$ & $6682(121)$ & $8.03\left({ }_{-0.08}^{+0.08}\right)$ & $0.59\left({ }_{-0.05}^{+0.05}\right)$ & $-6.44(0.07)$ & & $121.4(4.3)$ \\
\hline $\mathrm{J} 1542+4329$ & $9799(287)$ & $8.49\left({ }_{-0.08}^{+0.09}\right)$ & $0.89\left(_{-0.06}^{+0.06}\right)$ & $-3.61(0.16)$ & & $175.2(9.1)$ \\
\hline $\mathrm{J} 1545+2854$ & $7260(156)$ & $7.94\left({ }_{-0.09}^{+0.10}\right)$ & $0.54\left({ }_{-0.05}^{+0.06}\right)$ & $-5.81(0.13)$ & & $158.4(7.2)$ \\
\hline $\mathrm{J} 1548+5626$ & $7911(208)$ & $8.03\left({ }_{-0.08}^{+0.08}\right)$ & $0.60\left({ }_{-0.05}^{+0.05}\right)$ & $-5.42(0.12)$ & & $145.9(4.3)$ \\
\hline
\end{tabular}


Table 7. Atmospheric parameters for DQ stars - continued.

\begin{tabular}{|c|c|c|c|c|c|c|}
\hline $\mathrm{J}$ Name & $T_{\text {eff }}(\mathrm{K})$ & $\log g$ & $M / M_{\odot}$ & $\log \mathrm{C} / \mathrm{He}$ & $\log \mathrm{H} / \mathrm{He}$ & $D(\mathrm{pc})$ \\
\hline $\mathrm{J} 1551+0824$ & $6768(109)$ & $7.98\left({ }_{-0.05}^{+0.05}\right)$ & $0.56\left({ }_{-0.03}^{+0.03}\right)$ & $-6.28(0.03)$ & & $71.9(0.7)$ \\
\hline $\mathrm{J} 1552+1148$ & $7764(205)$ & $8.00\left({ }_{-0.10}^{+0.10}\right)$ & $0.58\left({ }_{-0.06}^{+0.06}\right)$ & $-5.54(0.10)$ & & $140.0(6.3)$ \\
\hline $\mathrm{J} 1552+3910$ & $7570(169)$ & $8.04\left({ }_{-0.06}^{+0.07}\right)$ & $0.60\left({ }_{-0.04}^{+0.04}\right)$ & $-5.67(0.08)$ & & $113.7(2.3)$ \\
\hline $\mathrm{J} 1554+0336$ & $6626(117)$ & $7.94\left({ }_{-0.07}^{+0.07}\right)$ & $0.54\left({ }_{-0.04}^{+0.04}\right)$ & $-6.78(0.13)$ & & $102.5(2.7)$ \\
\hline $\mathrm{J} 1555+3123$ & $8227(156)$ & $8.26\left({ }_{-0.06}^{+0.06}\right)$ & $0.74\left({ }_{-0.04}^{+0.04}\right)$ & $-4.29(0.04)$ & & $128.6(3.7)$ \\
\hline $\mathrm{J} 1555+3219$ & $9195(239)$ & $8.59\left({ }_{-0.11}^{+0.11}\right)$ & $0.96\left(_{-0.07}^{+0.07}\right)$ & $-3.74(0.31)$ & & $184.2(15.1)$ \\
\hline $\mathrm{J} 1609+0655$ & $6828(333)$ & $7.76\left({ }_{-0.11}^{+0.11}\right)$ & $0.44\left(\begin{array}{l}+0.06 \\
-0.05\end{array}\right)$ & $-6.73(0.07)$ & & $85.6(0.9)$ \\
\hline $\mathrm{J} 1610+3036$ & $8054(207)$ & $7.97\left({ }_{-0.09}^{+0.09}\right)$ & $0.56\left({ }_{-0.05}^{+0.05}\right)$ & $-5.27(0.06)$ & & $159.5(5.8)$ \\
\hline $\mathrm{J} 1613+5116$ & $7979(226)$ & $8.00\left({ }_{-0.10}^{+0.10}\right)$ & $0.58\left({ }_{-0.06}^{+0.06}\right)$ & $-5.42(0.13)$ & & $161.3(6.8)$ \\
\hline $\mathrm{J} 1616+3924$ & $7225(139)$ & $7.95\left({ }_{-0.05}^{+0.06}\right)$ & $0.55\left({ }_{-0.03}^{+0.03}\right)$ & $-6.07(0.05)$ & & $93.6(1.0)$ \\
\hline $\mathrm{J} 1620+1809$ & $7763(180)$ & $7.88\left({ }_{-0.07}^{+0.07}\right)$ & $0.51\left({ }_{-0.04}^{+0.04}\right)$ & $-5.79(0.06)$ & & $100.8(1.6)$ \\
\hline $\mathrm{J} 1621+2253$ & $7839(209)$ & $7.89\left({ }_{-0.08}^{+0.08}\right)$ & $0.51\left({ }_{-0.04}^{+0.05}\right)$ & $-5.82(0.10)$ & & $123.3(2.5)$ \\
\hline $\mathrm{J} 1634+5710$ & $6006(119)$ & $7.97\left({ }_{-0.06}^{+0.06}\right)$ & $0.55\left({ }_{-0.03}^{+0.03}\right)$ & $-7.28(0.03)$ & & $14.9(0.0)$ \\
\hline $\mathrm{J} 1638+1244$ & $8532(204)$ & $7.90\left({ }_{-0.06}^{+0.06}\right)$ & $0.52\left({ }_{-0.03}^{+0.04}\right)$ & $-5.07(0.04)$ & & $101.4(1.4)$ \\
\hline $\mathrm{J} 1640+7310$ & $8426(275)$ & $7.95\left({ }_{-0.07}^{+0.07}\right)$ & $0.55\left({ }_{-0.04}^{+0.04}\right)$ & $-4.98(0.05)$ & & $53.7(0.1)$ \\
\hline $\mathrm{J} 1641+4833$ & $7652(172)$ & $8.01\left({ }_{-0.06}^{+0.06}\right)$ & $0.58\left({ }_{-0.03}^{+0.04}\right)$ & $-5.80(0.04)$ & & $91.4(1.0)$ \\
\hline $\mathrm{J} 1643+4002$ & $7352(169)$ & $8.11\left({ }_{-0.08}^{+0.09}\right)$ & $0.64\left(\begin{array}{l}+0.06 \\
-0.05\end{array}\right)$ & $-6.05(0.17)$ & & $137.4(5.3)$ \\
\hline $\mathrm{J} 1643+4129$ & $7224(310)$ & $8.43\left({ }_{-0.36}^{+0.40}\right)$ & $0.86\left(_{-0.23}^{+0.25}\right)$ & $-6.02(0.14)$ & & $219.6(60.1)$ \\
\hline $\mathrm{J} 1647+4350$ & $9041(238)$ & $7.88\left({ }_{-0.07}^{+0.07}\right)$ & $0.51\left({ }_{-0.04}^{+0.04}\right)$ & $-4.83(0.11)$ & & $157.5(3.6)$ \\
\hline $\mathrm{J} 1647+5119$ & $8091(222)$ & $7.89\left({ }_{-0.09}^{+0.09}\right)$ & $0.51\left({ }_{-0.05}^{+0.05}\right)$ & $-5.61(0.11)$ & & $150.5(4.6)$ \\
\hline $\mathrm{J} 1653+1113$ & $6690(160)$ & $8.08\left({ }_{-0.12}^{+0.12}\right)$ & $0.63\left({ }_{-0.07}^{+0.08}\right)$ & $-6.58(0.10)$ & & $143.0(9.6)$ \\
\hline $\mathrm{J} 1654+3157$ & $7281(140)$ & $8.01\left({ }_{-0.05}^{+0.05}\right)$ & $0.58\left({ }_{-0.03}^{+0.03}\right)$ & $-5.89(0.03)$ & & $63.4(0.3)$ \\
\hline $\mathrm{J} 1655+3722$ & $8831(220)$ & $7.91\left({ }_{-0.07}^{+0.07}\right)$ & $0.53\left({ }_{-0.04}^{+0.04}\right)$ & $-4.79(0.07)$ & & $147.3(3.5)$ \\
\hline $\mathrm{J} 1712+3406$ & $7982(213)$ & $7.90\left({ }_{-0.10}^{+0.11}\right)$ & $0.52\left({ }_{-0.06}^{+0.06}\right)$ & $-5.47(0.10)$ & & $180.5(8.4)$ \\
\hline $\mathrm{J} 1713+3240$ & $7908(182)$ & $7.97\left({ }_{-0.06}^{+0.06}\right)$ & $0.56\left({ }_{-0.03}^{+0.04}\right)$ & $-5.38(0.03)$ & & $69.6(0.3)$ \\
\hline $\mathrm{J} 1724+5333$ & $6660(164)$ & $8.13\left({ }_{-0.15}^{+0.16}\right)$ & $0.66\left({ }_{-0.09}^{+0.10}\right)$ & $-6.35(0.22)$ & & $178.2(16.4)$ \\
\hline $\mathrm{J} 1728+5558$ & $14453(683)$ & $8.90\left({ }_{-0.06}^{+0.06}\right)$ & $1.14\left({ }_{-0.03}^{+0.03}\right)$ & $-1.37(0.07)$ & $-3.00^{*}$ & $47.2(0.1)$ \\
\hline $\mathrm{J} 1833+1945$ & $7124(133)$ & $7.97\left({ }_{-0.05}^{+0.05}\right)$ & $0.56\left({ }_{-0.03}^{+0.03}\right)$ & $-6.20(0.03)$ & & $39.3(0.1)$ \\
\hline $\mathrm{J} 1838+4046$ & $7126(229)$ & $8.43\left({ }_{-0.29}^{+0.31}\right)$ & $0.86\left({ }_{-0.19}^{+0.20}\right)$ & $-5.76(0.13)$ & & $199.5(43.6)$ \\
\hline $\mathrm{J} 2046-0715$ & $8025(210)$ & $7.75\left({ }_{-0.10}^{+0.10}\right)$ & $0.44\left({ }_{-0.05}^{+0.05}\right)$ & $-5.45(0.16)$ & & $162.7(6.6)$ \\
\hline $\mathrm{J} 2053-0702$ & $6584(80)$ & $8.04\left({ }_{-0.06}^{+0.06}\right)$ & $0.60\left({ }_{-0.04}^{+0.04}\right)$ & $-5.27(0.04)$ & & $98.1(2.9)$ \\
\hline $\mathrm{J} 2101+3148$ & $9100(348)$ & $7.86\left({ }_{-0.07}^{+0.07}\right)$ & $0.50\left({ }_{-0.04}^{+0.04}\right)$ & $-4.97(0.08)$ & & $32.2(0.0)$ \\
\hline $\mathrm{J} 2111-0036$ & $7199(141)$ & $7.91\left({ }_{-0.06}^{+0.06}\right)$ & $0.52\left({ }_{-0.04}^{+0.04}\right)$ & $-6.17(0.08)$ & & $94.5(1.9)$ \\
\hline $\mathrm{J} 2135+0003$ & $6427(110)$ & $7.93\left({ }_{-0.20}^{+0.21}\right)$ & $0.53\left({ }_{-0.11}^{+0.13}\right)$ & $-6.53(0.08)$ & & $120.7(14.5)$ \\
\hline $\mathrm{J} 2140-0045$ & $7524(192)$ & $8.02\left({ }_{-0.12}^{+0.12}\right)$ & $0.58\left({ }_{-0.07}^{+0.08}\right)$ & $-5.90(0.07)$ & & $154.3(9.9)$ \\
\hline $\mathrm{J} 2142+0908$ & $7211(169)$ & $8.14\left({ }_{-0.17}^{+0.18}\right)$ & $0.66\left({ }_{-0.11}^{+0.12}\right)$ & $-5.92(0.06)$ & & $131.0(14.6)$ \\
\hline $\mathrm{J} 2142+2059$ & $7515(262)$ & $7.86\left({ }_{-0.08}^{+0.08}\right)$ & $0.50\left({ }_{-0.04}^{+0.04}\right)$ & $-6.29(0.06)$ & & $11.0(0.0)$ \\
\hline $\mathrm{J} 2149+2039$ & $8663(238)$ & $7.63\left({ }_{-0.18}^{+0.20}\right)$ & $0.39\left({ }_{-0.07}^{+0.10}\right)$ & $-4.72(0.08)$ & & $211.5(22.0)$ \\
\hline
\end{tabular}


Analysis of Polluted Helium-Rich White Dwarfs

Table 7. Atmospheric parameters for DQ stars - continued.

\begin{tabular}{|c|c|c|c|c|c|c|}
\hline J Name & $T_{\text {eff }}(\mathrm{K})$ & $\log g$ & $M / M_{\odot}$ & $\log \mathrm{C} / \mathrm{He}$ & $\log \mathrm{H} / \mathrm{He}$ & $D(\mathrm{pc})$ \\
\hline $\mathrm{J} 2150-0113$ & $8785(213)$ & $7.94\left({ }_{-0.06}^{+0.06}\right)$ & $0.54\left({ }_{-0.04}^{+0.04}\right)$ & $-5.06(0.06)$ & & $90.4(1.5)$ \\
\hline $\mathrm{J} 2156+0559$ & $6911(127)$ & $8.07\left(\begin{array}{l}+0.06 \\
-0.06\end{array}\right)$ & $0.62\left(\begin{array}{l}+0.04 \\
-0.04\end{array}\right)$ & $-6.38(0.06)$ & & $97.2(2.3)$ \\
\hline $\mathrm{J} 2157+1137$ & $8522(206)$ & $7.80\left(\begin{array}{l}+0.10 \\
-0.09\end{array}\right)$ & $0.47\left(\begin{array}{l}+0.05 \\
-0.05\end{array}\right)$ & $-4.99(0.06)$ & & $151.9(6.7)$ \\
\hline $\mathrm{J} 2207+0135$ & $6514(131)$ & $8.12\left({ }_{-0.09}^{+0.09}\right)$ & $0.65\left(_{-0.05}^{+0.06}\right)$ & $-6.67(0.08)$ & & $115.6(5.1)$ \\
\hline $\mathrm{J} 2218+2123$ & $8120(188)$ & $7.95\left({ }_{-0.06}^{+0.06}\right)$ & $0.55\left({ }_{-0.04}^{+0.04}\right)$ & $-5.29(0.04)$ & & $96.5(1.5)$ \\
\hline $\mathrm{J} 2220+1420$ & $8542(228)$ & $8.03\left({ }_{-0.09}^{+0.09}\right)$ & $0.60\left(\begin{array}{l}+0.06 \\
-0.05\end{array}\right)$ & $-4.98(0.05)$ & & $134.6(5.6)$ \\
\hline $\mathrm{J} 2225+1251$ & $6388(176)$ & $7.82\left({ }_{-0.22}^{+0.24}\right)$ & $0.47\left({ }_{-0.11}^{+0.14}\right)$ & $-6.86(0.08)$ & & $155.6(19.5)$ \\
\hline $\mathrm{J} 2239+2303$ & $8116(239)$ & $7.99\left(\begin{array}{l}+0.14 \\
-0.13\end{array}\right)$ & $0.57\left(\begin{array}{l}+0.09 \\
-0.08\end{array}\right)$ & $-5.51(0.14)$ & & $177.6(12.9)$ \\
\hline $\mathrm{J} 2248+2826$ & $9390(311)$ & $7.98\left({ }_{-0.16}^{+0.16}\right)$ & $0.57\left({ }_{-0.09}^{+0.10}\right)$ & $-4.21(0.12)$ & $-3.00^{*}$ & $233.7(21.1)$ \\
\hline $\mathrm{J} 2250+1240$ & $9801(292)$ & $8.50\left({ }_{-0.13}^{+0.13}\right)$ & $0.90\left(\begin{array}{l}+0.08 \\
-0.08\end{array}\right)$ & $-3.66(0.15)$ & & $173.1(15.9)$ \\
\hline $\mathrm{J} 2302+2430$ & $6816(116)$ & $8.06\left({ }_{-0.05}^{+0.05}\right)$ & $0.61\left({ }_{-0.03}^{+0.03}\right)$ & $-6.54(0.04)$ & & $72.5(0.9)$ \\
\hline $\mathrm{J} 2310-0057$ & $7647(138)$ & $7.94\left({ }_{-0.11}^{+0.12}\right)$ & $0.54\left({ }_{-0.06}^{+0.07}\right)$ & $-4.63(0.02)$ & & $143.9(9.2)$ \\
\hline $\mathrm{J} 2314-0632$ & $7352(197)$ & $7.96\left({ }_{-0.06}^{+0.07}\right)$ & $0.55\left(_{-0.04}^{+0.04}\right)$ & $-6.06(0.03)$ & & $25.9(0.0)$ \\
\hline $\mathrm{J} 2341-0101$ & $8499(220)$ & $7.93\left({ }_{-0.08}^{+0.08}\right)$ & $0.54\left({ }_{-0.04}^{+0.05}\right)$ & $-5.07(0.10)$ & & $122.6(3.6)$ \\
\hline $\mathrm{J} 2354+4027$ & $7555(232)$ & $7.93\left({ }_{-0.07}^{+0.07}\right)$ & $0.53\left({ }_{-0.04}^{+0.04}\right)$ & $-5.75(0.04)$ & & $22.1(0.0)$ \\
\hline
\end{tabular}


9. APPENDIX I 

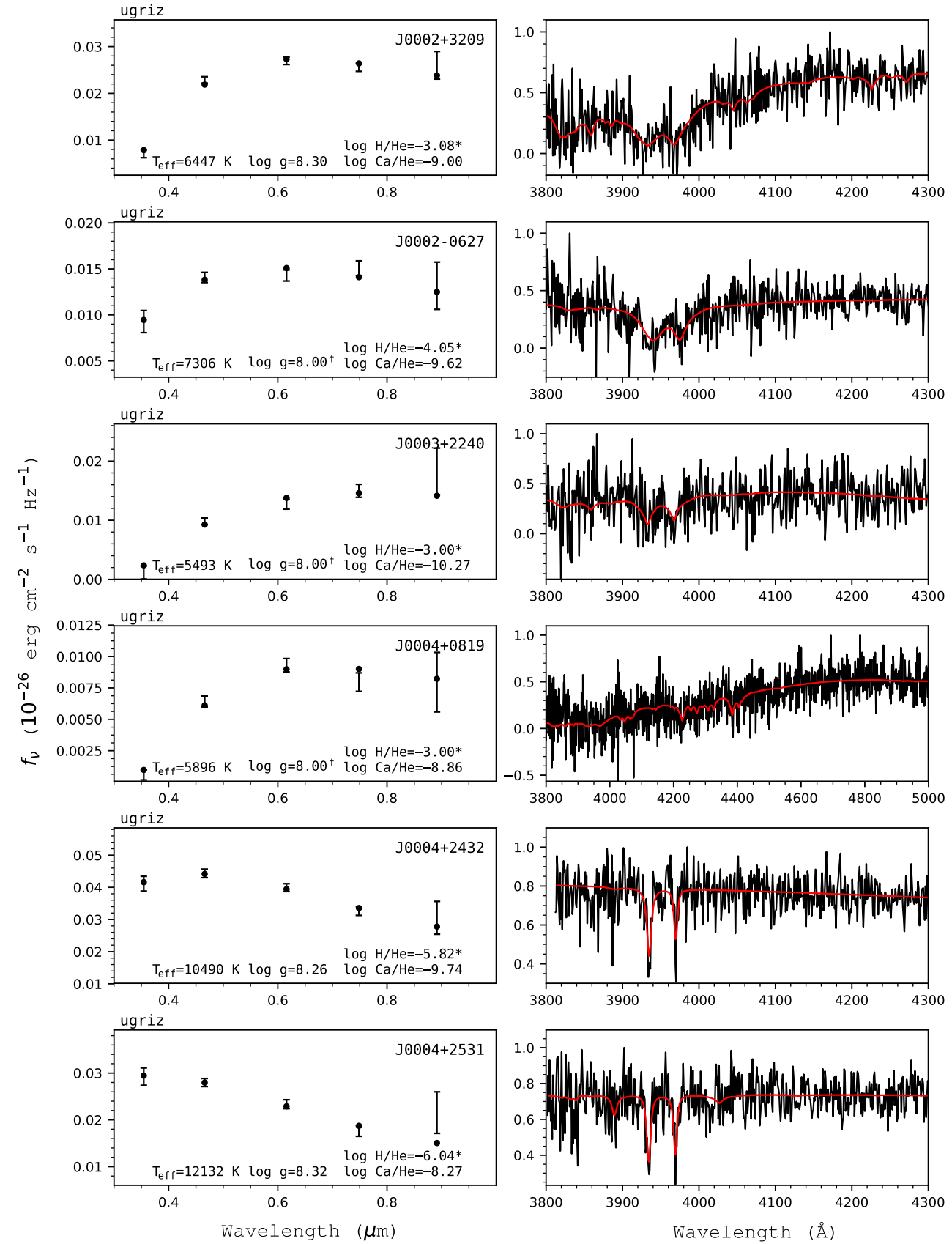

Figure 20. Fits to our sample of $\mathrm{DBZ} / \mathrm{DZ}(\mathrm{A})$ white dwarfs. Left panels: Photometric fits where error bars represent the observed data, while filled circles correspond to average model fluxes. A dagger symbol indicates that $\log g$ is fixed at 8.0 (no parallax measurement available), while a star symbol indicates a value of $\log \mathrm{H} / \mathrm{He}$ fixed at the visibility limit. Right panels: Spectroscopic fits (red) to the normalized observed spectra (black). The inset shows the fit to H $\alpha$ when present. 

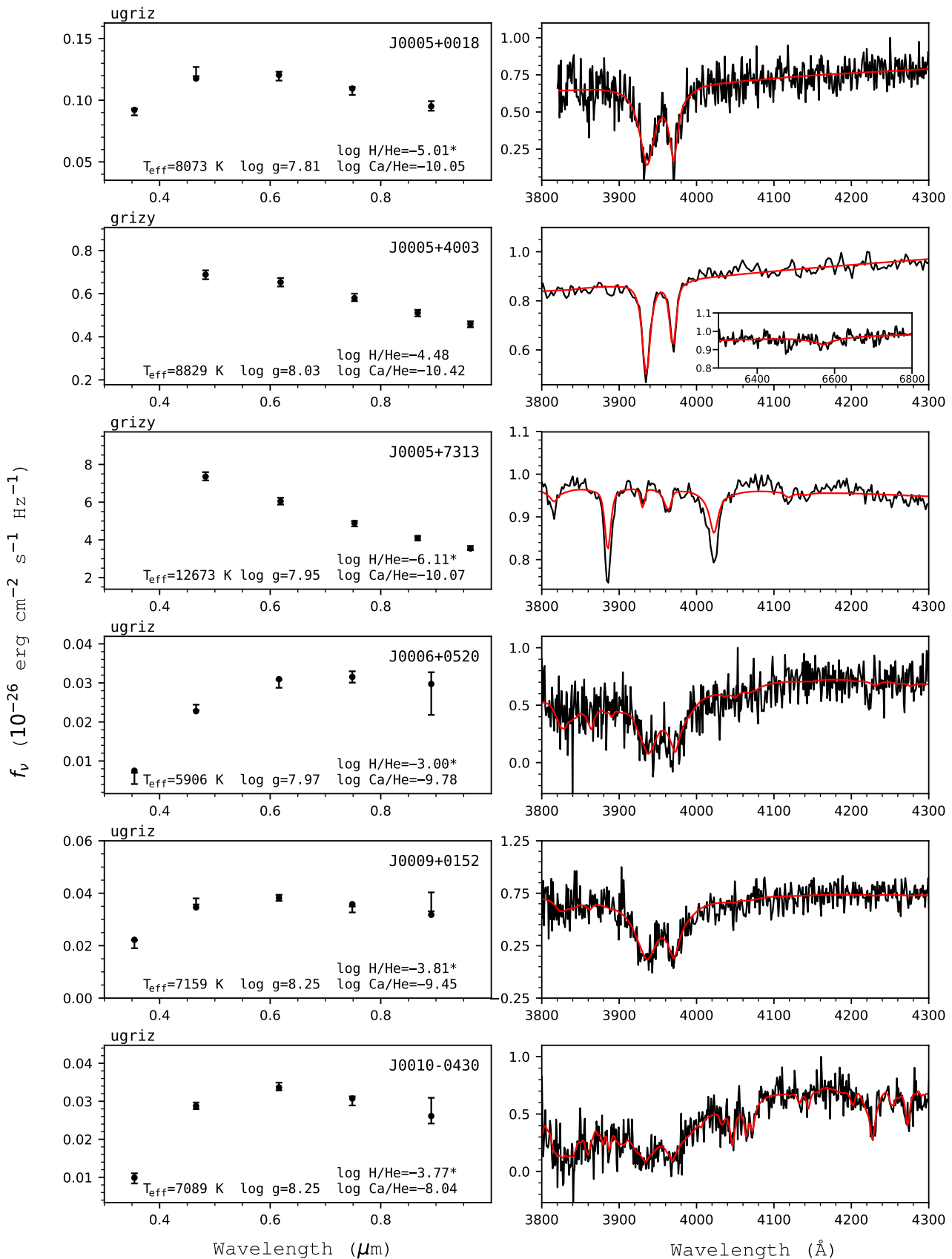

Figure 21. Fits to the DBZ/DZ(A) white dwarfs - continued. 

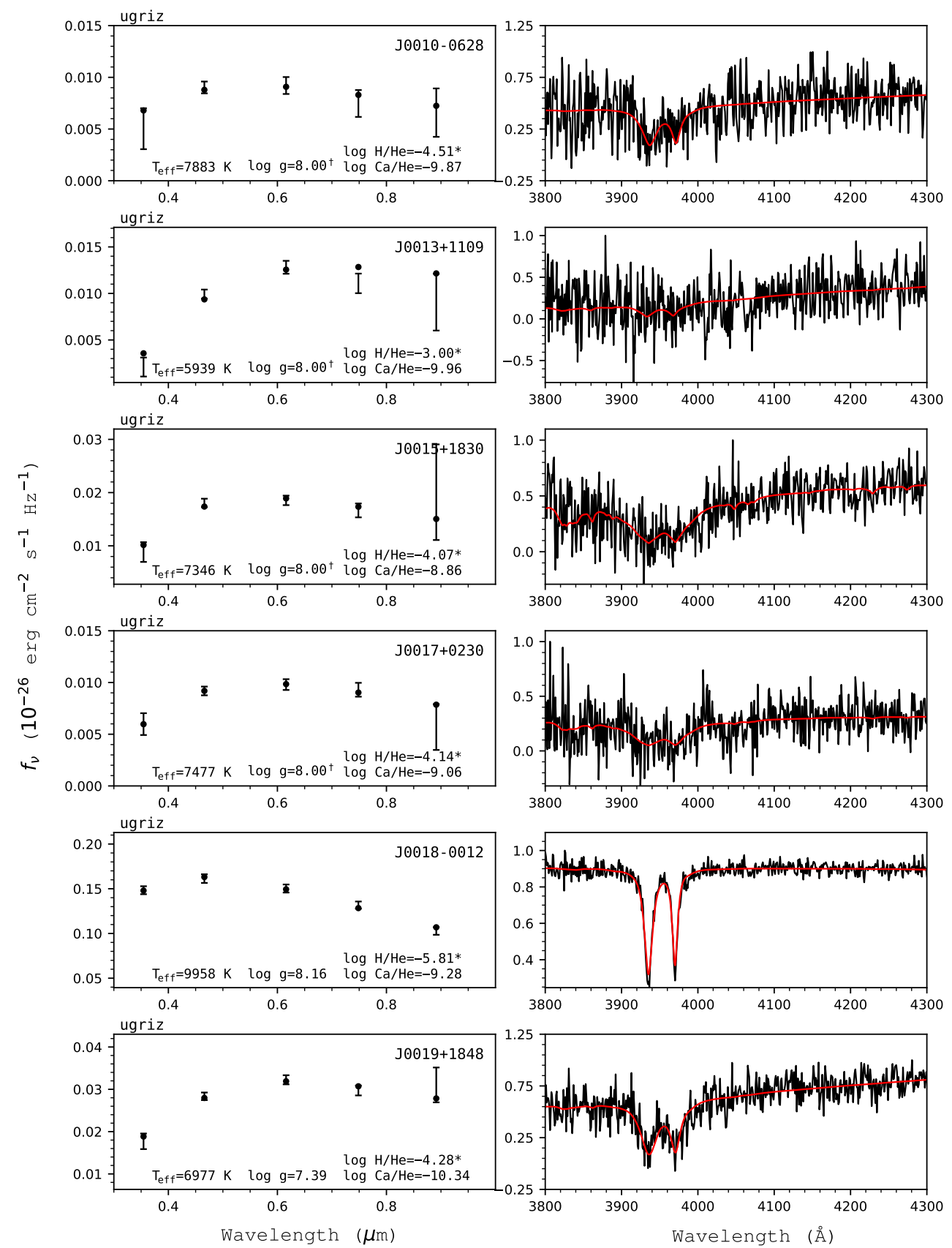

Figure 22. Fits to the DBZ/DZ(A) white dwarfs - continued. 


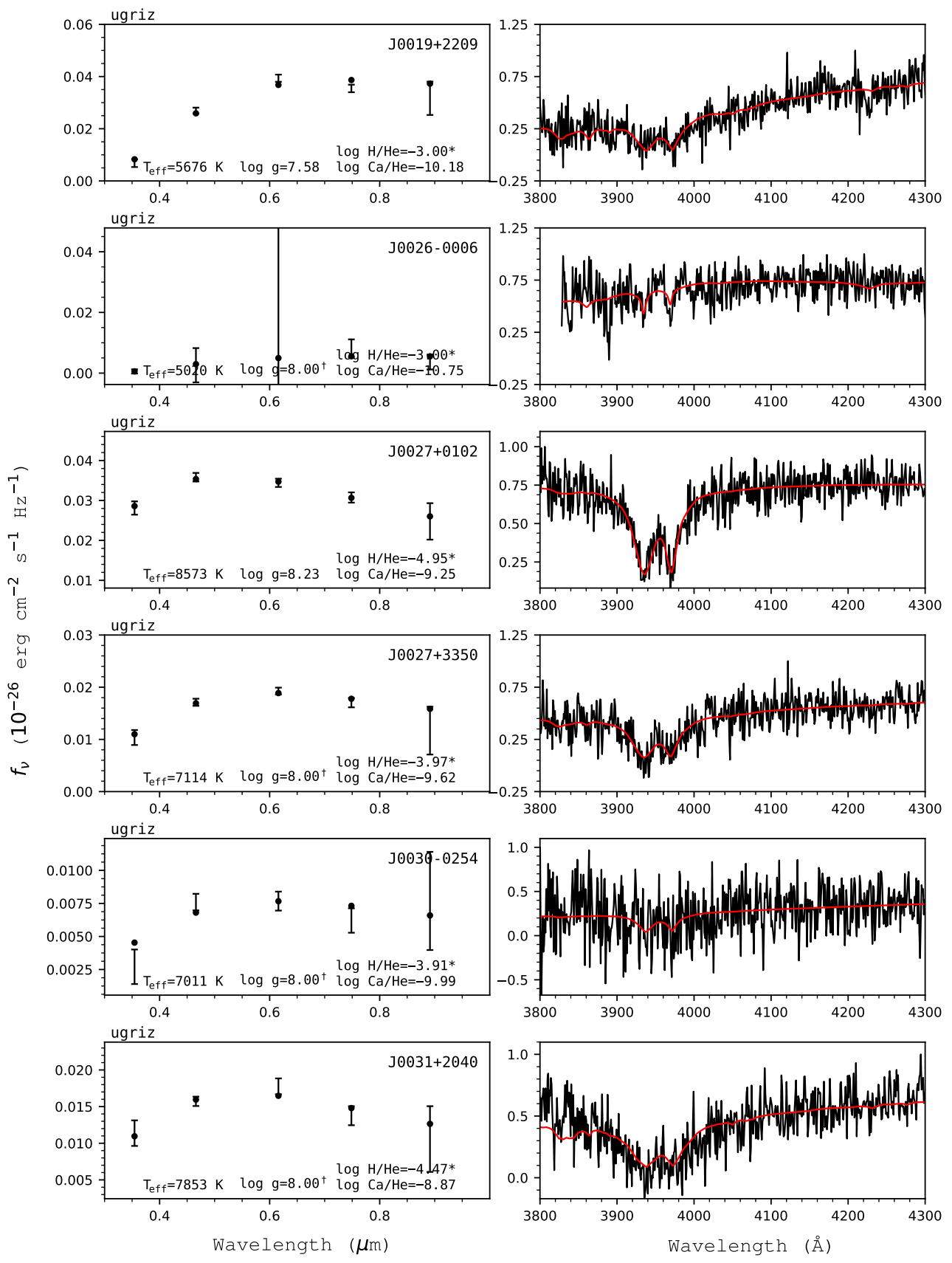

Figure 23. Fits to the DBZ/DZ(A) white dwarfs - continued. 

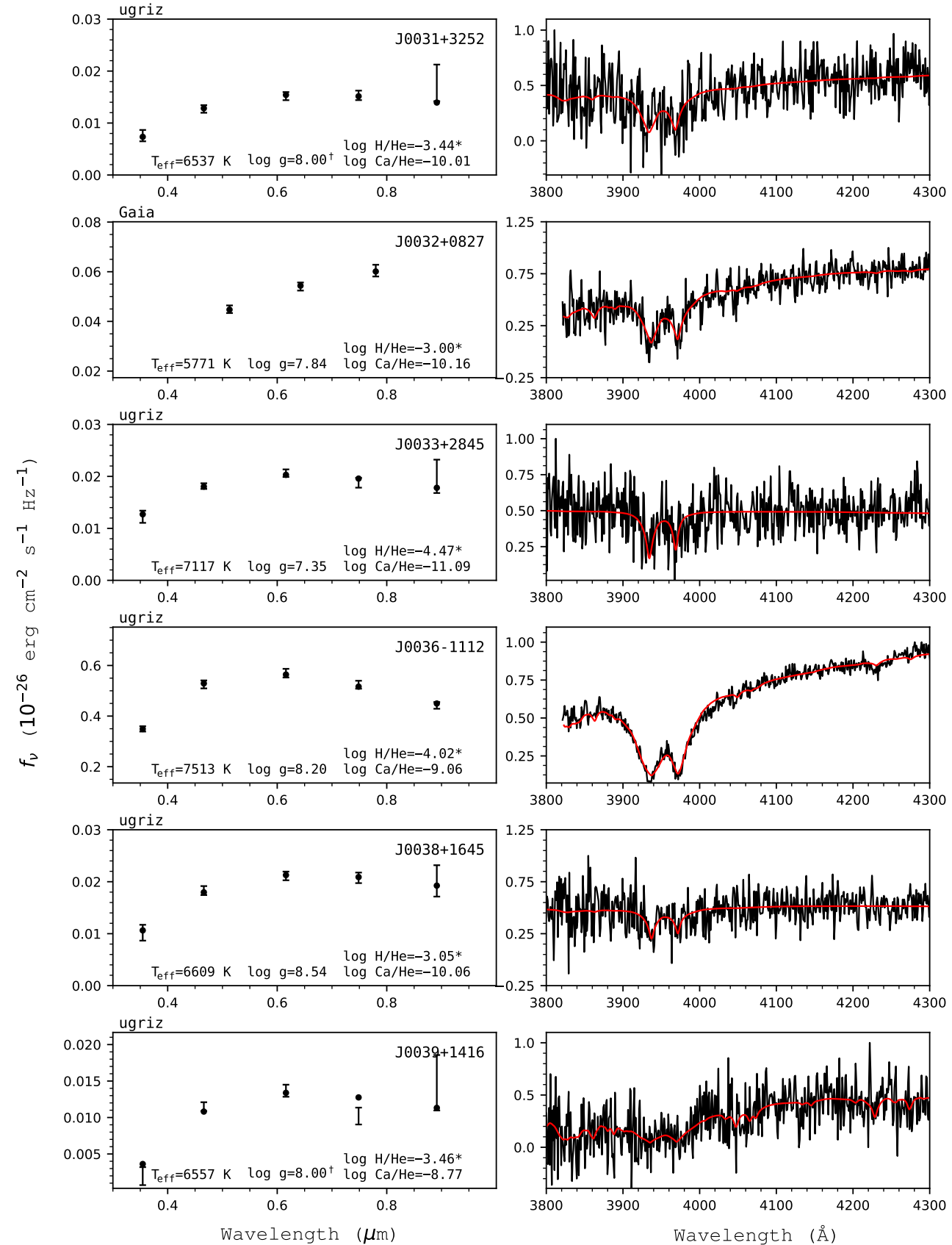

Figure 24. Fits to the DBZ/DZ(A) white dwarfs - continued. 

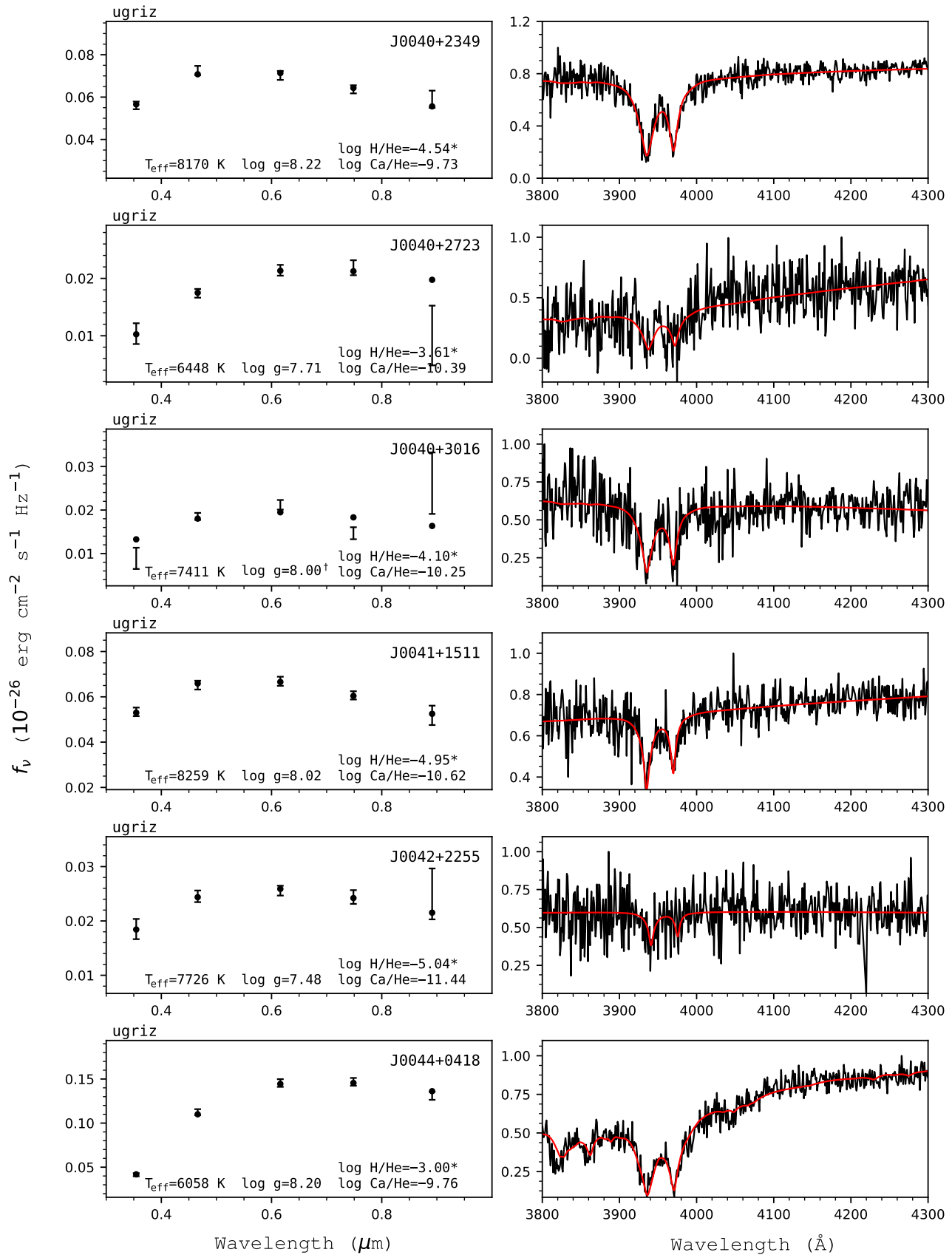

Figure 25. Fits to the DBZ/DZ(A) white dwarfs - continued. 

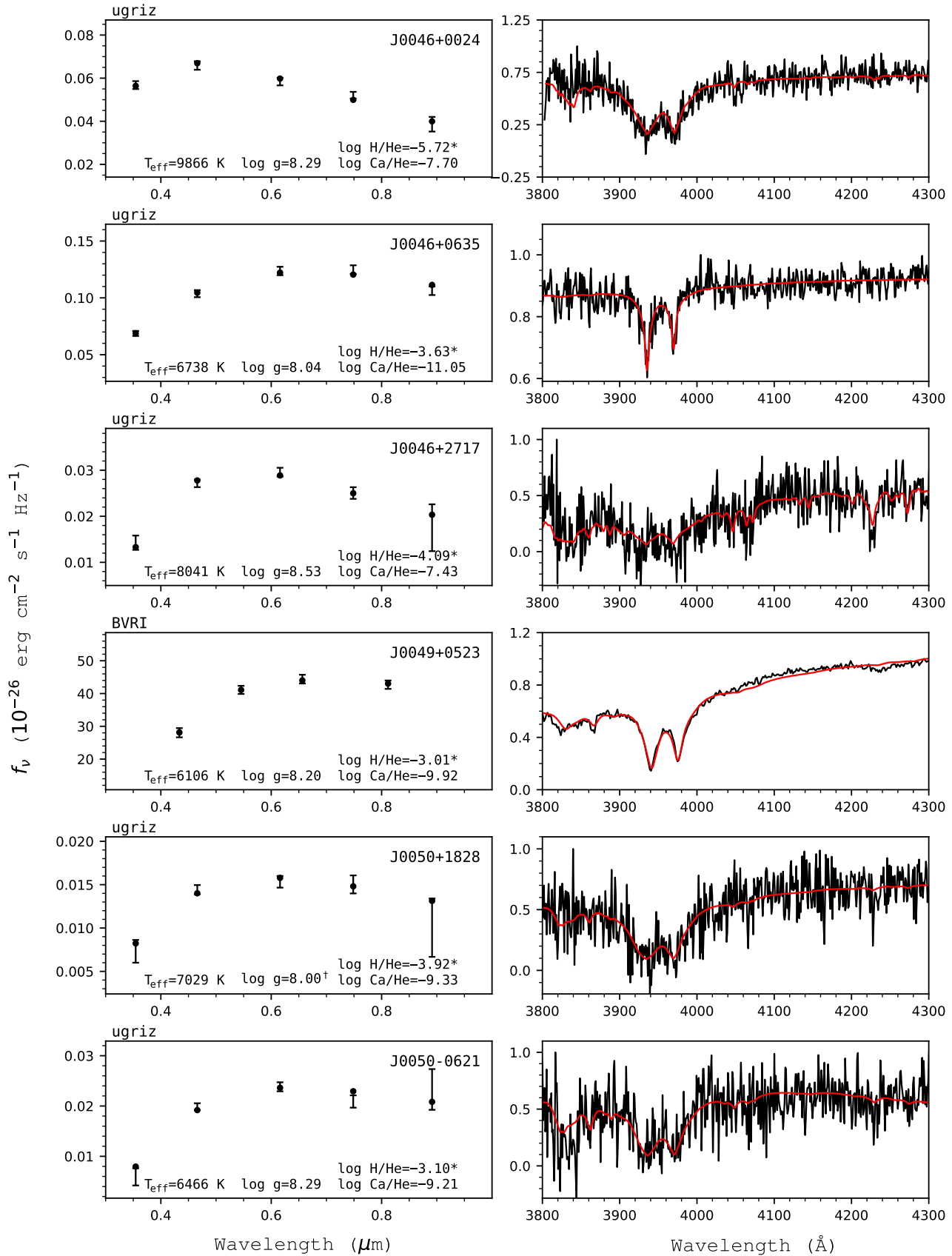

Figure 26. Fits to the DBZ/DZ(A) white dwarfs - continued. 

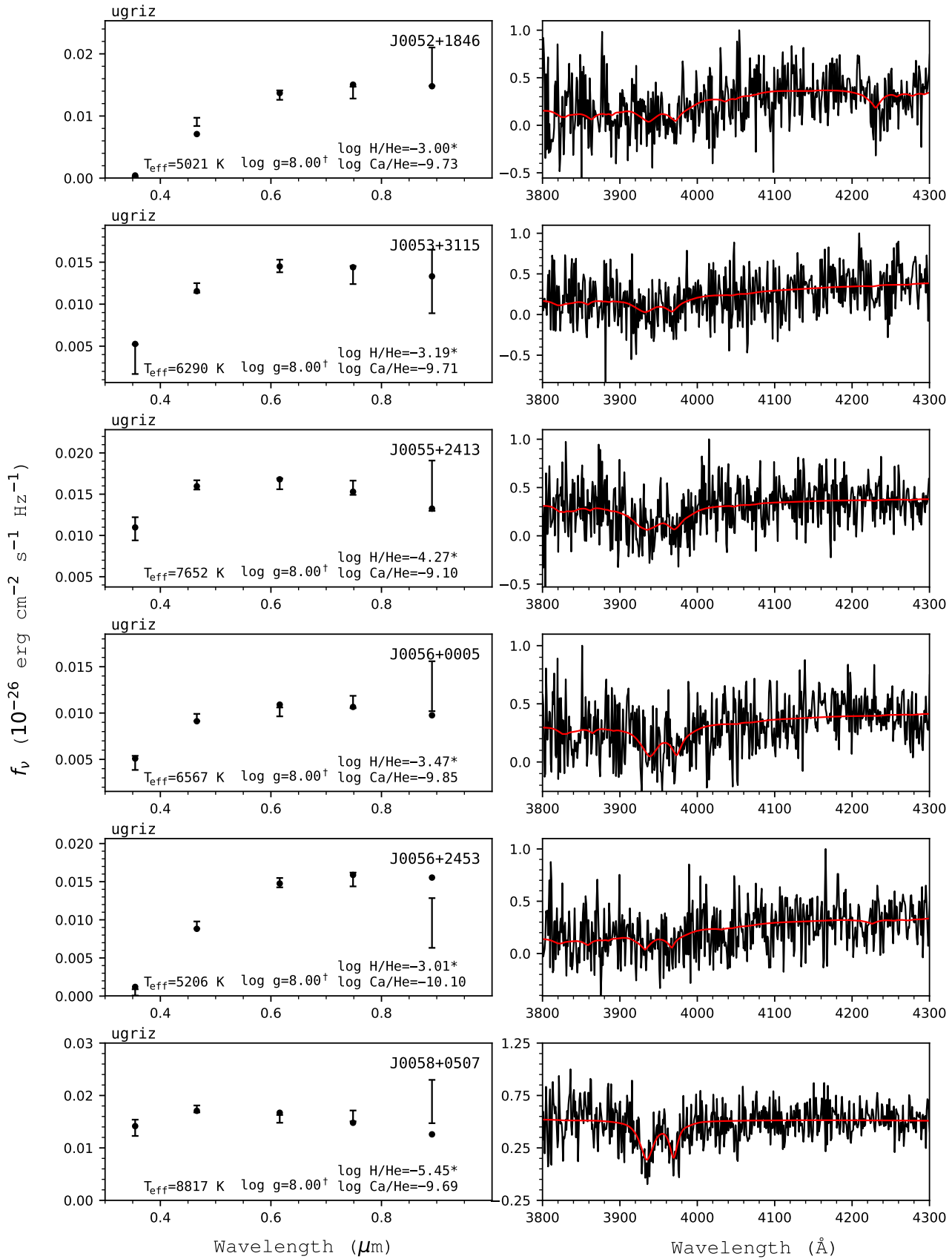

Figure 27. Fits to the DBZ/DZ(A) white dwarfs - continued. 

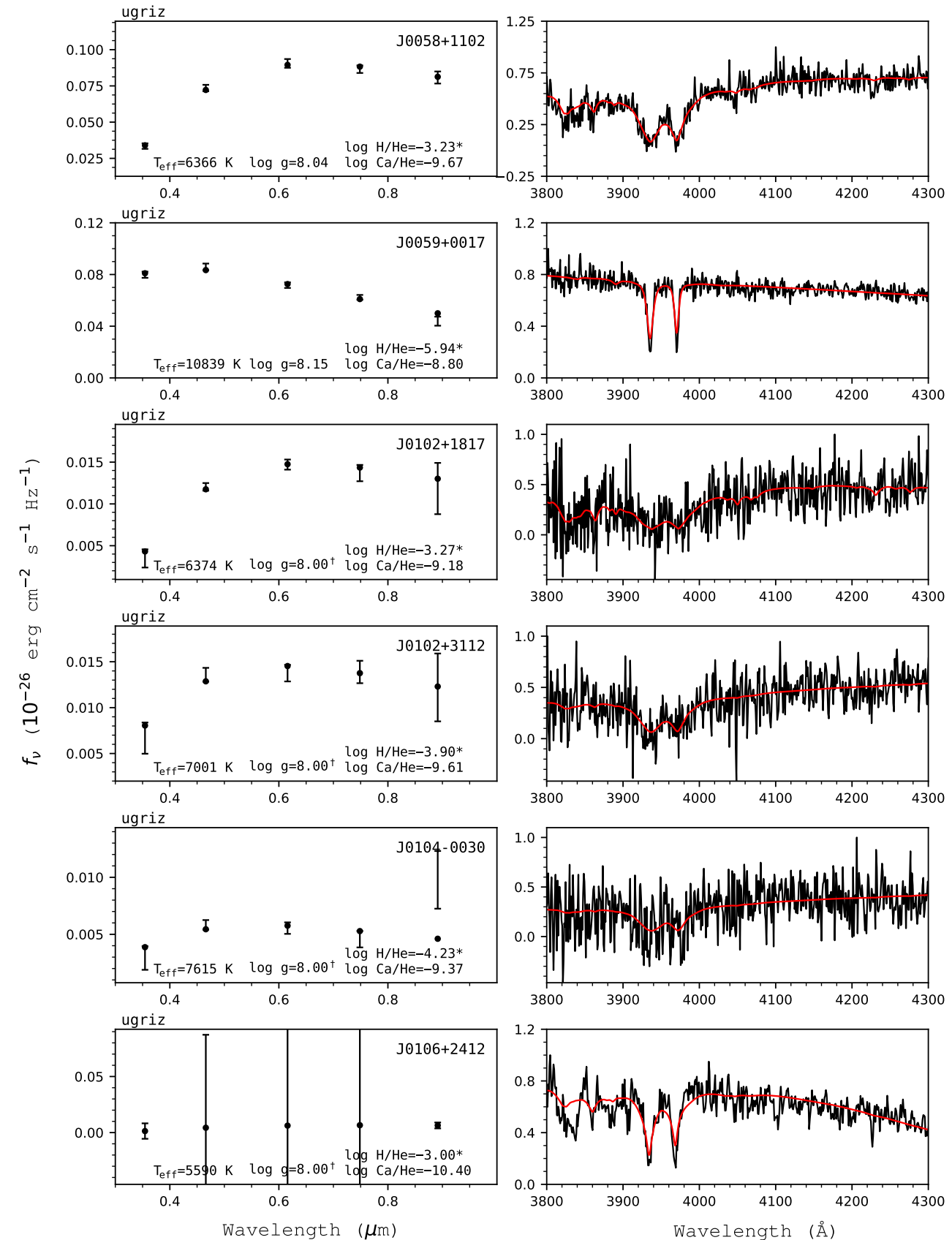

Figure 28. Fits to the DBZ/DZ(A) white dwarfs - continued. 

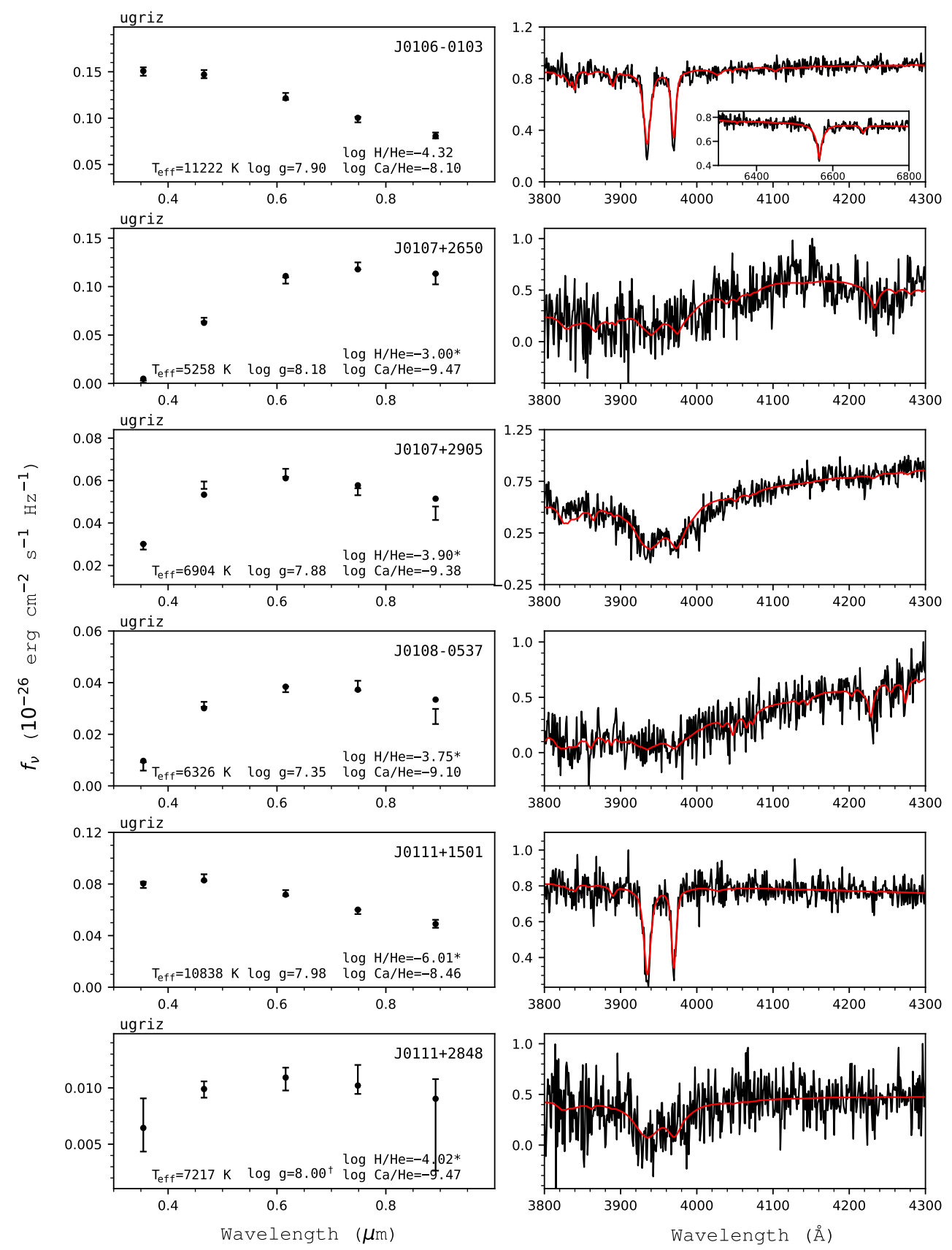

Figure 29. Fits to the DBZ/DZ(A) white dwarfs - continued. 

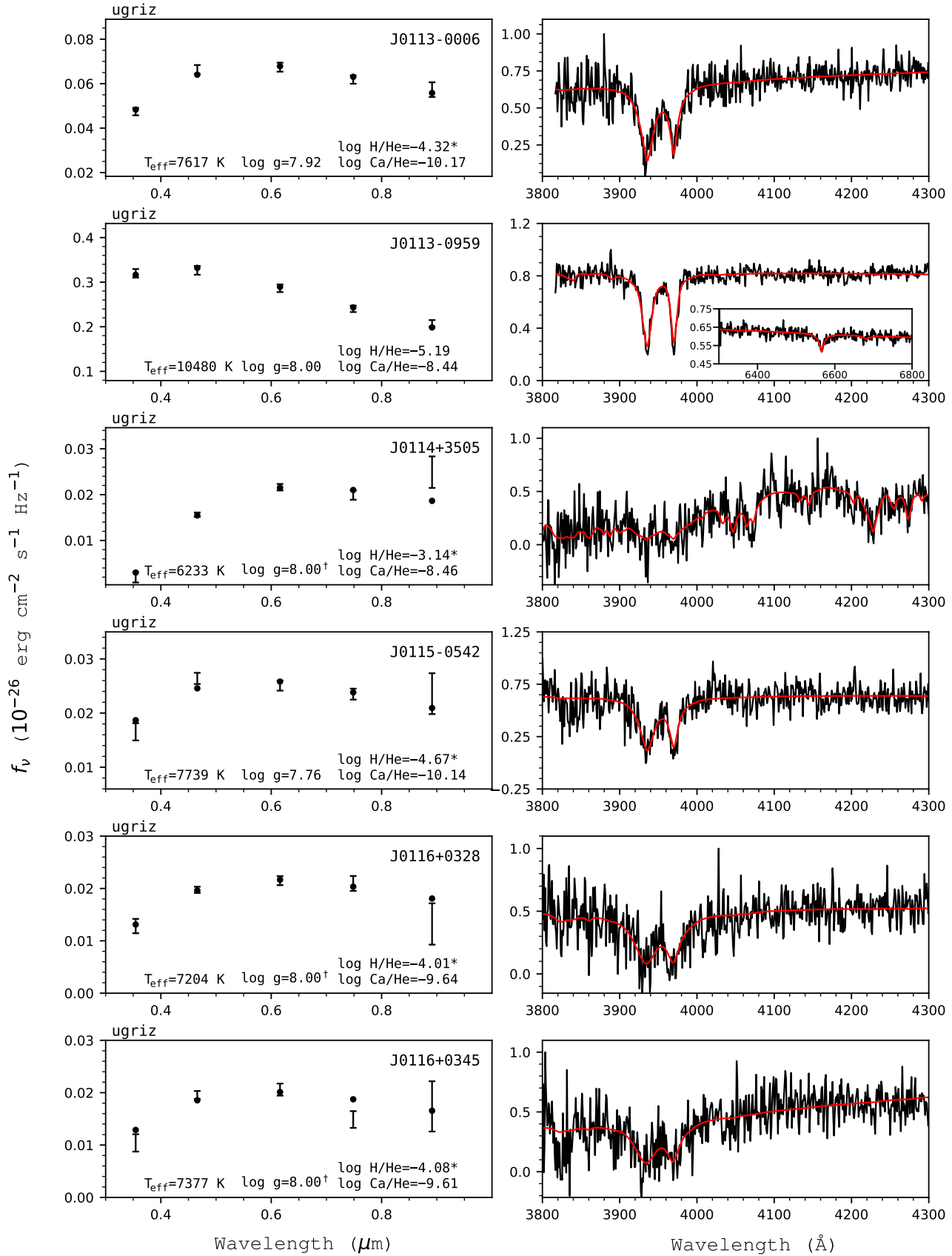

Figure 30. Fits to the DBZ/DZ(A) white dwarfs - continued. 

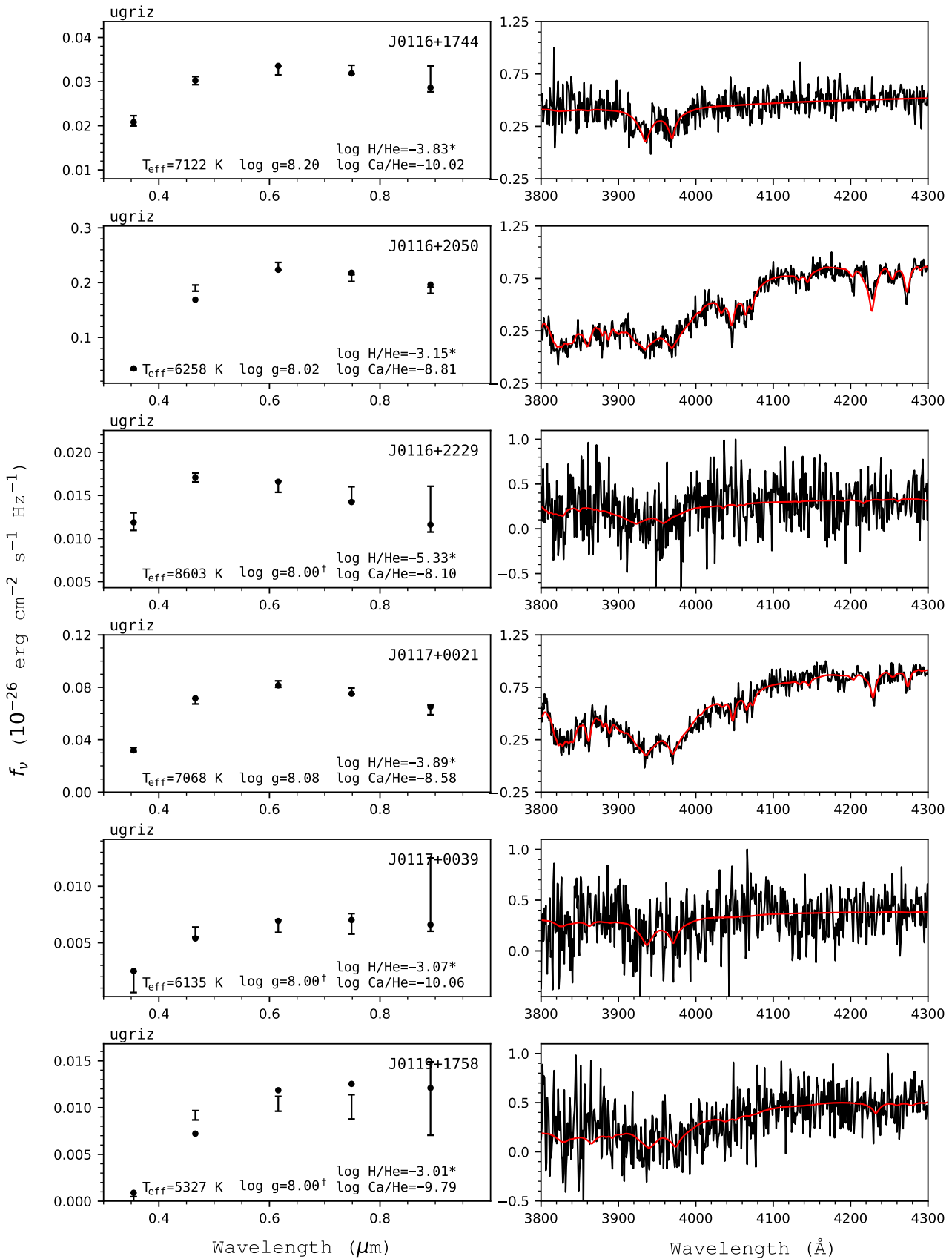

Figure 31. Fits to the DBZ/DZ(A) white dwarfs - continued. 

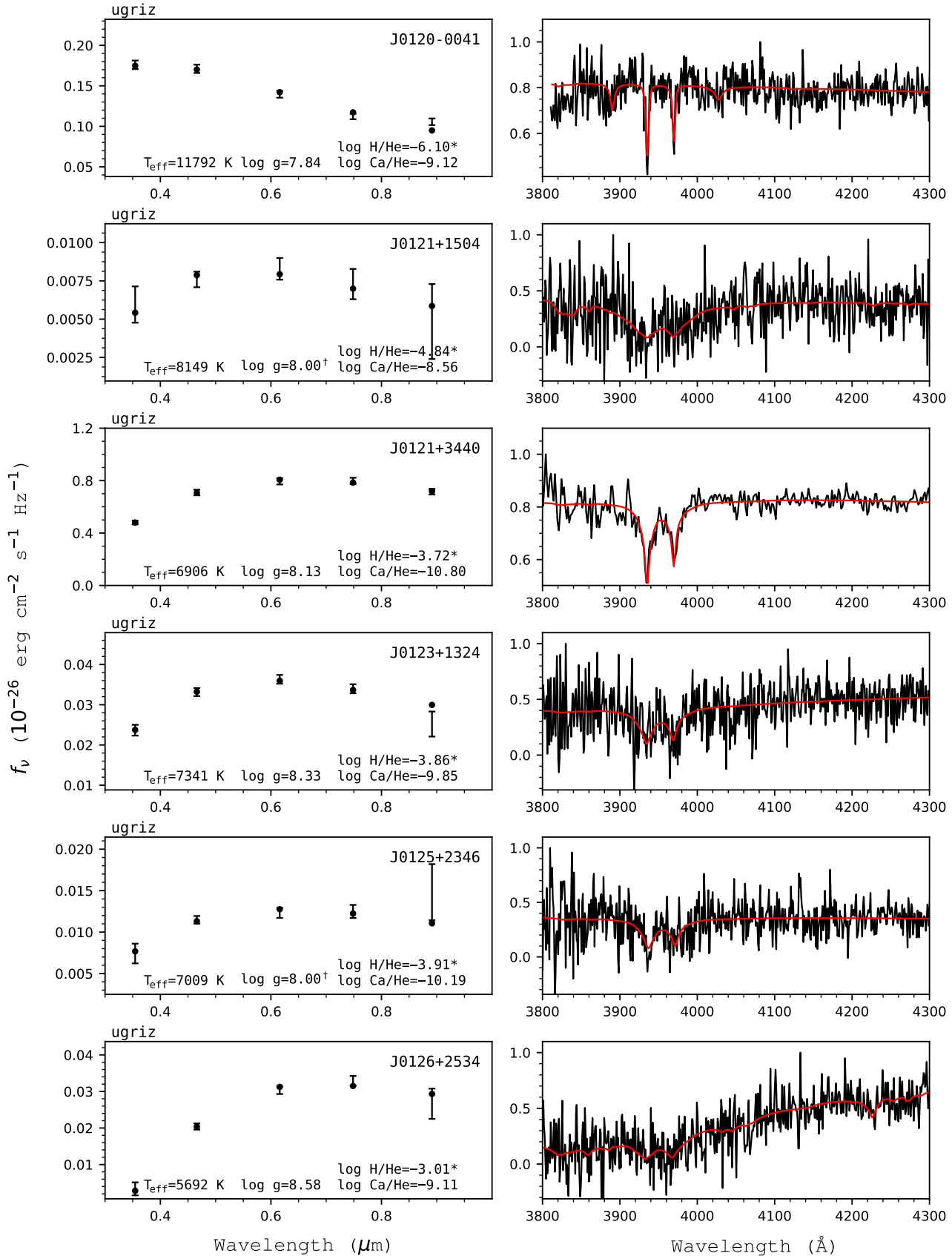

Figure 32. Fits to the DBZ/DZ(A) white dwarfs - continued. 

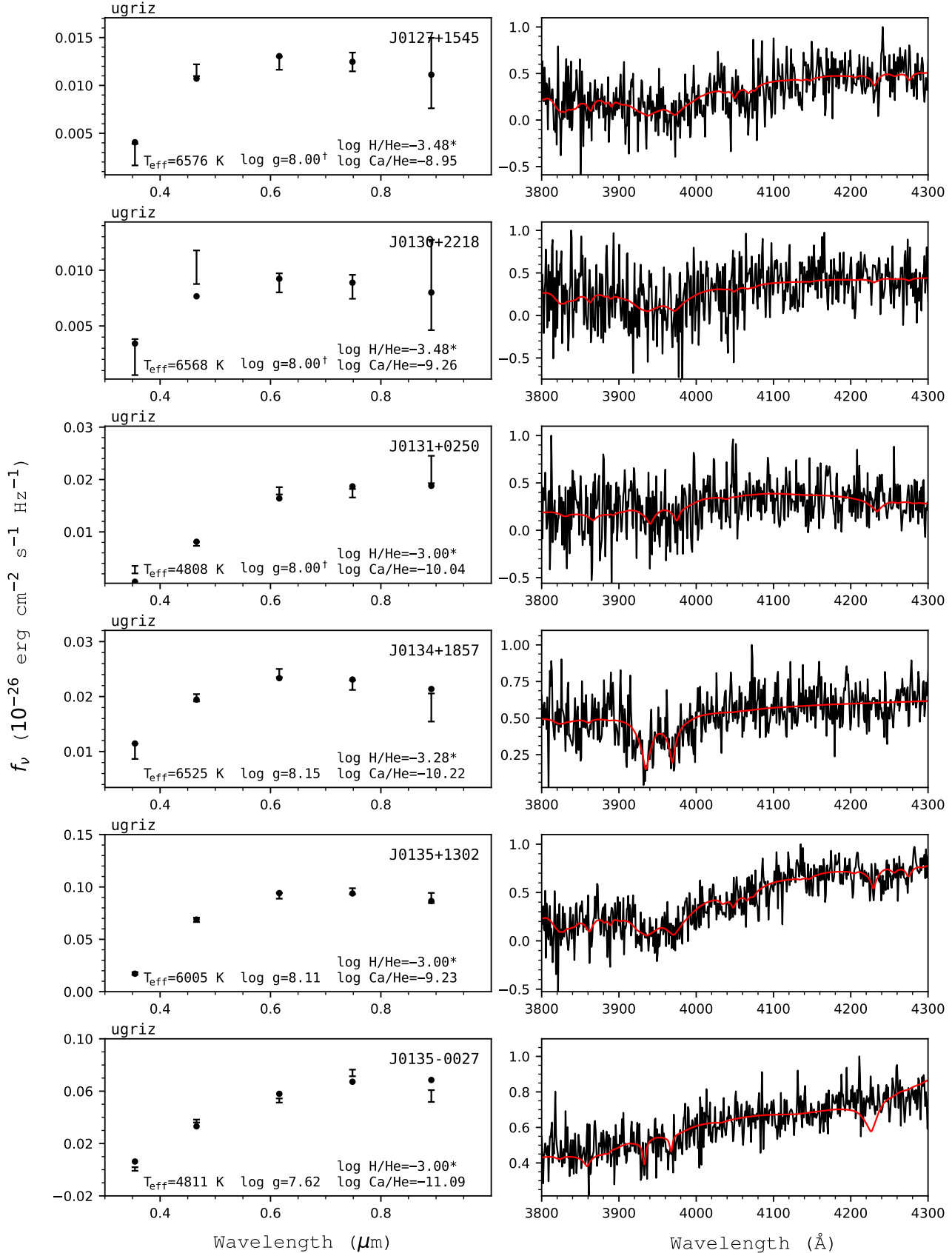

Figure 33. Fits to the DBZ/DZ(A) white dwarfs - continued. 

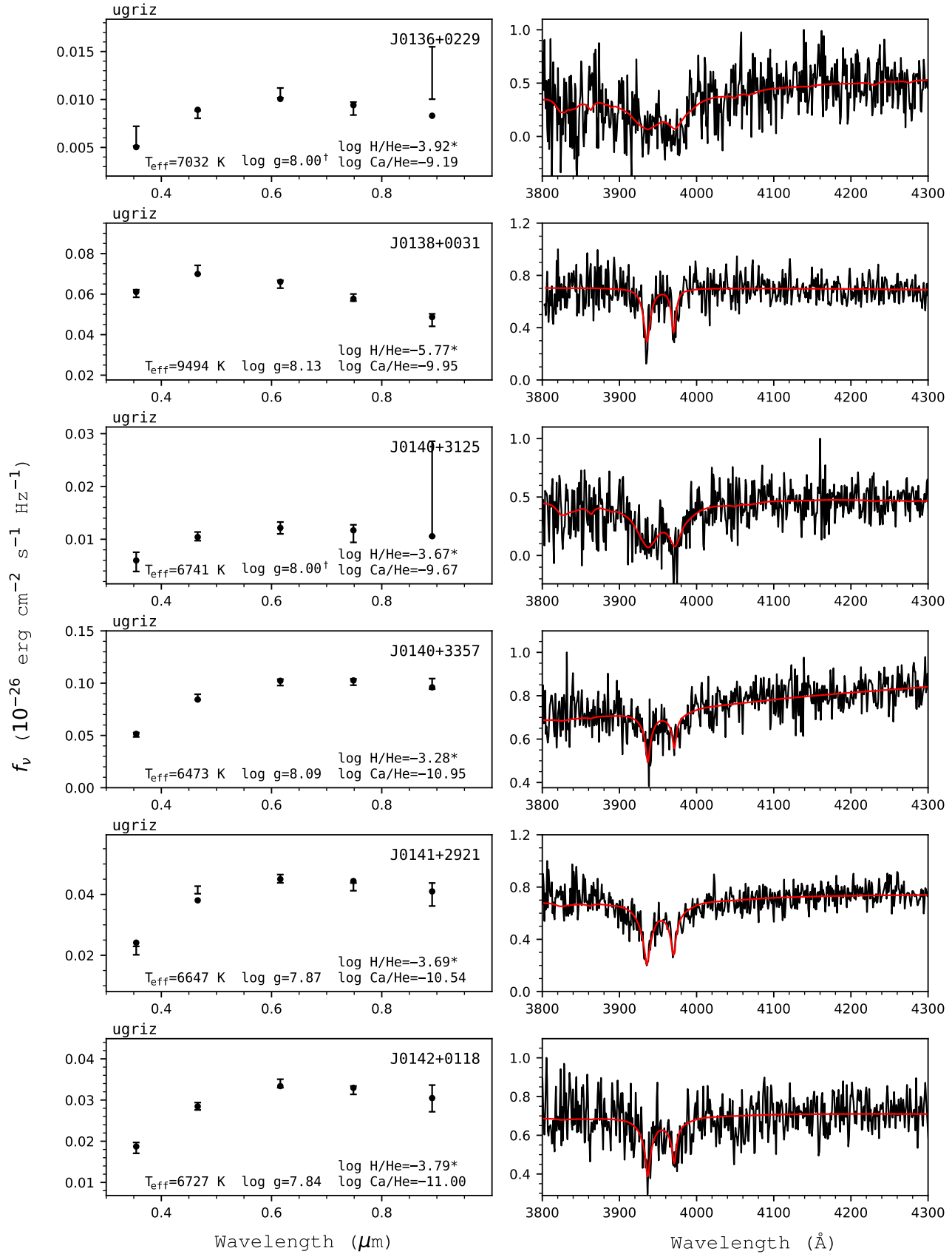

Figure 34. Fits to the DBZ/DZ(A) white dwarfs - continued. 

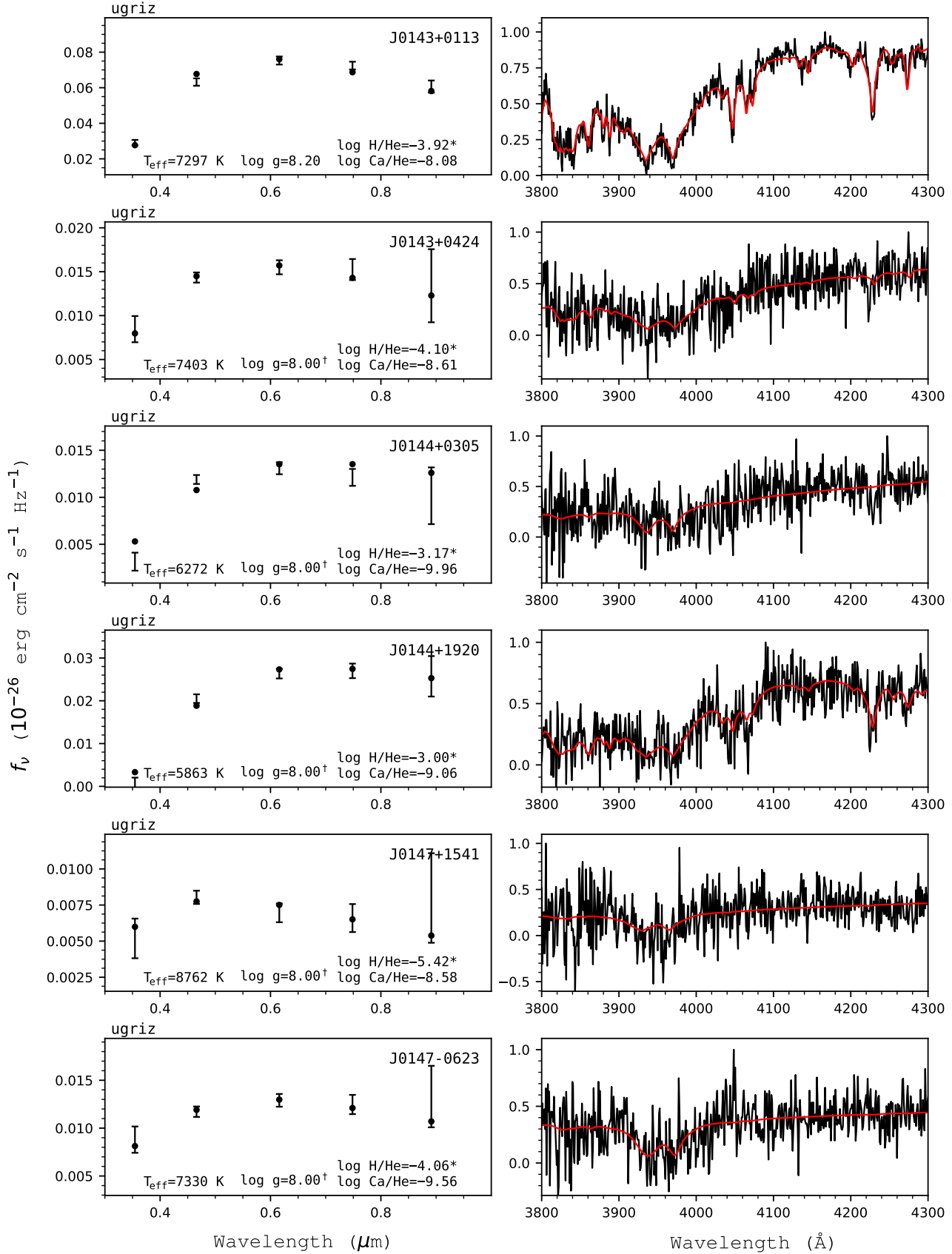

Figure 35. Fits to the DBZ/DZ(A) white dwarfs - continued. 

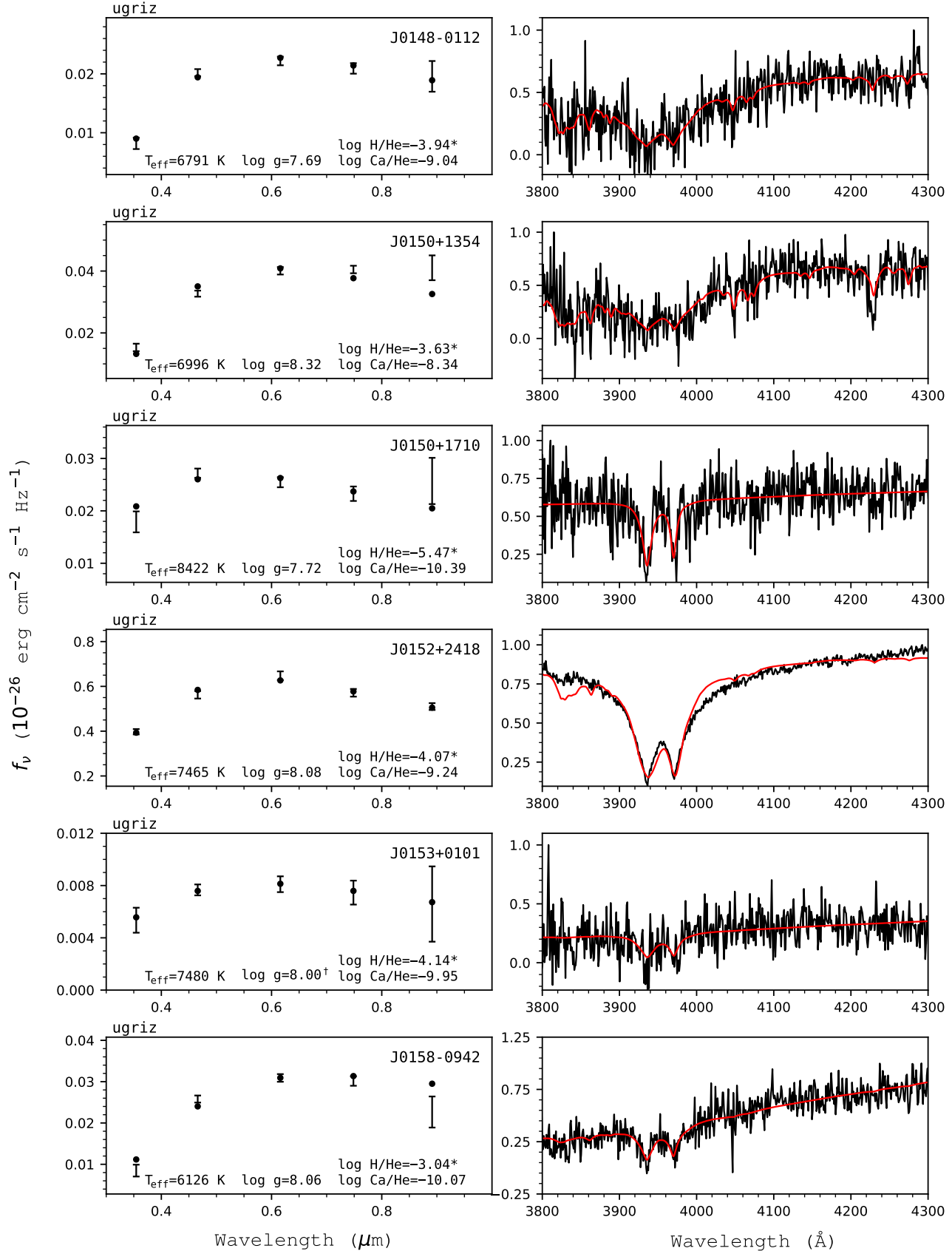

Figure 36. Fits to the DBZ/DZ(A) white dwarfs - continued. 

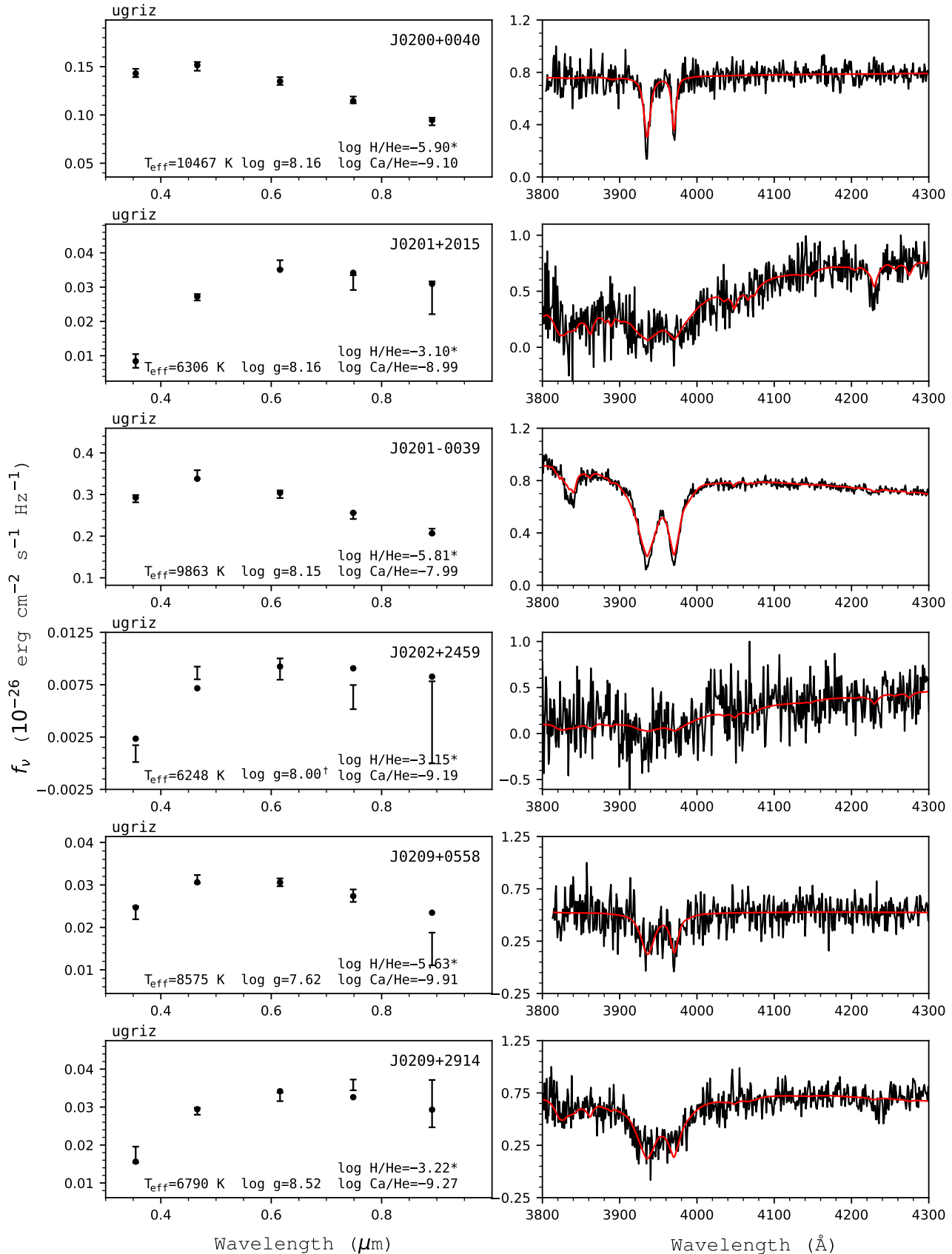

Figure 37. Fits to the DBZ/DZ(A) white dwarfs - continued. 

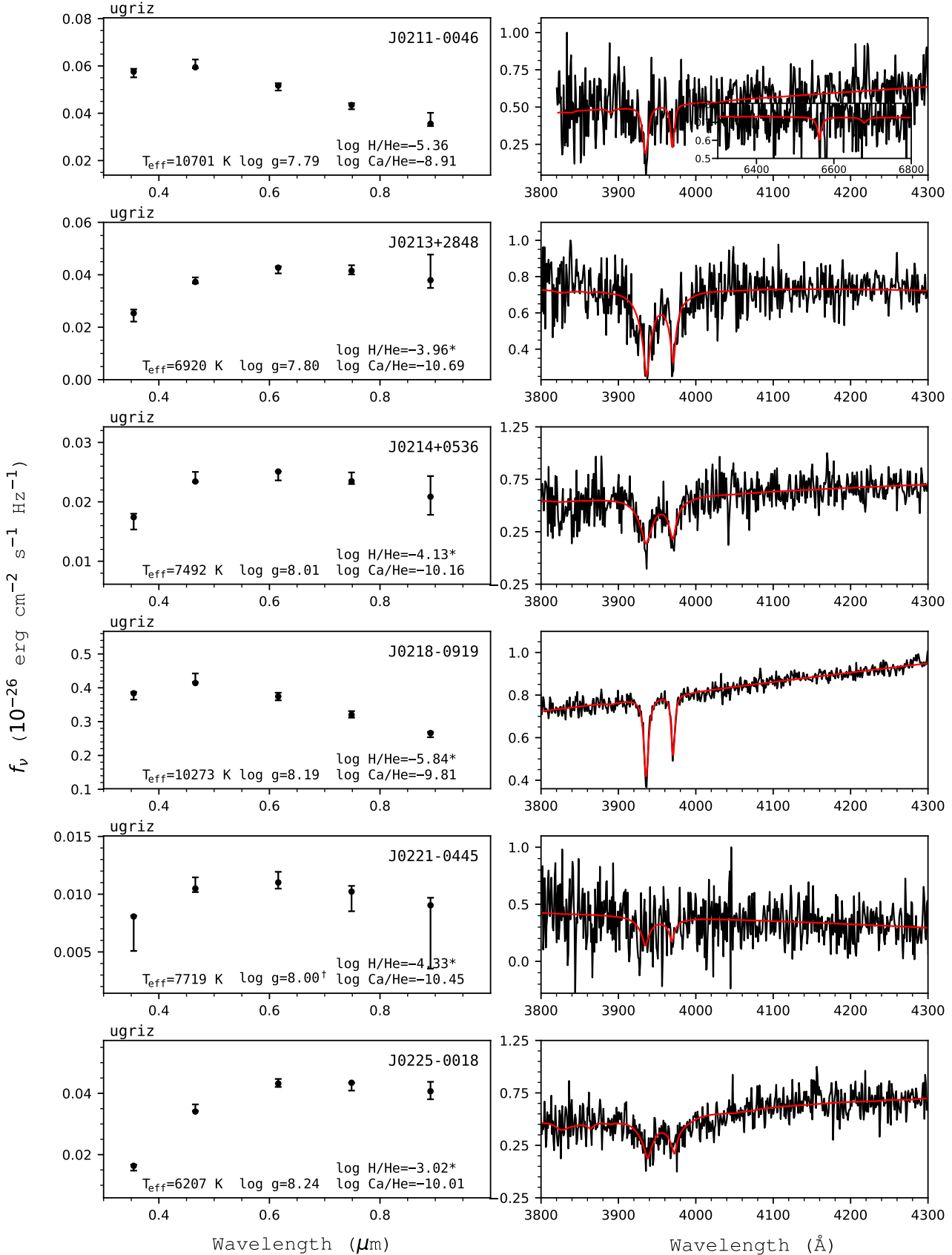

Figure 38. Fits to the DBZ/DZ(A) white dwarfs - continued. 

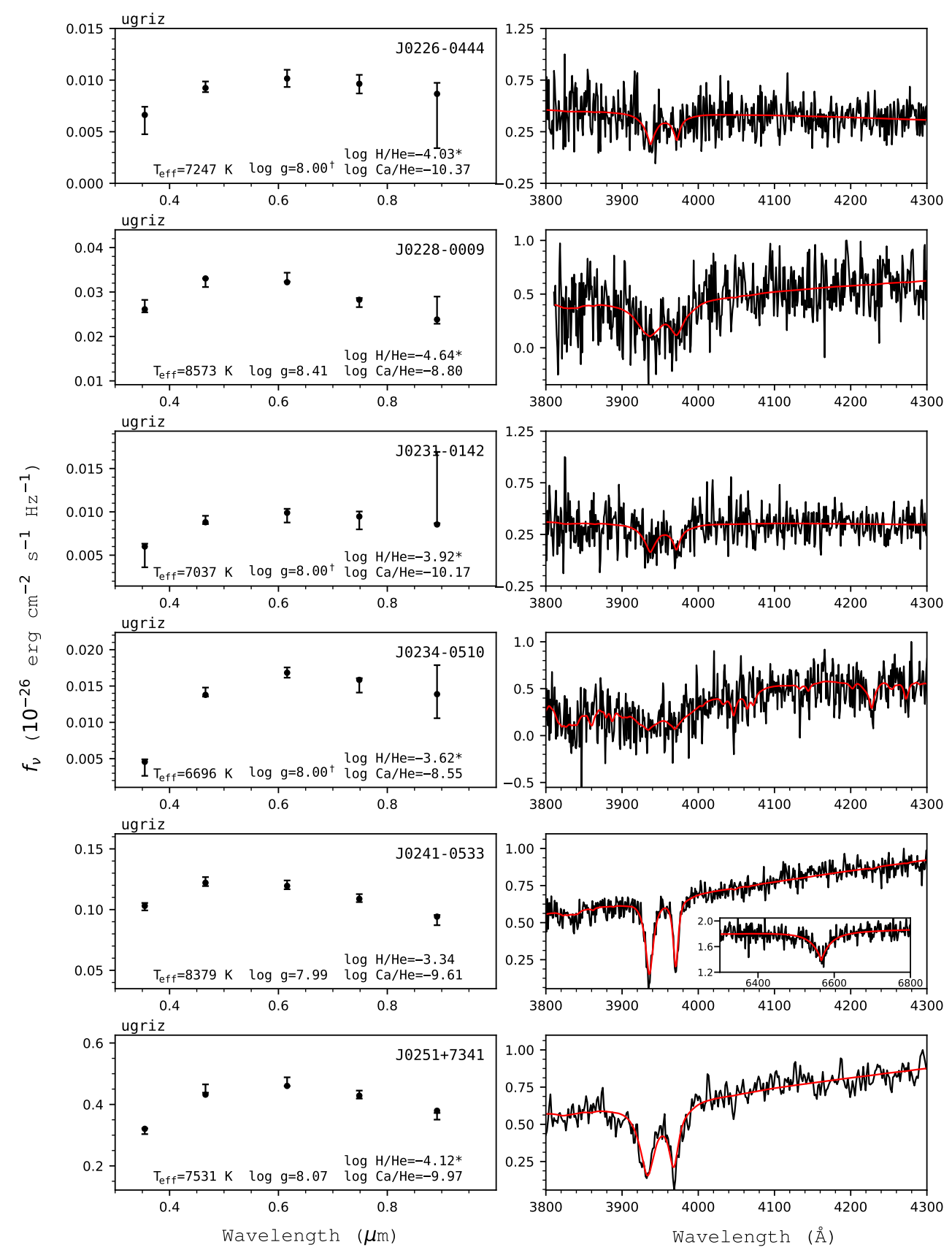

Figure 39. Fits to the DBZ/DZ(A) white dwarfs - continued. 

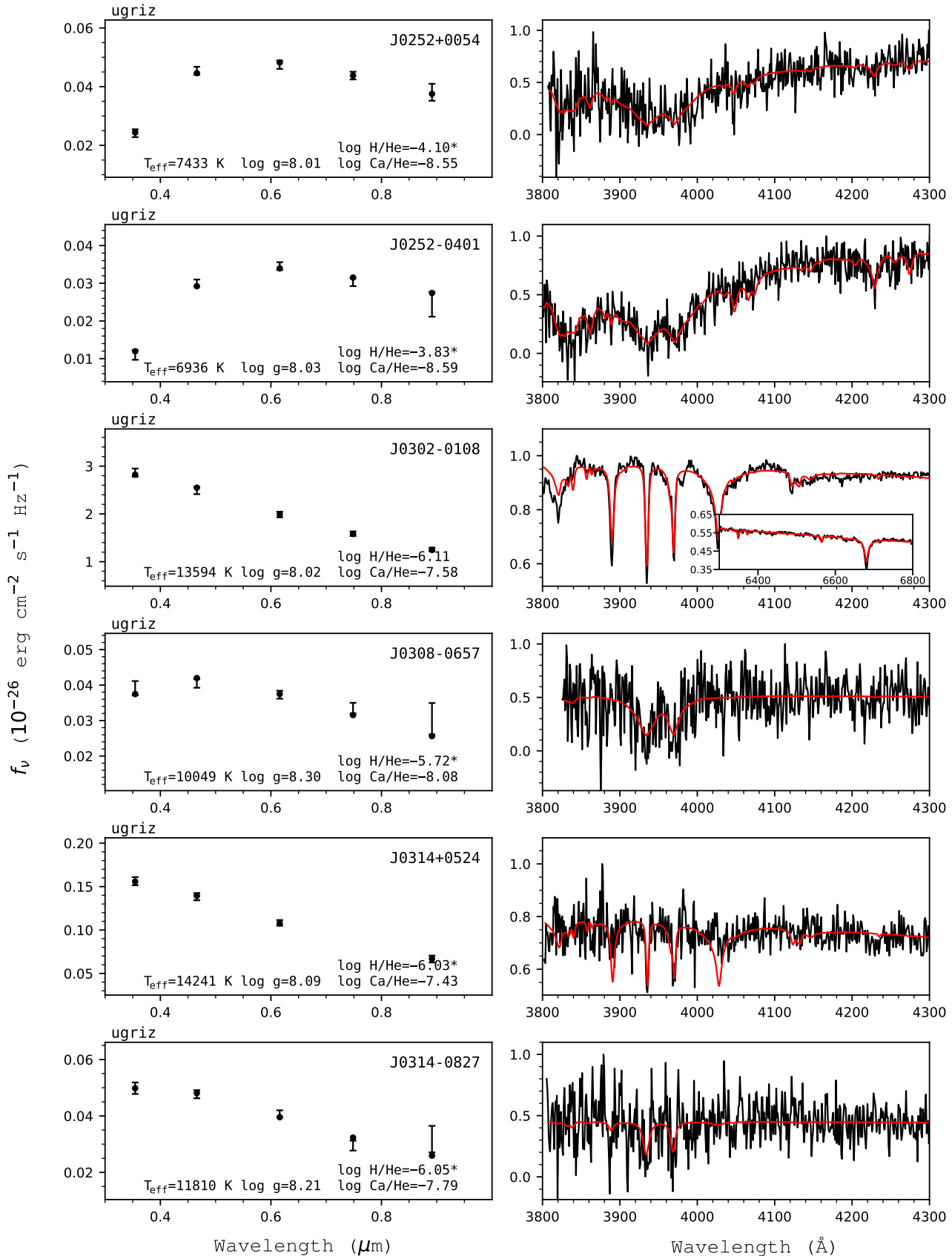

Figure 40. Fits to the DBZ/DZ(A) white dwarfs - continued. 


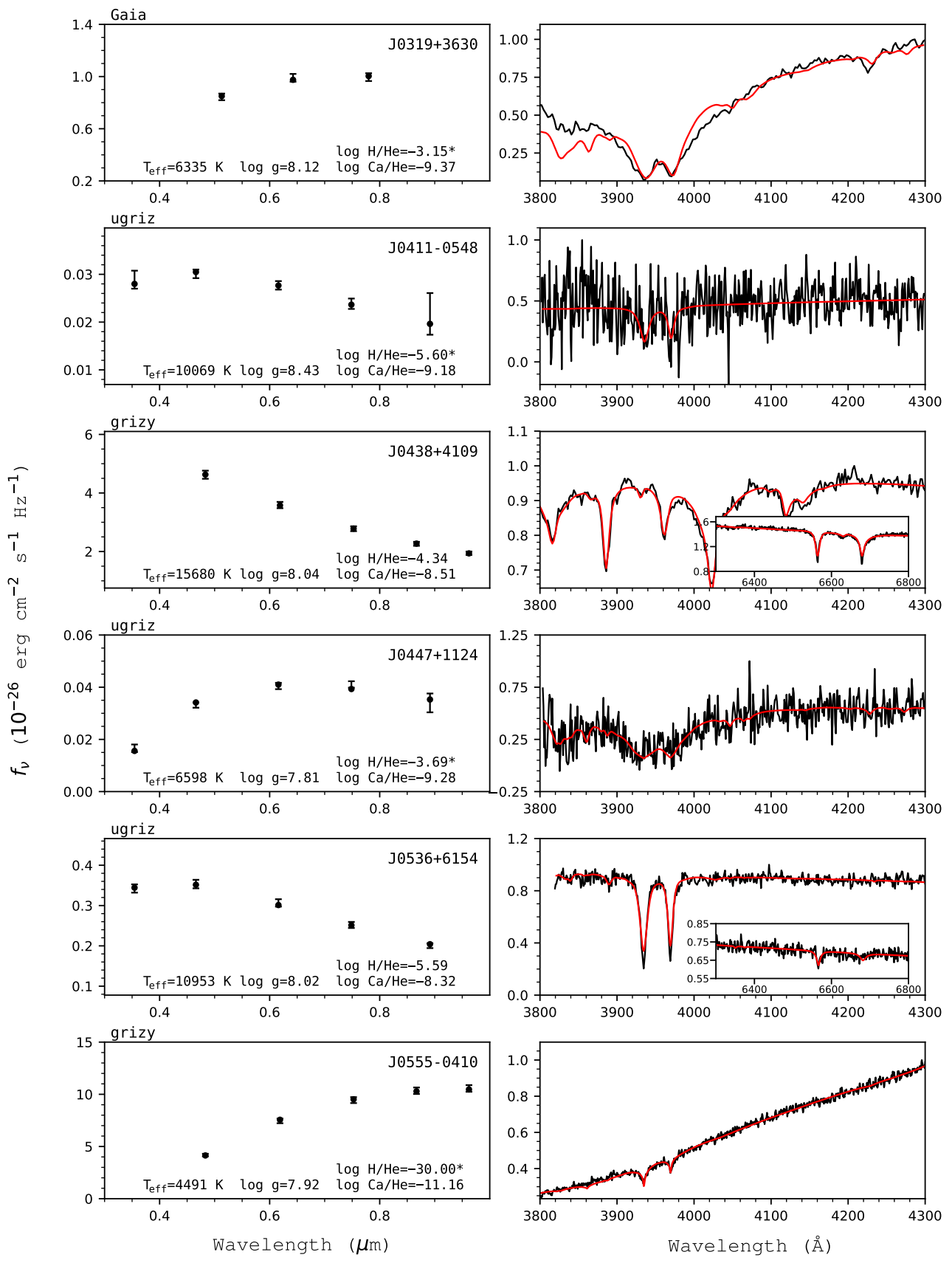

Figure 41. Fits to the DBZ/DZ(A) white dwarfs - continued. 

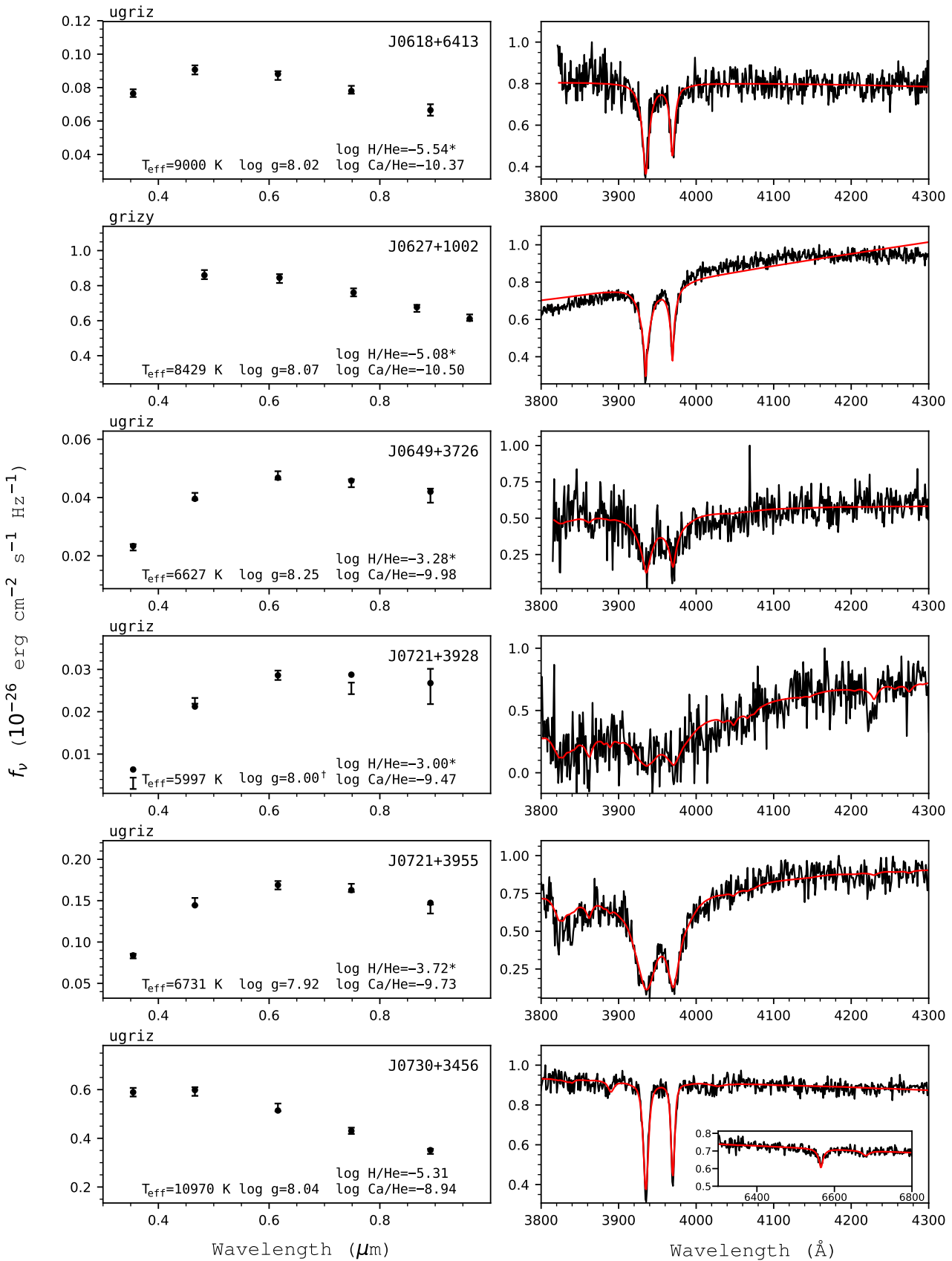

Figure 42. Fits to the DBZ/DZ(A) white dwarfs - continued. 

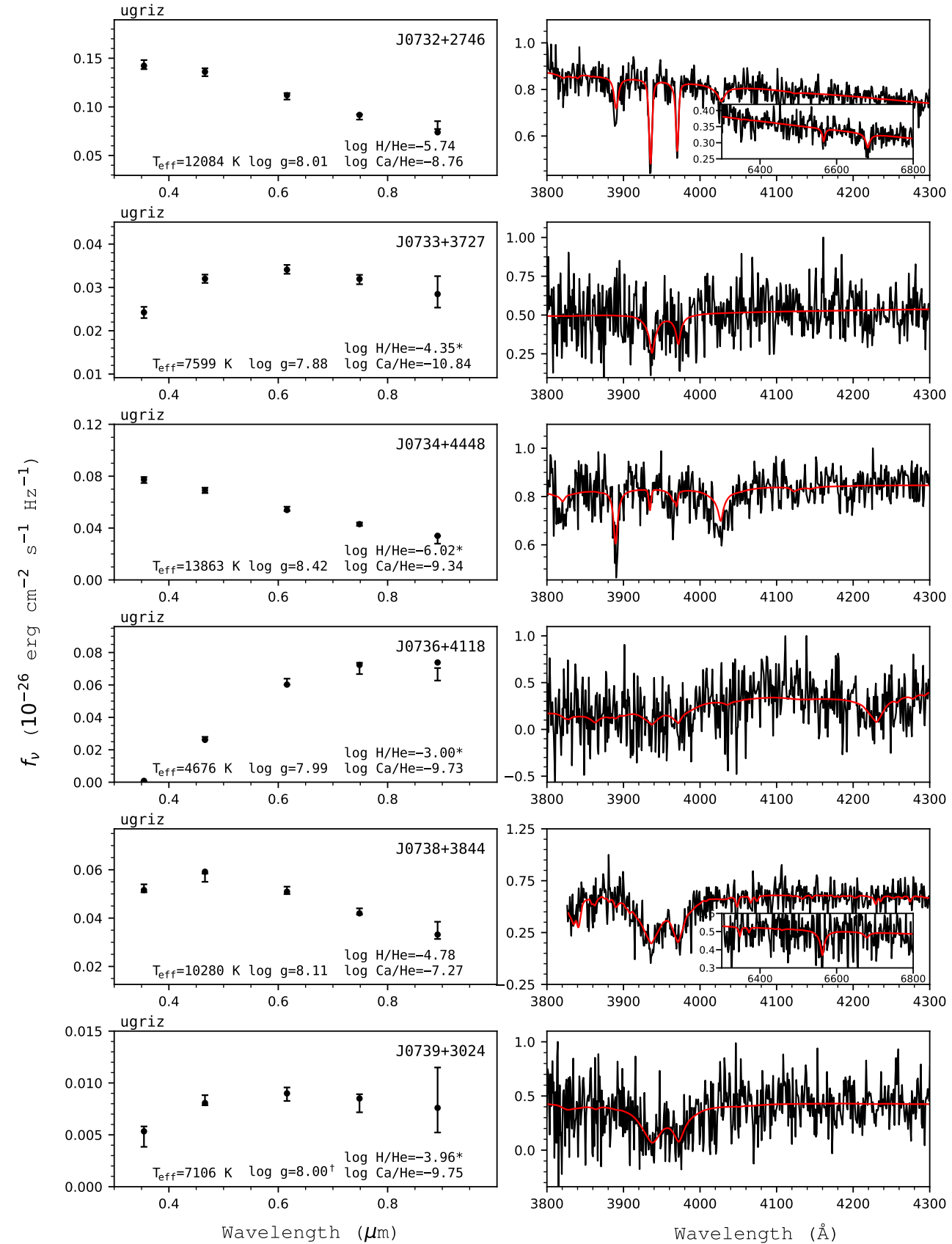

Figure 43. Fits to the DBZ/DZ(A) white dwarfs - continued. 

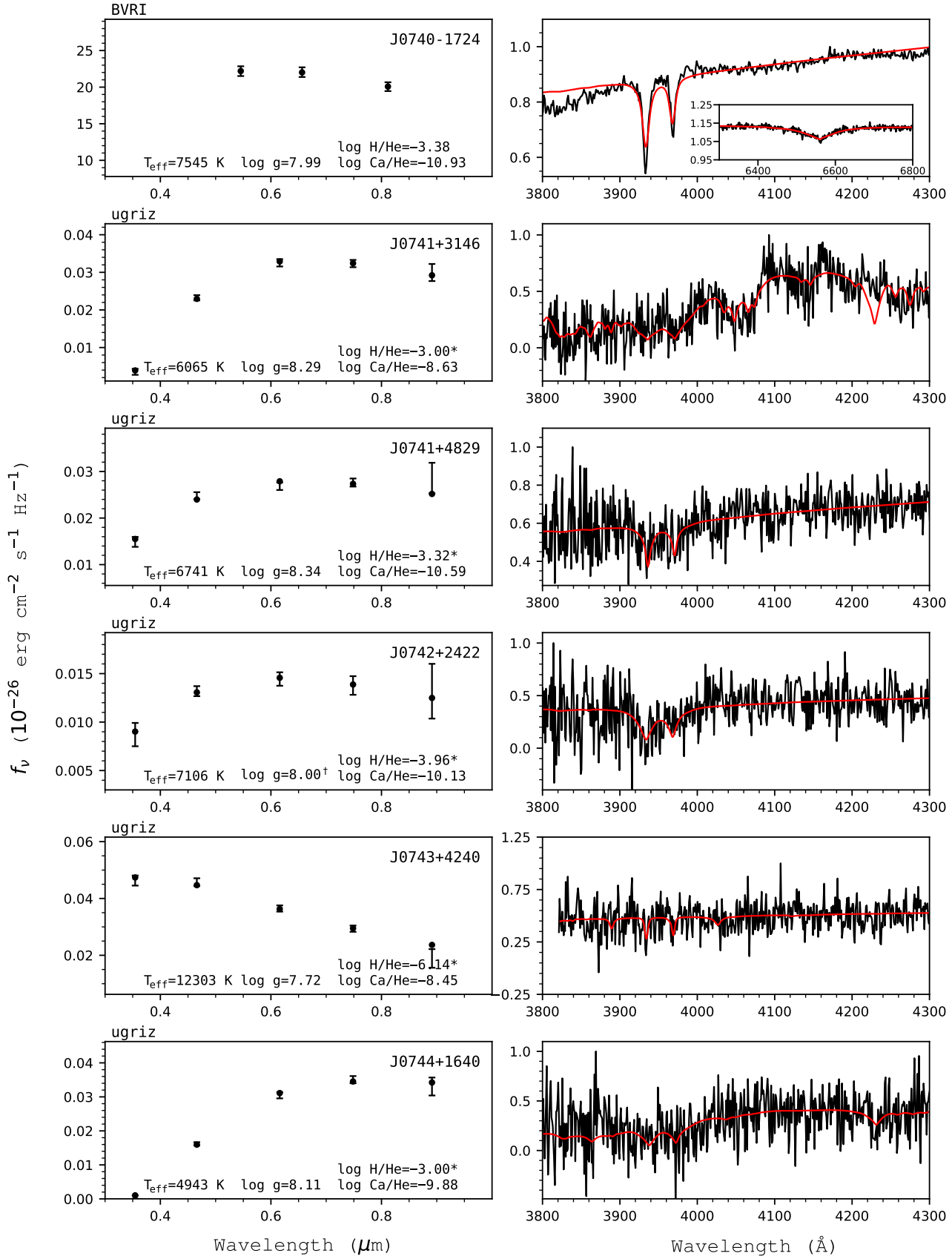

Figure 44. Fits to the DBZ/DZ(A) white dwarfs - continued. 

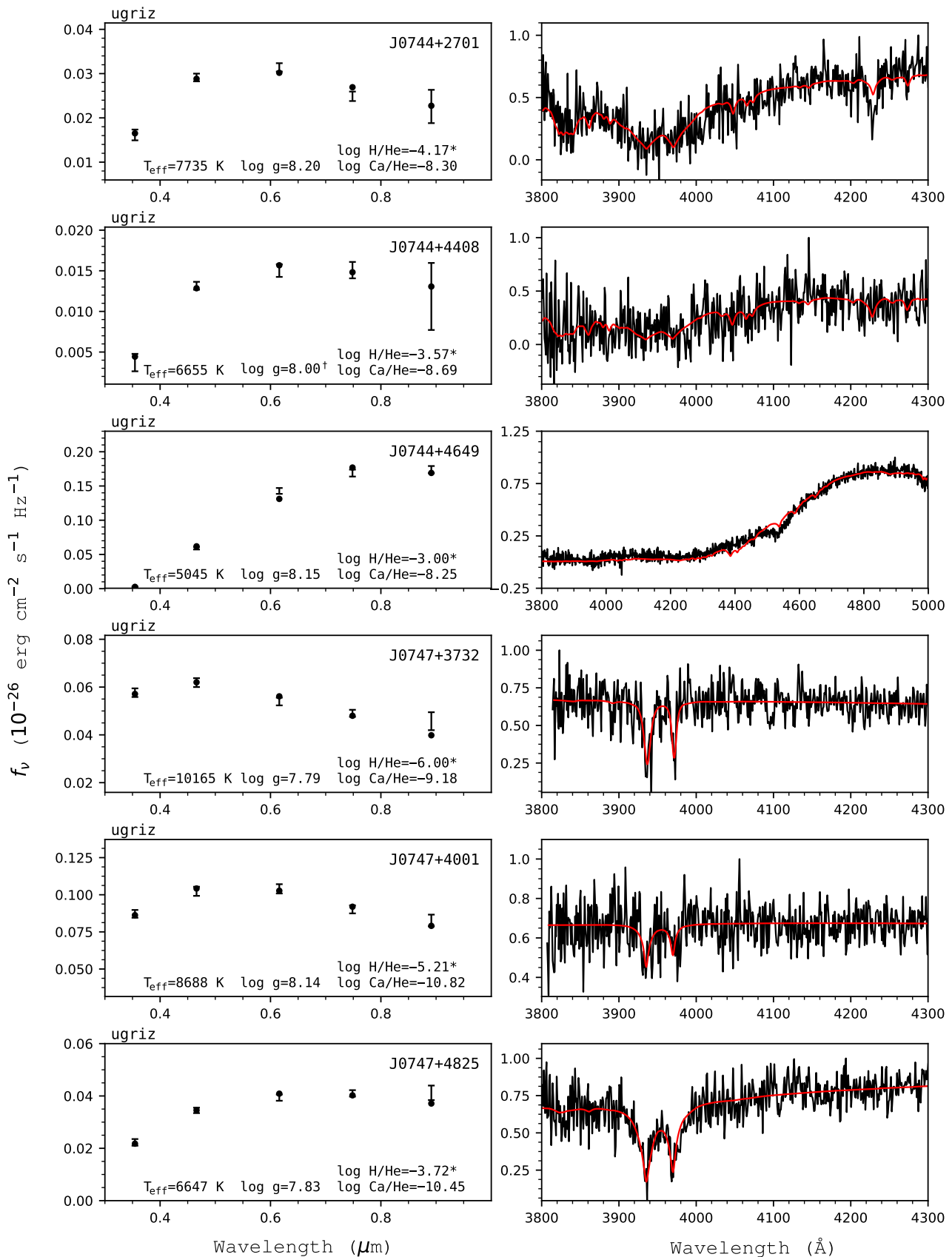

Figure 45. Fits to the DBZ/DZ(A) white dwarfs - continued. 

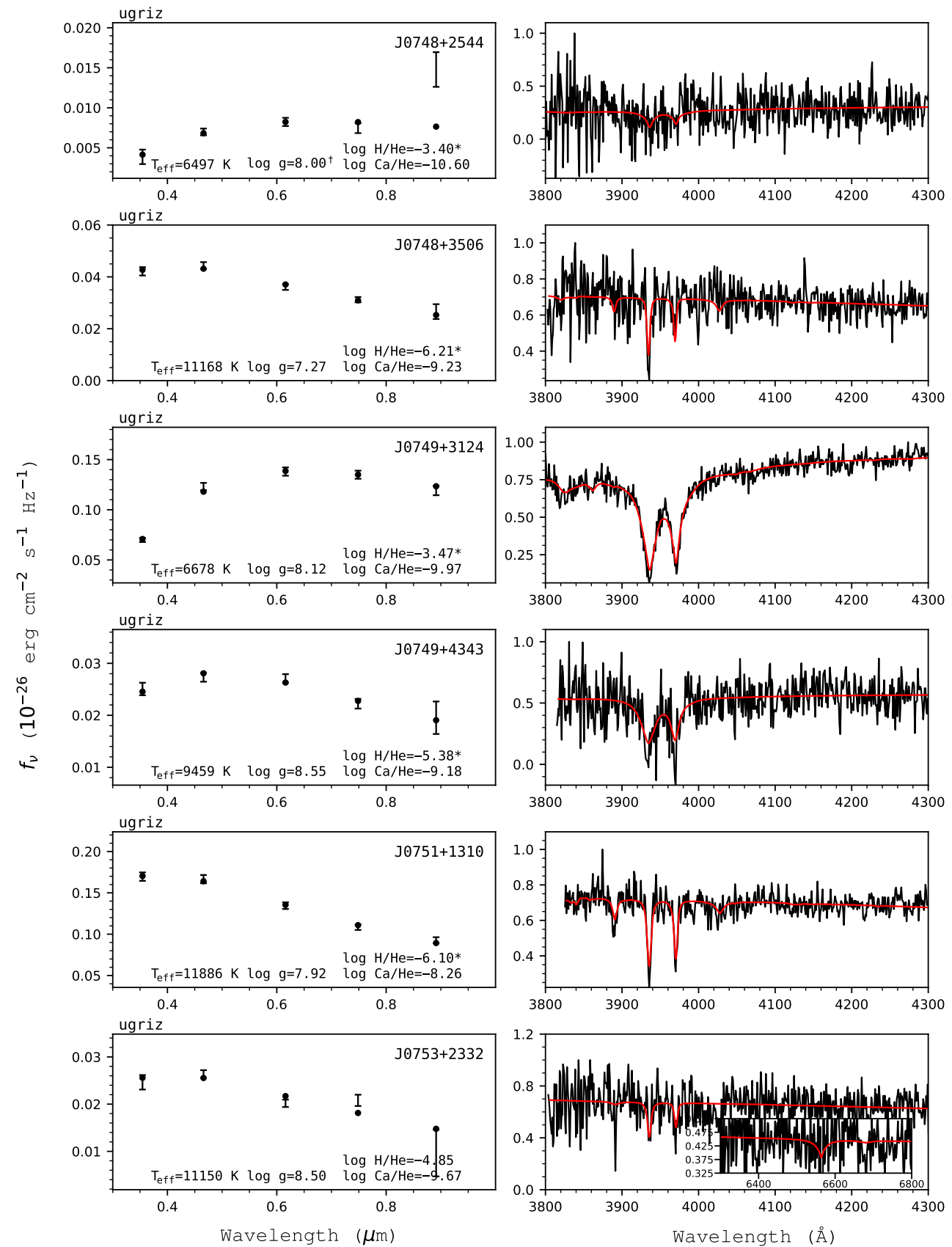

Figure 46. Fits to the DBZ/DZ(A) white dwarfs - continued. 

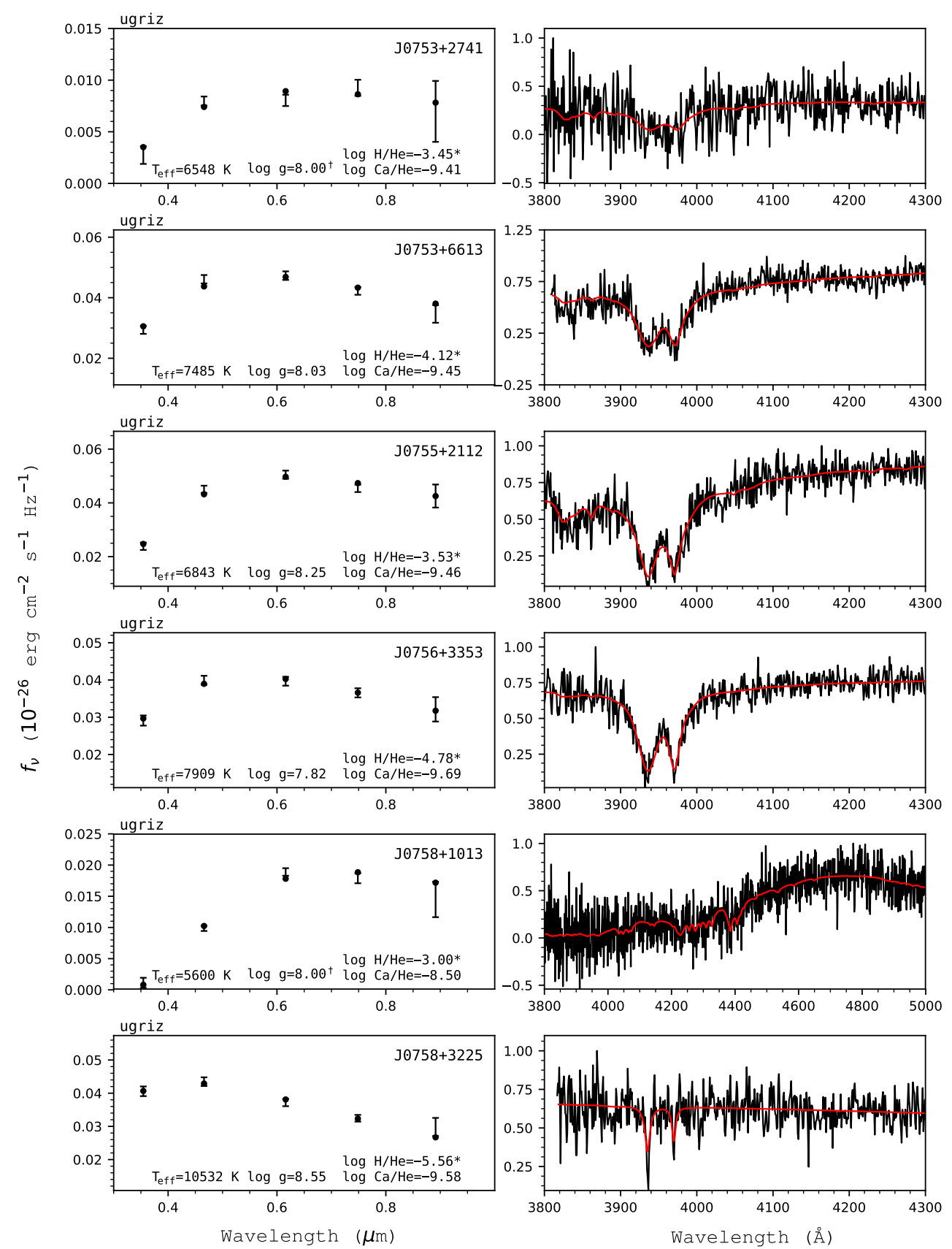

Figure 47. Fits to the DBZ/DZ(A) white dwarfs - continued. 

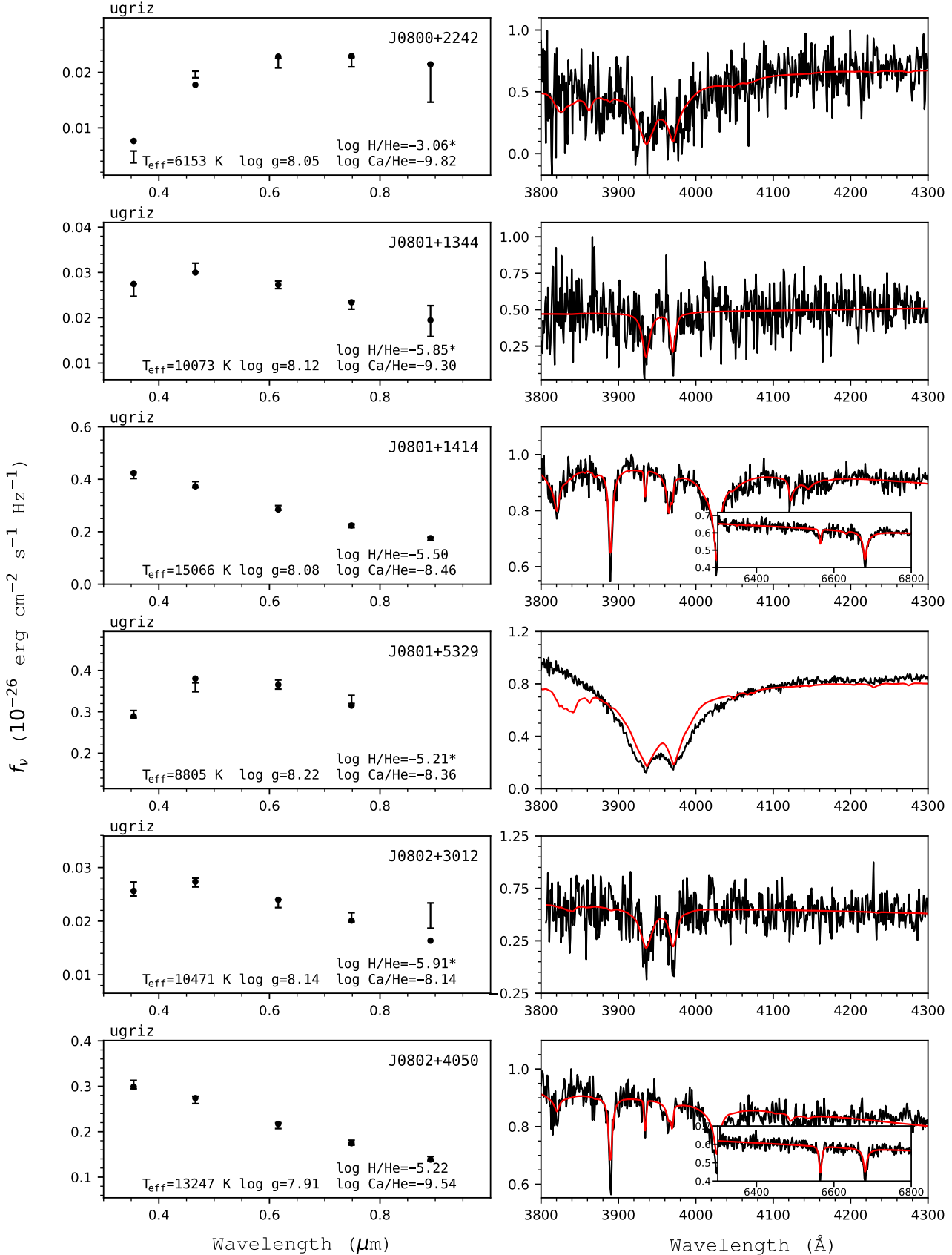

Figure 48. Fits to the DBZ/DZ(A) white dwarfs - continued. 

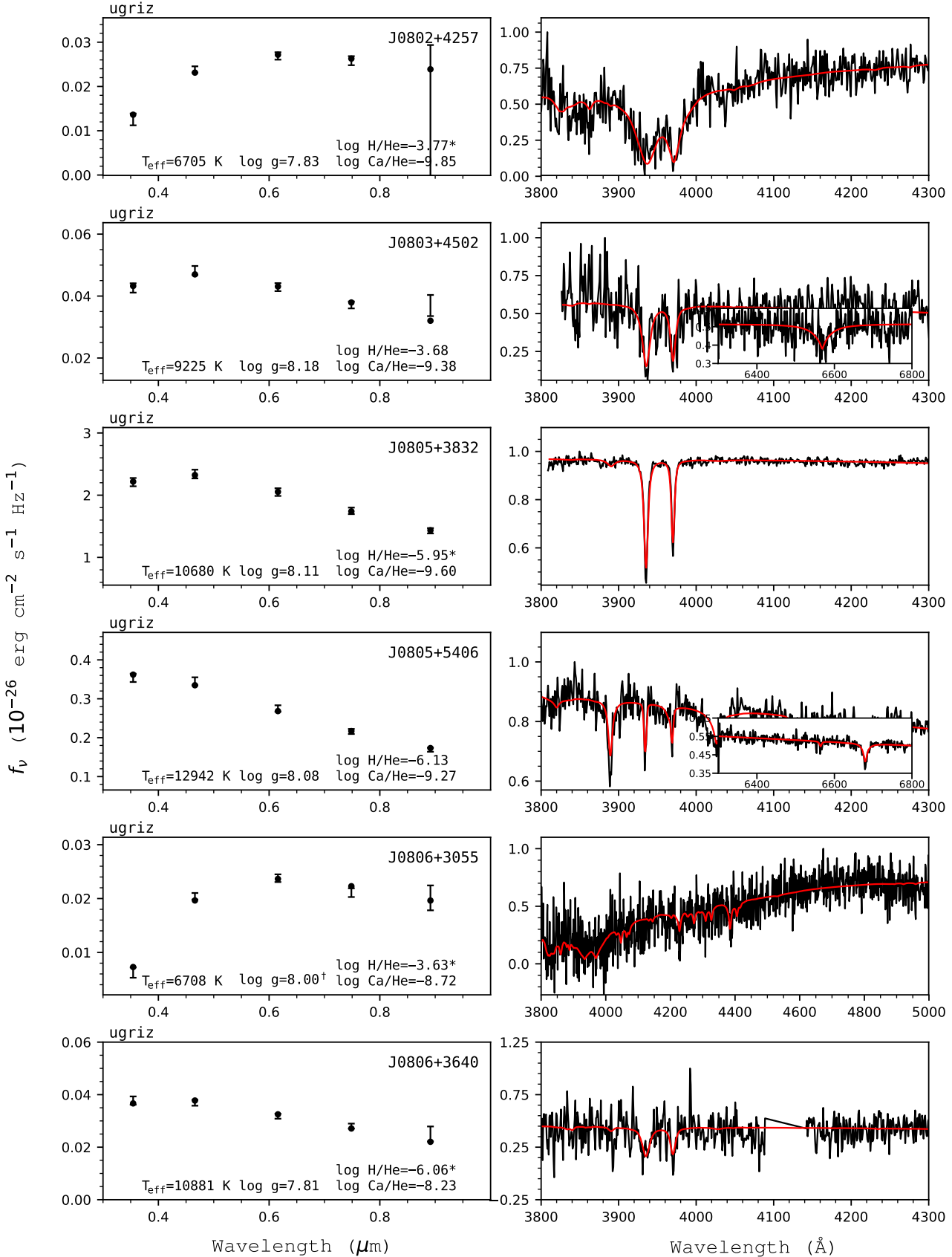

Figure 49. Fits to the DBZ/DZ(A) white dwarfs - continued. 

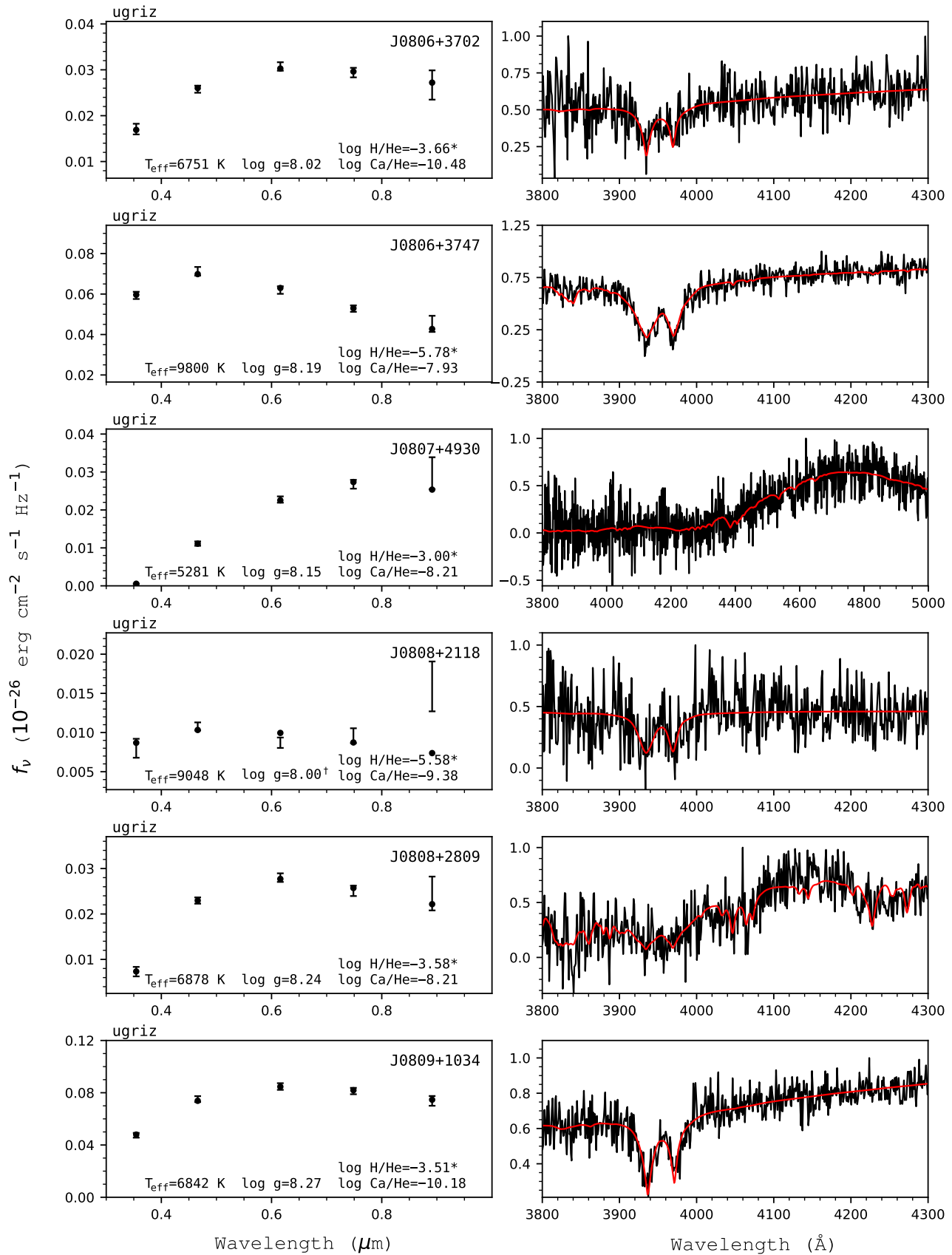

Figure 50. Fits to the DBZ/DZ(A) white dwarfs - continued. 

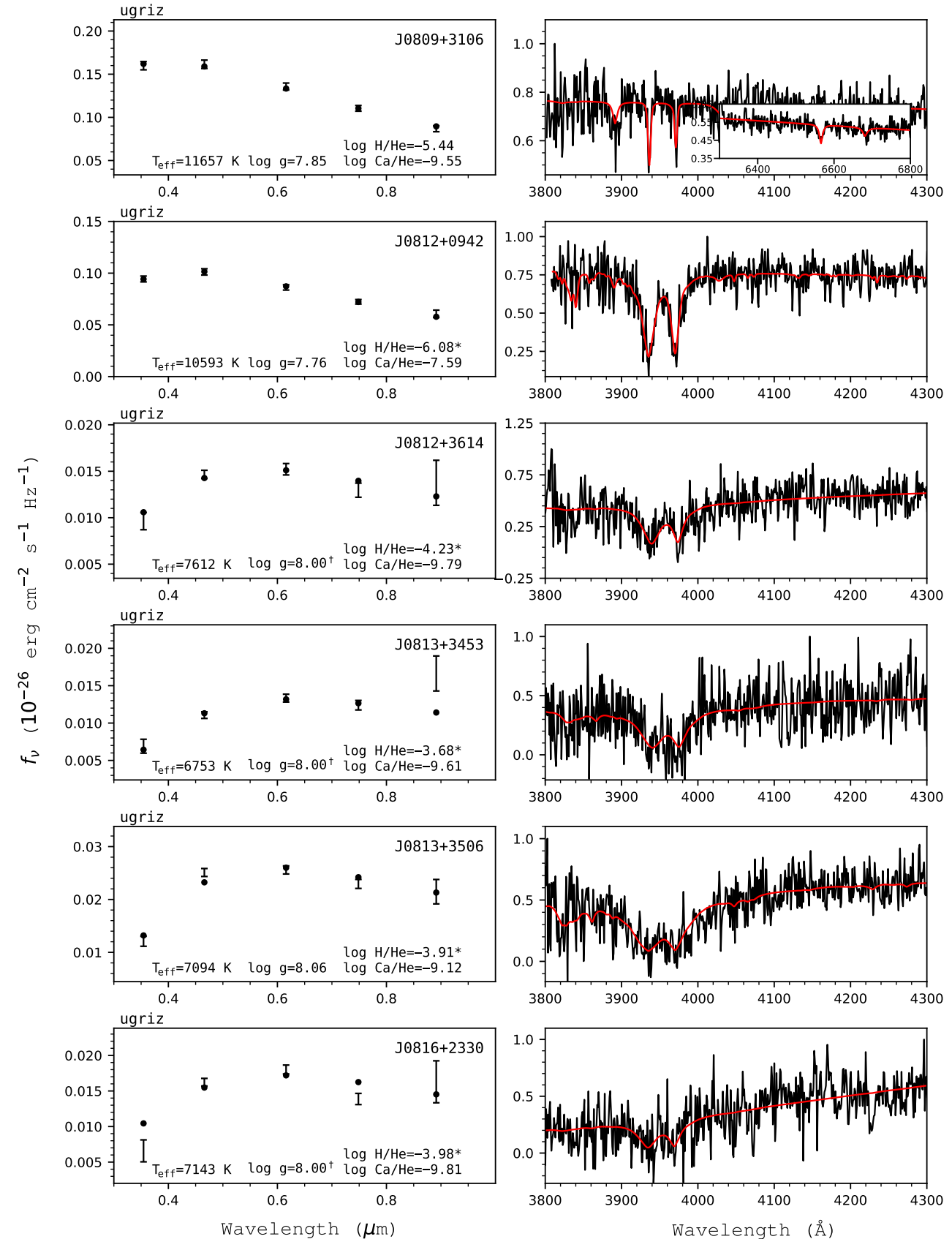

Figure 51. Fits to the DBZ/DZ(A) white dwarfs - continued. 

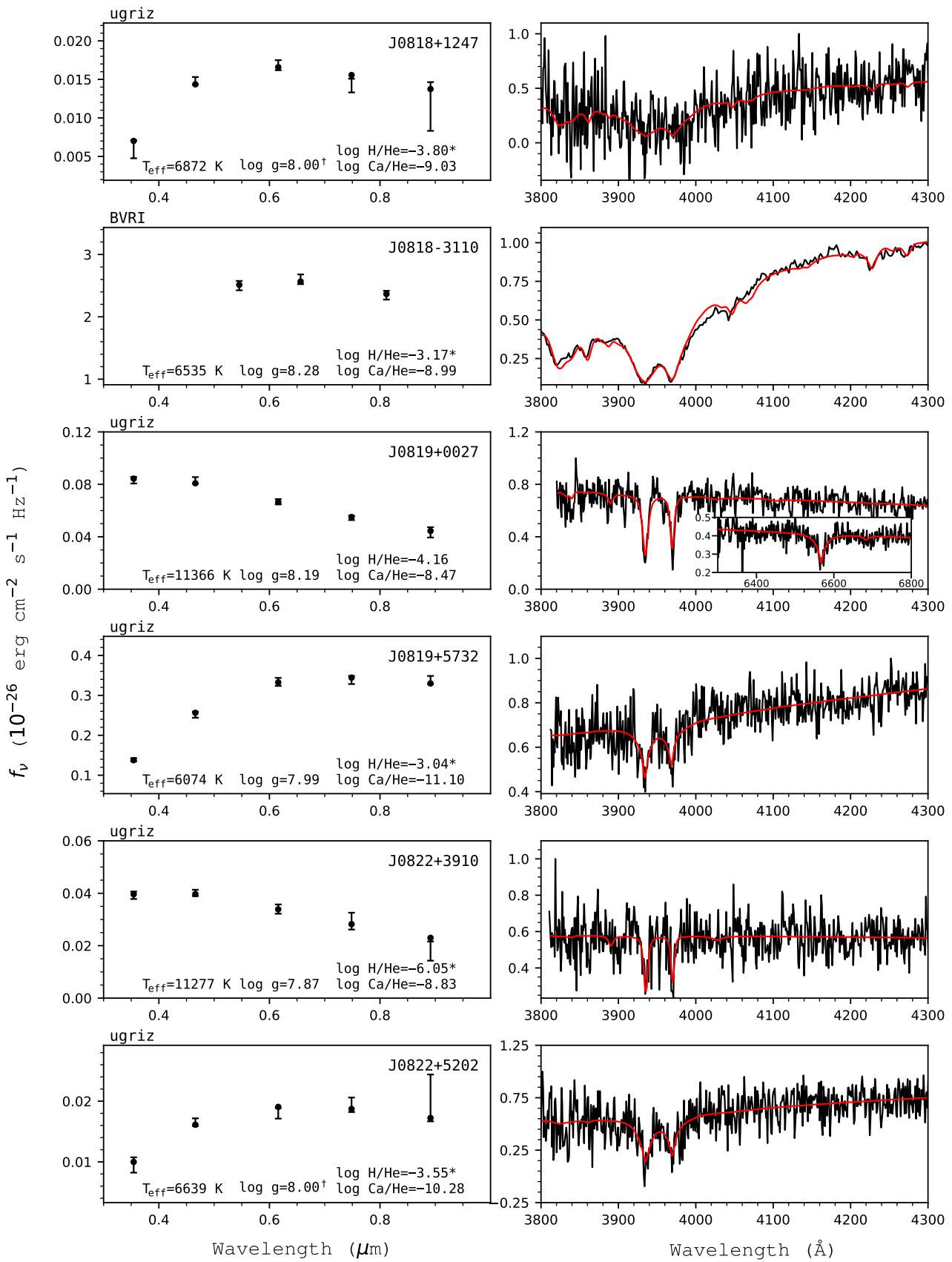

Figure 52. Fits to the DBZ/DZ(A) white dwarfs - continued. 

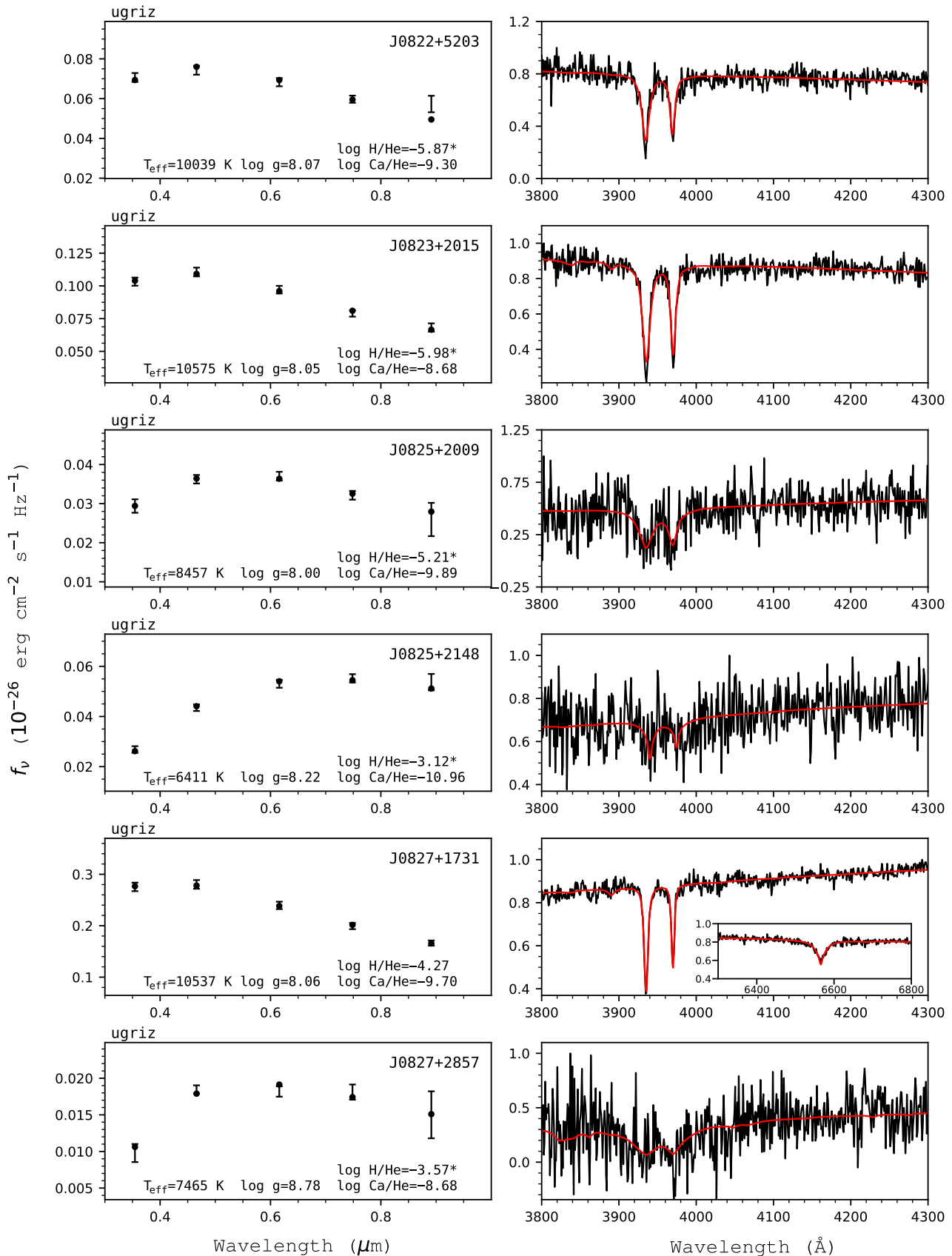

Figure 53. Fits to the DBZ/DZ(A) white dwarfs - continued. 

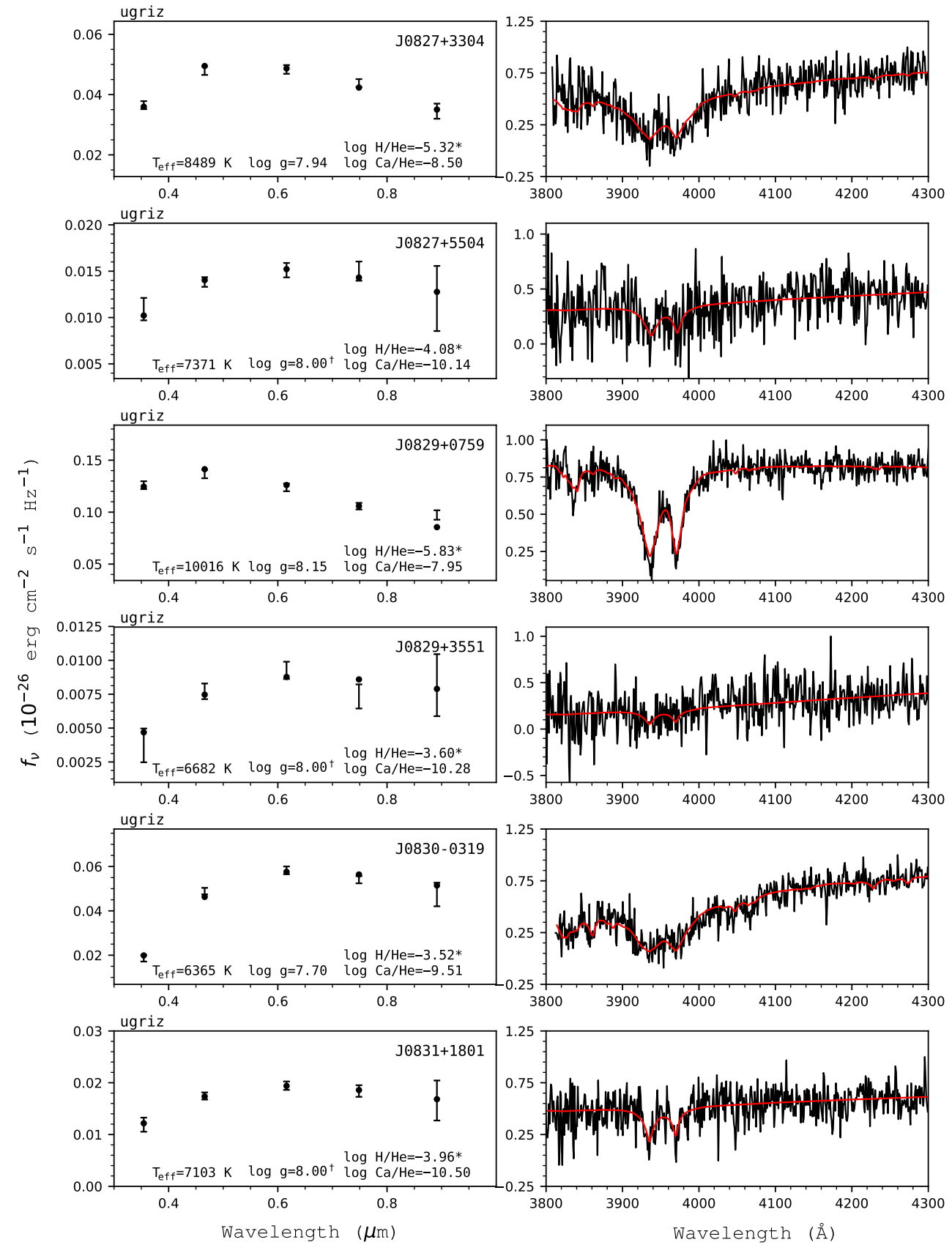

Figure 54. Fits to the DBZ/DZ(A) white dwarfs - continued. 

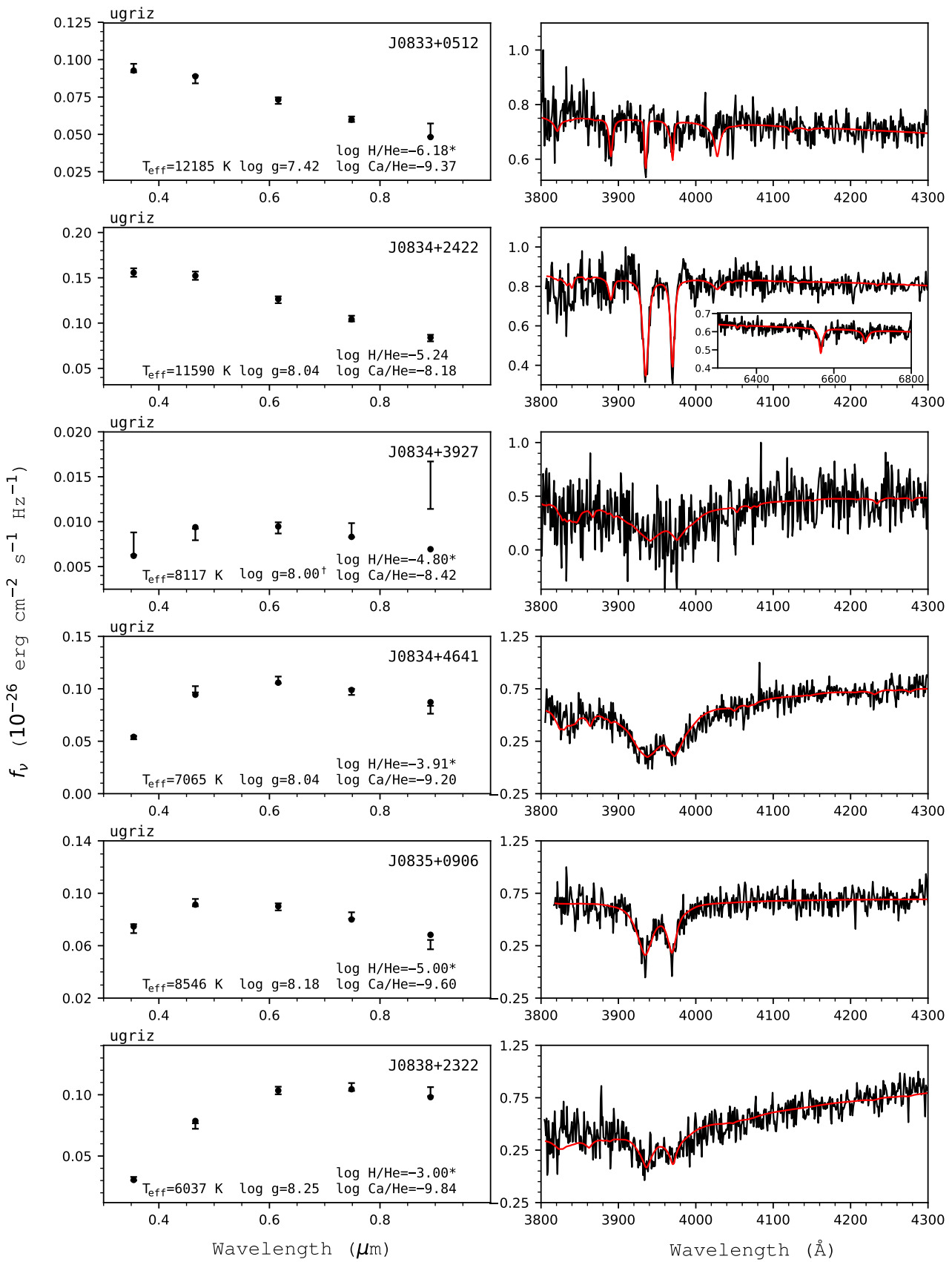

Figure 55. Fits to the DBZ/DZ(A) white dwarfs - continued. 

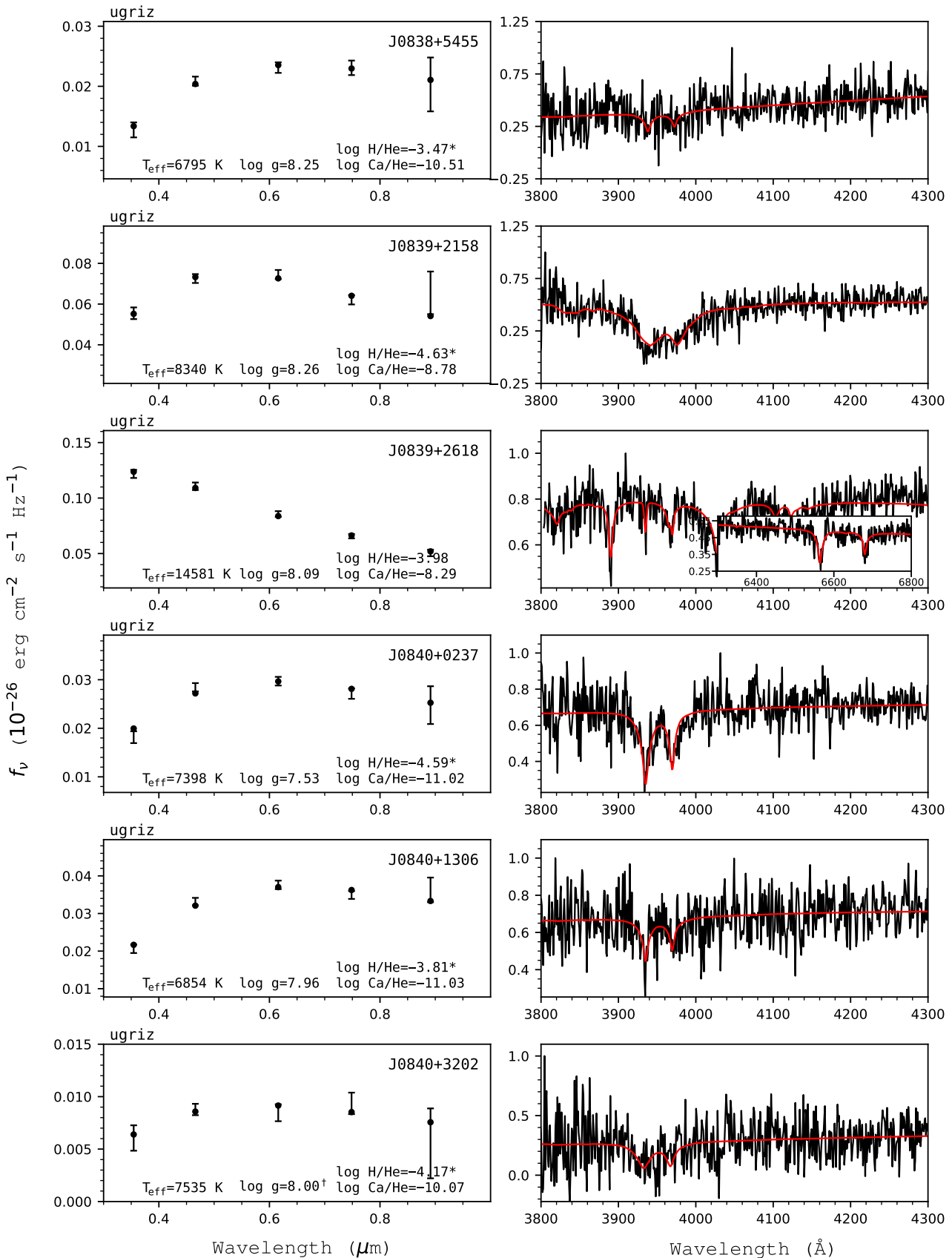

Figure 56. Fits to the DBZ/DZ(A) white dwarfs - continued. 

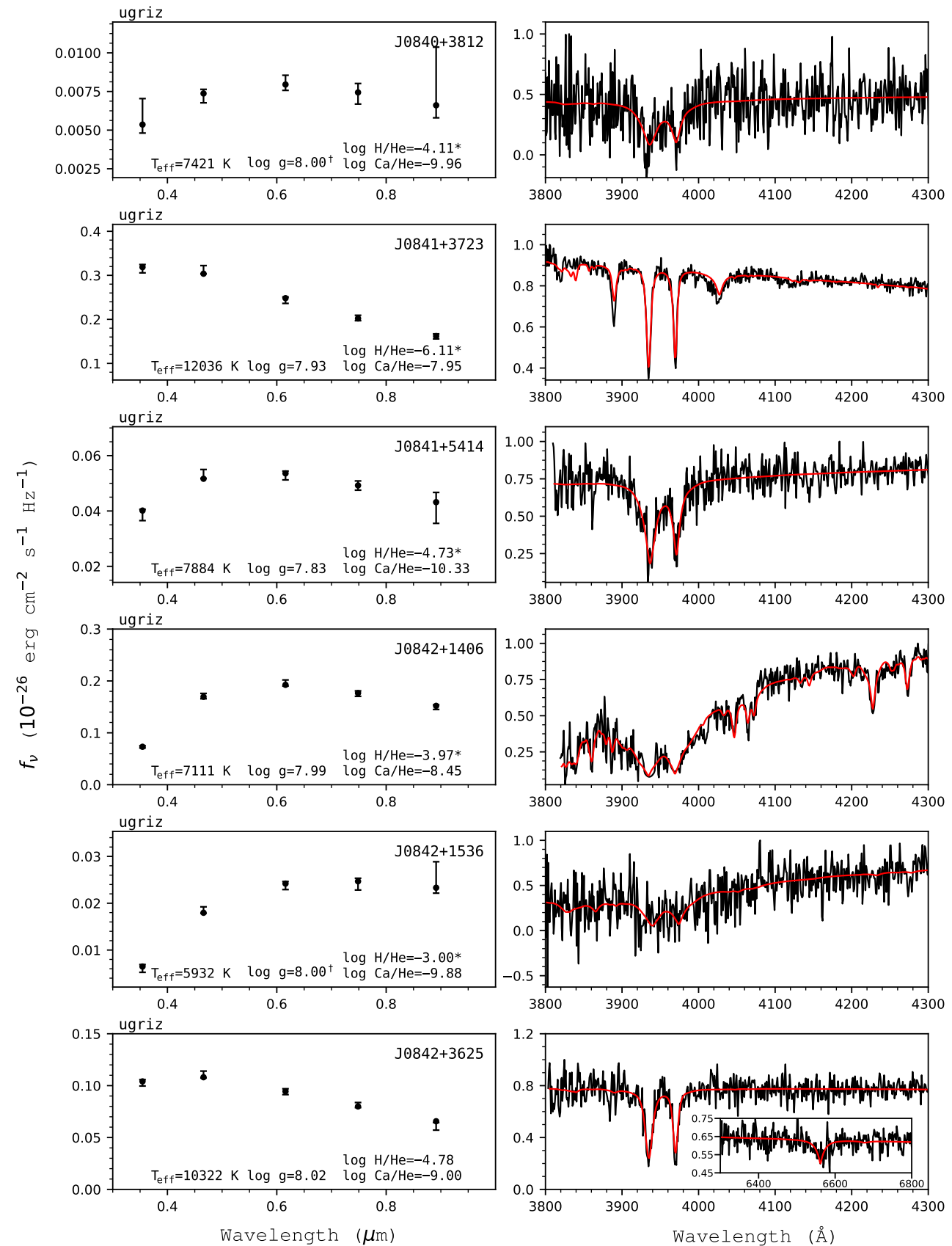

Figure 57. Fits to the DBZ/DZ(A) white dwarfs - continued. 

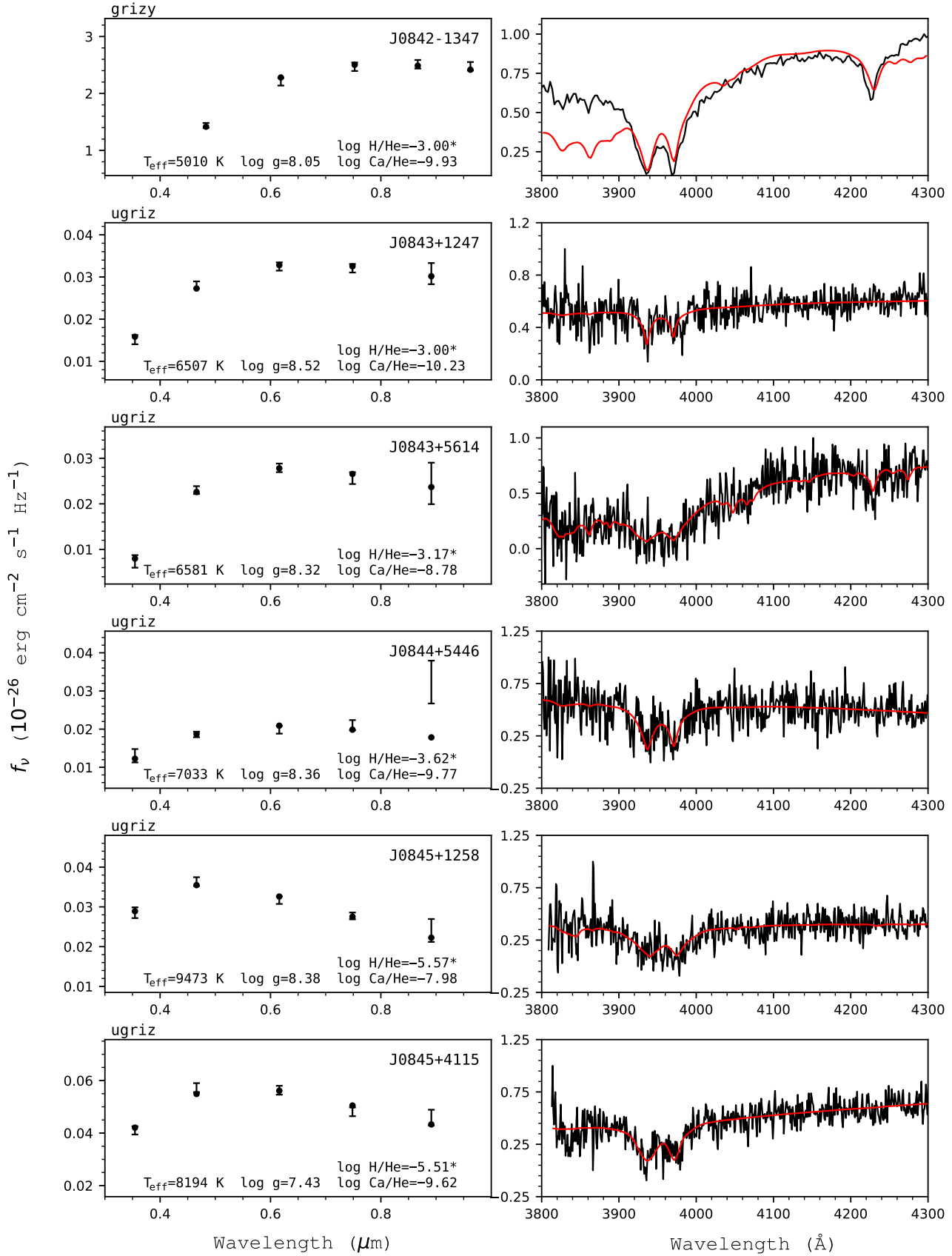

Figure 58. Fits to the DBZ/DZ(A) white dwarfs - continued. 

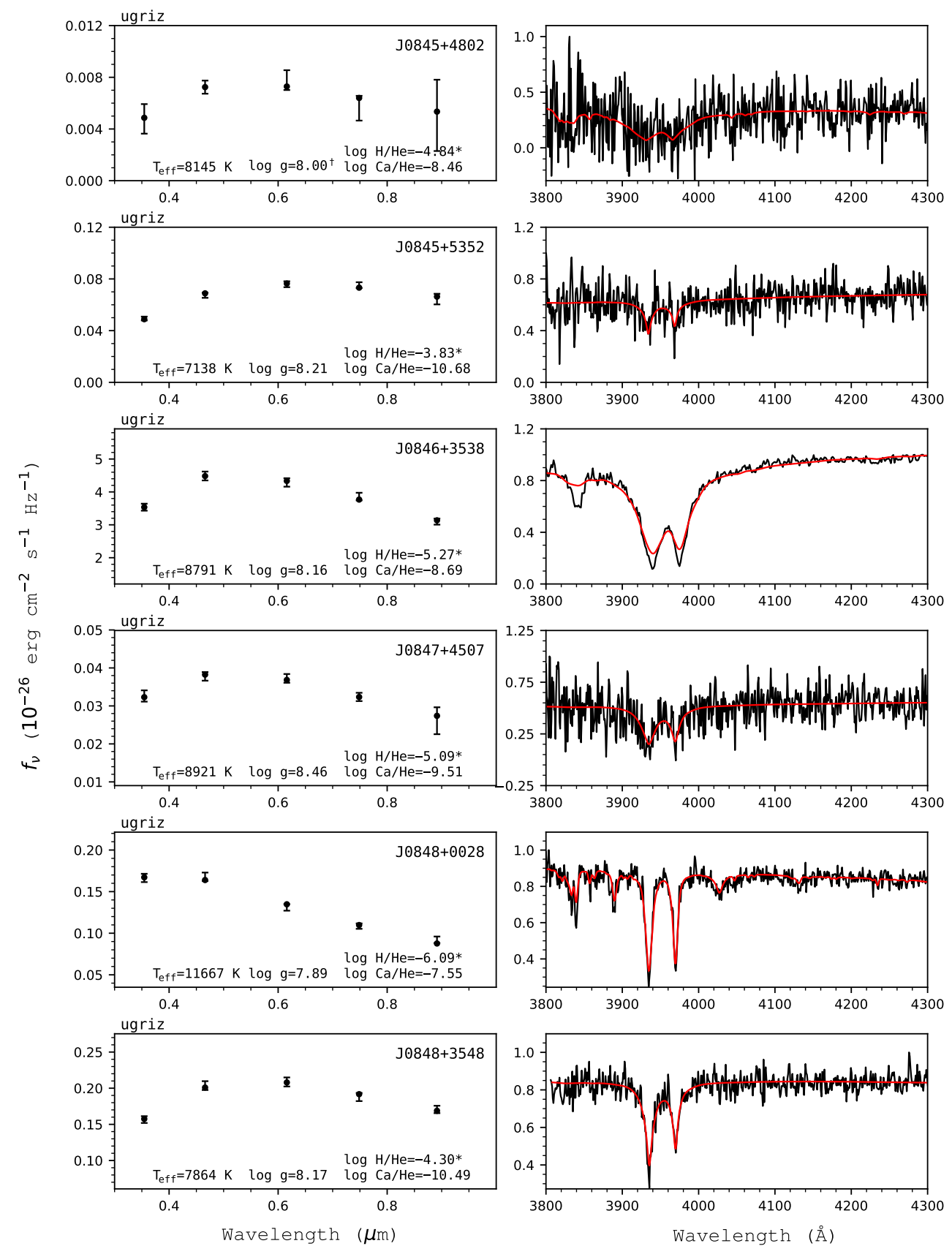

Figure 59. Fits to the DBZ/DZ(A) white dwarfs - continued. 

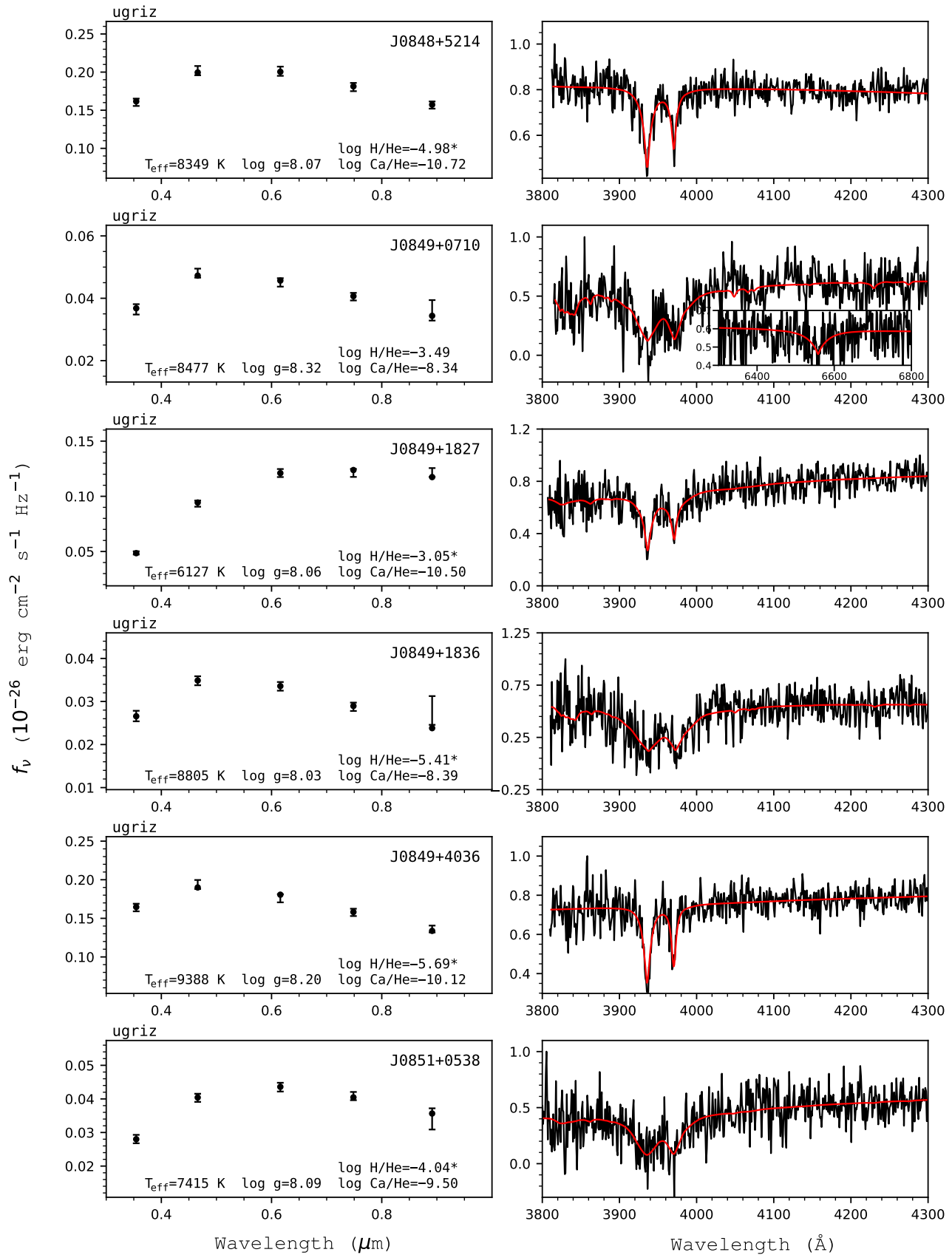

Figure 60. Fits to the DBZ/DZ(A) white dwarfs - continued. 

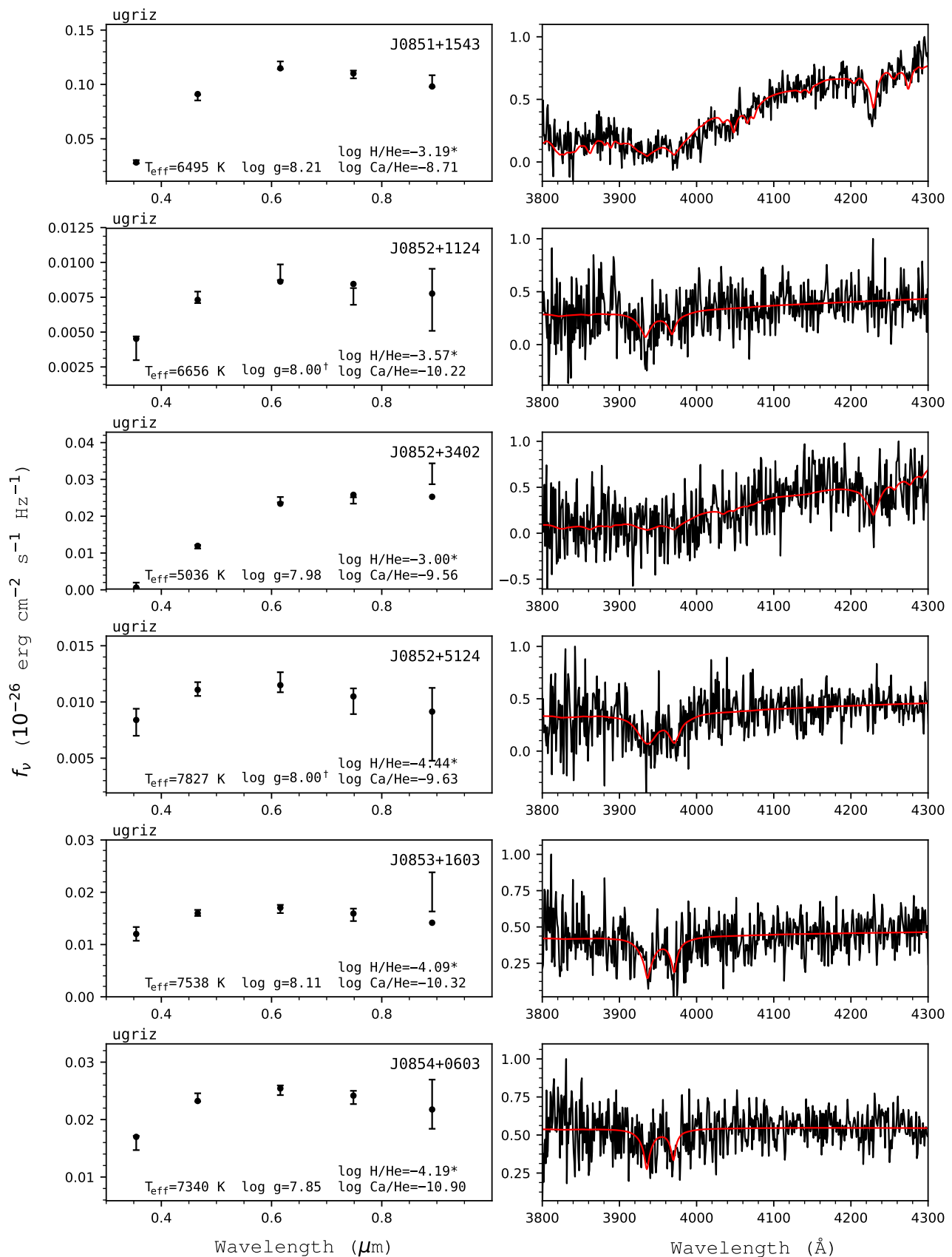

Figure 61. Fits to the DBZ/DZ(A) white dwarfs - continued. 

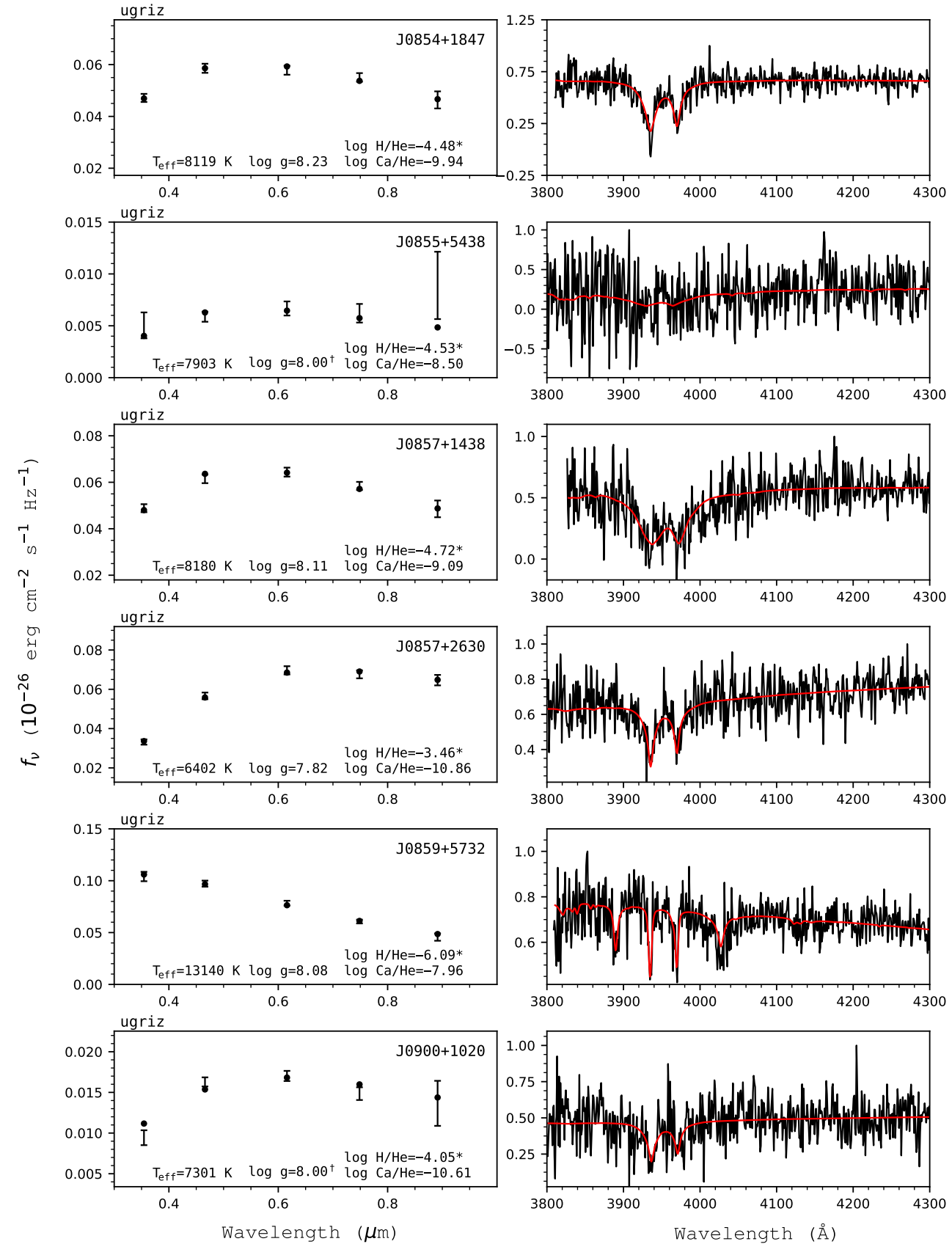

Figure 62. Fits to the DBZ/DZ(A) white dwarfs - continued. 

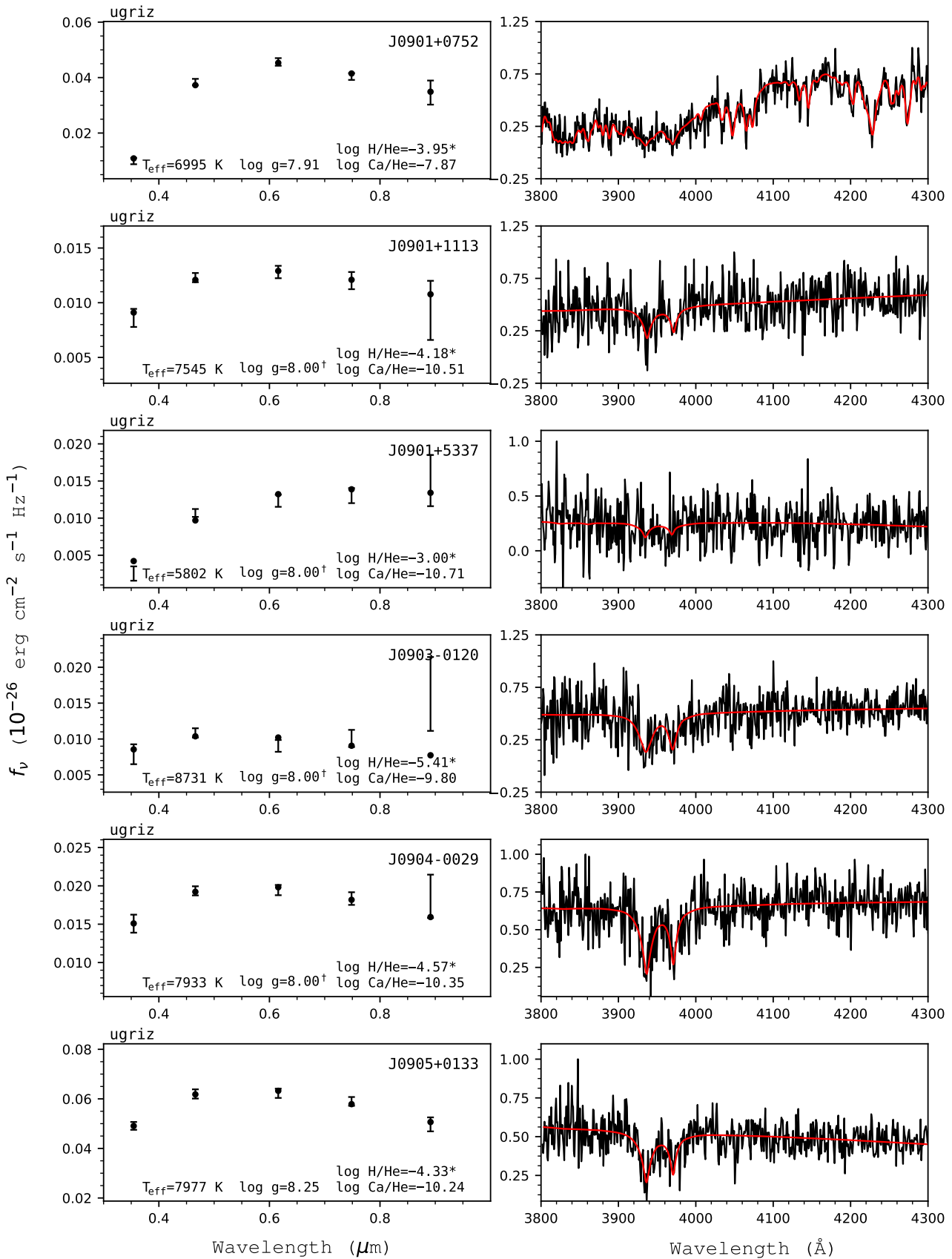

Figure 63. Fits to the DBZ/DZ(A) white dwarfs - continued. 

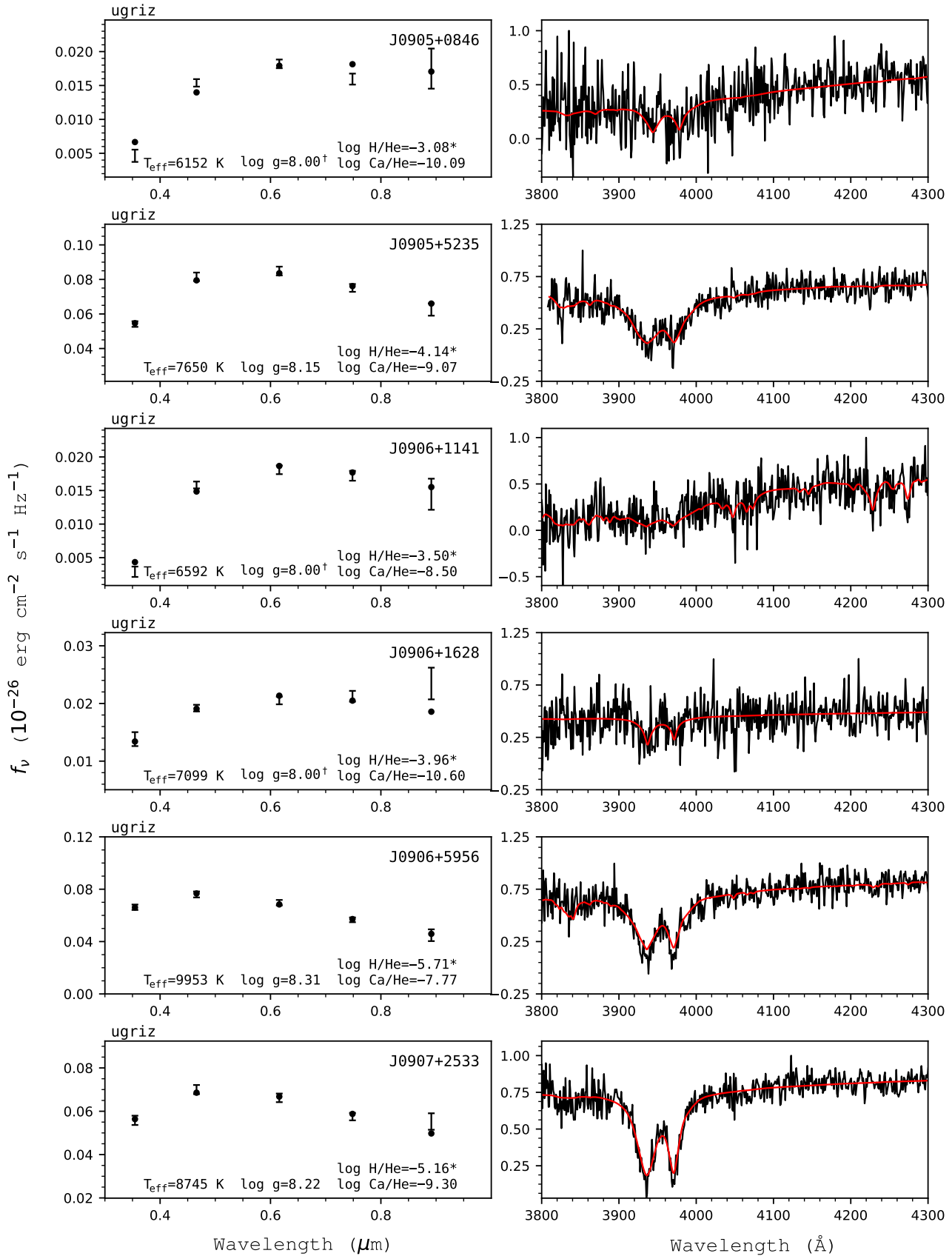

Figure 64. Fits to the DBZ/DZ(A) white dwarfs - continued. 

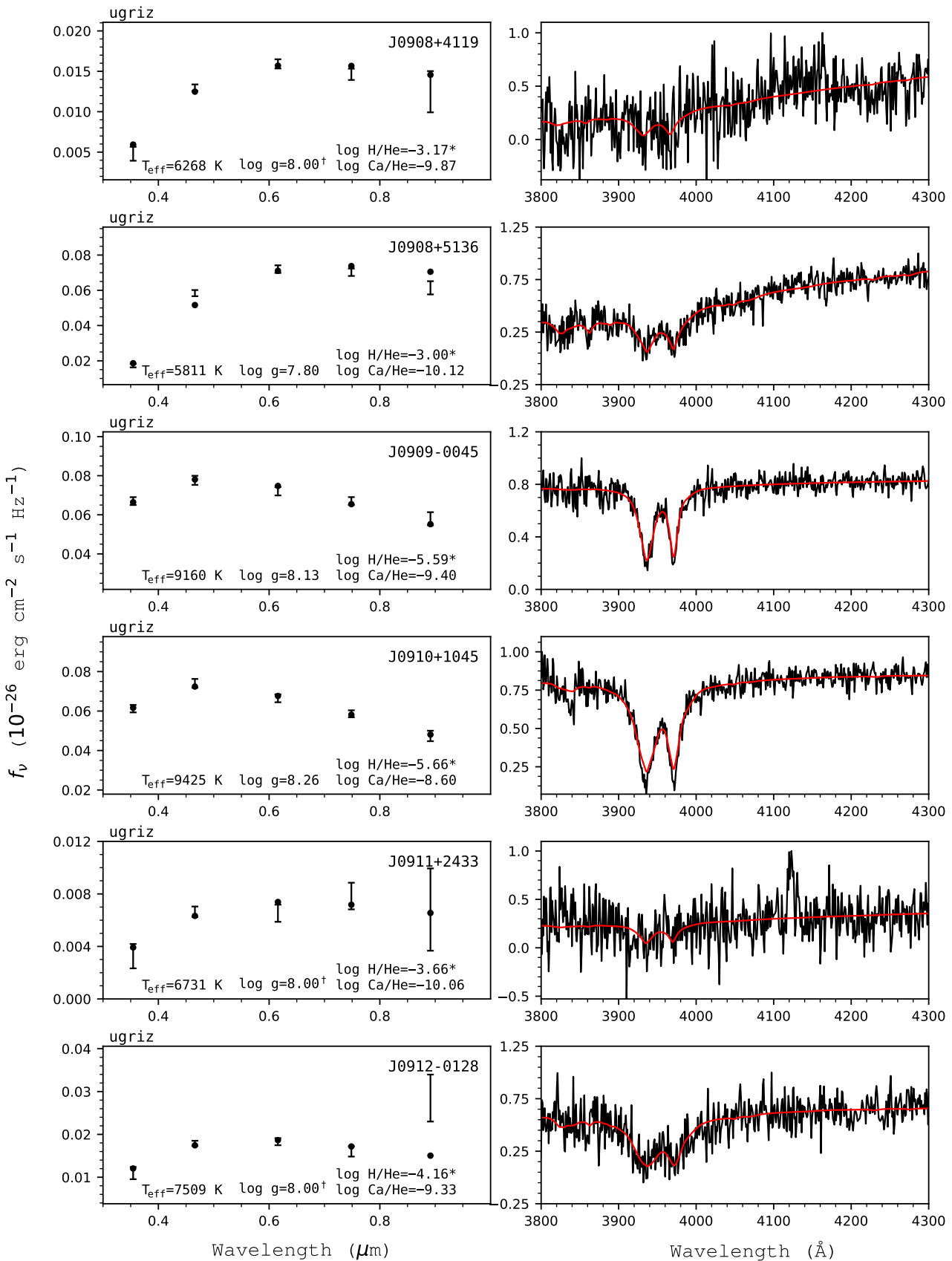

Figure 65. Fits to the DBZ/DZ(A) white dwarfs - continued. 

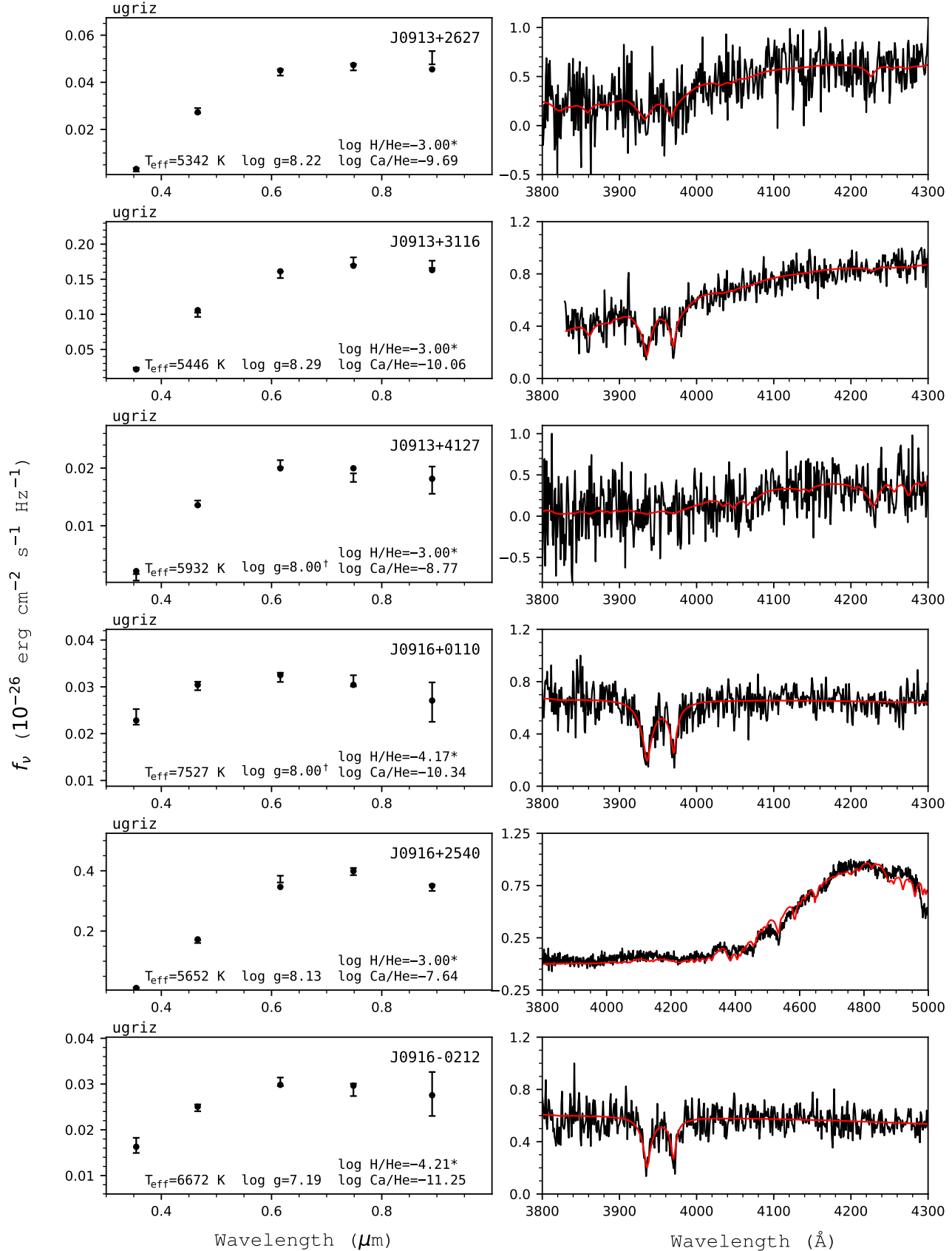

Figure 66. Fits to the DBZ/DZ(A) white dwarfs - continued. 

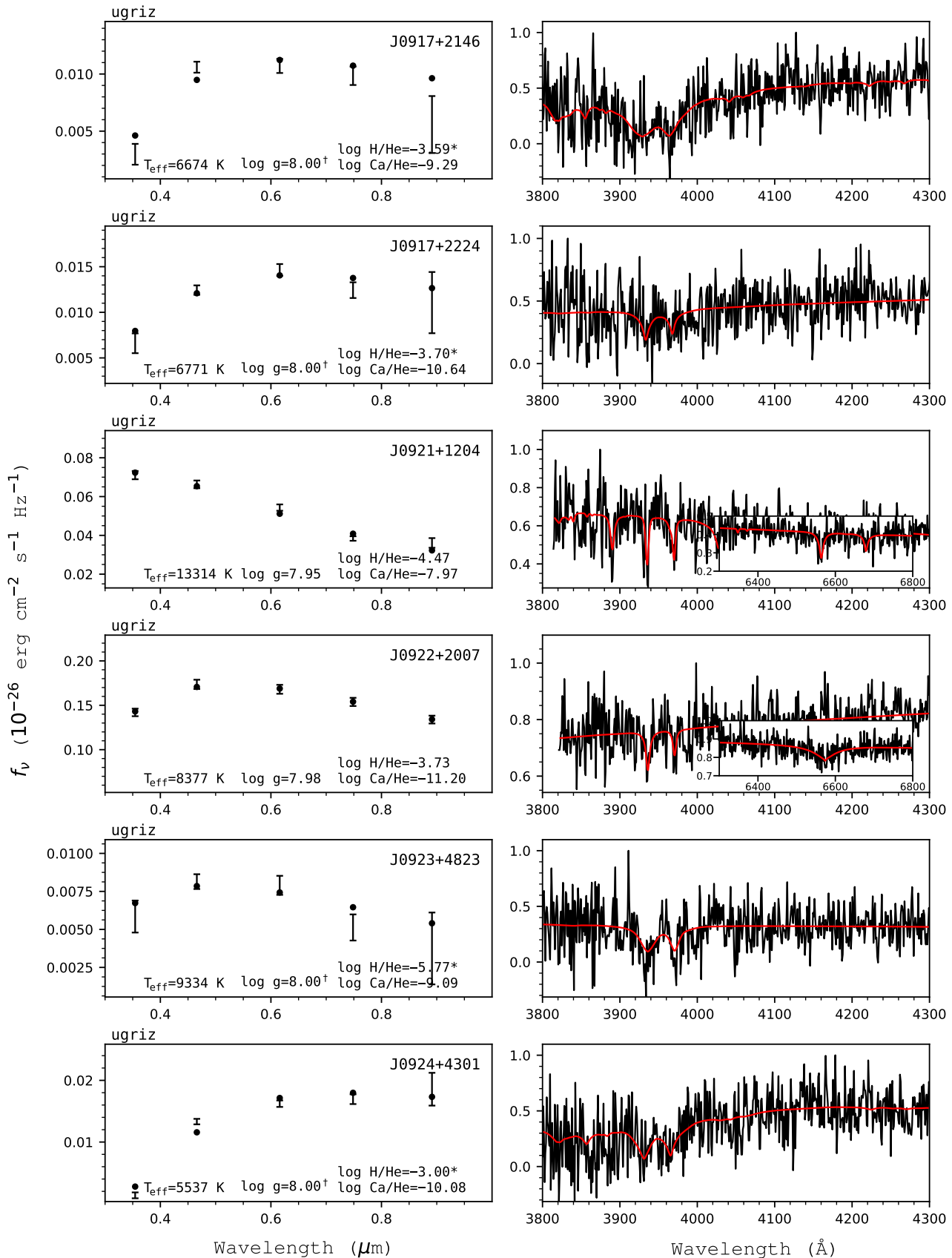

Figure 67. Fits to the DBZ/DZ(A) white dwarfs - continued. 

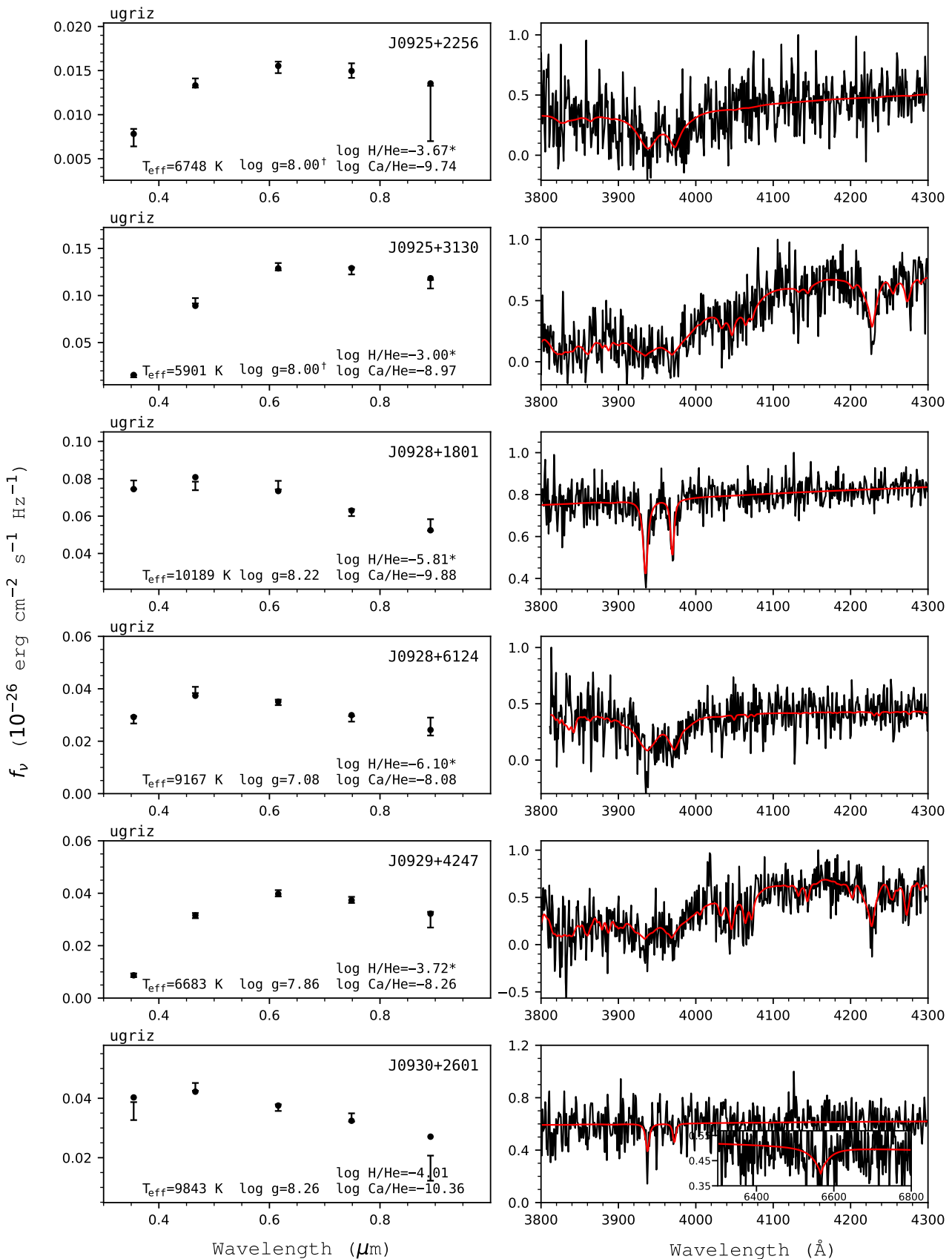

Figure 68. Fits to the DBZ/DZ(A) white dwarfs - continued. 

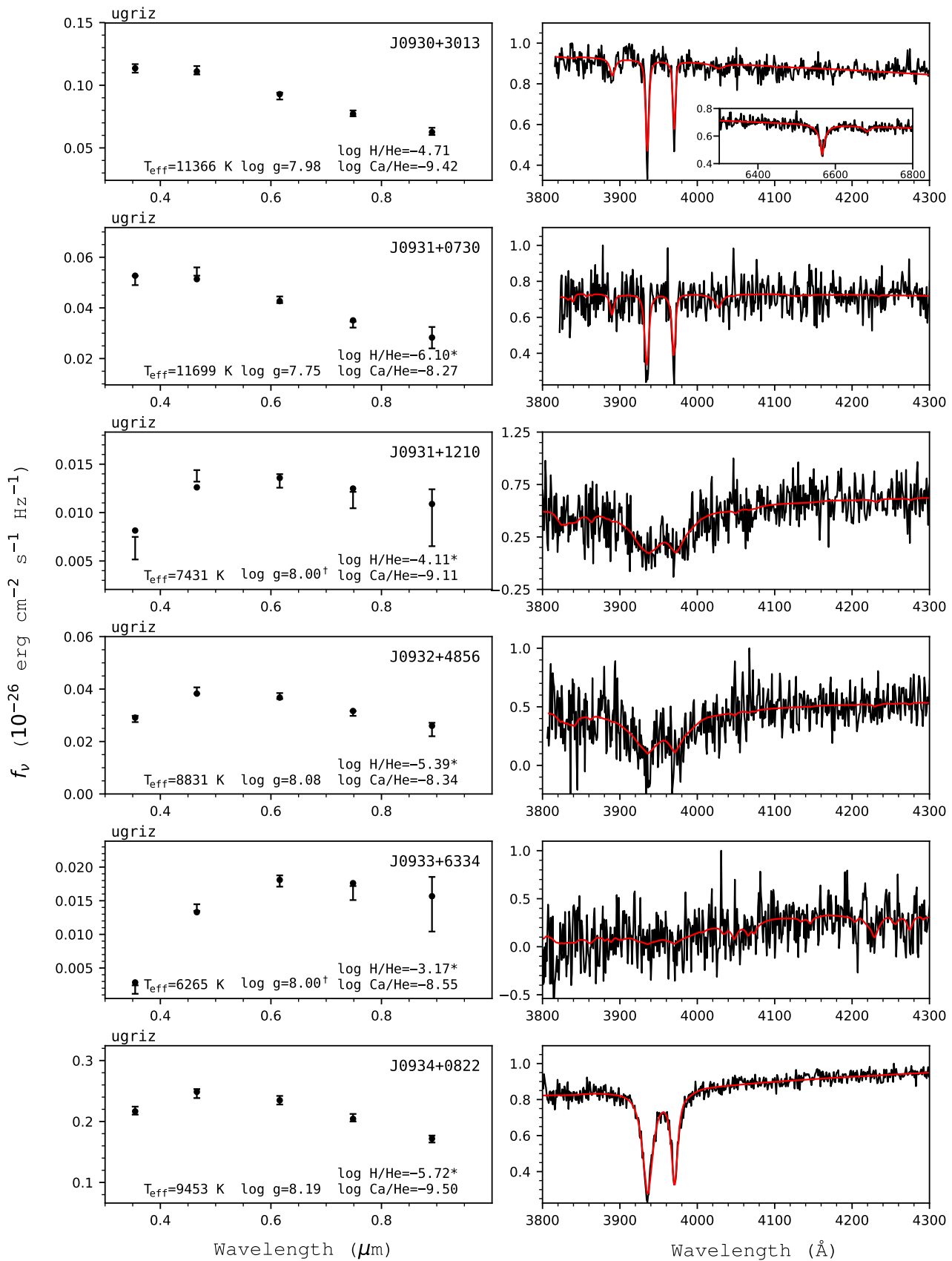

Figure 69. Fits to the DBZ/DZ(A) white dwarfs - continued. 

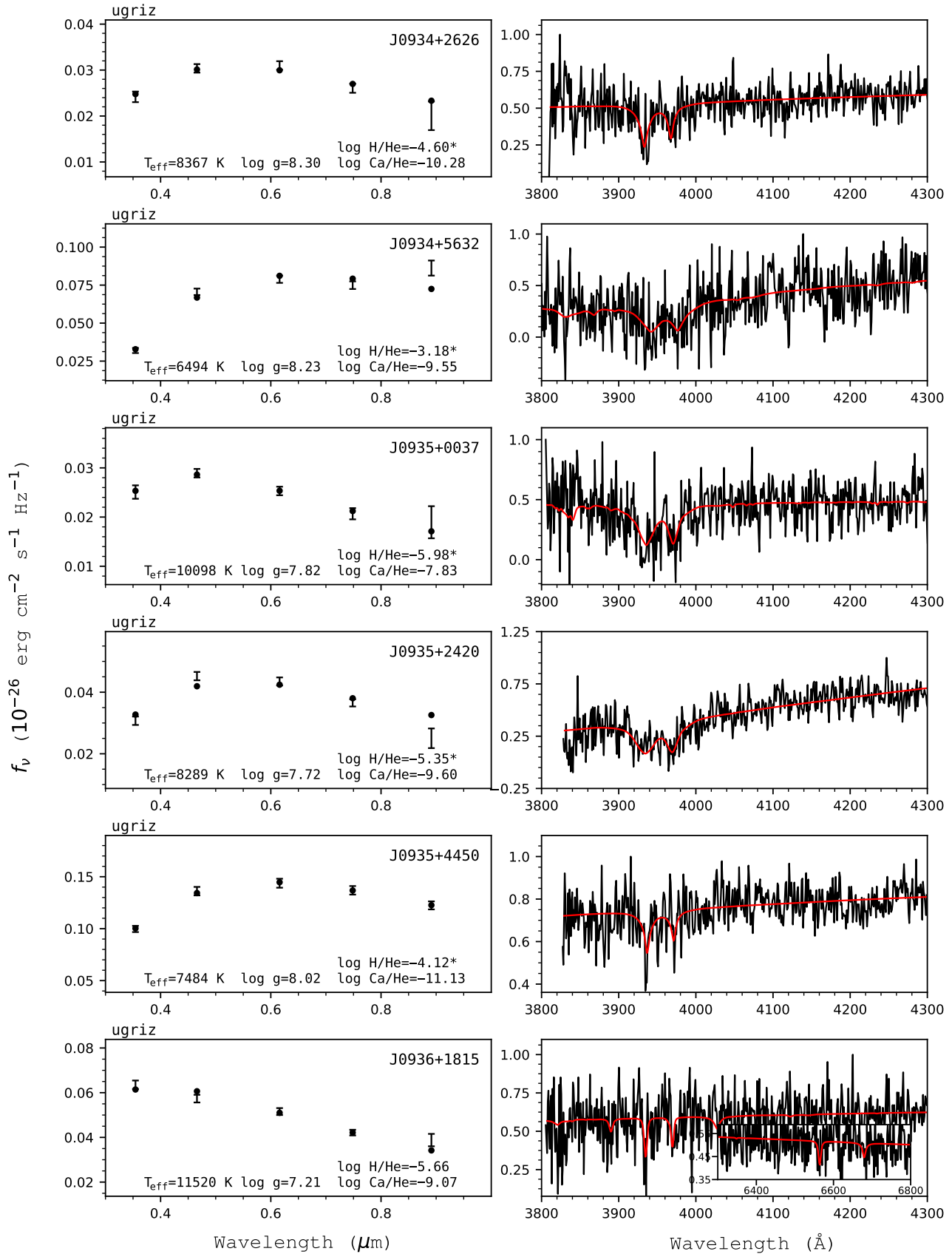

Figure 70. Fits to the DBZ/DZ(A) white dwarfs - continued. 

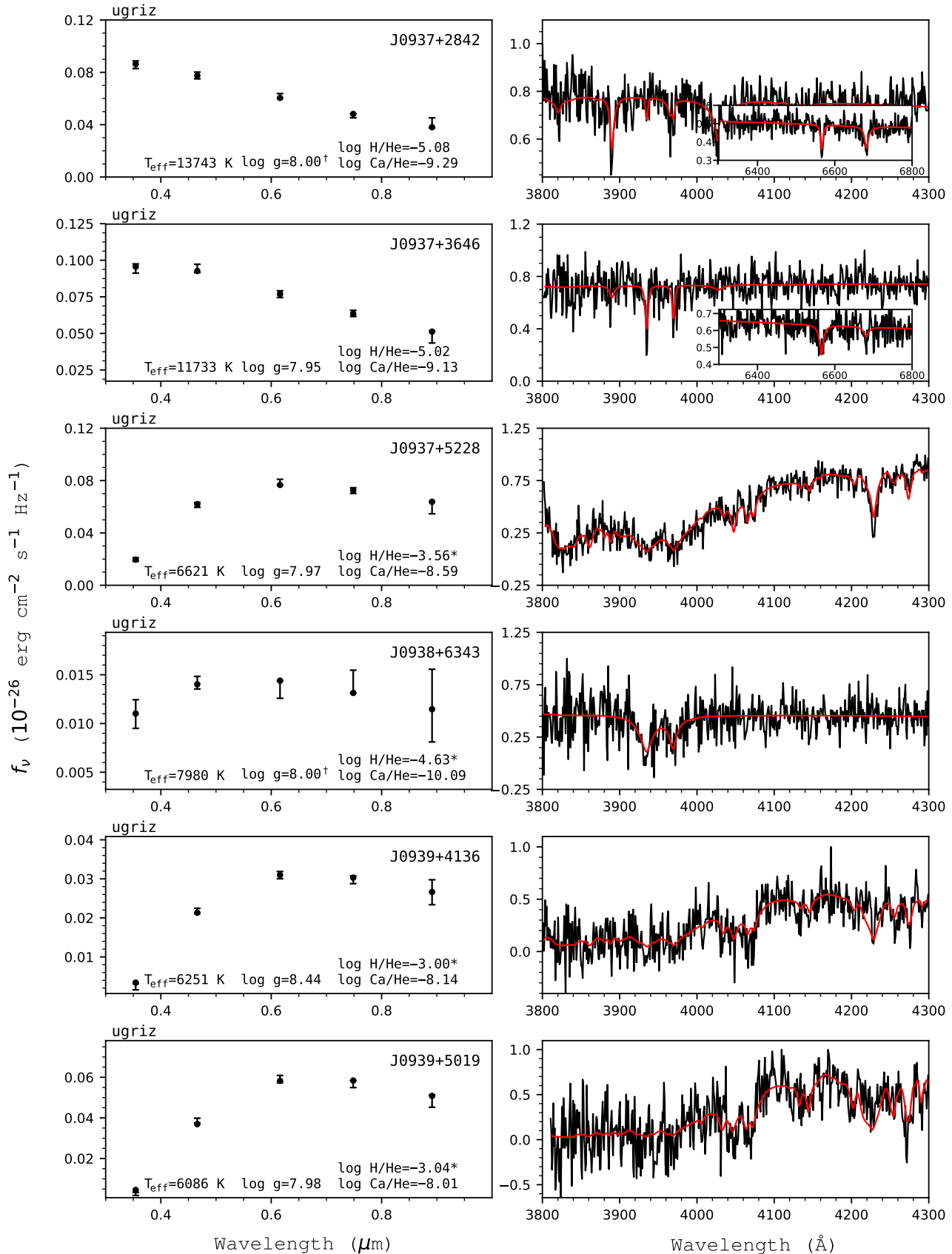

Figure 71. Fits to the DBZ/DZ(A) white dwarfs - continued. 

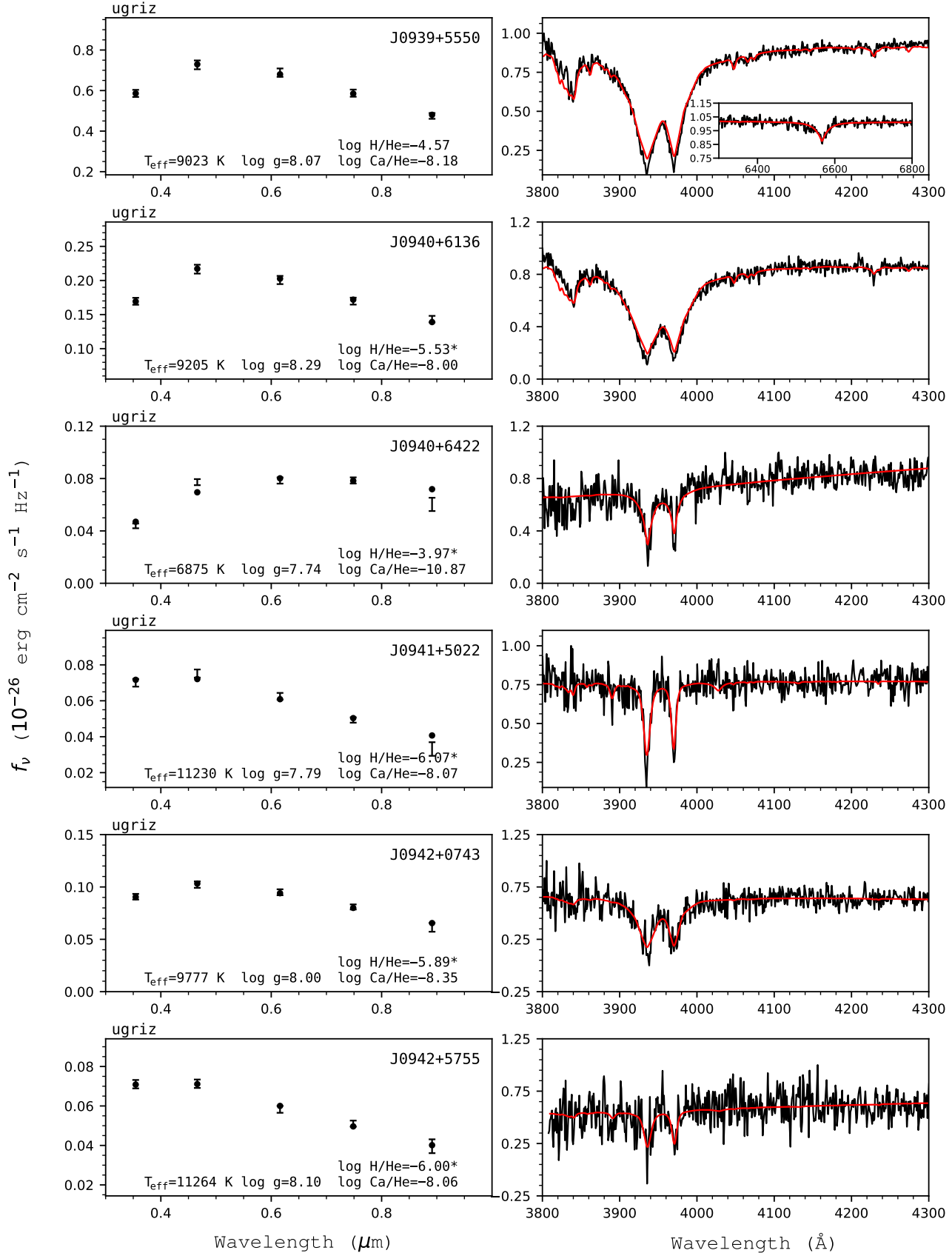

Figure 72. Fits to the DBZ/DZ(A) white dwarfs - continued. 

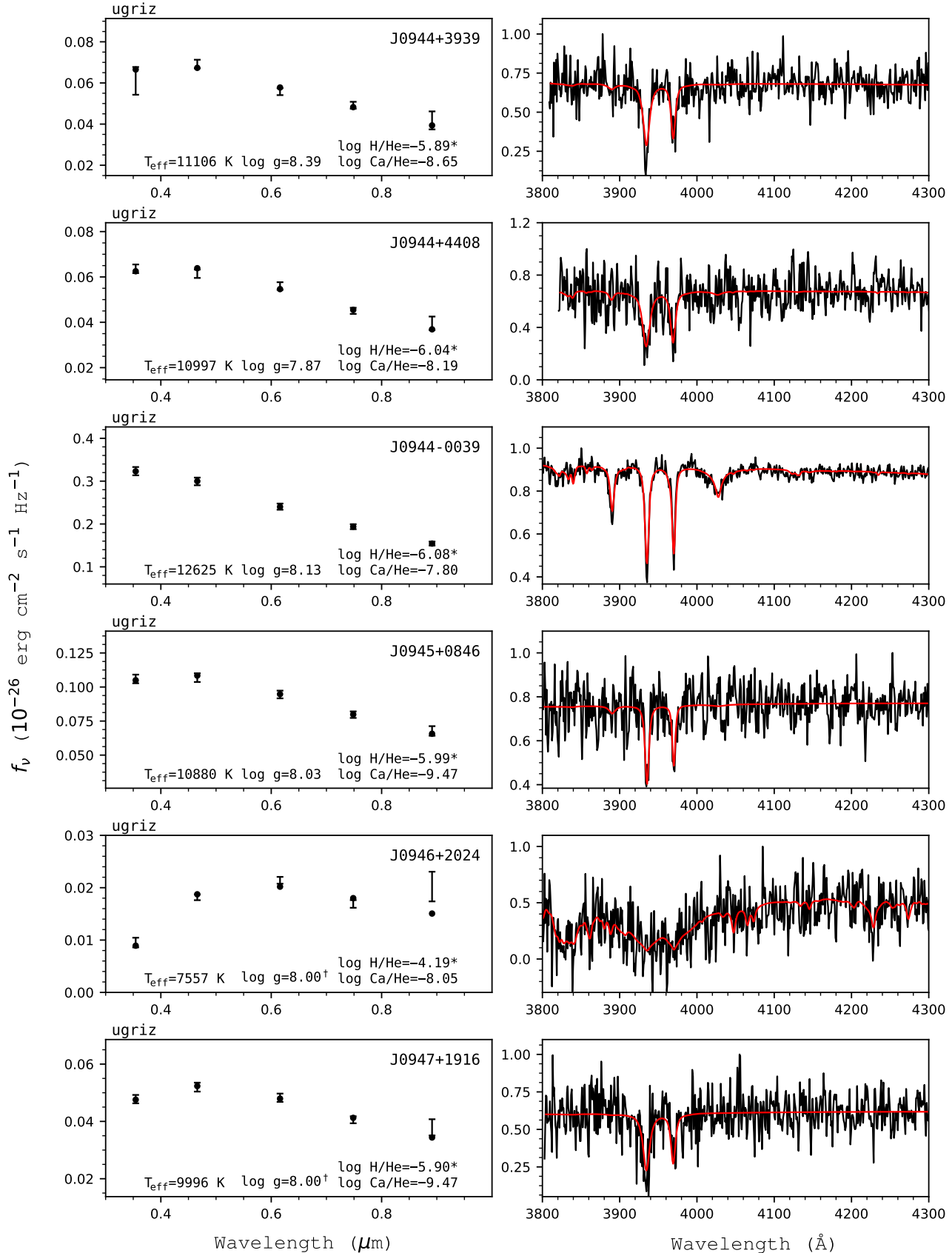

Figure 73. Fits to the DBZ/DZ(A) white dwarfs - continued. 

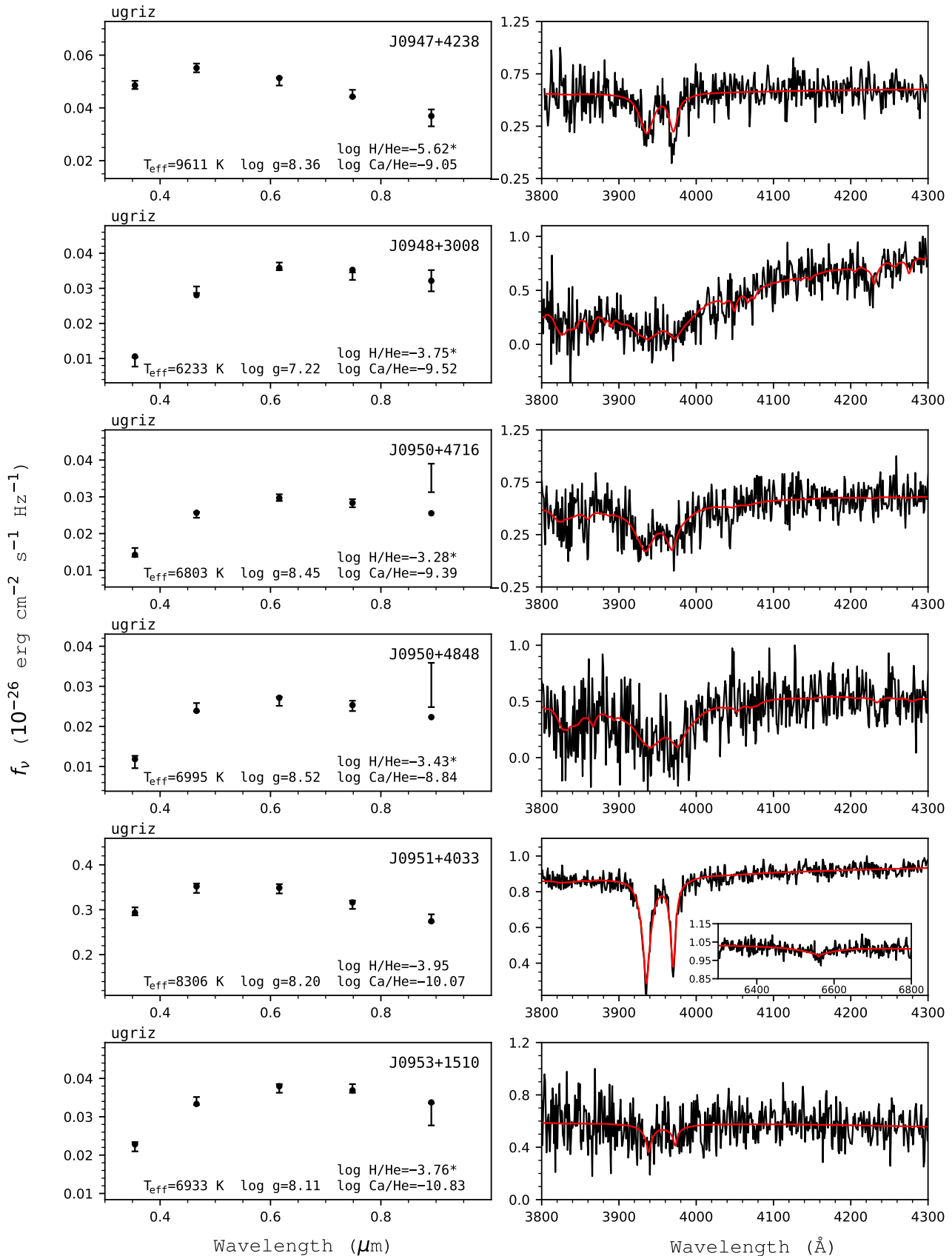

Figure 74. Fits to the DBZ/DZ(A) white dwarfs - continued. 

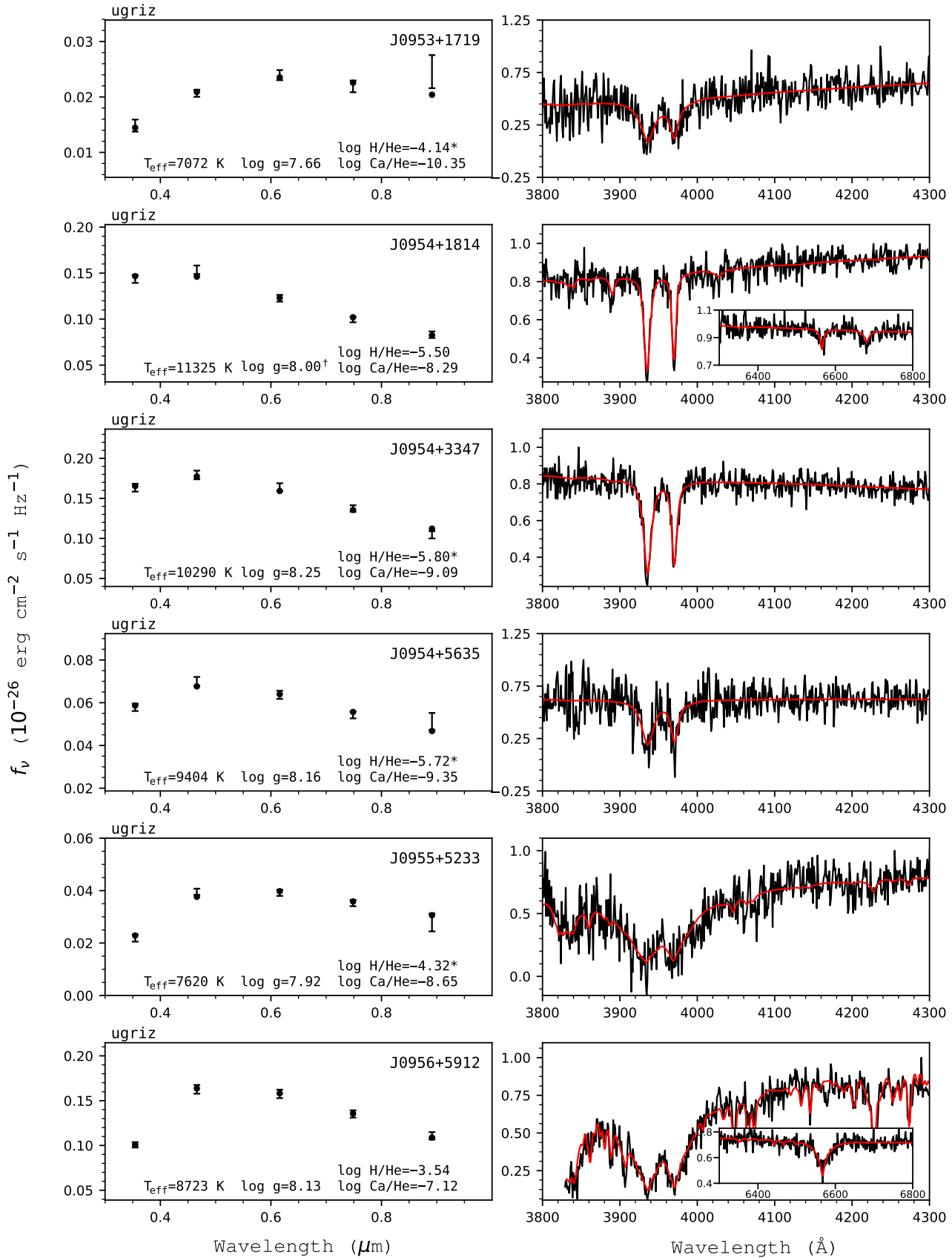

Figure 75. Fits to the DBZ/DZ(A) white dwarfs - continued. 

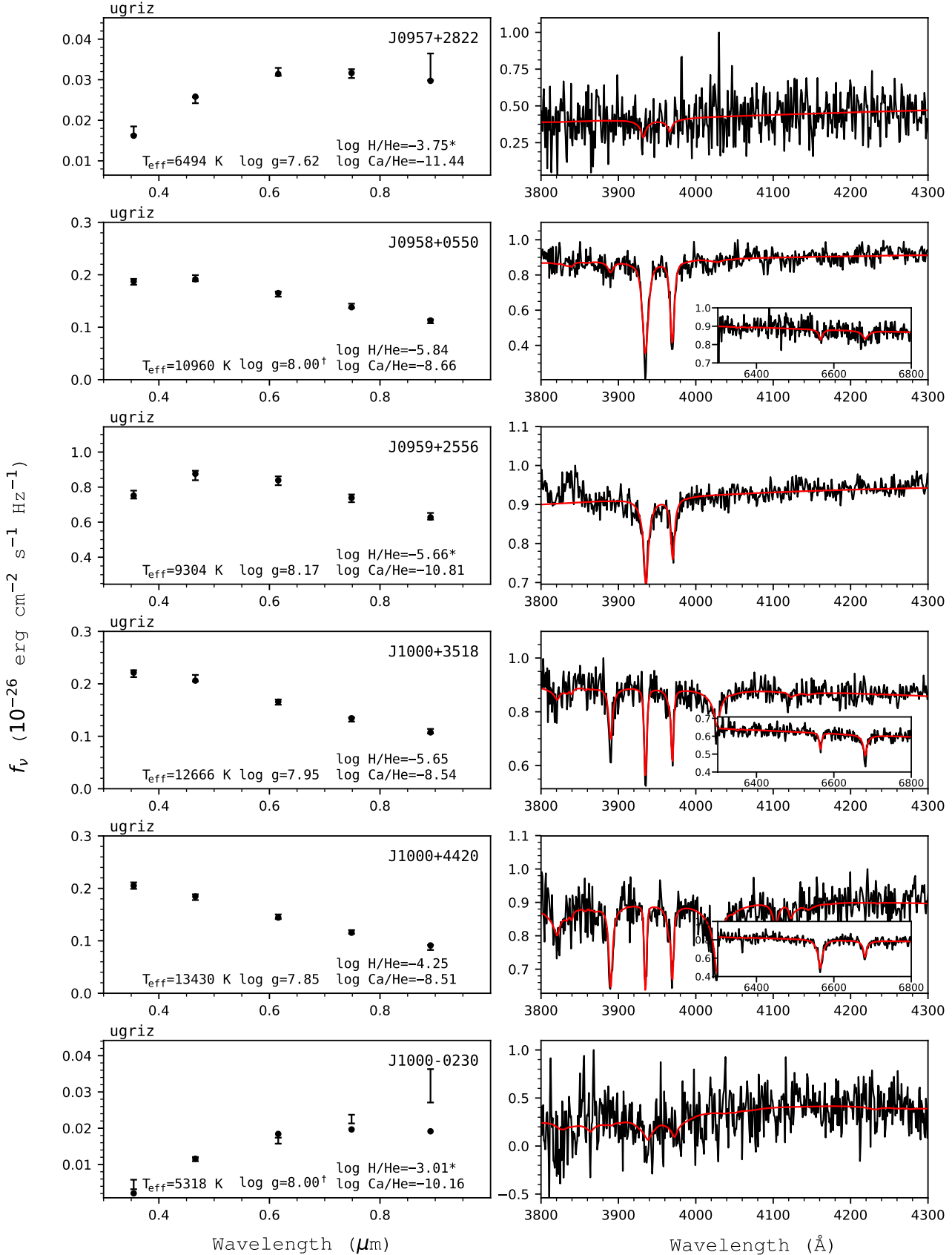

Figure 76. Fits to the DBZ/DZ(A) white dwarfs - continued. 

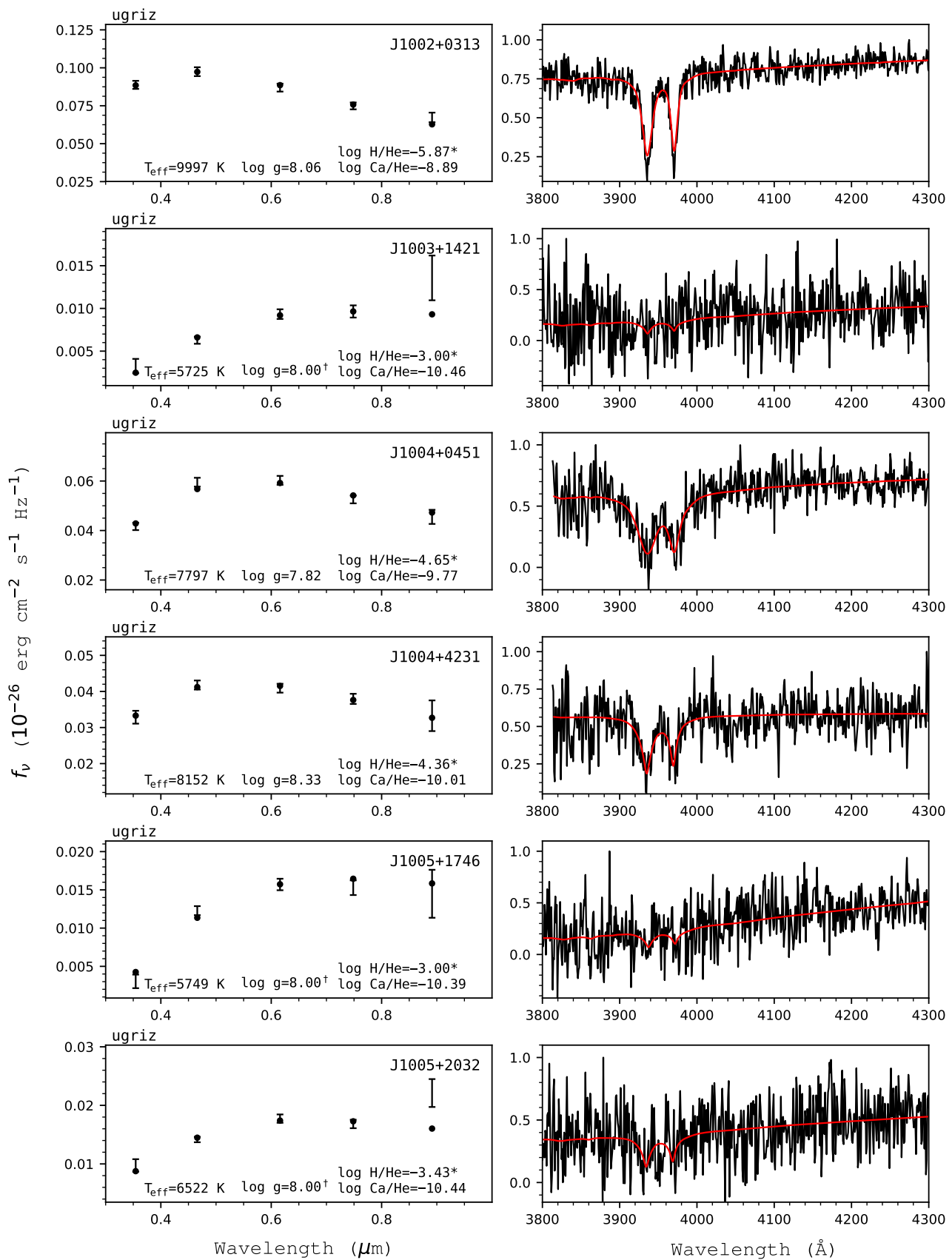

Figure 77. Fits to the DBZ/DZ(A) white dwarfs - continued. 

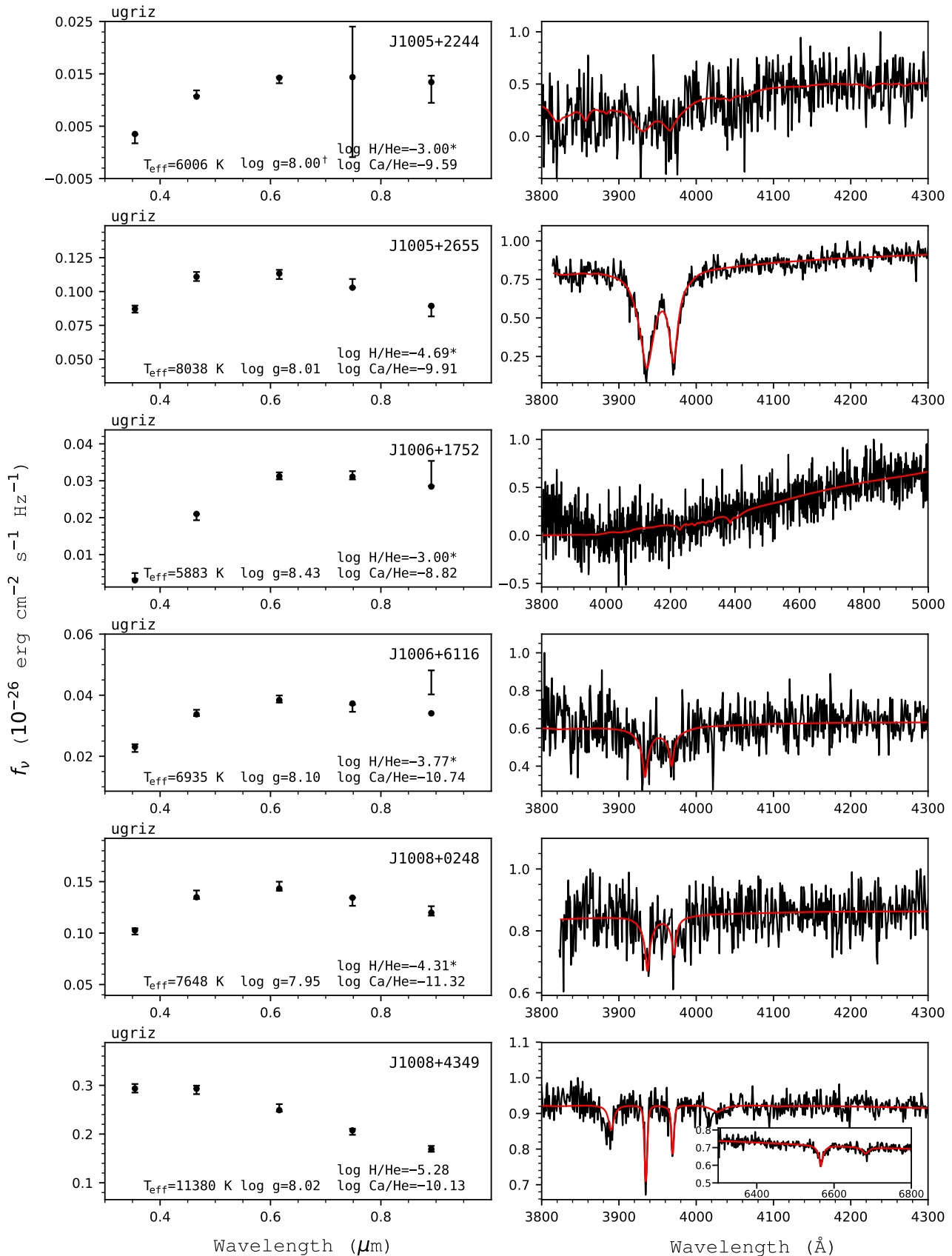

Figure 78. Fits to the DBZ/DZ(A) white dwarfs - continued. 

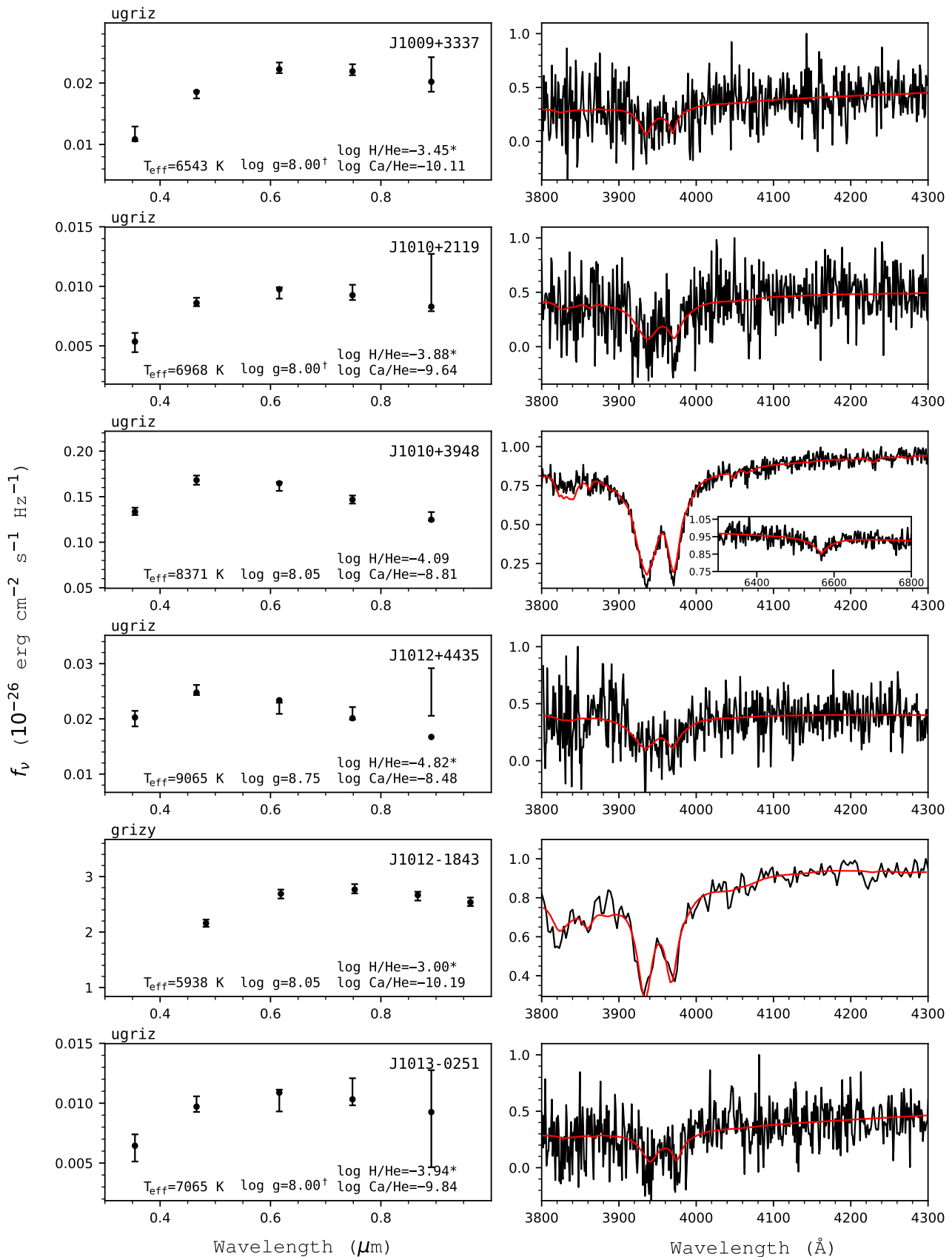

Figure 79. Fits to the DBZ/DZ(A) white dwarfs - continued. 

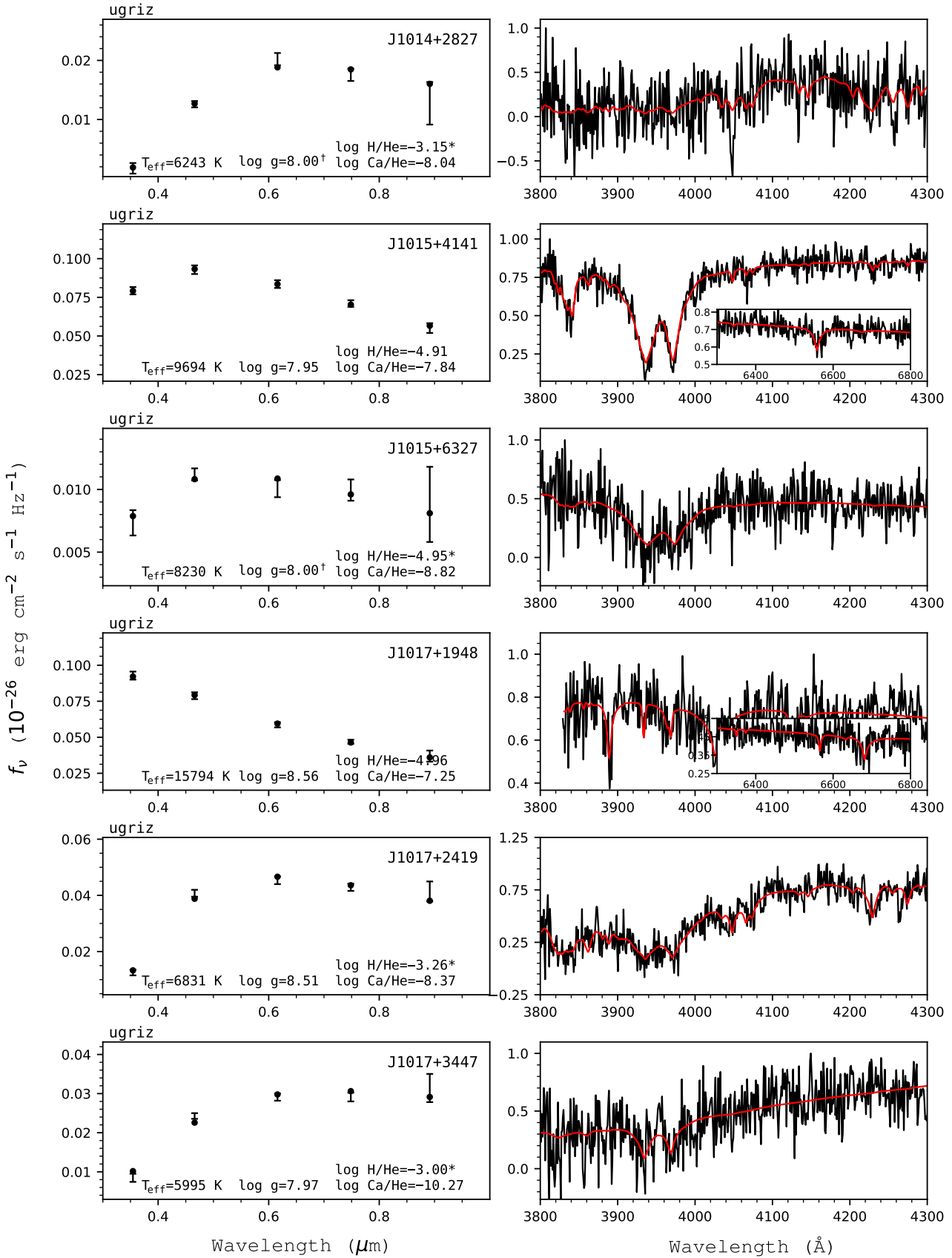

Figure 80. Fits to the DBZ/DZ(A) white dwarfs - continued. 

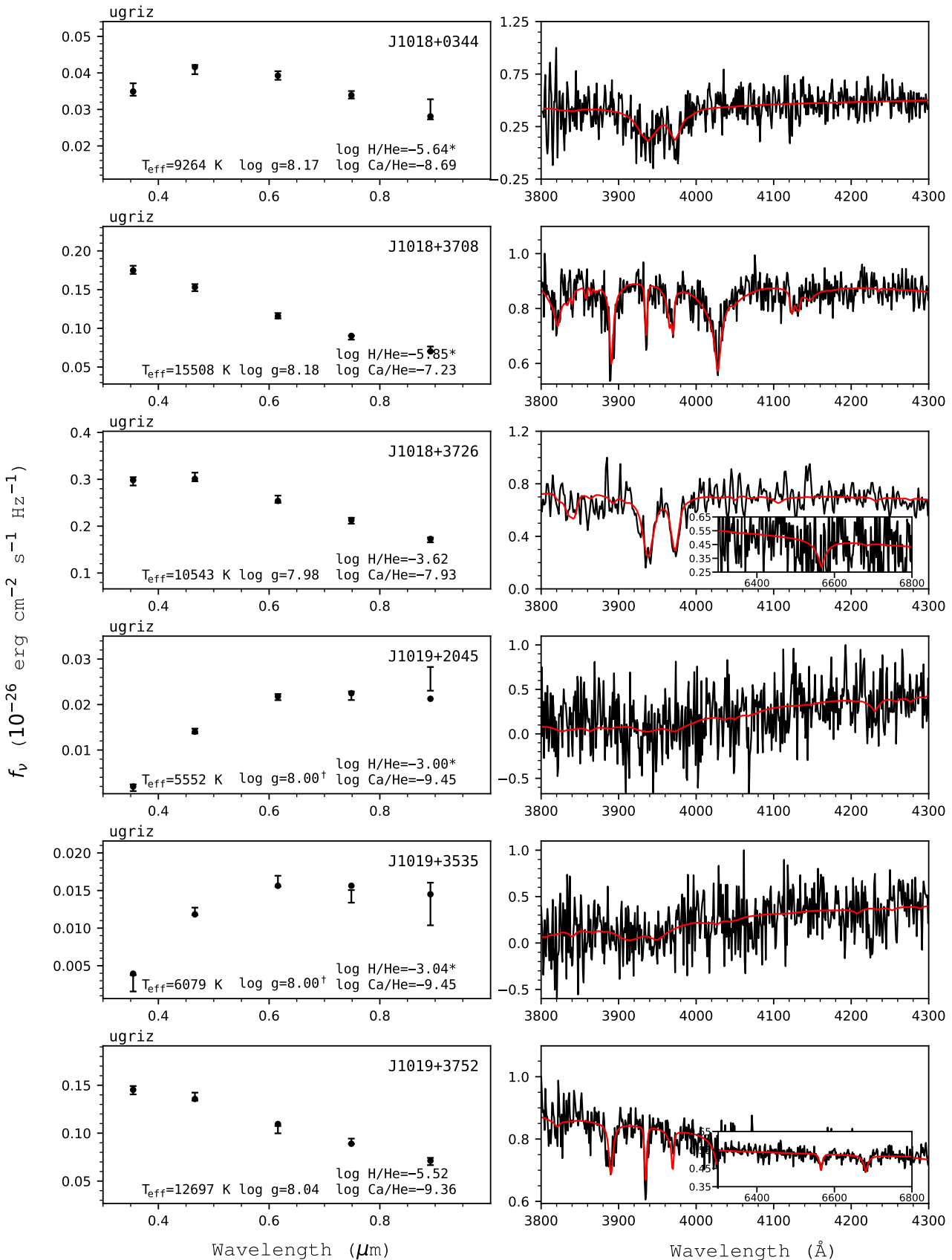

Figure 81. Fits to the DBZ/DZ(A) white dwarfs - continued. 

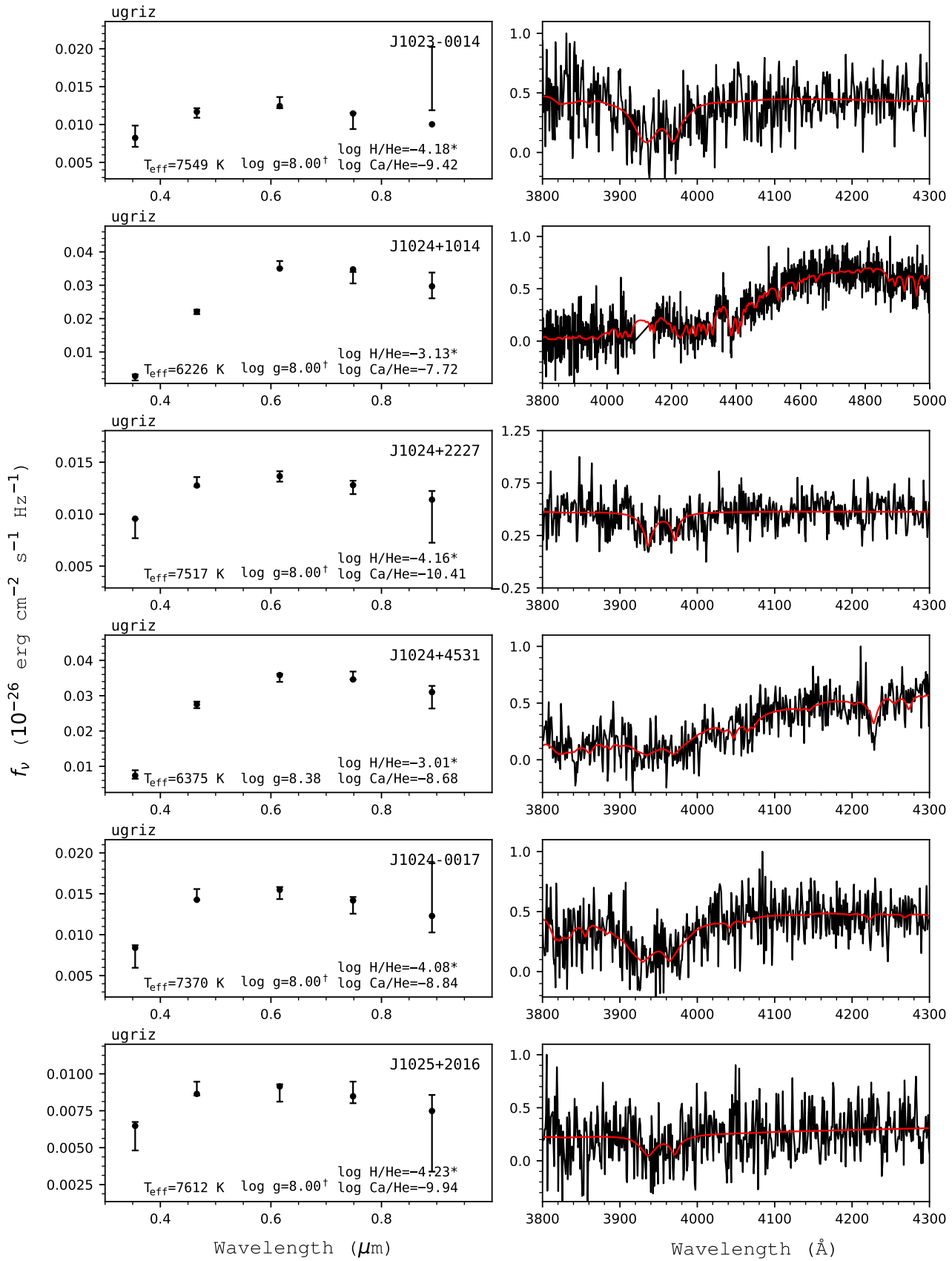

Figure 82. Fits to the DBZ/DZ(A) white dwarfs - continued. 

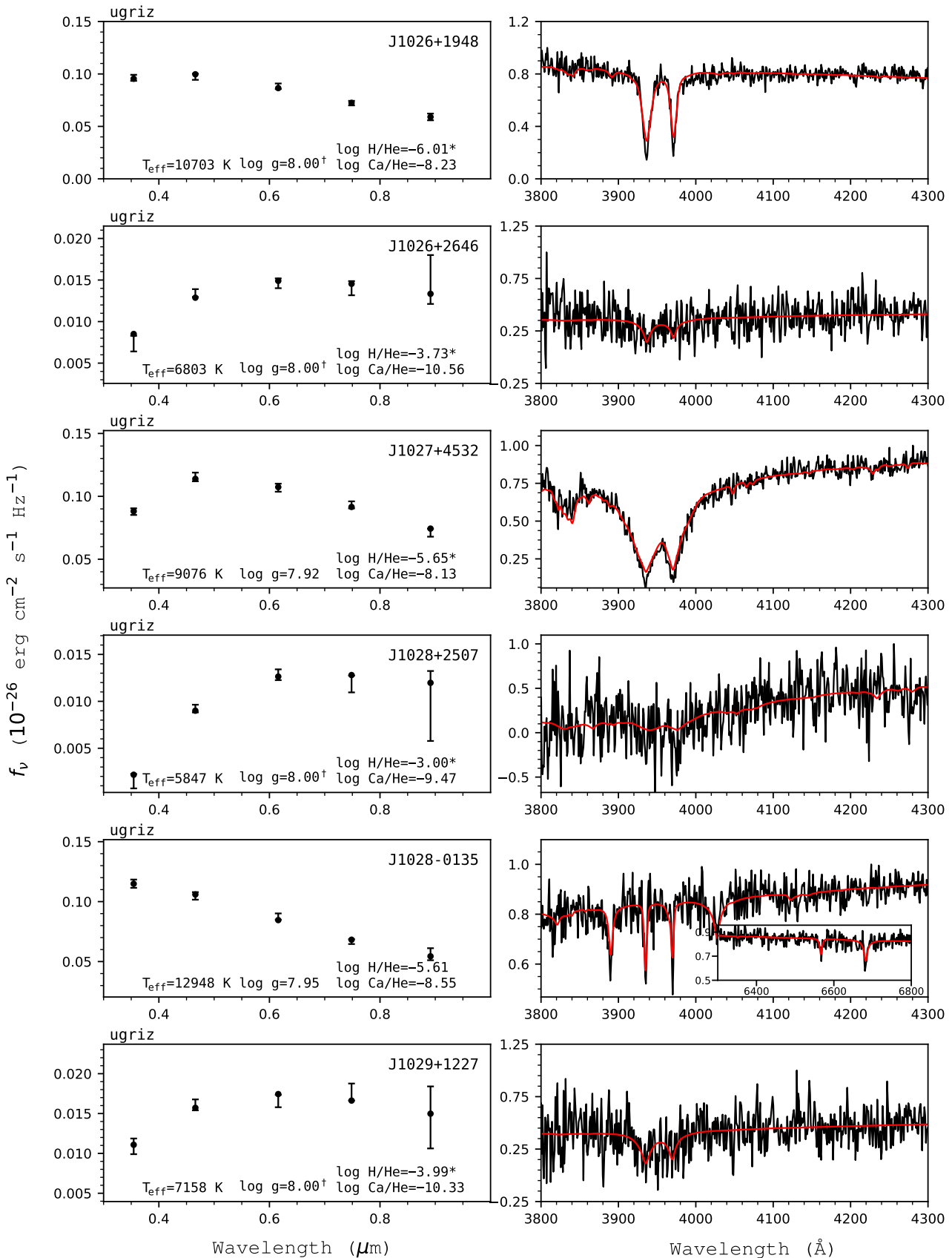

Figure 83. Fits to the DBZ/DZ(A) white dwarfs - continued. 

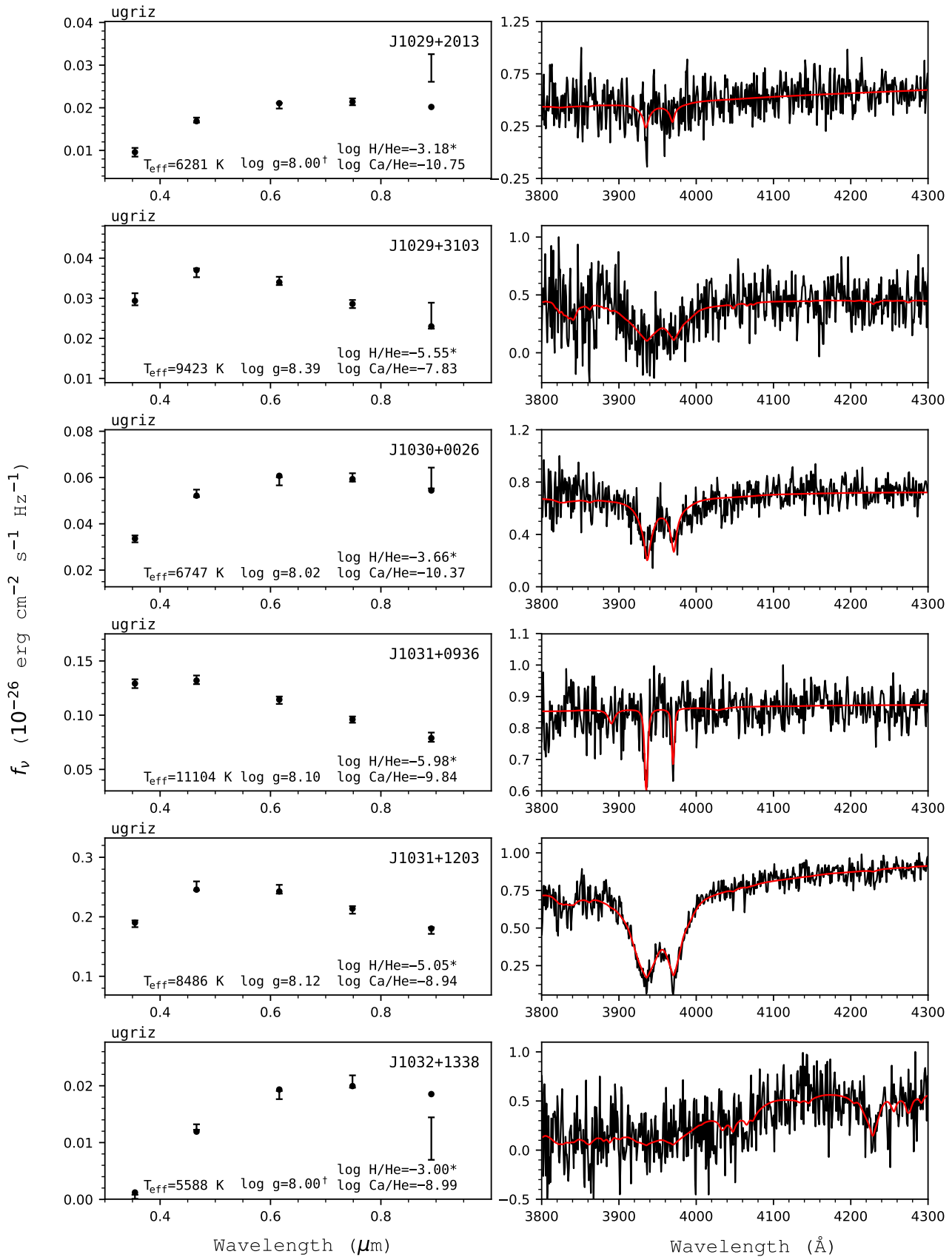

Figure 84. Fits to the DBZ/DZ(A) white dwarfs - continued. 

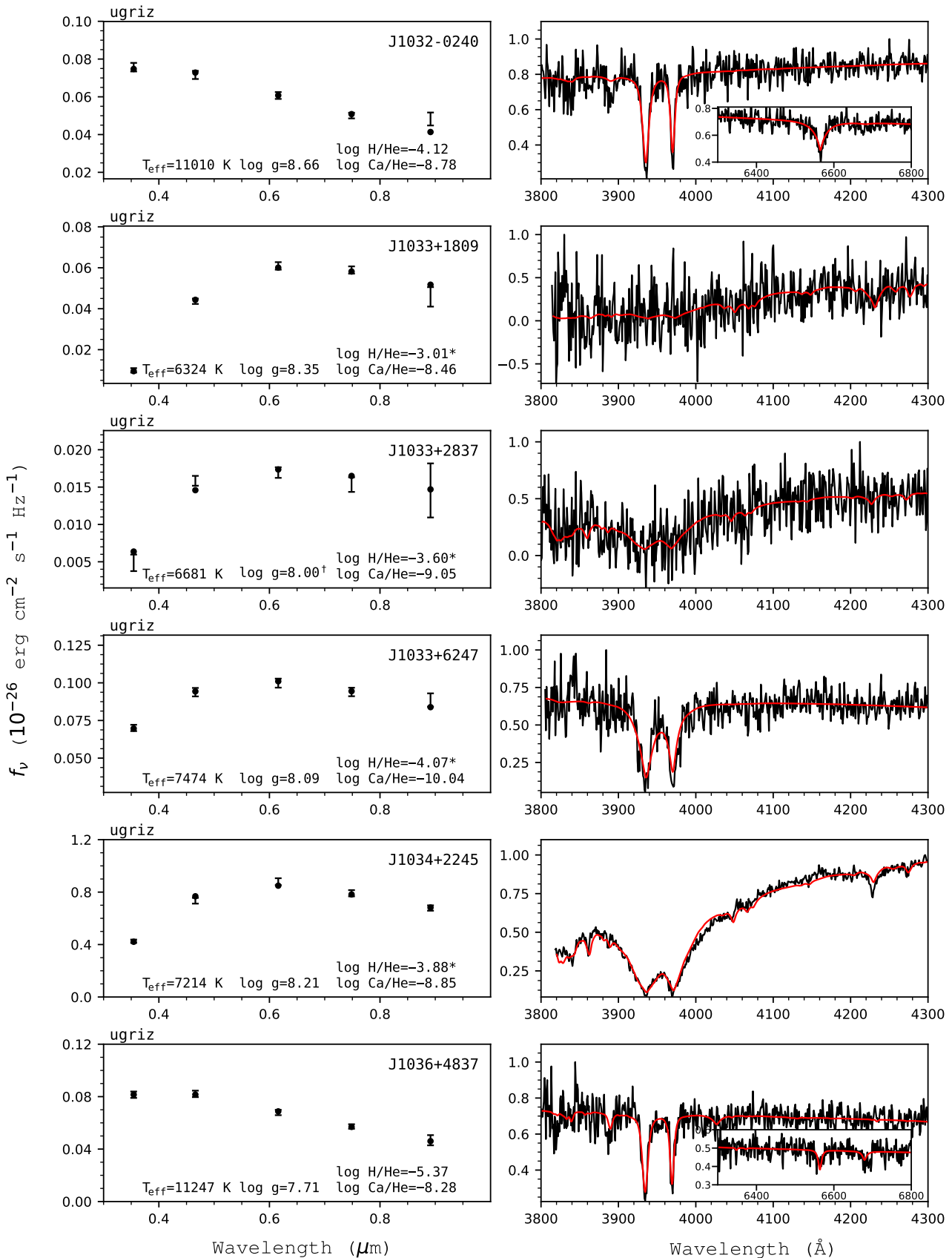

Figure 85. Fits to the DBZ/DZ(A) white dwarfs - continued. 

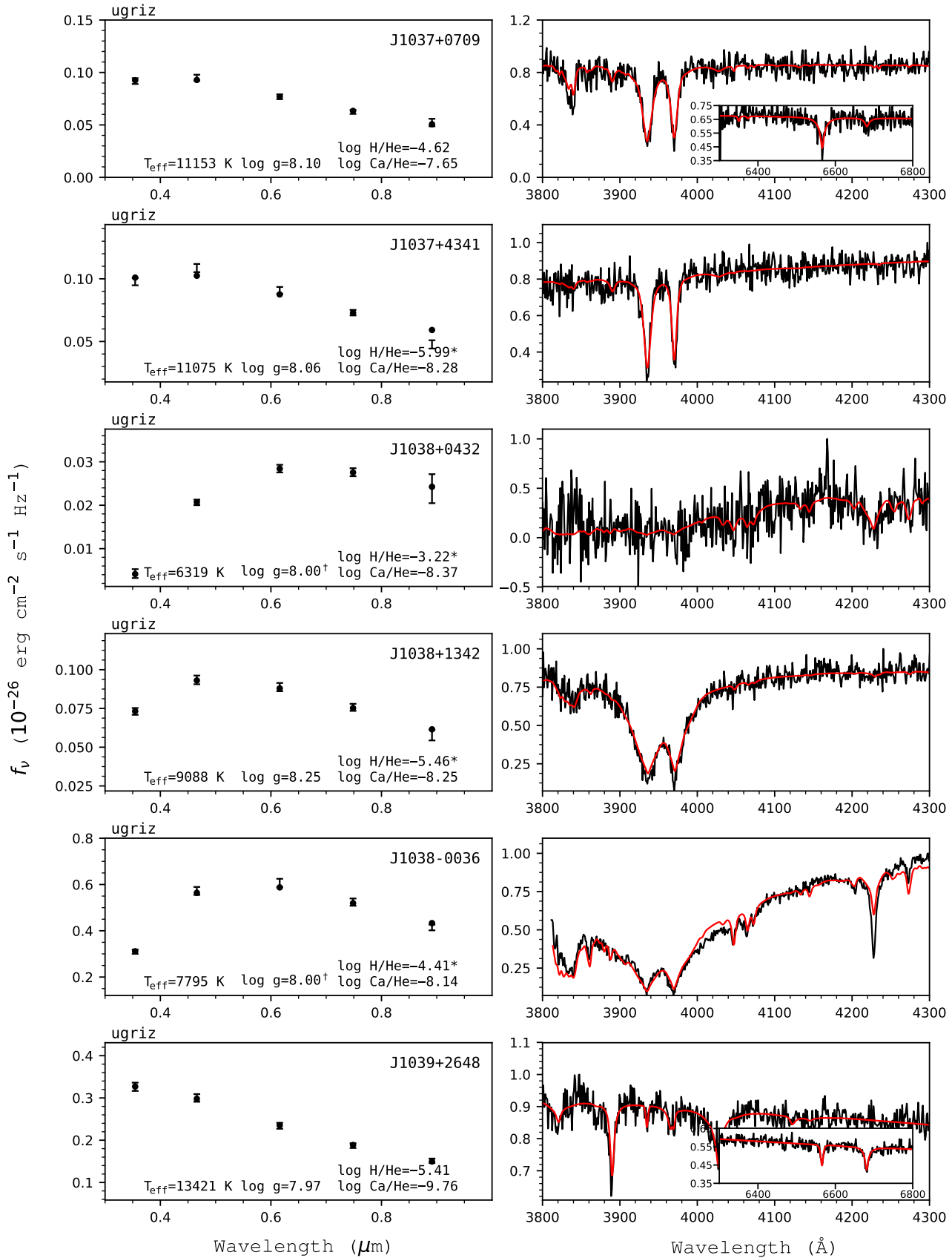

Figure 86. Fits to the DBZ/DZ(A) white dwarfs - continued. 

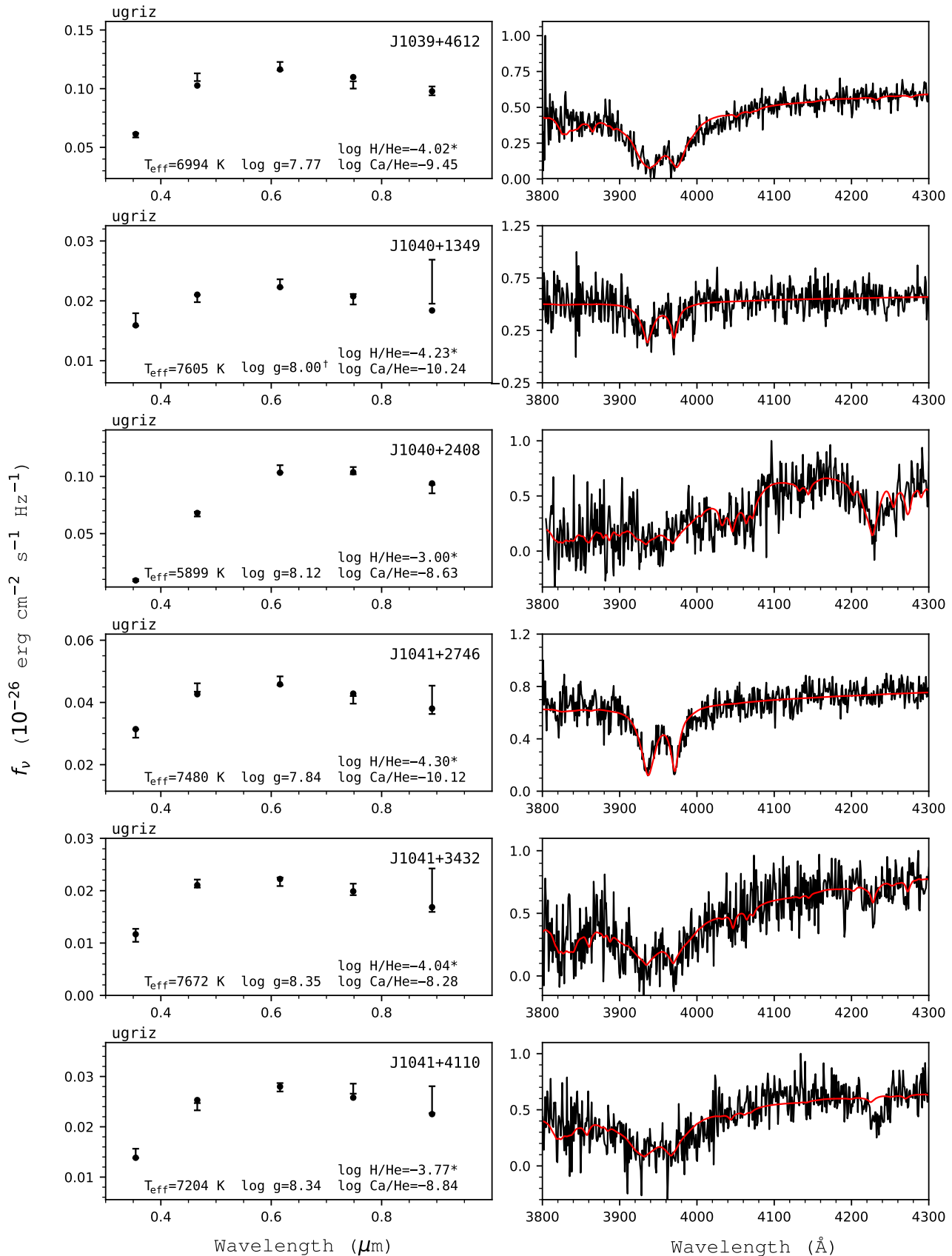

Figure 87. Fits to the DBZ/DZ(A) white dwarfs - continued. 

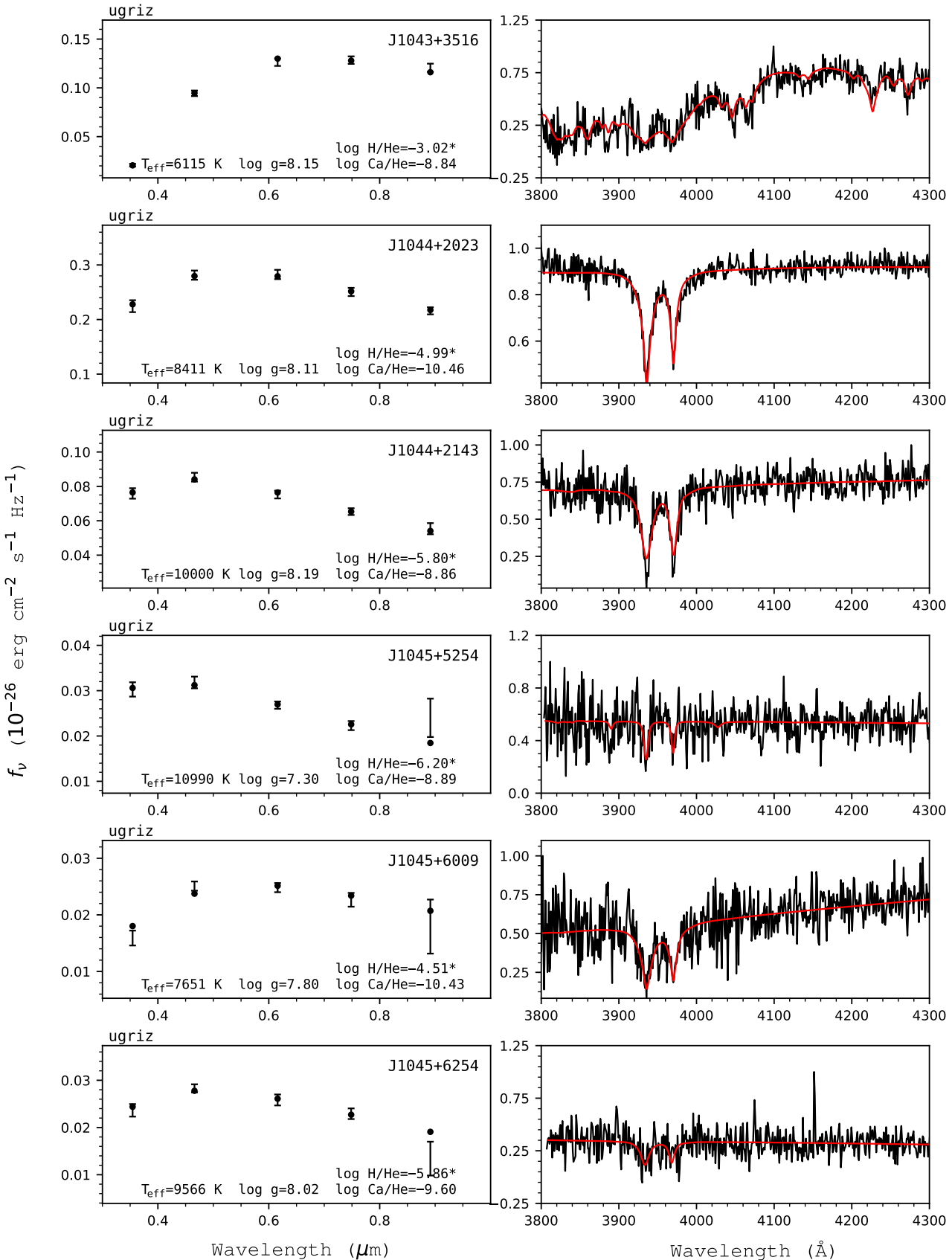

Figure 88. Fits to the DBZ/DZ(A) white dwarfs - continued. 

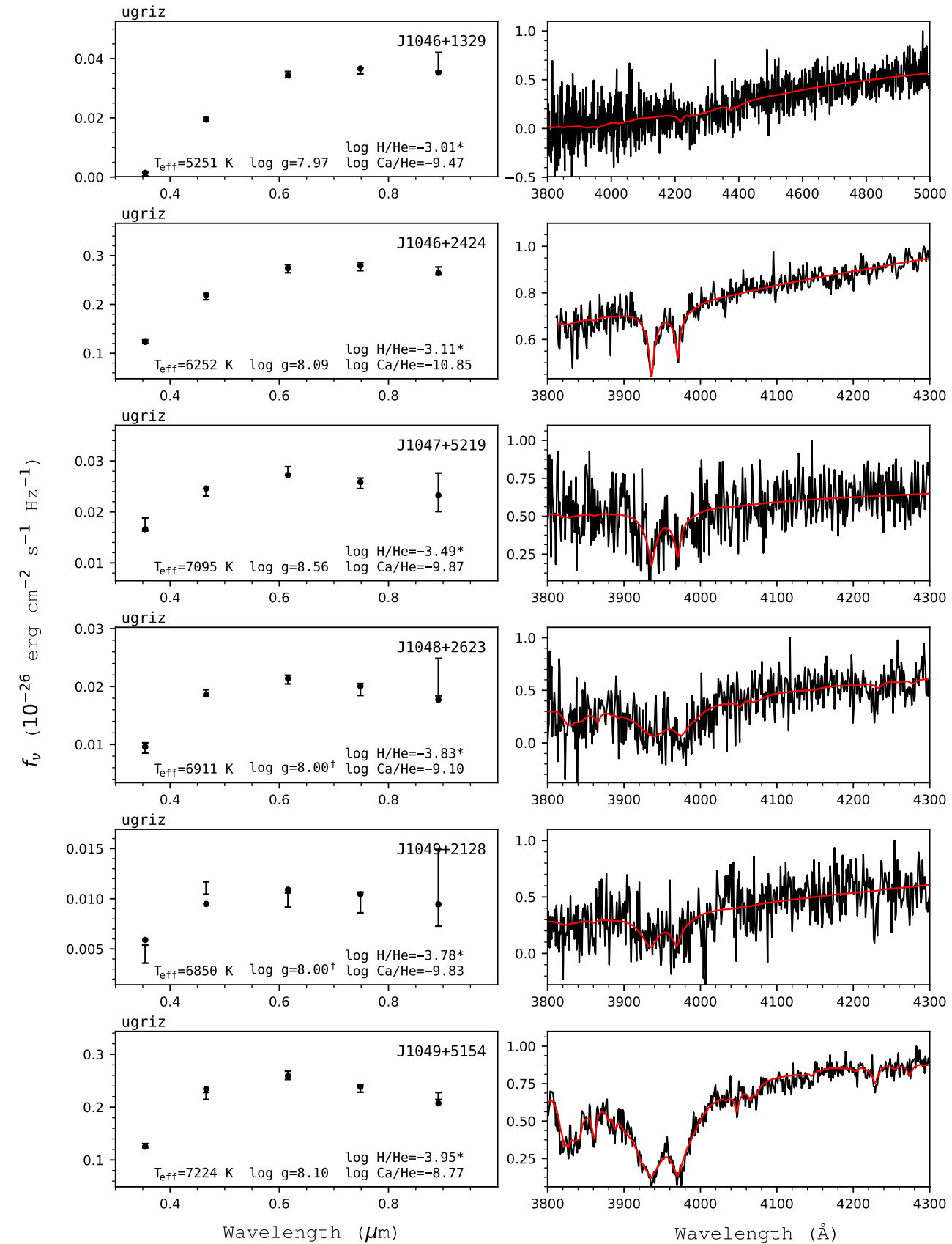

Figure 89. Fits to the DBZ/DZ(A) white dwarfs - continued. 

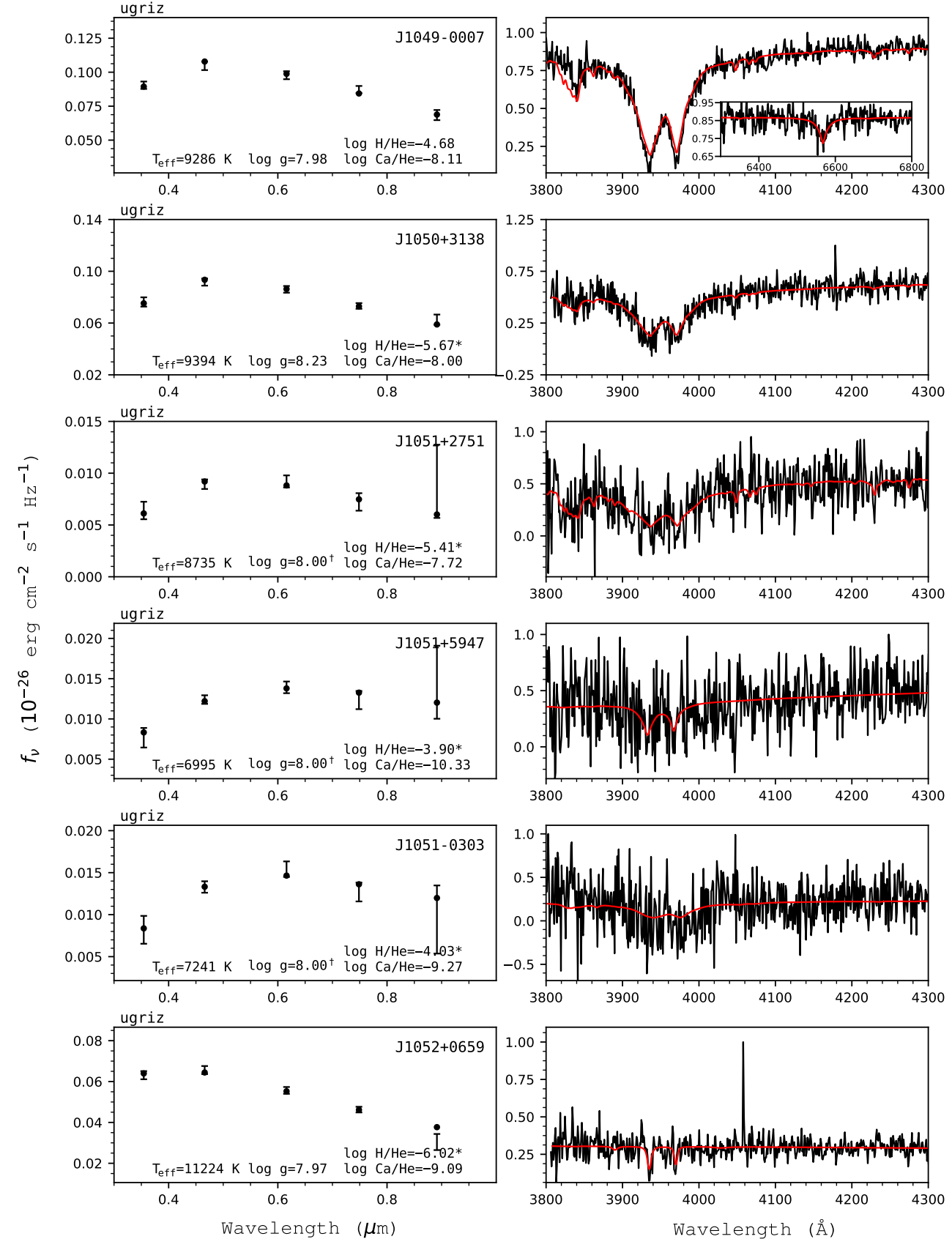

Figure 90. Fits to the DBZ/DZ(A) white dwarfs - continued. 

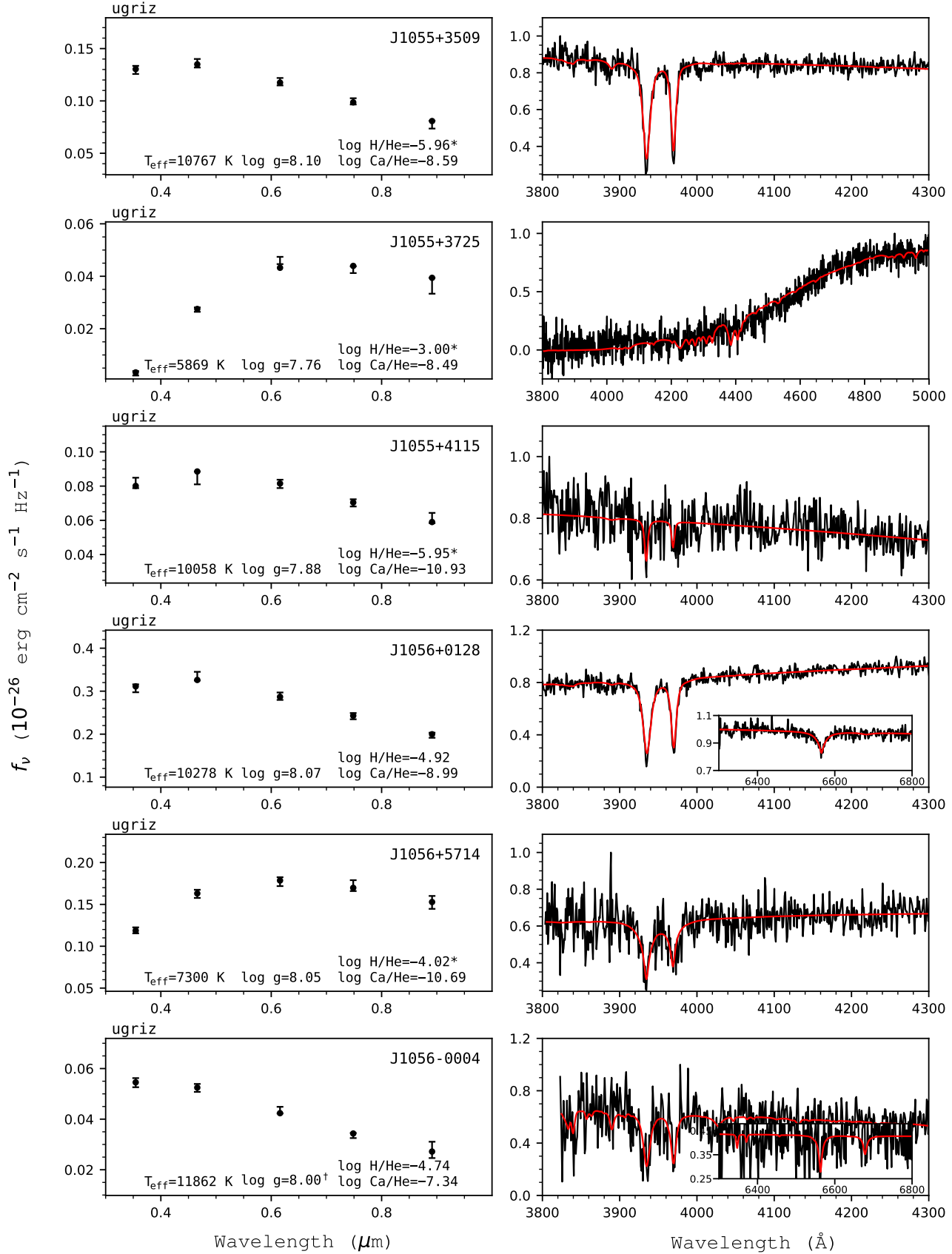

Figure 91. Fits to the DBZ/DZ(A) white dwarfs - continued. 

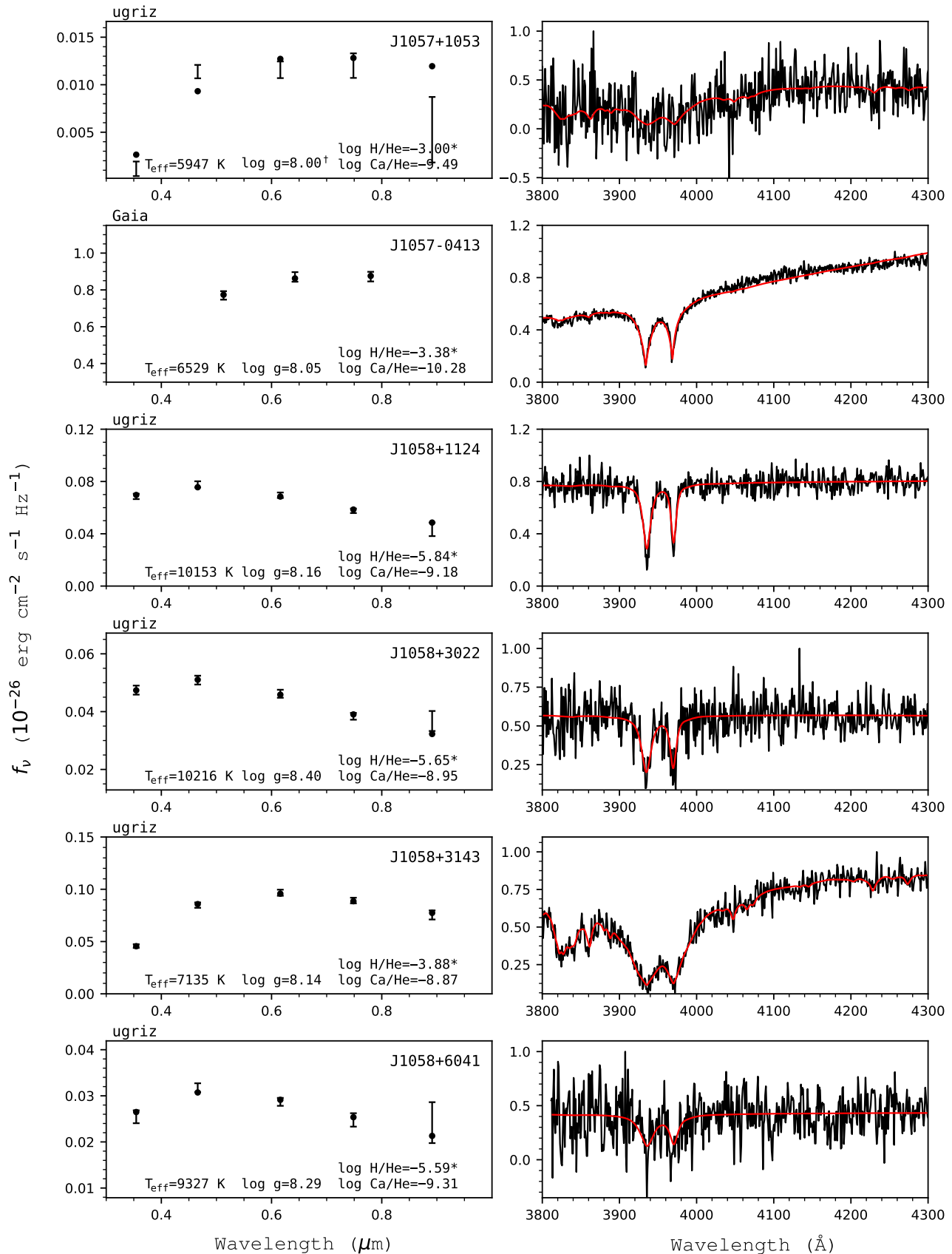

Figure 92. Fits to the DBZ/DZ(A) white dwarfs - continued. 

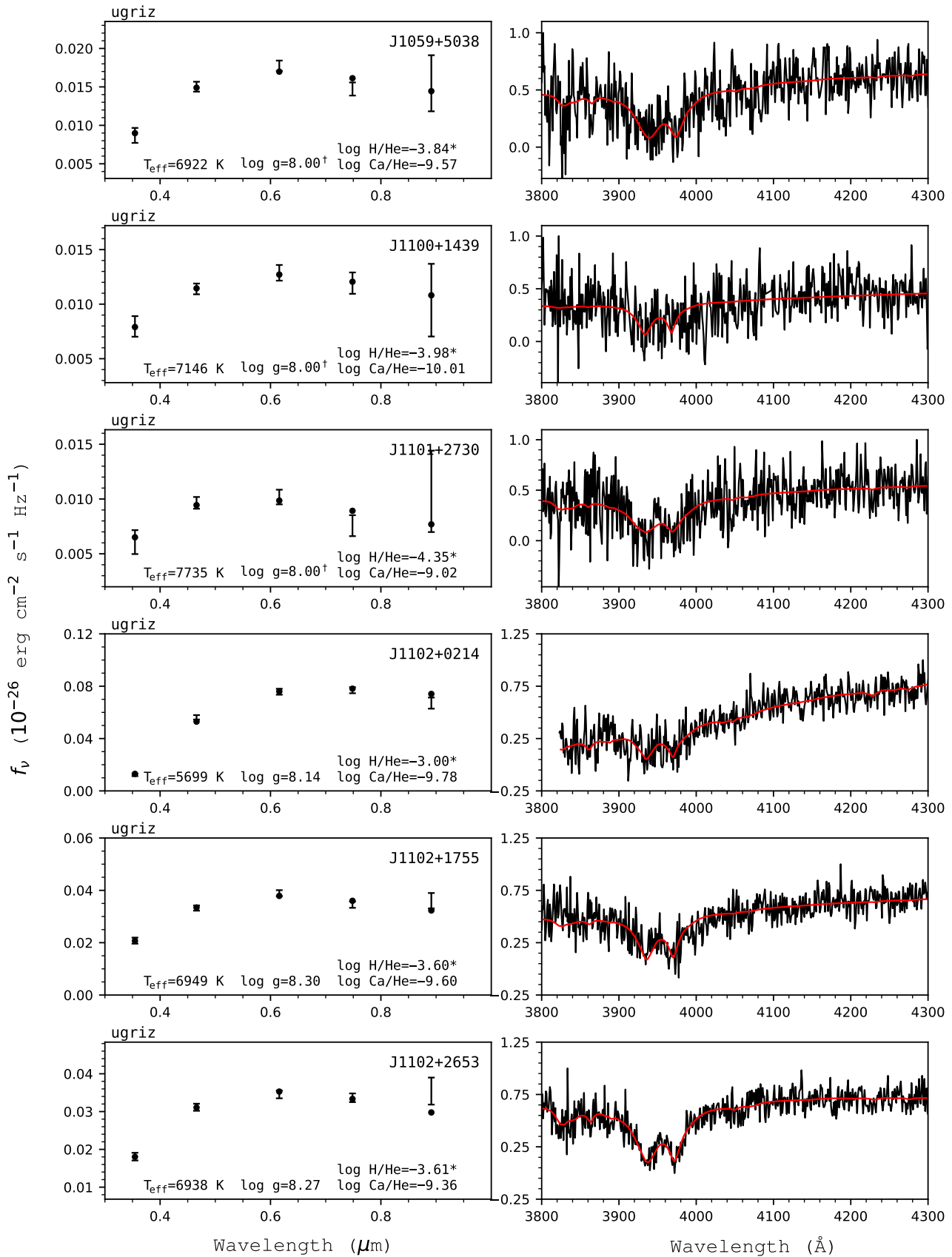

Figure 93. Fits to the DBZ/DZ(A) white dwarfs - continued. 

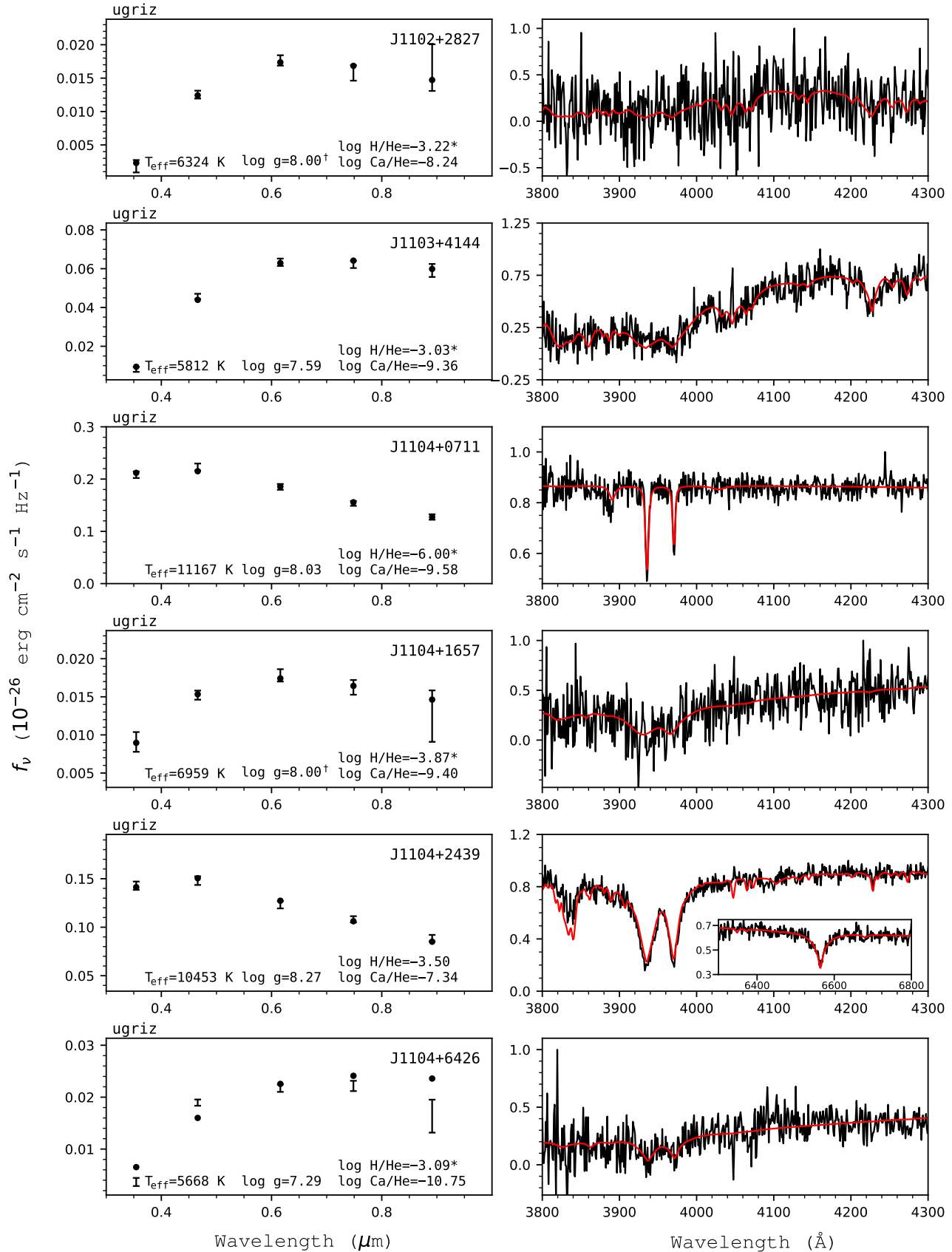

Figure 94. Fits to the DBZ/DZ(A) white dwarfs - continued. 

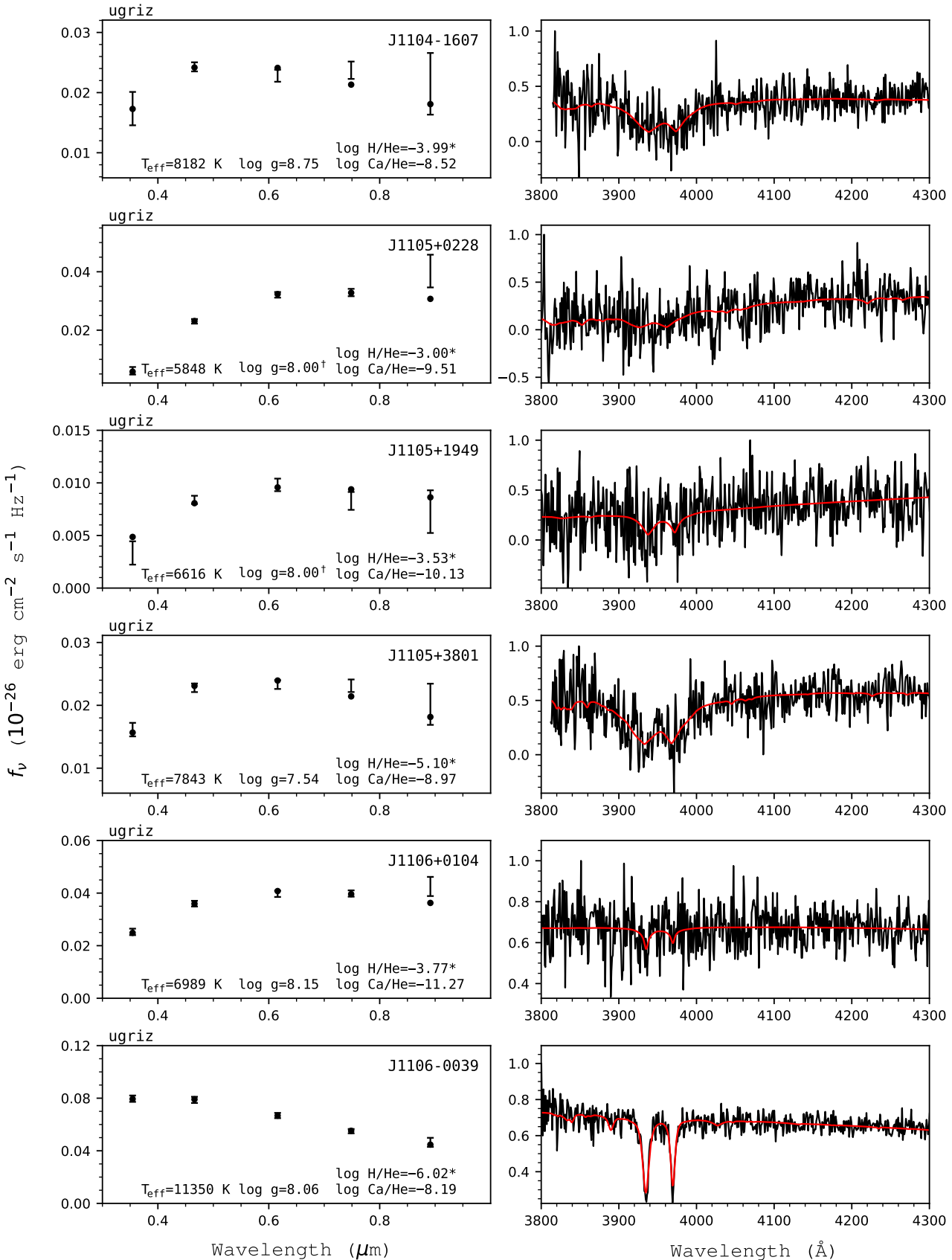

Figure 95. Fits to the DBZ/DZ(A) white dwarfs - continued. 

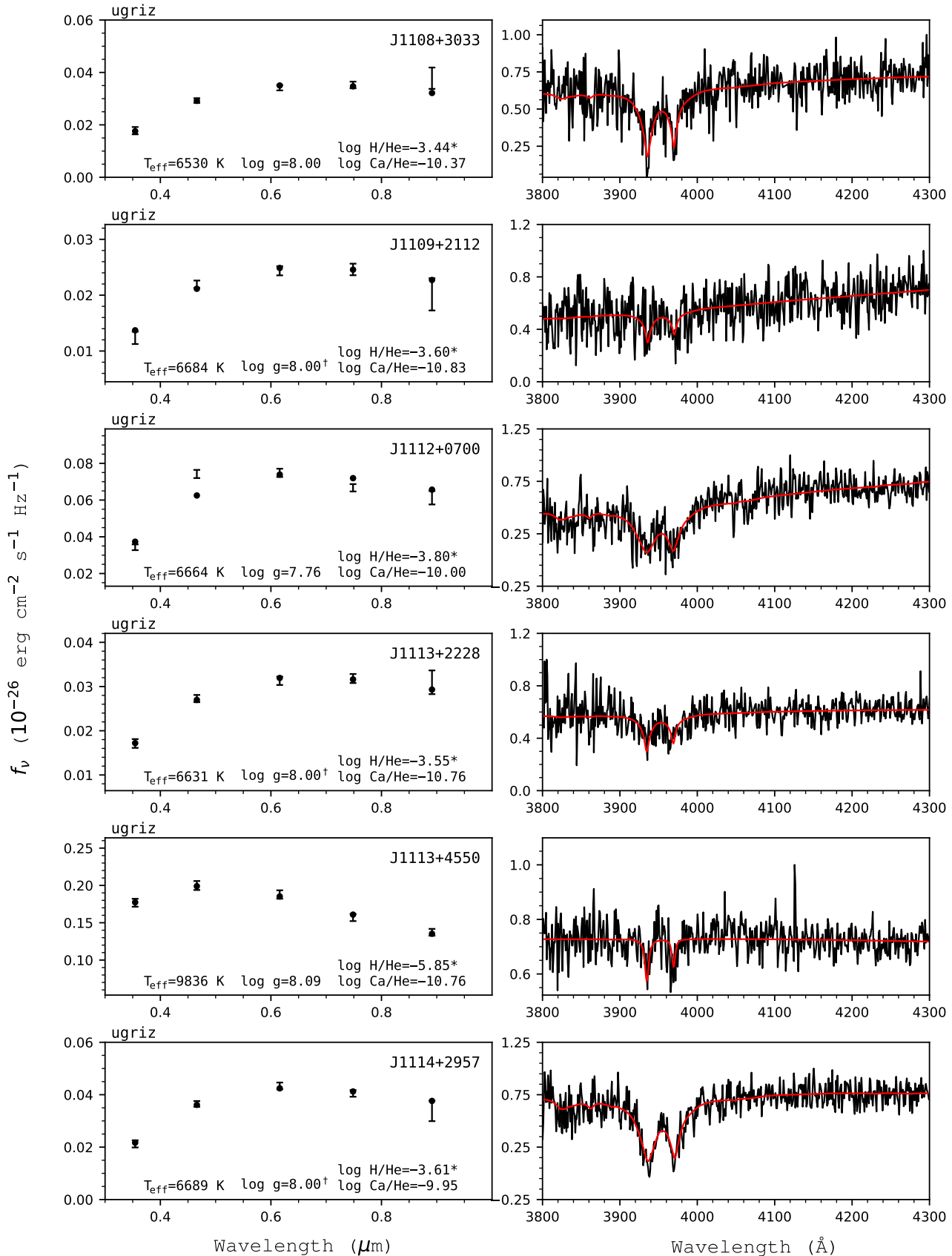

Figure 96. Fits to the DBZ/DZ(A) white dwarfs - continued. 

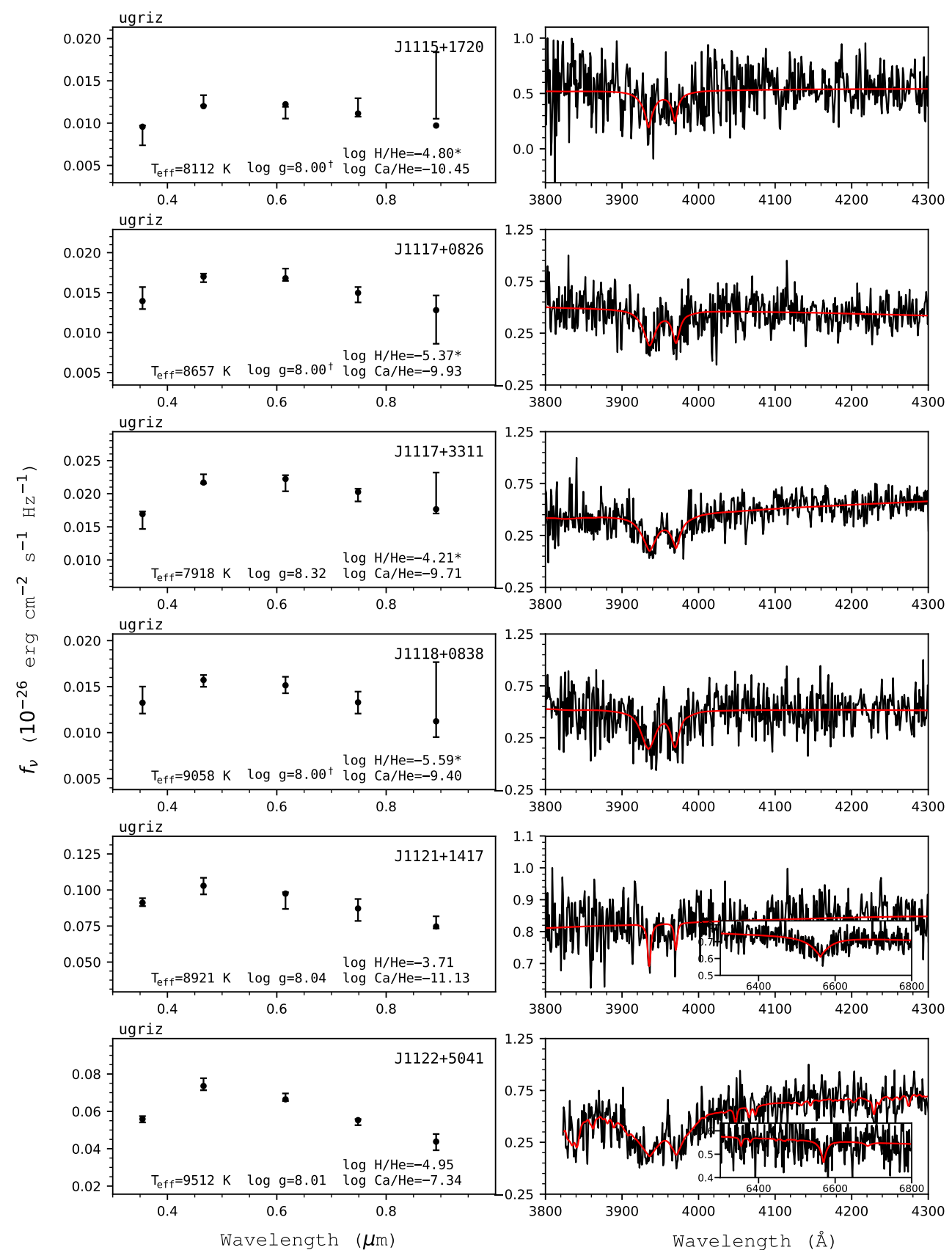

Figure 97. Fits to the DBZ/DZ(A) white dwarfs - continued. 

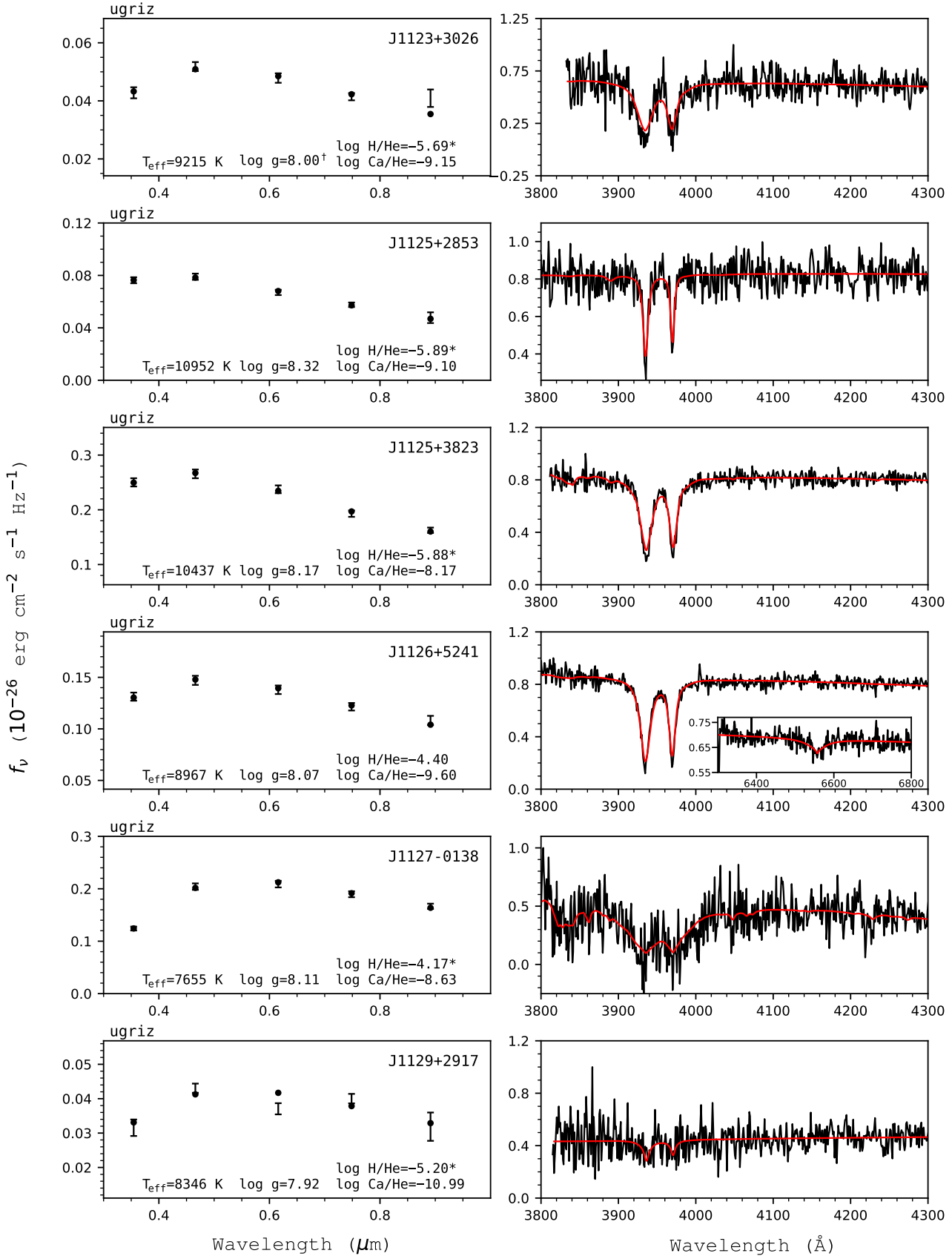

Figure 98. Fits to the DBZ/DZ(A) white dwarfs - continued. 

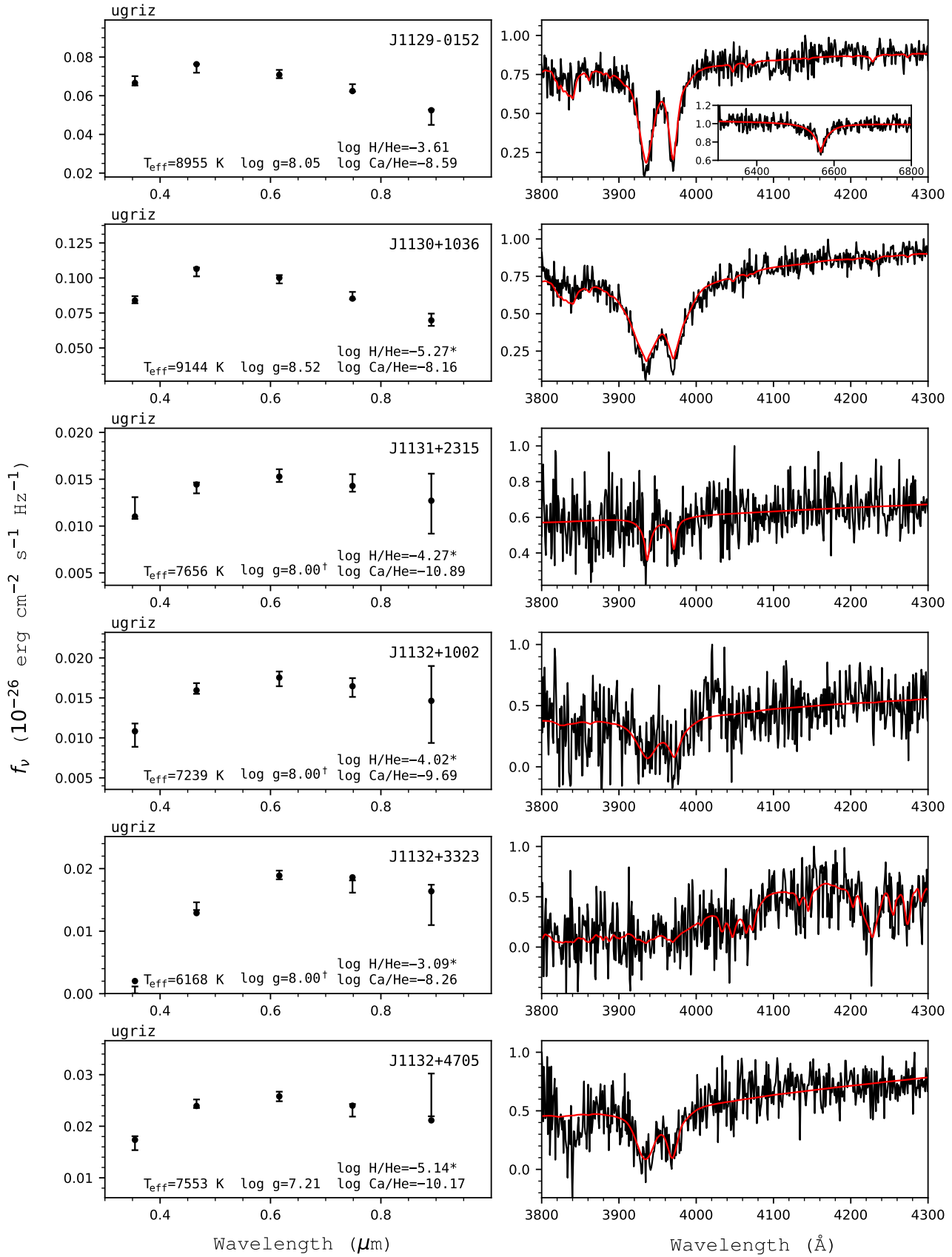

Figure 99. Fits to the DBZ/DZ(A) white dwarfs - continued. 

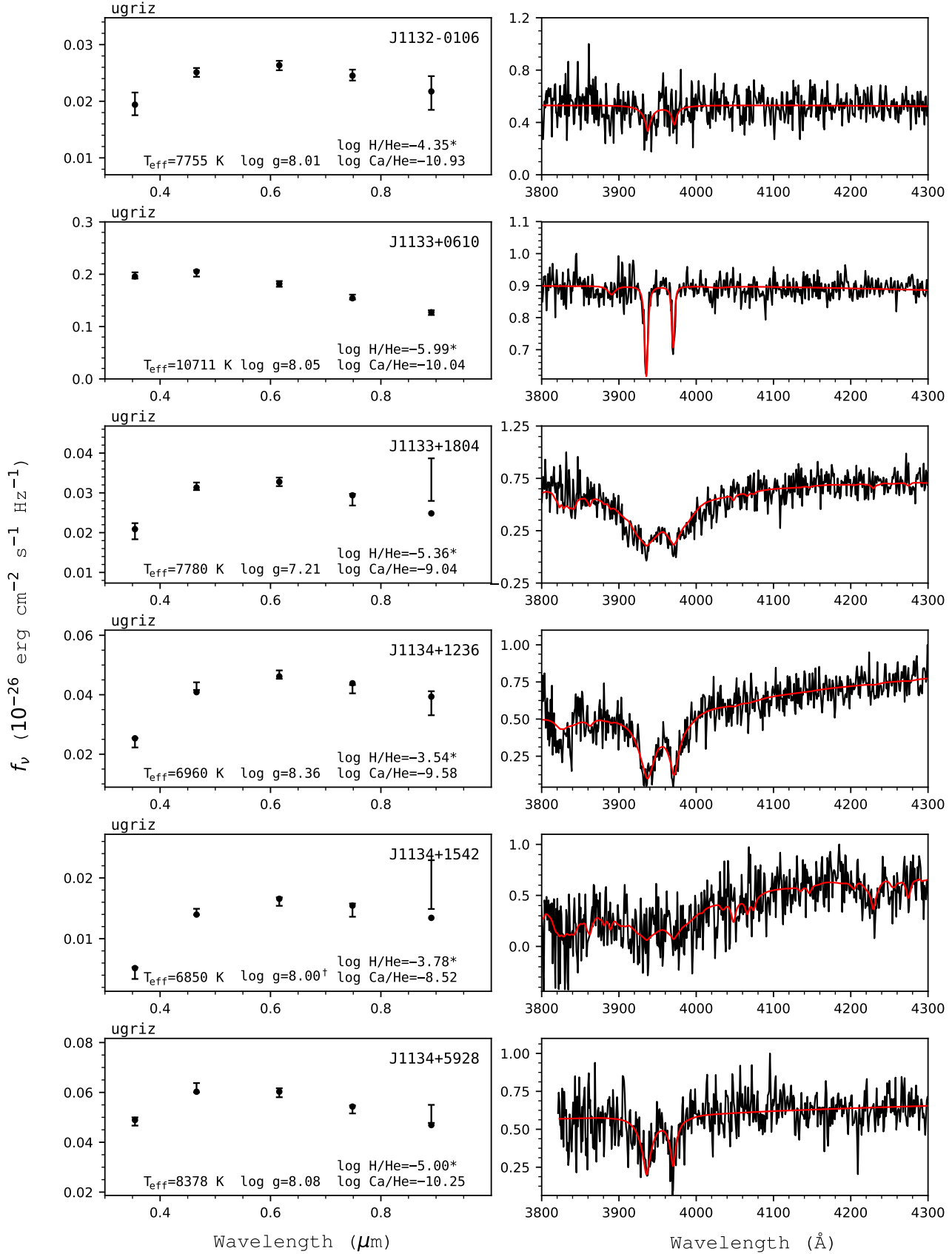

Figure 100. Fits to the DBZ/DZ(A) white dwarfs - continued. 

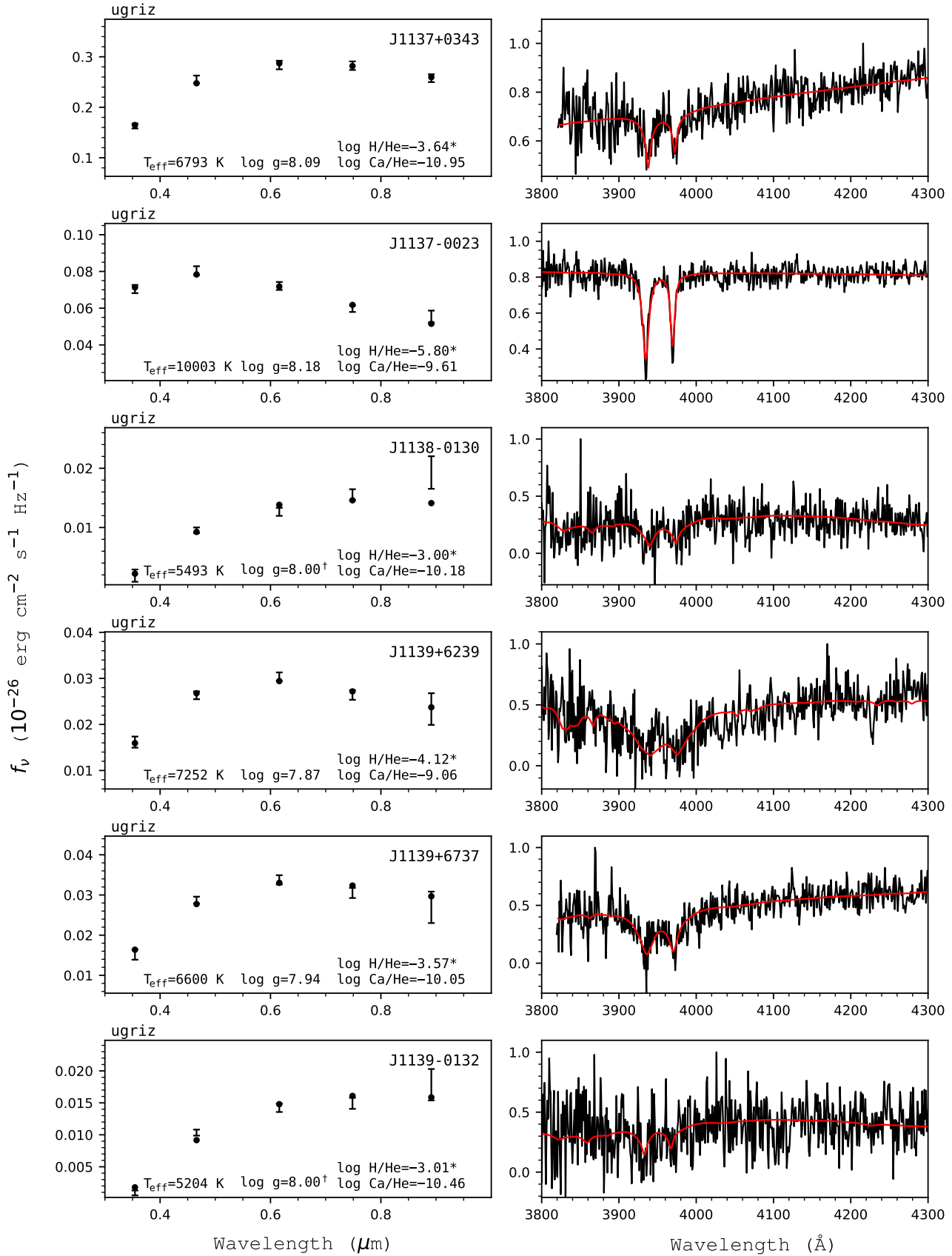

Figure 101. Fits to the DBZ/DZ(A) white dwarfs - continued. 

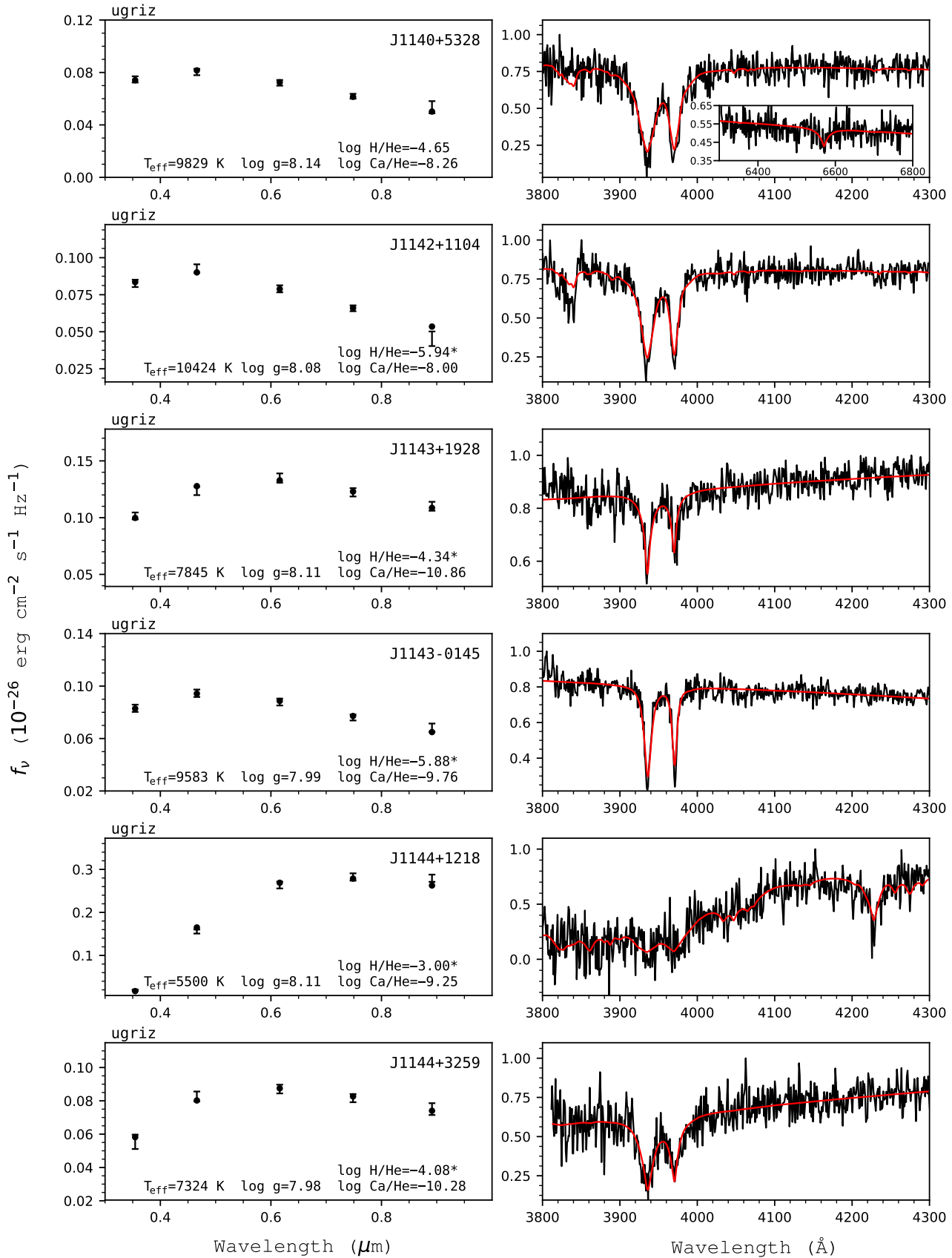

Figure 102. Fits to the DBZ/DZ(A) white dwarfs - continued. 


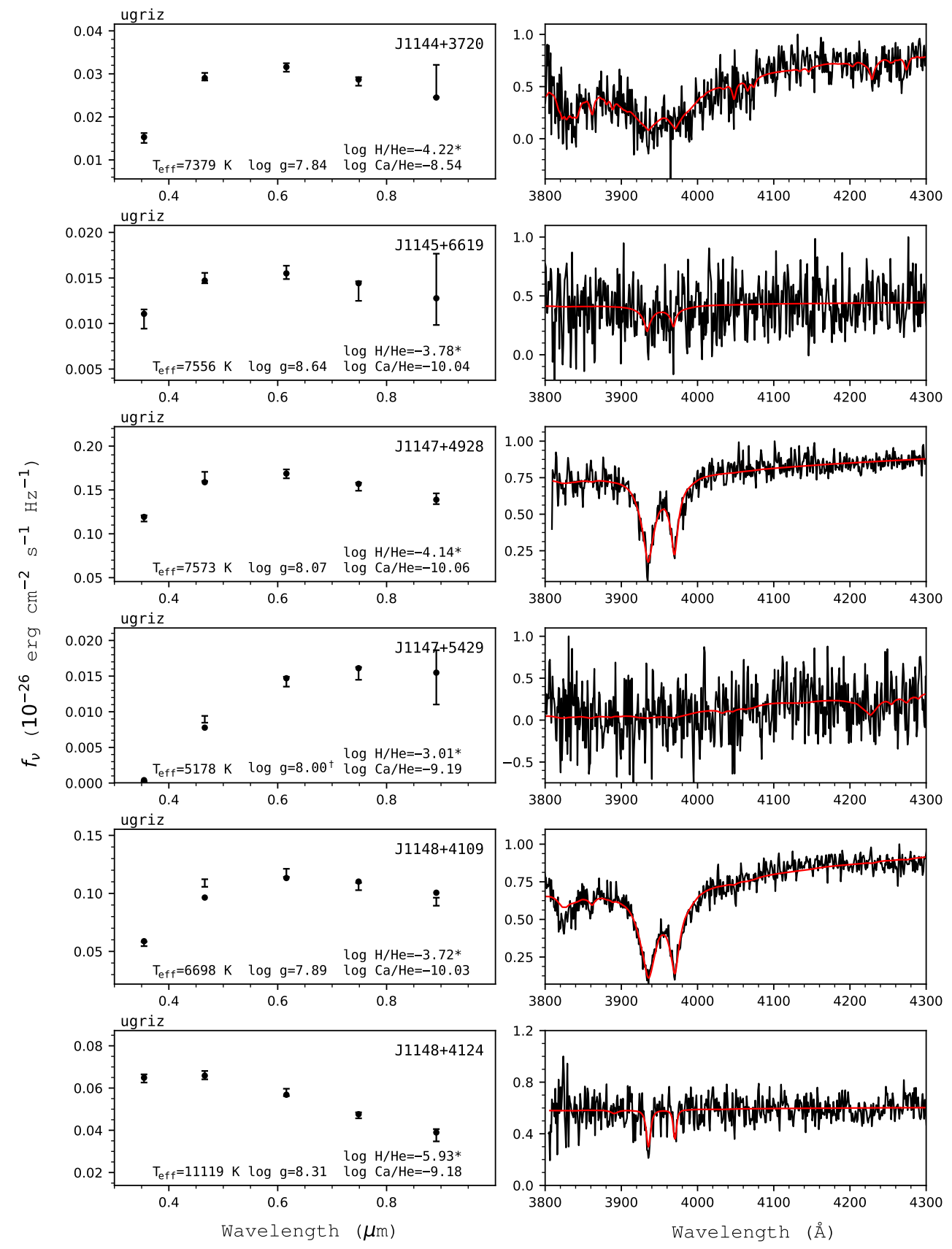

Figure 103. Fits to the DBZ/DZ(A) white dwarfs - continued. 

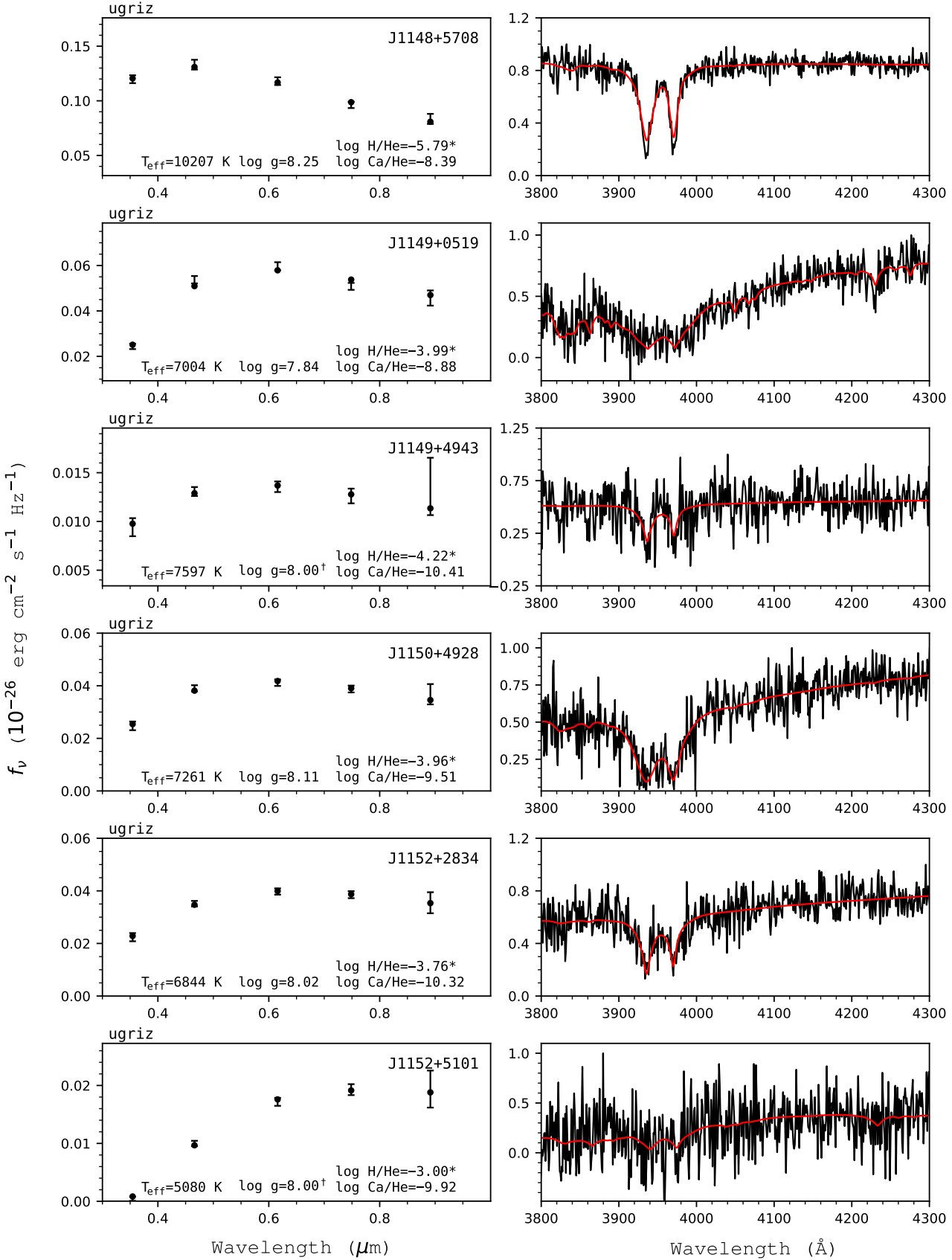

Figure 104. Fits to the DBZ/DZ(A) white dwarfs - continued. 

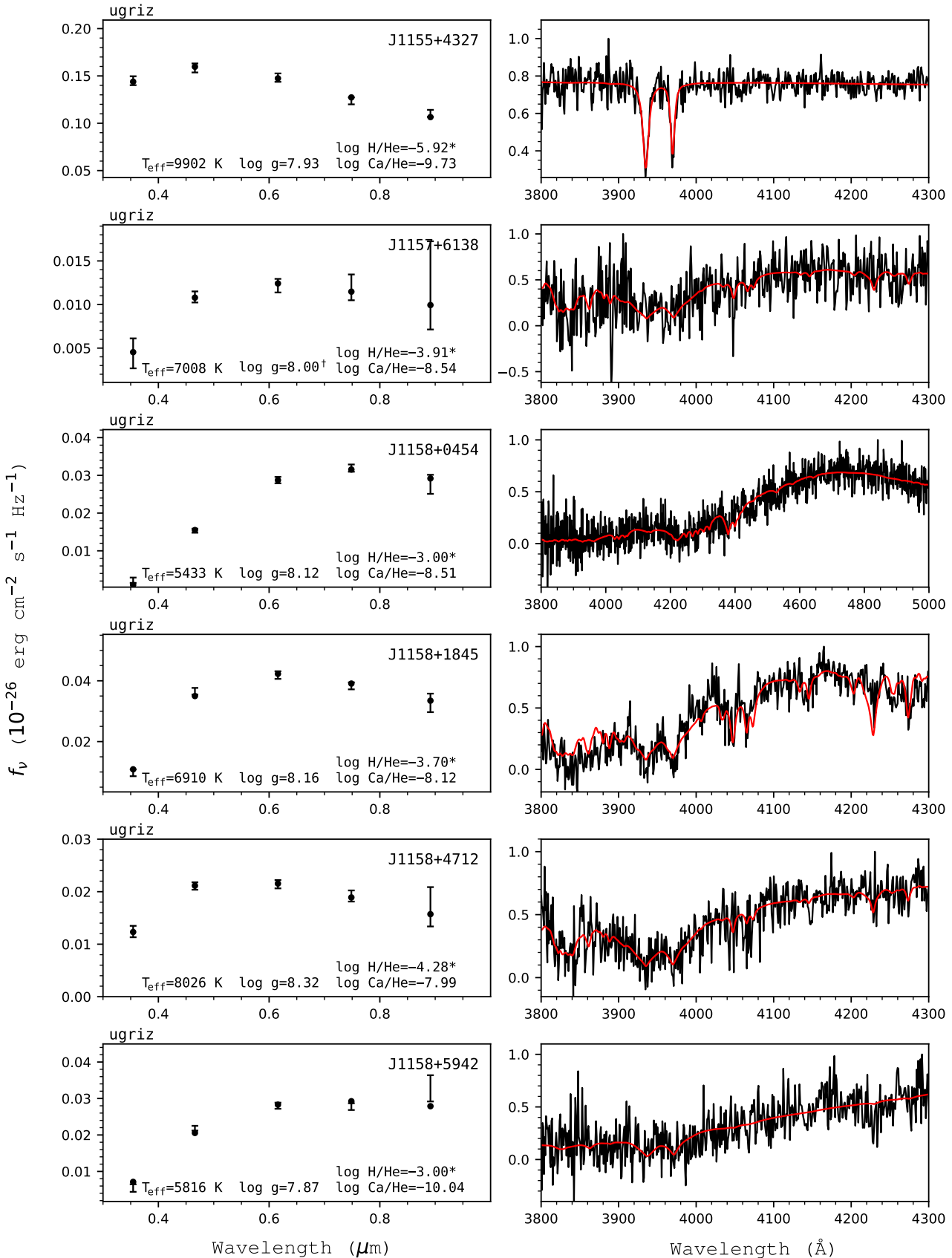

Figure 105. Fits to the DBZ/DZ(A) white dwarfs - continued. 

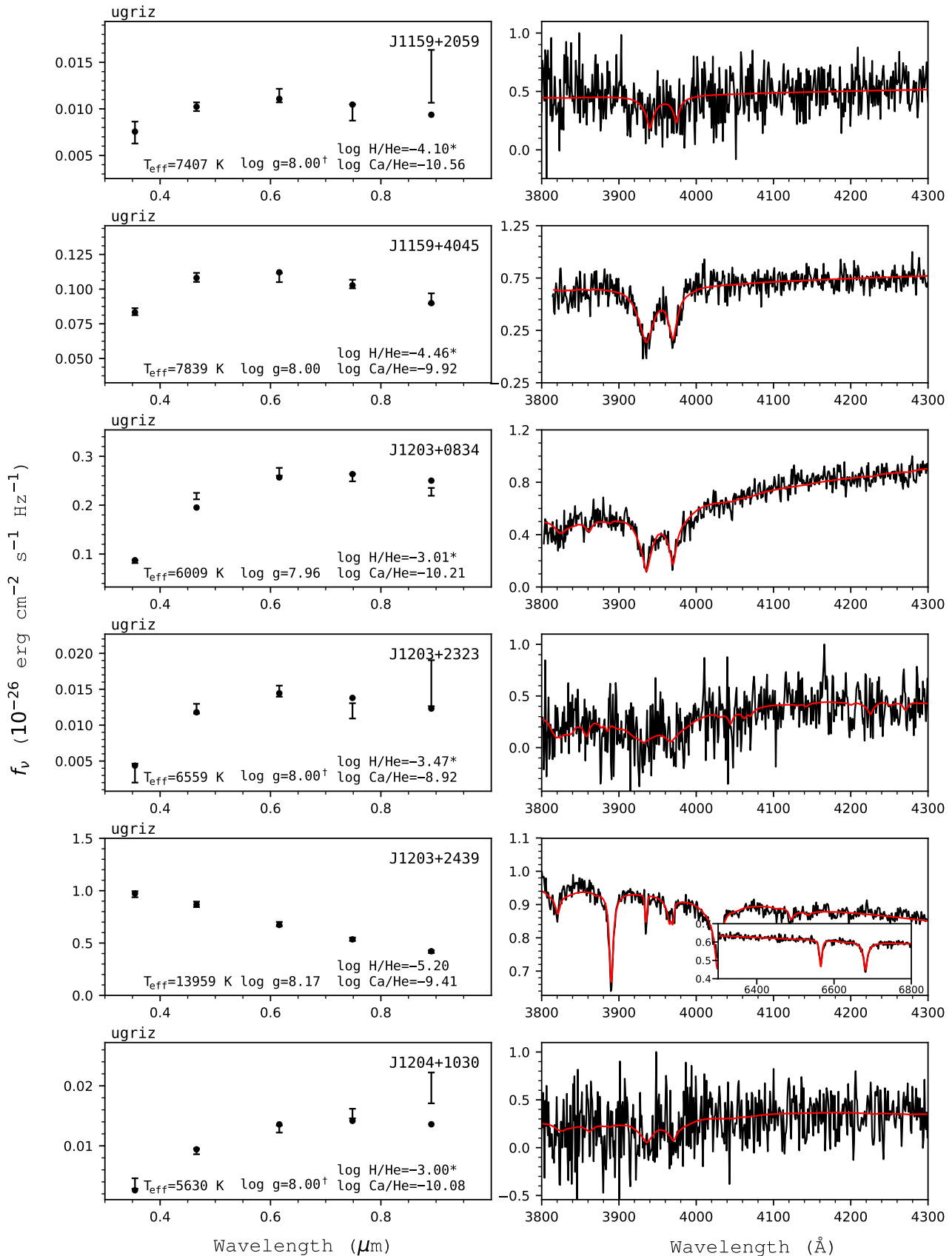

Figure 106. Fits to the DBZ/DZ(A) white dwarfs - continued. 


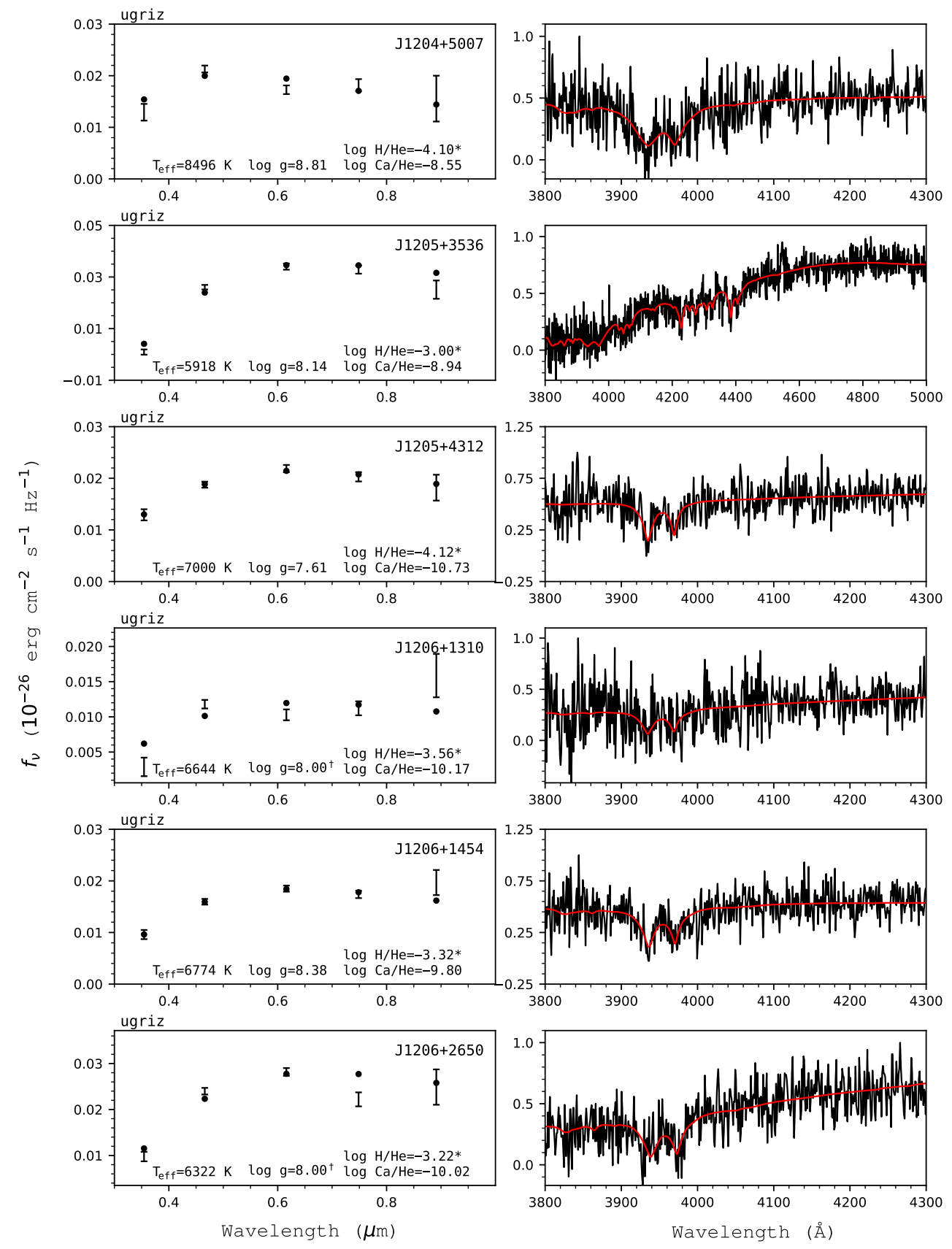

Figure 107. Fits to the DBZ/DZ(A) white dwarfs - continued. 

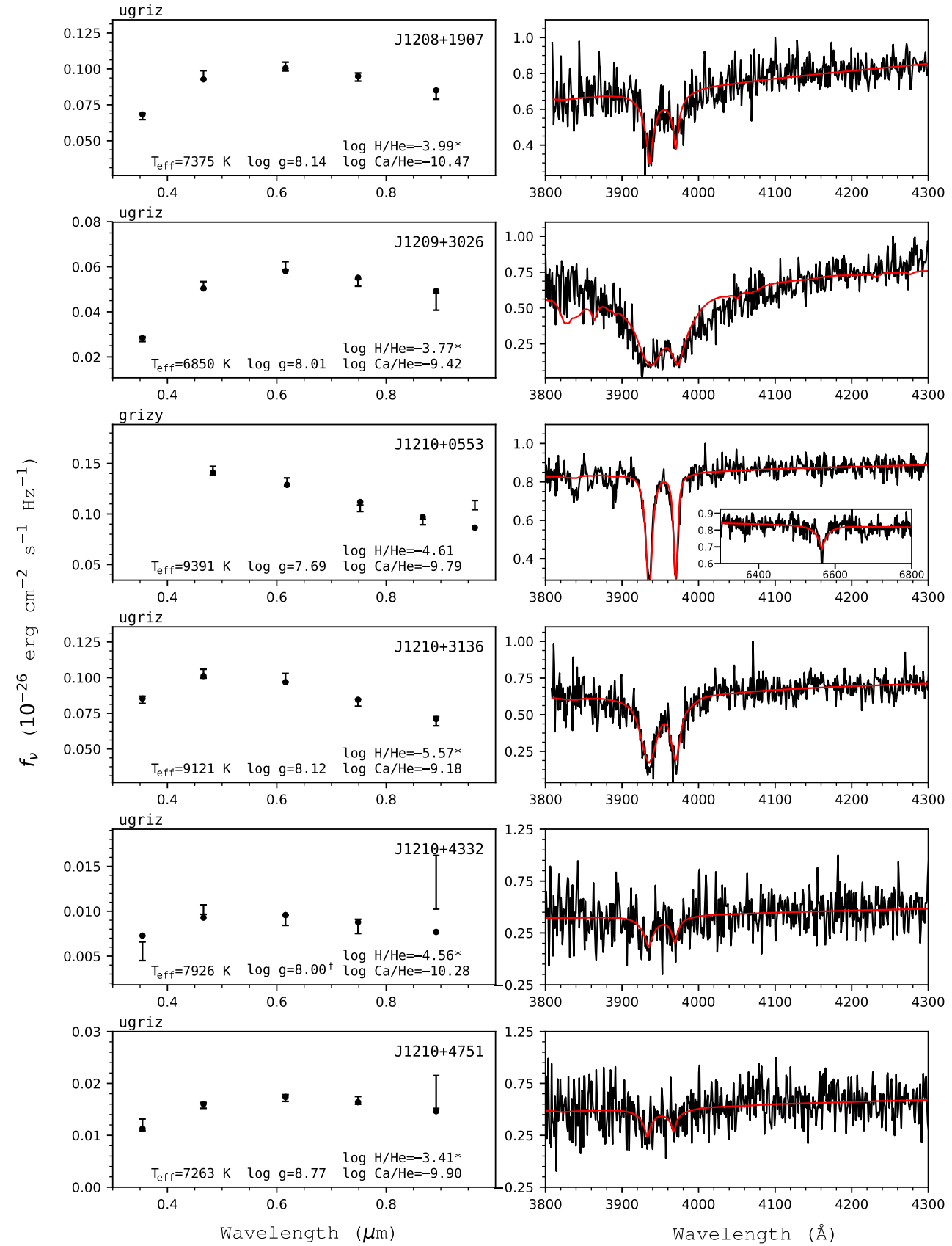

Figure 108. Fits to the DBZ/DZ(A) white dwarfs - continued. 

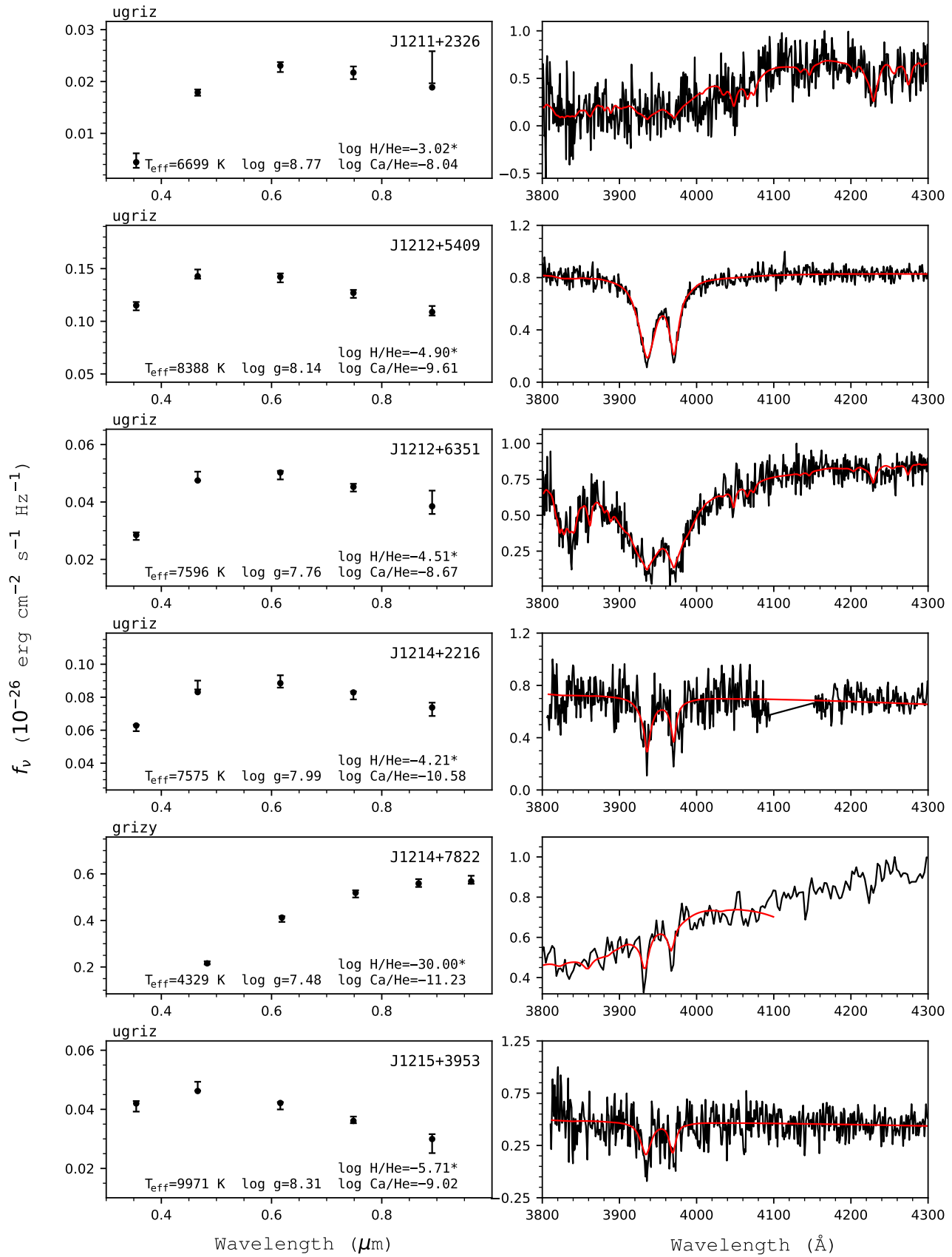

Figure 109. Fits to the DBZ/DZ(A) white dwarfs - continued. 

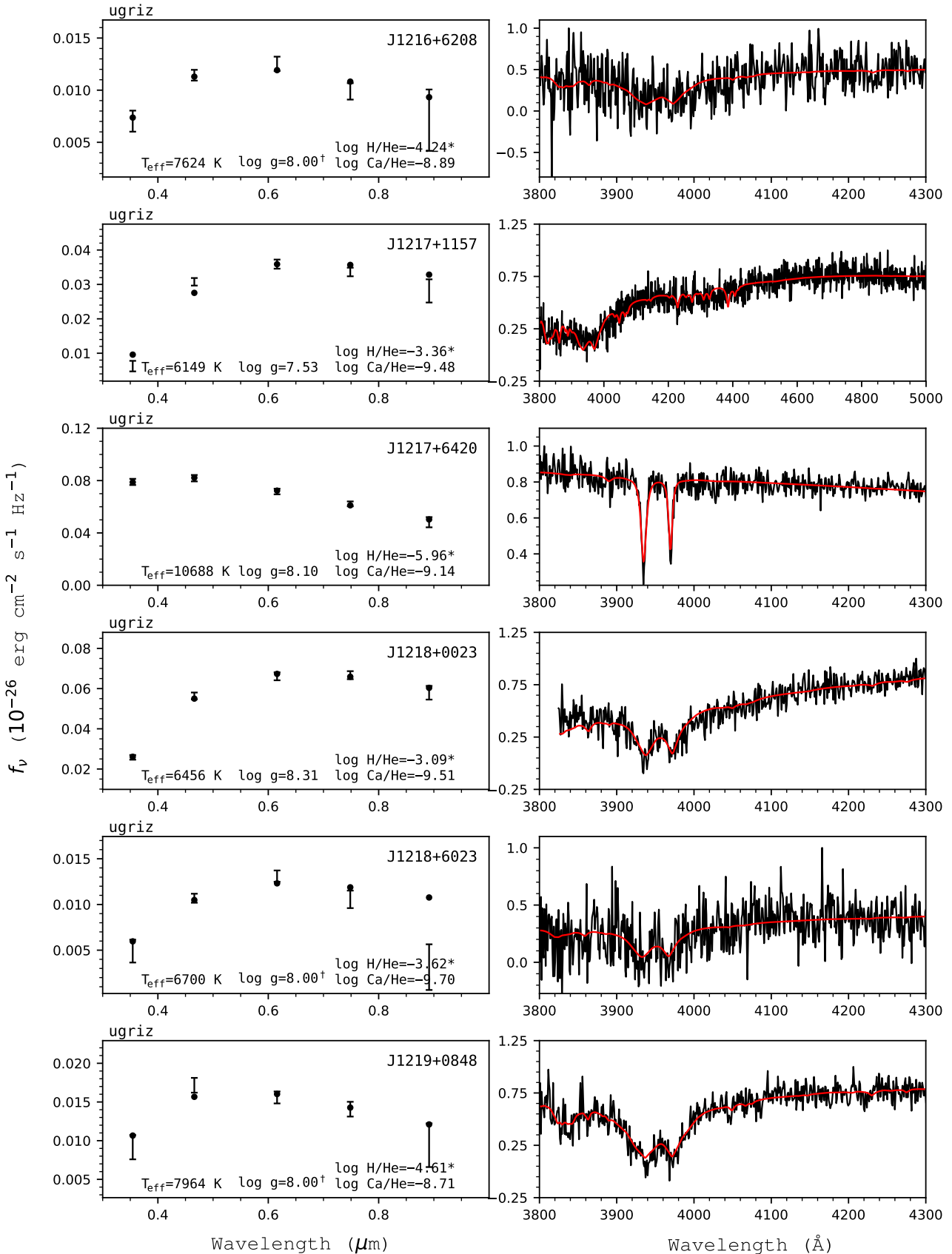

Figure 110. Fits to the DBZ/DZ(A) white dwarfs - continued. 

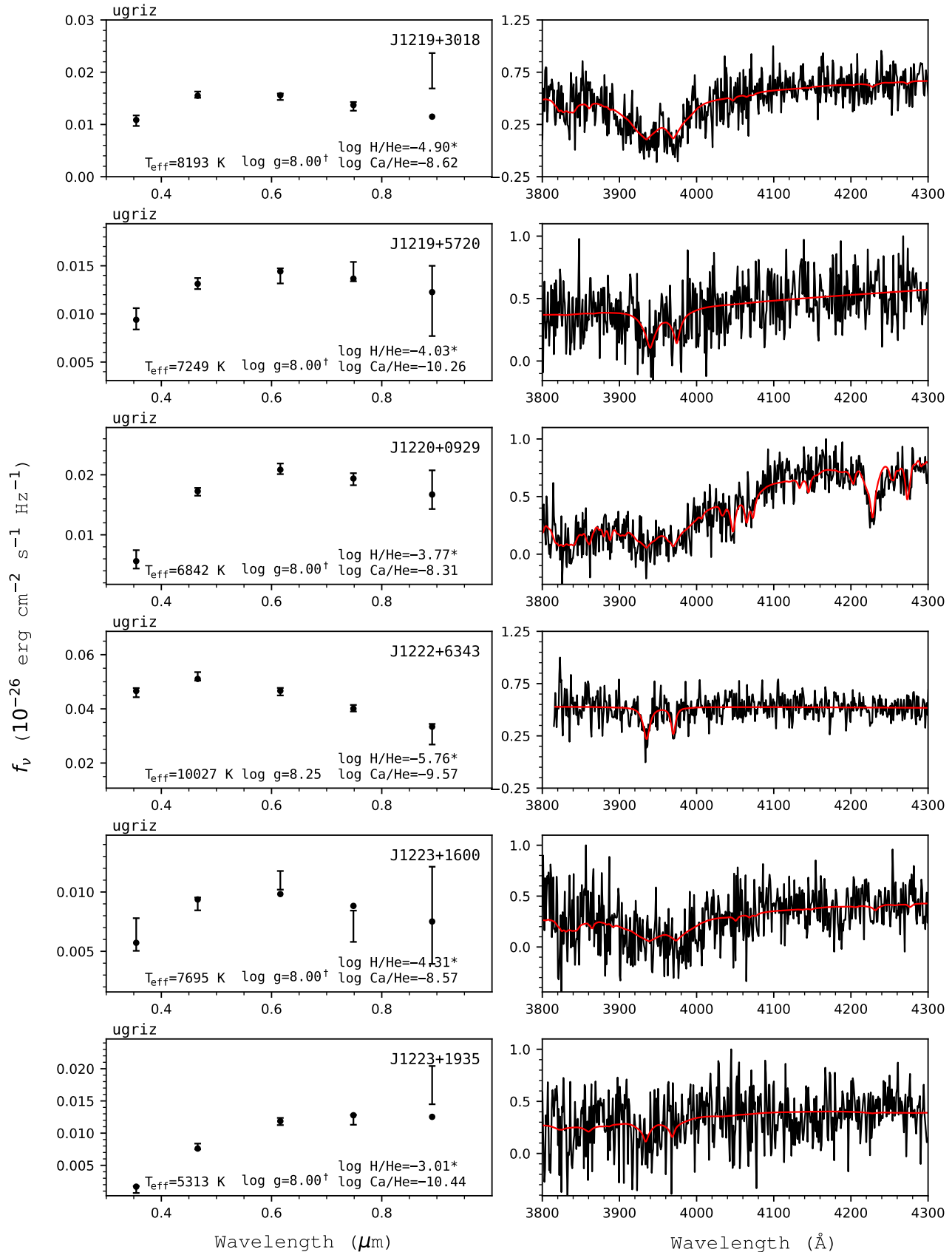

Figure 111. Fits to the DBZ/DZ(A) white dwarfs - continued. 

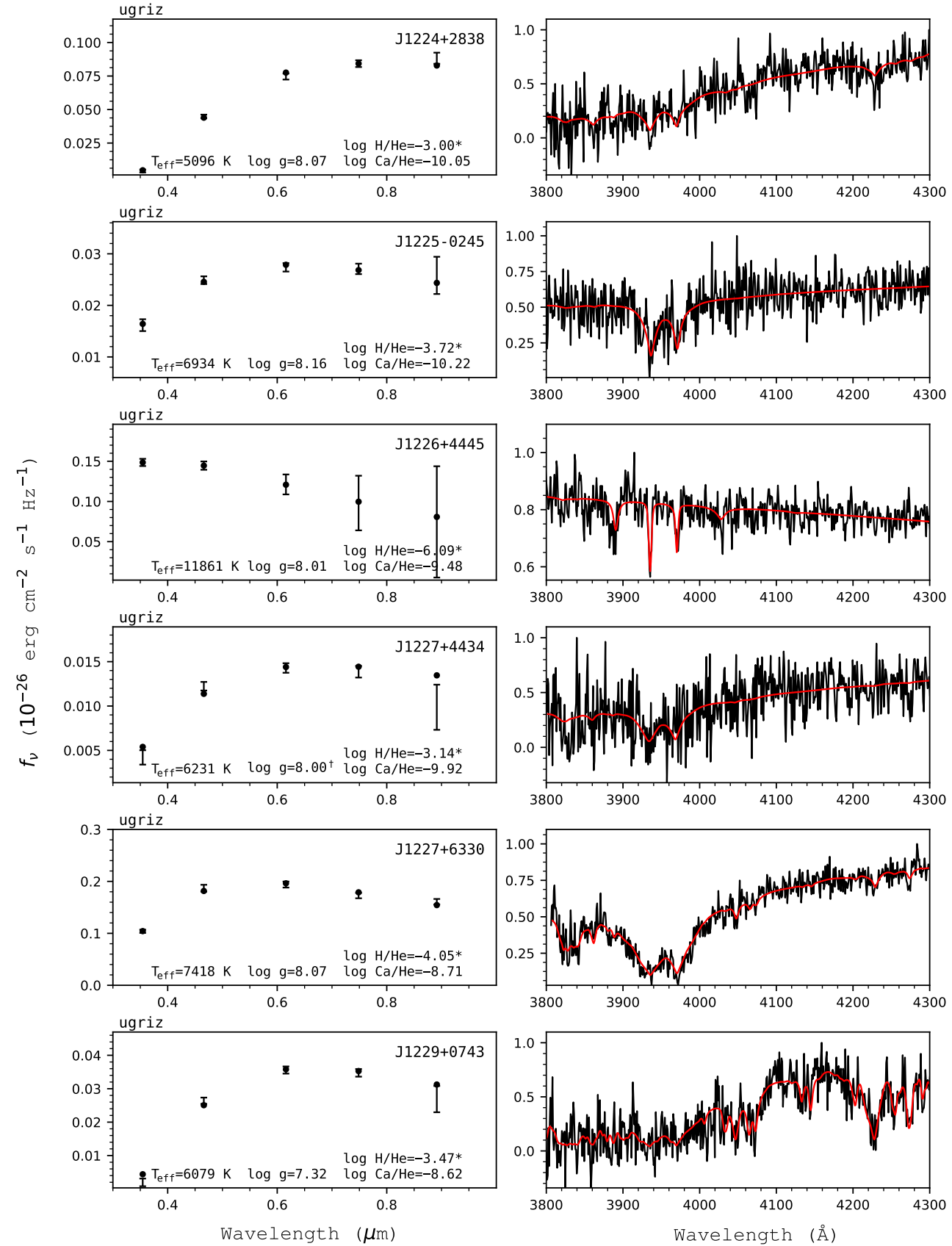

Figure 112. Fits to the DBZ/DZ(A) white dwarfs - continued. 

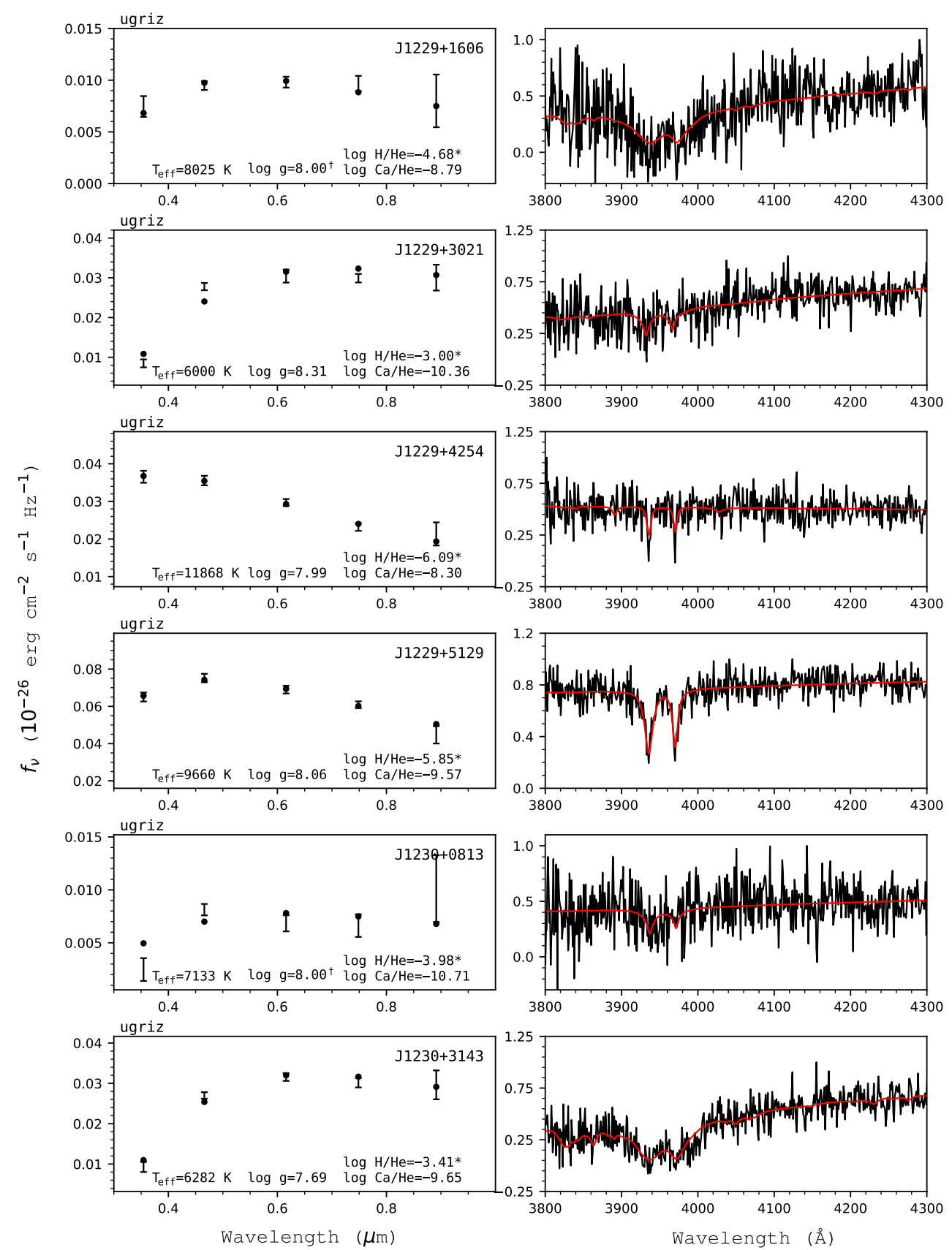

Figure 113. Fits to the DBZ/DZ(A) white dwarfs - continued. 

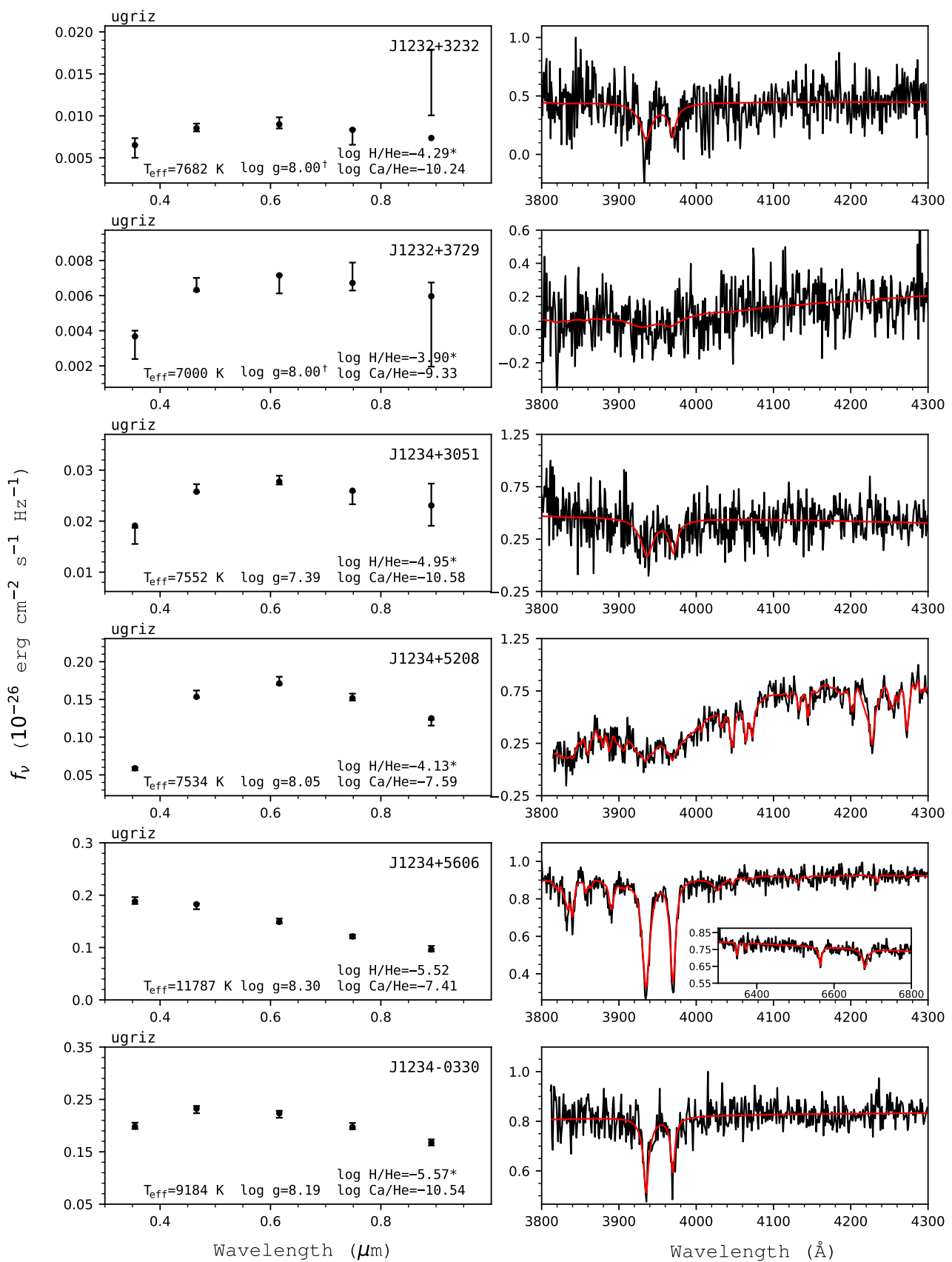

Figure 114. Fits to the DBZ/DZ(A) white dwarfs - continued. 

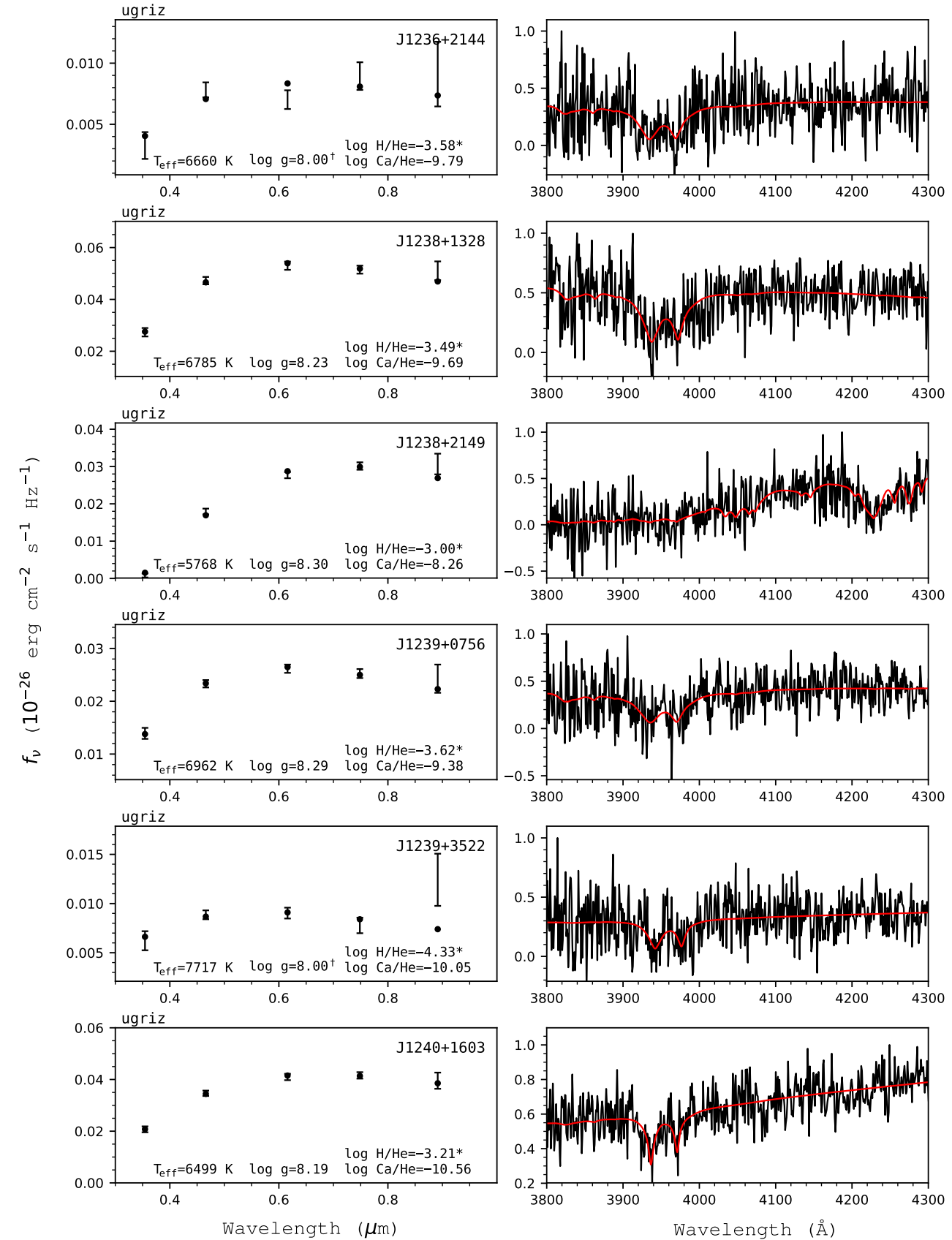

Figure 115. Fits to the DBZ/DZ(A) white dwarfs - continued. 

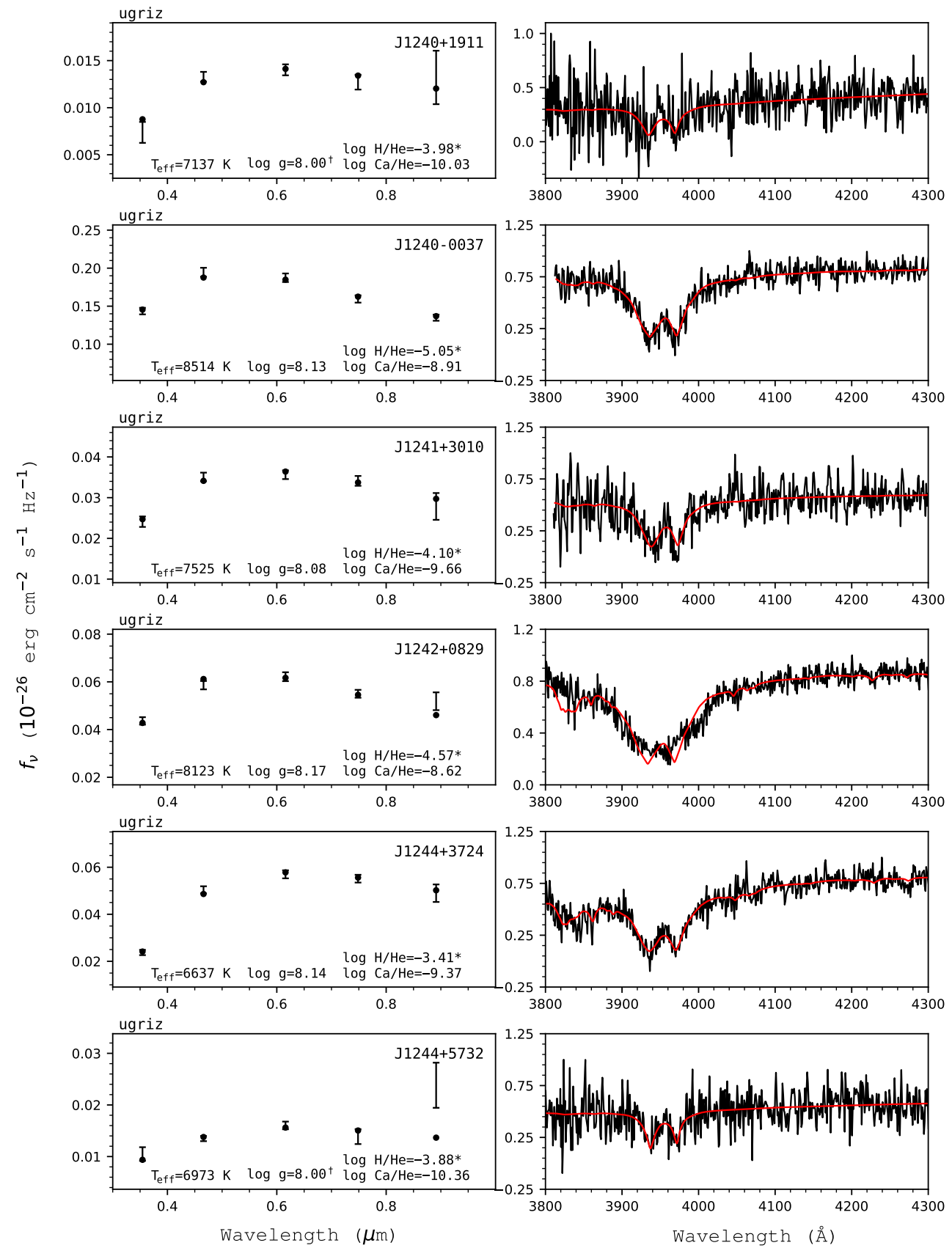

Figure 116. Fits to the DBZ/DZ(A) white dwarfs - continued. 

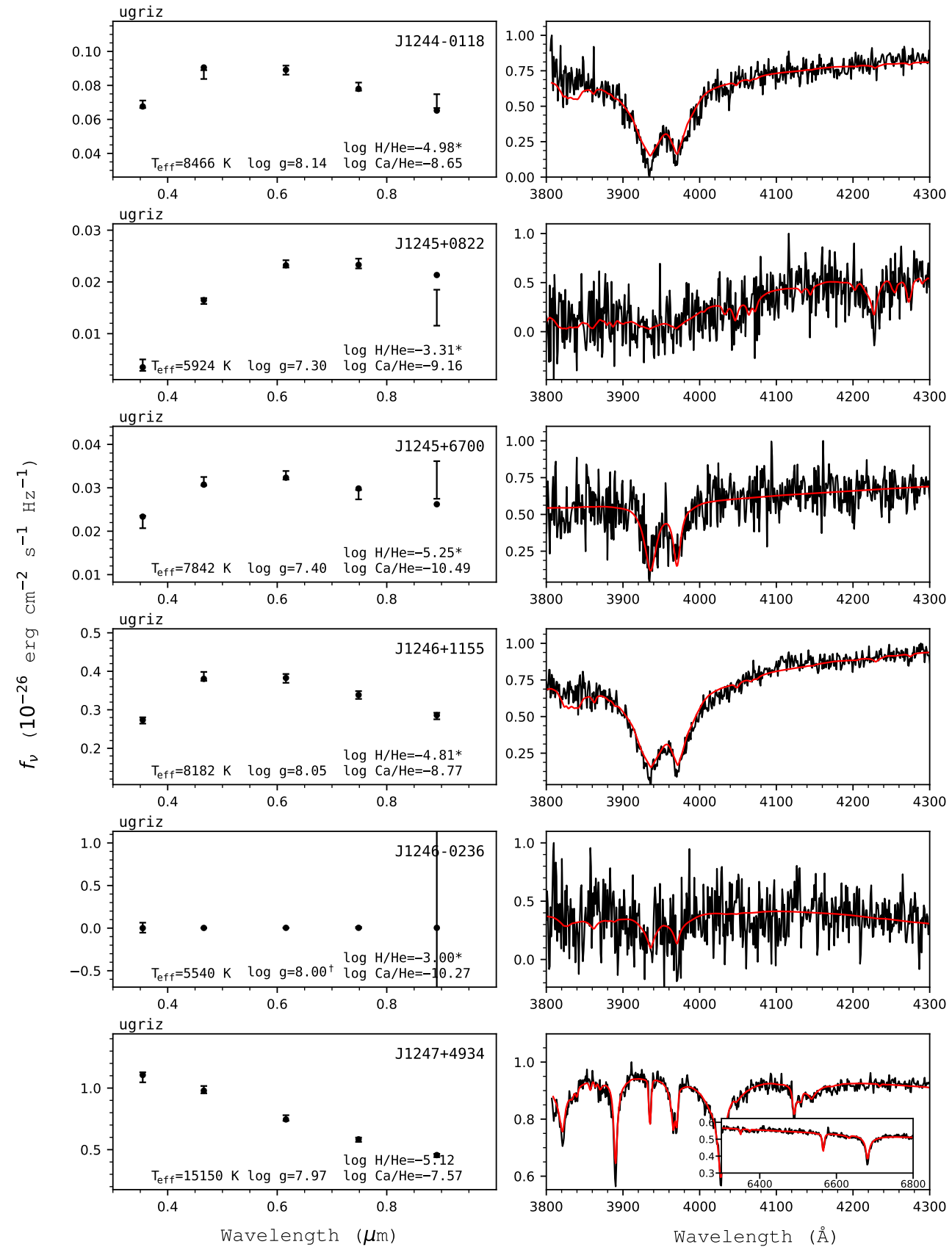

Figure 117. Fits to the DBZ/DZ(A) white dwarfs - continued. 

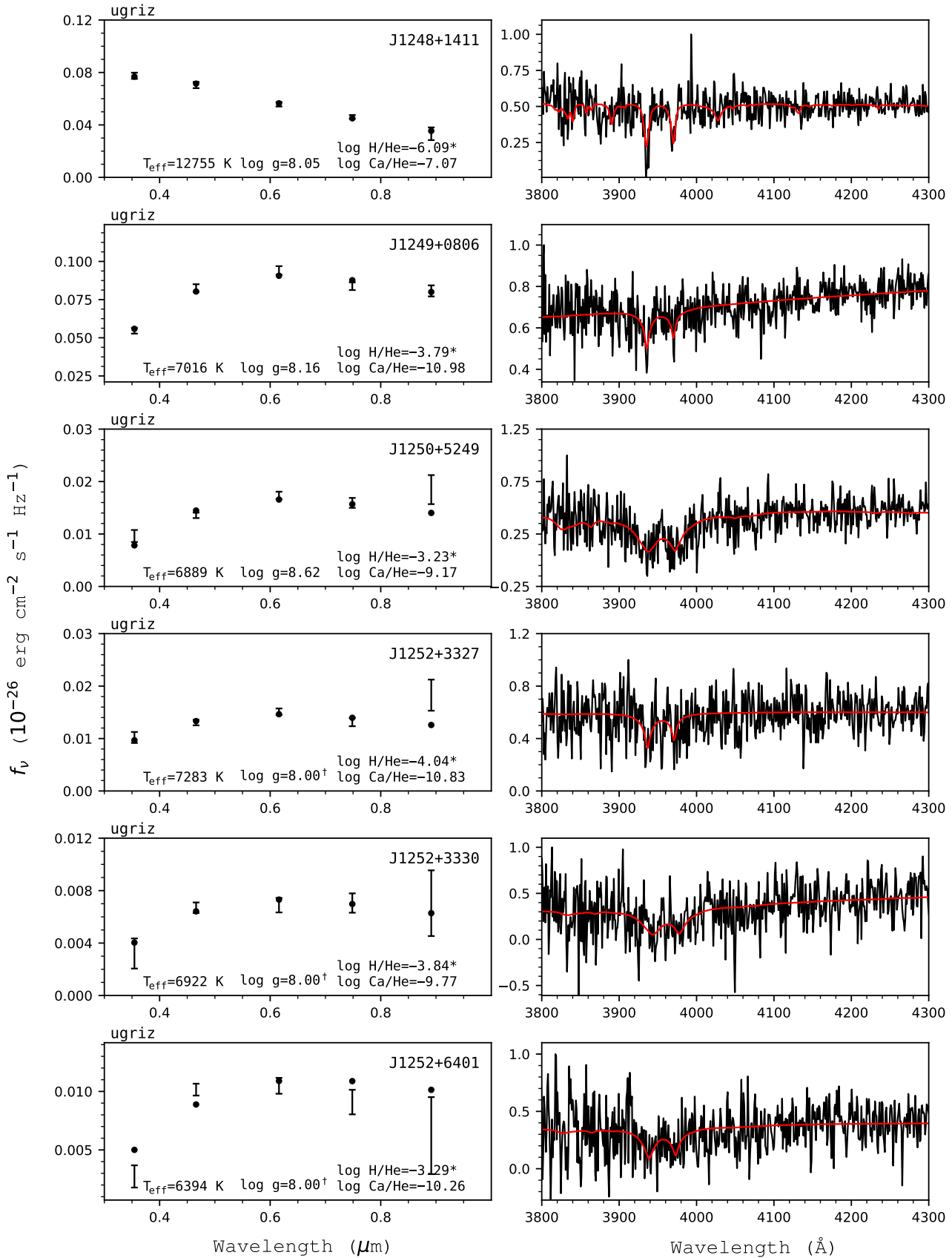

Figure 118. Fits to the DBZ/DZ(A) white dwarfs - continued. 

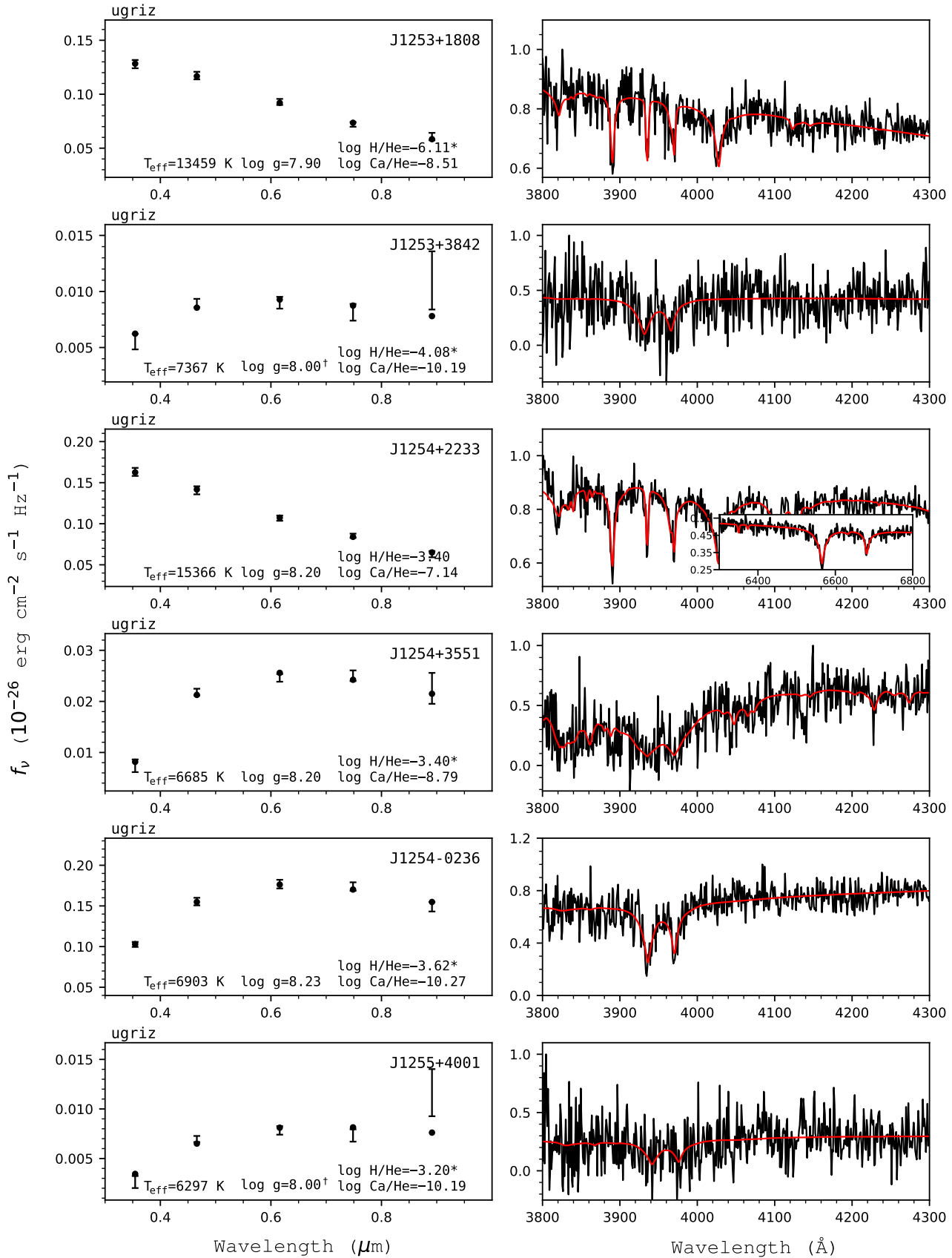

Figure 119. Fits to the DBZ/DZ(A) white dwarfs - continued. 

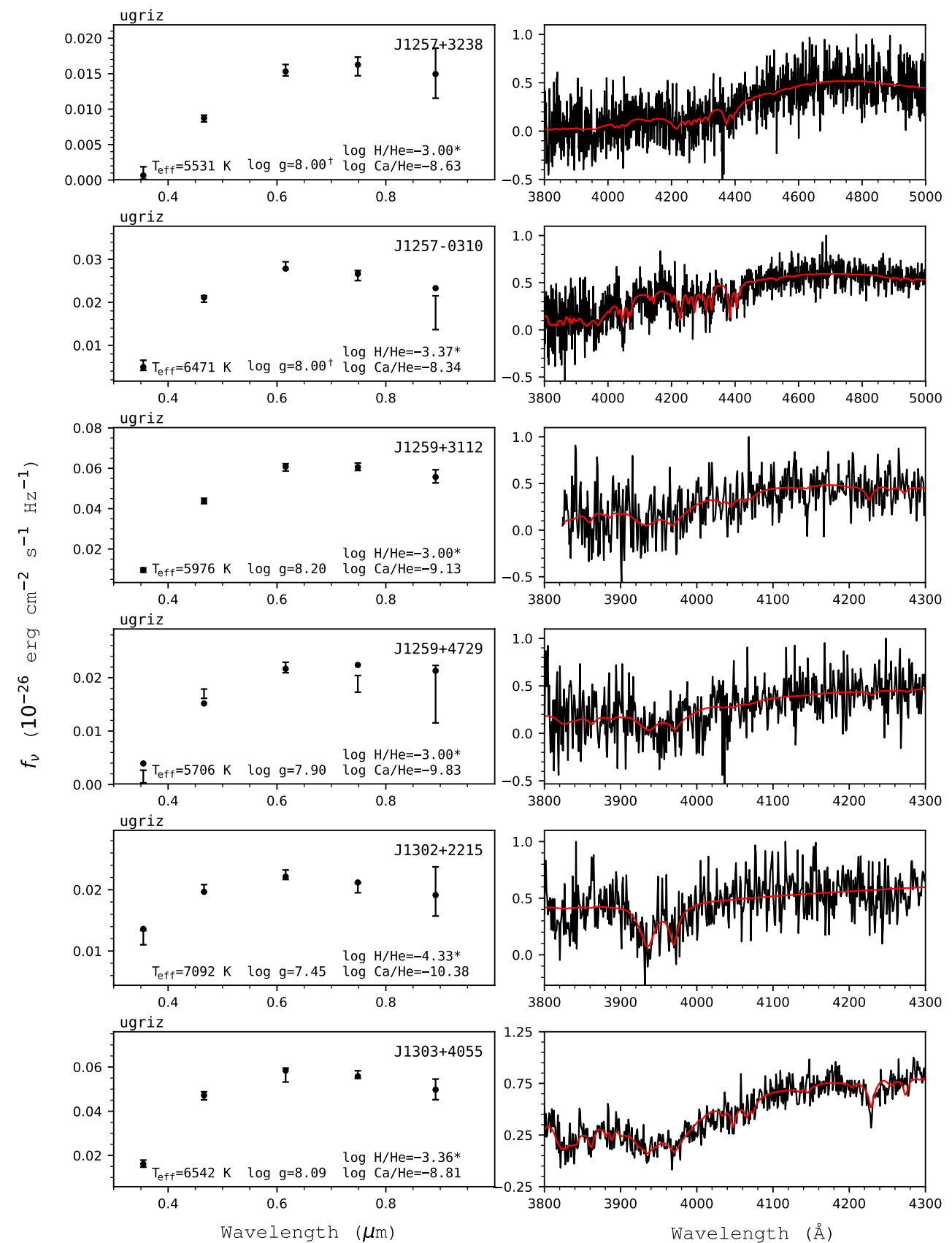

Figure 120. Fits to the DBZ/DZ(A) white dwarfs - continued. 

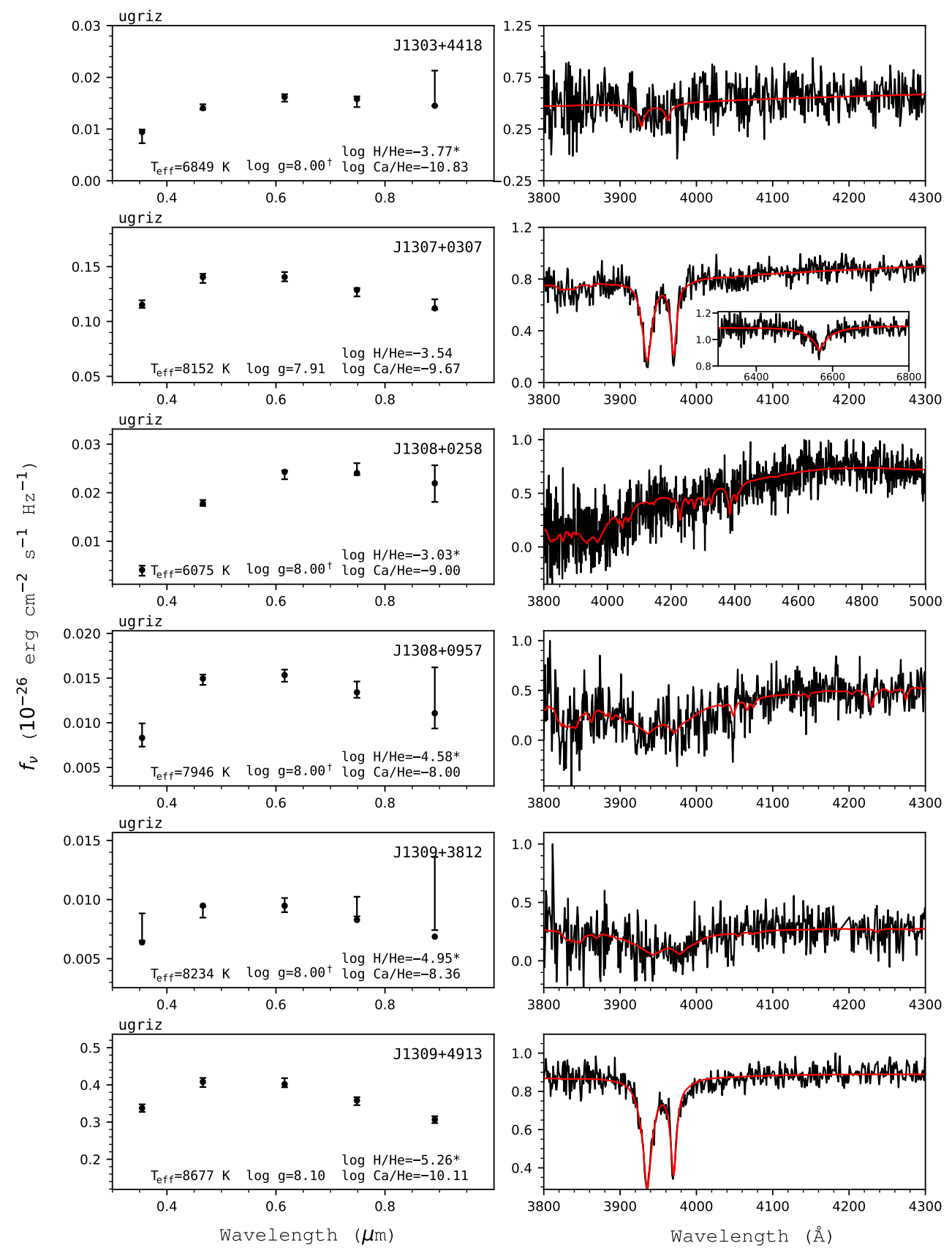

Figure 121. Fits to the DBZ/DZ(A) white dwarfs - continued. 

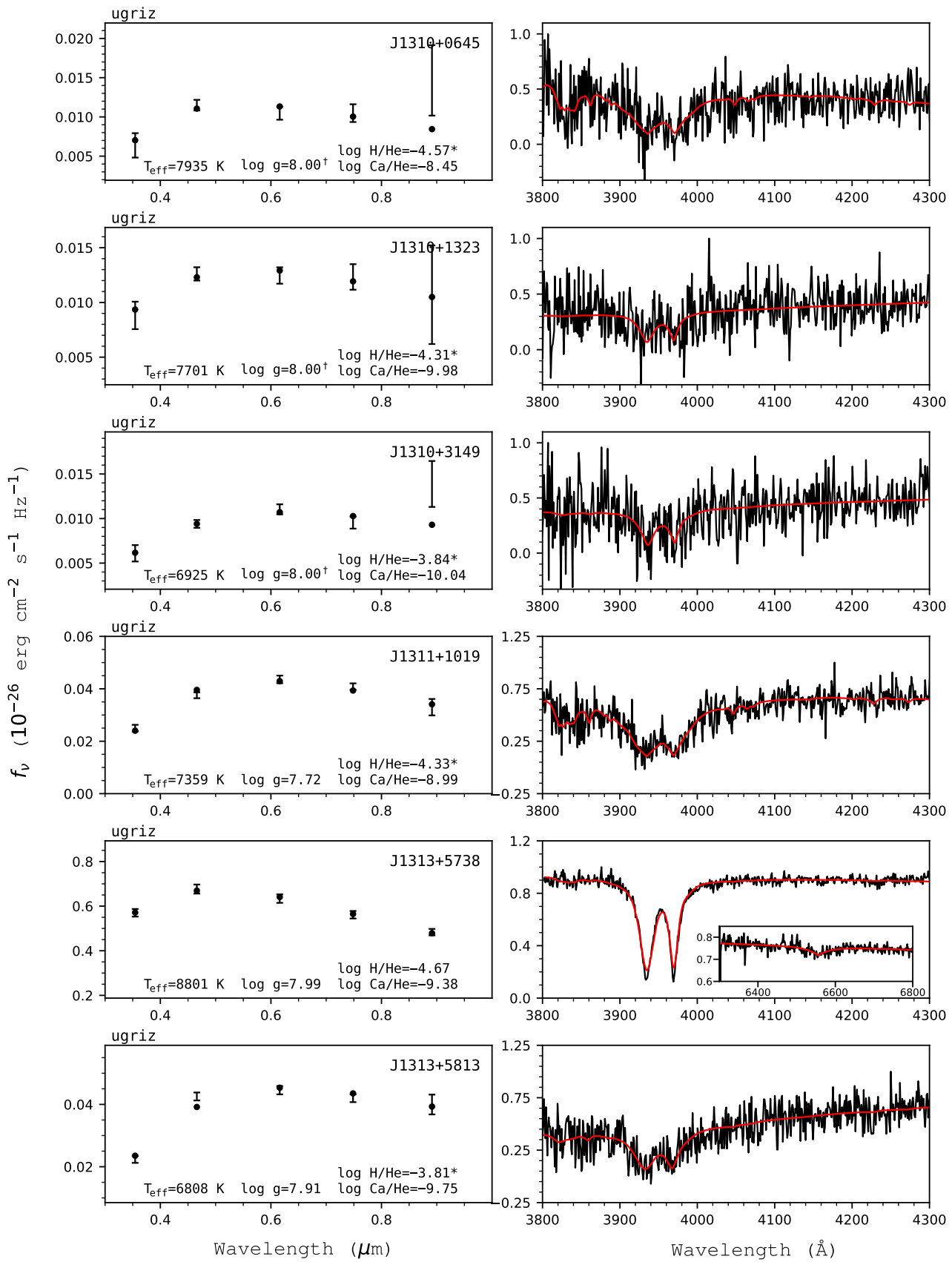

Figure 122. Fits to the DBZ/DZ(A) white dwarfs - continued. 

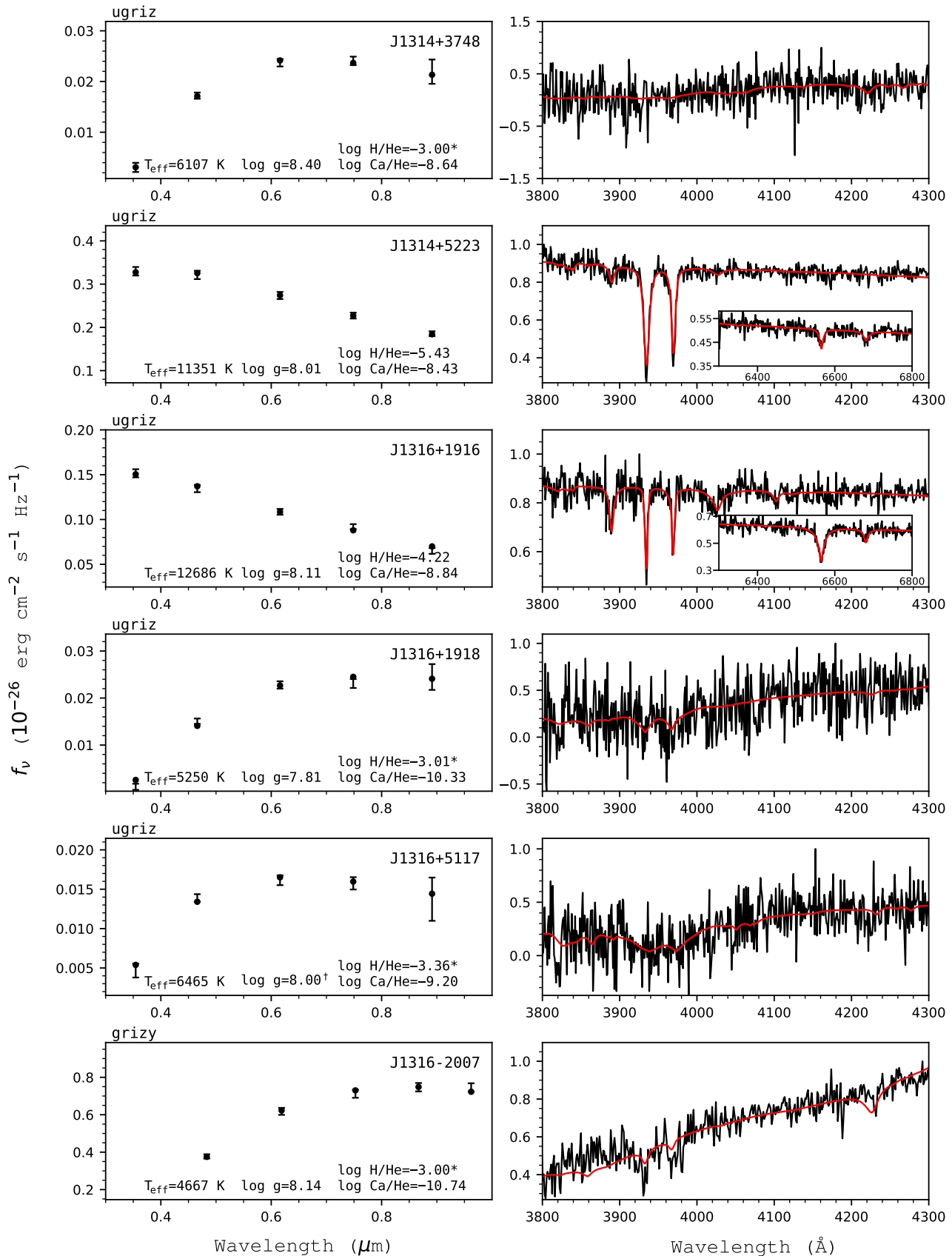

Figure 123. Fits to the DBZ/DZ(A) white dwarfs - continued. 

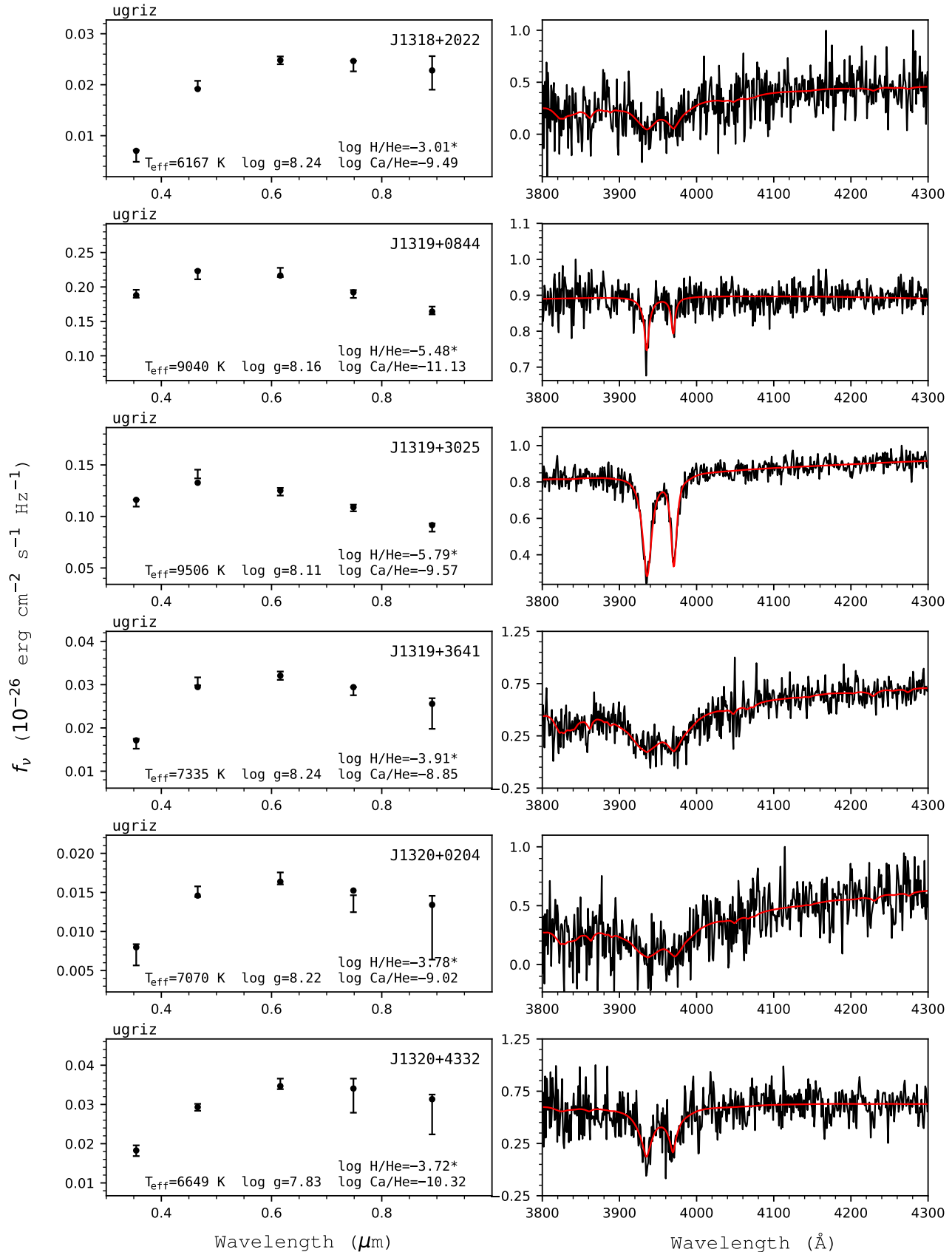

Figure 124. Fits to the DBZ/DZ(A) white dwarfs - continued. 


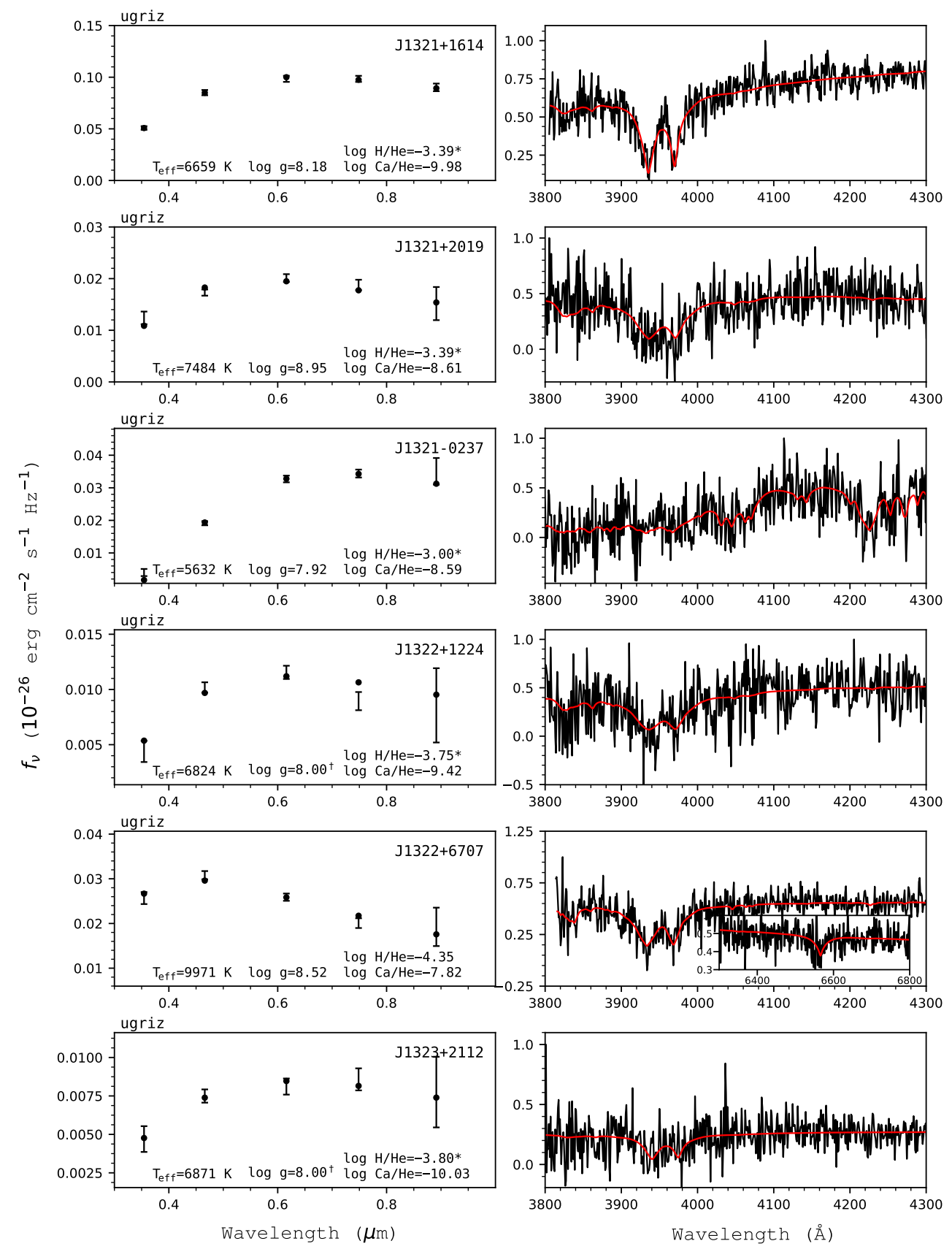

Figure 125. Fits to the DBZ/DZ(A) white dwarfs - continued. 

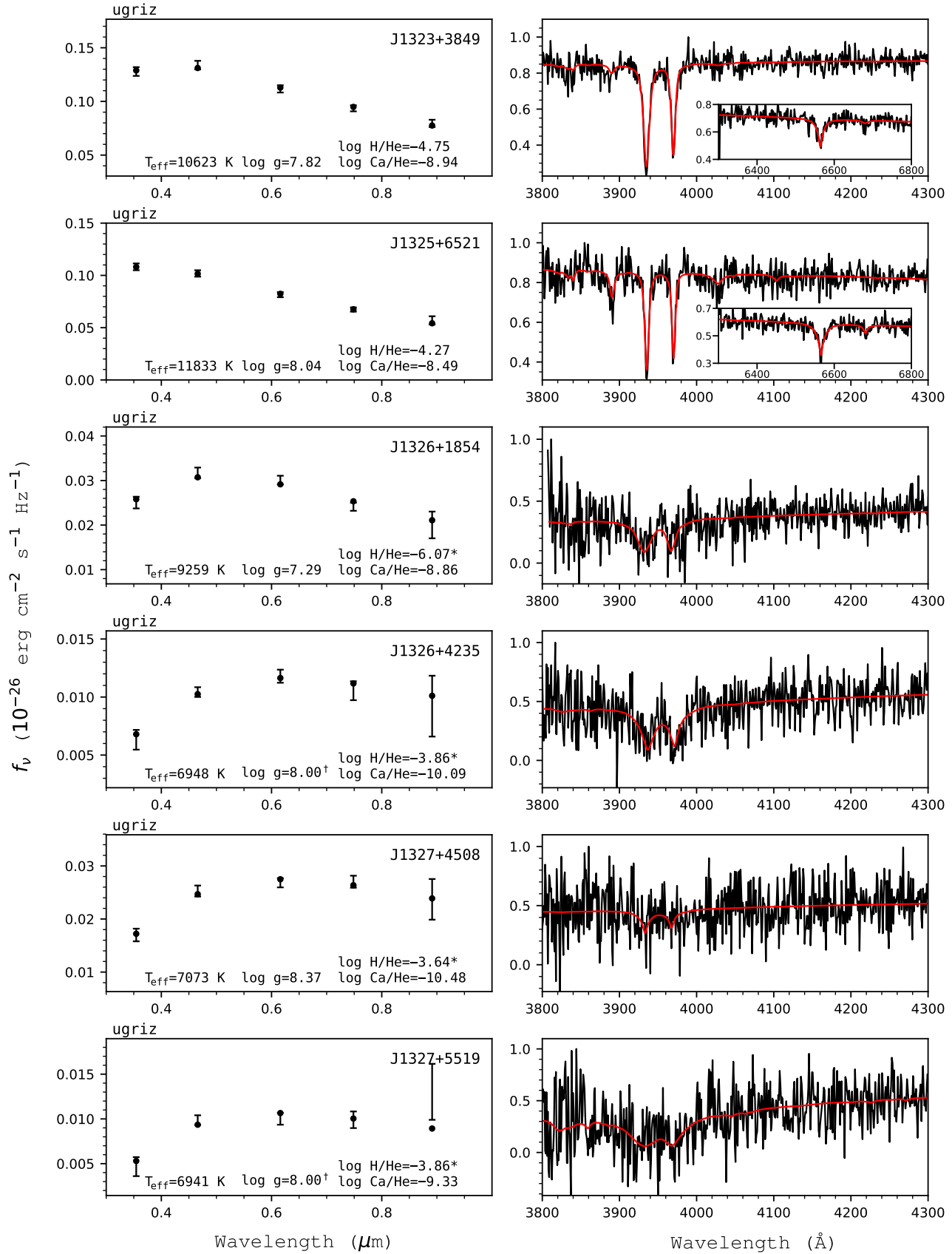

Figure 126. Fits to the DBZ/DZ(A) white dwarfs - continued. 

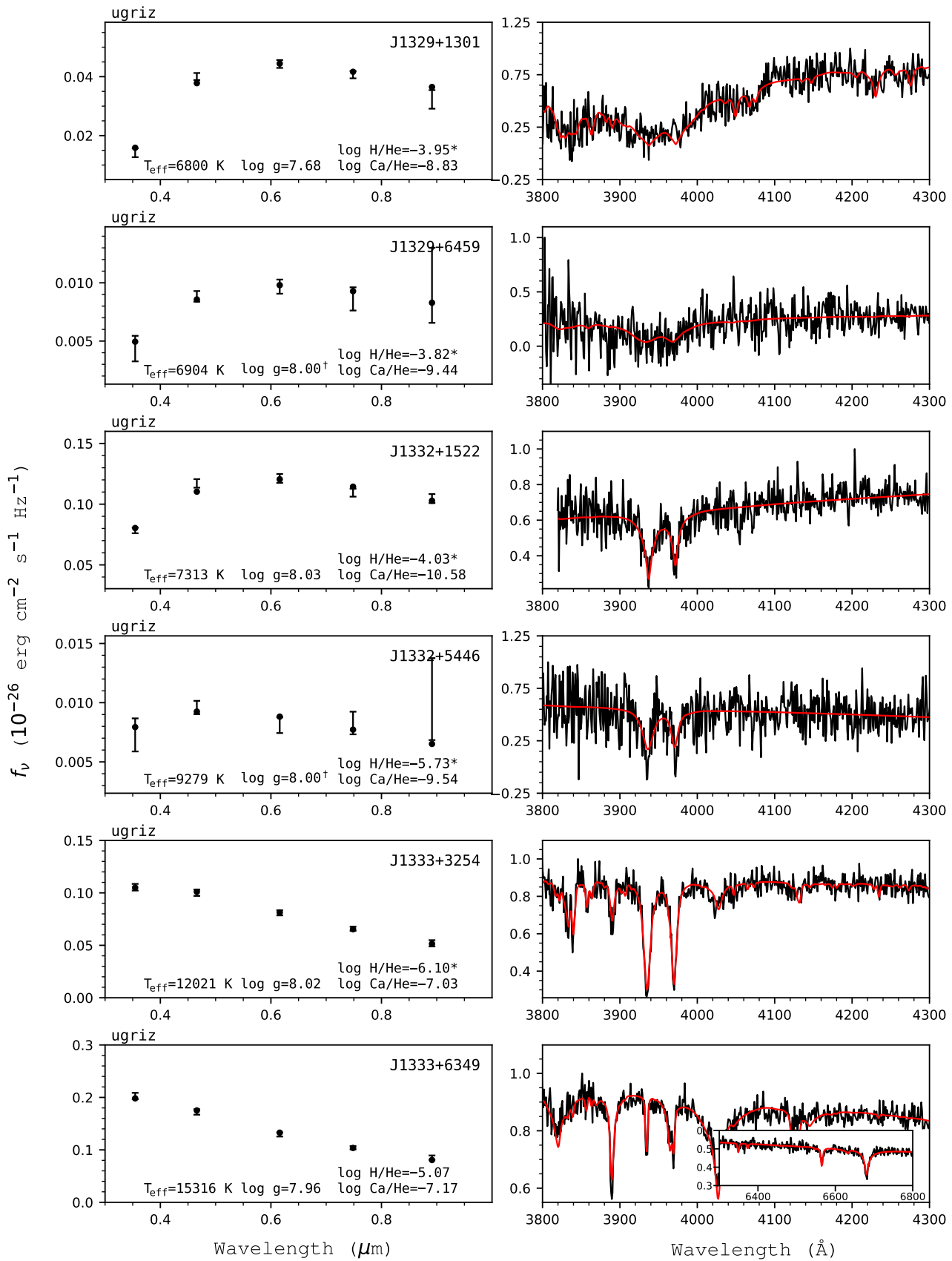

Figure 127. Fits to the DBZ/DZ(A) white dwarfs - continued. 

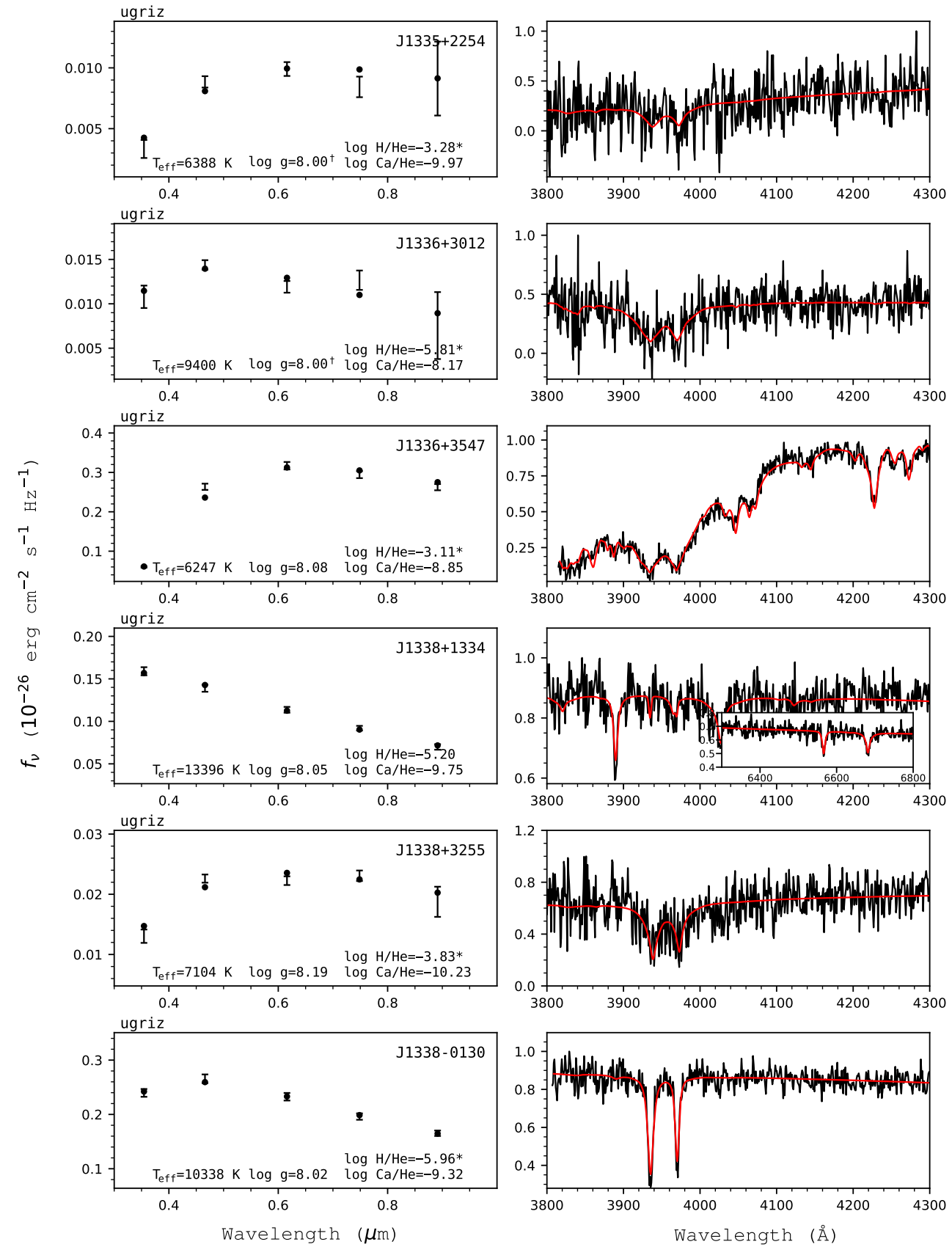

Figure 128. Fits to the DBZ/DZ(A) white dwarfs - continued. 

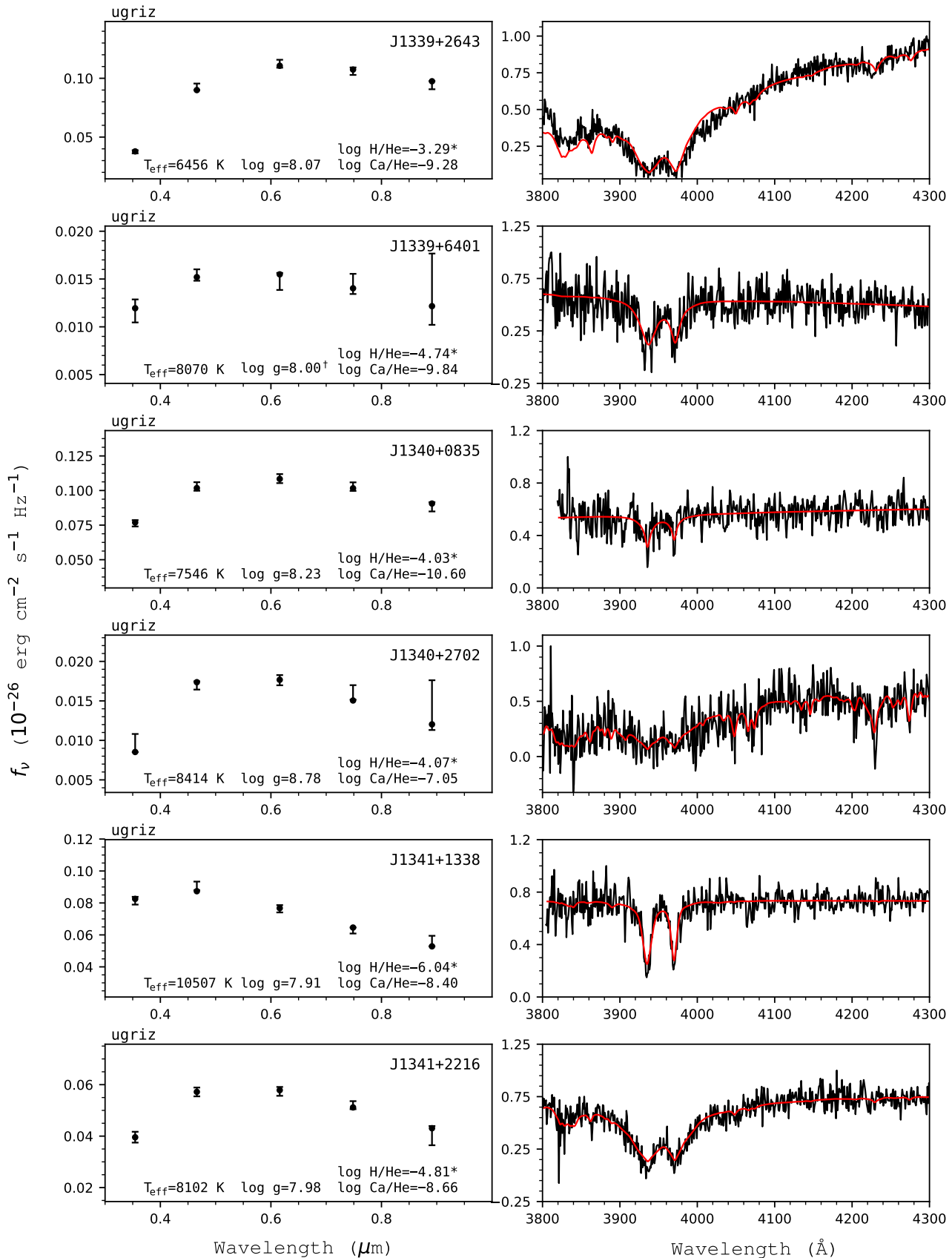

Figure 129. Fits to the DBZ/DZ(A) white dwarfs - continued. 

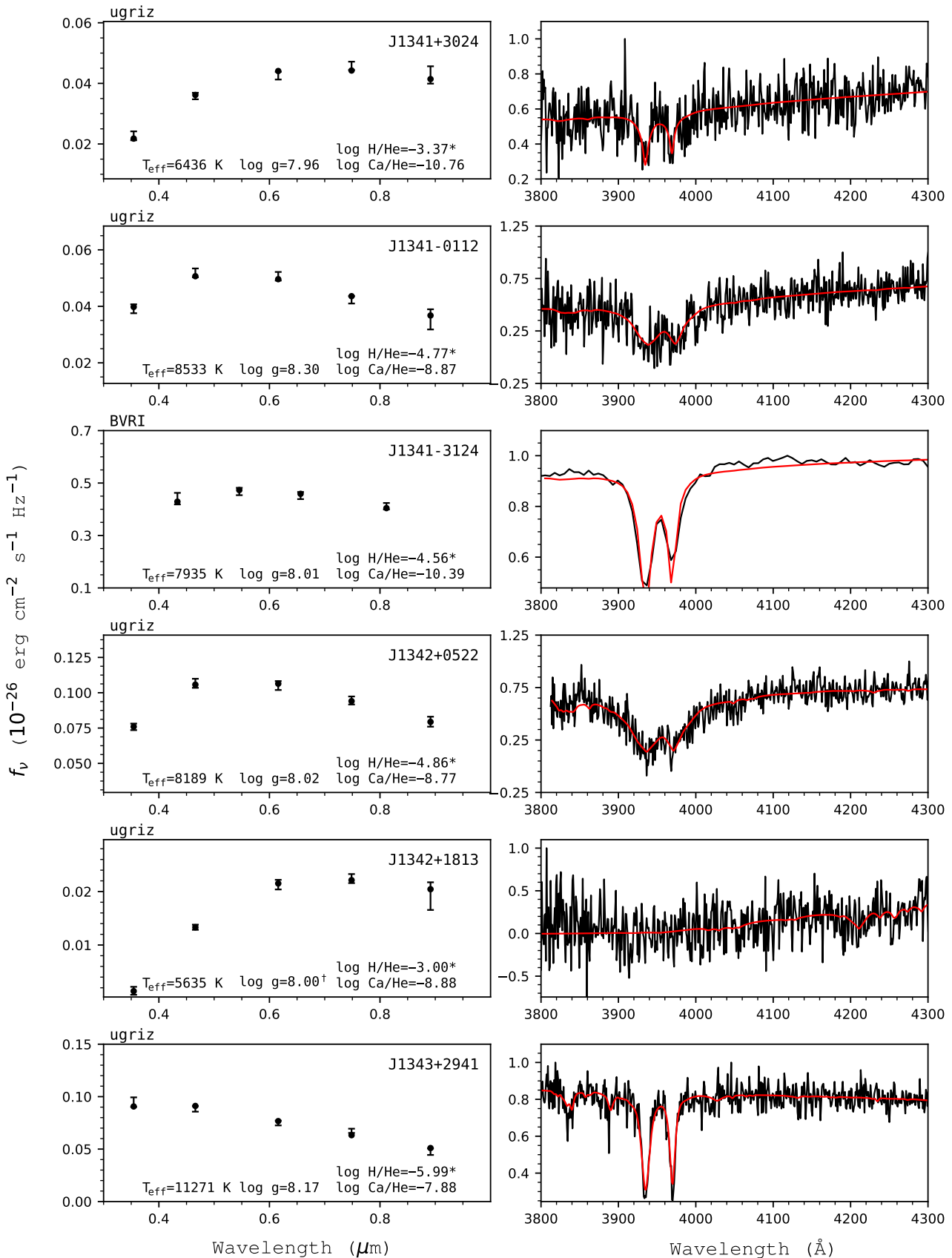

Figure 130. Fits to the DBZ/DZ(A) white dwarfs - continued. 


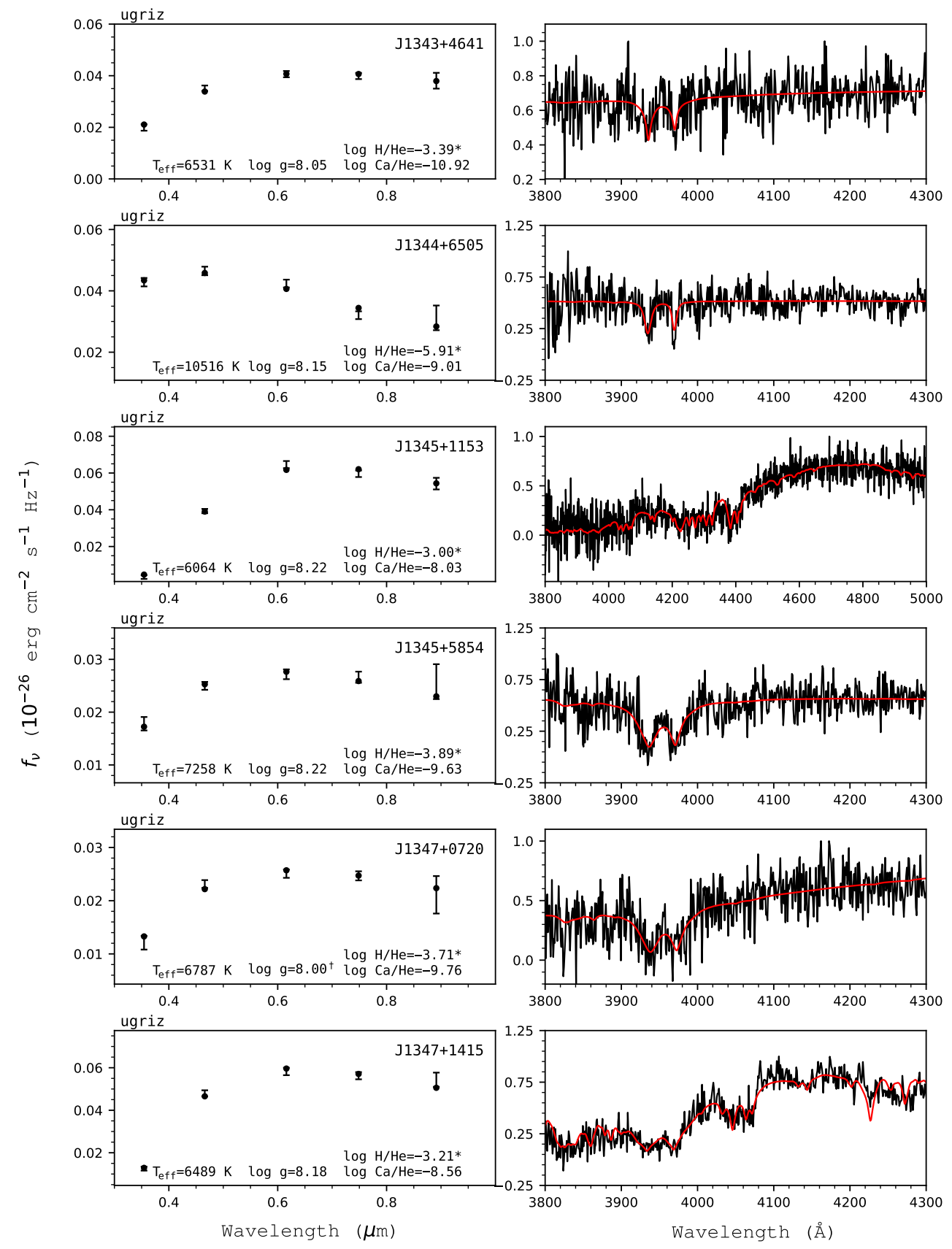

Figure 131. Fits to the DBZ/DZ(A) white dwarfs - continued. 

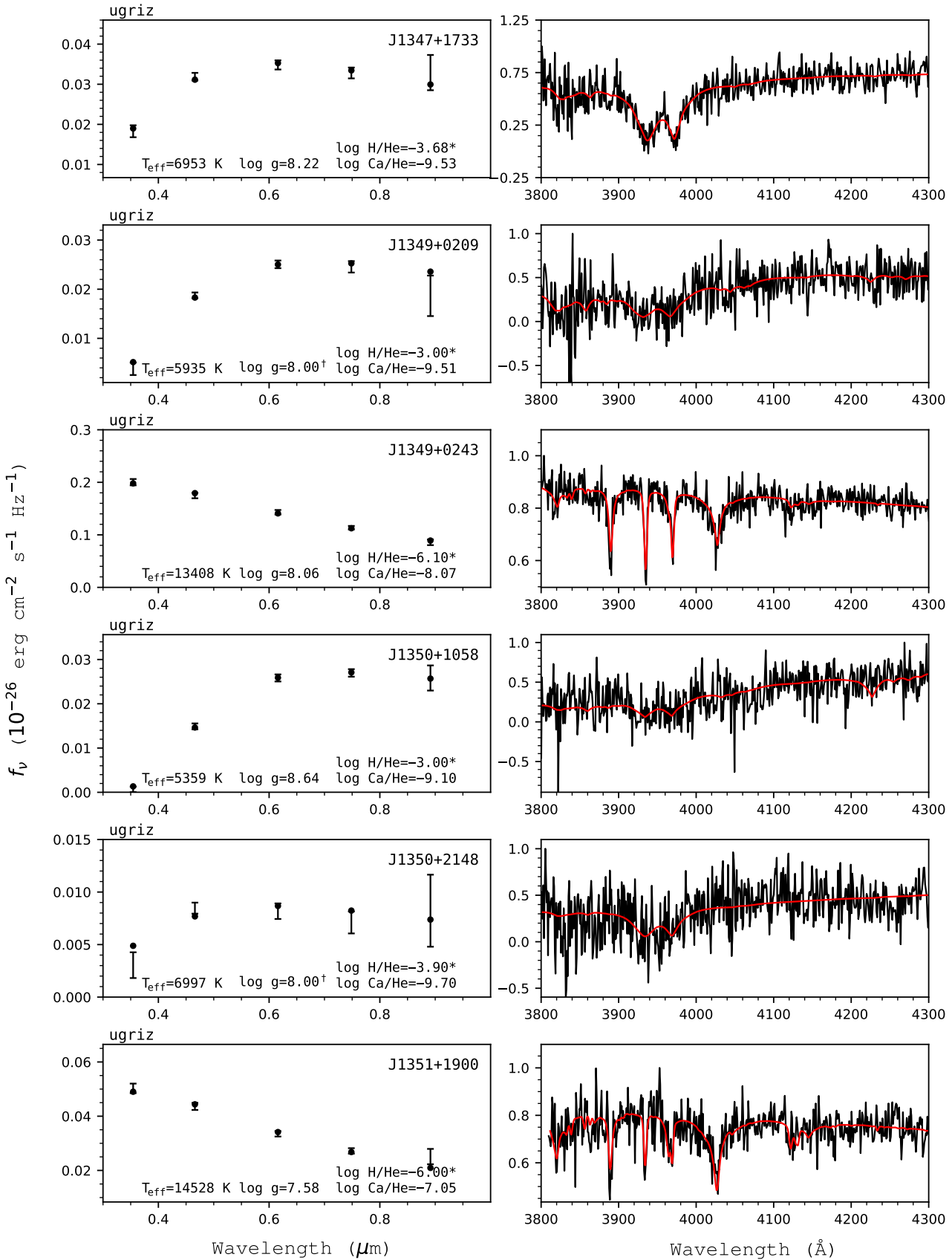

Figure 132. Fits to the DBZ/DZ(A) white dwarfs - continued. 

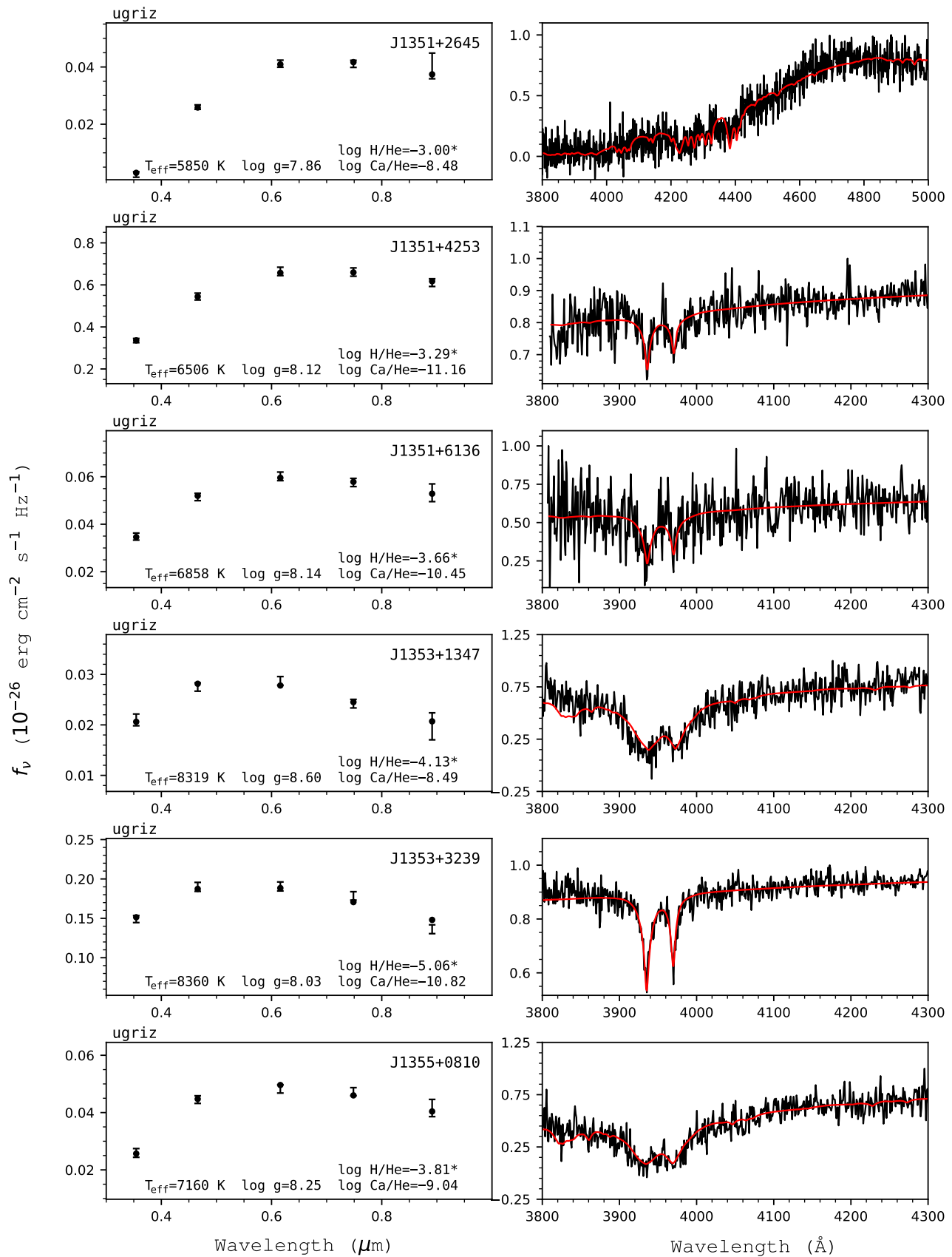

Figure 133. Fits to the DBZ/DZ(A) white dwarfs - continued. 

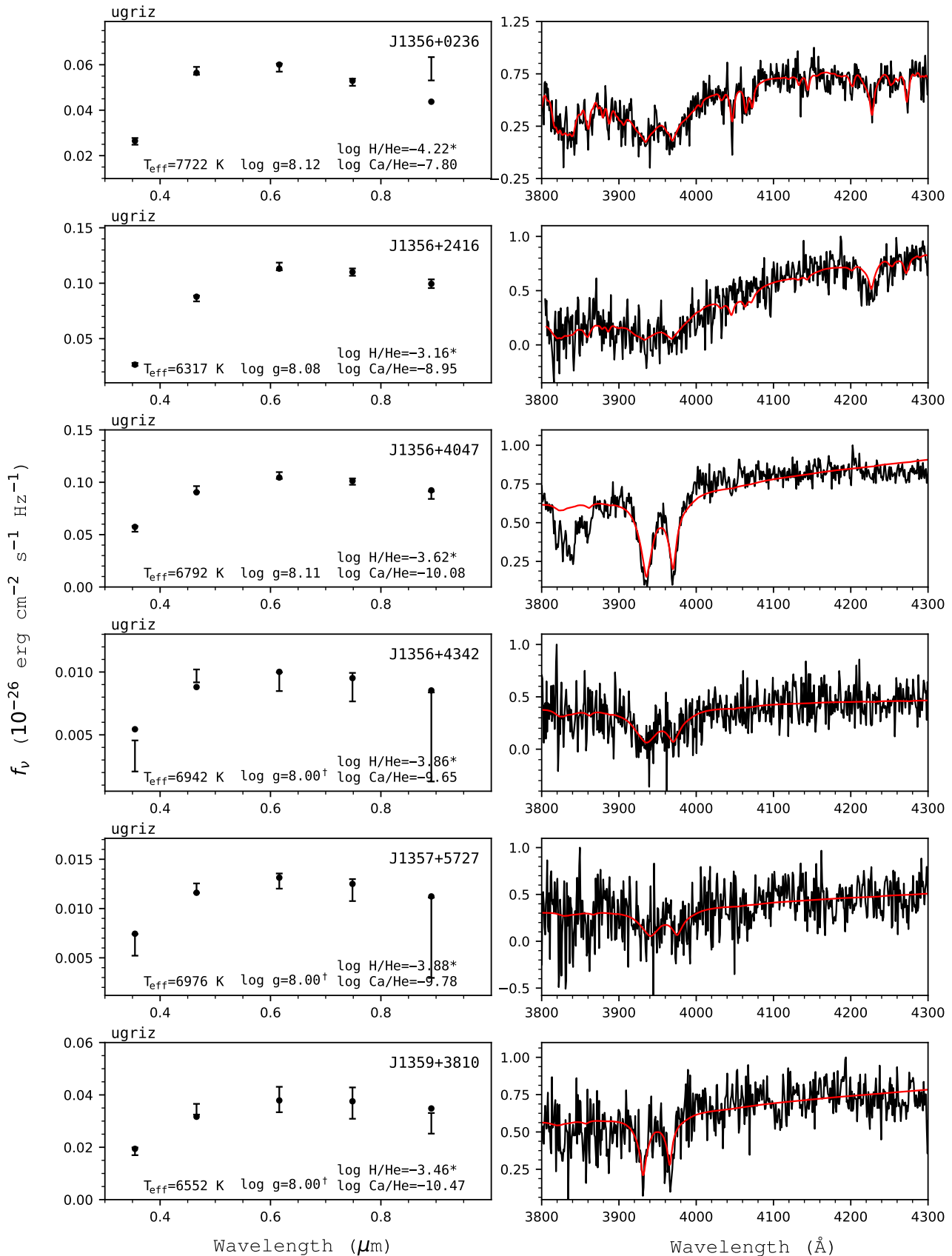

Figure 134. Fits to the DBZ/DZ(A) white dwarfs - continued. 

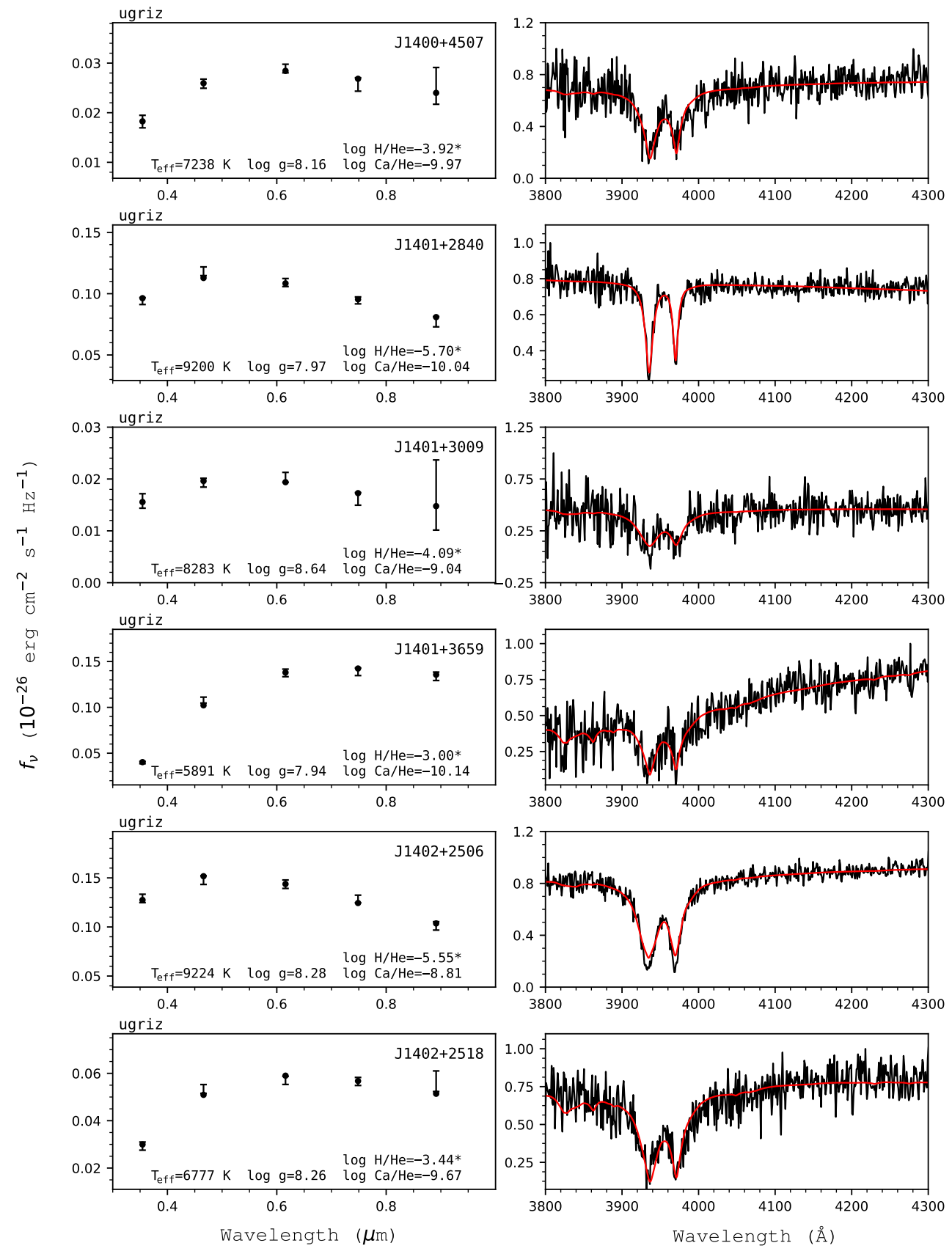

Figure 135. Fits to the DBZ/DZ(A) white dwarfs - continued. 

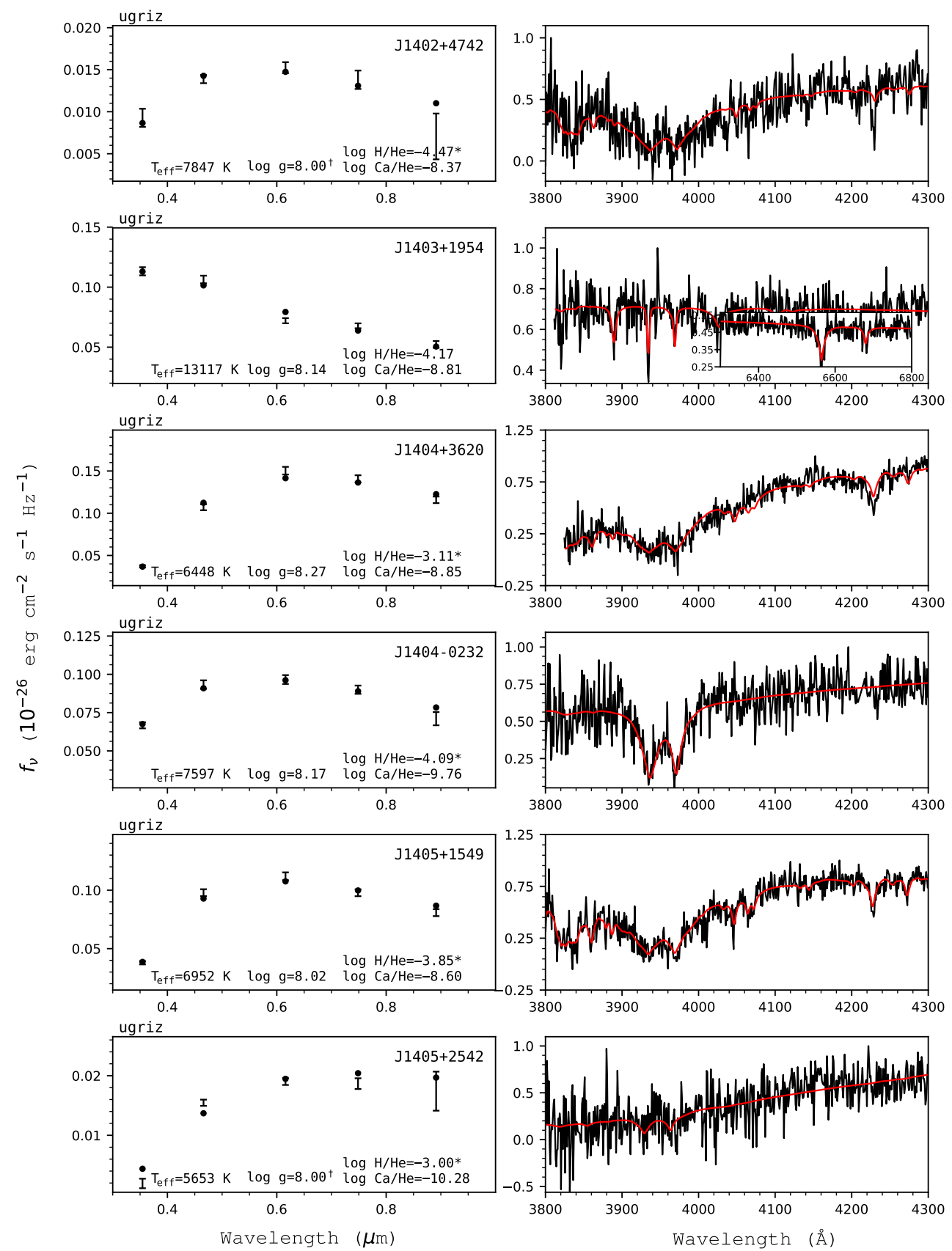

Figure 136. Fits to the DBZ/DZ(A) white dwarfs - continued. 

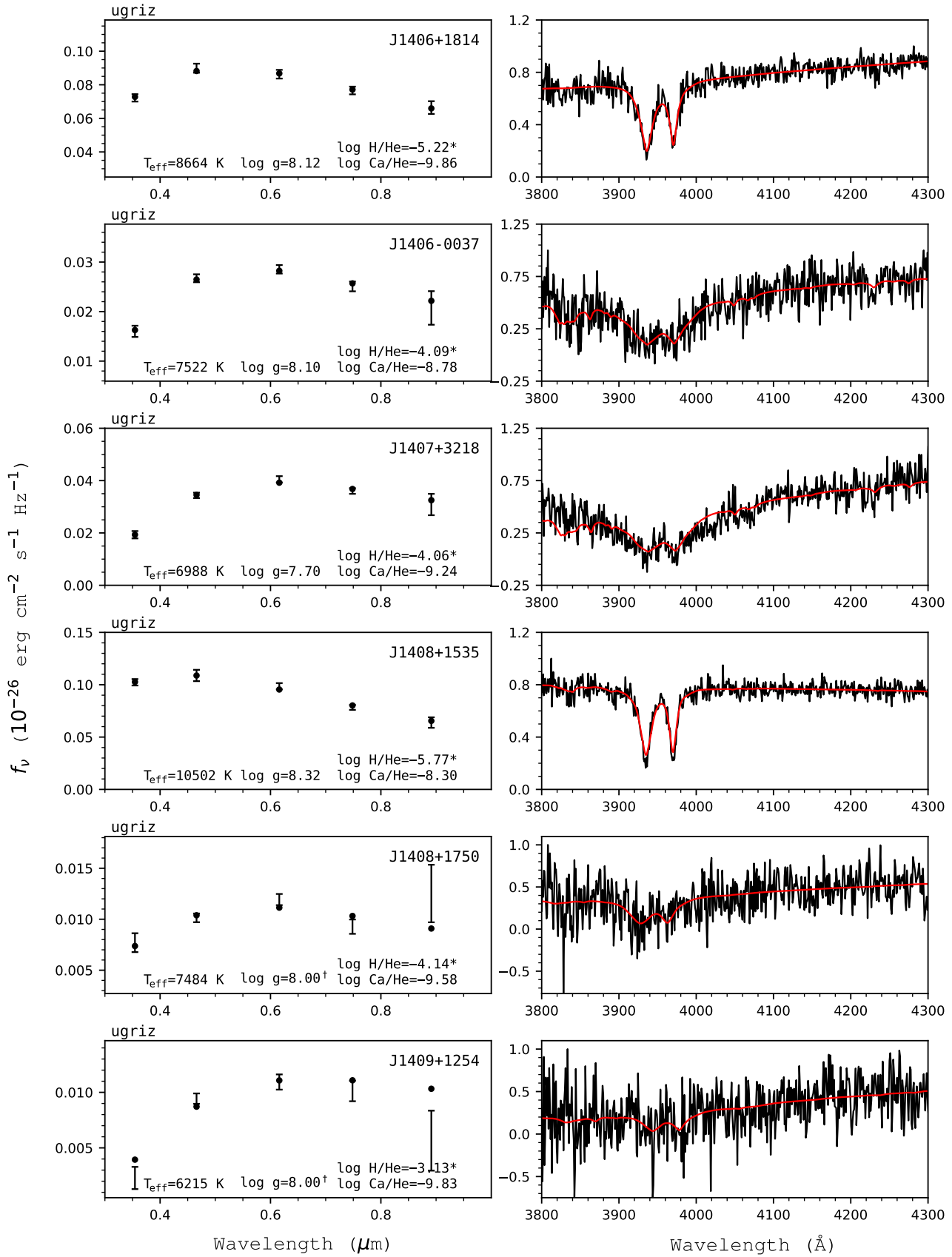

Figure 137. Fits to the DBZ/DZ(A) white dwarfs - continued. 

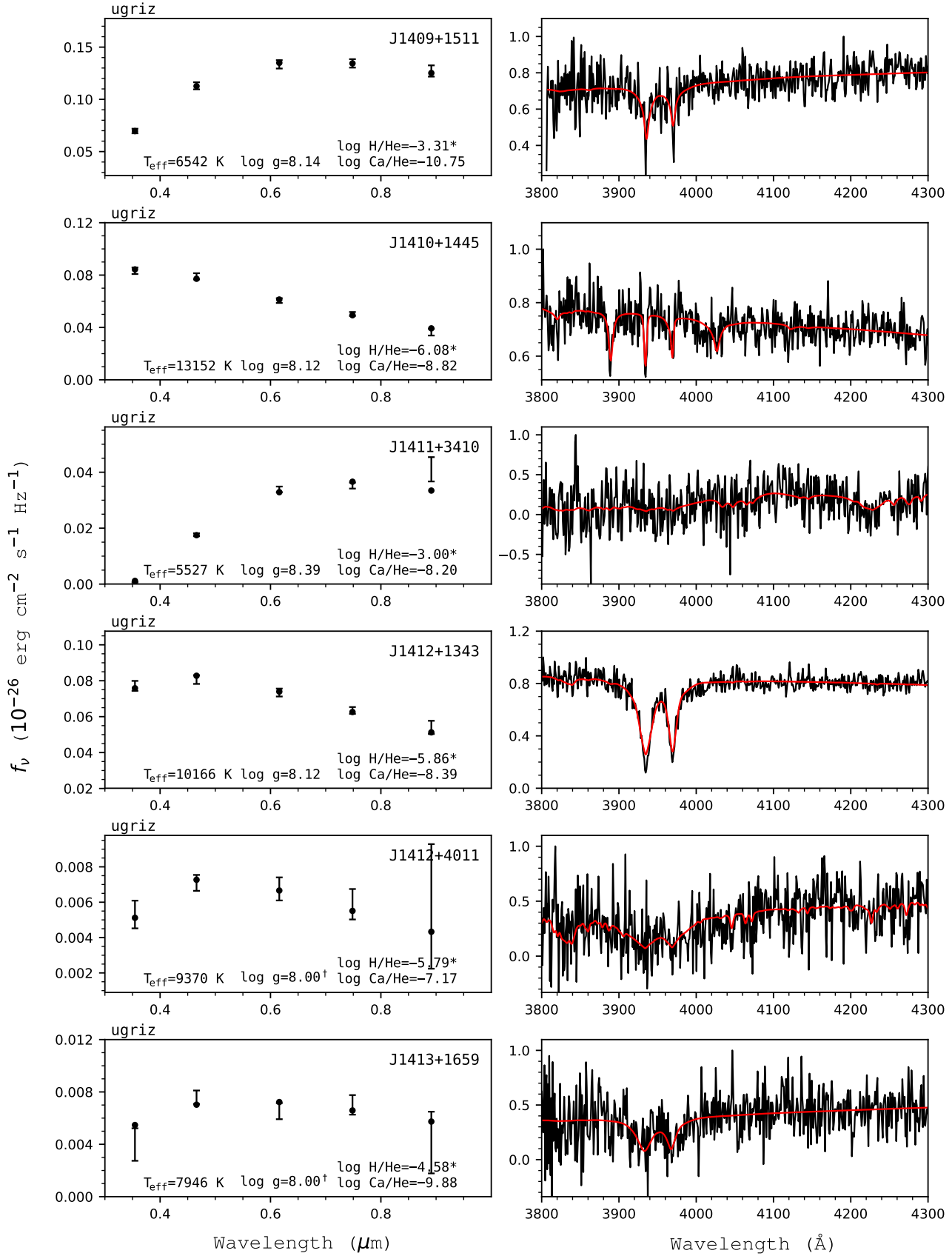

Figure 138. Fits to the DBZ/DZ(A) white dwarfs - continued. 


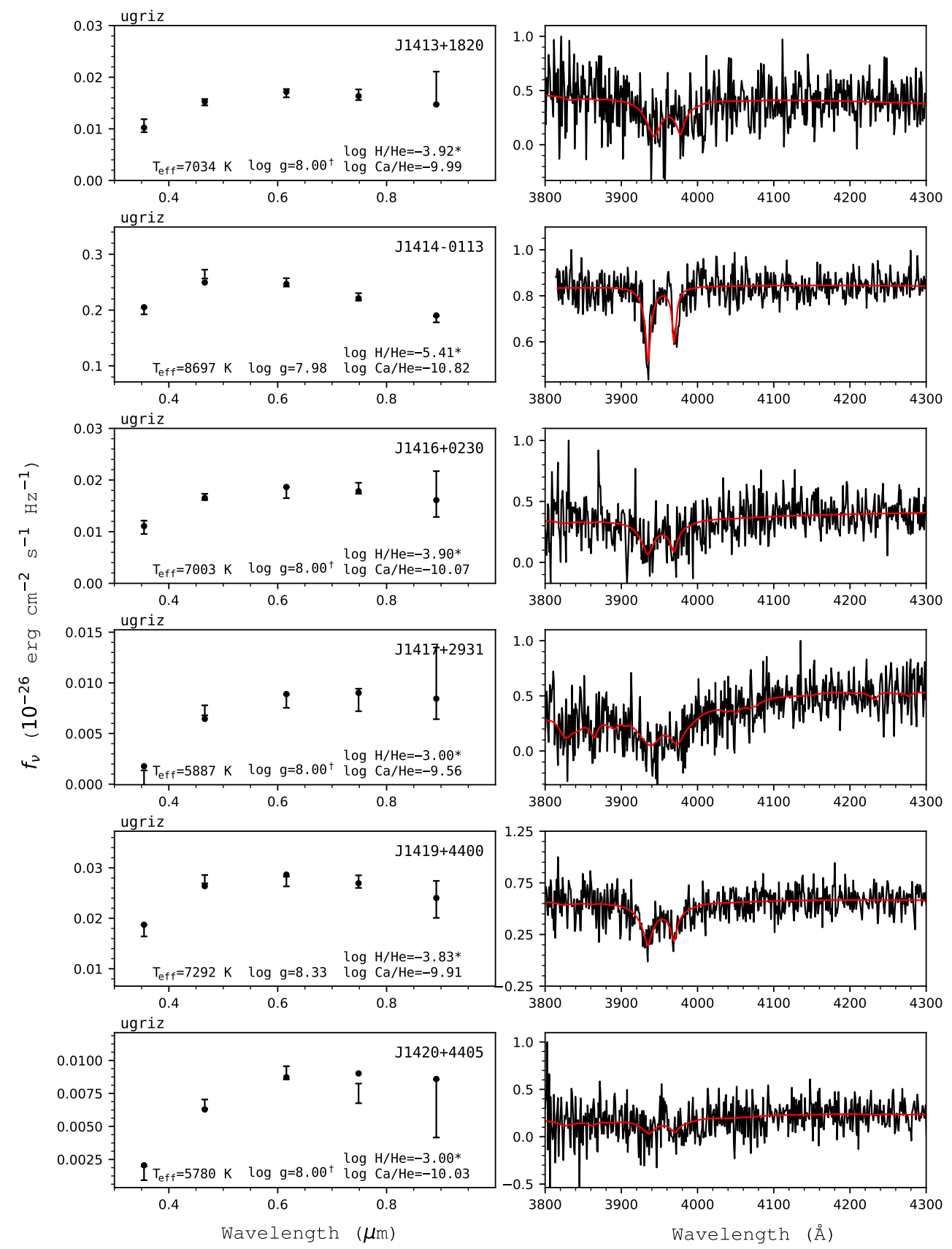

Figure 139. Fits to the DBZ/DZ(A) white dwarfs - continued. 

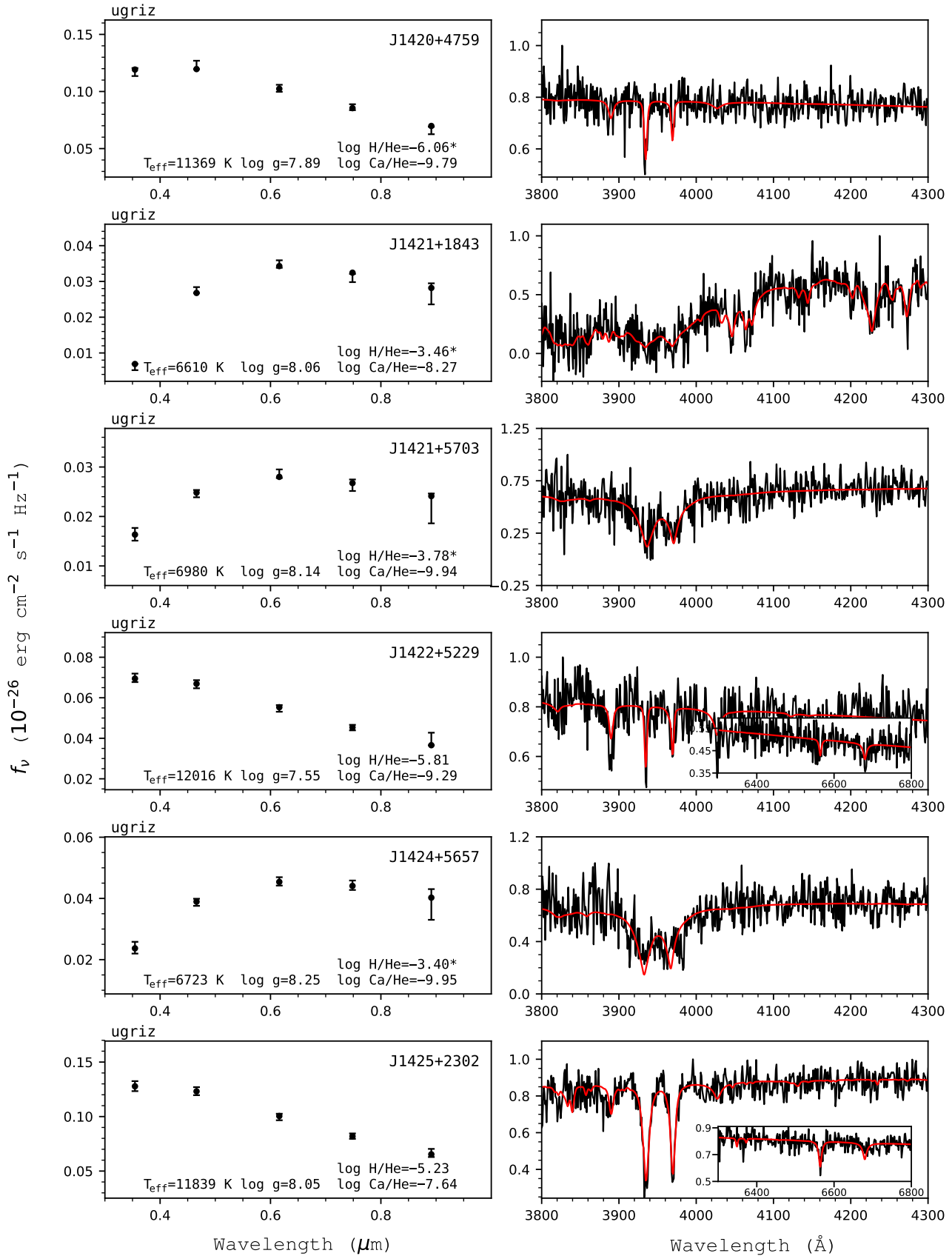

Figure 140. Fits to the DBZ/DZ(A) white dwarfs - continued. 


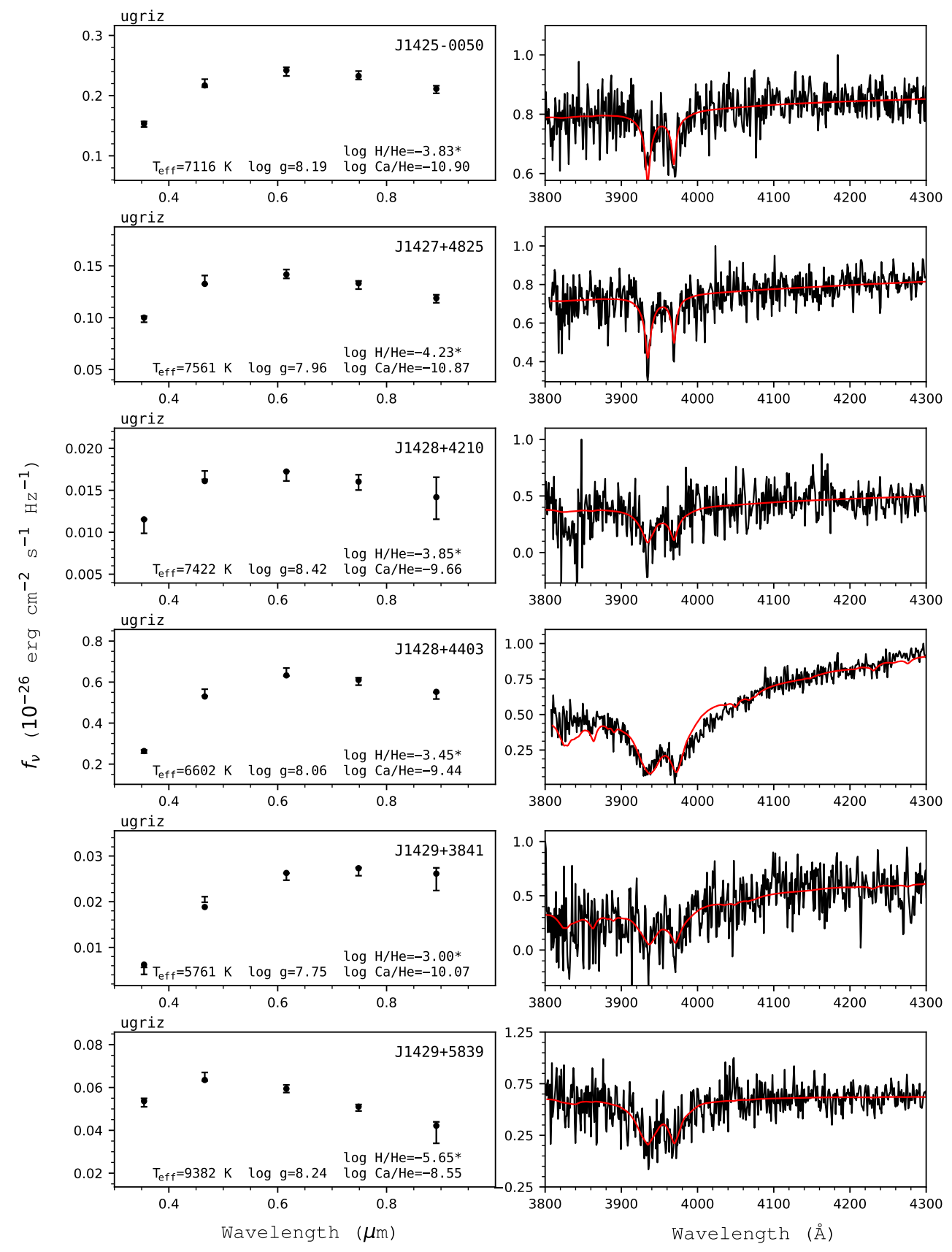

Figure 141. Fits to the DBZ/DZ(A) white dwarfs - continued. 

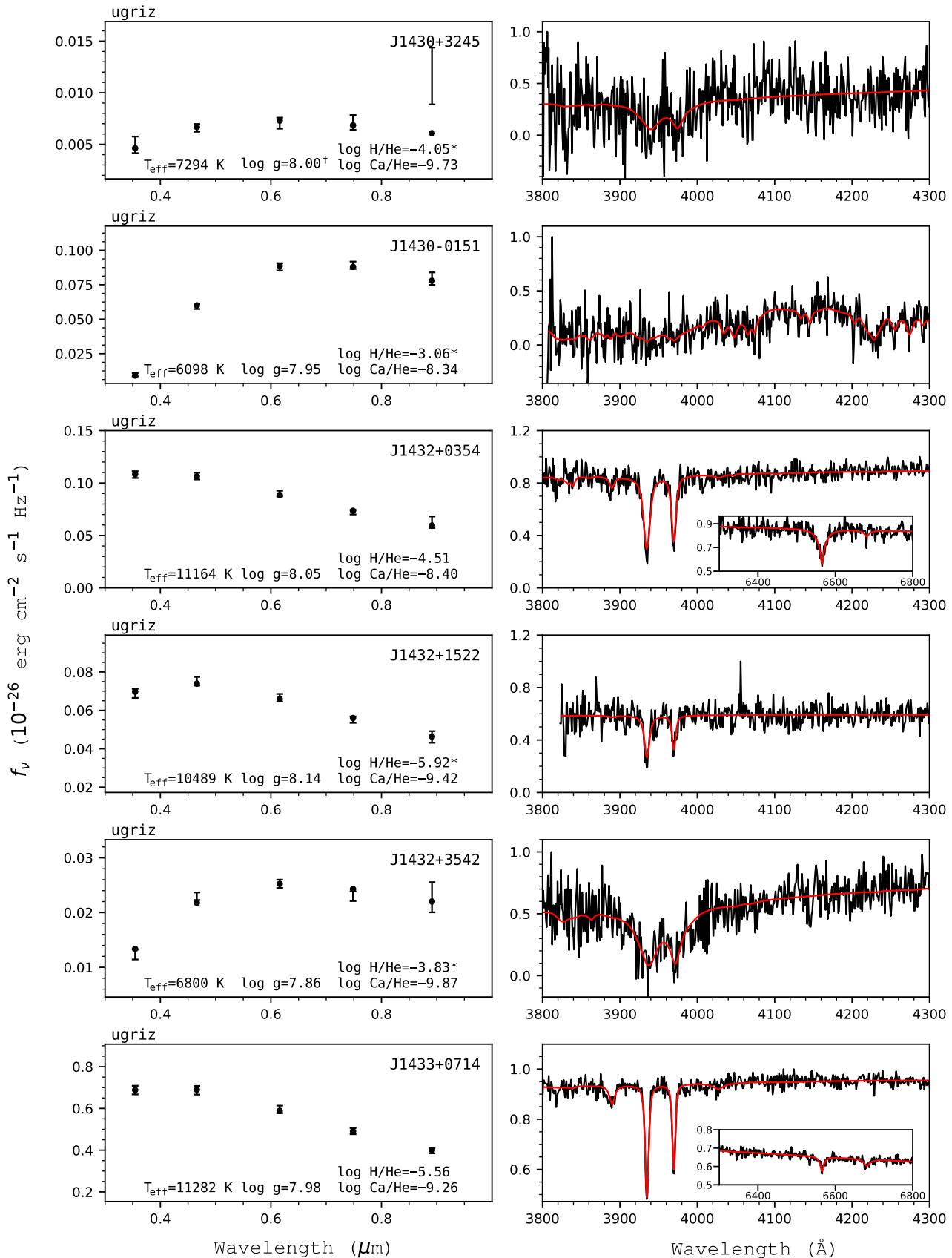

Figure 142. Fits to the DBZ/DZ(A) white dwarfs - continued. 

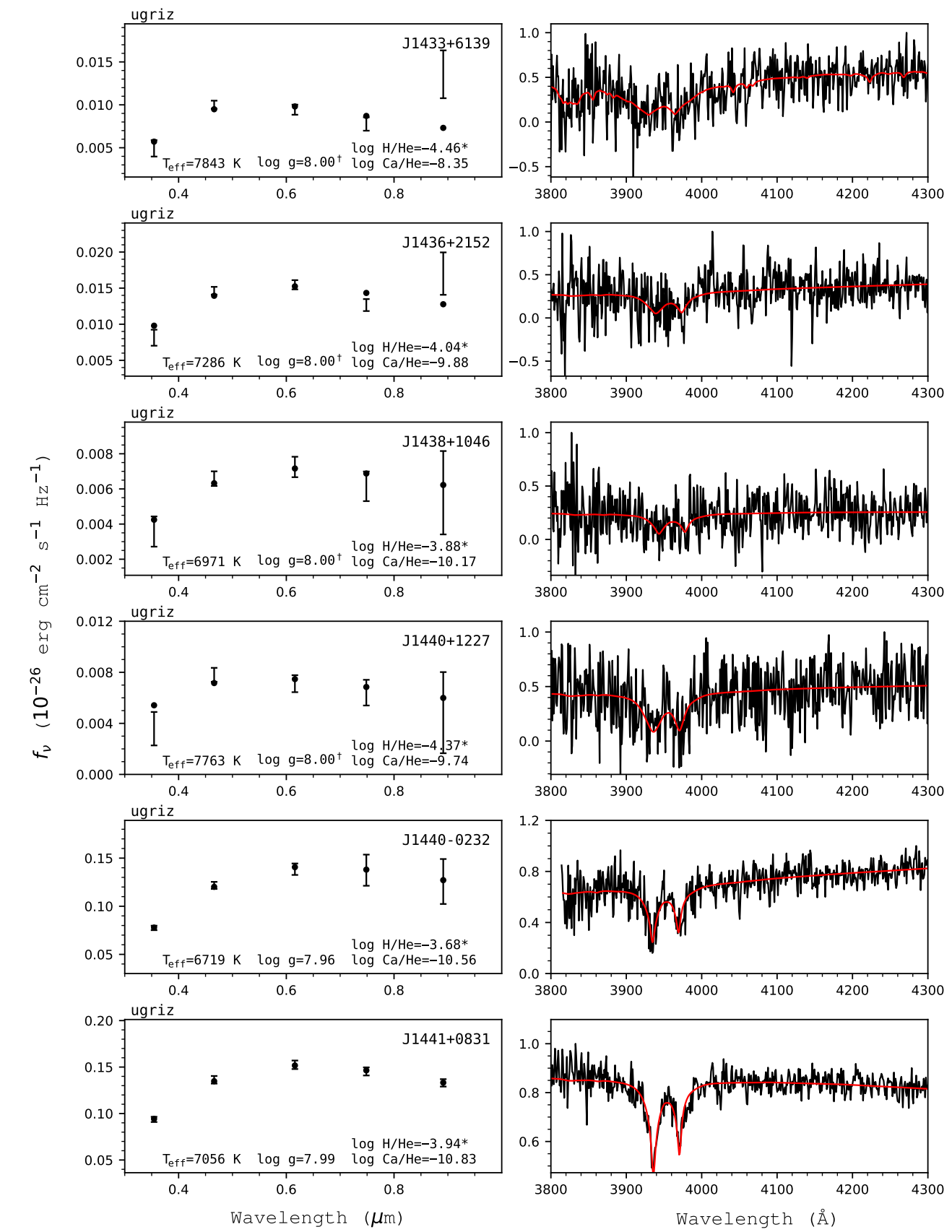

Figure 143. Fits to the DBZ/DZ(A) white dwarfs - continued. 

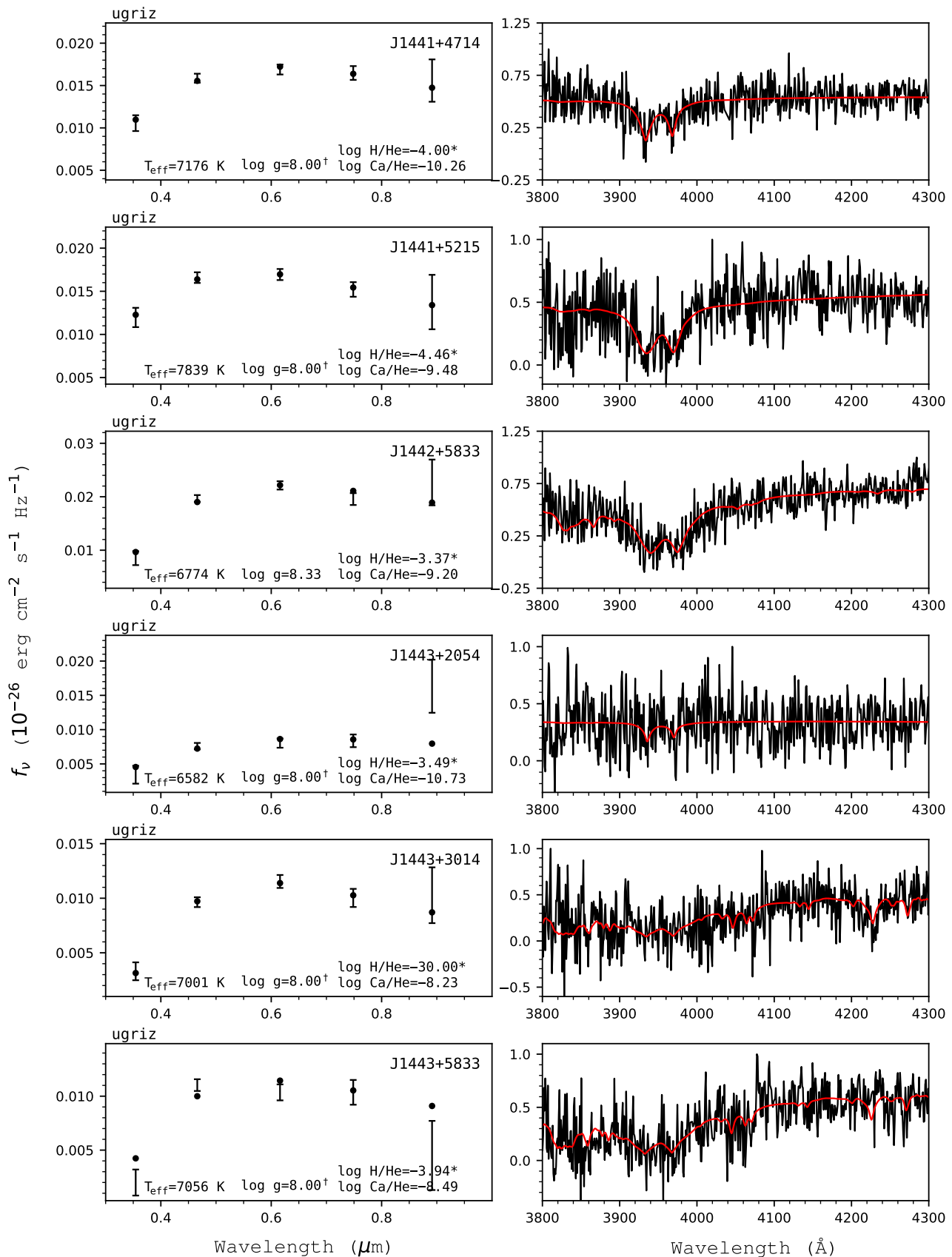

Figure 144. Fits to the DBZ/DZ(A) white dwarfs - continued. 

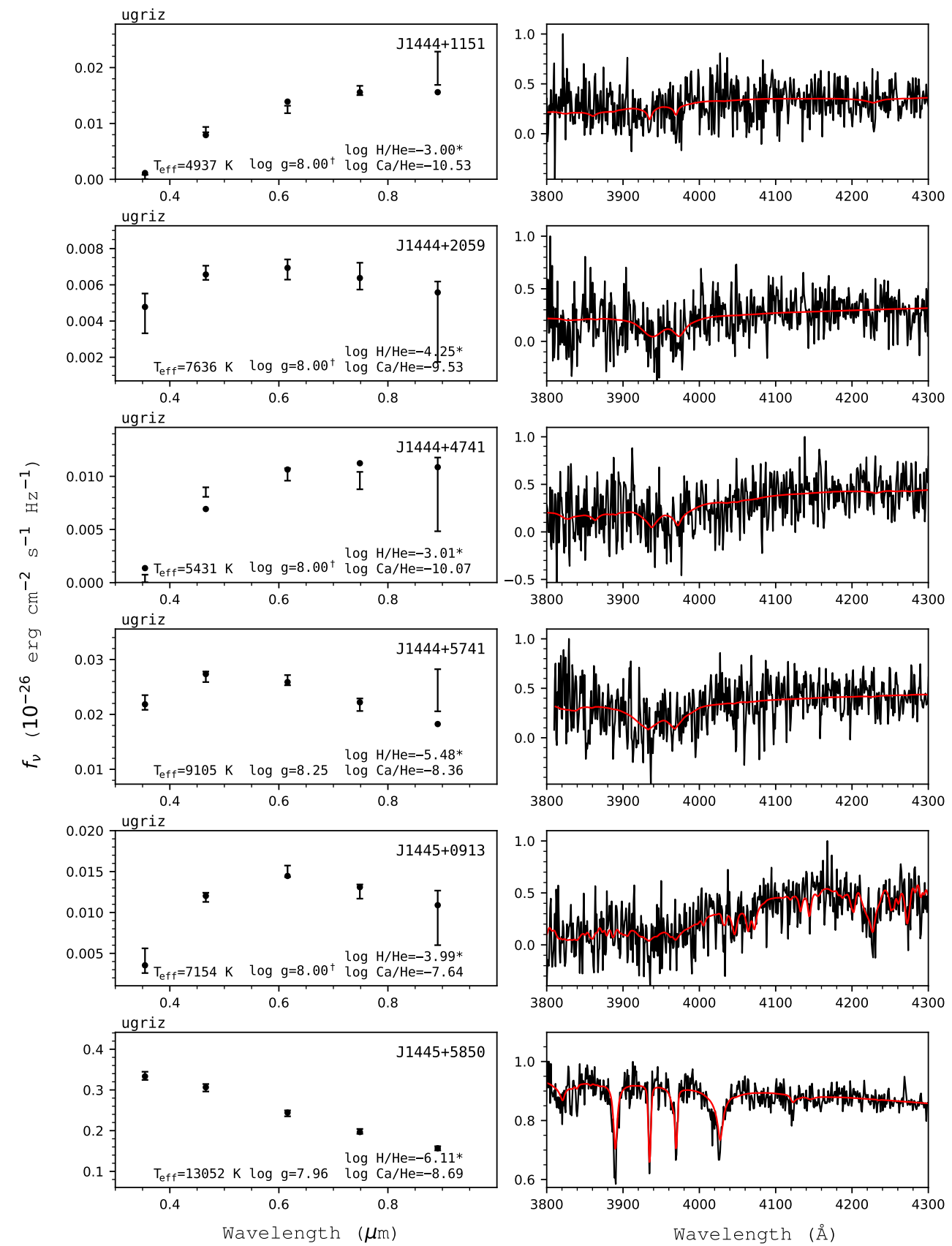

Figure 145. Fits to the DBZ/DZ(A) white dwarfs - continued. 

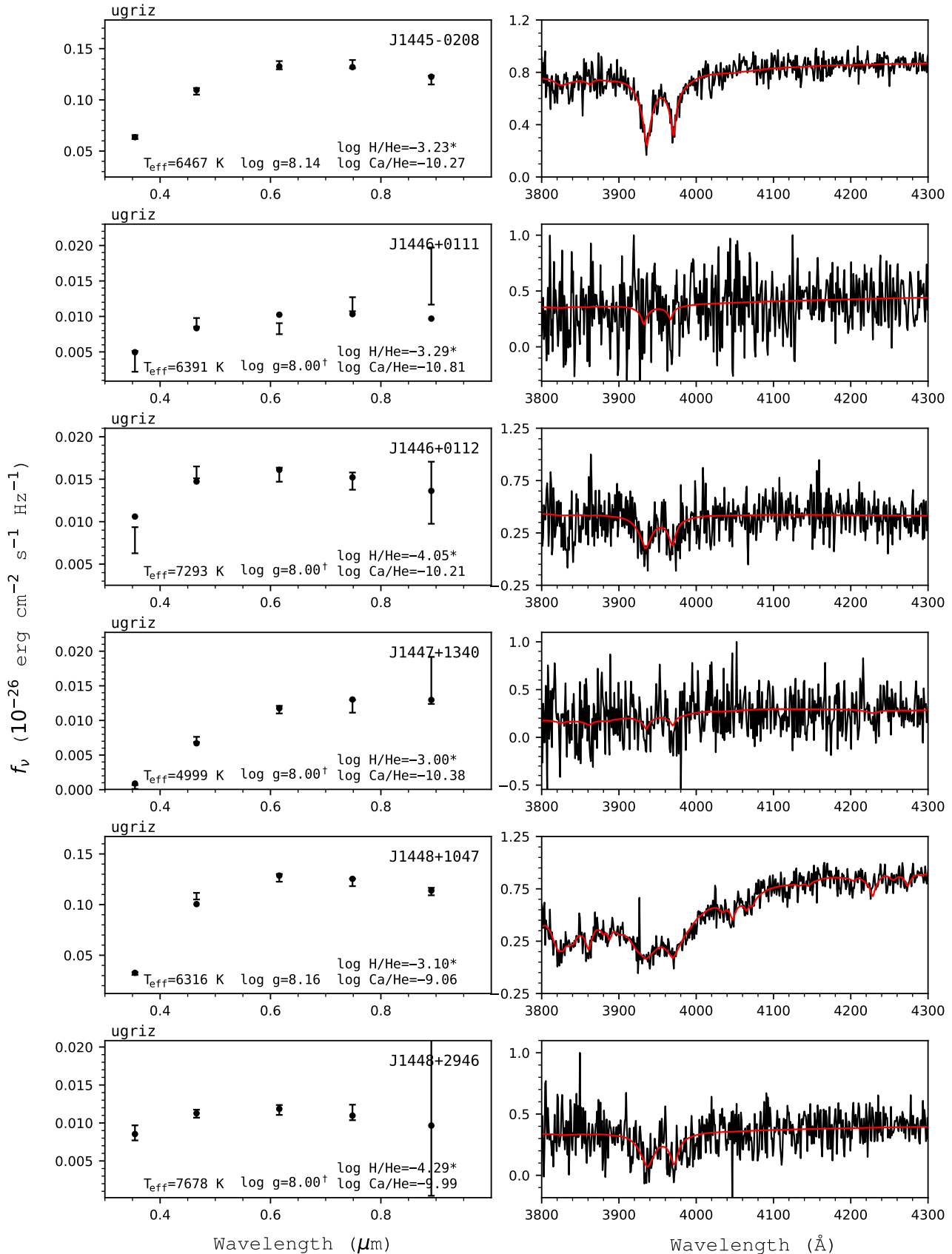

Figure 146. Fits to the DBZ/DZ(A) white dwarfs - continued. 

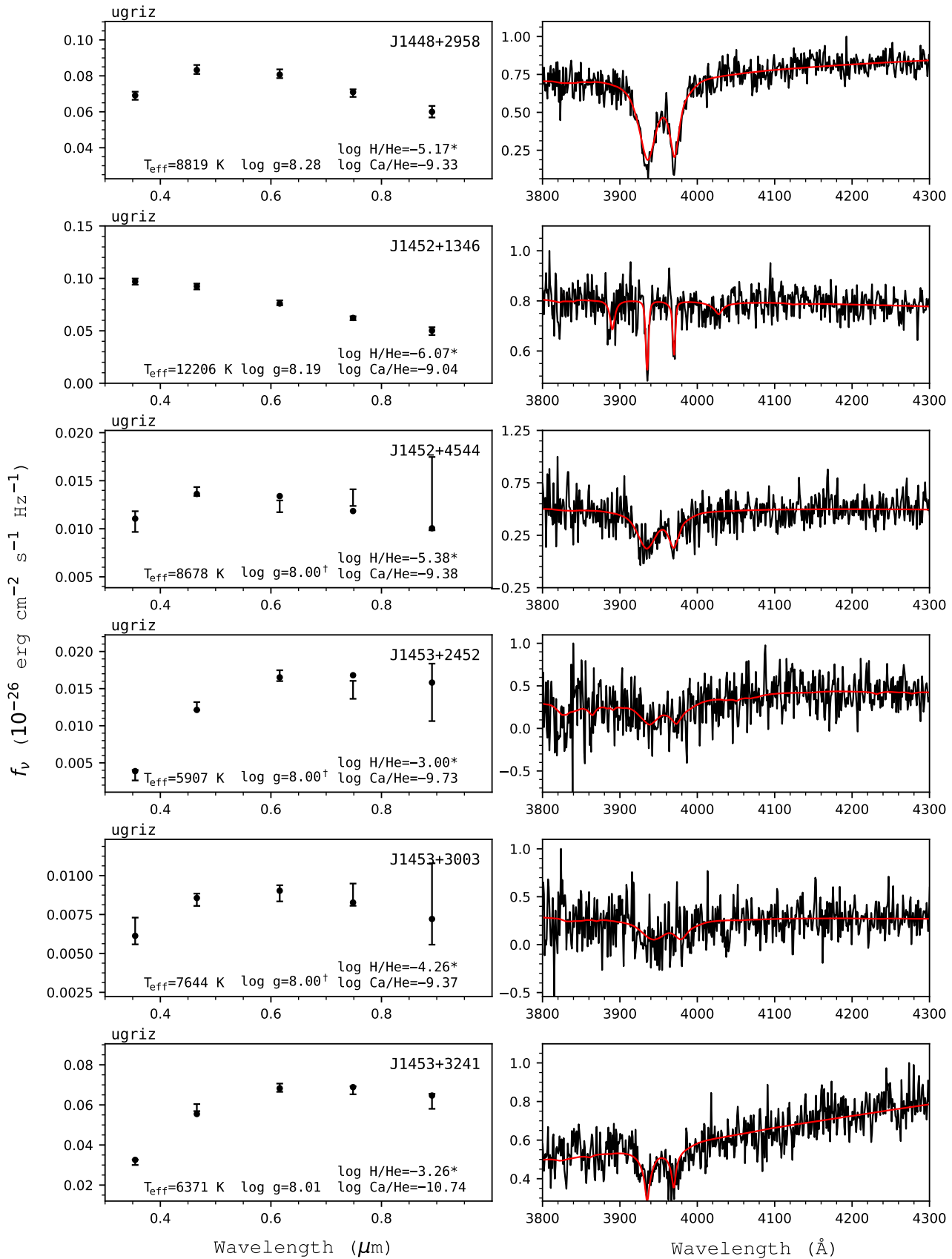

Figure 147. Fits to the DBZ/DZ(A) white dwarfs - continued. 

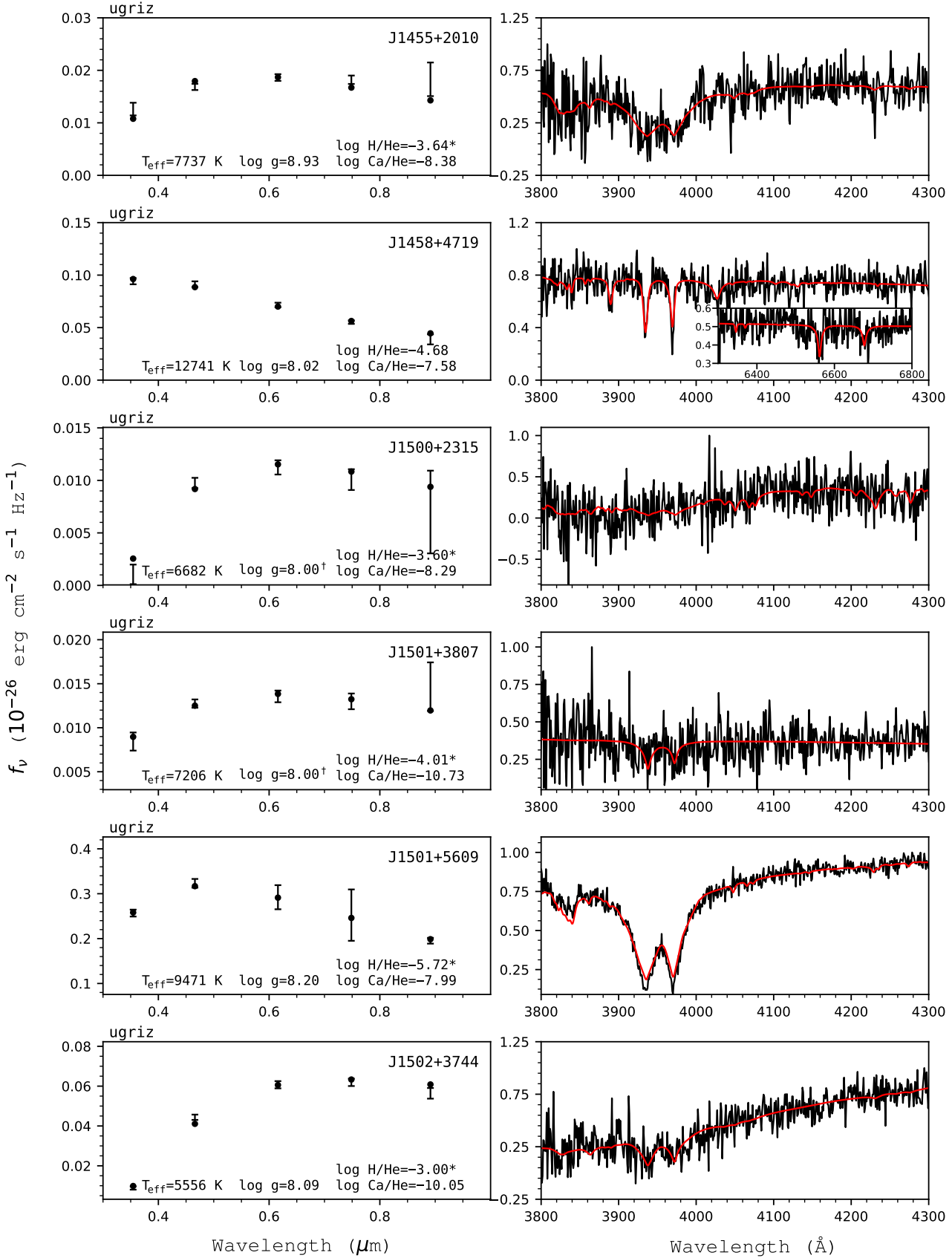

Figure 148. Fits to the DBZ/DZ(A) white dwarfs - continued. 

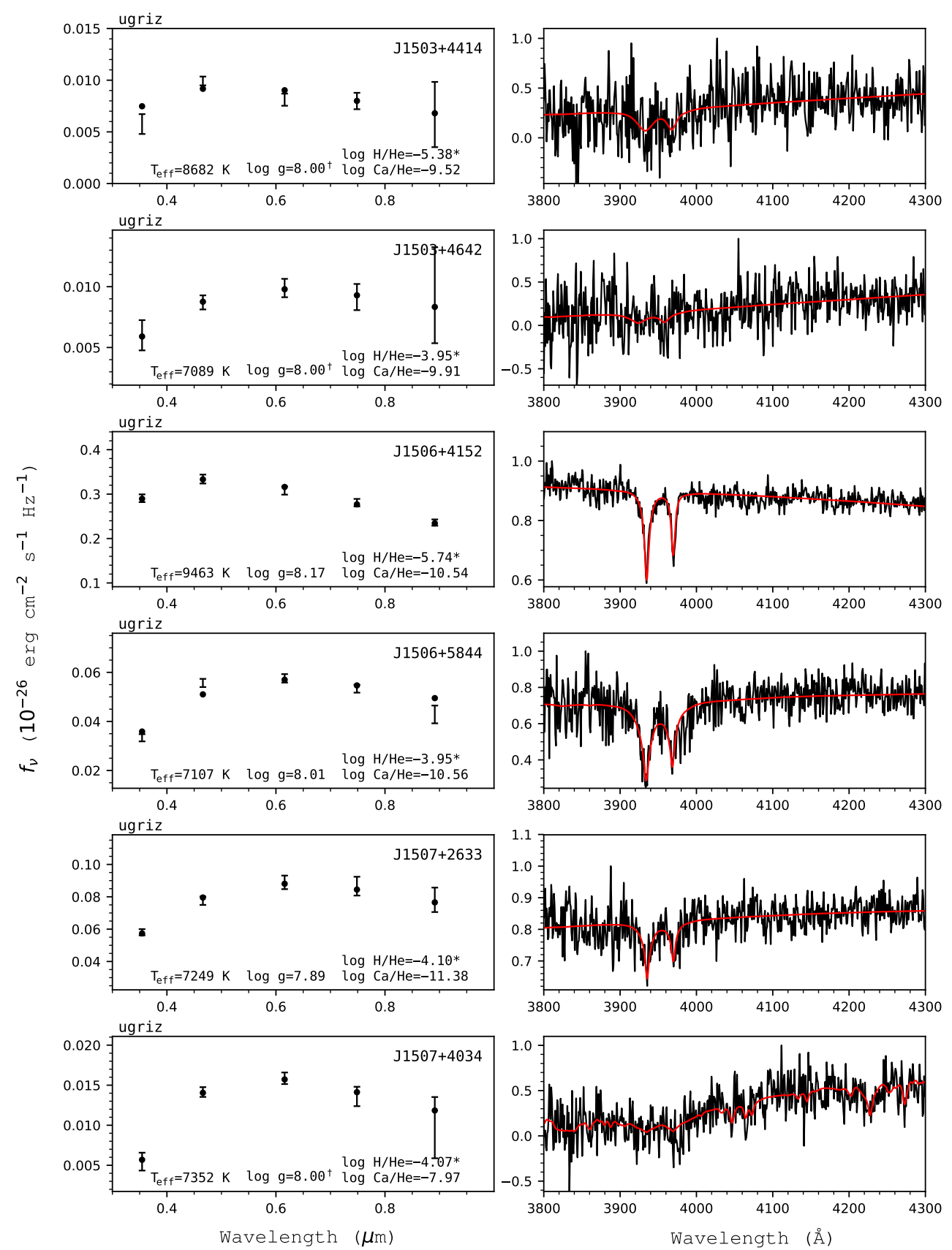

Figure 149. Fits to the DBZ/DZ(A) white dwarfs - continued. 

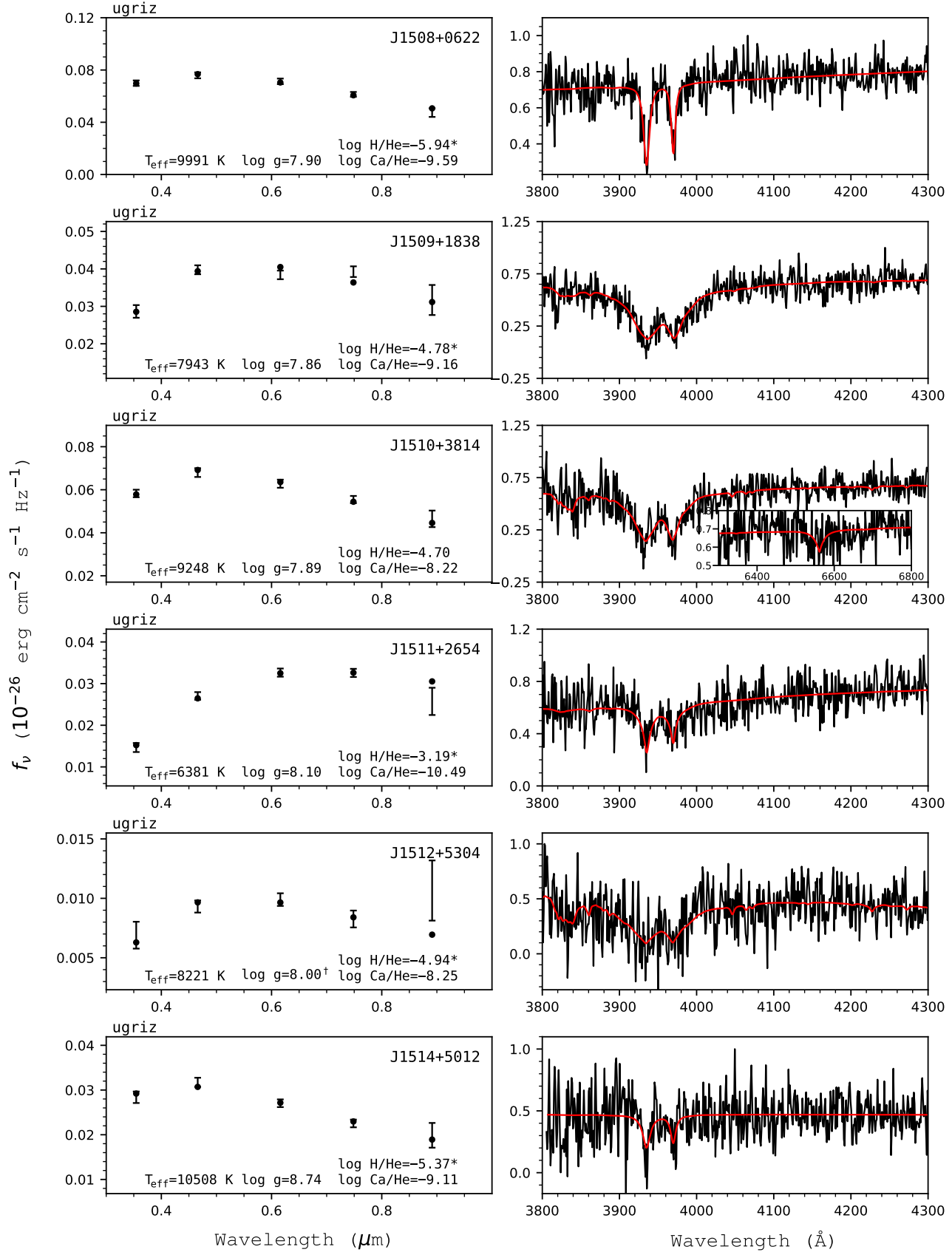

Figure 150. Fits to the DBZ/DZ(A) white dwarfs - continued. 

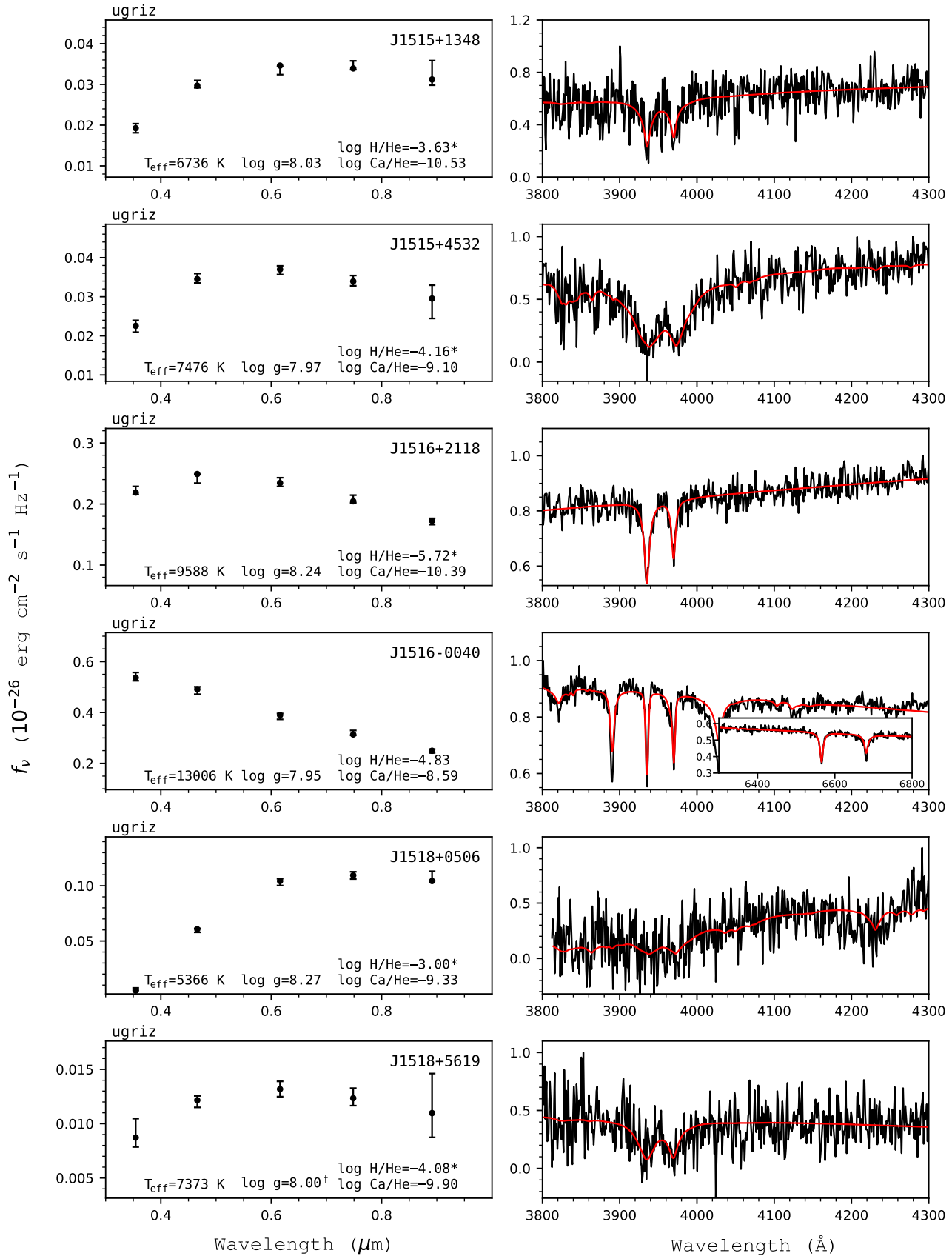

Figure 151. Fits to the DBZ/DZ(A) white dwarfs - continued. 

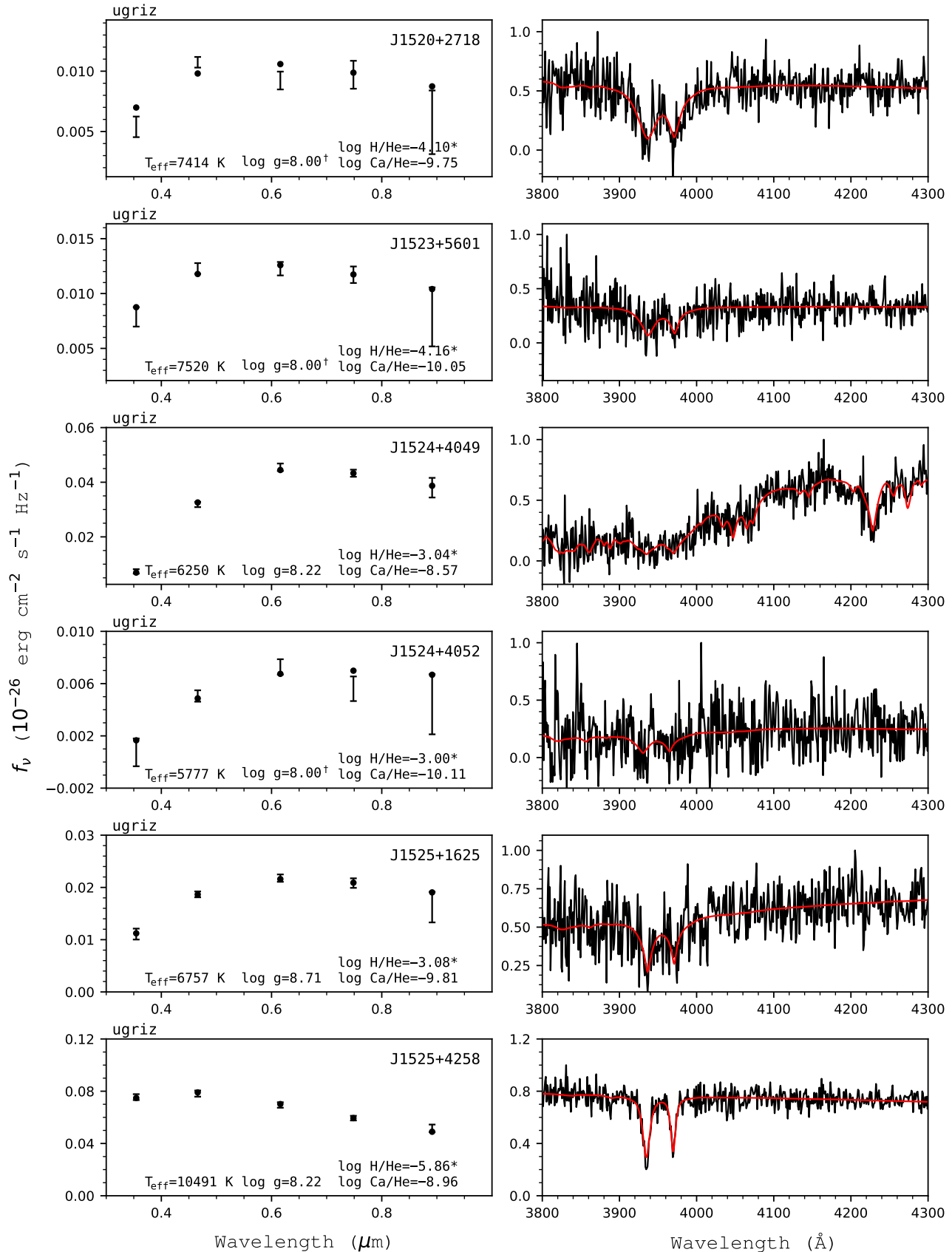

Figure 152. Fits to the DBZ/DZ(A) white dwarfs - continued. 

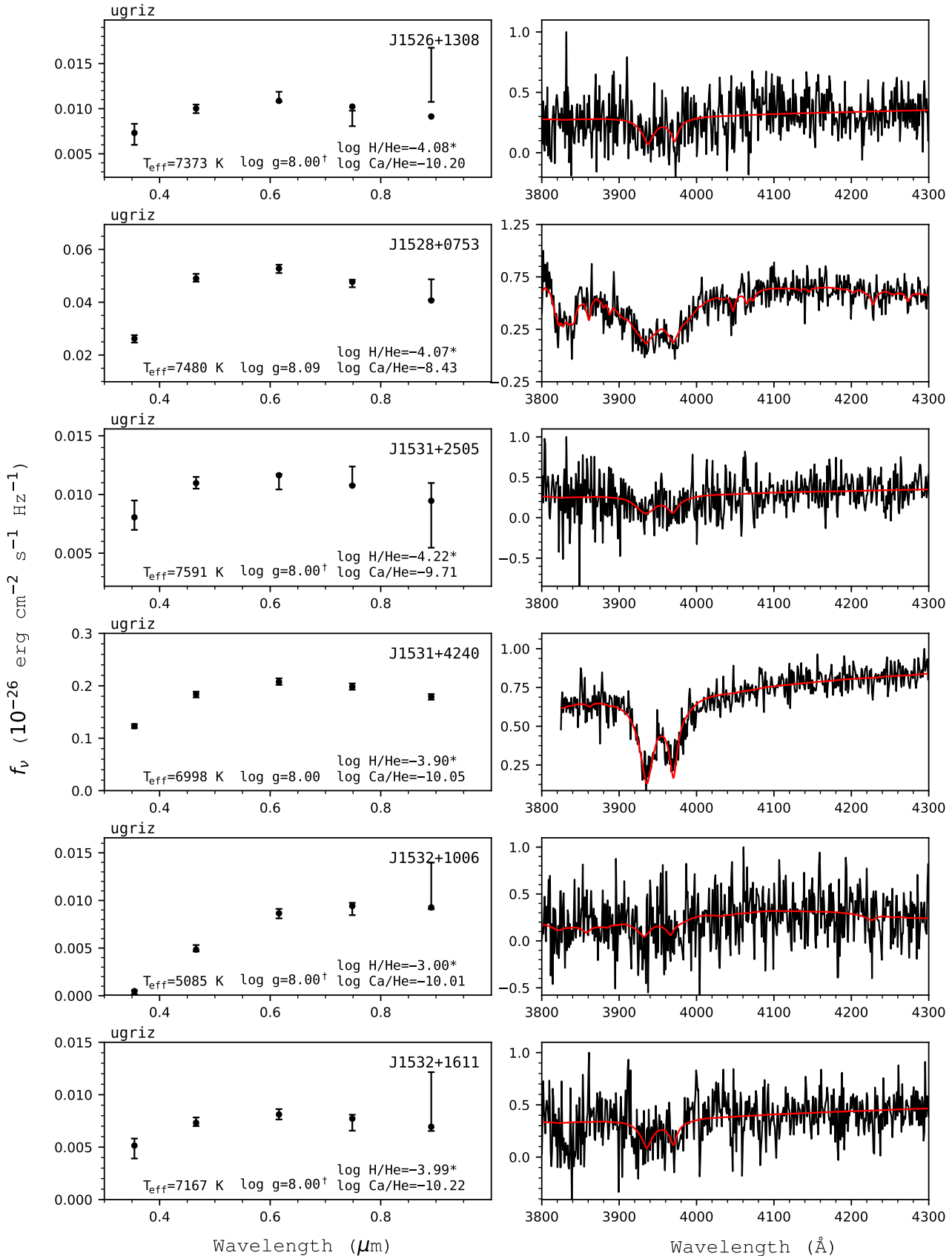

Figure 153. Fits to the DBZ/DZ(A) white dwarfs - continued. 

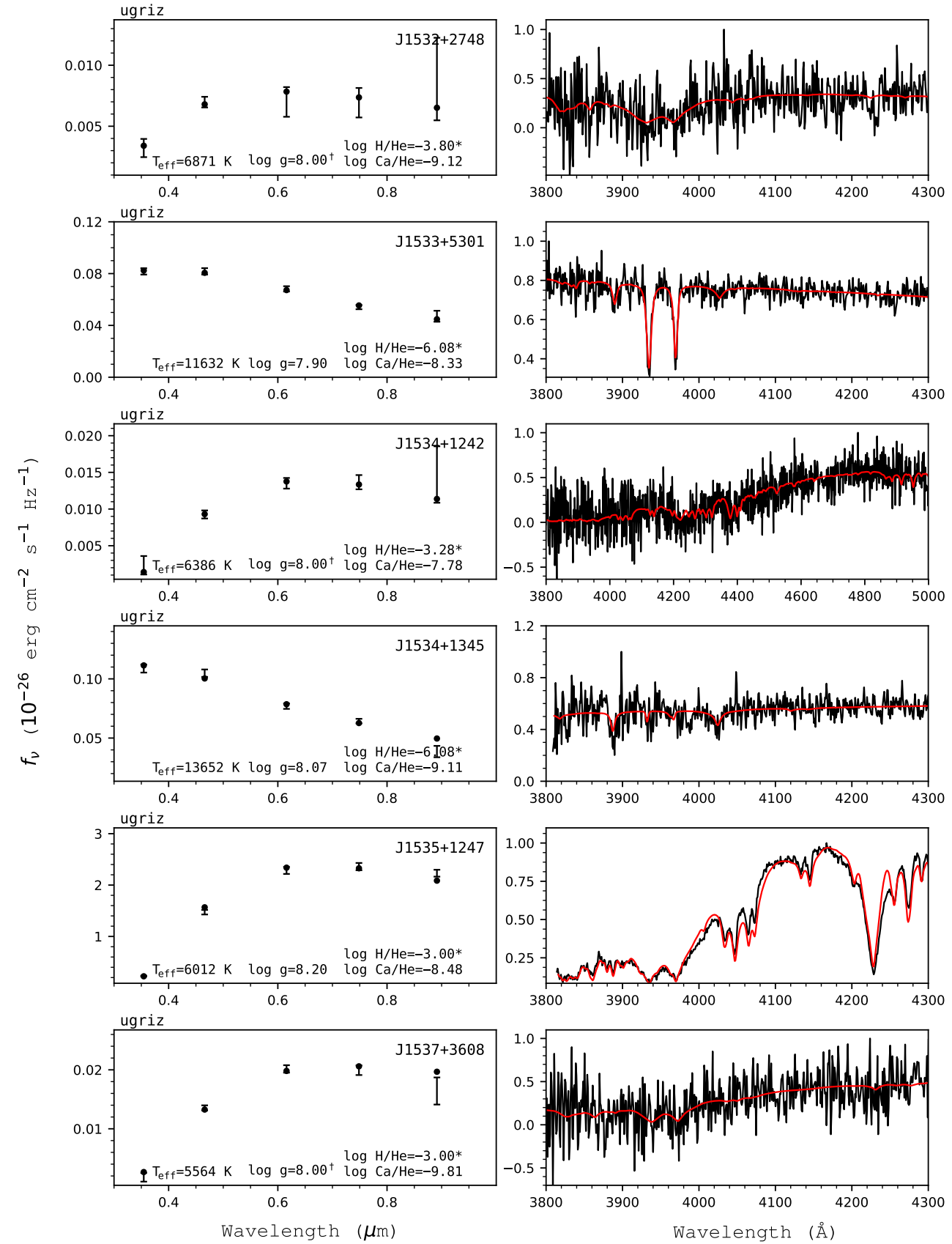

Figure 154. Fits to the DBZ/DZ(A) white dwarfs - continued. 

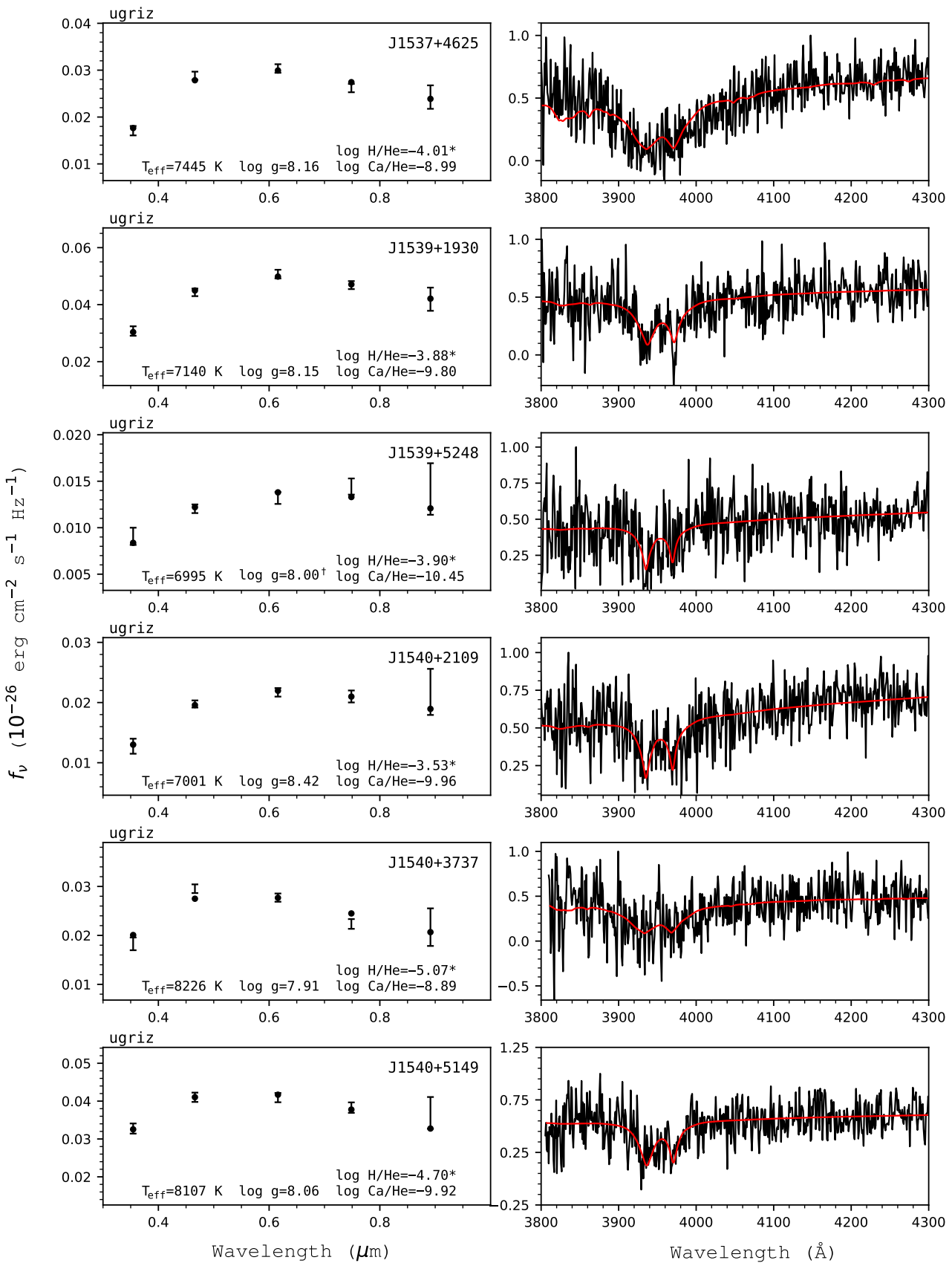

Figure 155. Fits to the DBZ/DZ(A) white dwarfs - continued. 

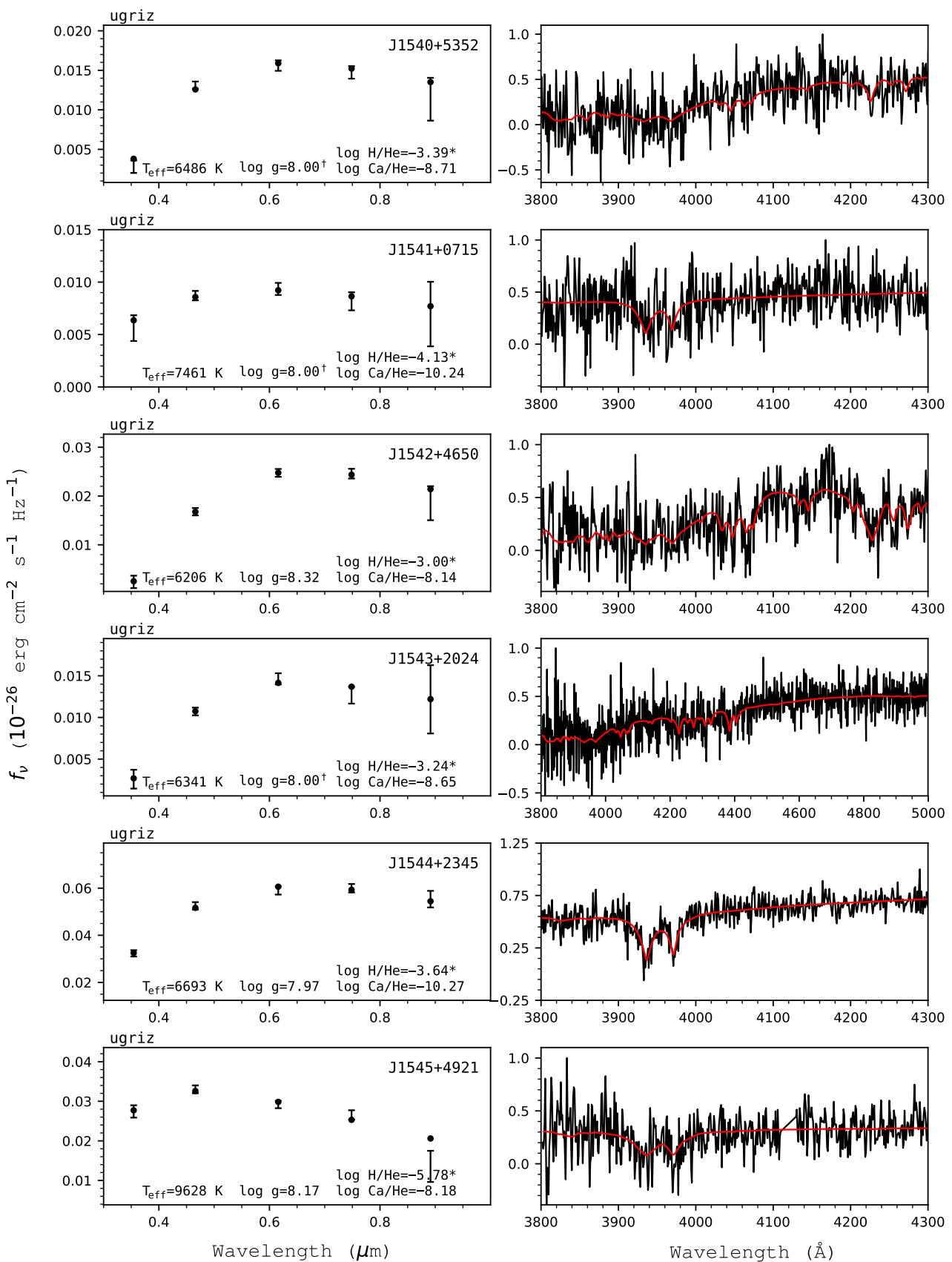

Figure 156. Fits to the DBZ/DZ(A) white dwarfs - continued. 

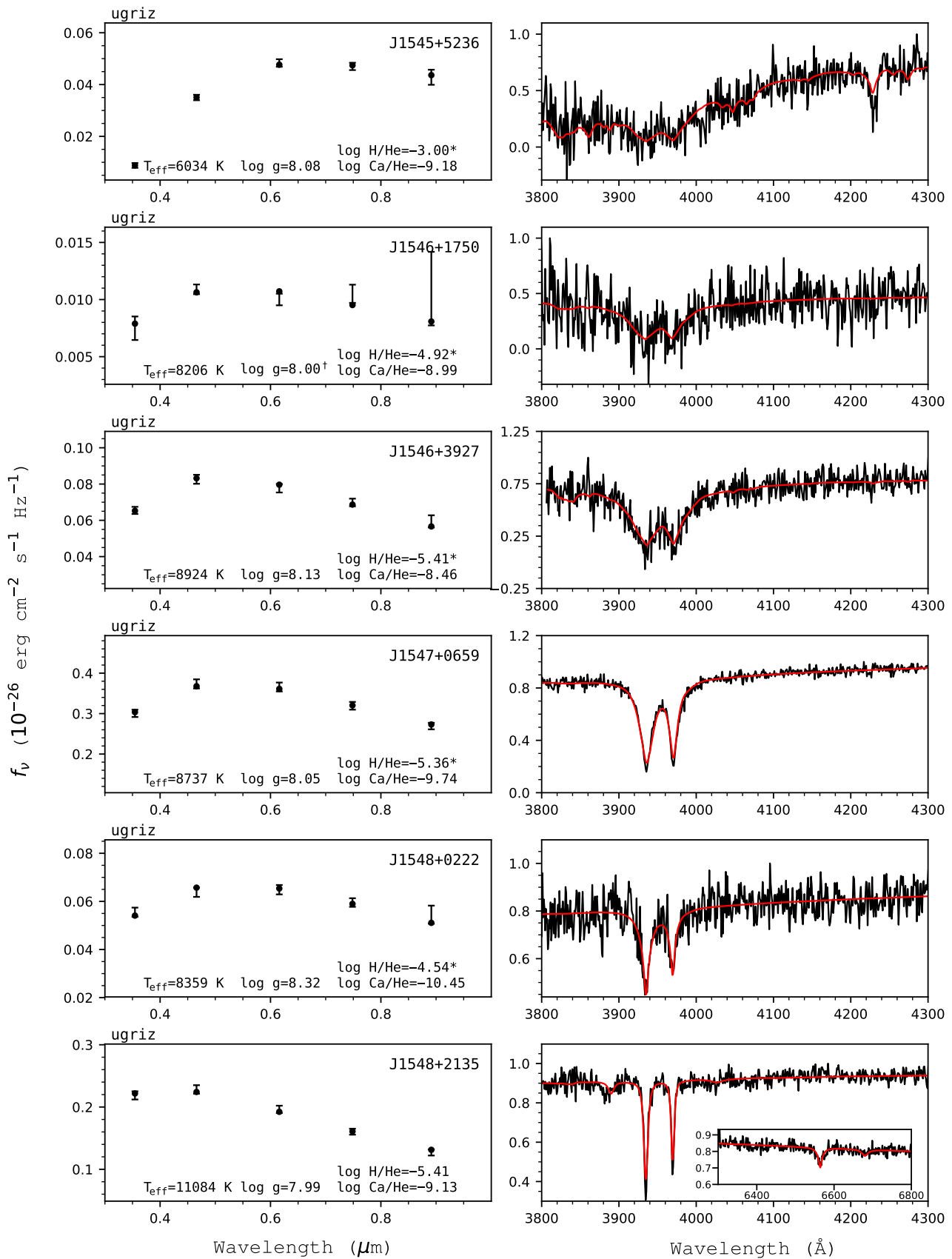

Figure 157. Fits to the DBZ/DZ(A) white dwarfs - continued. 

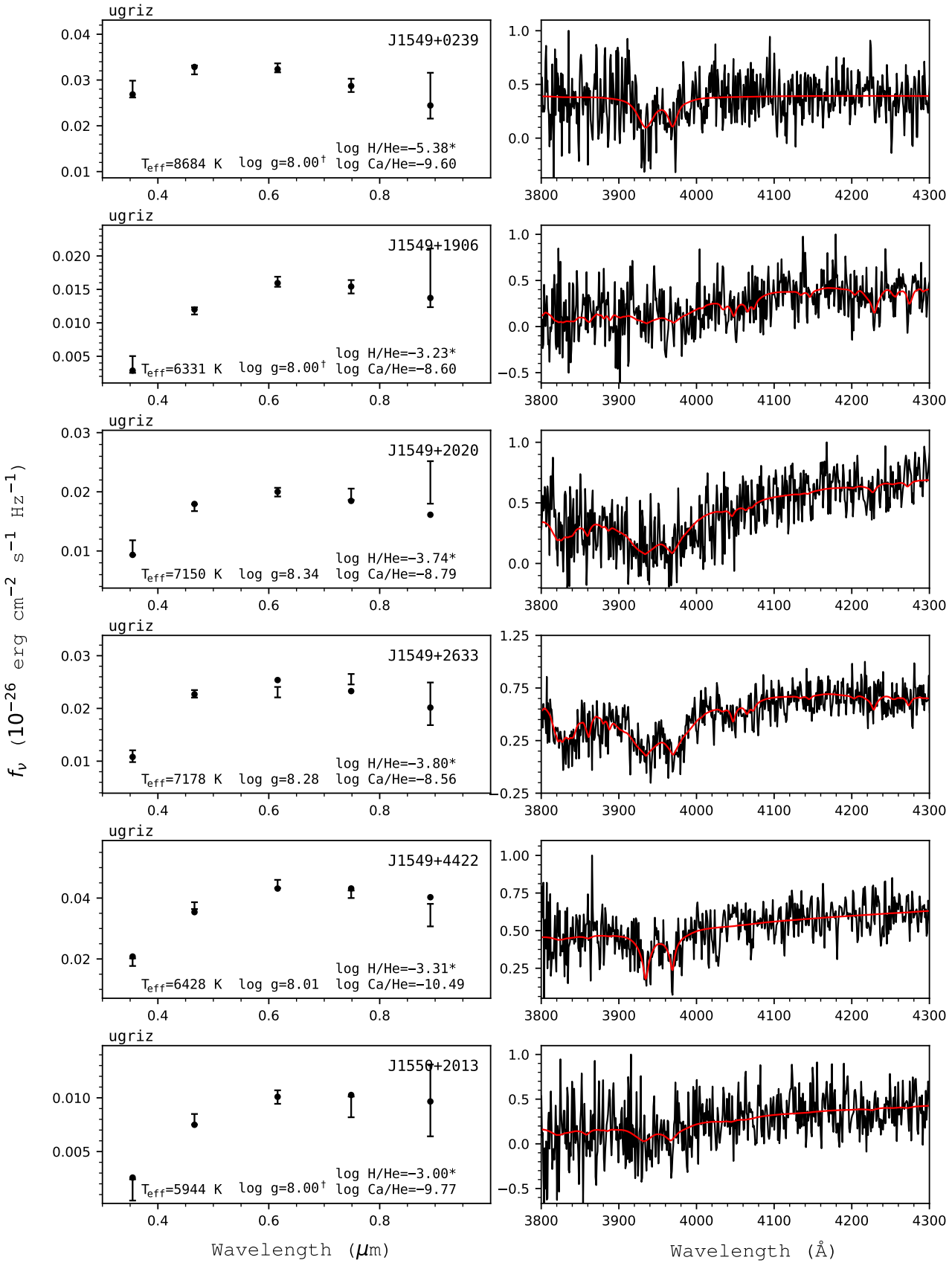

Figure 158. Fits to the DBZ/DZ(A) white dwarfs - continued. 

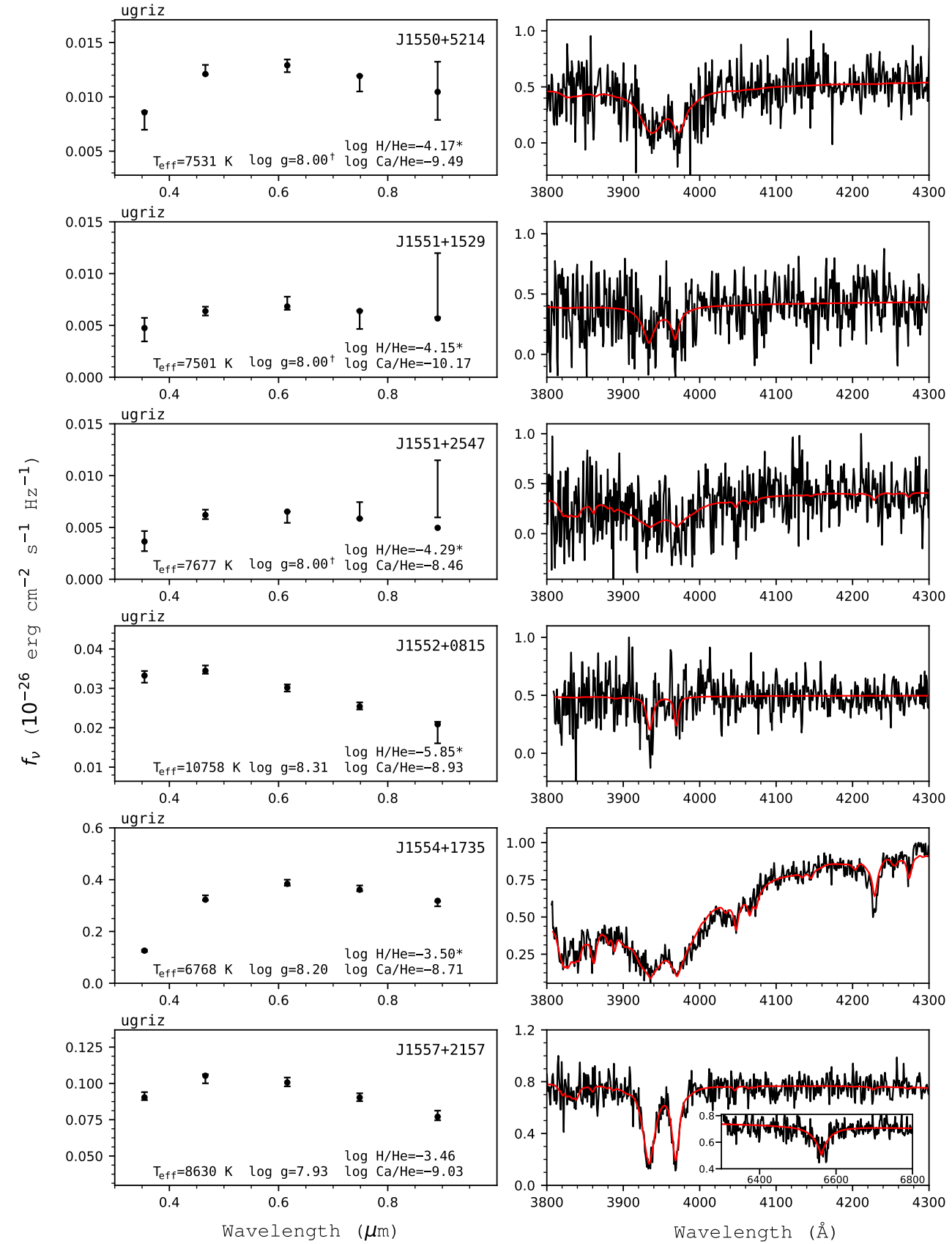

Figure 159. Fits to the DBZ/DZ(A) white dwarfs - continued. 

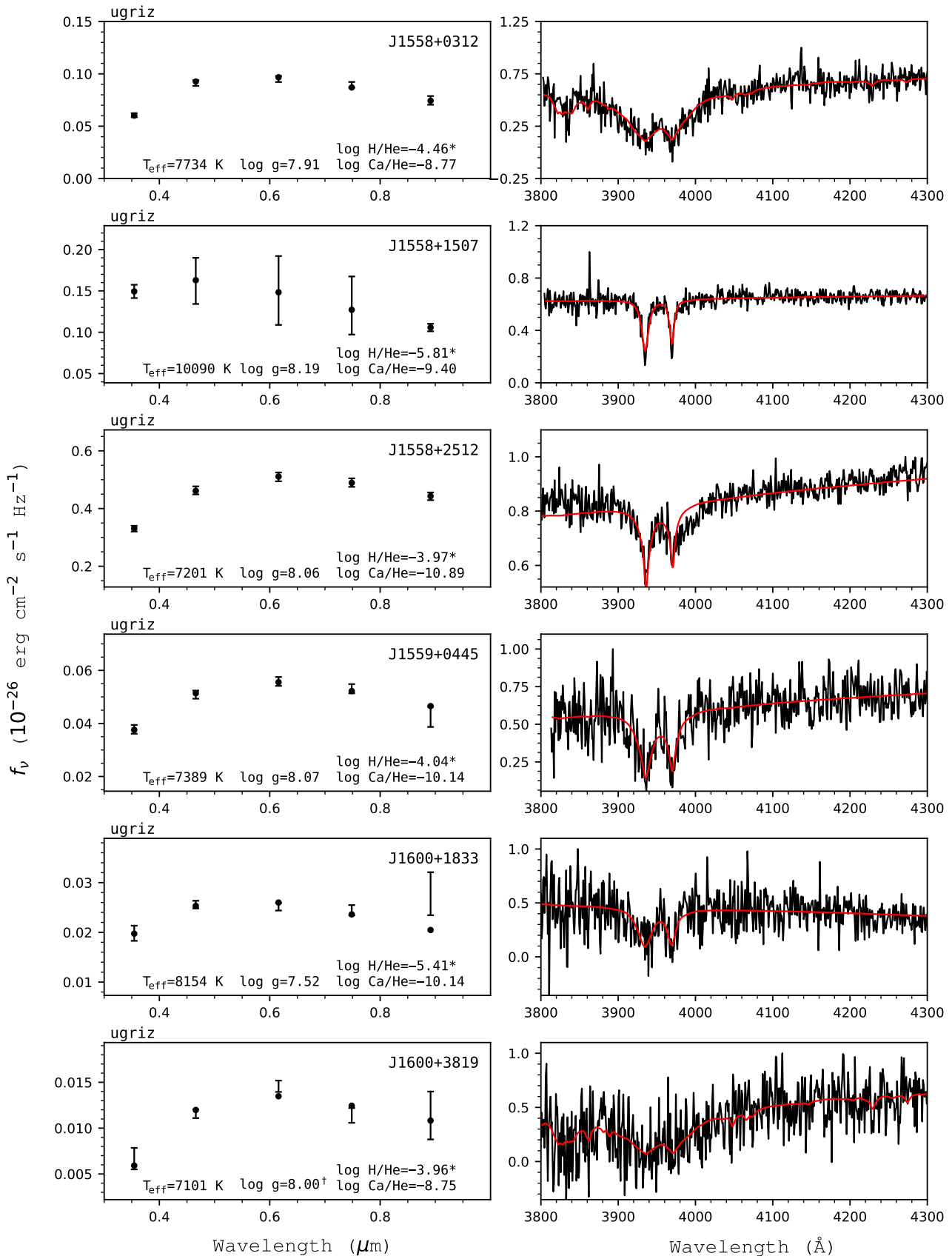

Figure 160. Fits to the DBZ/DZ(A) white dwarfs - continued. 

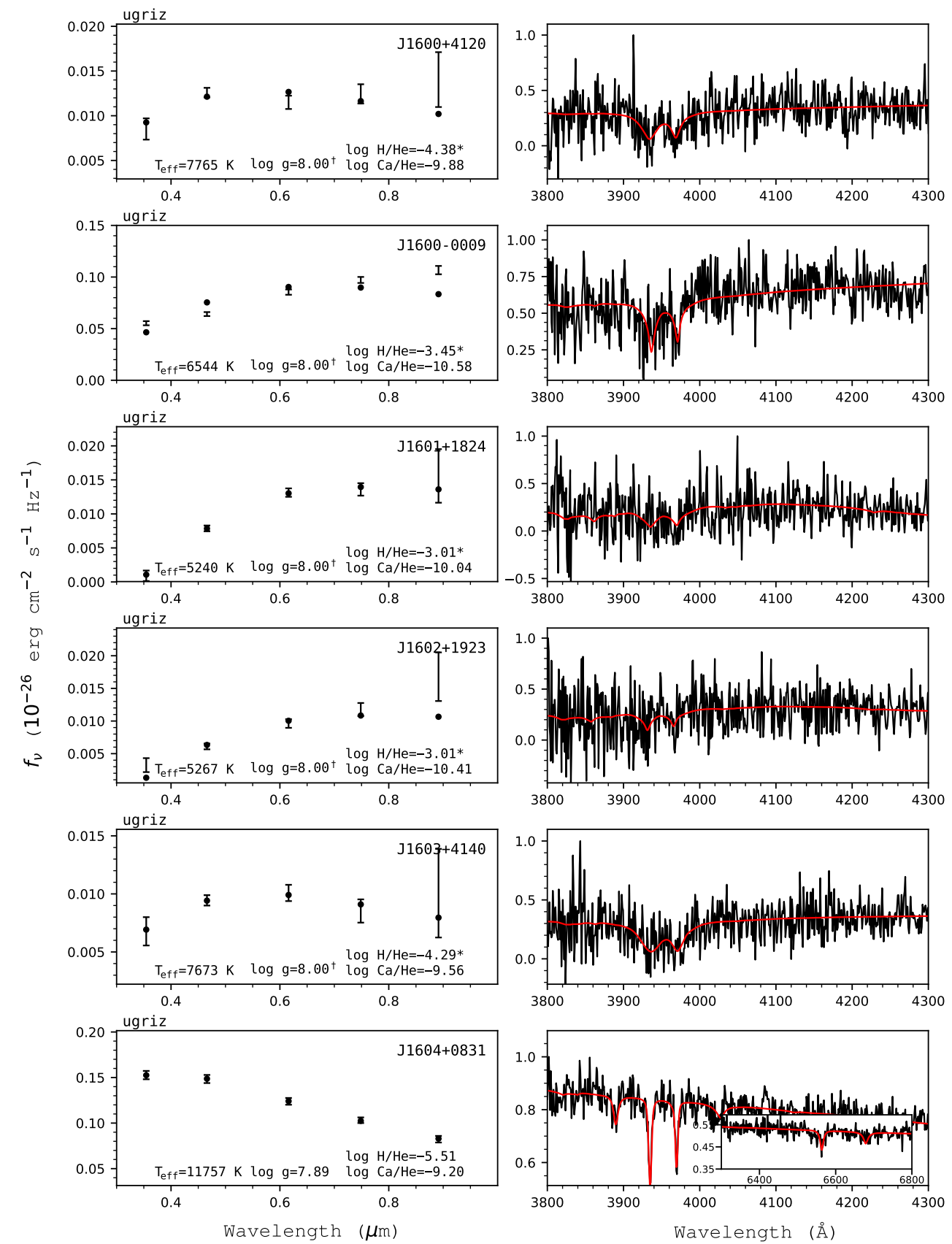

Figure 161. Fits to the DBZ/DZ(A) white dwarfs - continued. 

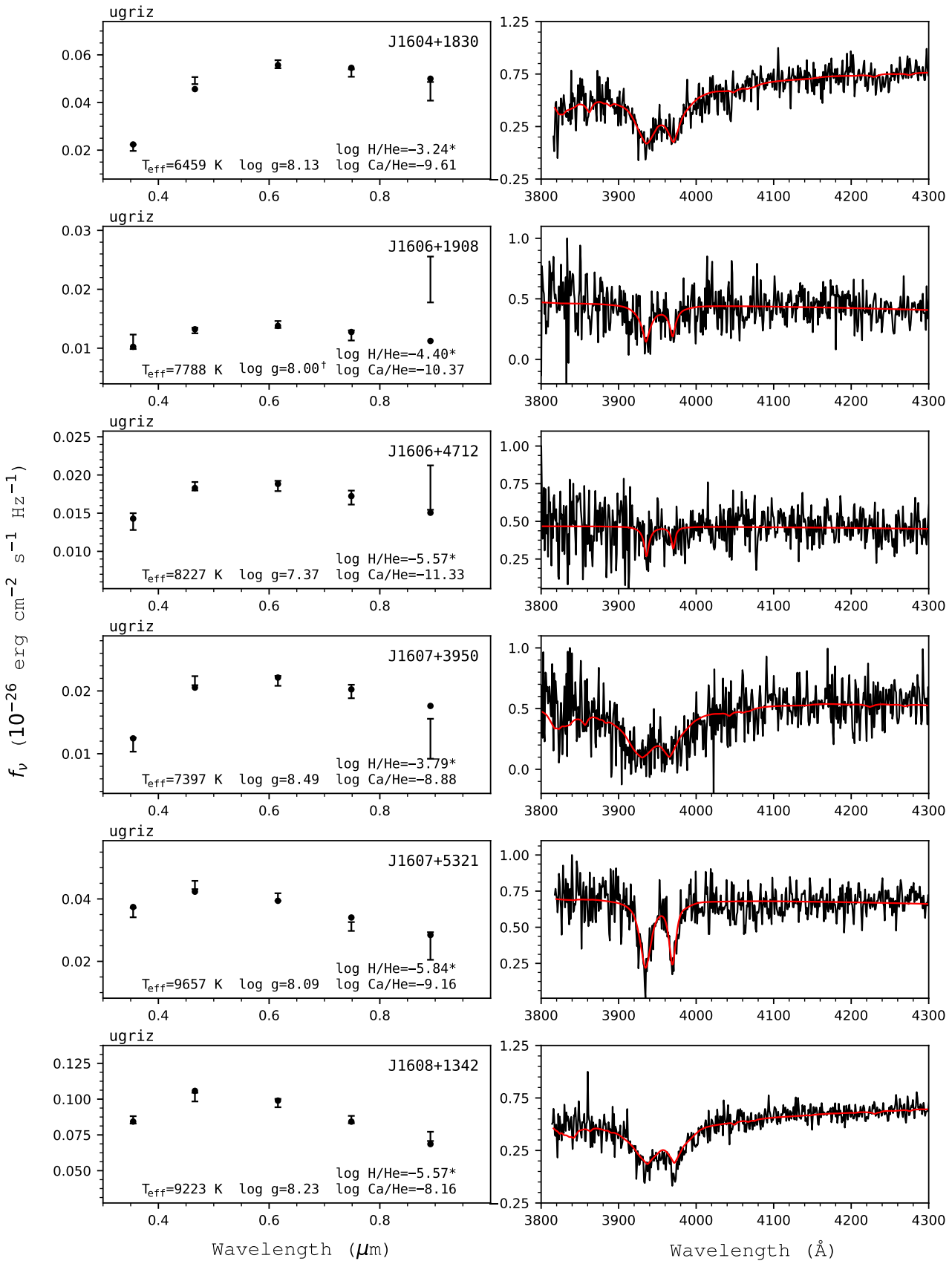

Figure 162. Fits to the DBZ/DZ(A) white dwarfs - continued. 

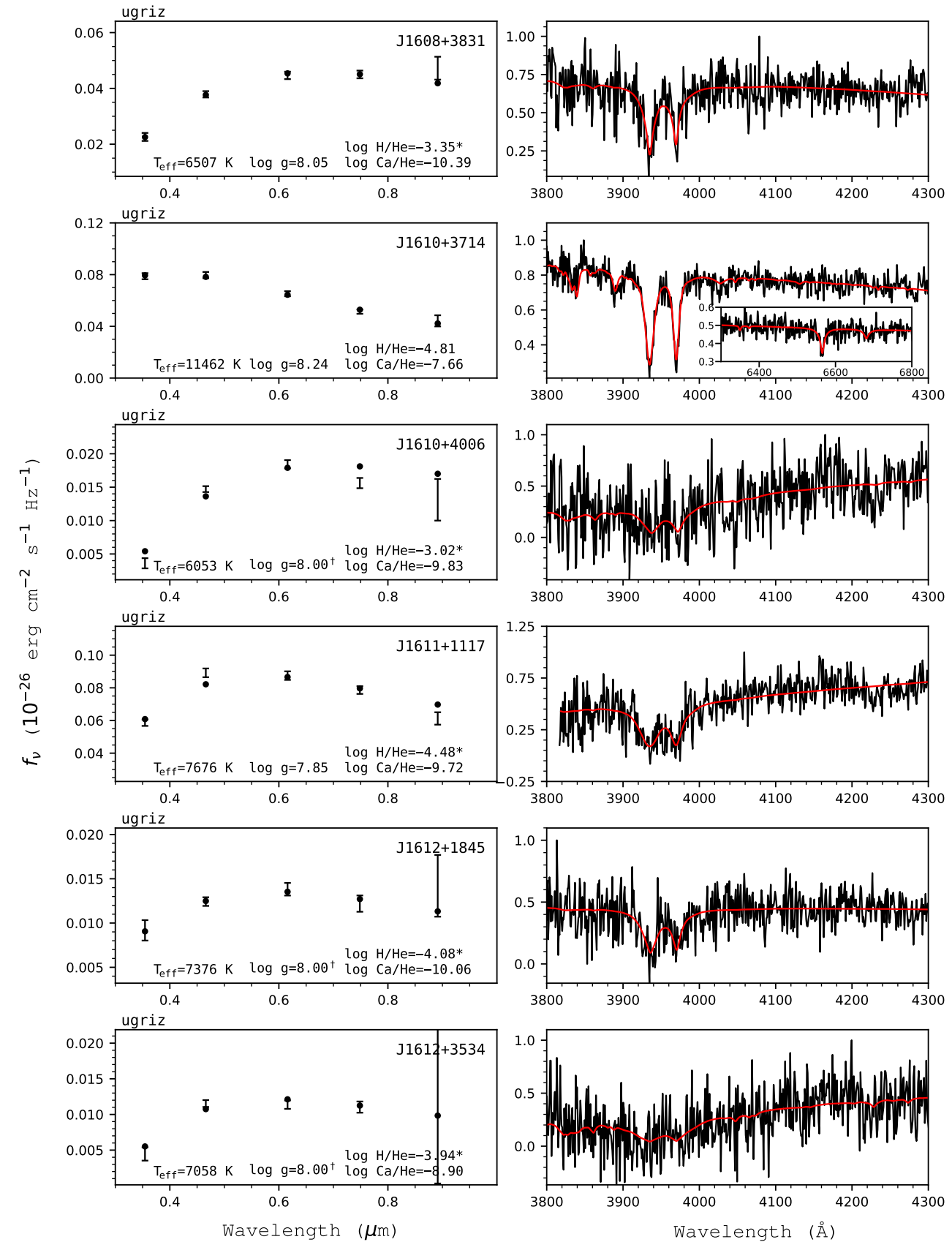

Figure 163. Fits to the DBZ/DZ(A) white dwarfs - continued. 

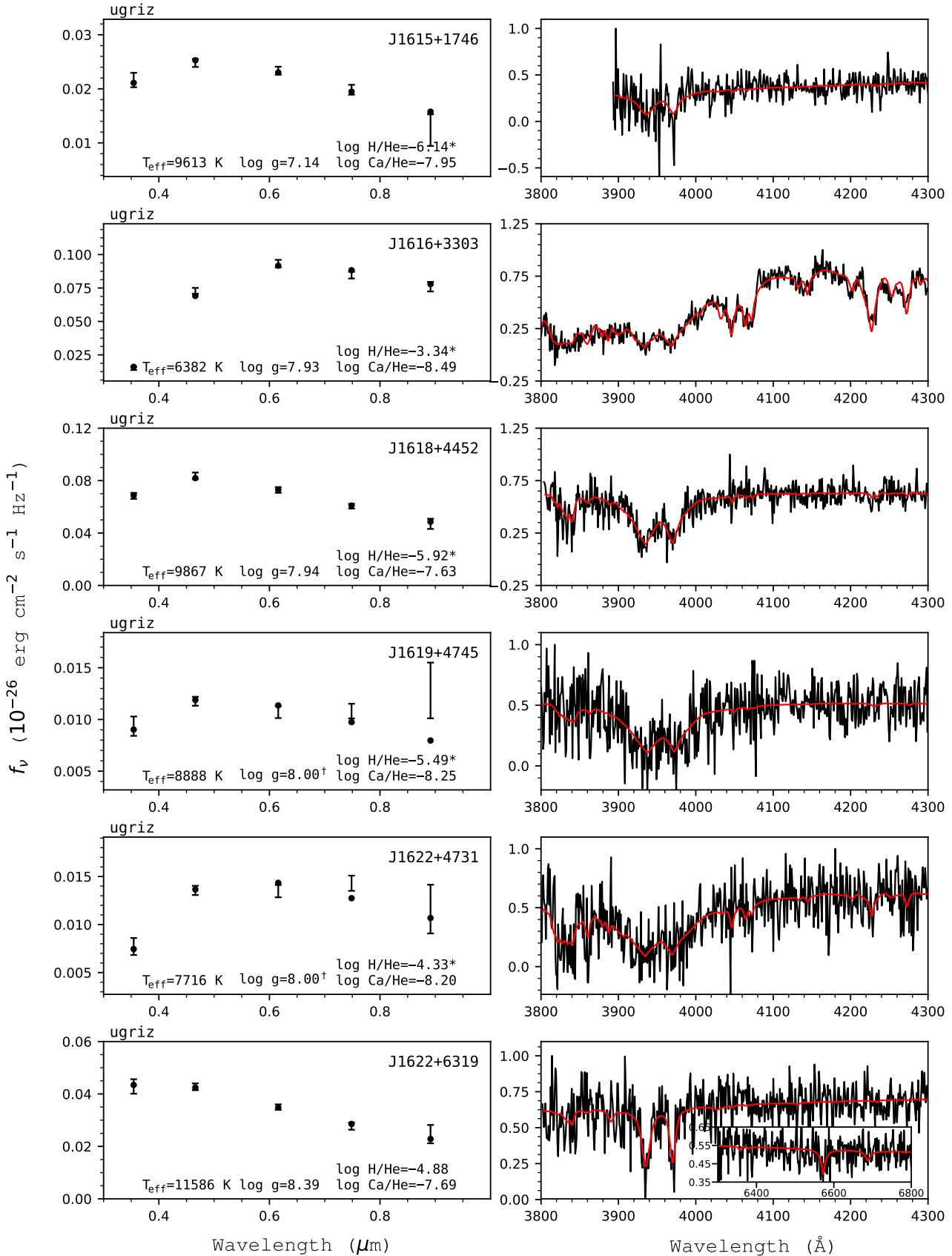

Figure 164. Fits to the DBZ/DZ(A) white dwarfs - continued. 

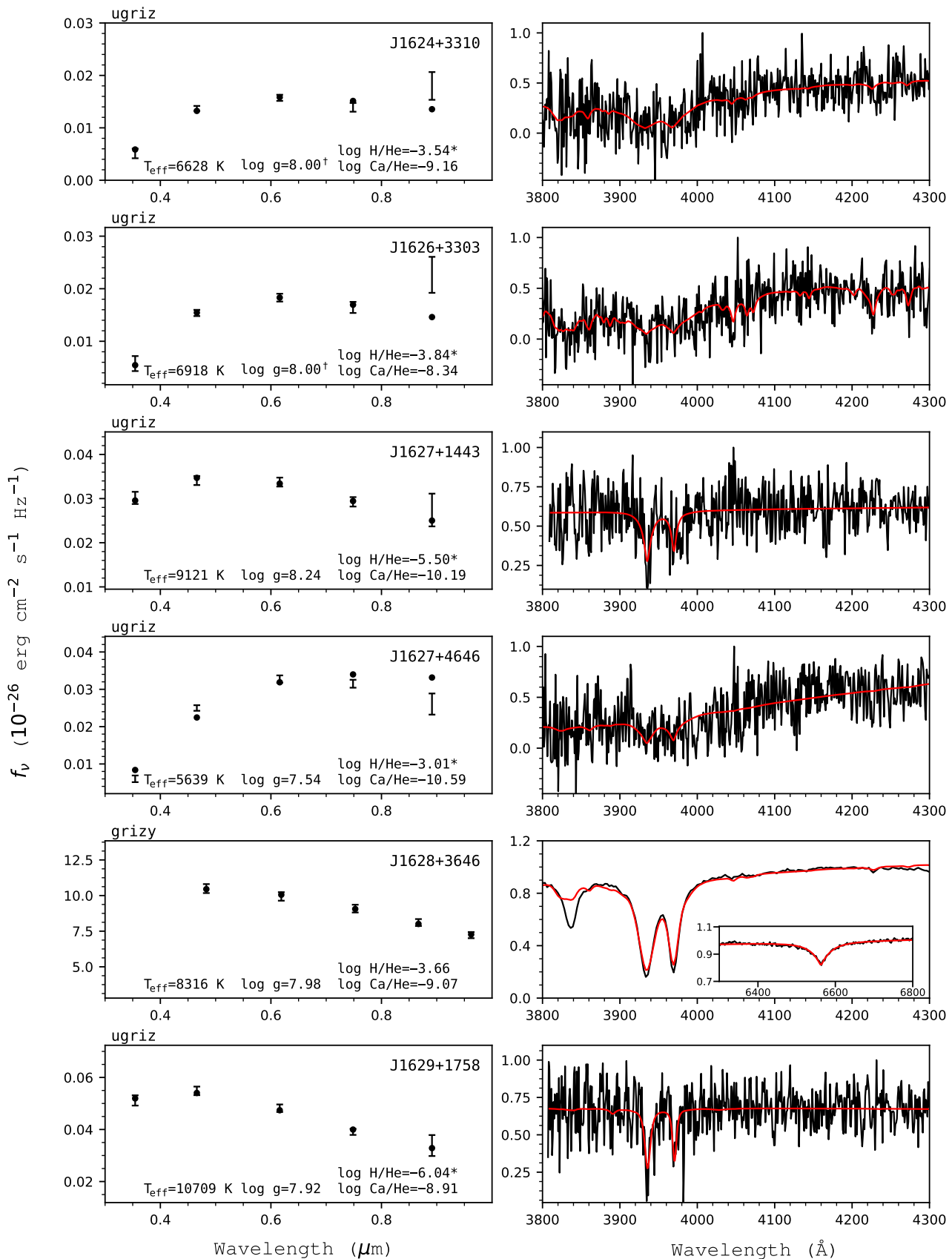

Figure 165. Fits to the DBZ/DZ(A) white dwarfs - continued. 

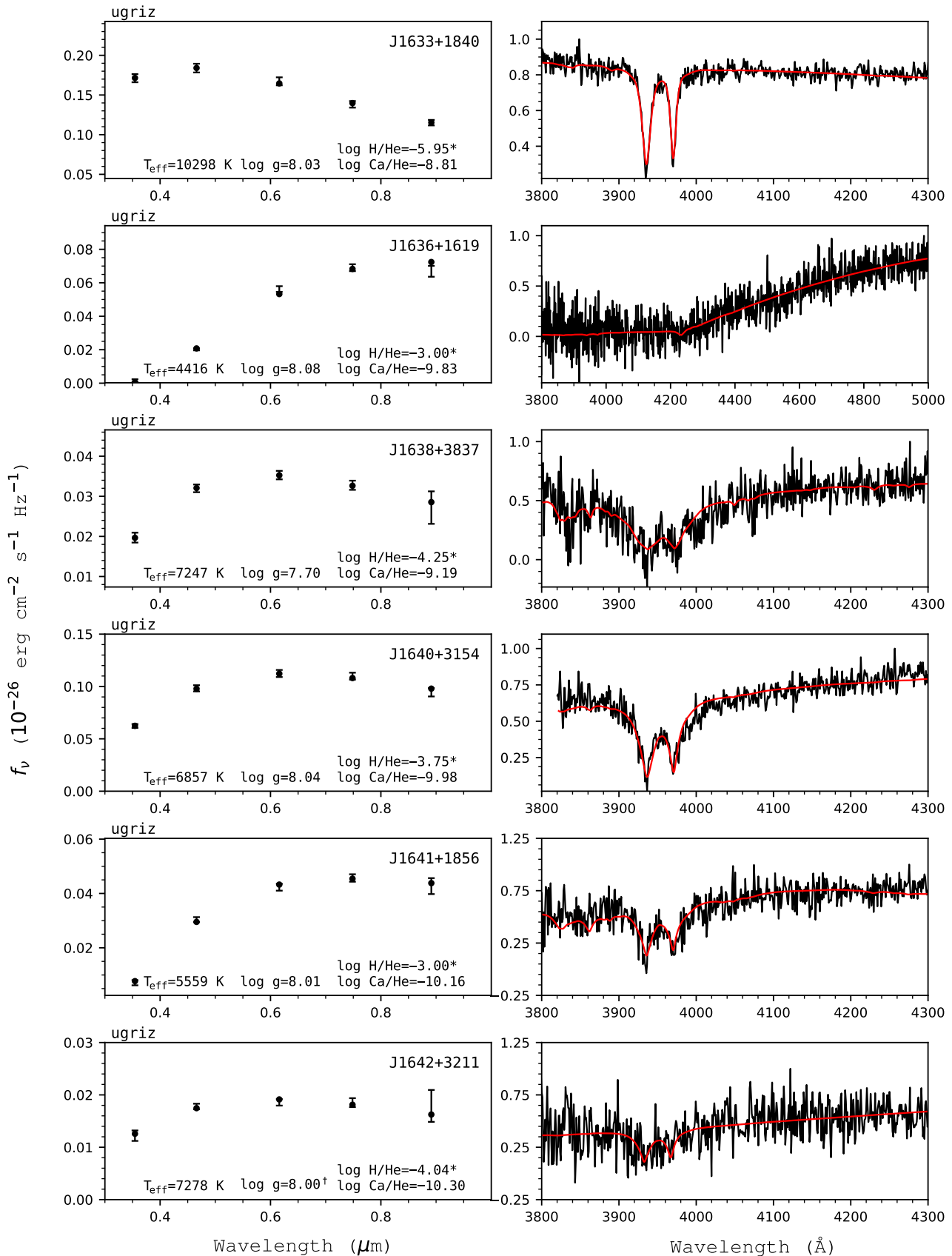

Figure 166. Fits to the DBZ/DZ(A) white dwarfs - continued. 

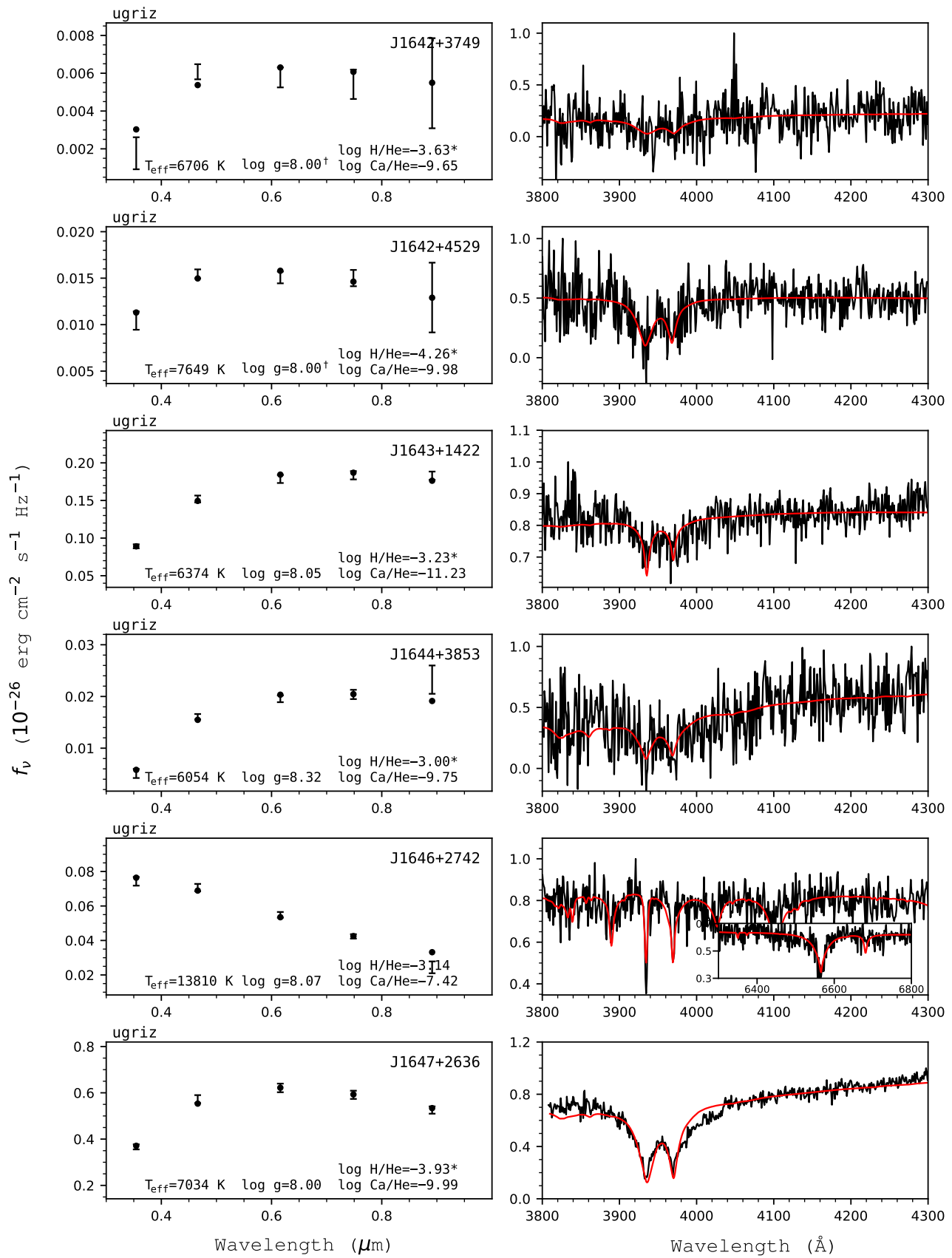

Figure 167. Fits to the DBZ/DZ(A) white dwarfs - continued. 

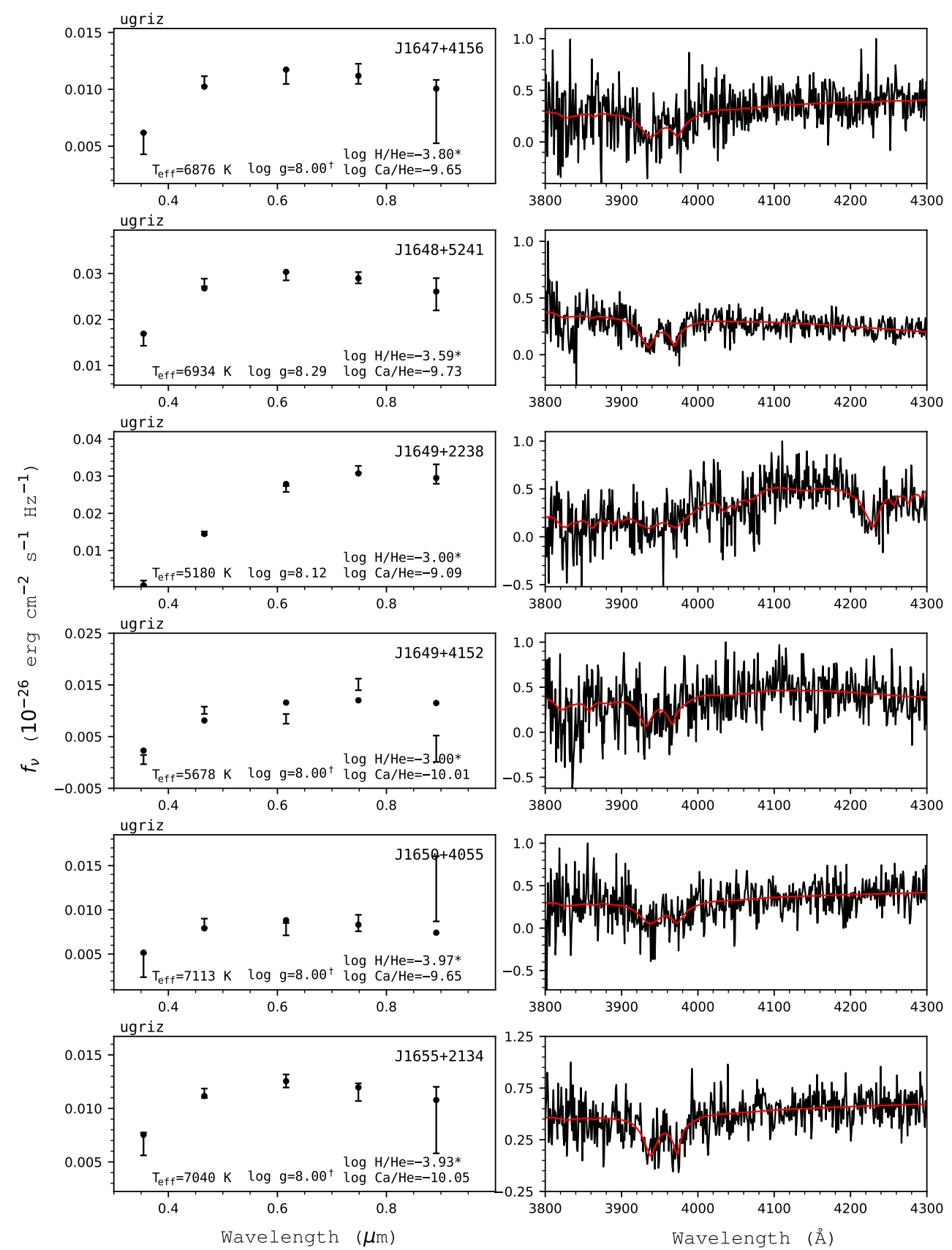

Figure 168. Fits to the DBZ/DZ(A) white dwarfs - continued. 

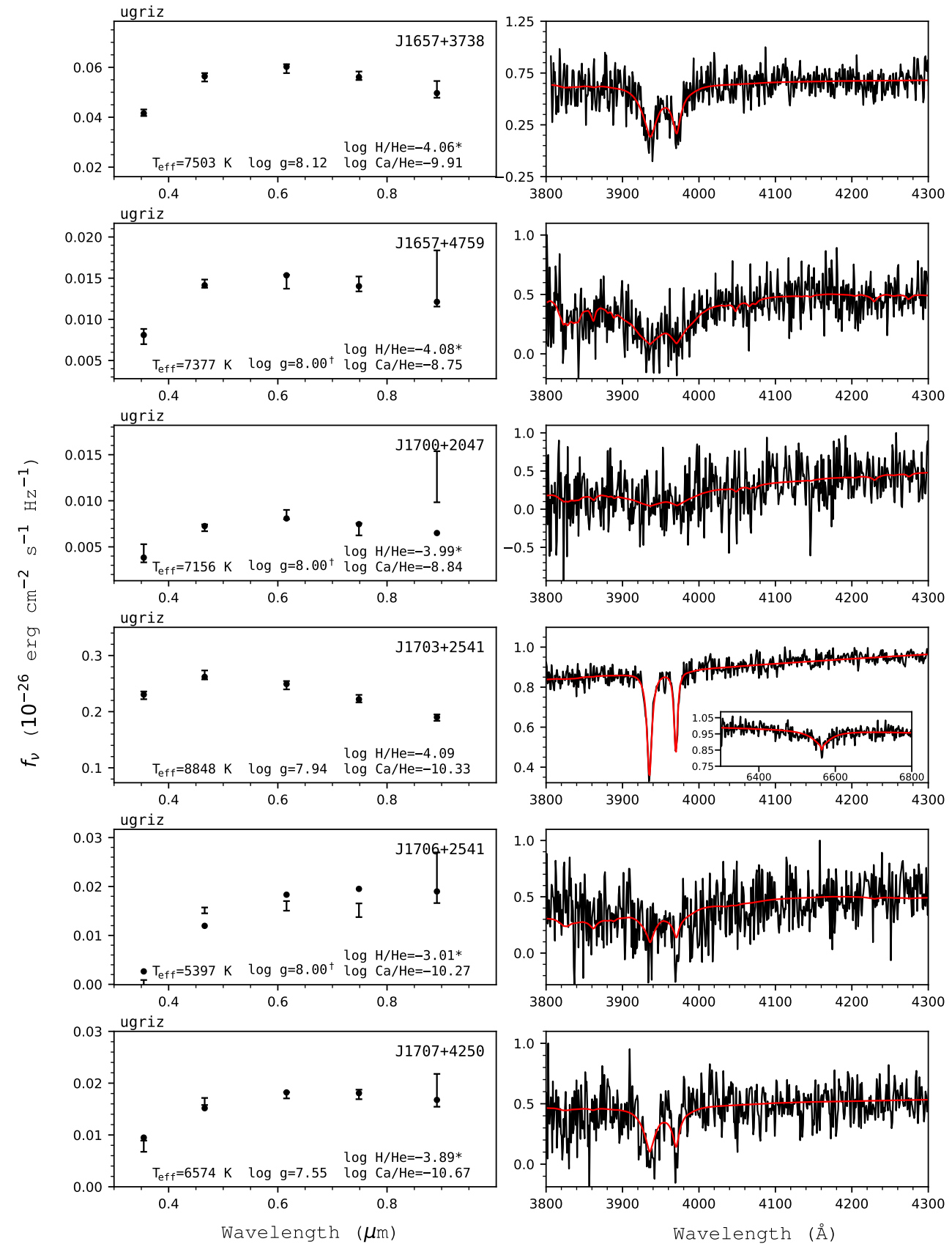

Figure 169. Fits to the DBZ/DZ(A) white dwarfs - continued. 

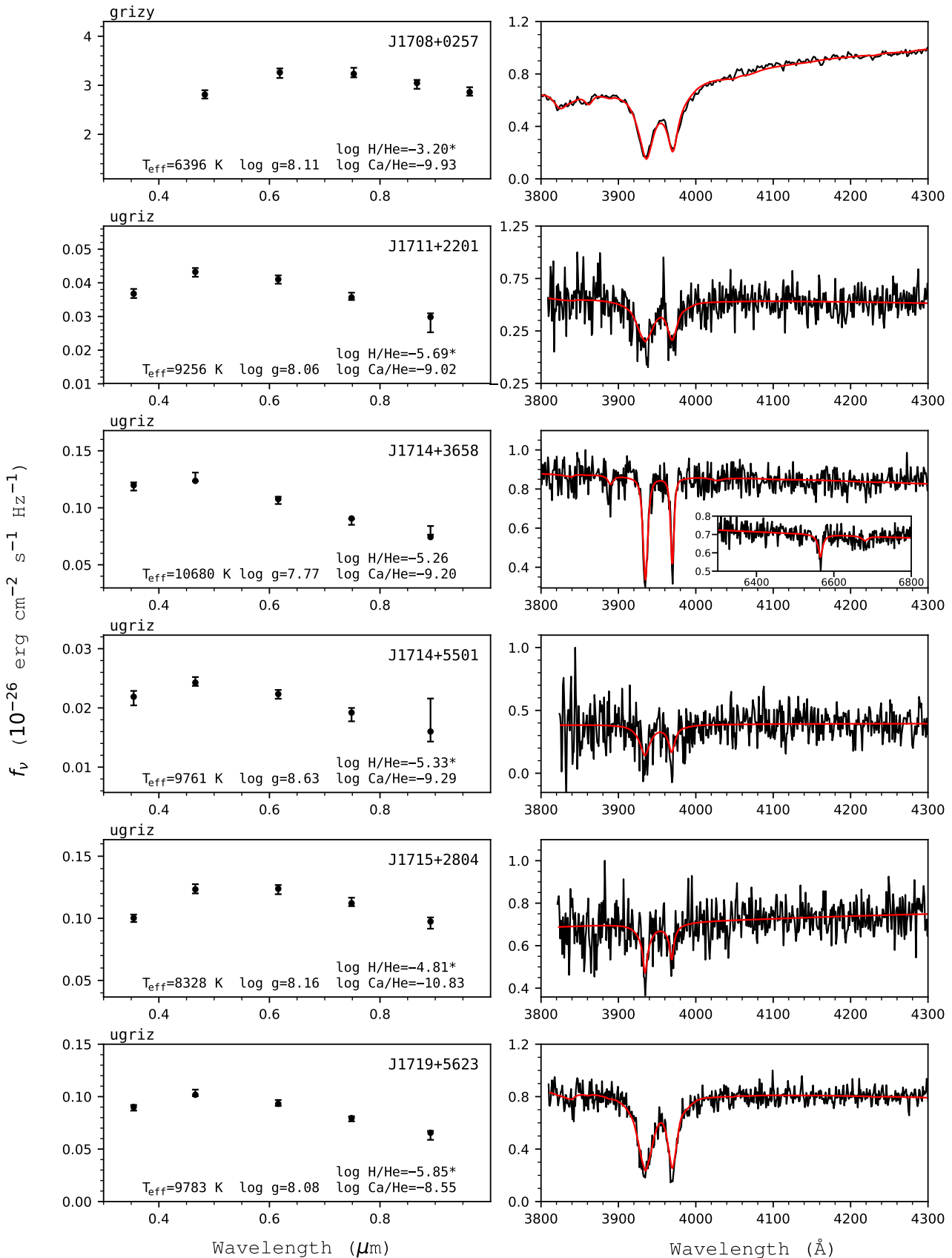

Figure 170. Fits to the DBZ/DZ(A) white dwarfs - continued. 

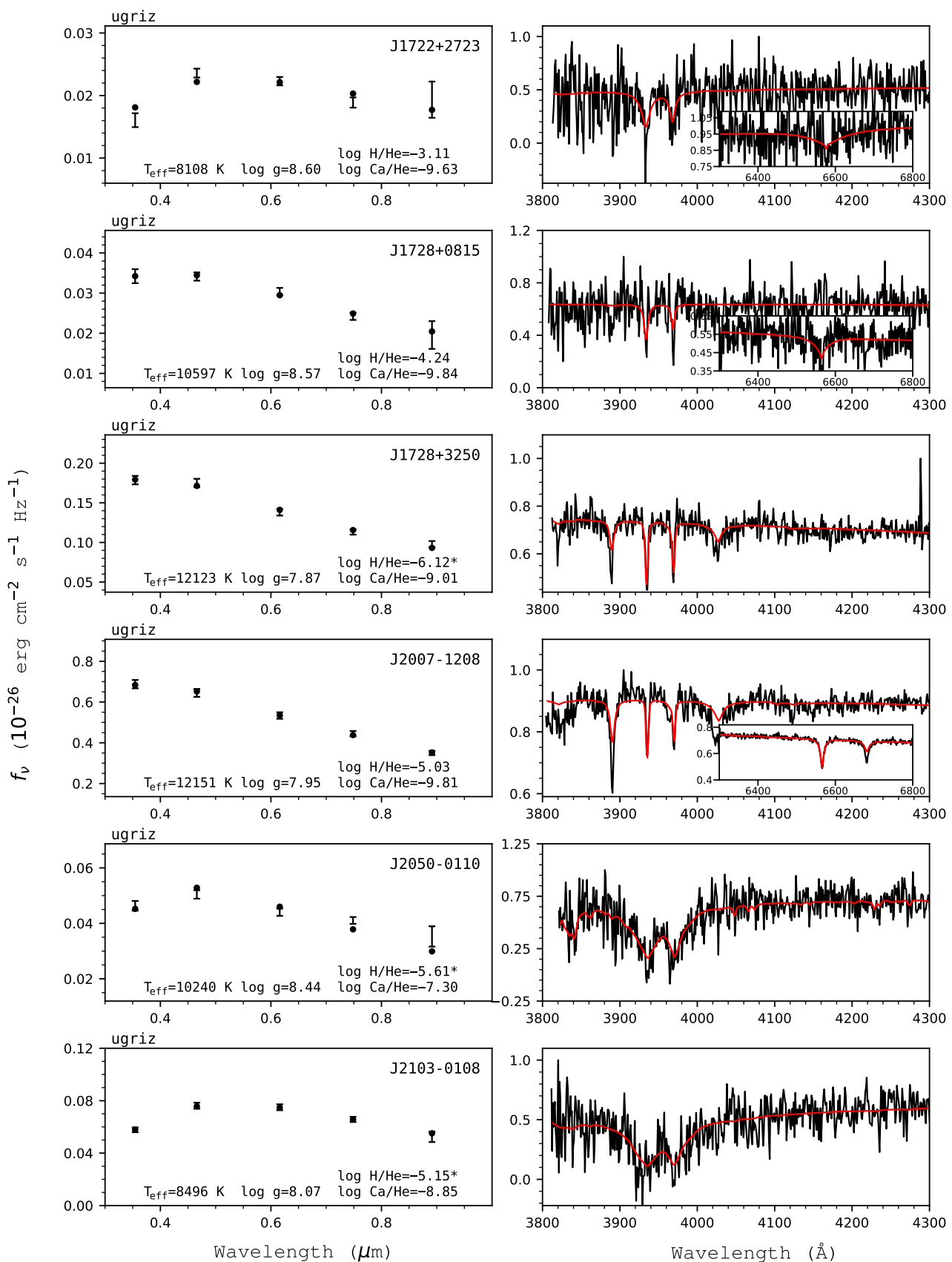

Figure 171. Fits to the DBZ/DZ(A) white dwarfs - continued. 

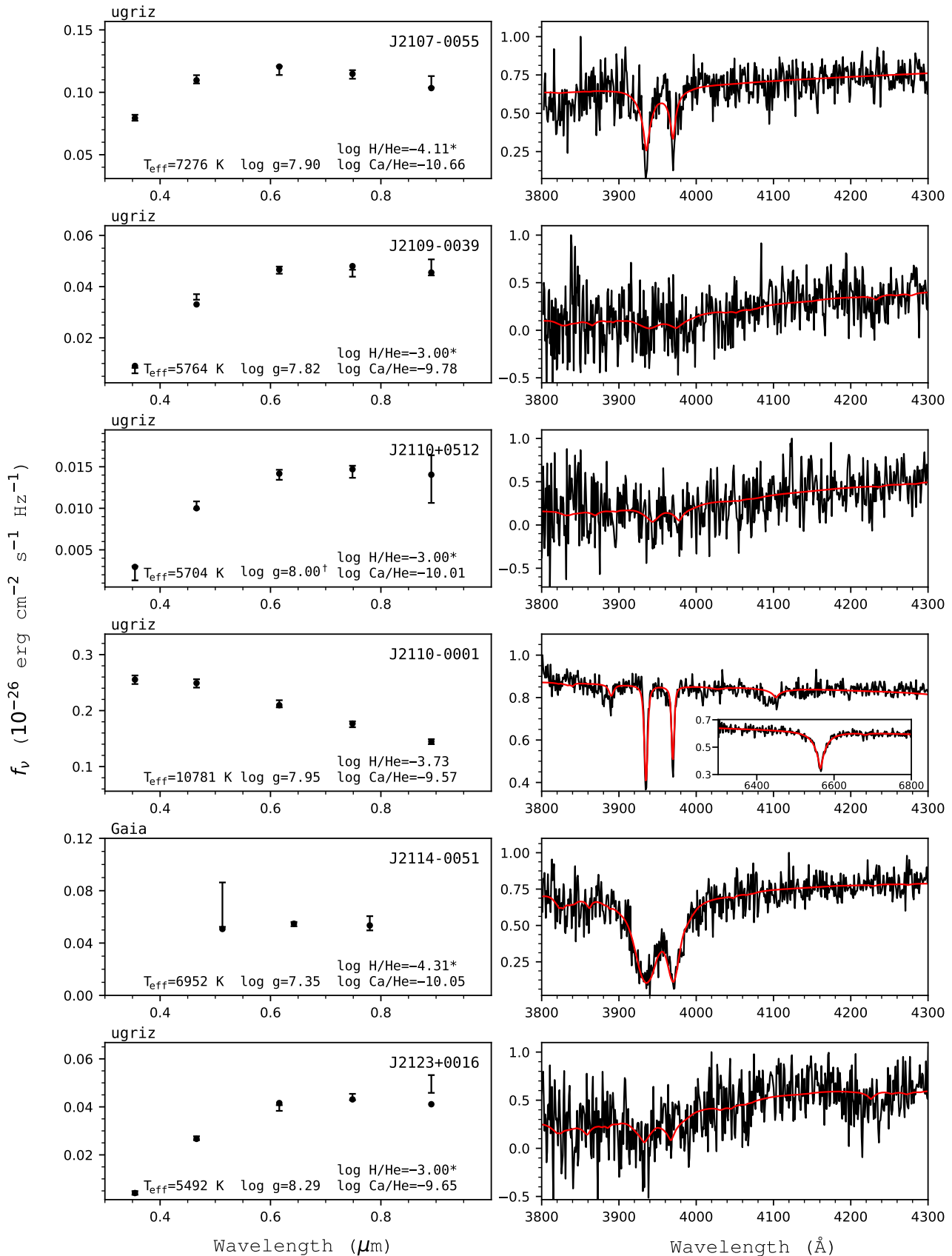

Figure 172. Fits to the DBZ/DZ(A) white dwarfs - continued. 

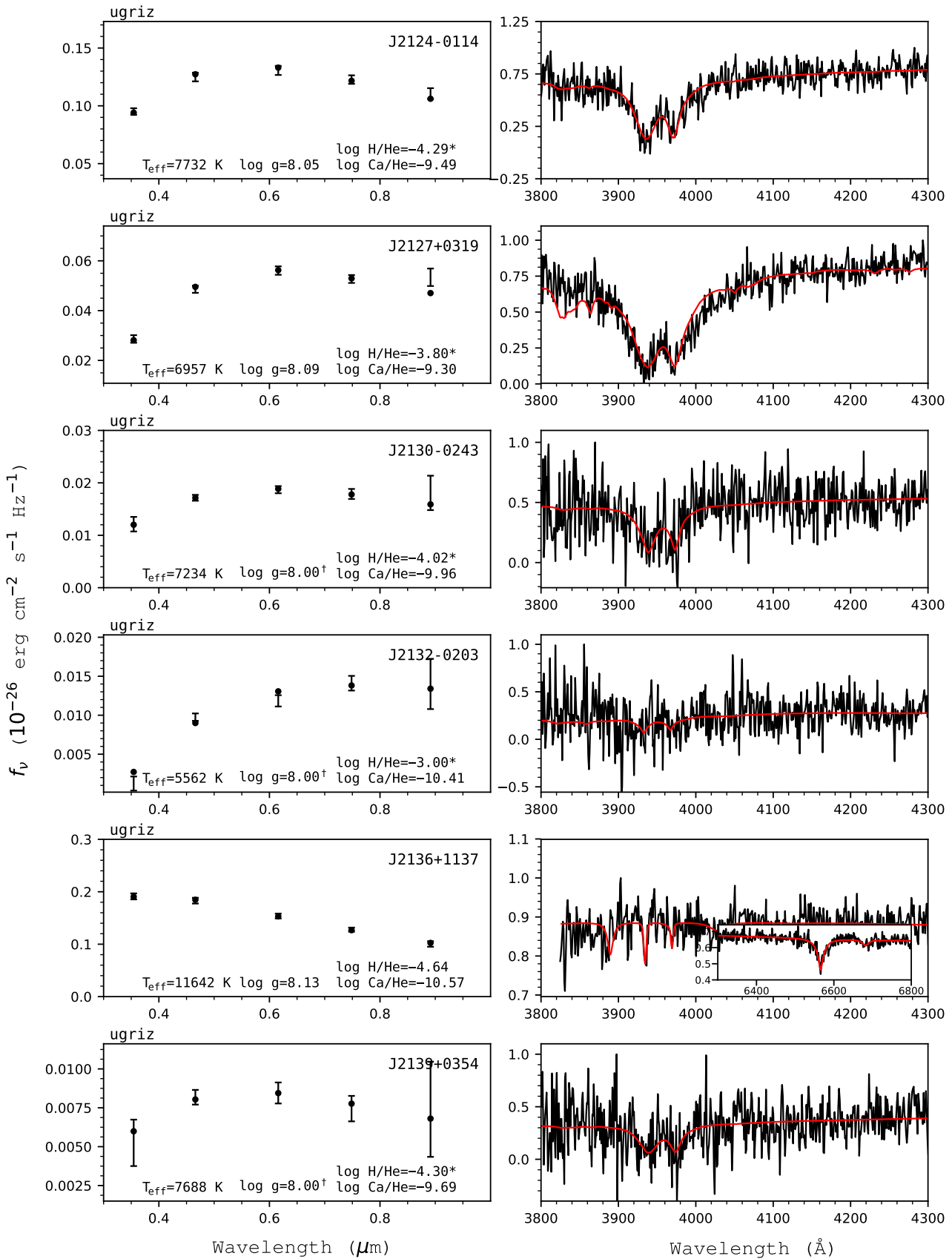

Figure 173. Fits to the DBZ/DZ(A) white dwarfs - continued. 

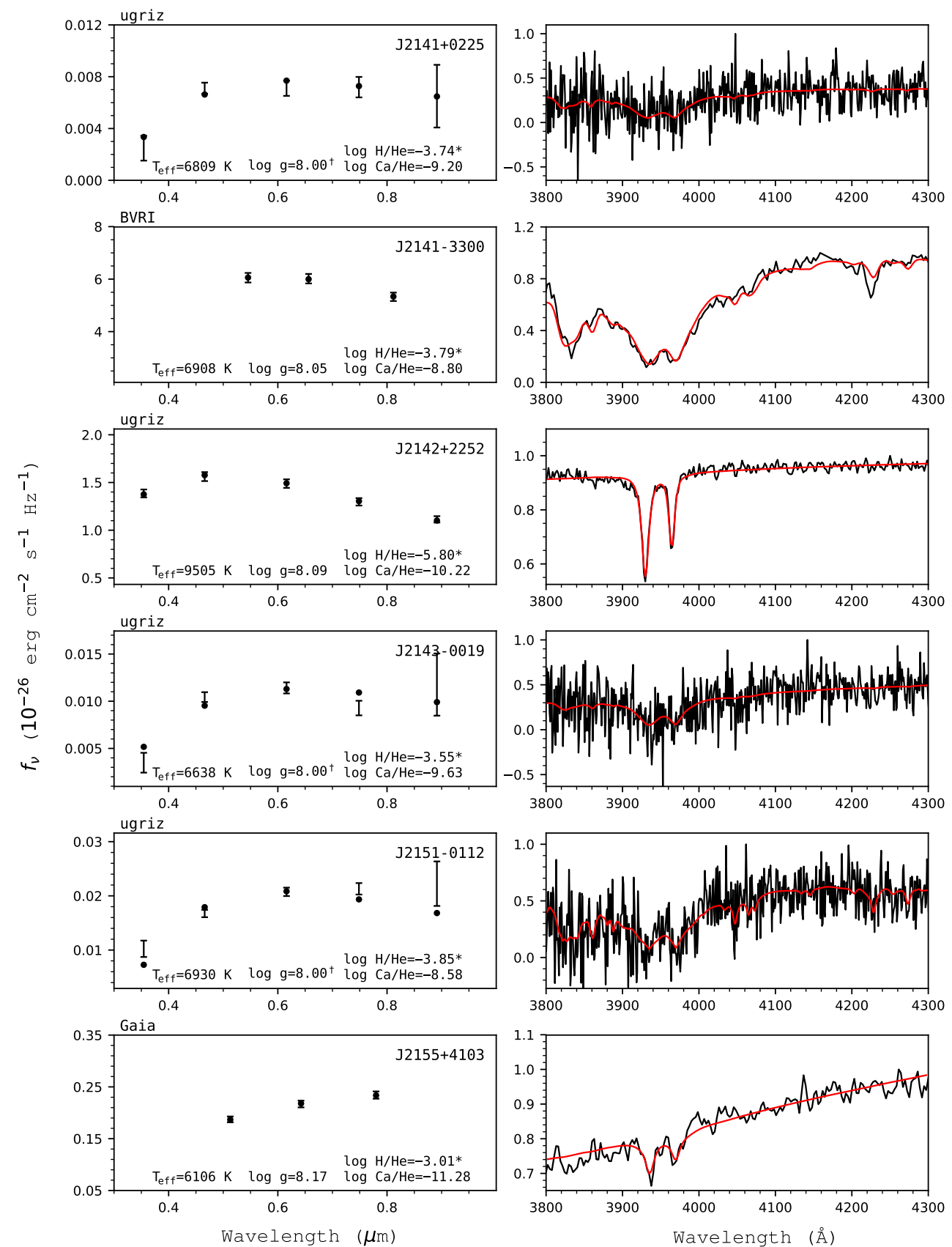

Figure 174. Fits to the DBZ/DZ(A) white dwarfs - continued. 

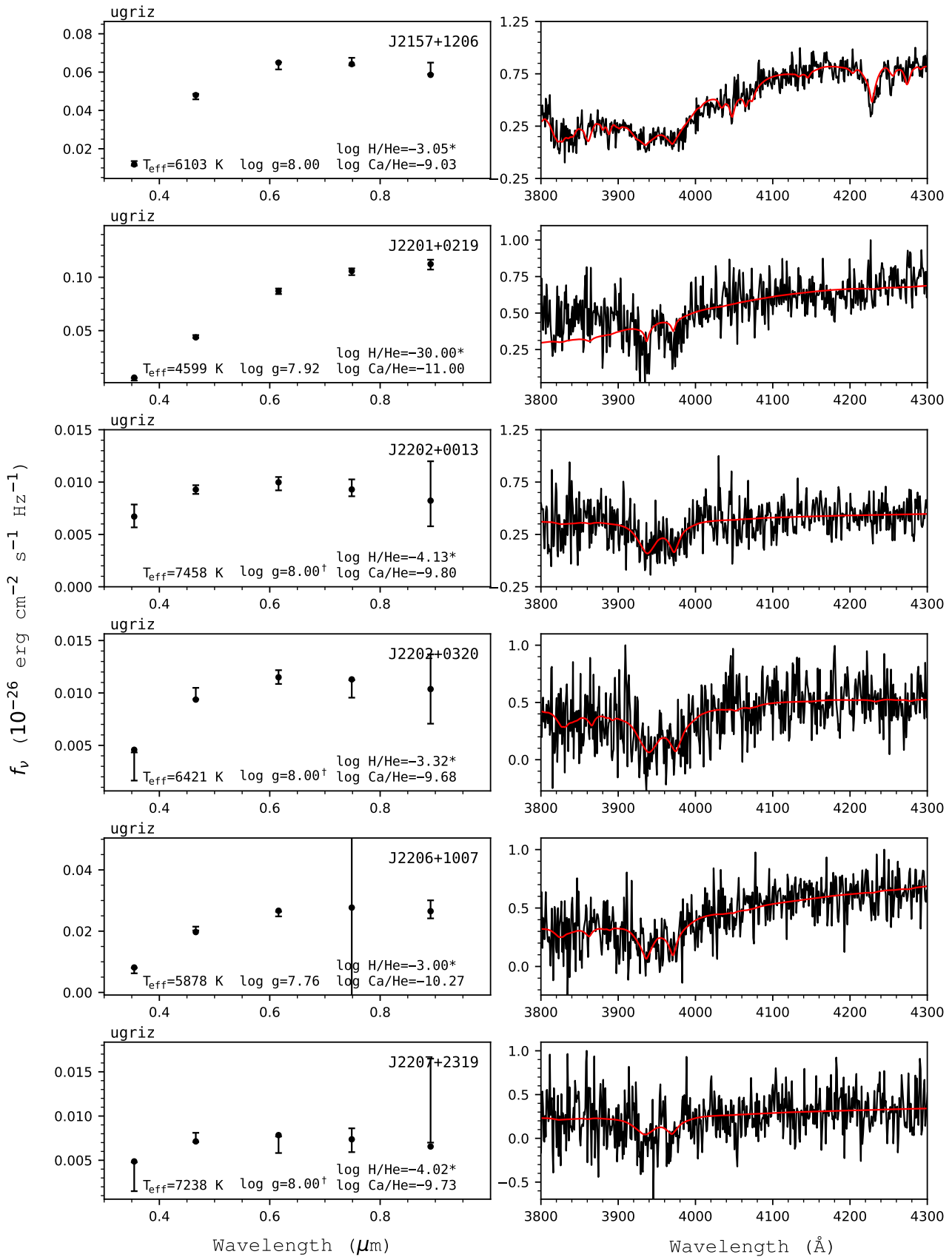

Figure 175. Fits to the DBZ/DZ(A) white dwarfs - continued. 

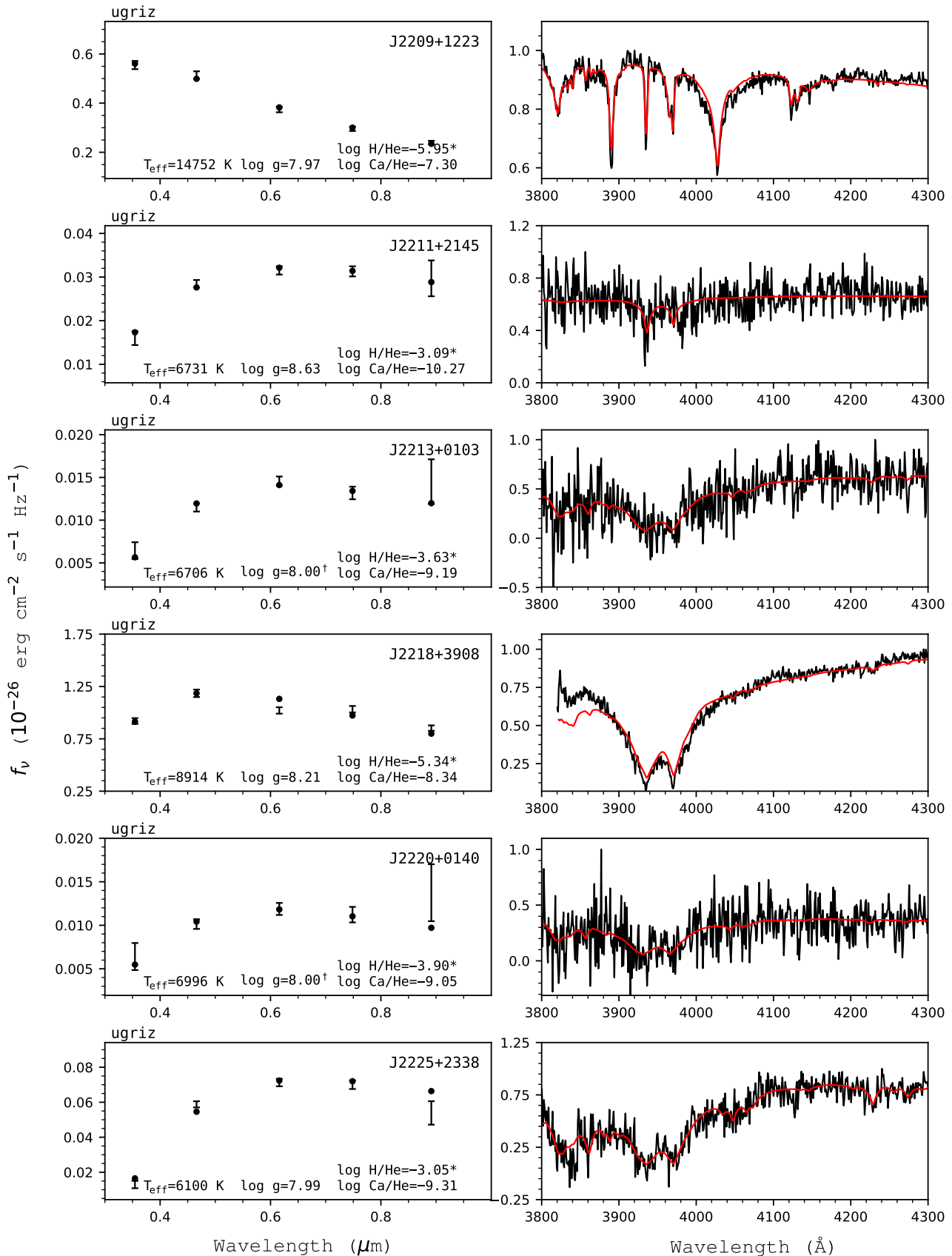

Figure 176. Fits to the DBZ/DZ(A) white dwarfs - continued. 

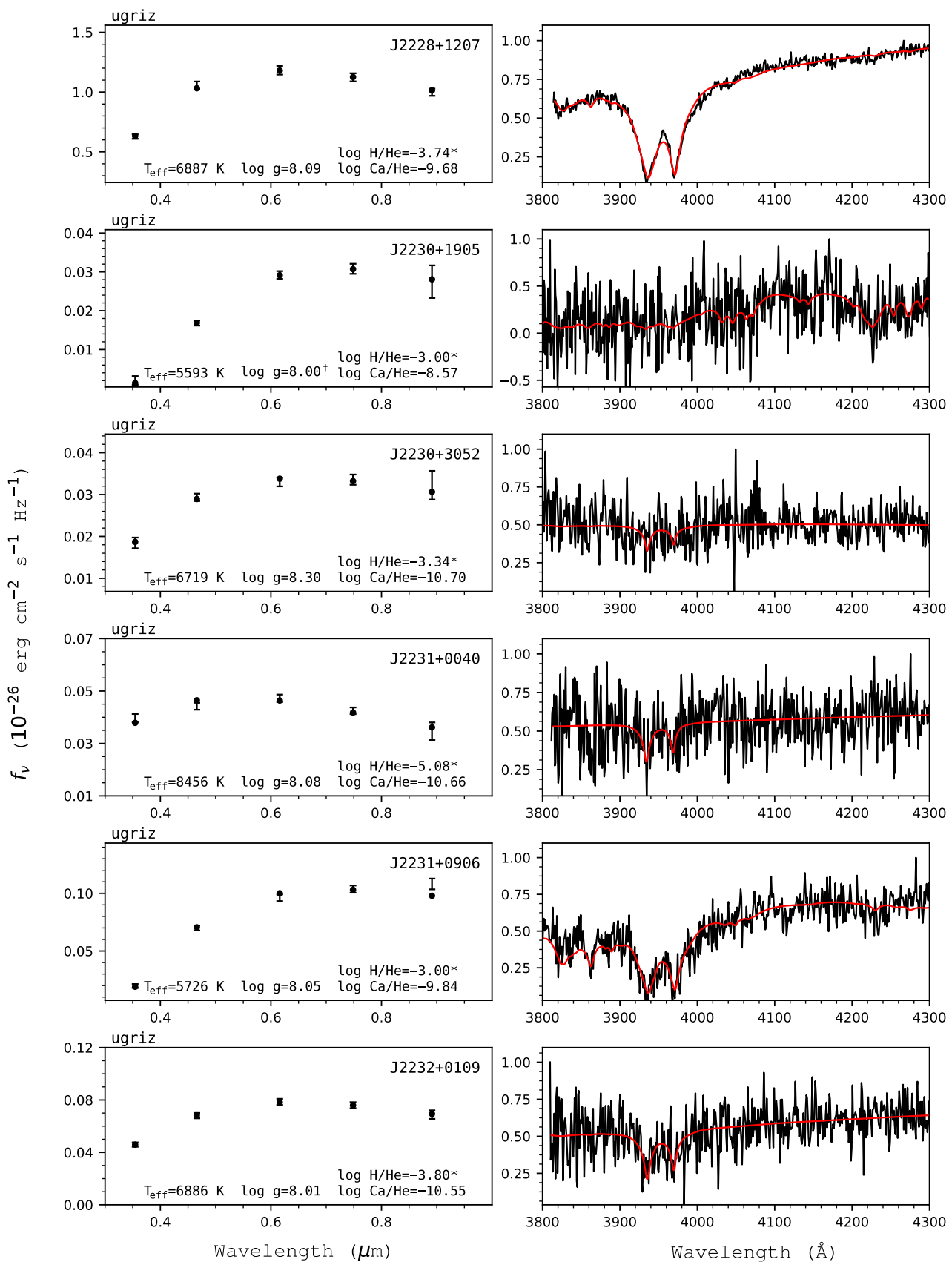

Figure 177. Fits to the DBZ/DZ(A) white dwarfs - continued. 

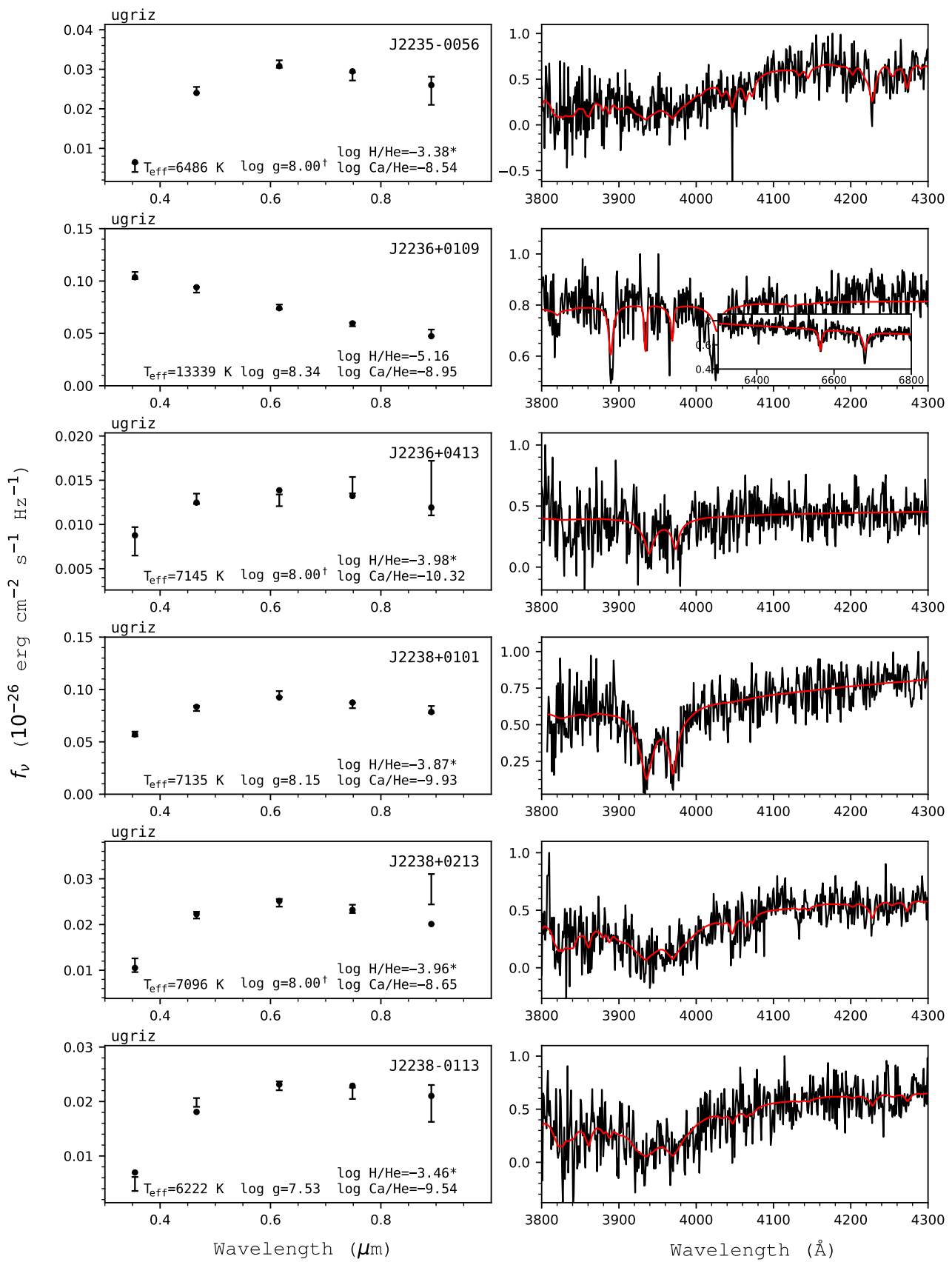

Figure 178. Fits to the DBZ/DZ(A) white dwarfs - continued. 

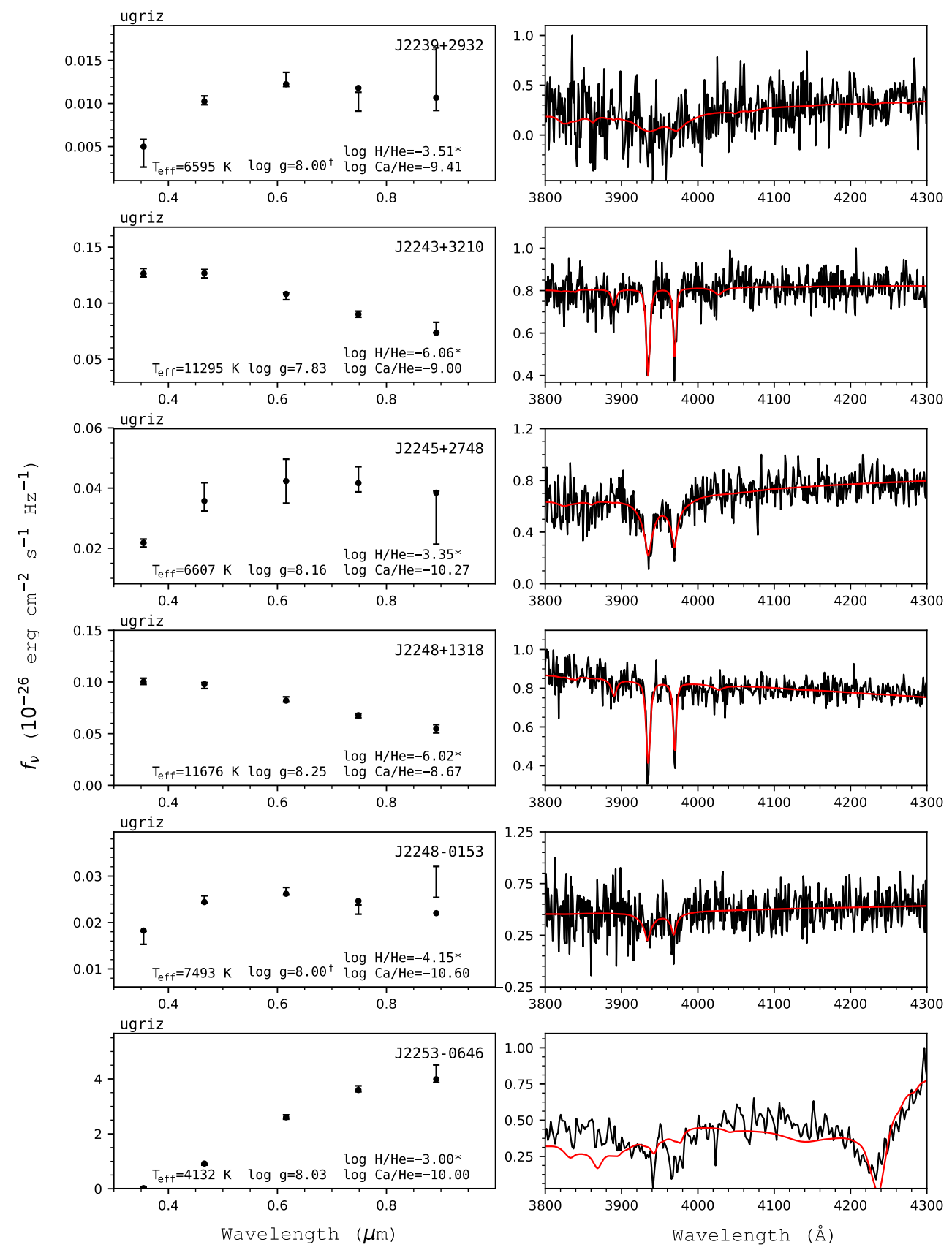

Figure 179. Fits to the DBZ/DZ(A) white dwarfs - continued. 

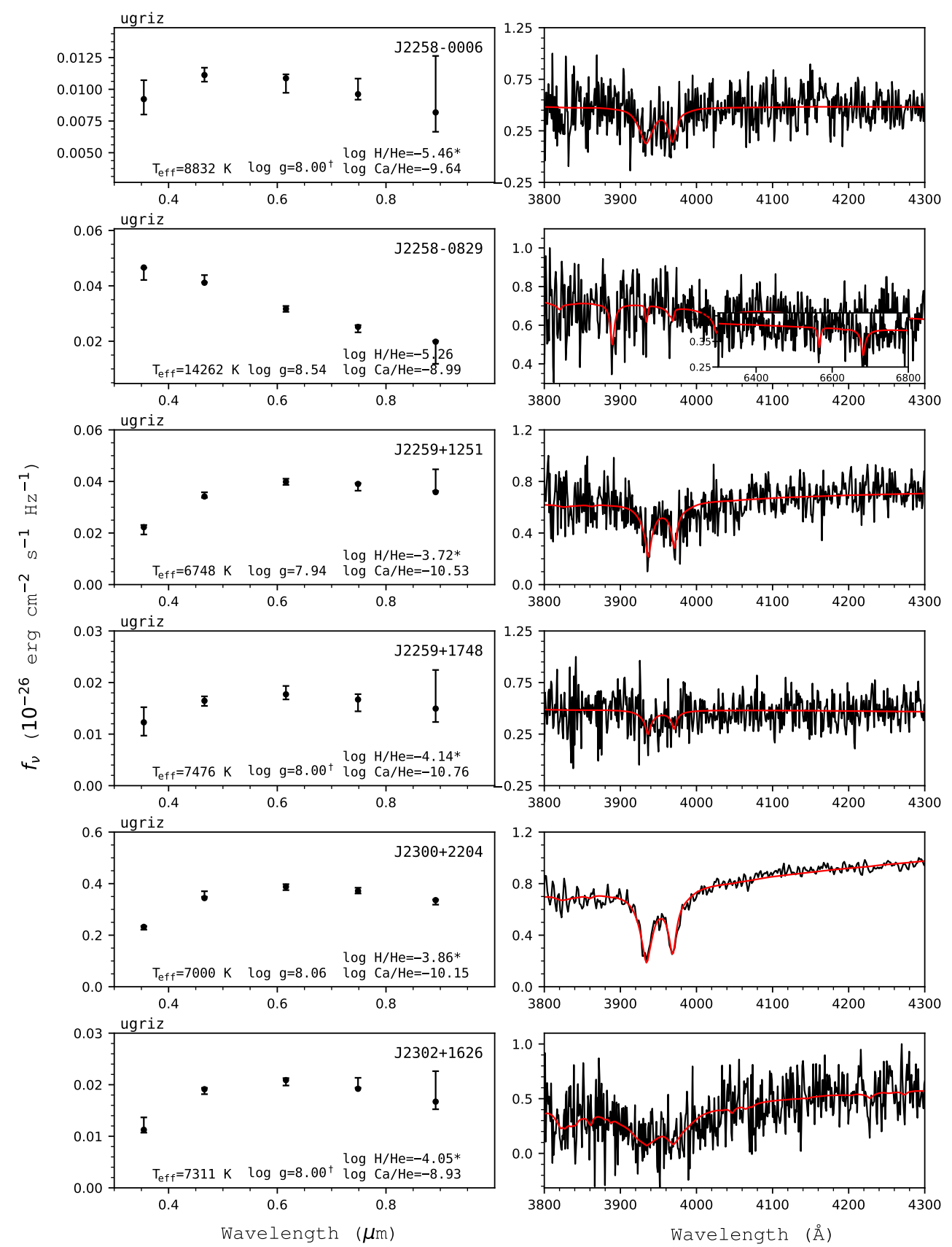

Figure 180. Fits to the DBZ/DZ(A) white dwarfs - continued. 


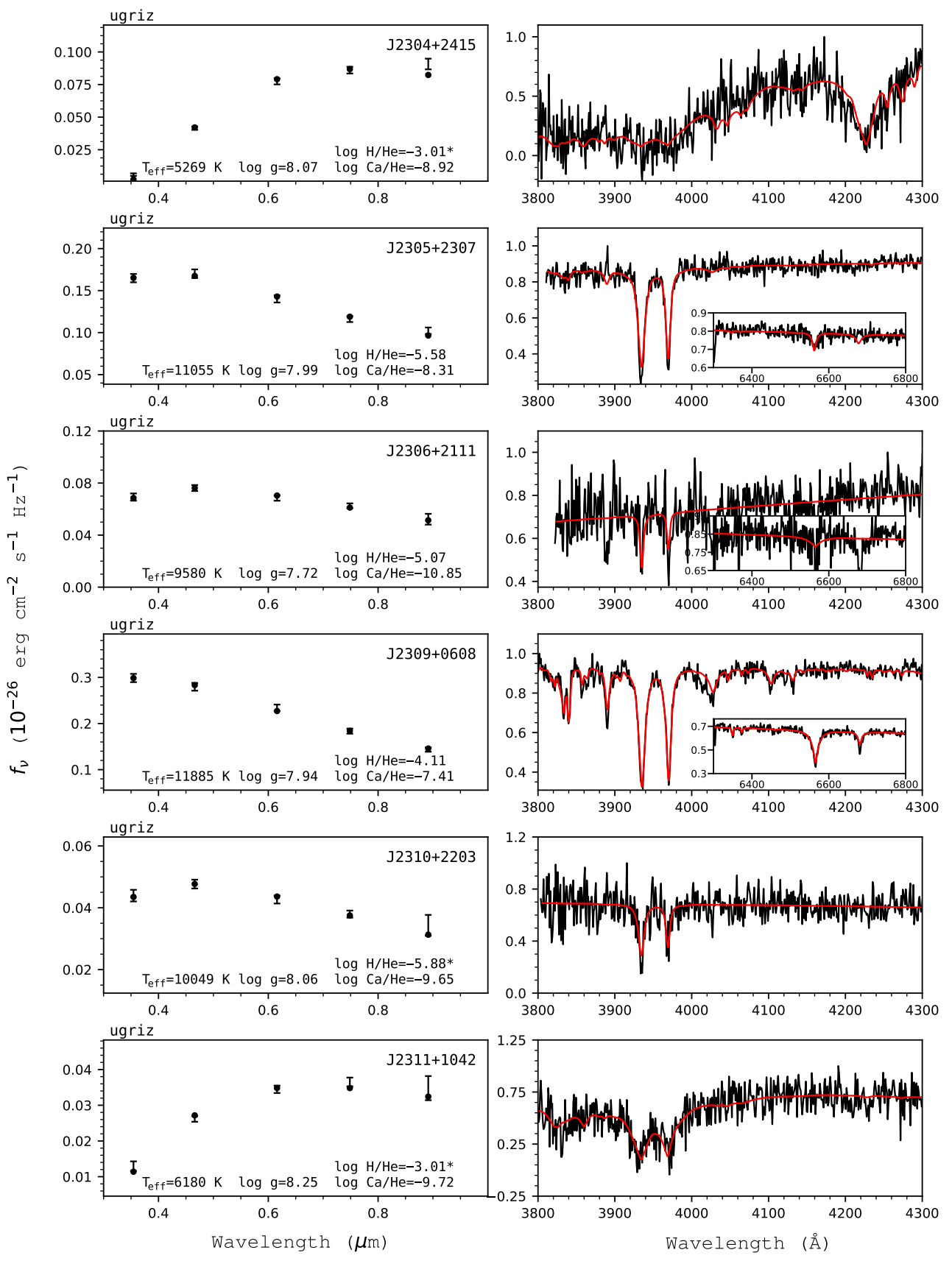

Figure 181. Fits to the DBZ/DZ(A) white dwarfs - continued. 

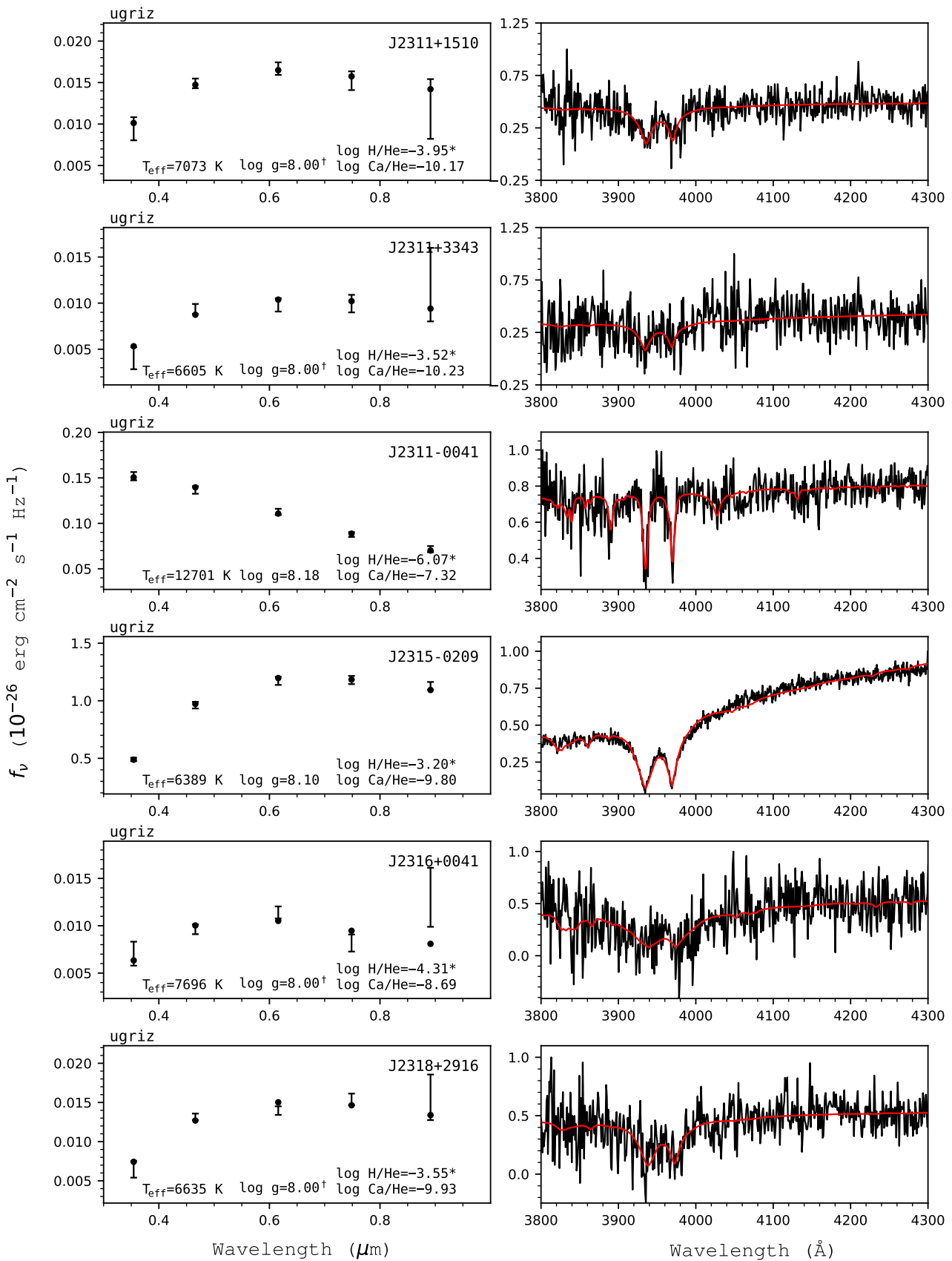

Figure 182. Fits to the DBZ/DZ(A) white dwarfs - continued. 


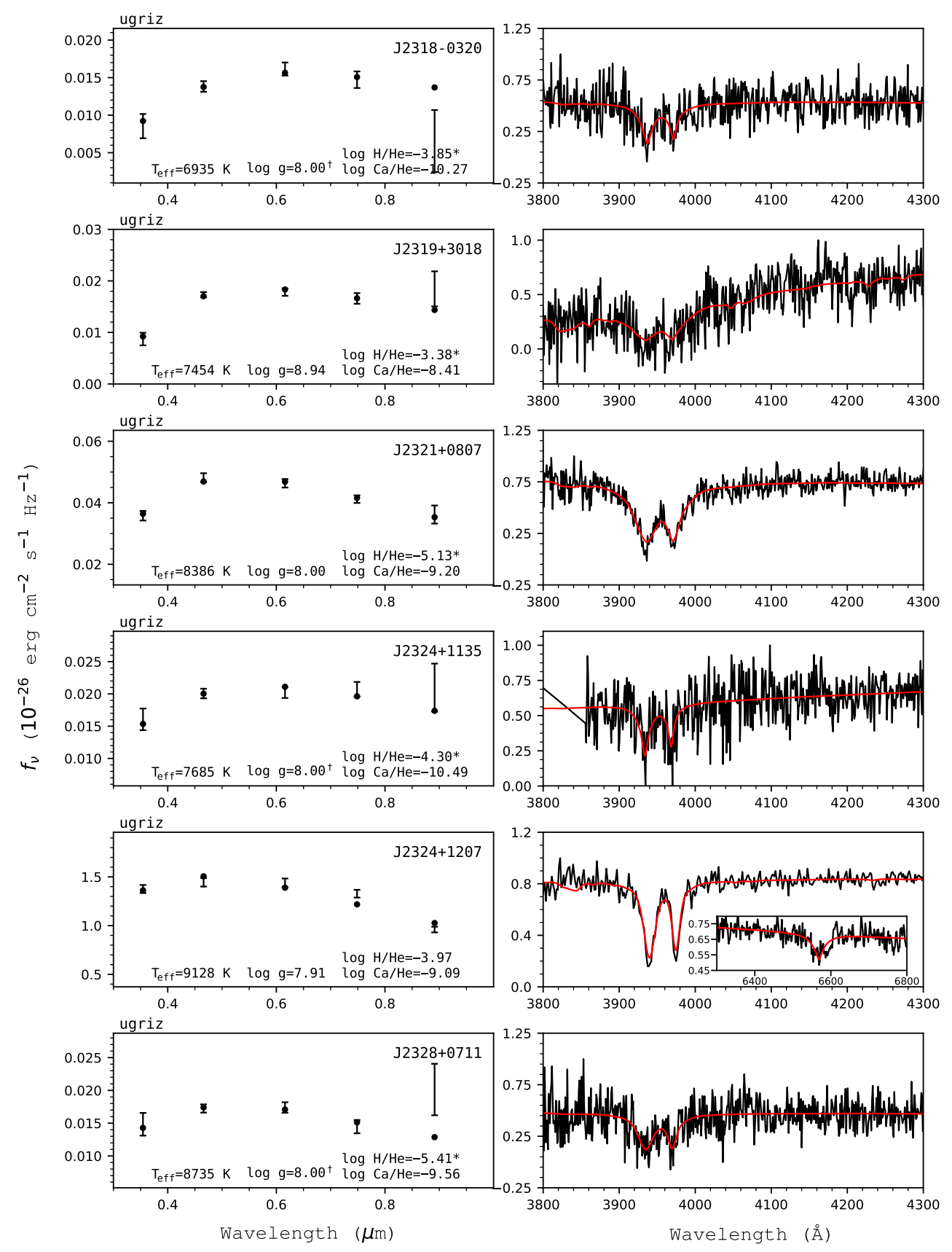

Figure 183. Fits to the DBZ/DZ(A) white dwarfs - continued. 

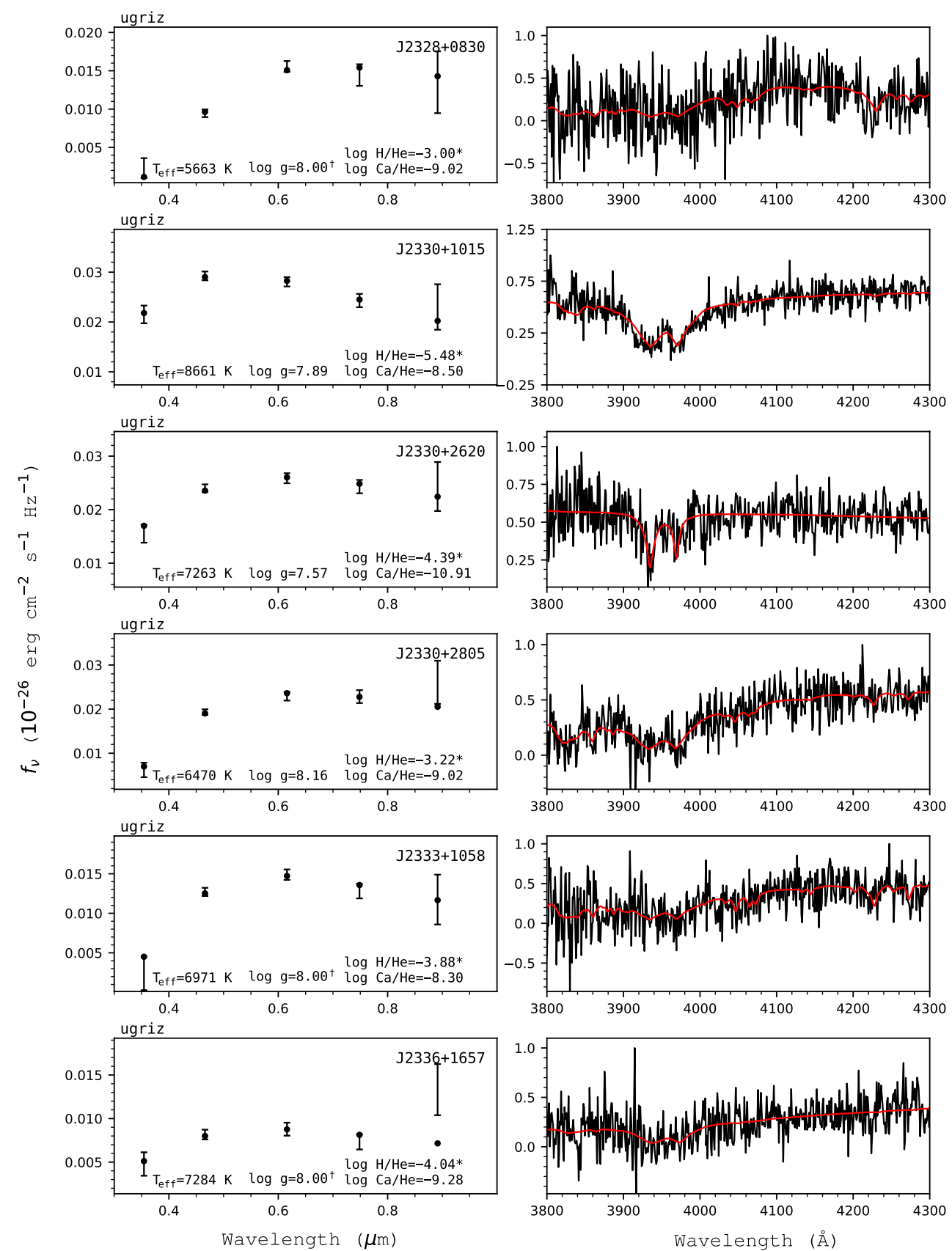

Figure 184. Fits to the DBZ/DZ(A) white dwarfs - continued. 

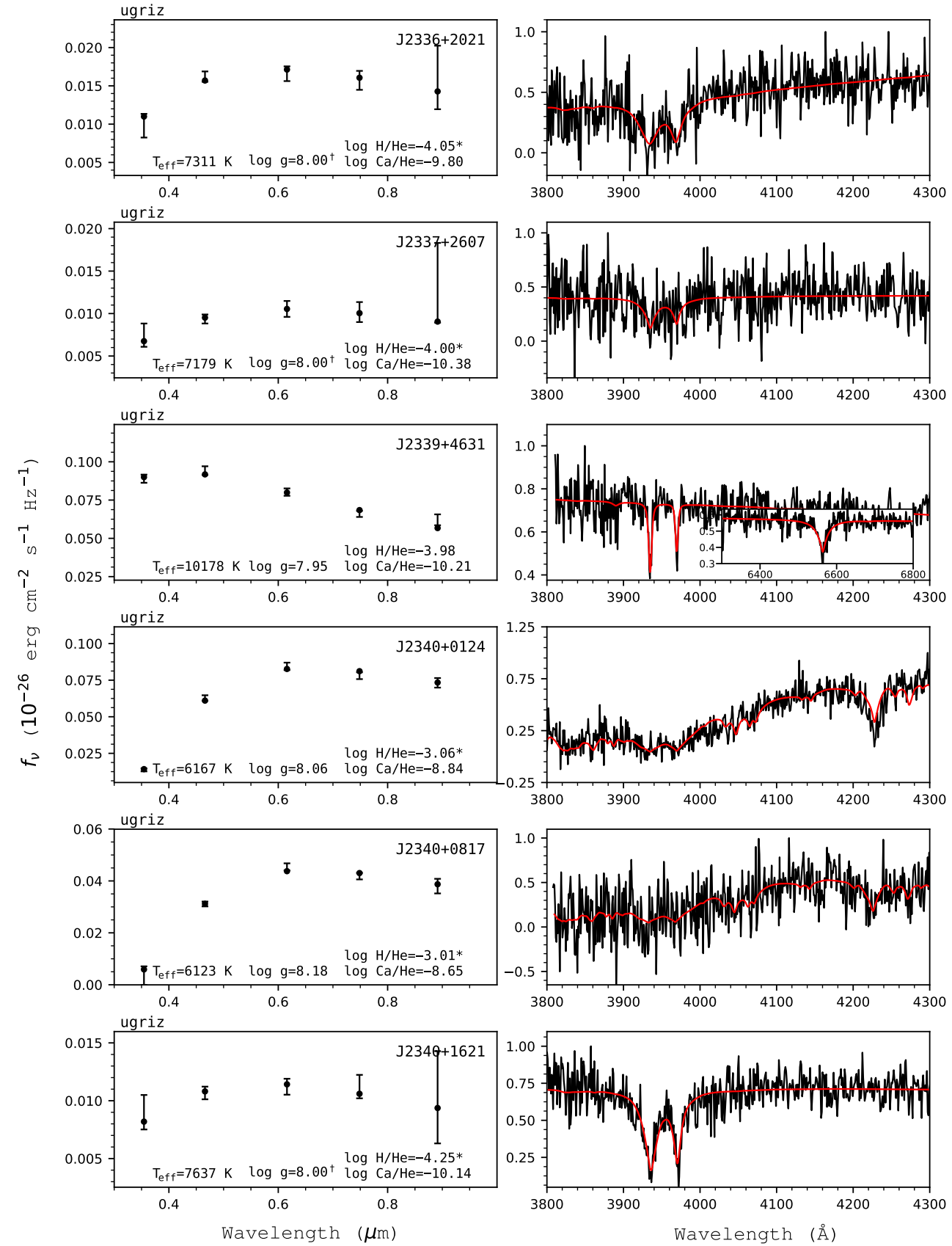

Figure 185. Fits to the DBZ/DZ(A) white dwarfs - continued. 

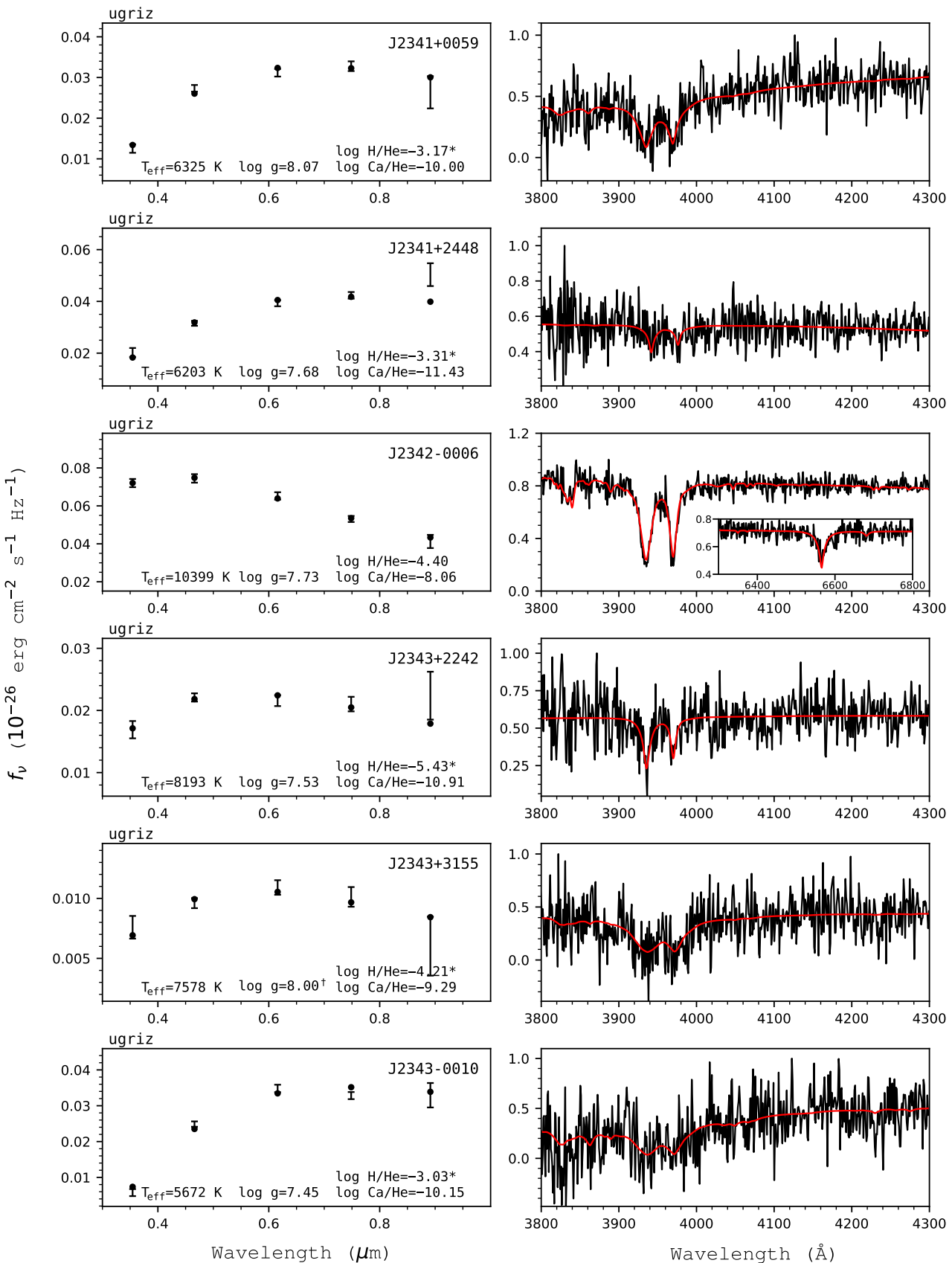

Figure 186. Fits to the DBZ/DZ(A) white dwarfs - continued. 

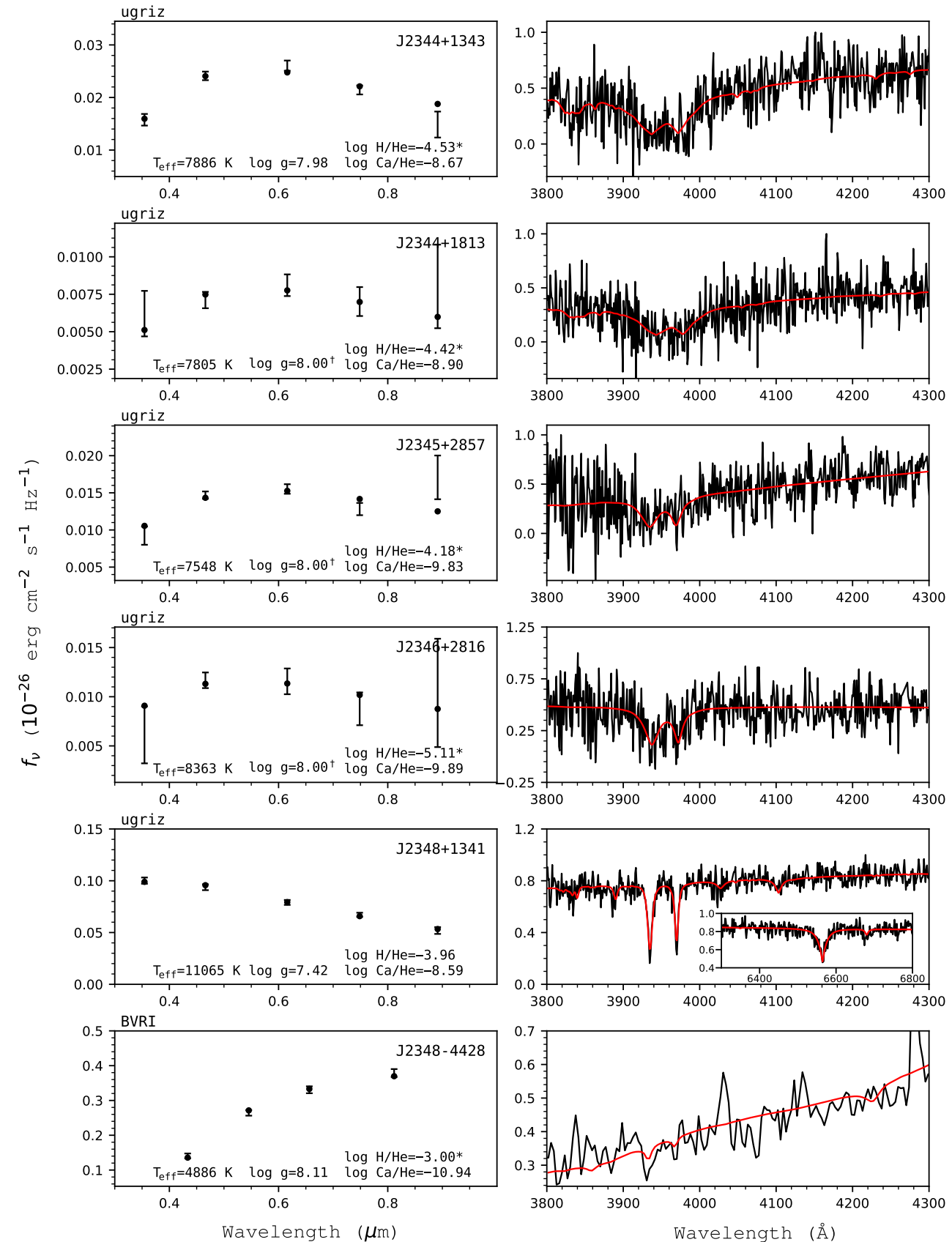

Figure 187. Fits to the DBZ/DZ(A) white dwarfs - continued. 

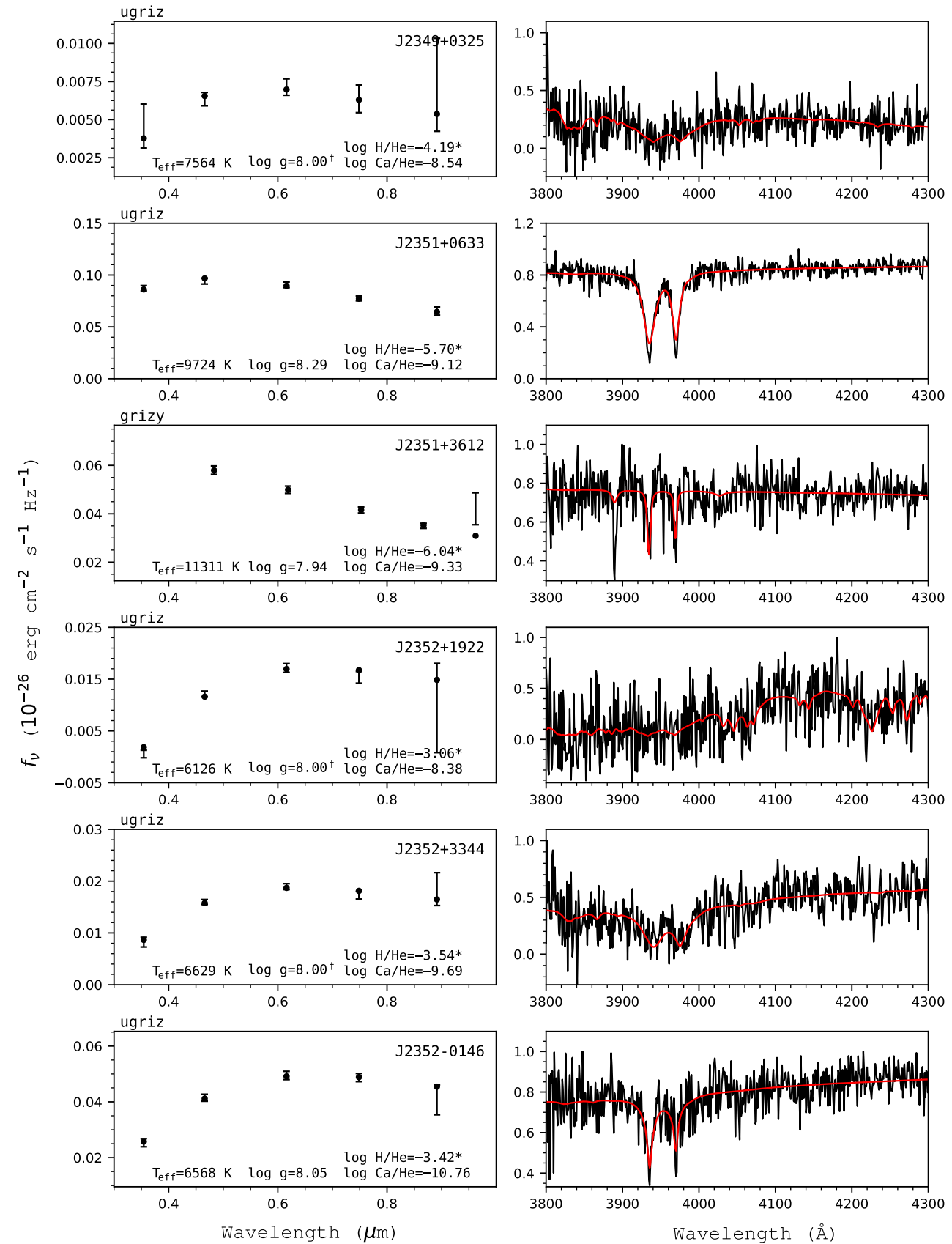

Figure 188. Fits to the DBZ/DZ(A) white dwarfs - continued. 

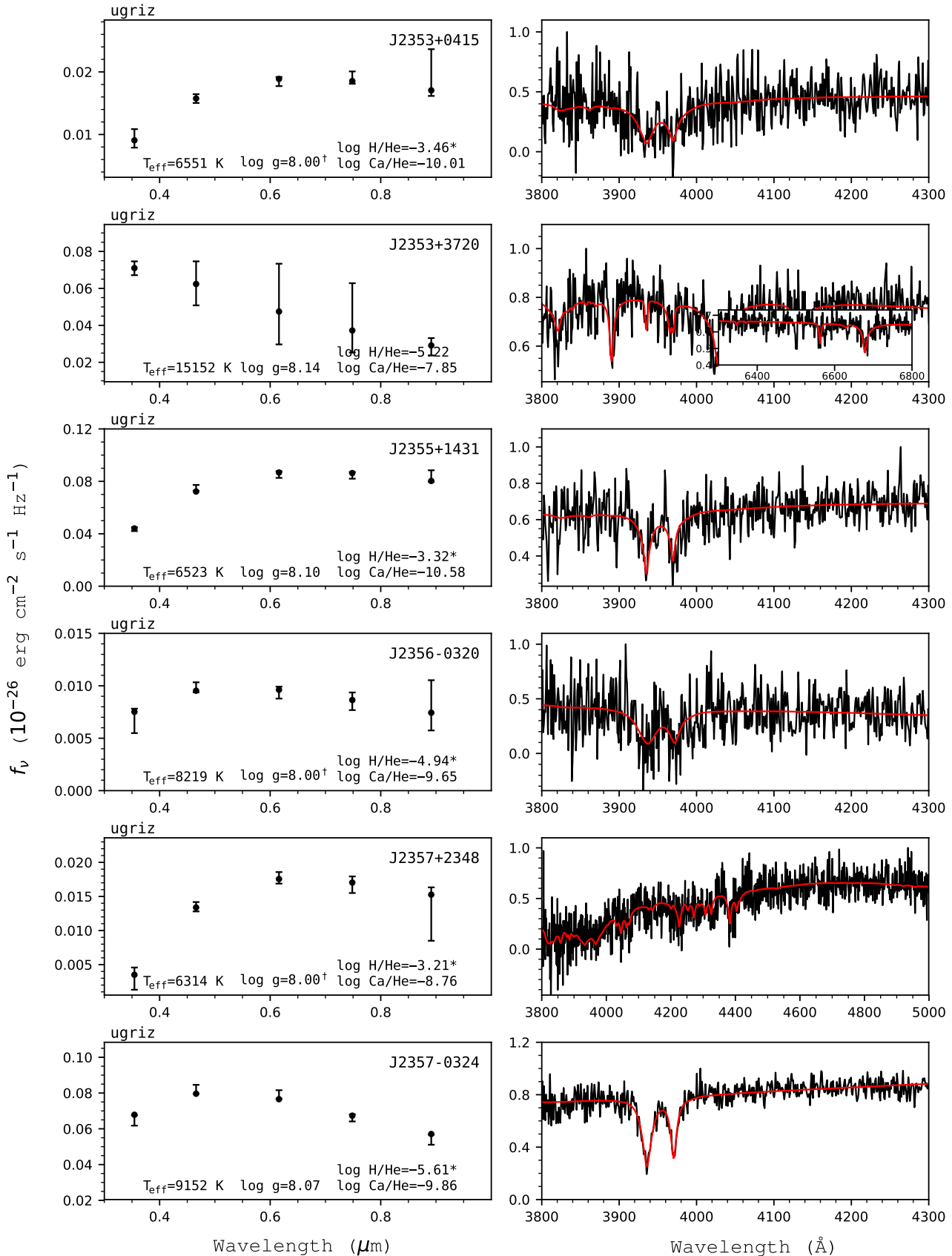

Figure 189. Fits to the DBZ/DZ(A) white dwarfs - continued. 

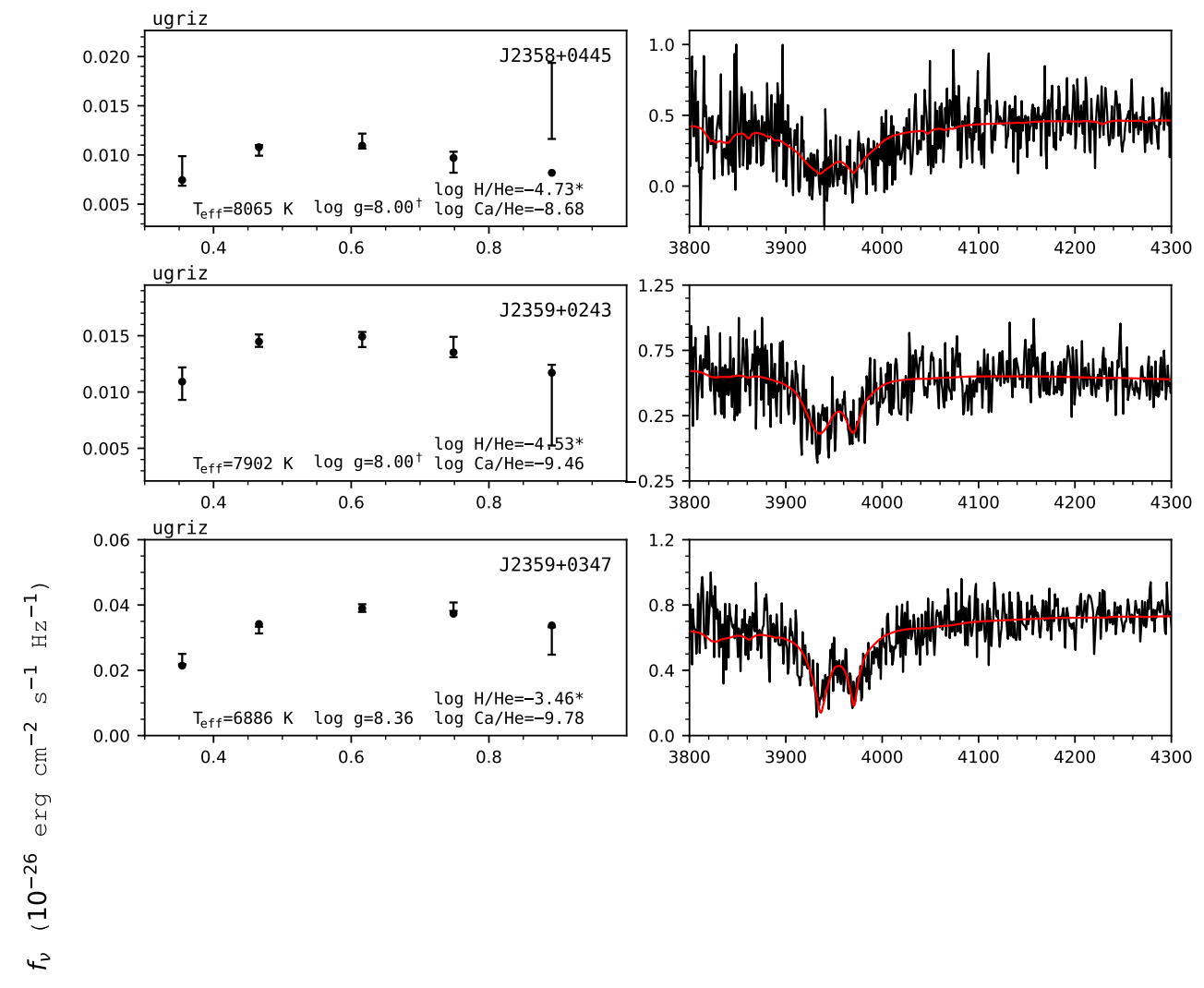

Figure 190. Fits to the DBZ/DZ(A) white dwarfs - continued. 

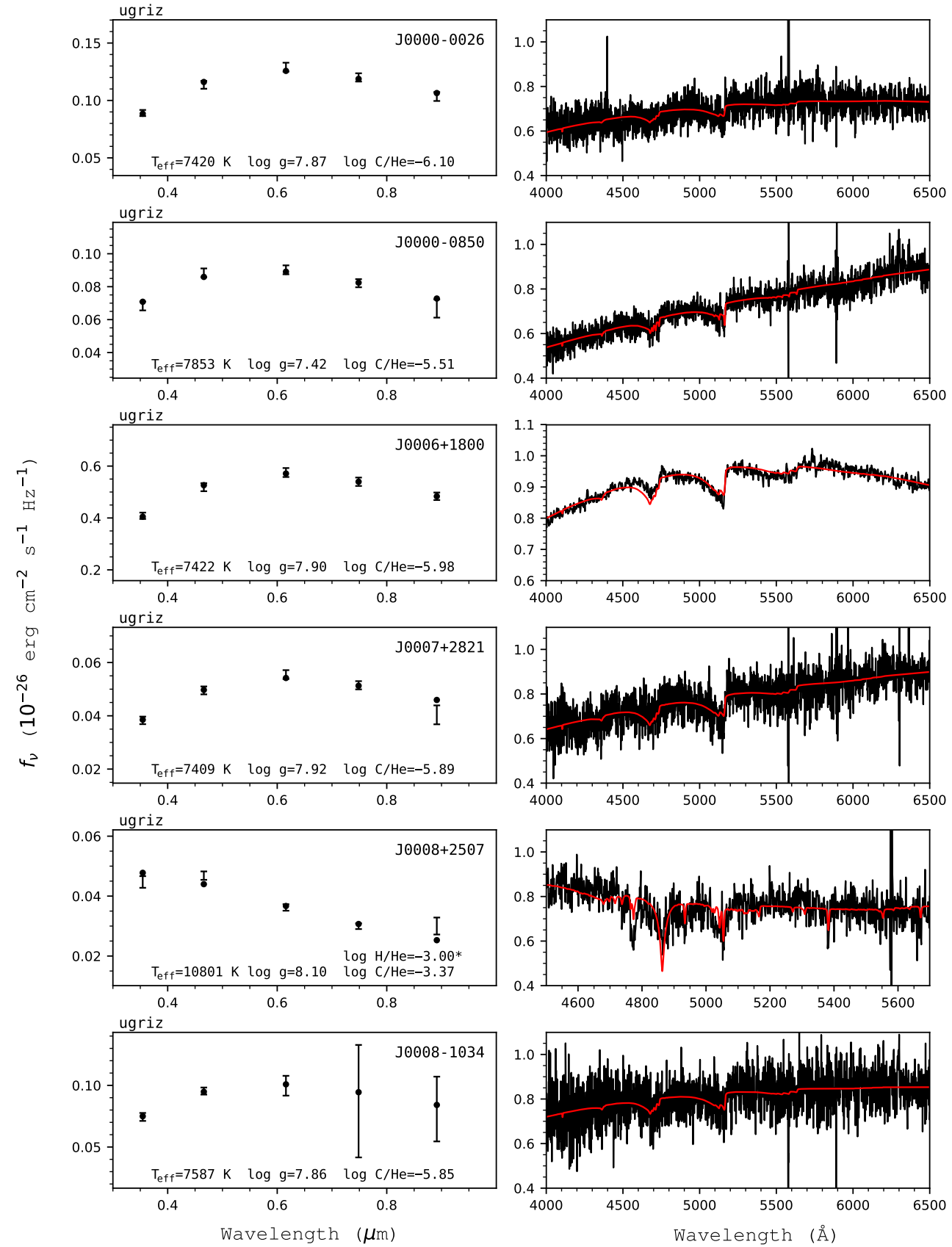

Figure 191. Fits to DQ white dwarfs in our sample. In the left panels, error bars represent the observed data, while filled circles correspond to our best fit model, with the atmospheric parameters given in each panel. The photometry used in the fit is indicated at the top left of each panel. A dagger symbol indicates that the $\log g$ value has been fixed at 8.0, when no trigonometric parallax is available. A star symbol indicates that the value of $\log \mathrm{H} / \mathrm{He}$ has been fixed rather than fitted. The right panels show our spectroscopic fits (red) to the normalized observed spectra (black). 

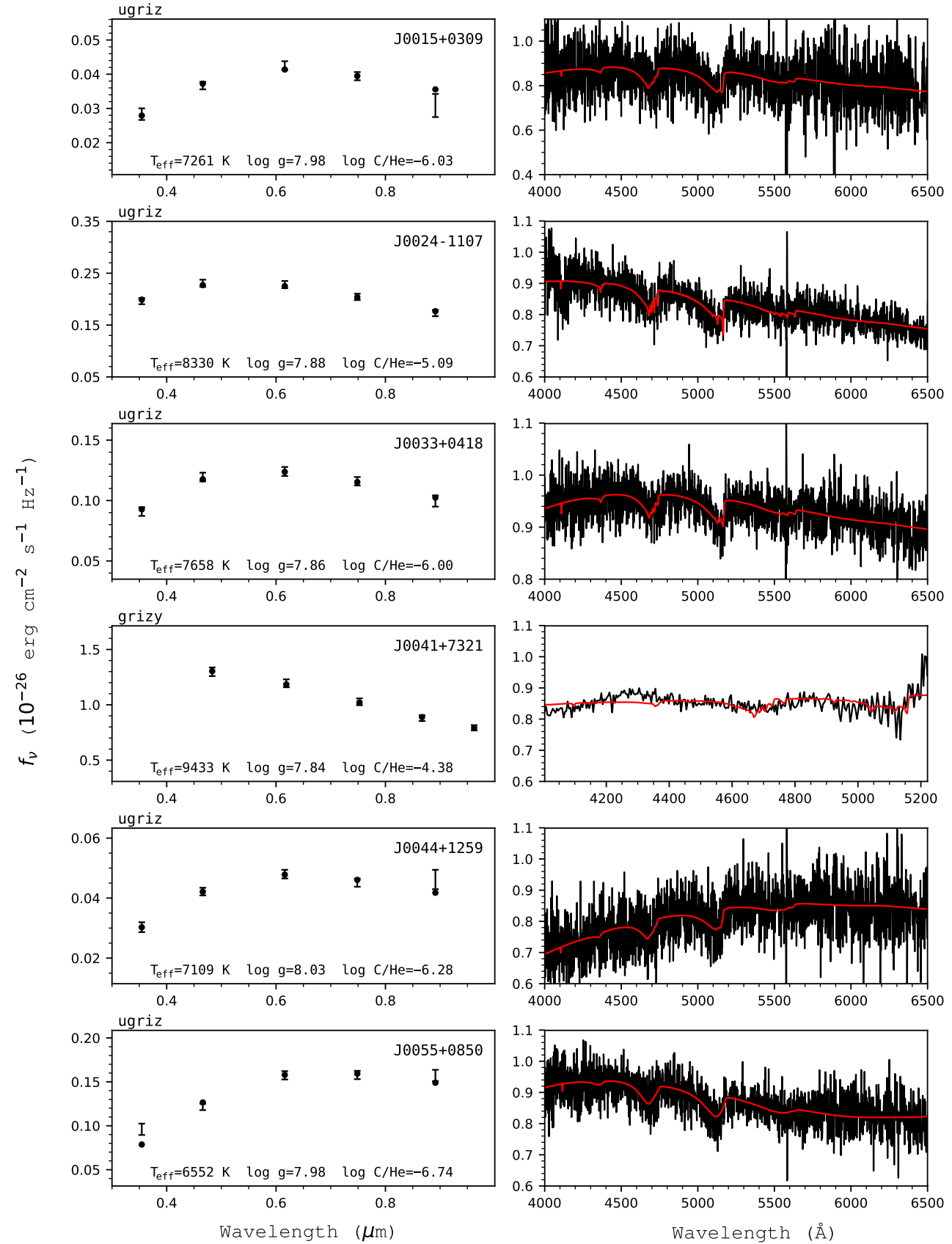

Figure 192. Fits to the DQ white dwarfs - continued. 

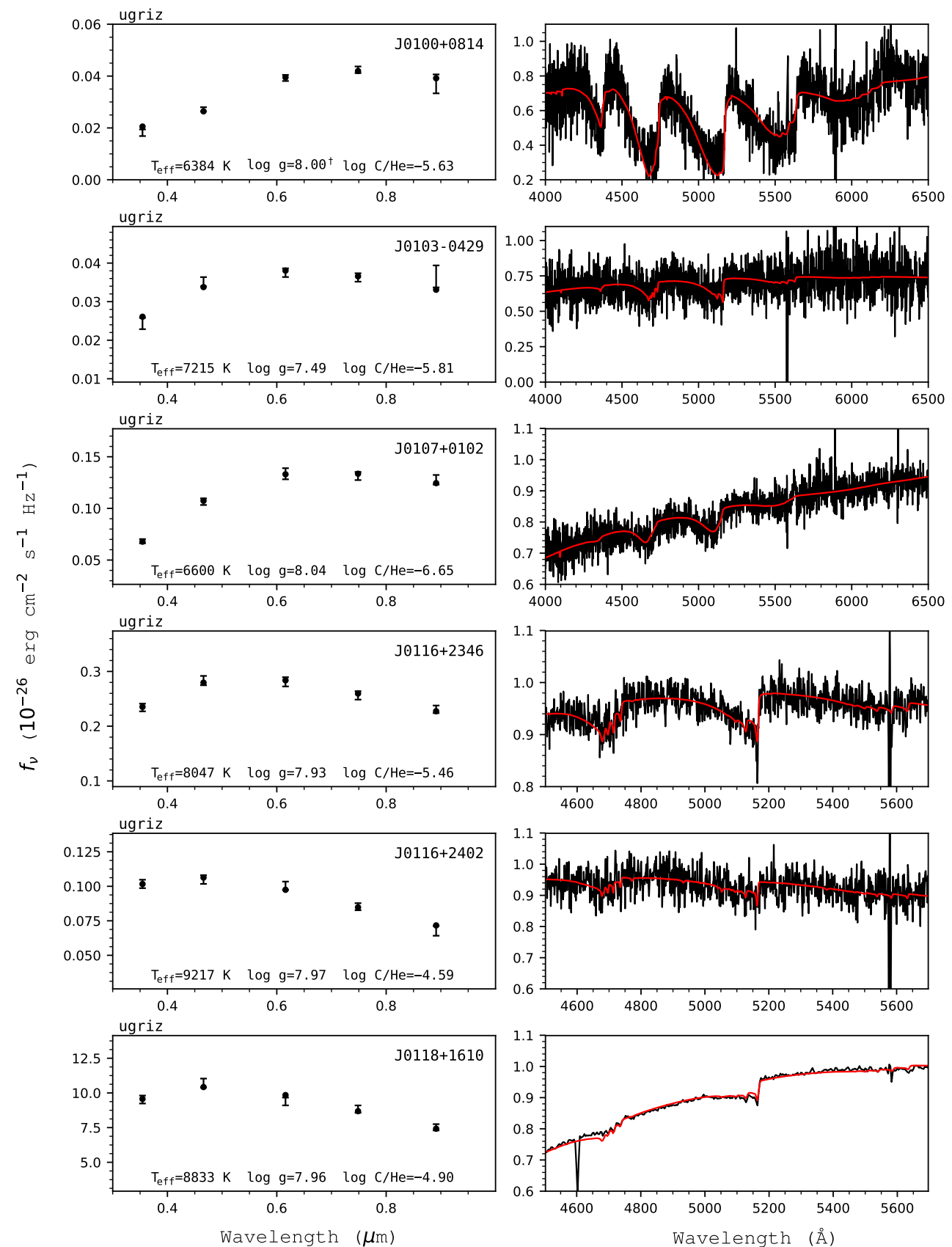

Figure 193. Fits to the DQ white dwarfs - continued. 

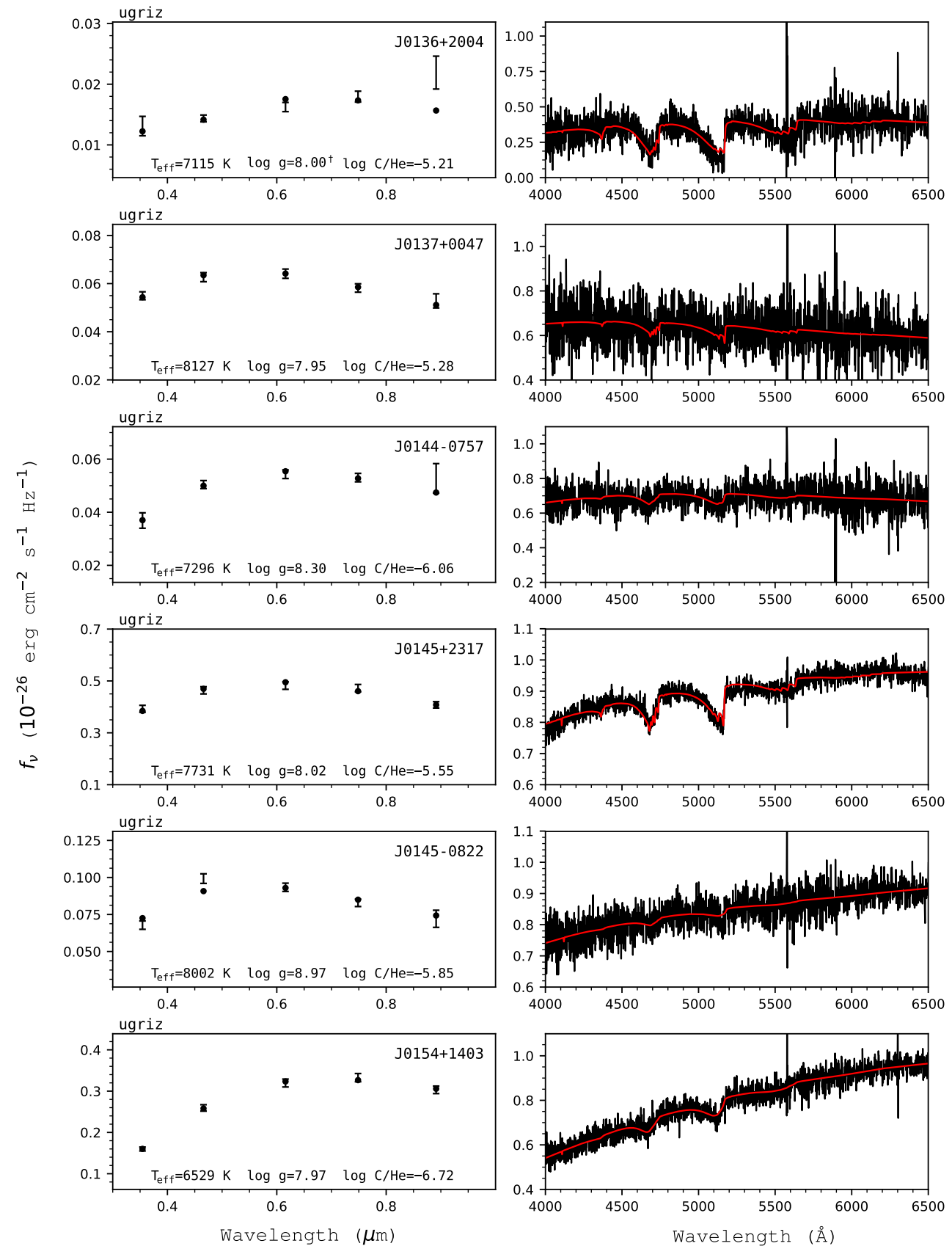

Figure 194. Fits to the DQ white dwarfs - continued. 

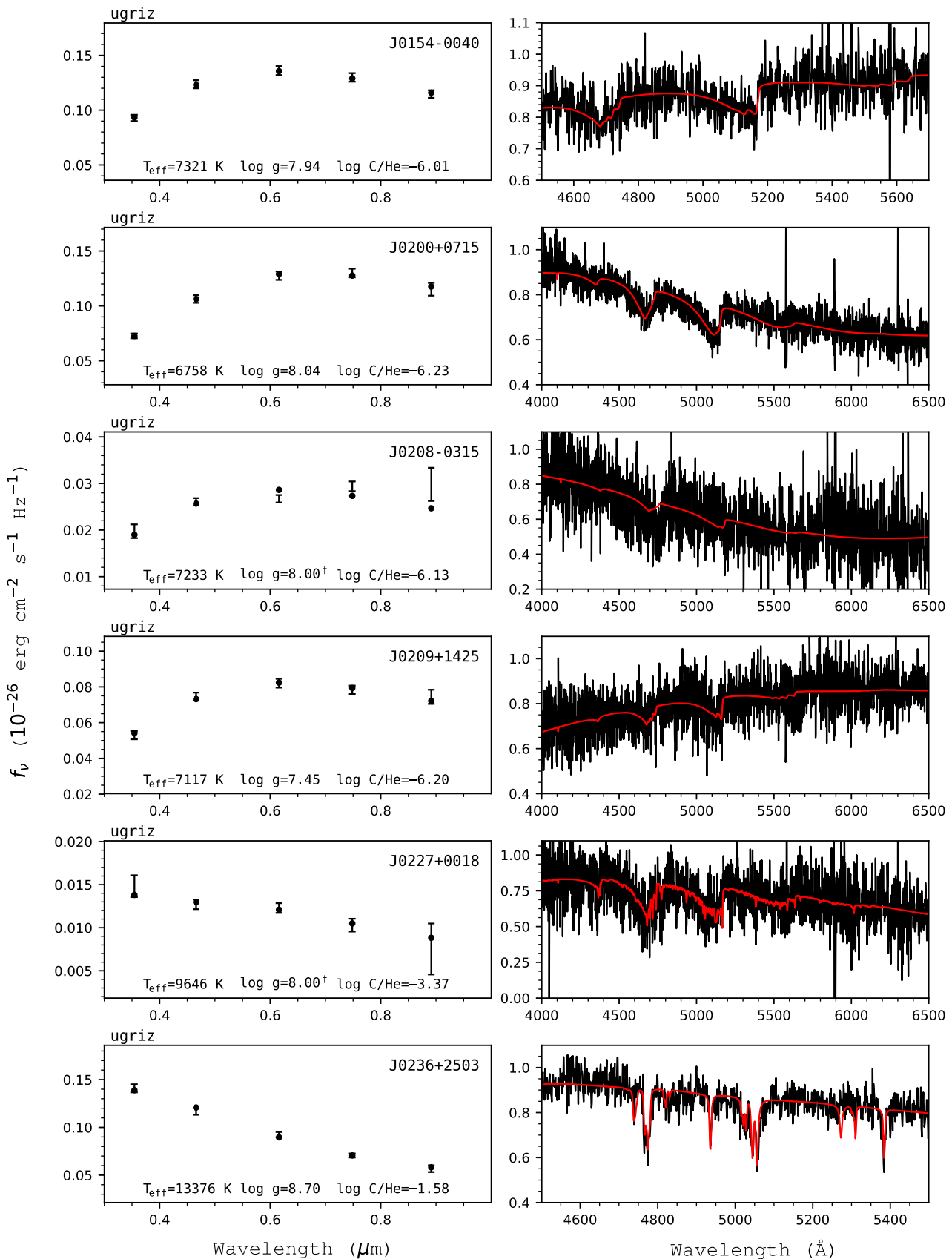

Figure 195. Fits to the DQ white dwarfs - continued. 

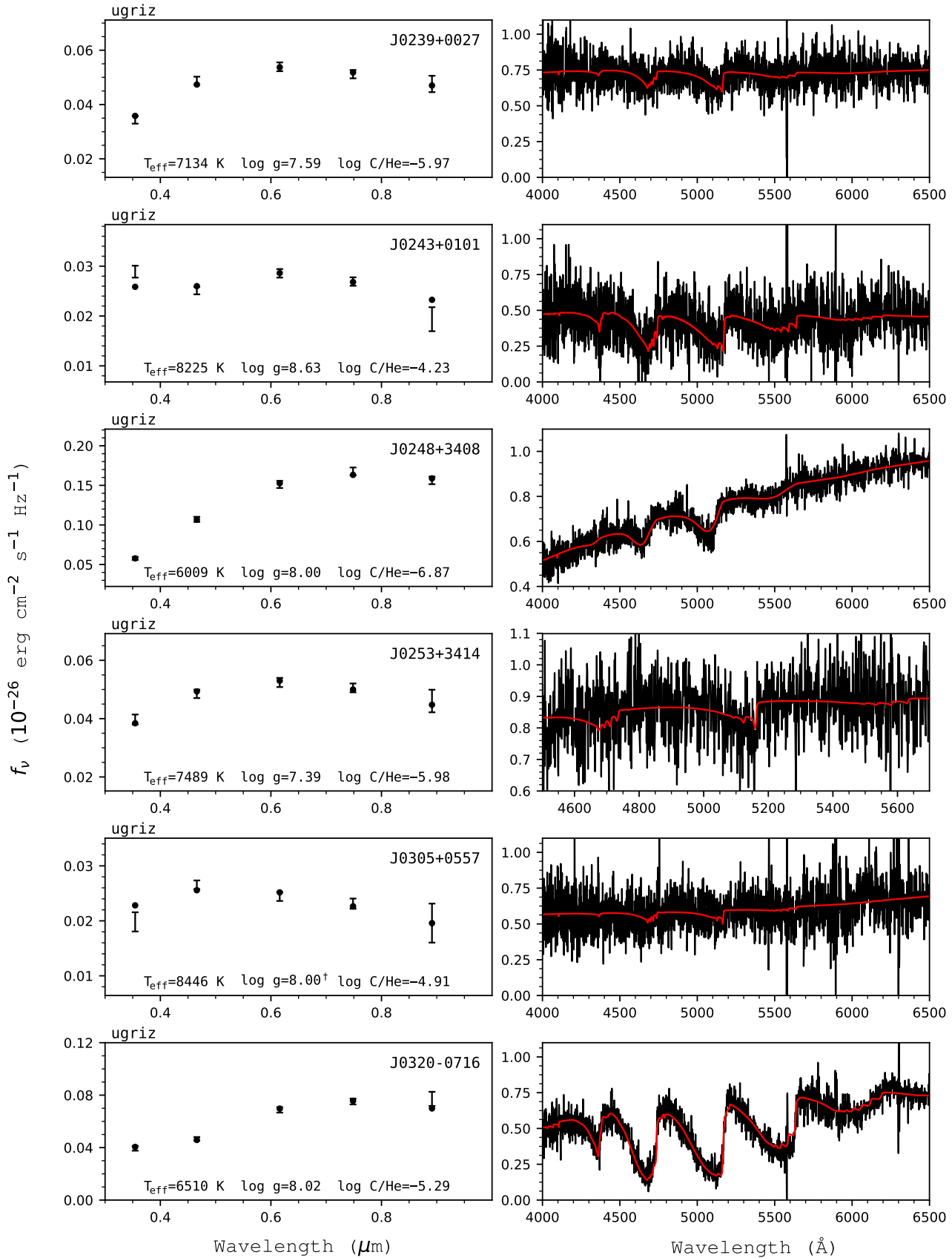

Figure 196. Fits to the DQ white dwarfs - continued. 

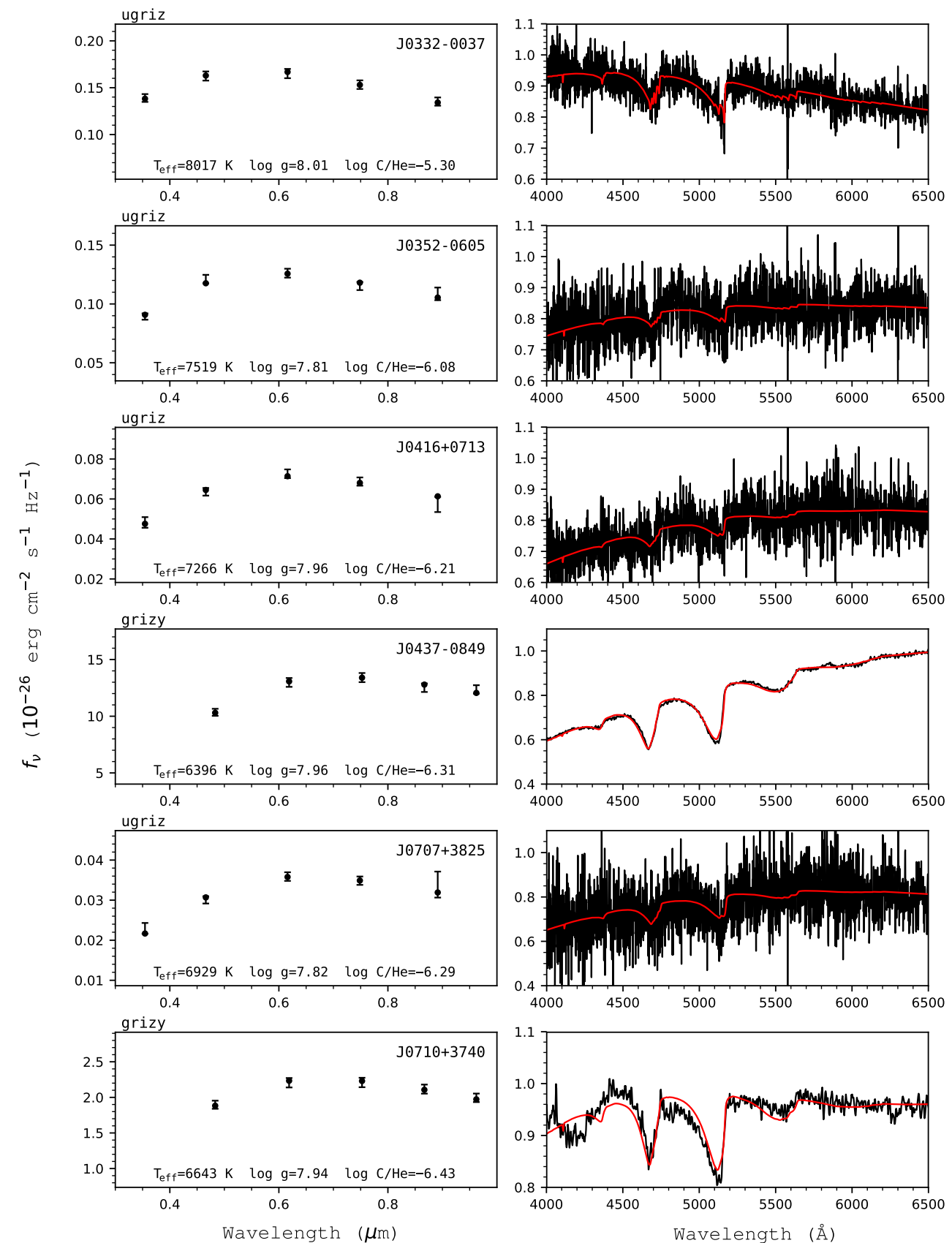

Figure 197. Fits to the DQ white dwarfs - continued. 

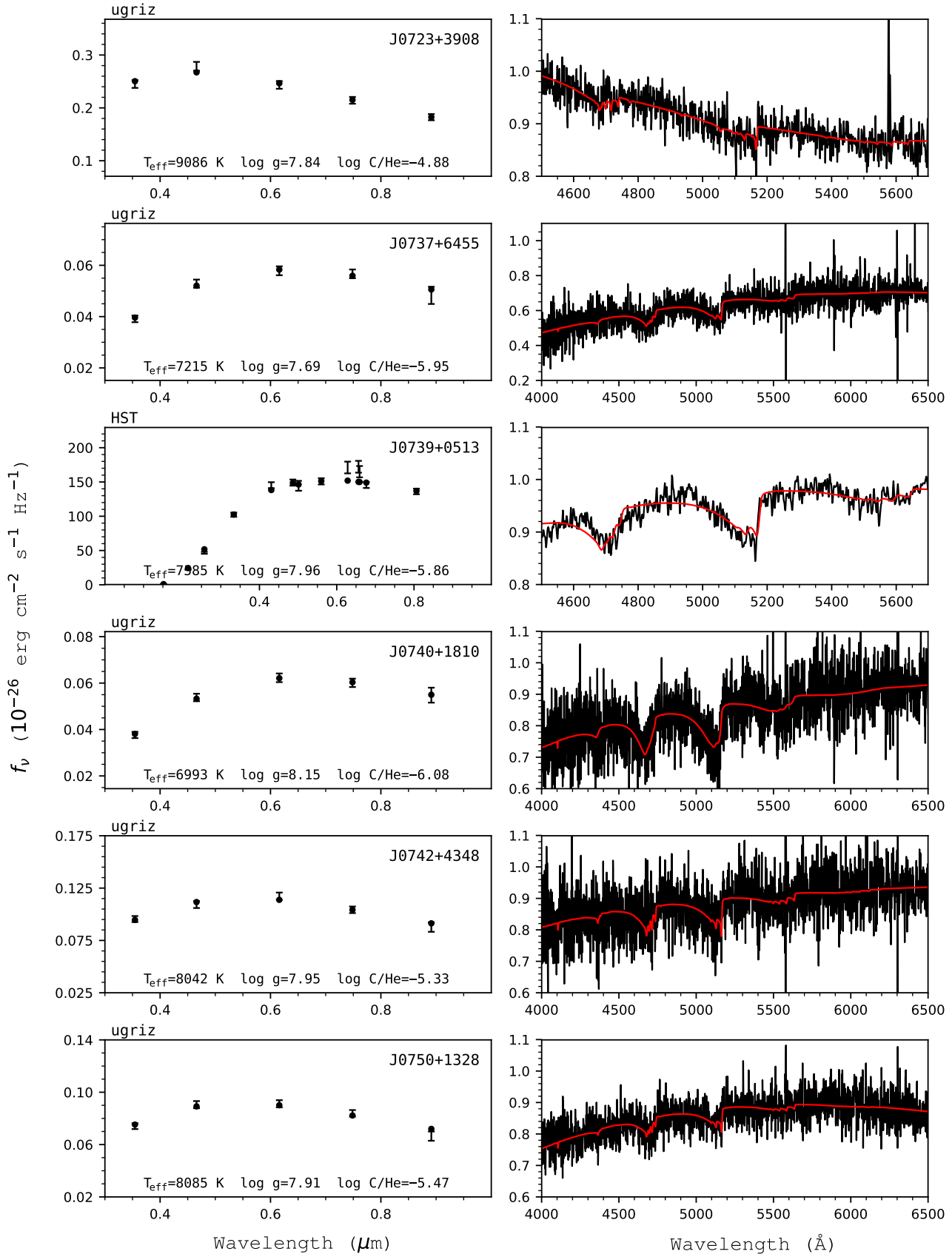

Figure 198. Fits to the DQ white dwarfs - continued. 

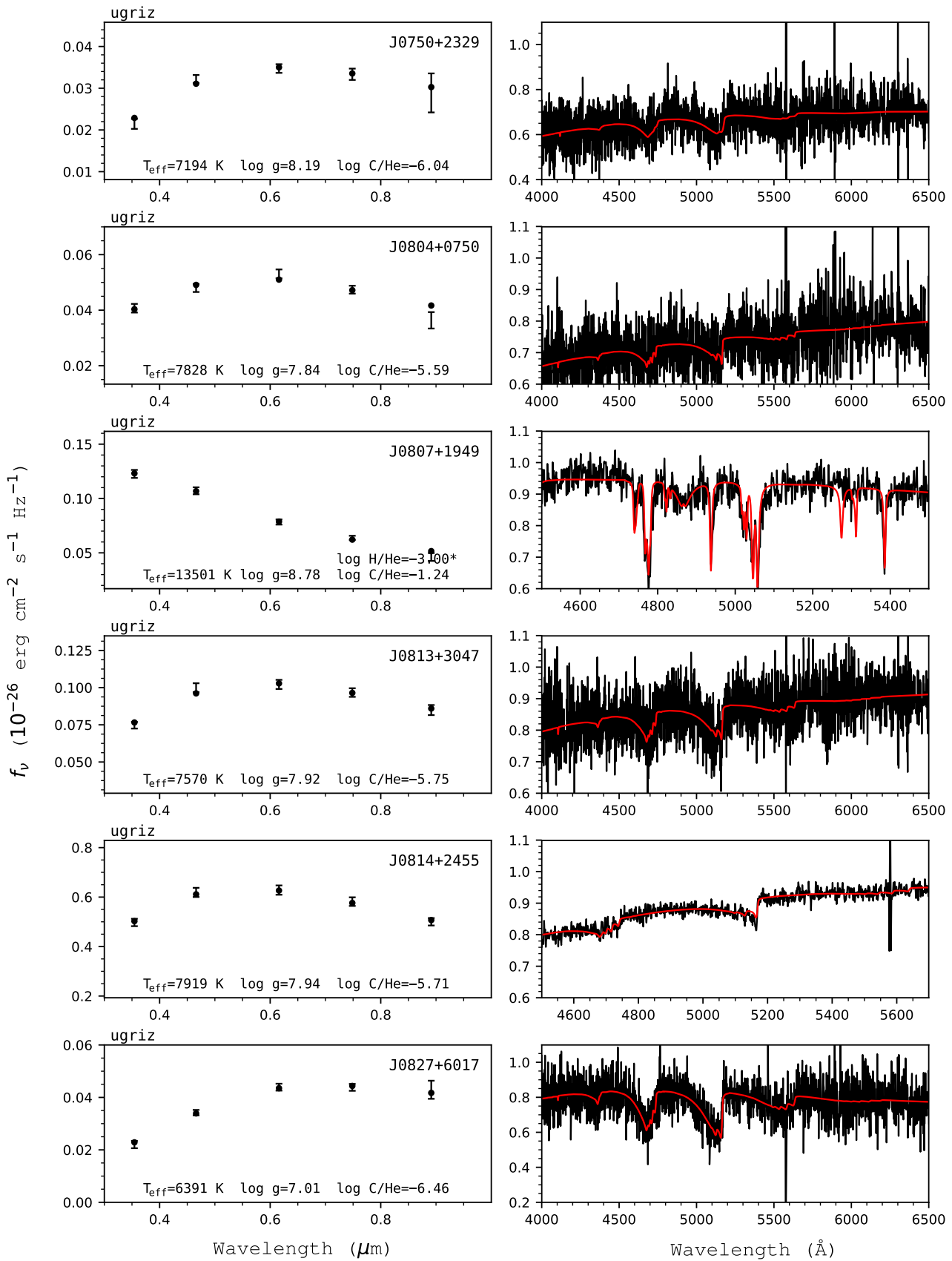

Figure 199. Fits to the DQ white dwarfs - continued. 

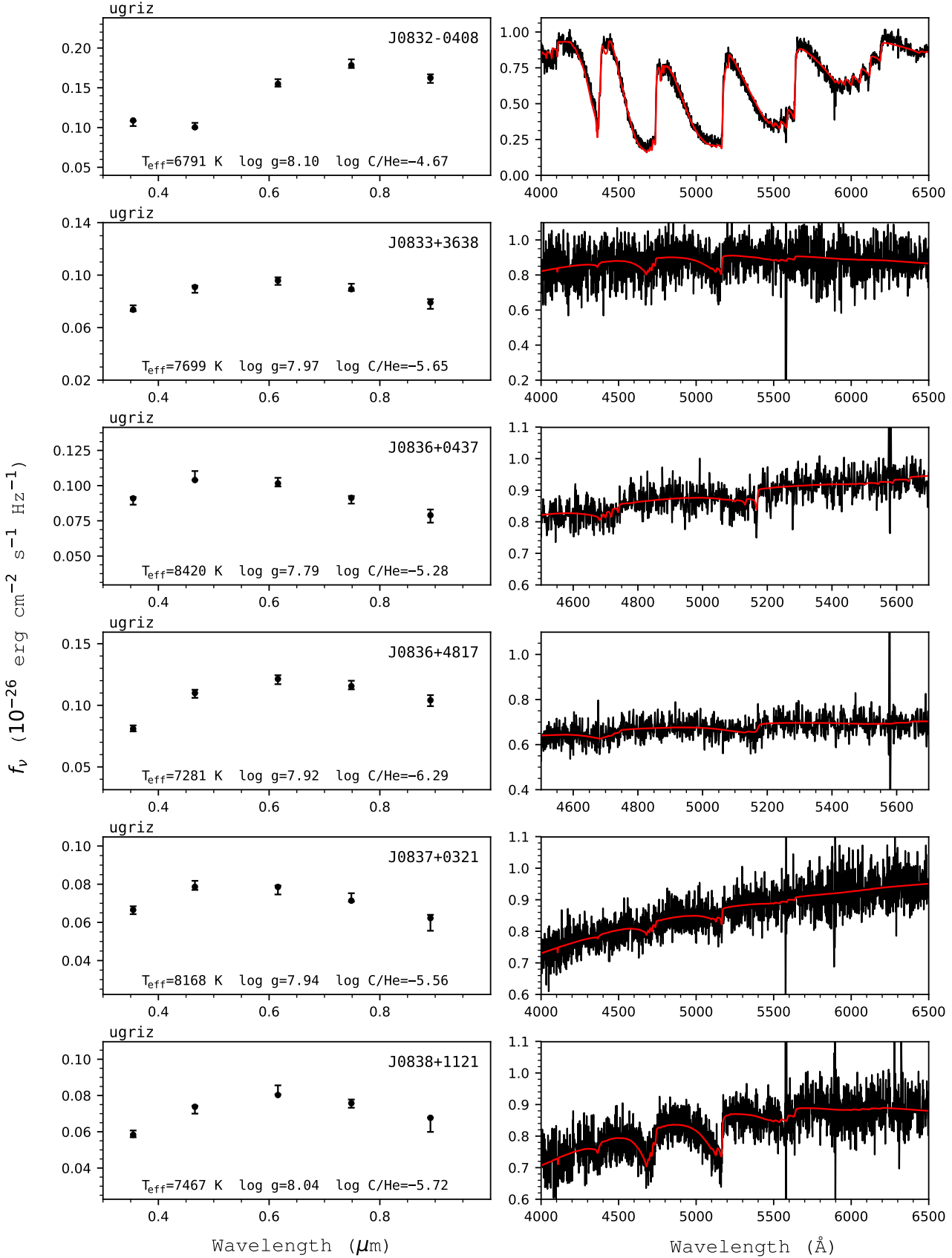

Figure 200. Fits to the DQ white dwarfs - continued. 

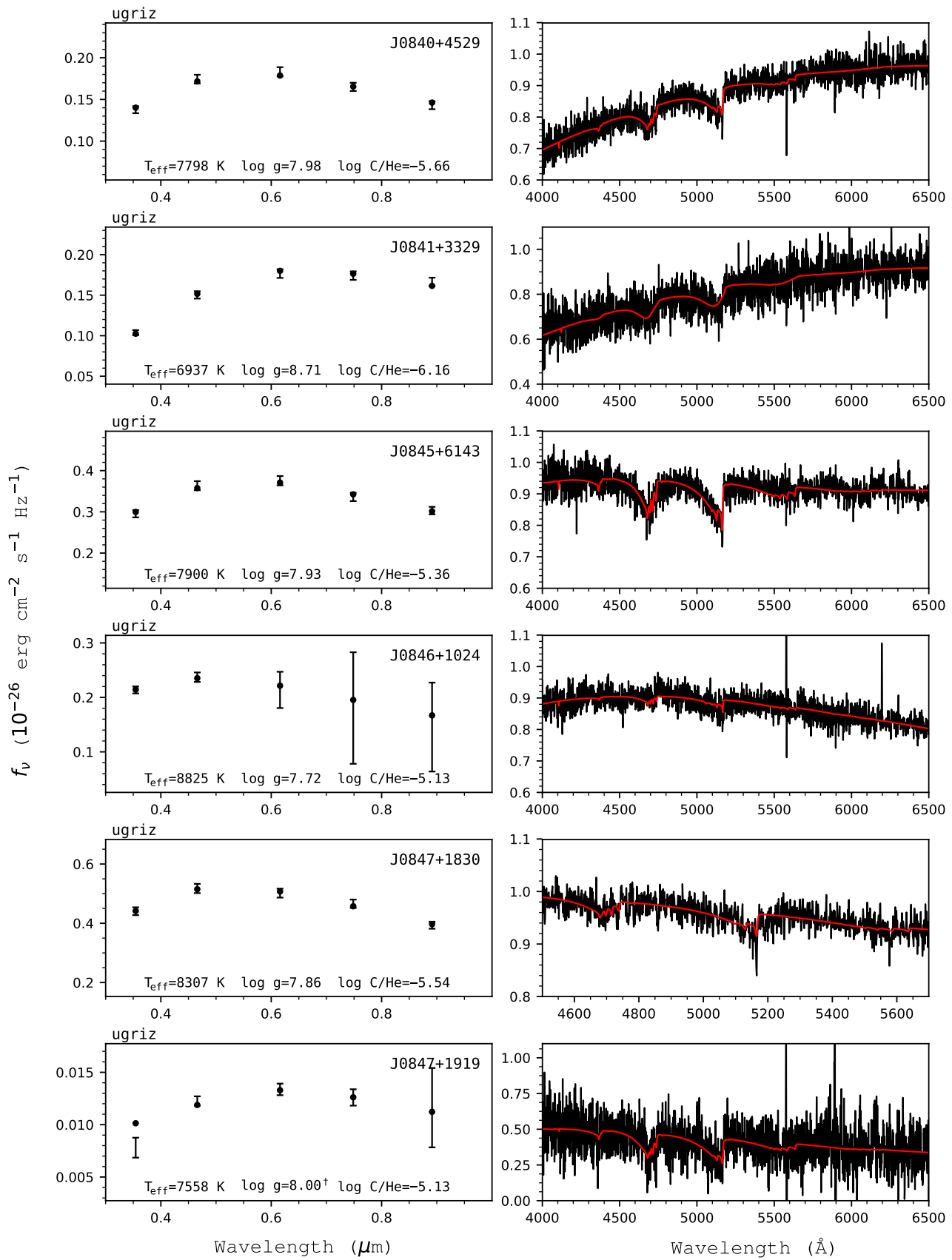

Figure 201. Fits to the DQ white dwarfs - continued. 

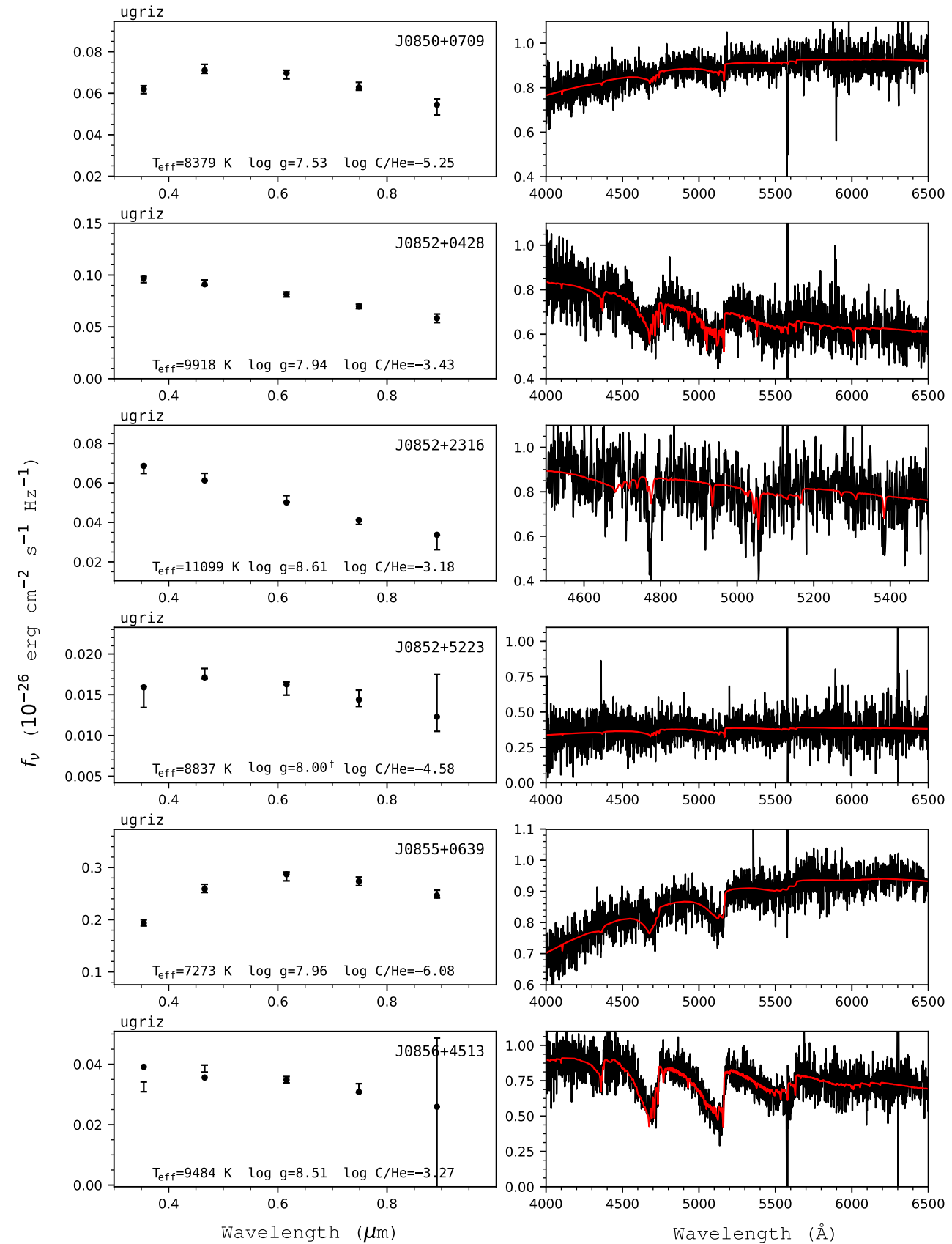

Figure 202. Fits to the DQ white dwarfs - continued. 

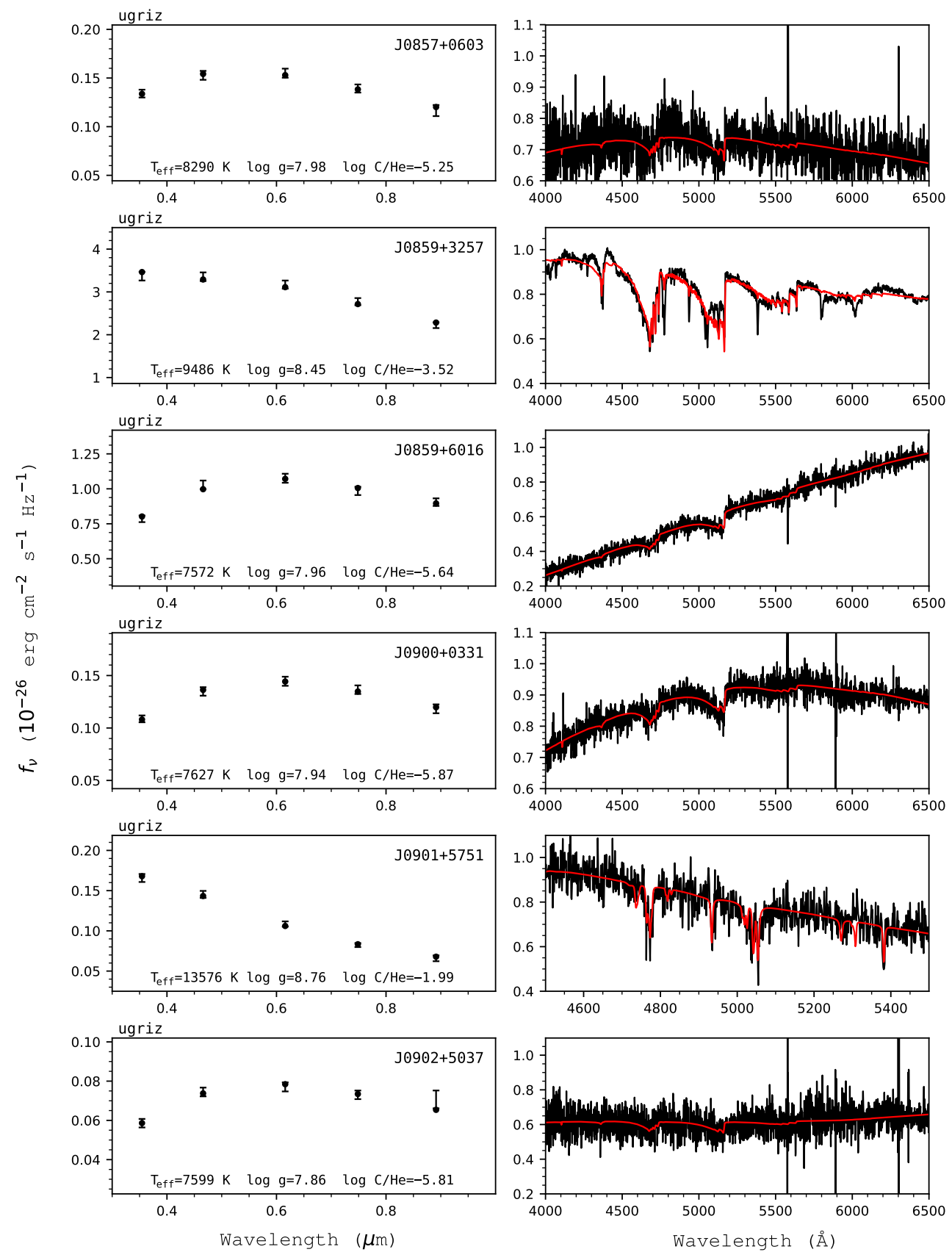

Figure 203. Fits to the DQ white dwarfs - continued. 

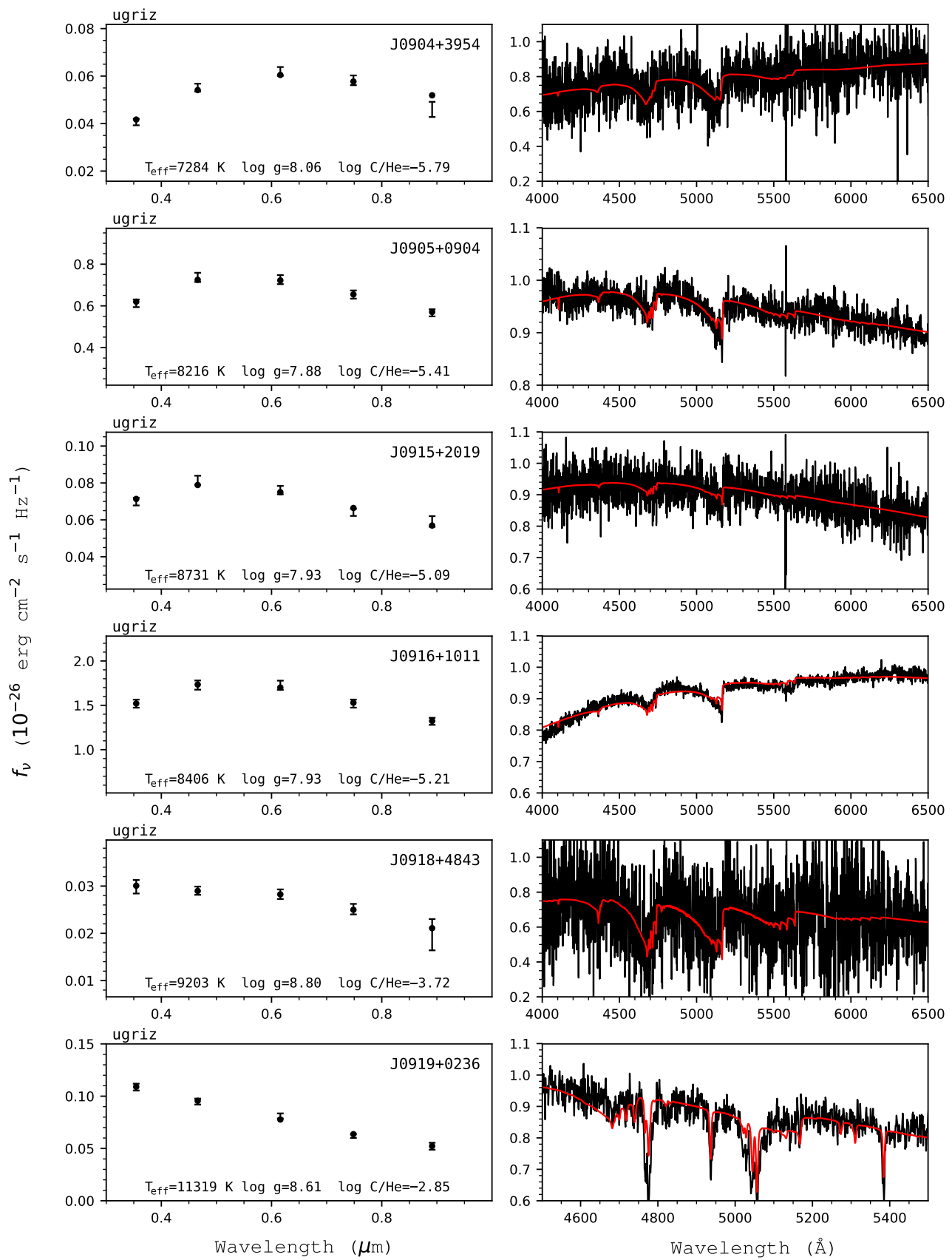

Figure 204. Fits to the DQ white dwarfs - continued. 

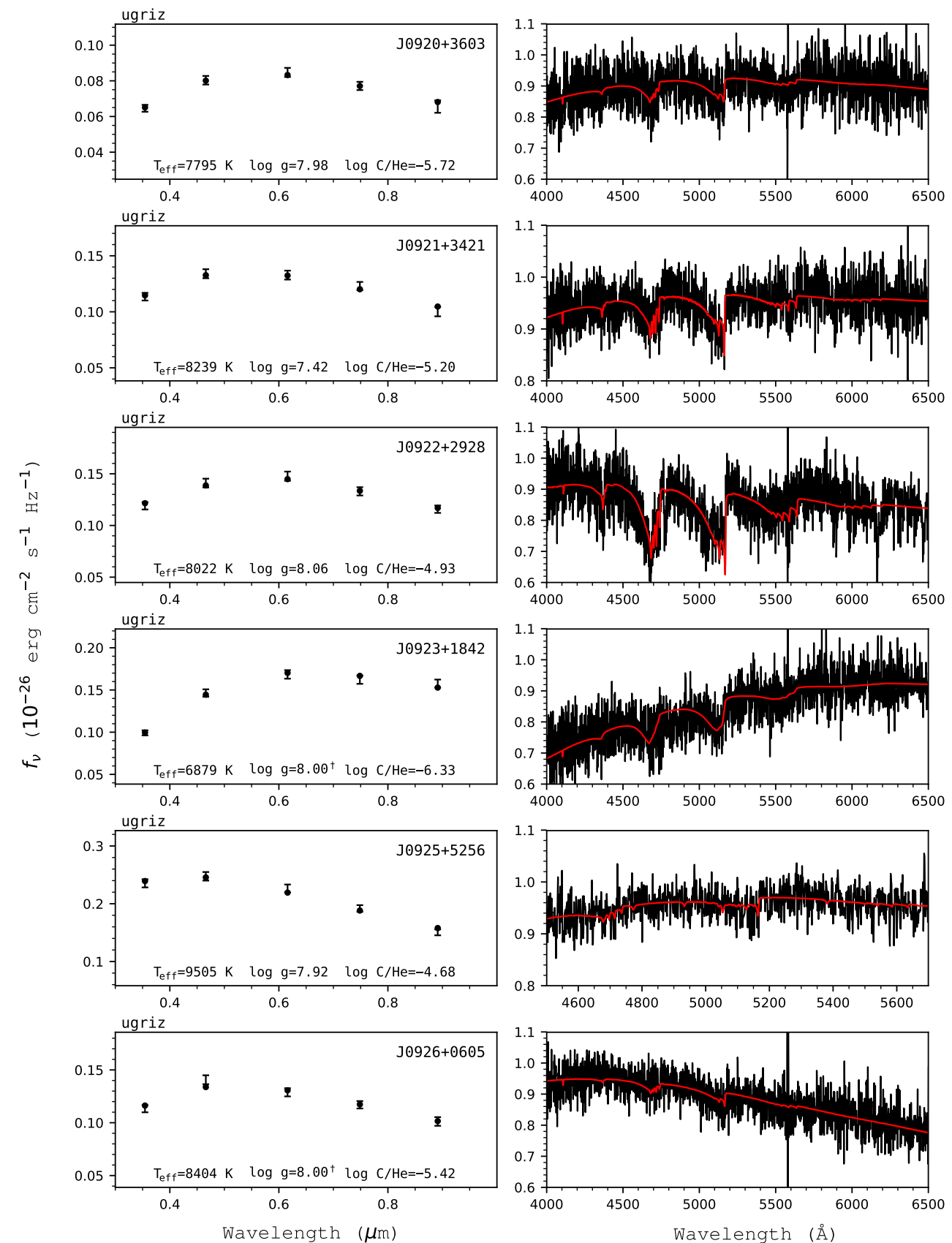

Figure 205. Fits to the DQ white dwarfs - continued. 

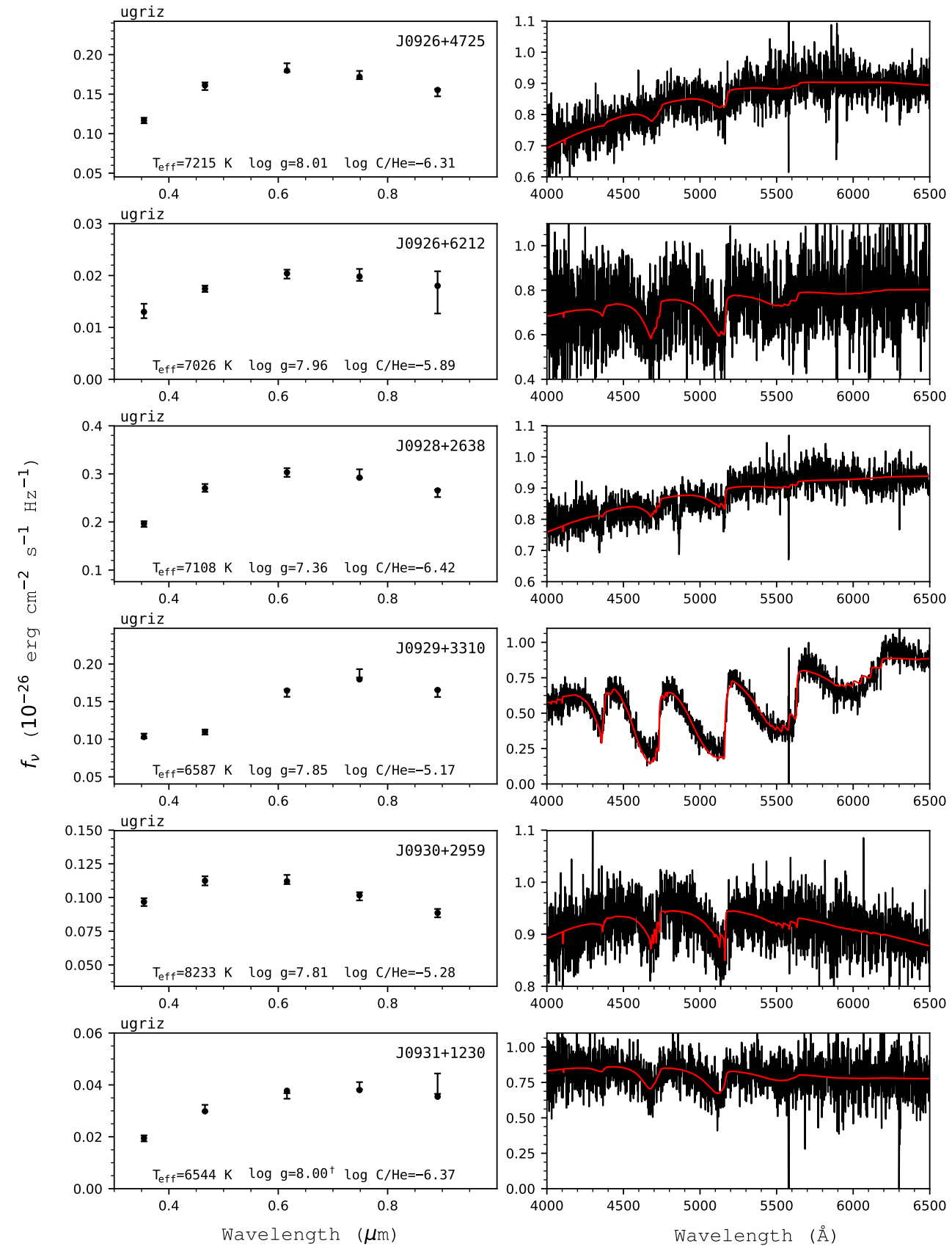

Figure 206. Fits to the DQ white dwarfs - continued. 

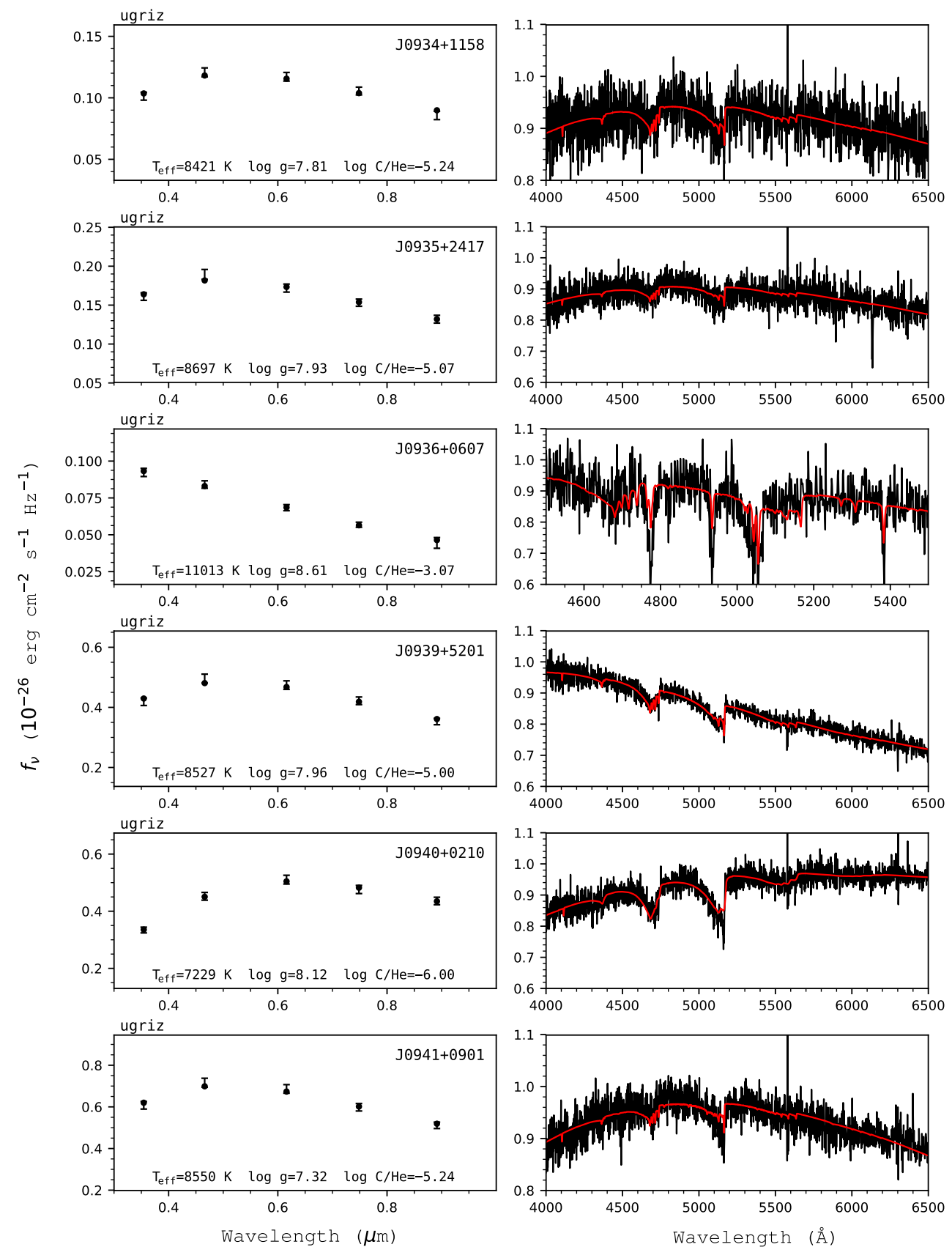

Figure 207. Fits to the DQ white dwarfs - continued. 

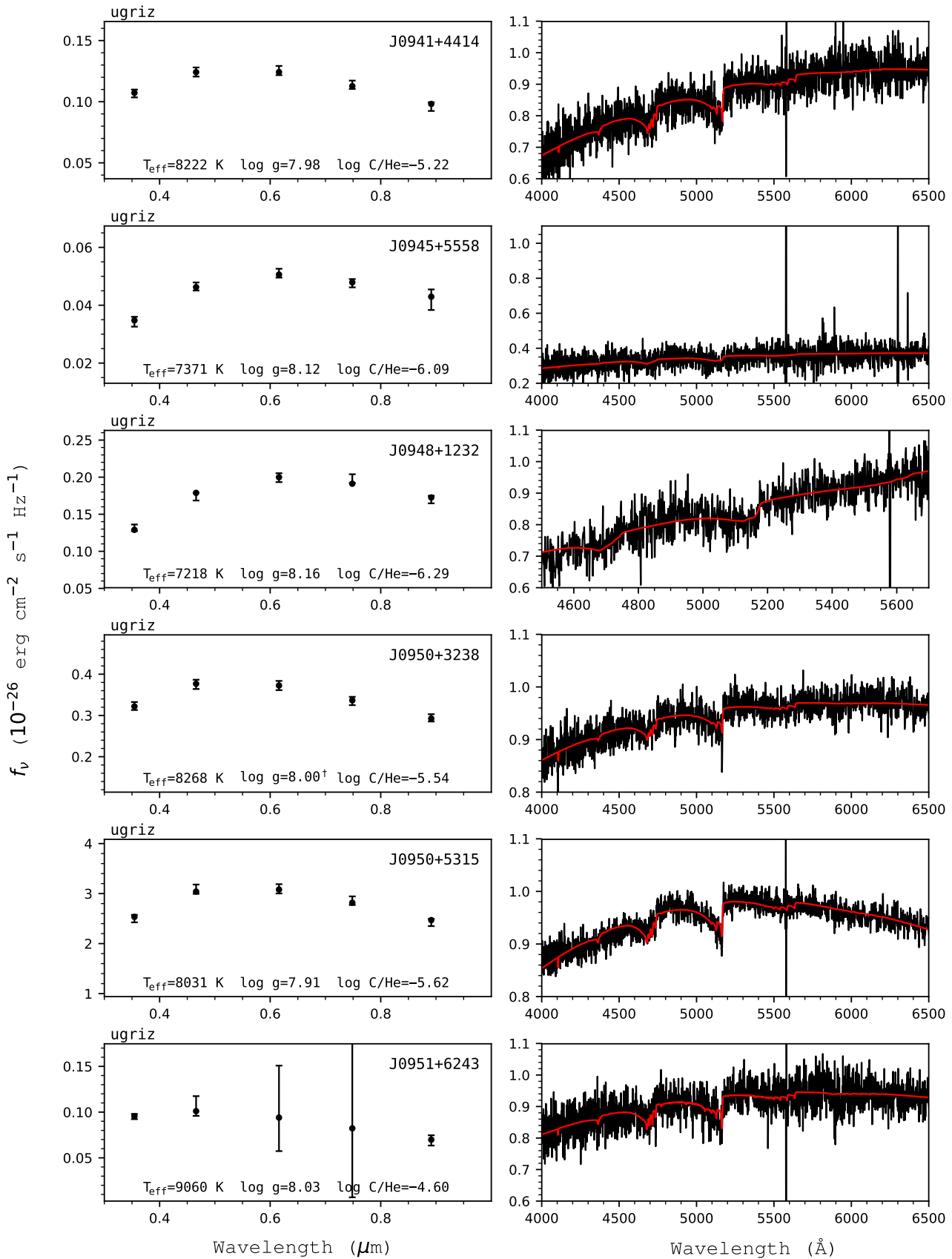

Figure 208. Fits to the DQ white dwarfs - continued. 

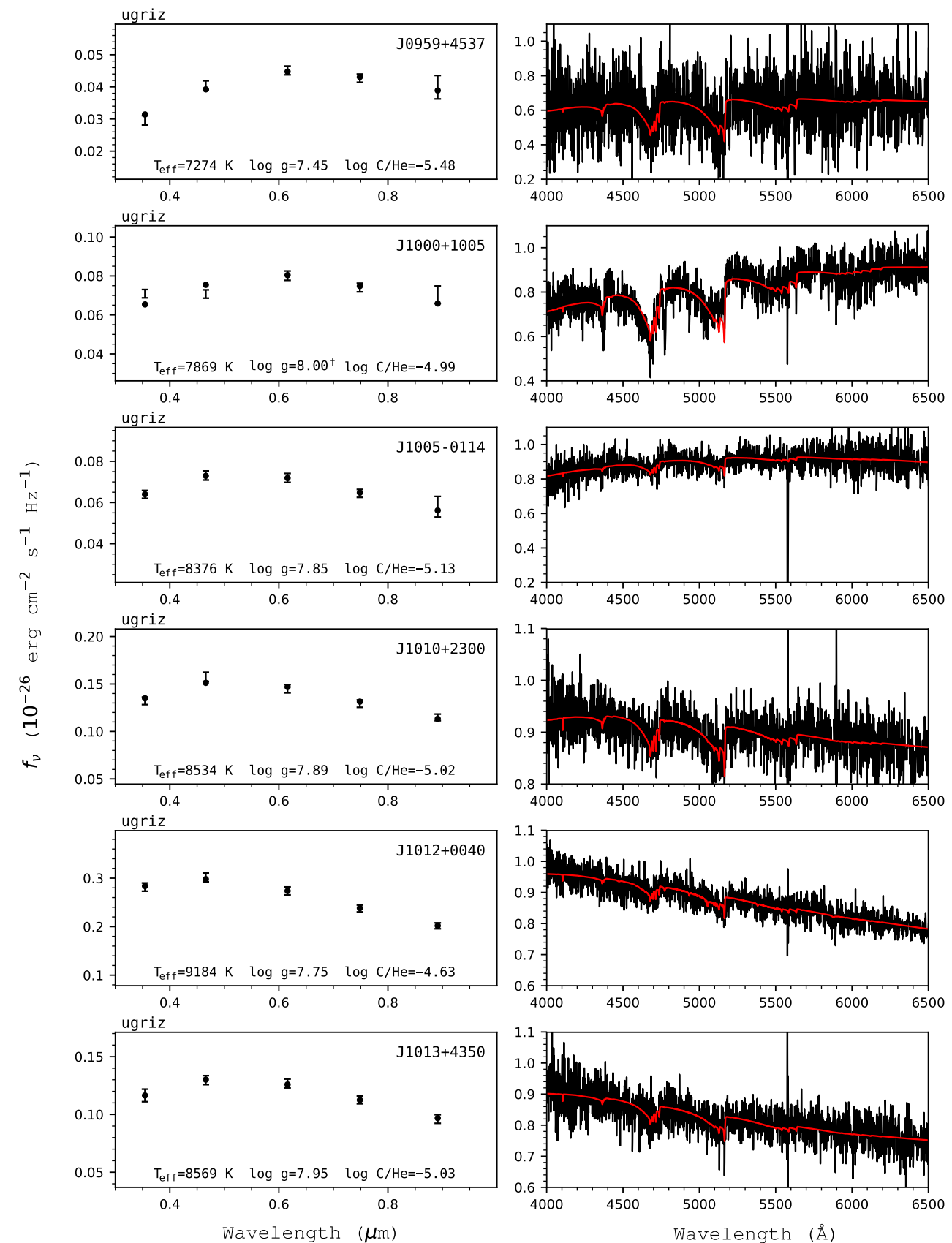

Figure 209. Fits to the DQ white dwarfs - continued. 

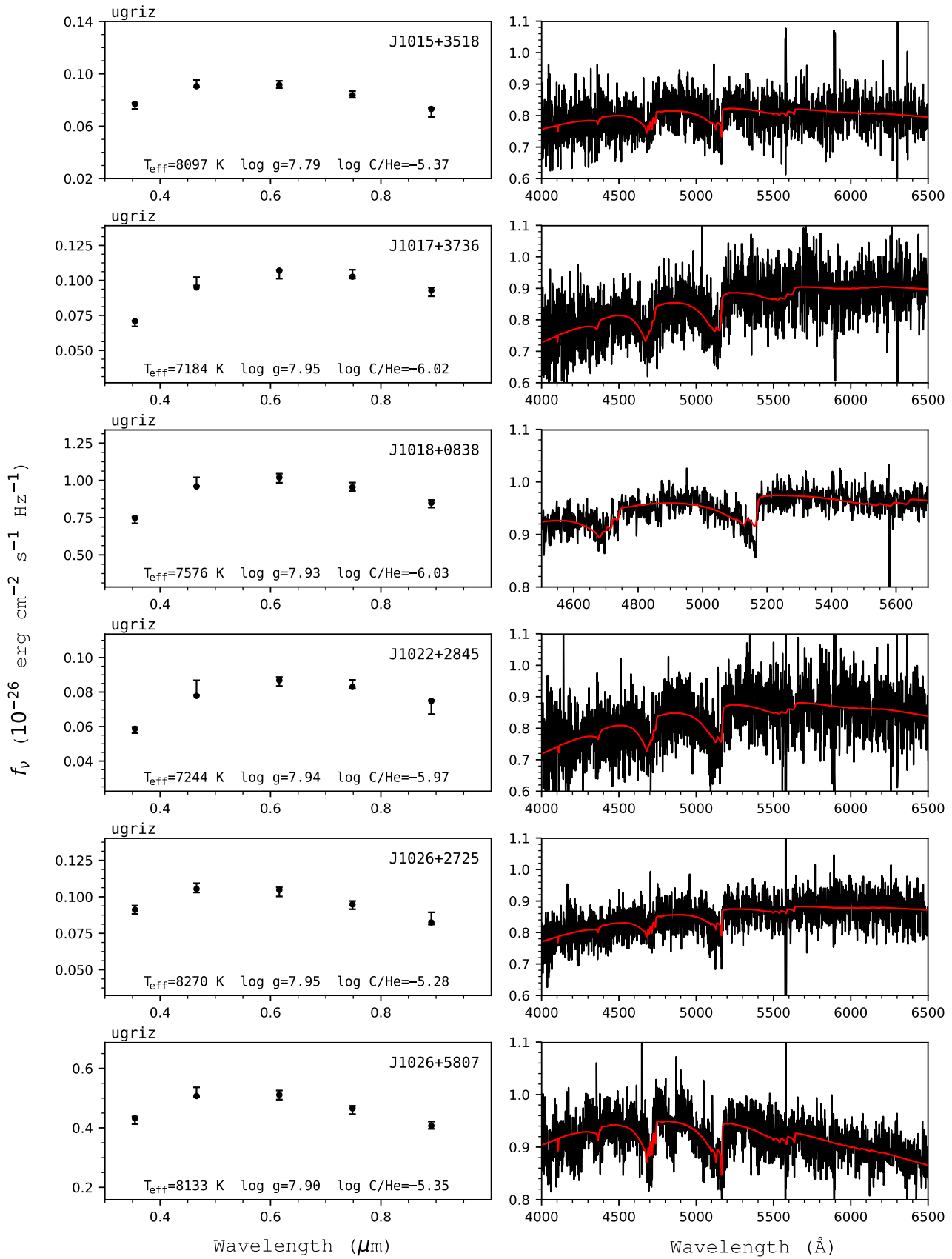

Figure 210. Fits to the DQ white dwarfs - continued. 

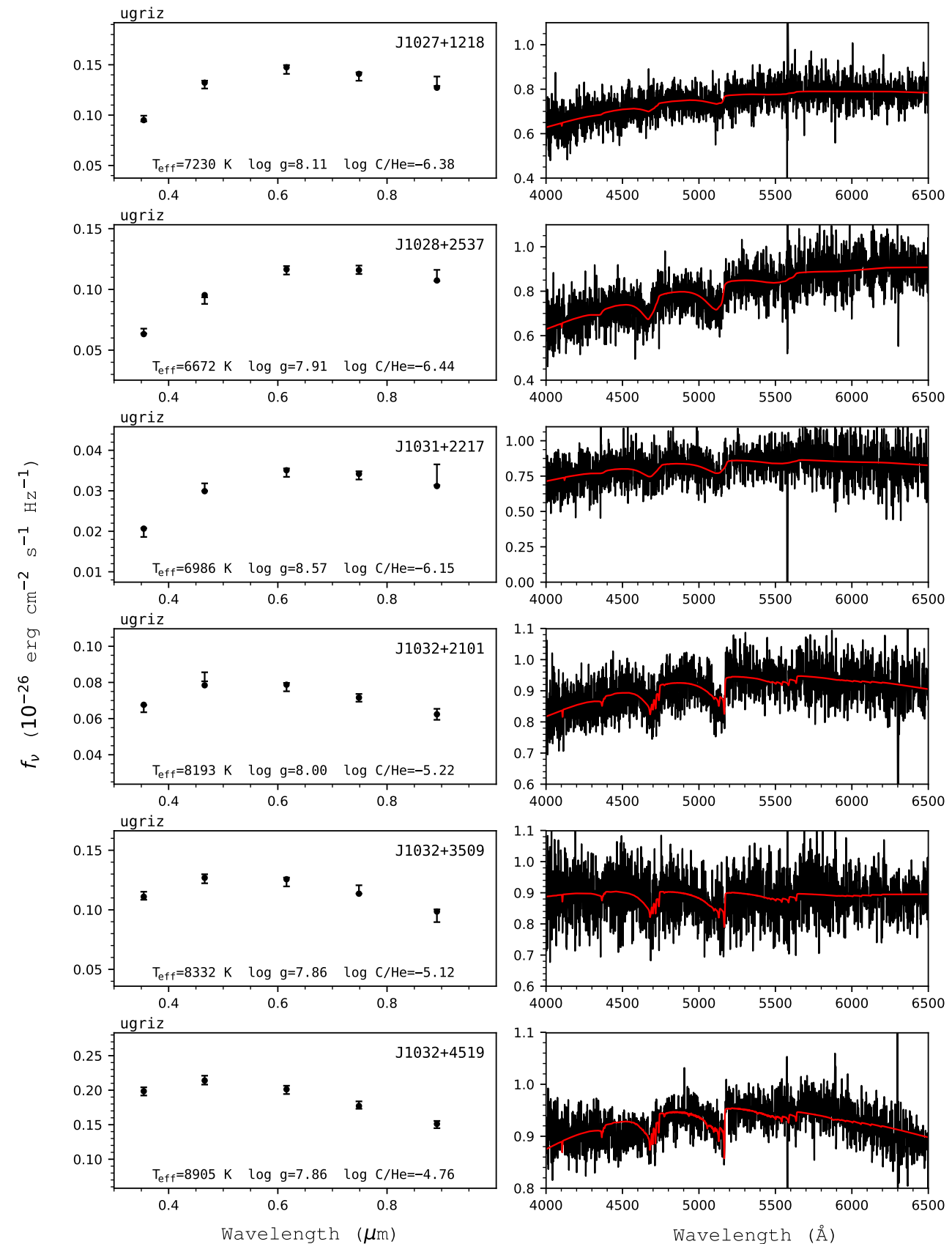

Figure 211. Fits to the DQ white dwarfs - continued. 

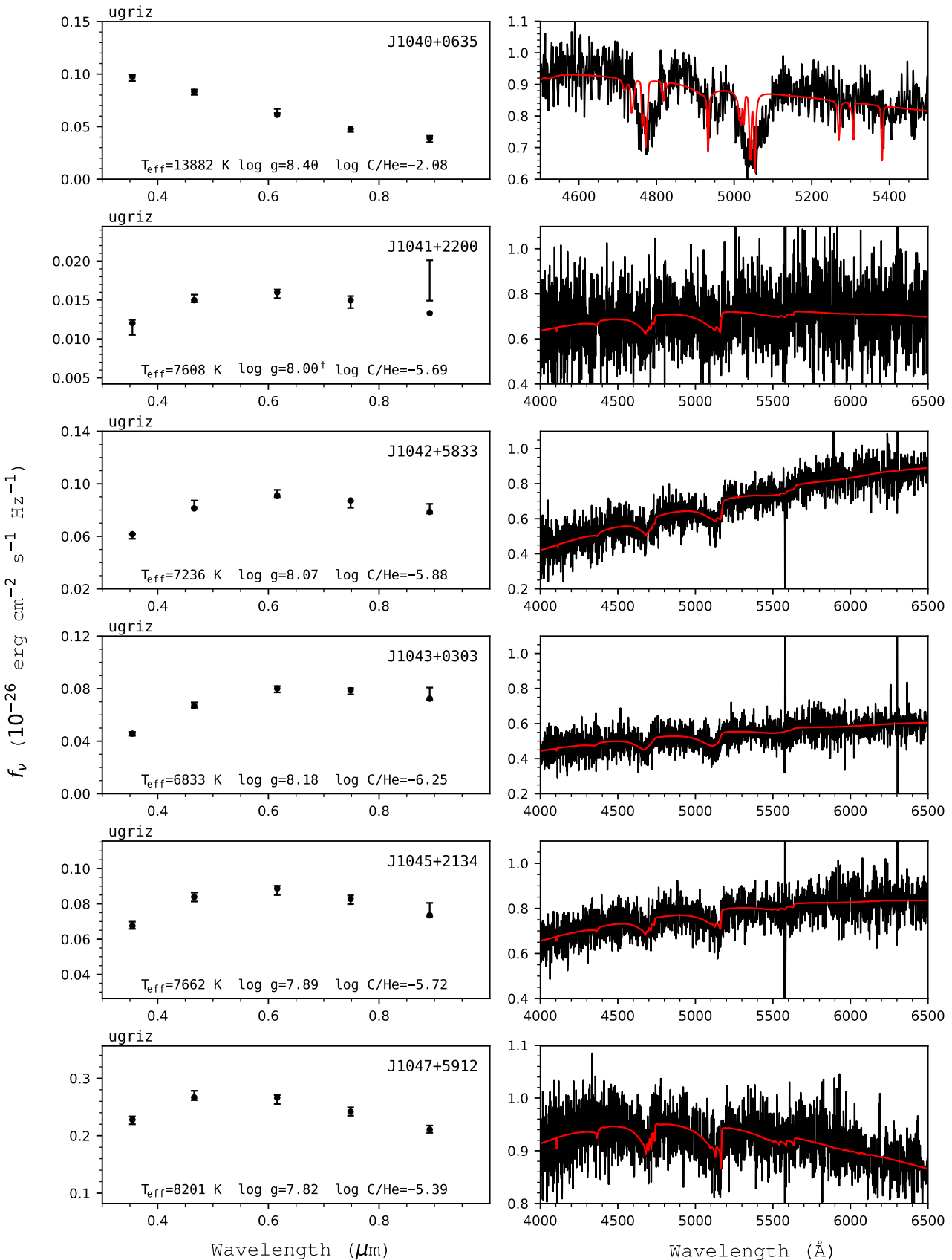

Figure 212. Fits to the DQ white dwarfs - continued. 

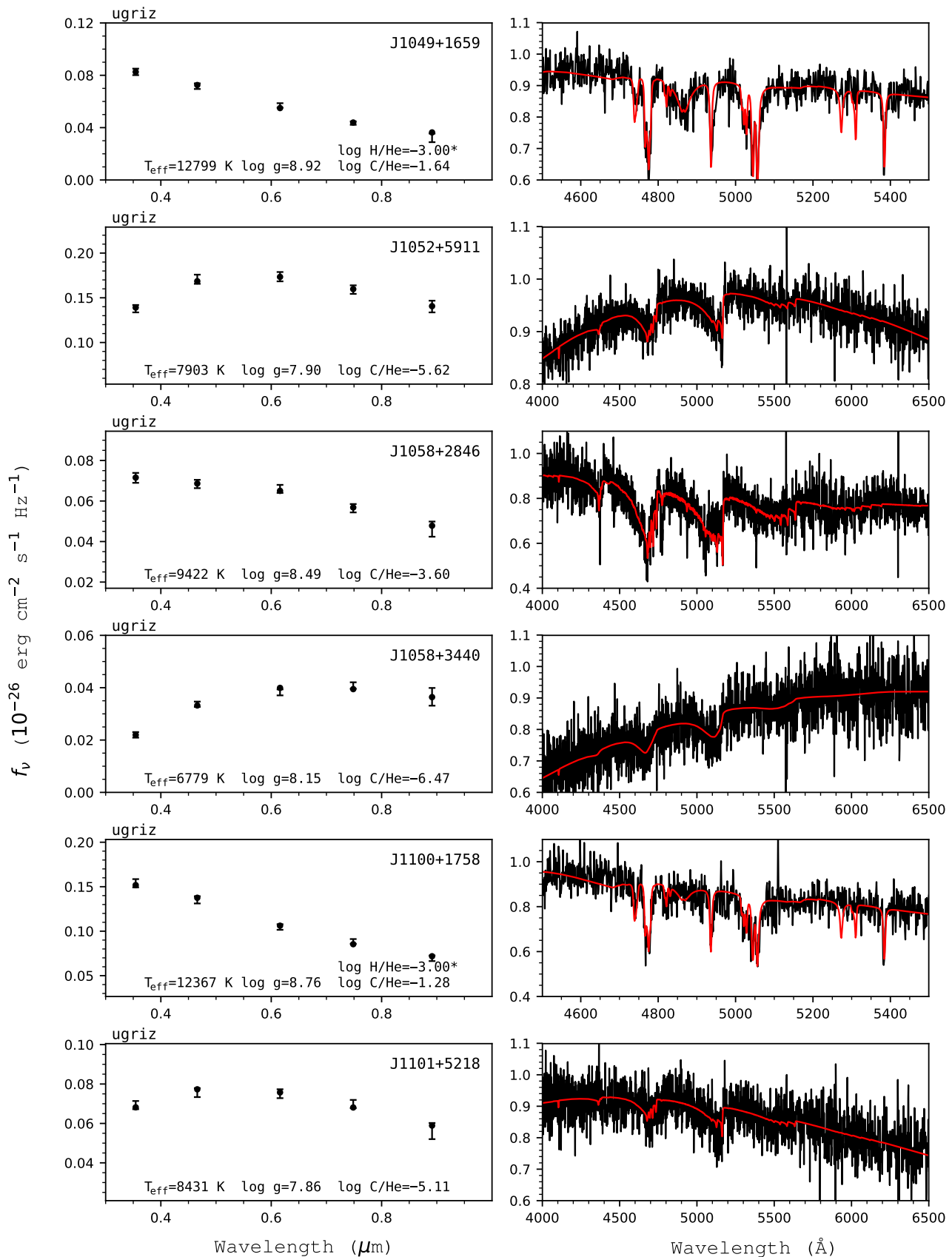

Figure 213. Fits to the DQ white dwarfs - continued. 

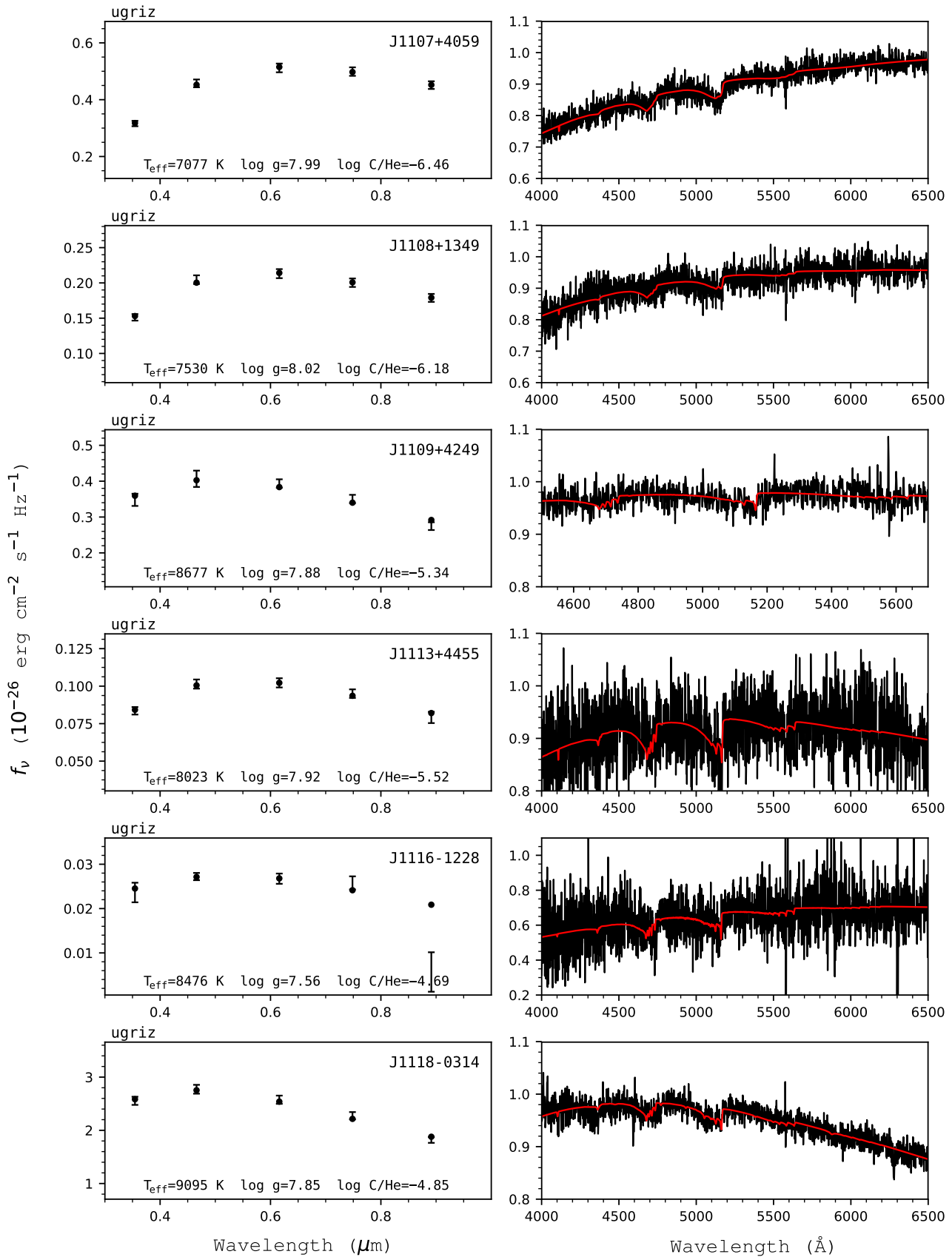

Figure 214. Fits to the DQ white dwarfs - continued. 

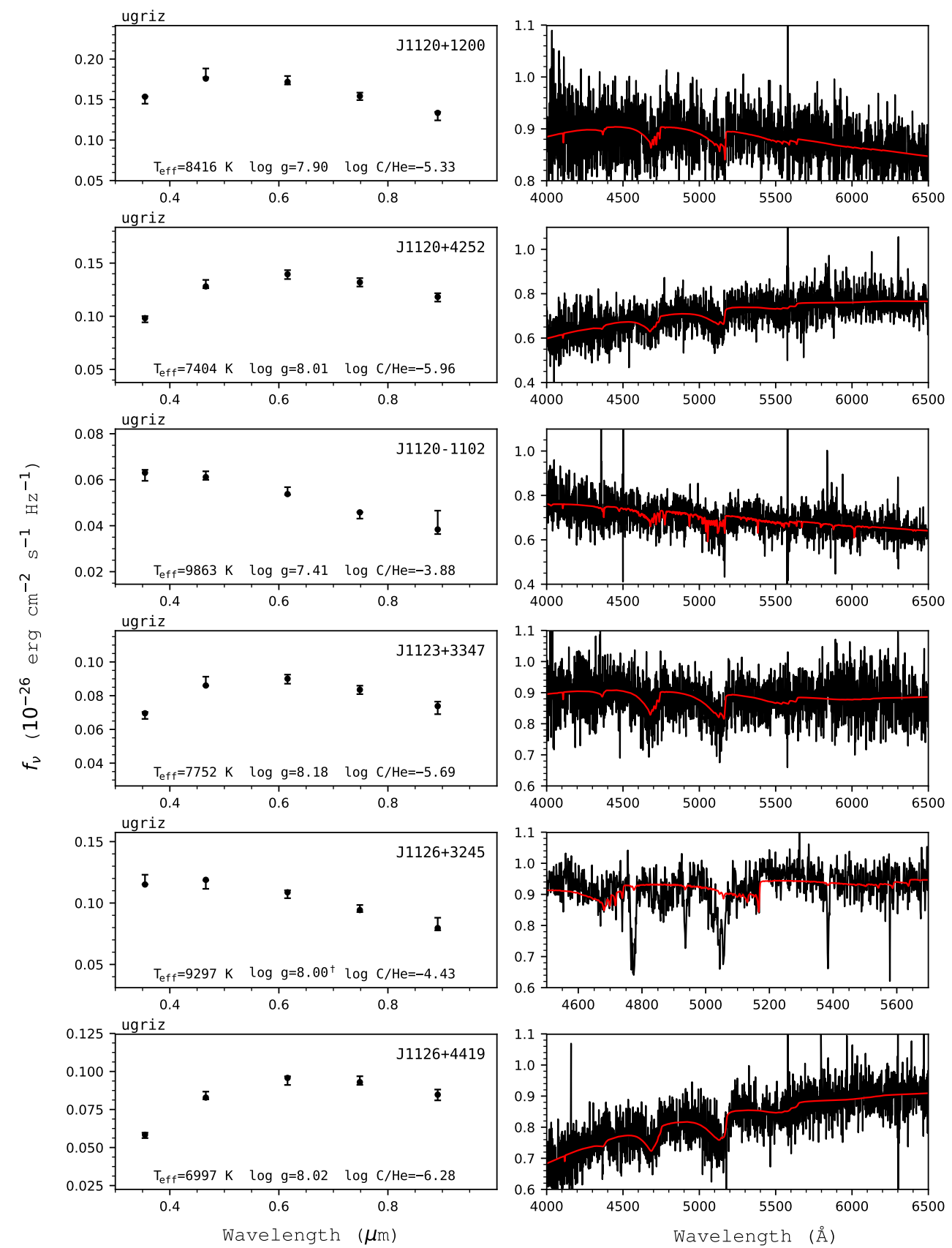

Figure 215. Fits to the DQ white dwarfs - continued. 

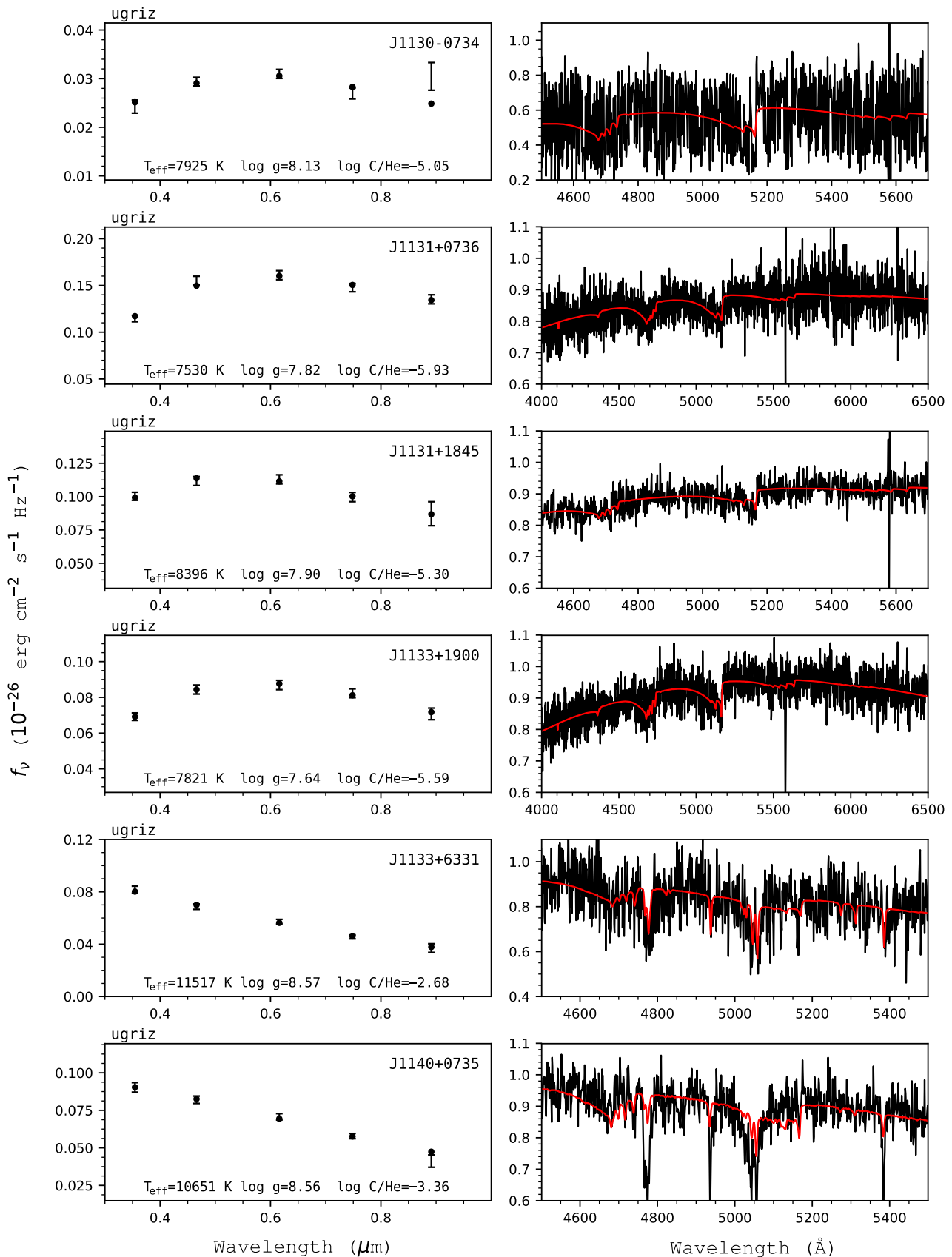

Figure 216. Fits to the DQ white dwarfs - continued. 

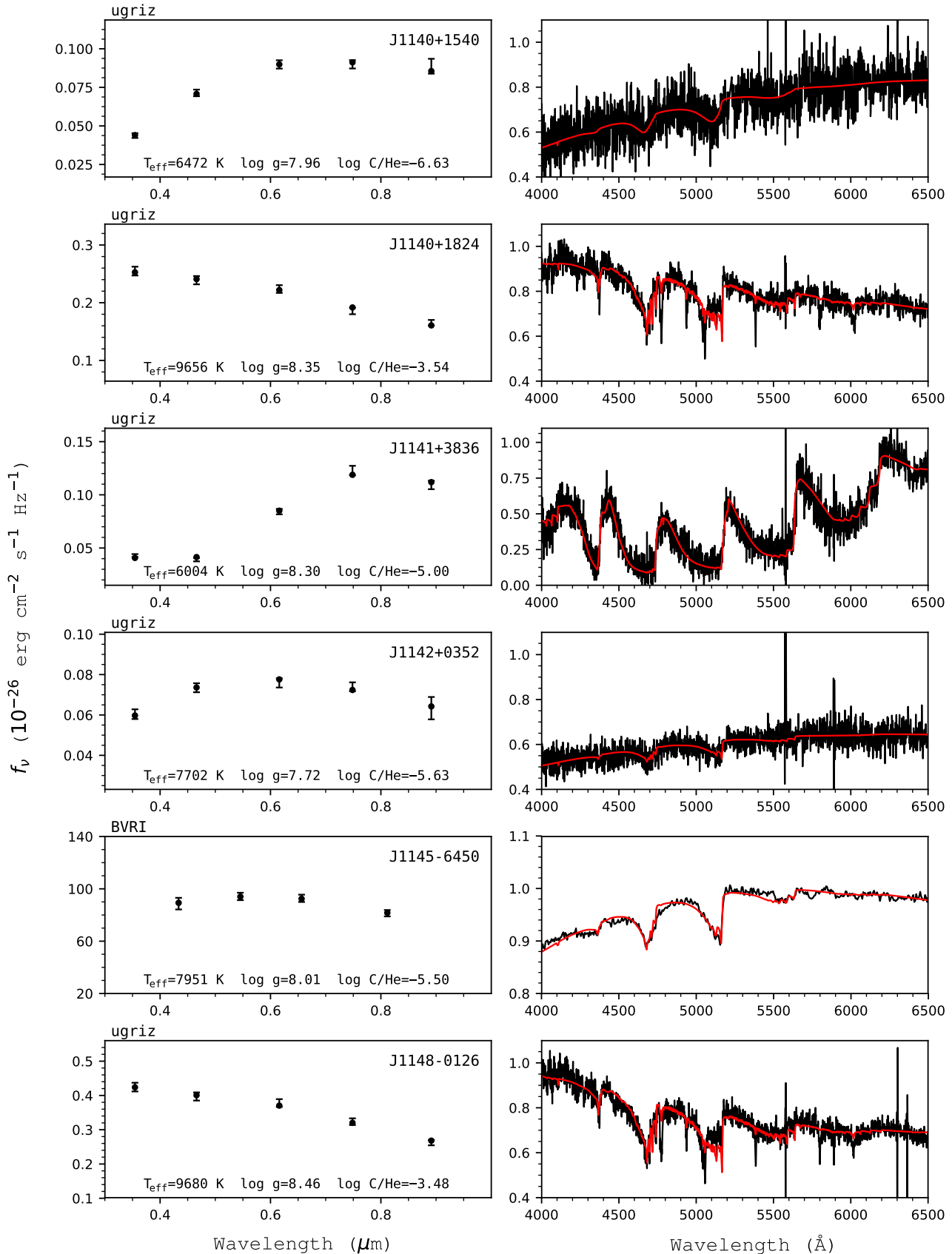

Figure 217. Fits to the DQ white dwarfs - continued. 

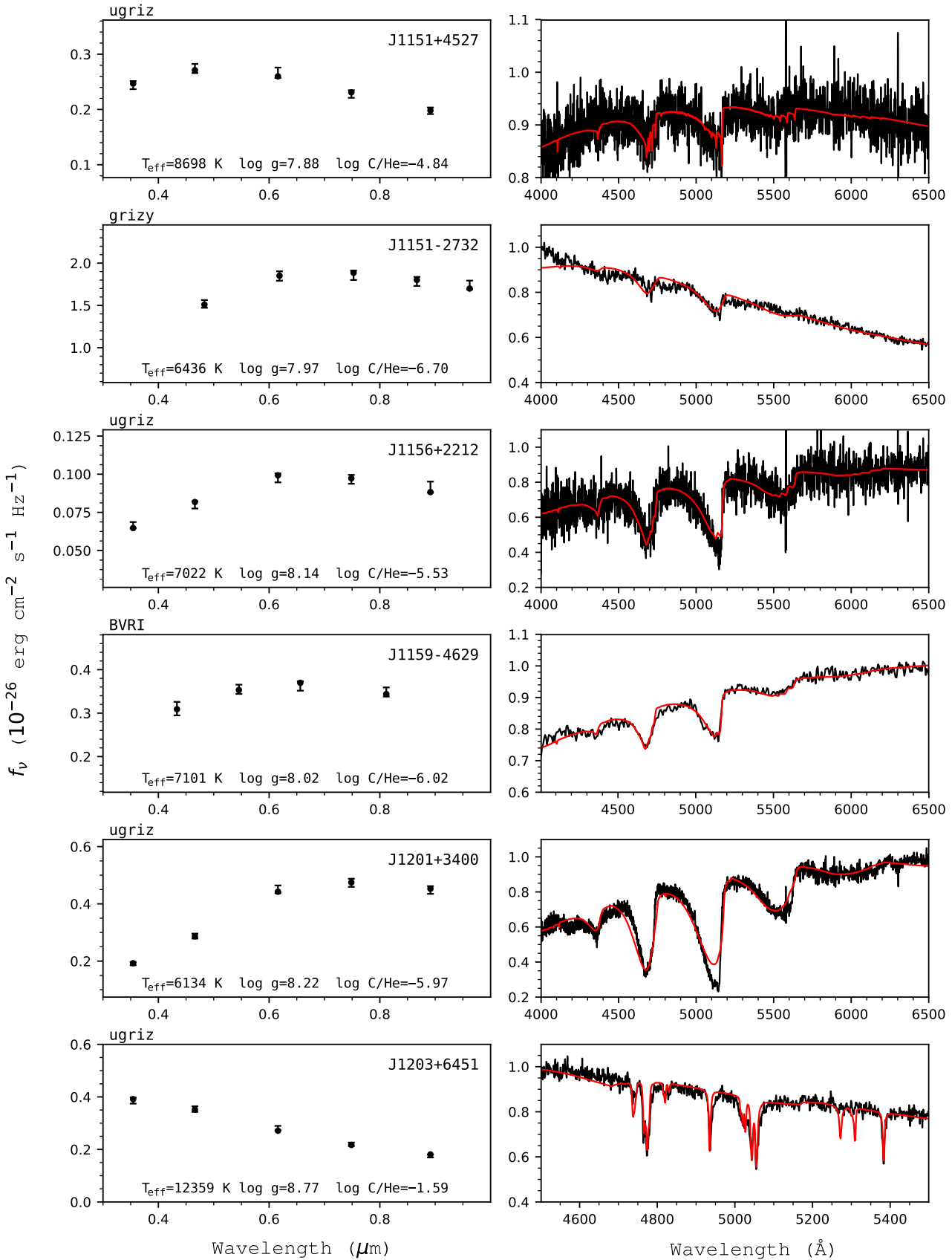

Figure 218. Fits to the DQ white dwarfs - continued. 

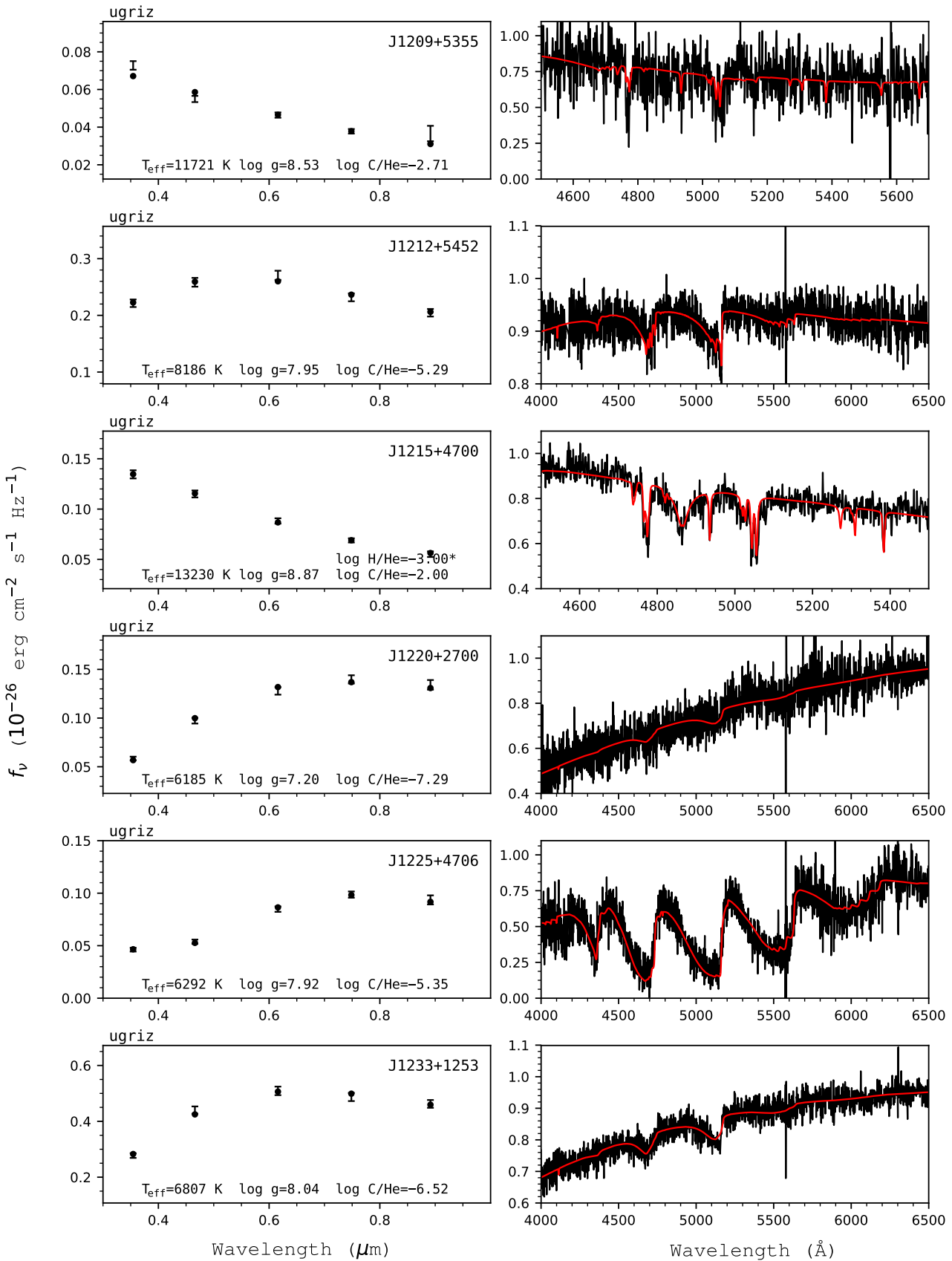

Figure 219. Fits to the DQ white dwarfs - continued. 

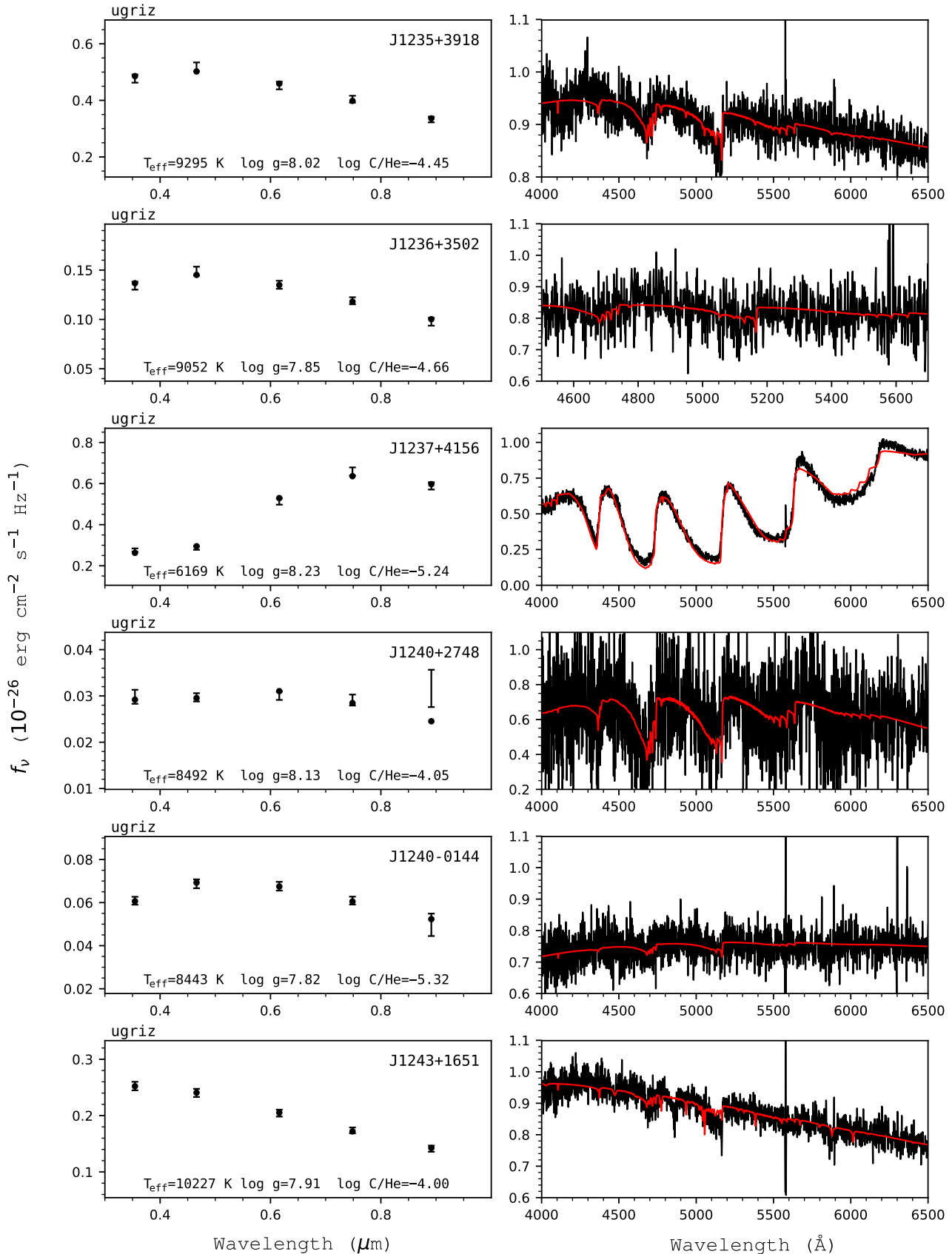

Figure 220. Fits to the DQ white dwarfs - continued. 

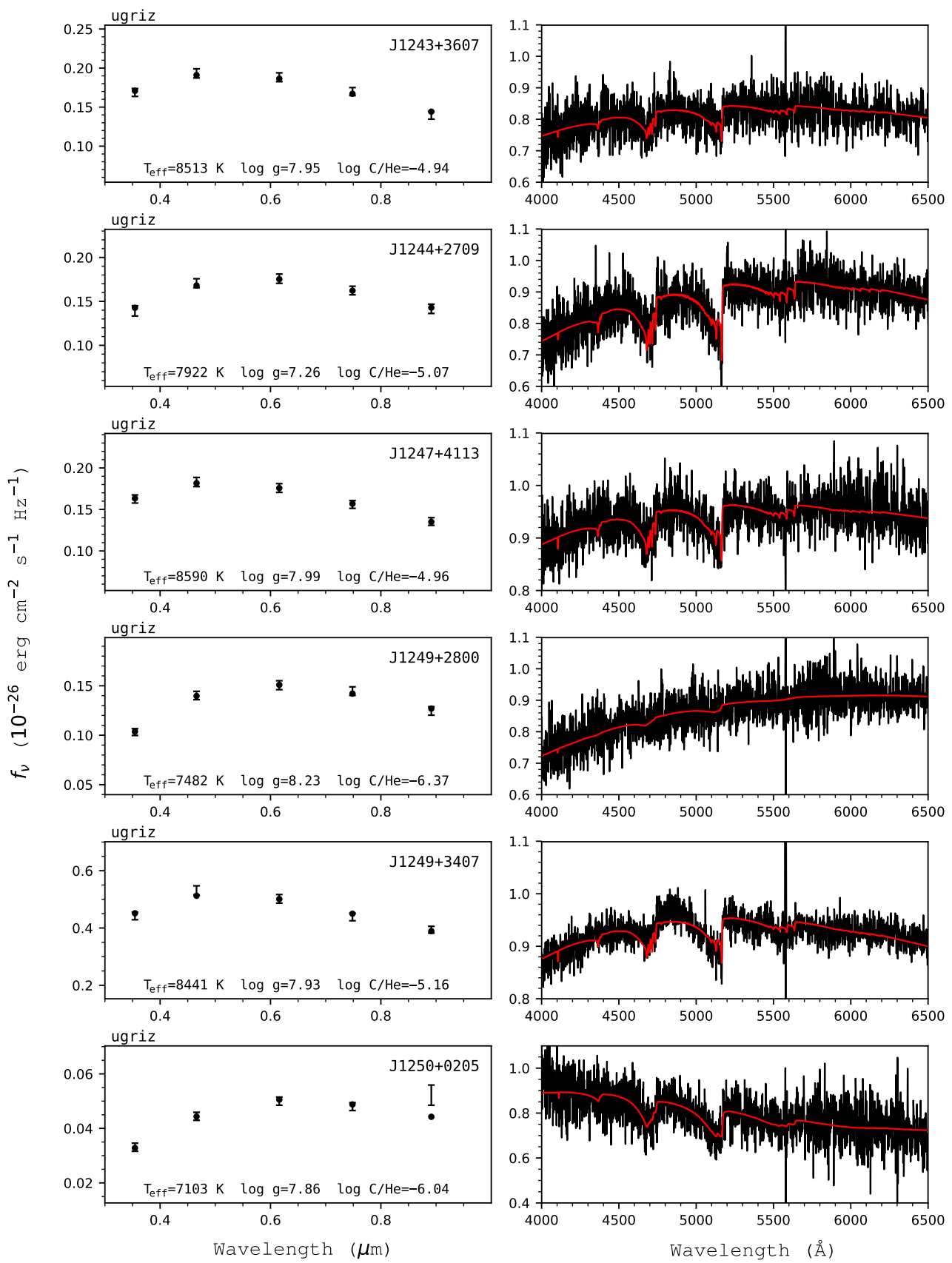

Figure 221. Fits to the DQ white dwarfs - continued. 

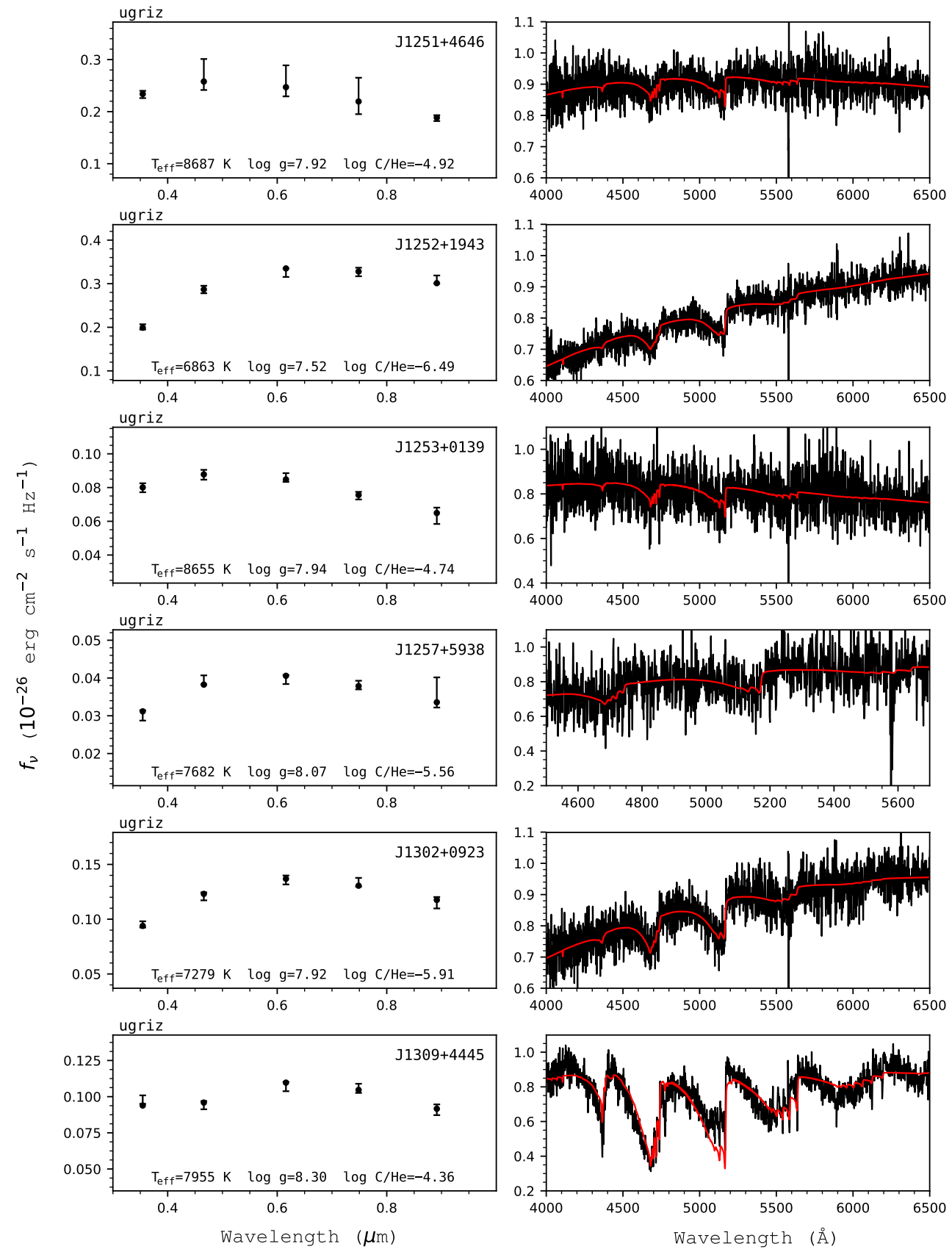

Figure 222. Fits to the DQ white dwarfs - continued. 

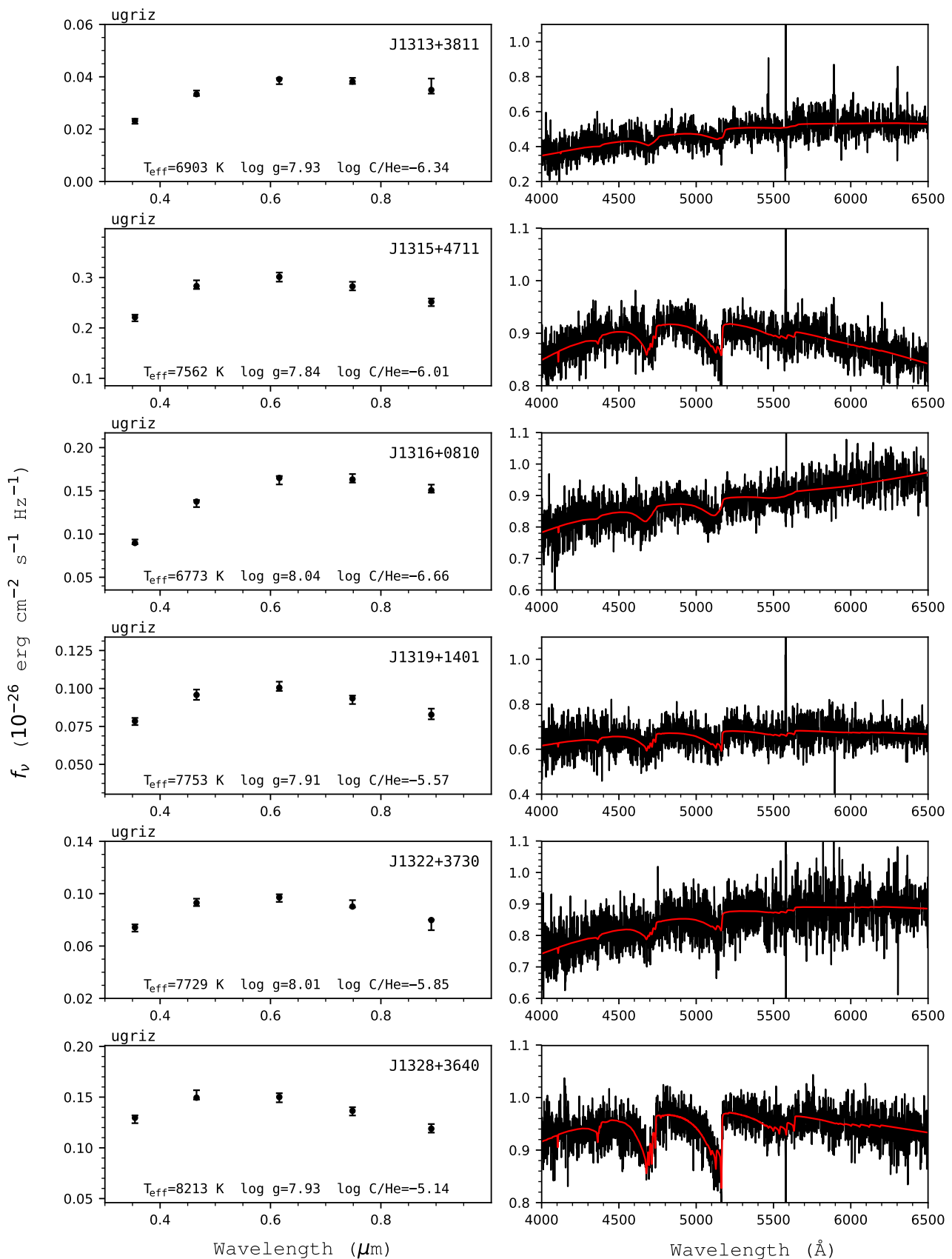

Figure 223. Fits to the DQ white dwarfs - continued. 

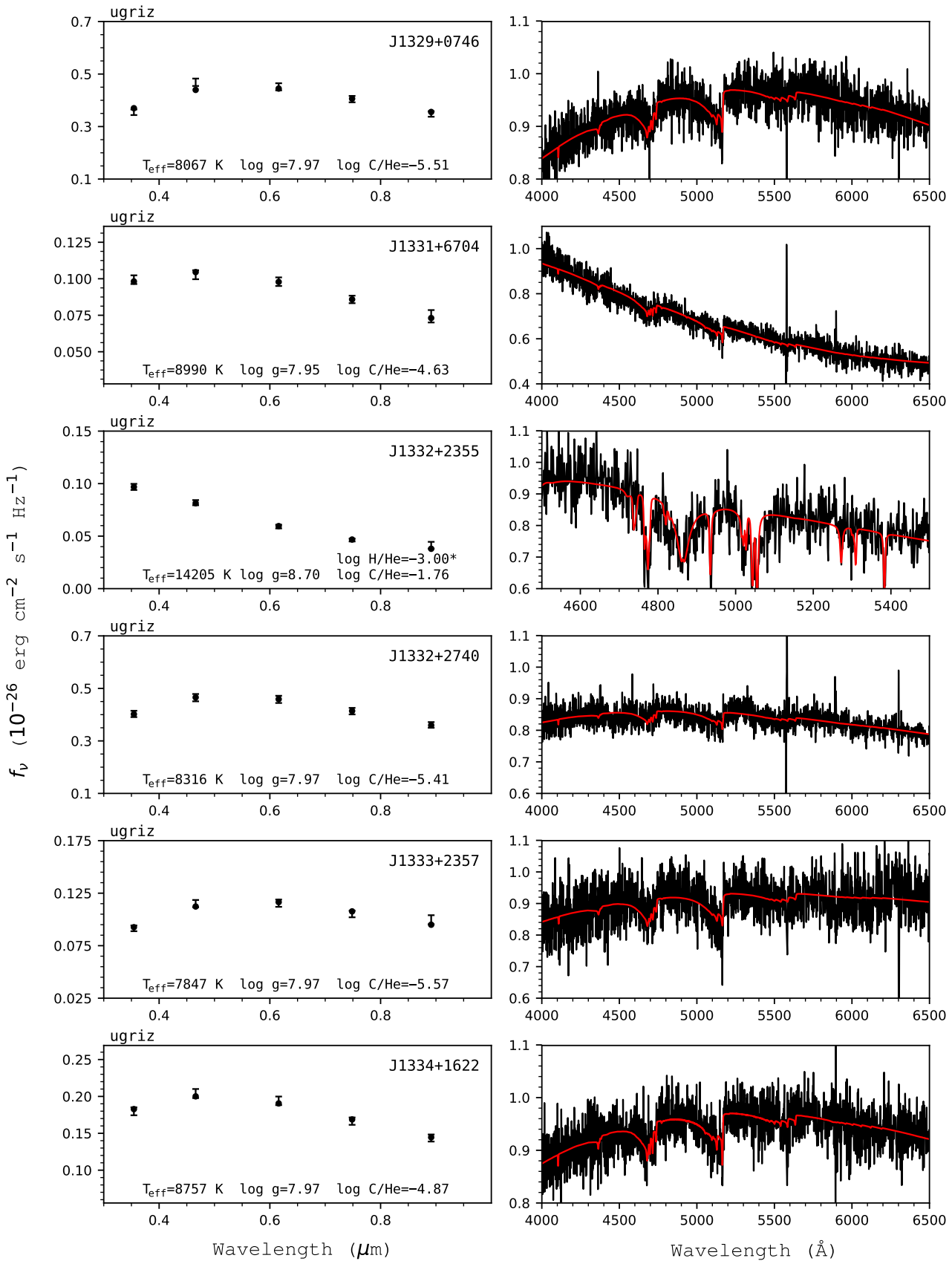

Figure 224. Fits to the DQ white dwarfs - continued. 

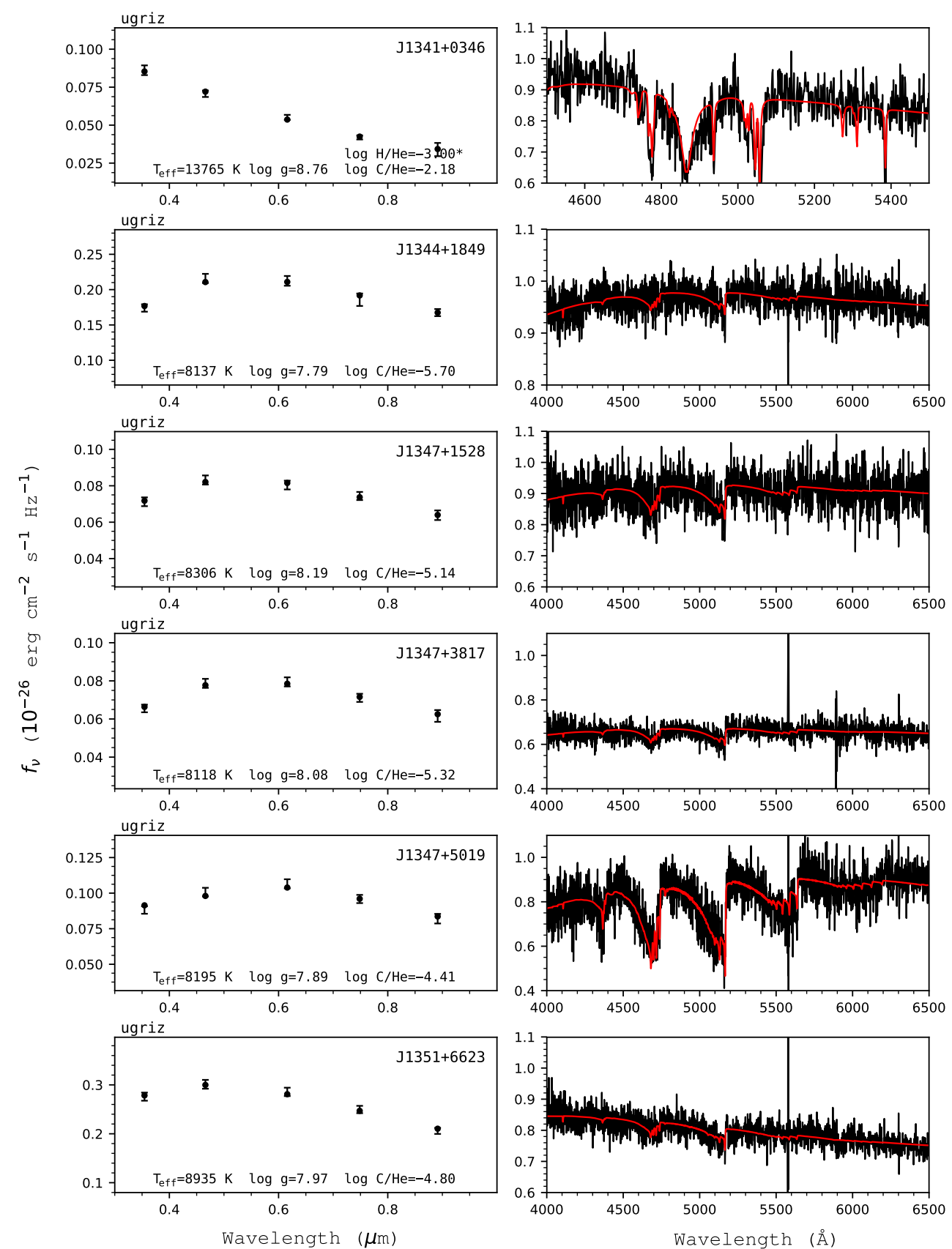

Figure 225. Fits to the DQ white dwarfs - continued. 

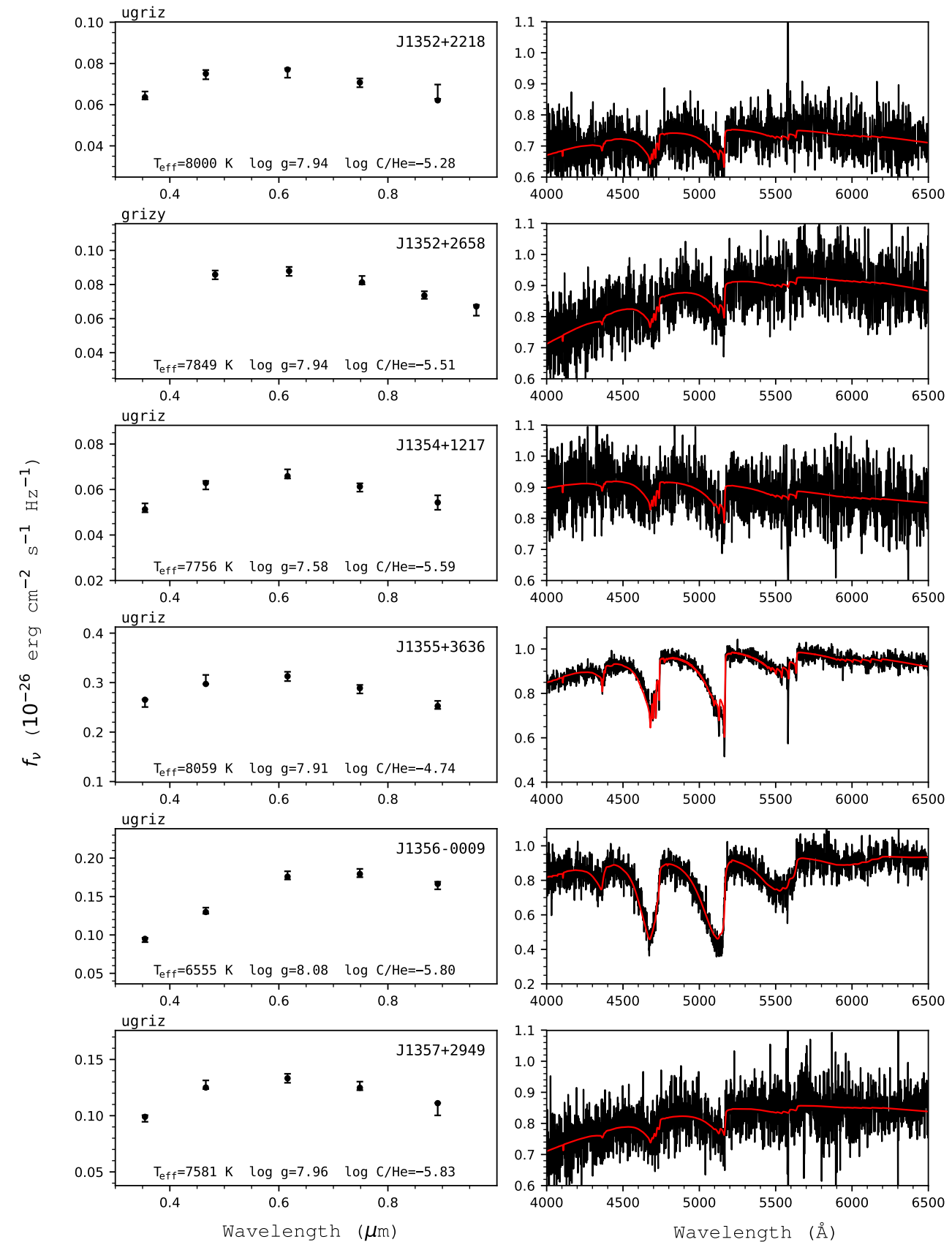

Figure 226. Fits to the DQ white dwarfs - continued. 

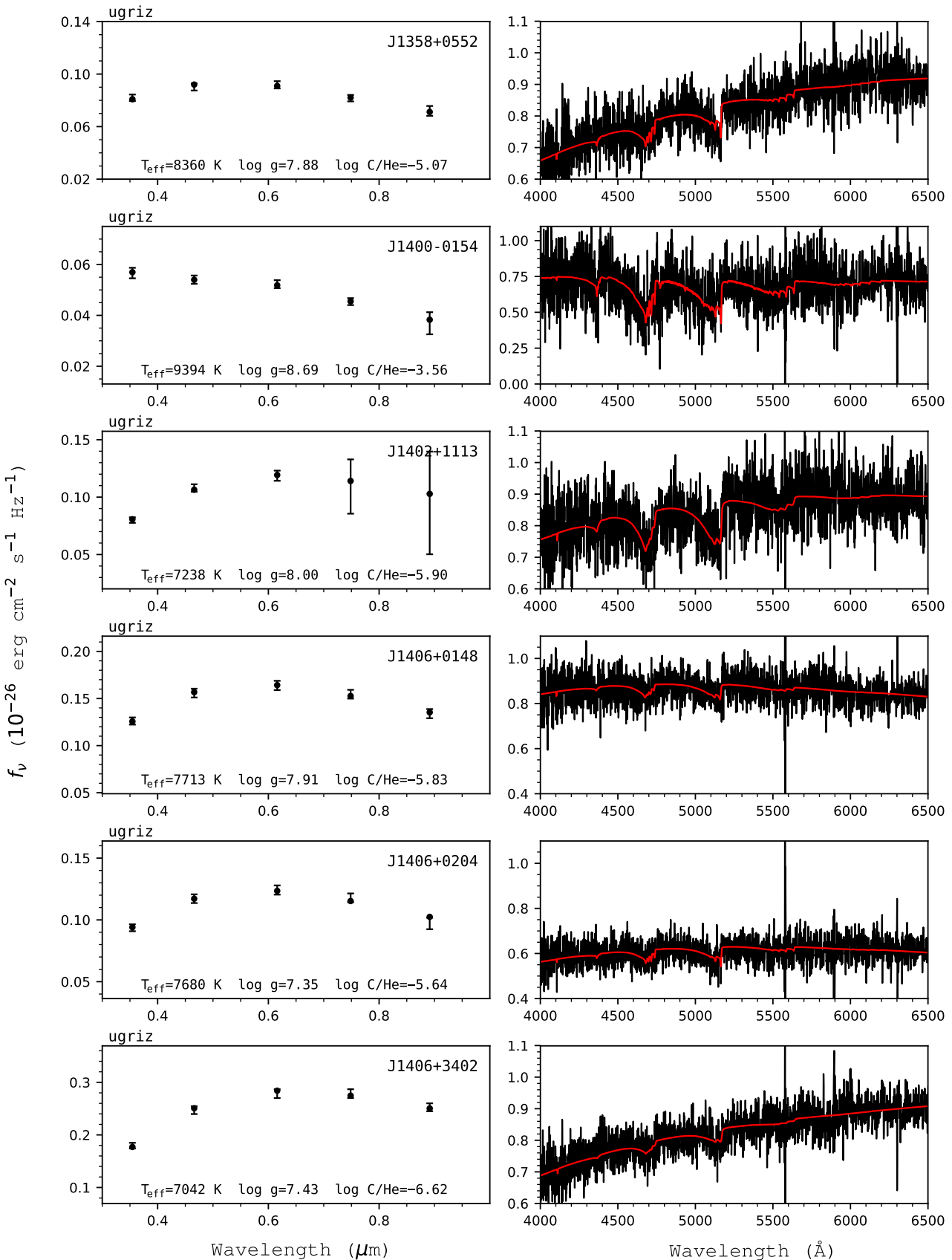

Figure 227. Fits to the DQ white dwarfs - continued. 

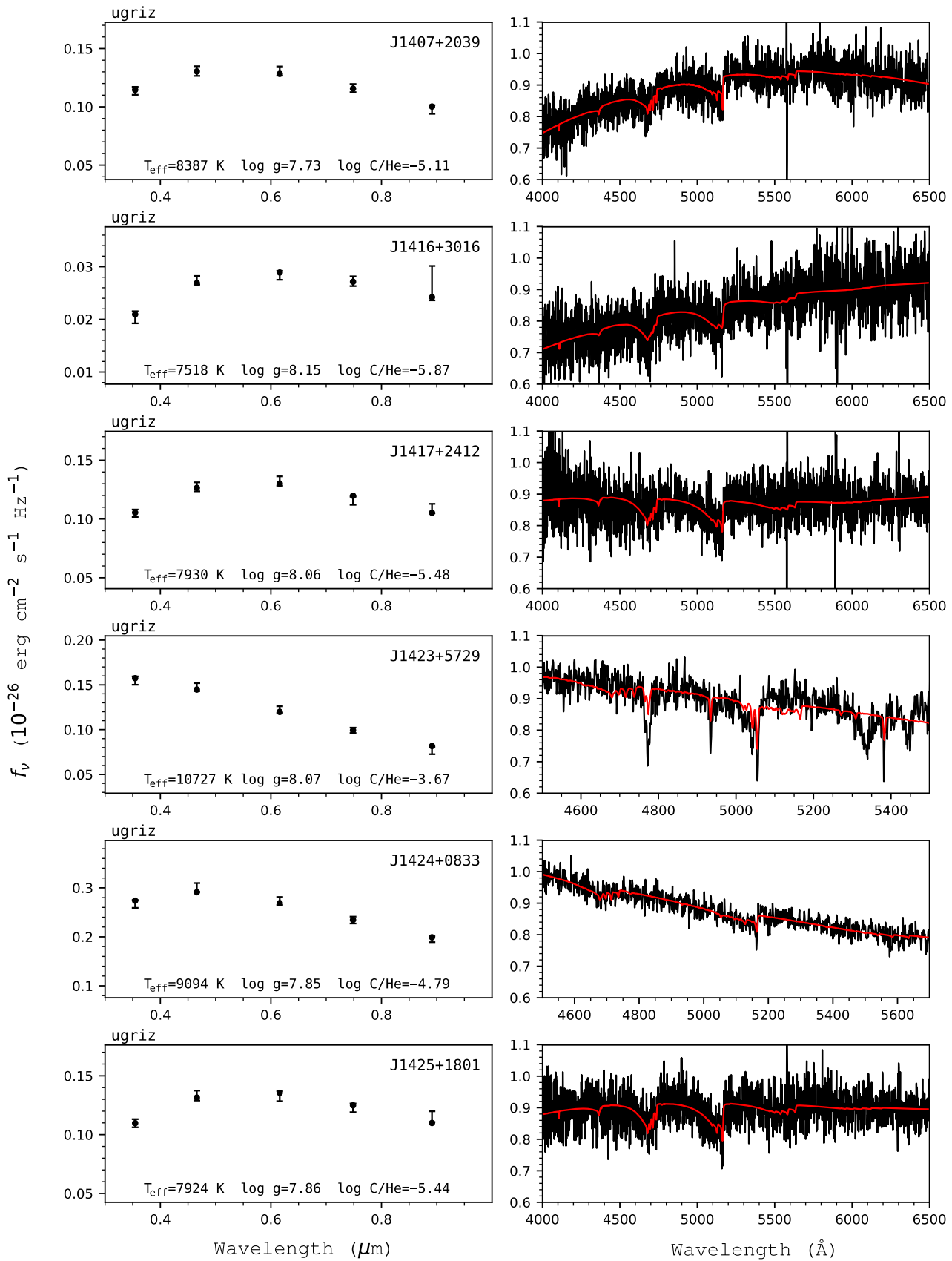

Figure 228. Fits to the DQ white dwarfs - continued. 

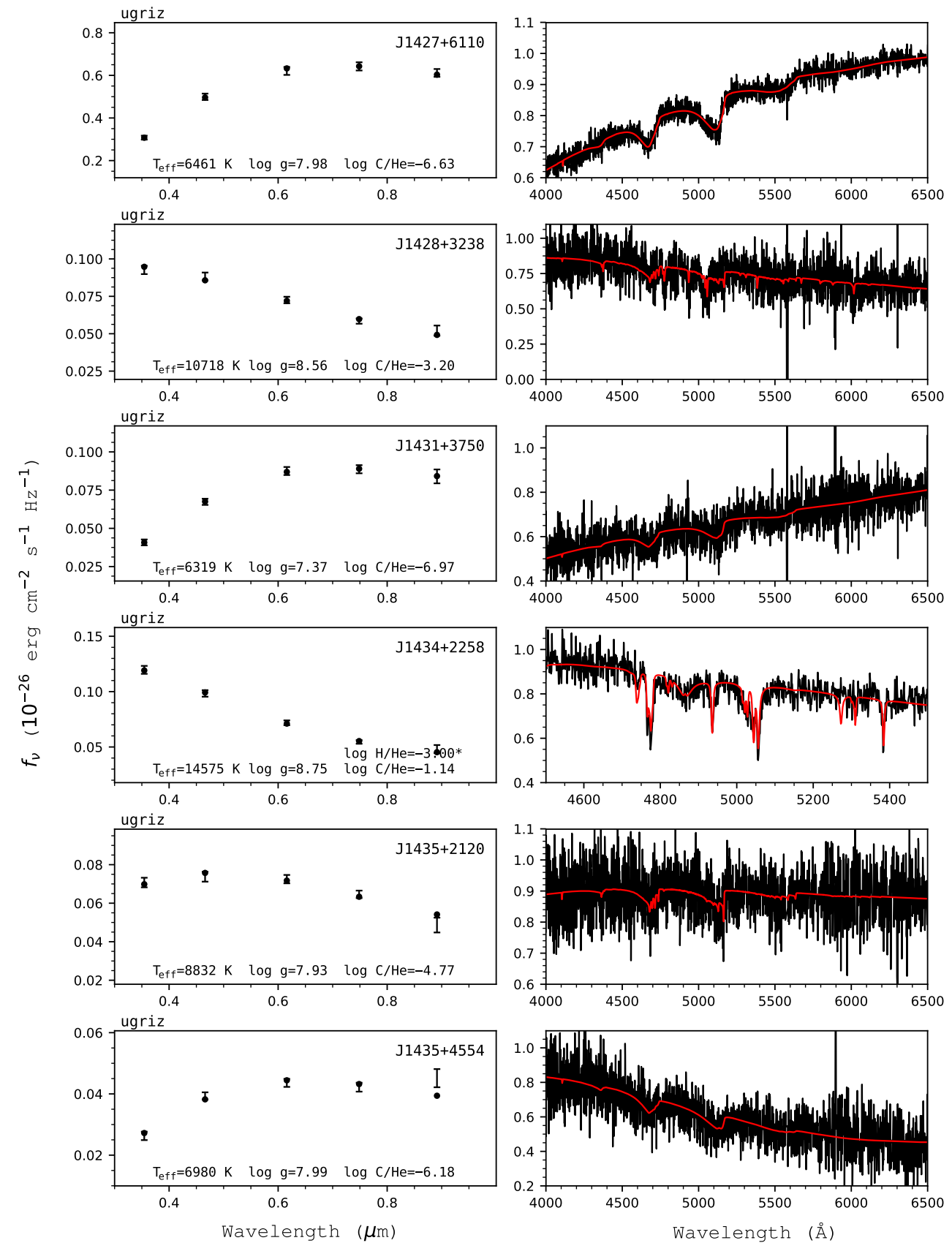

Figure 229. Fits to the DQ white dwarfs - continued. 

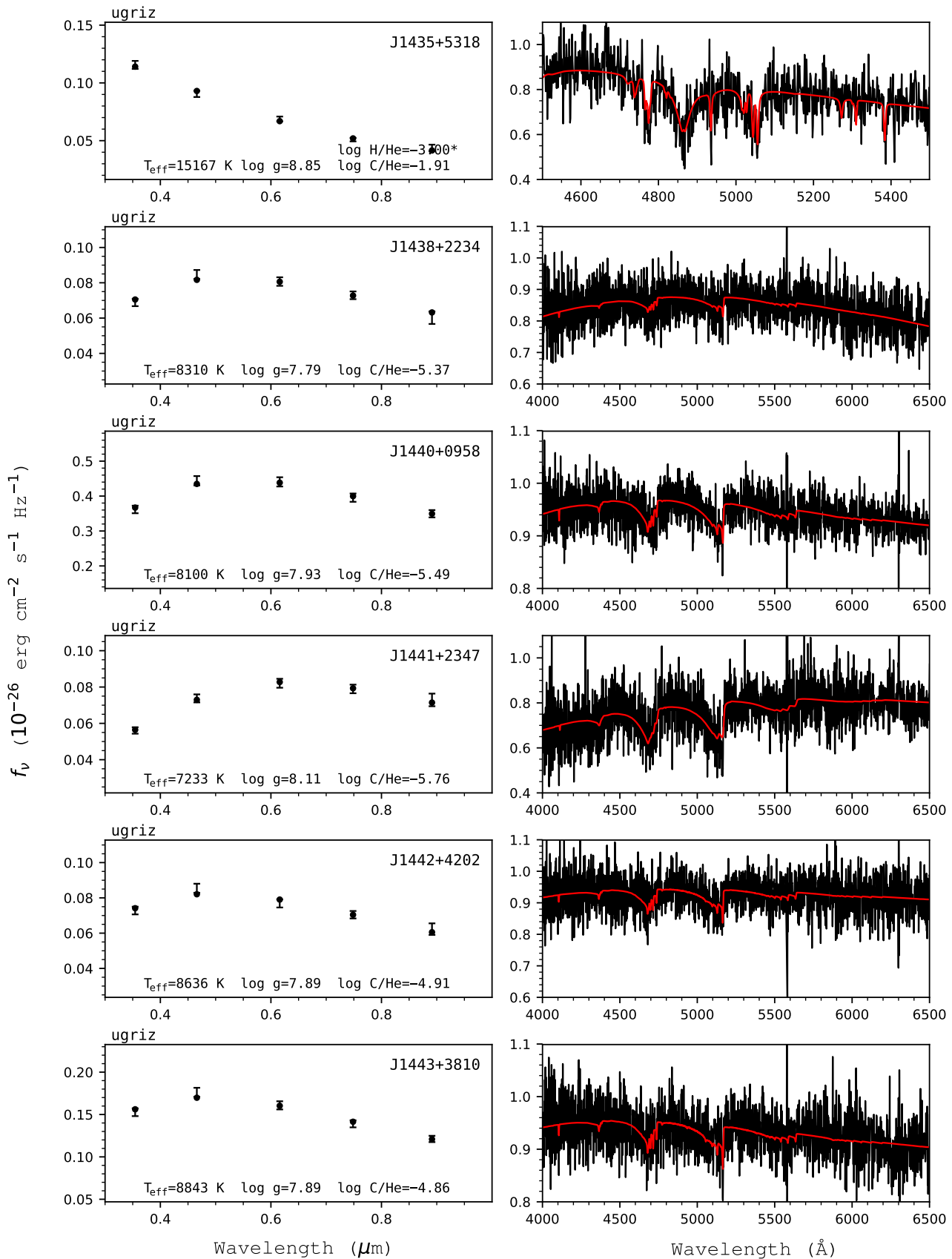

Figure 230. Fits to the DQ white dwarfs - continued. 

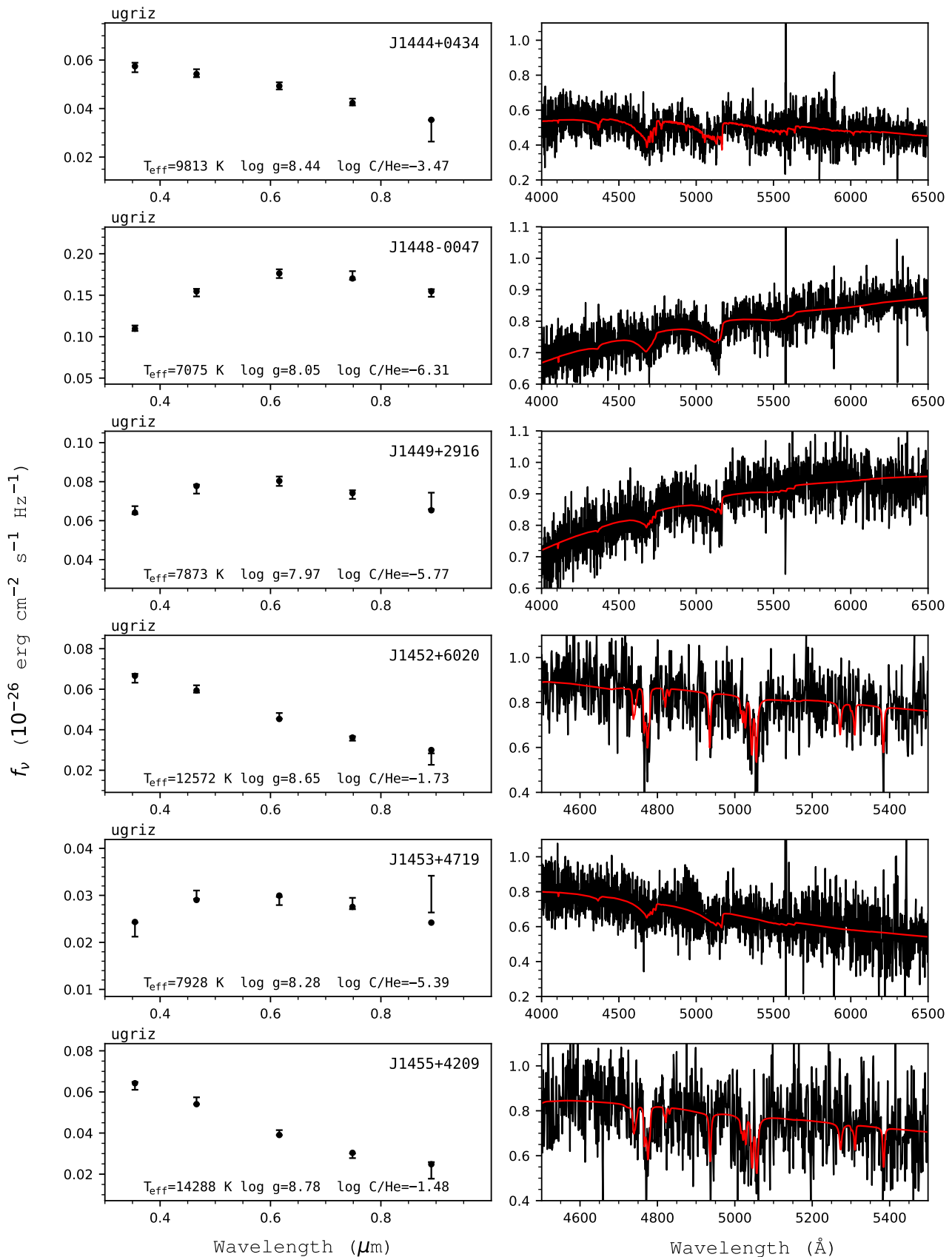

Figure 231. Fits to the DQ white dwarfs - continued. 

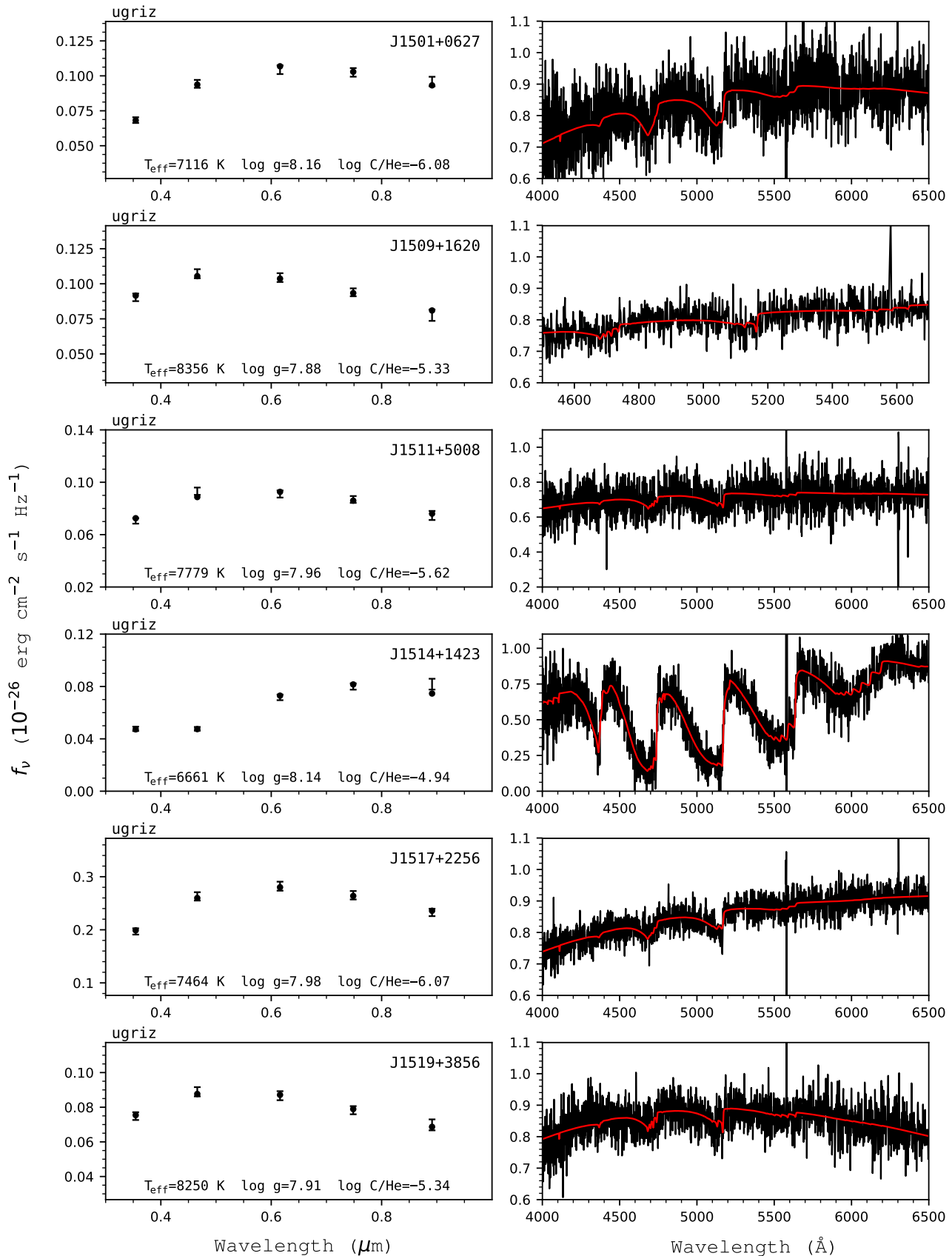

Figure 232. Fits to the DQ white dwarfs - continued. 

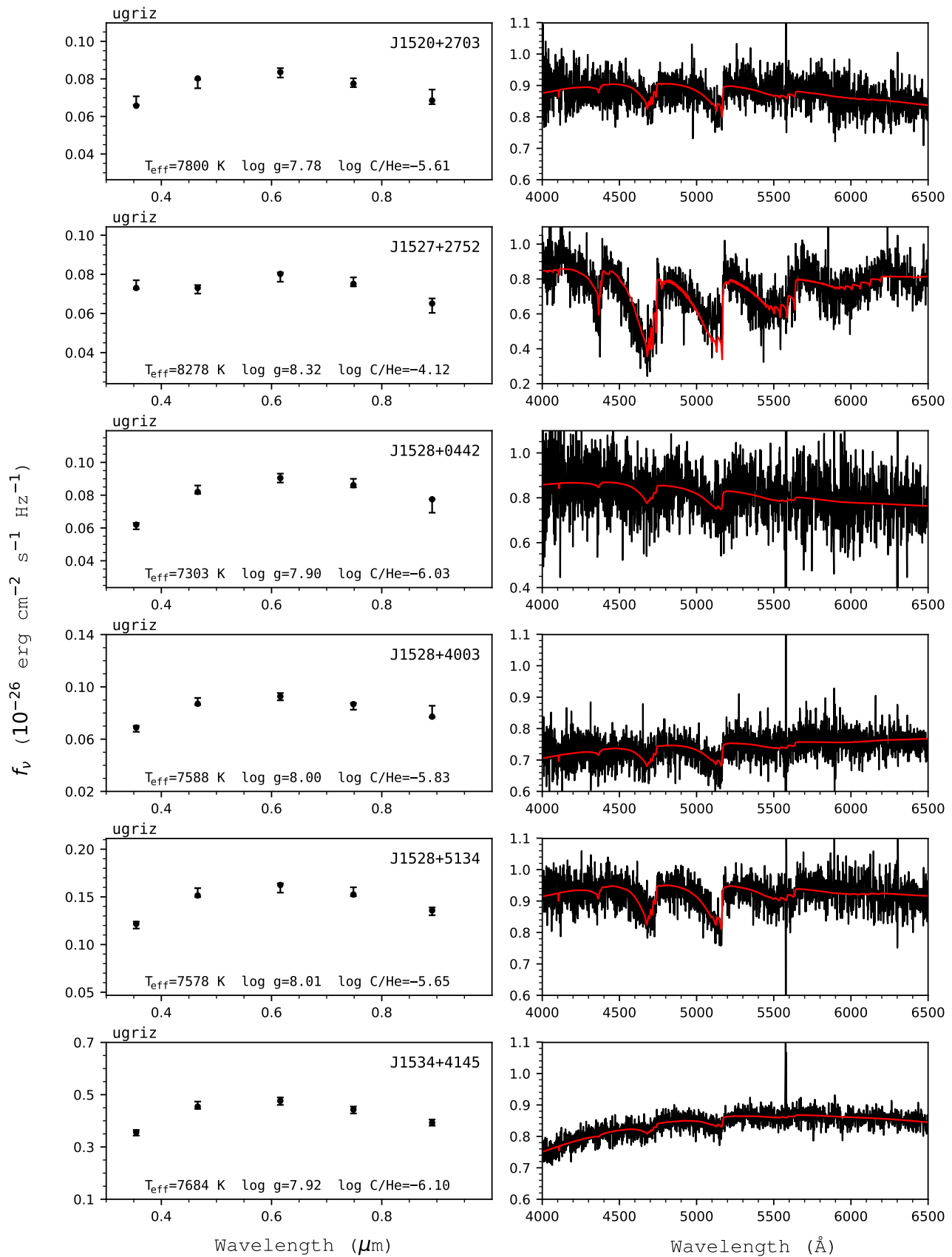

Figure 233. Fits to the DQ white dwarfs - continued. 

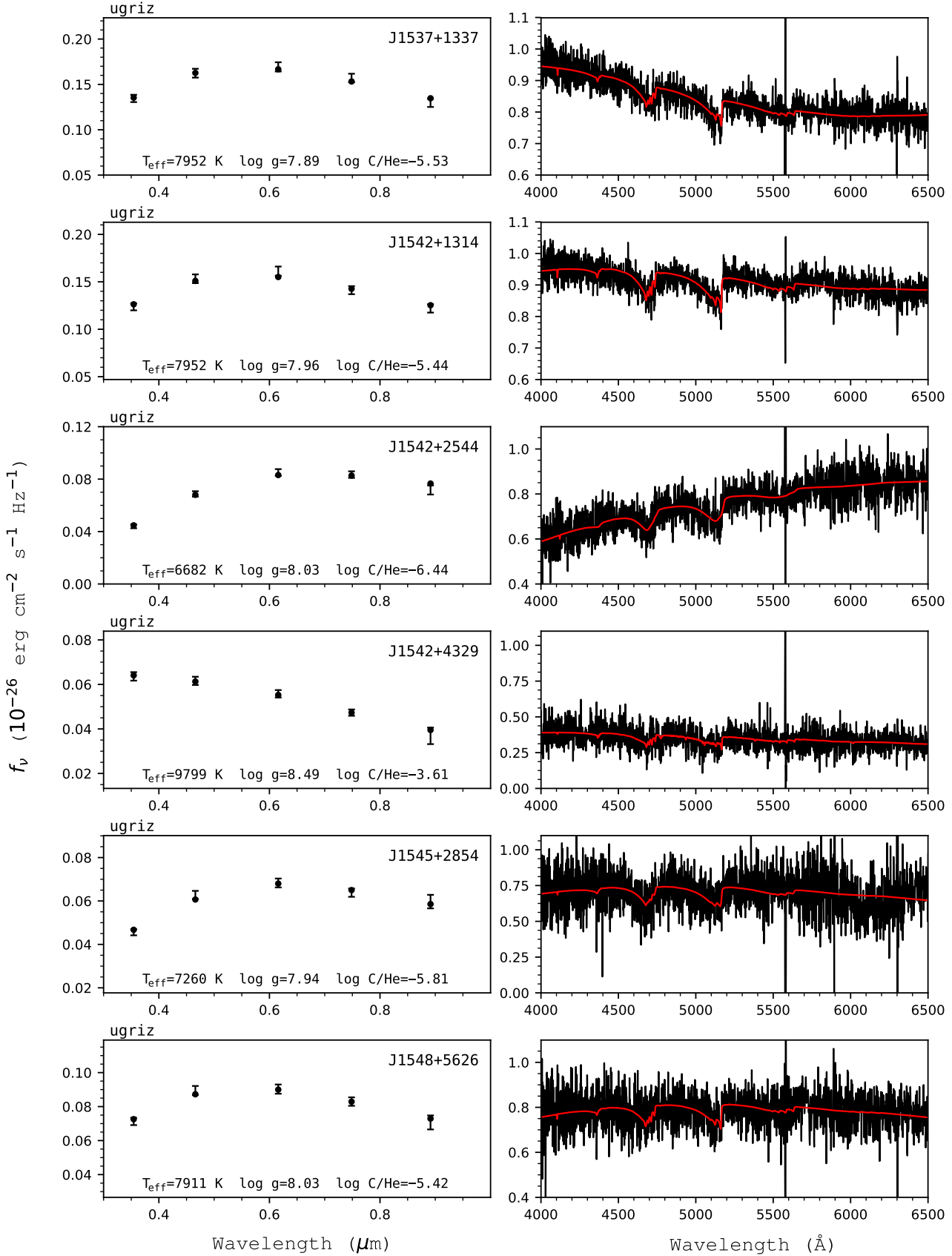

Figure 234. Fits to the DQ white dwarfs - continued. 

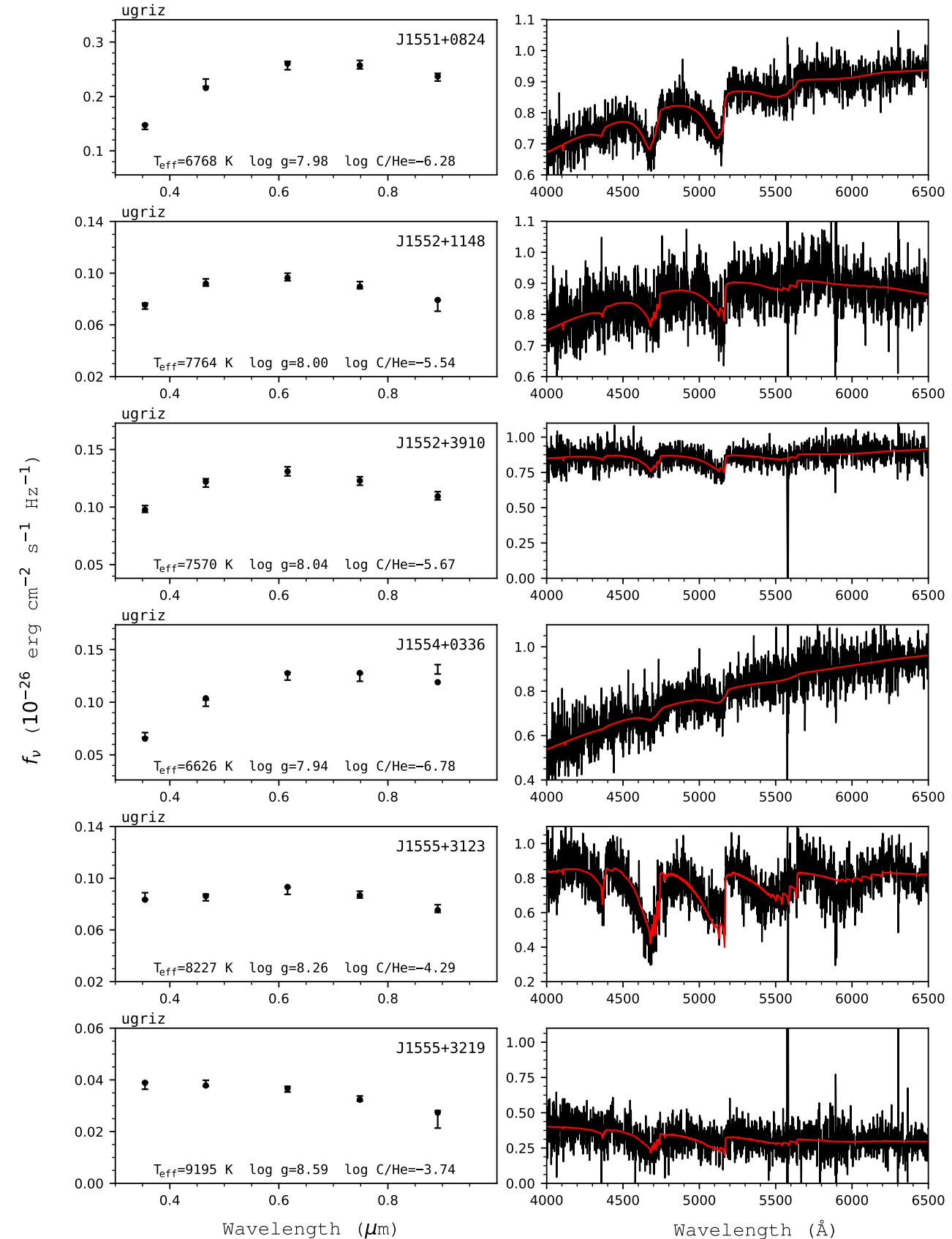

Figure 235. Fits to the DQ white dwarfs - continued. 

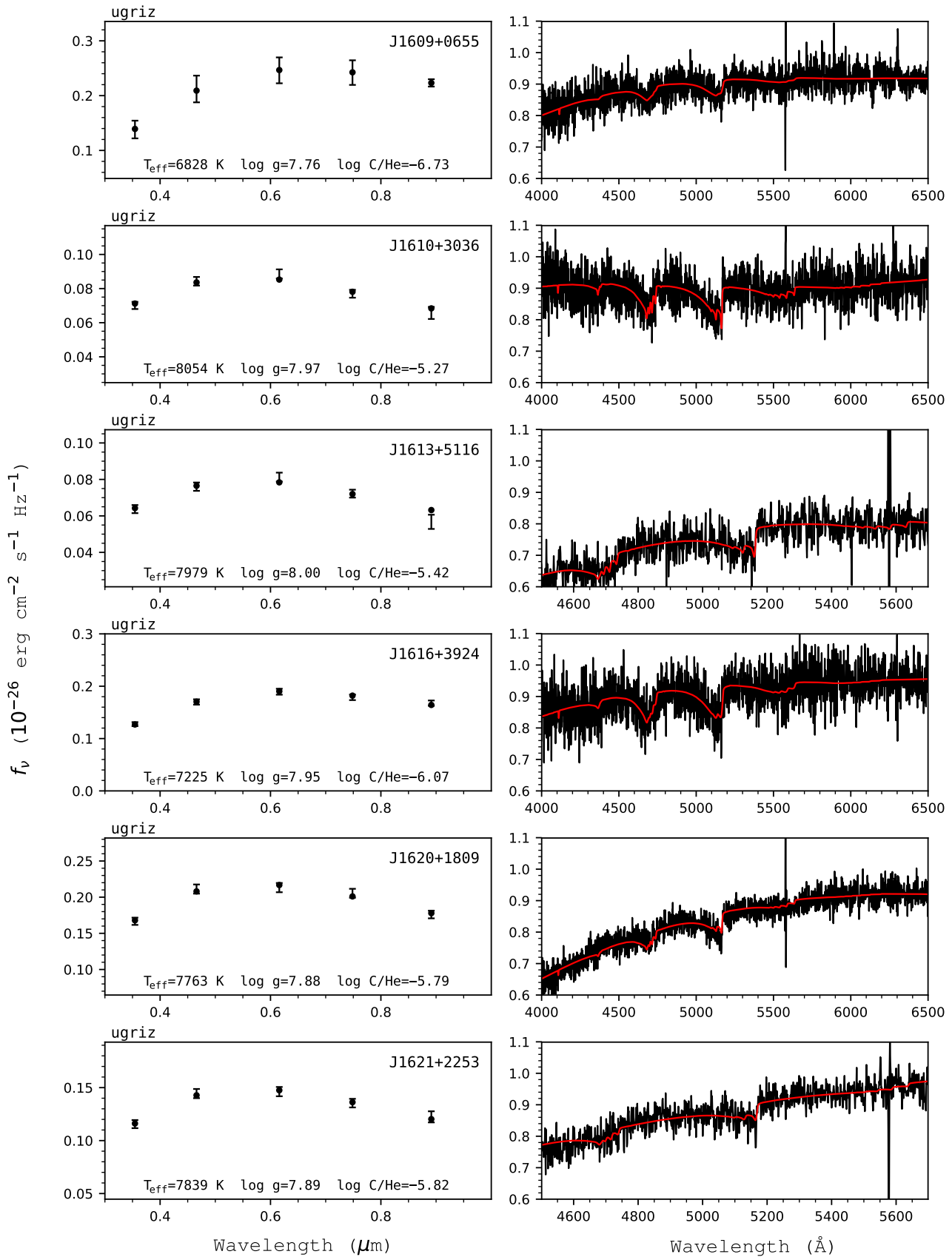

Figure 236. Fits to the DQ white dwarfs - continued. 

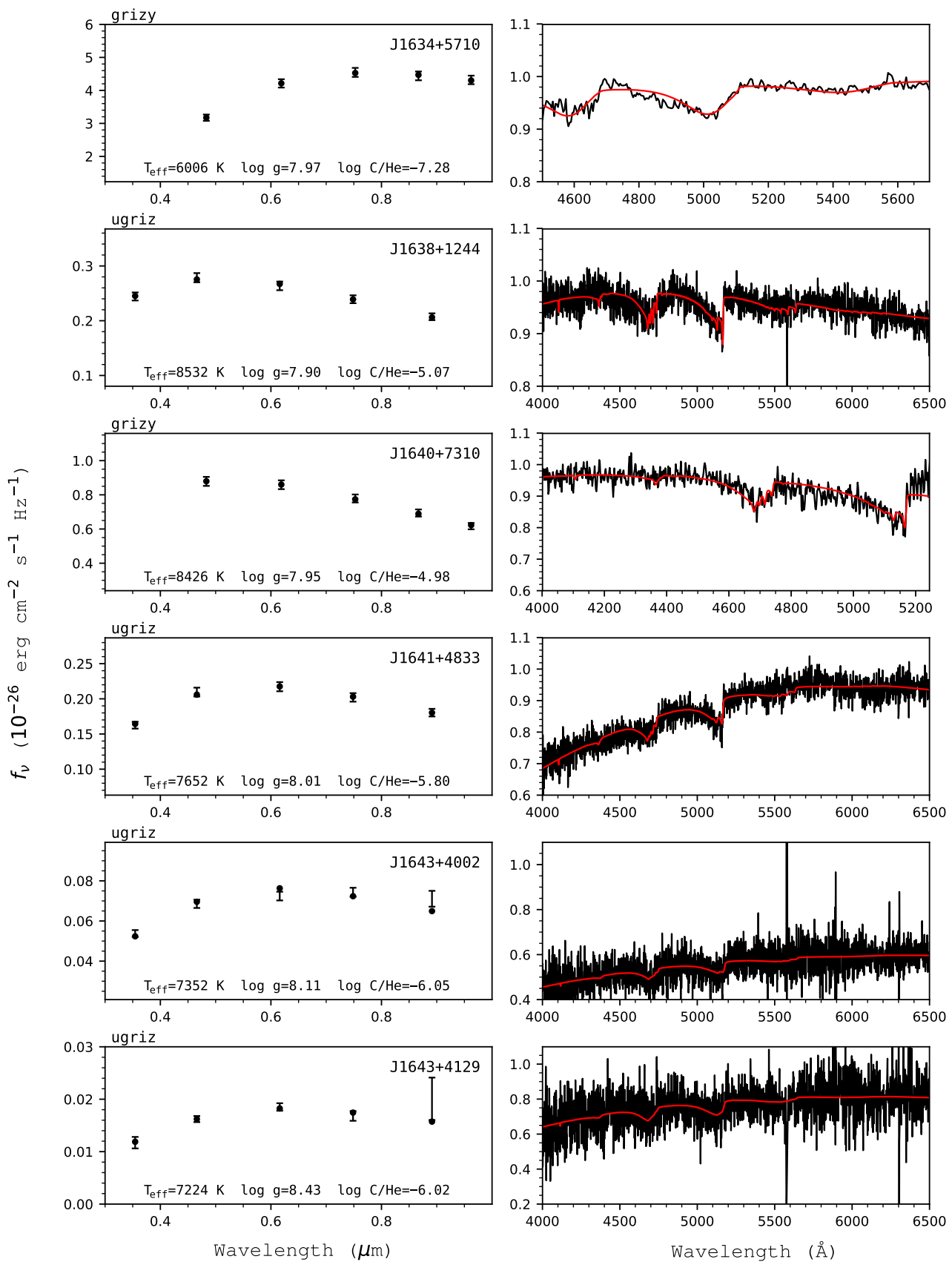

Figure 237. Fits to the DQ white dwarfs - continued. 

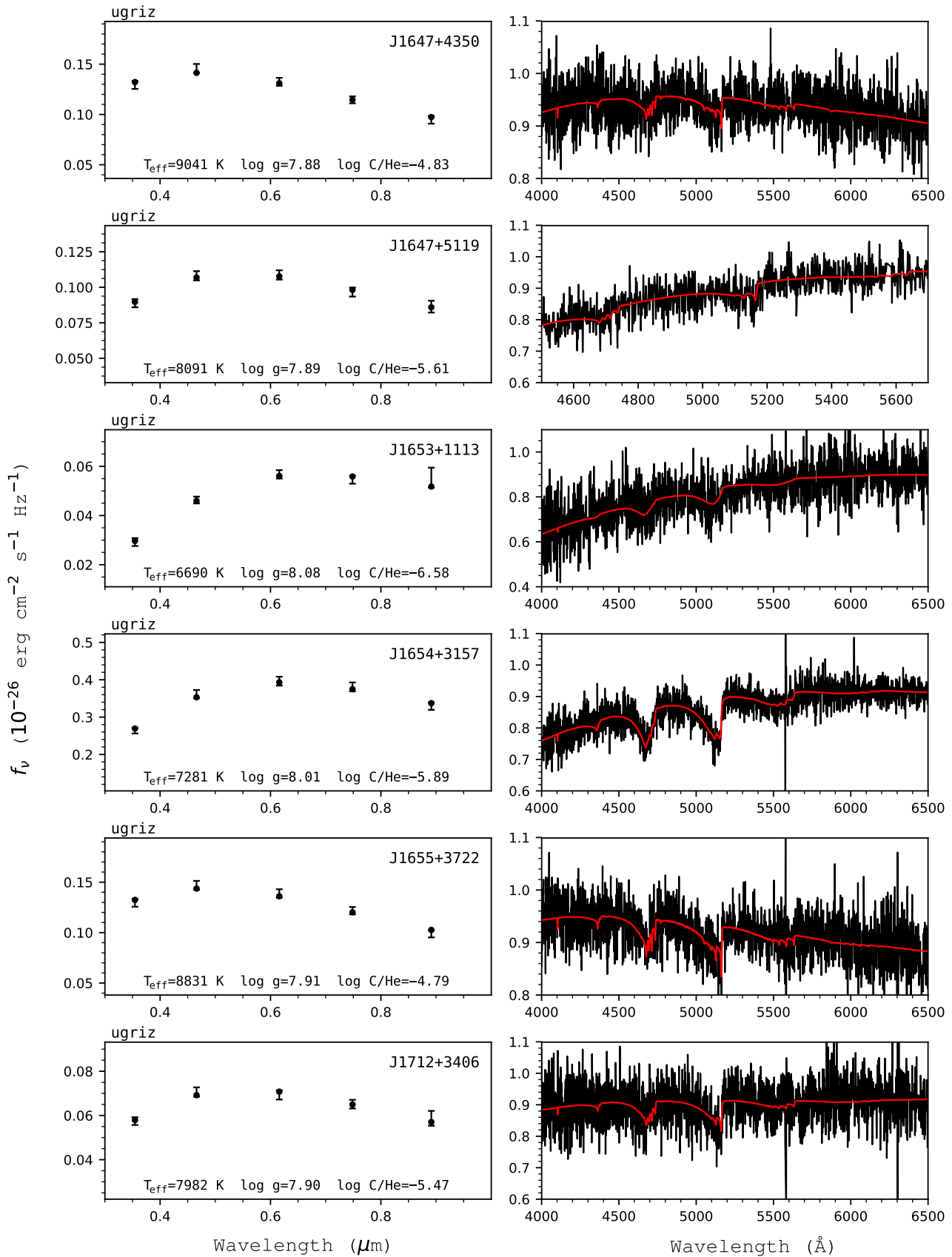

Figure 238. Fits to the DQ white dwarfs - continued. 

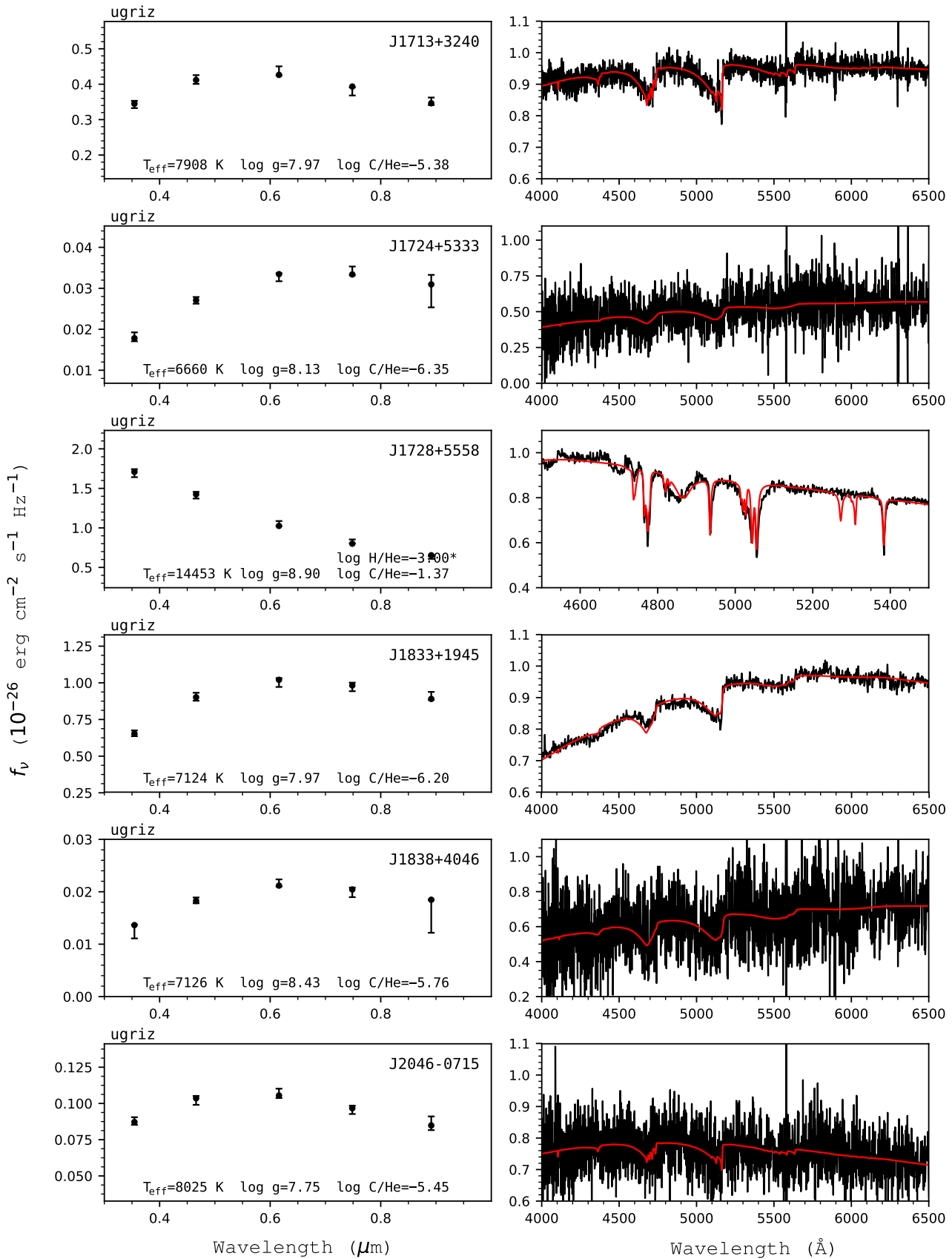

Figure 239. Fits to the DQ white dwarfs - continued. 

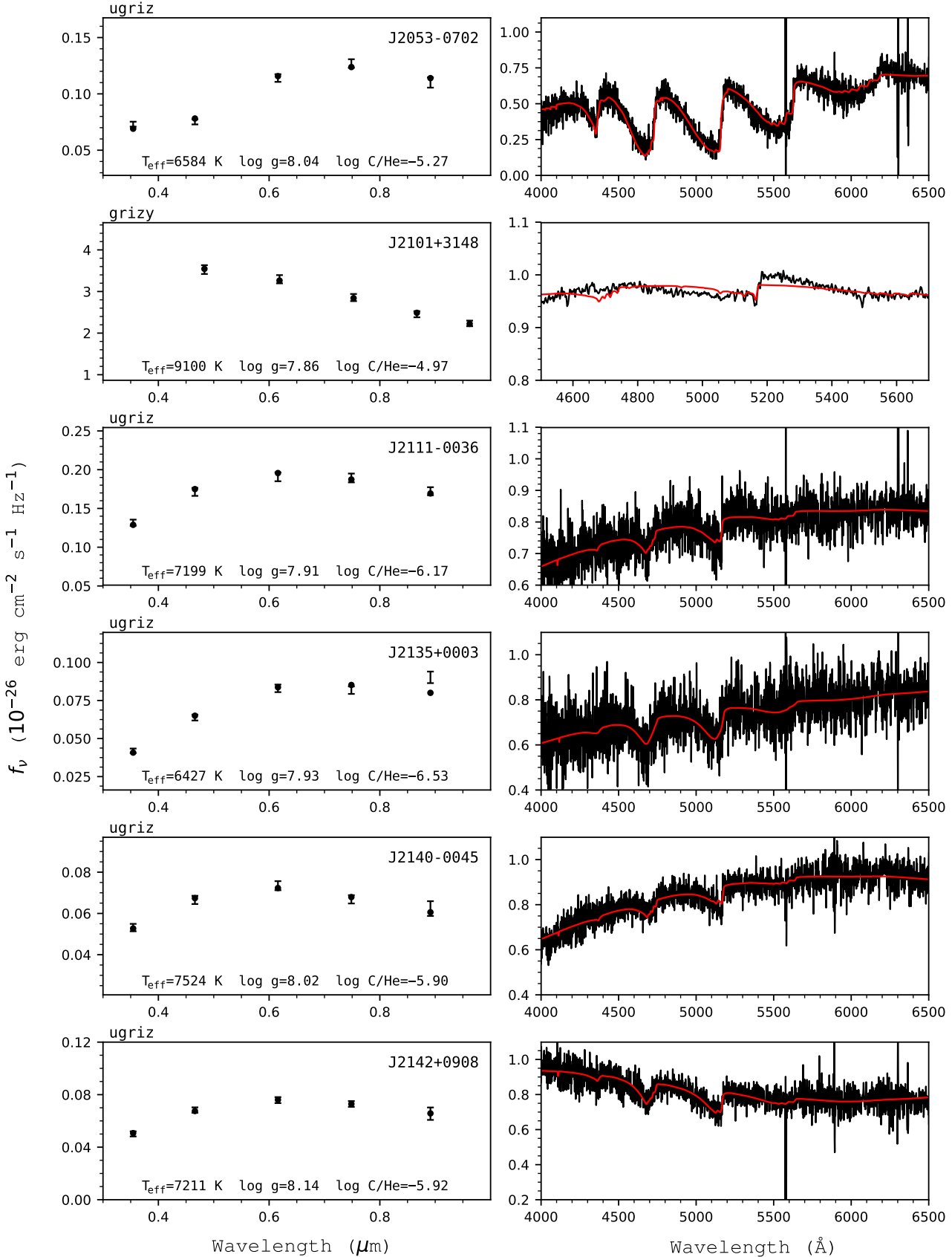

Figure 240. Fits to the DQ white dwarfs - continued. 

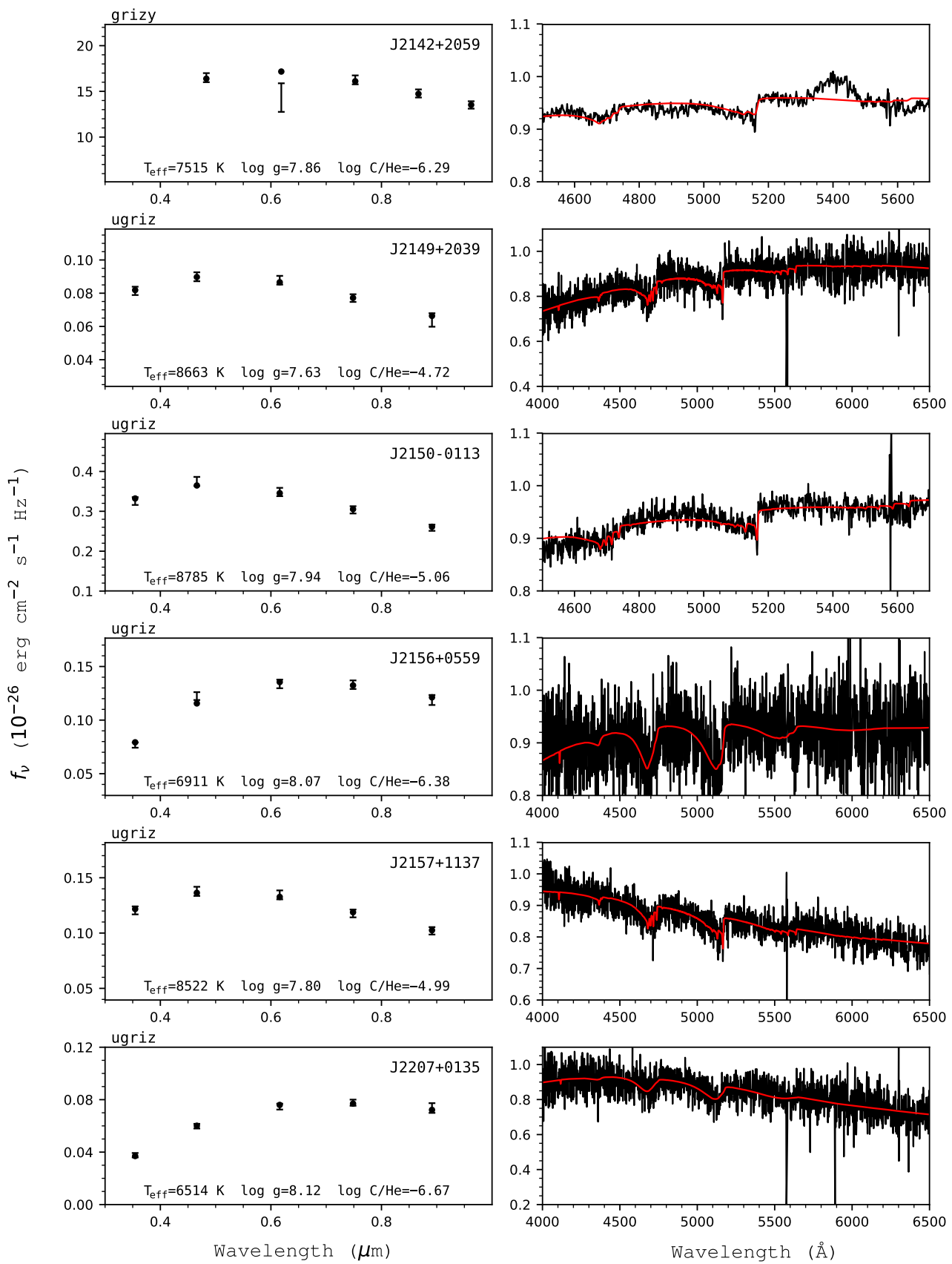

Figure 241. Fits to the DQ white dwarfs - continued. 

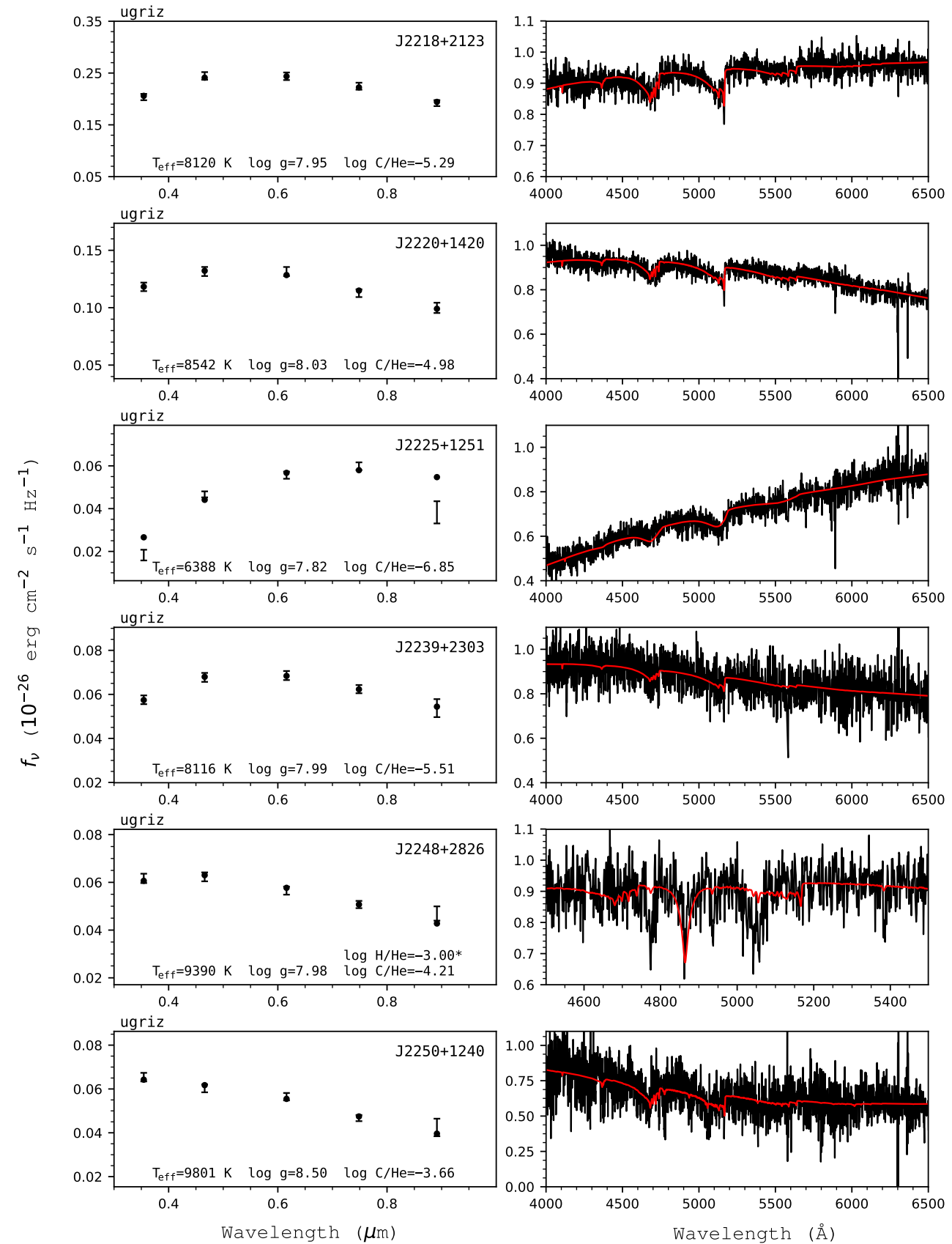

Figure 242. Fits to the DQ white dwarfs - continued. 

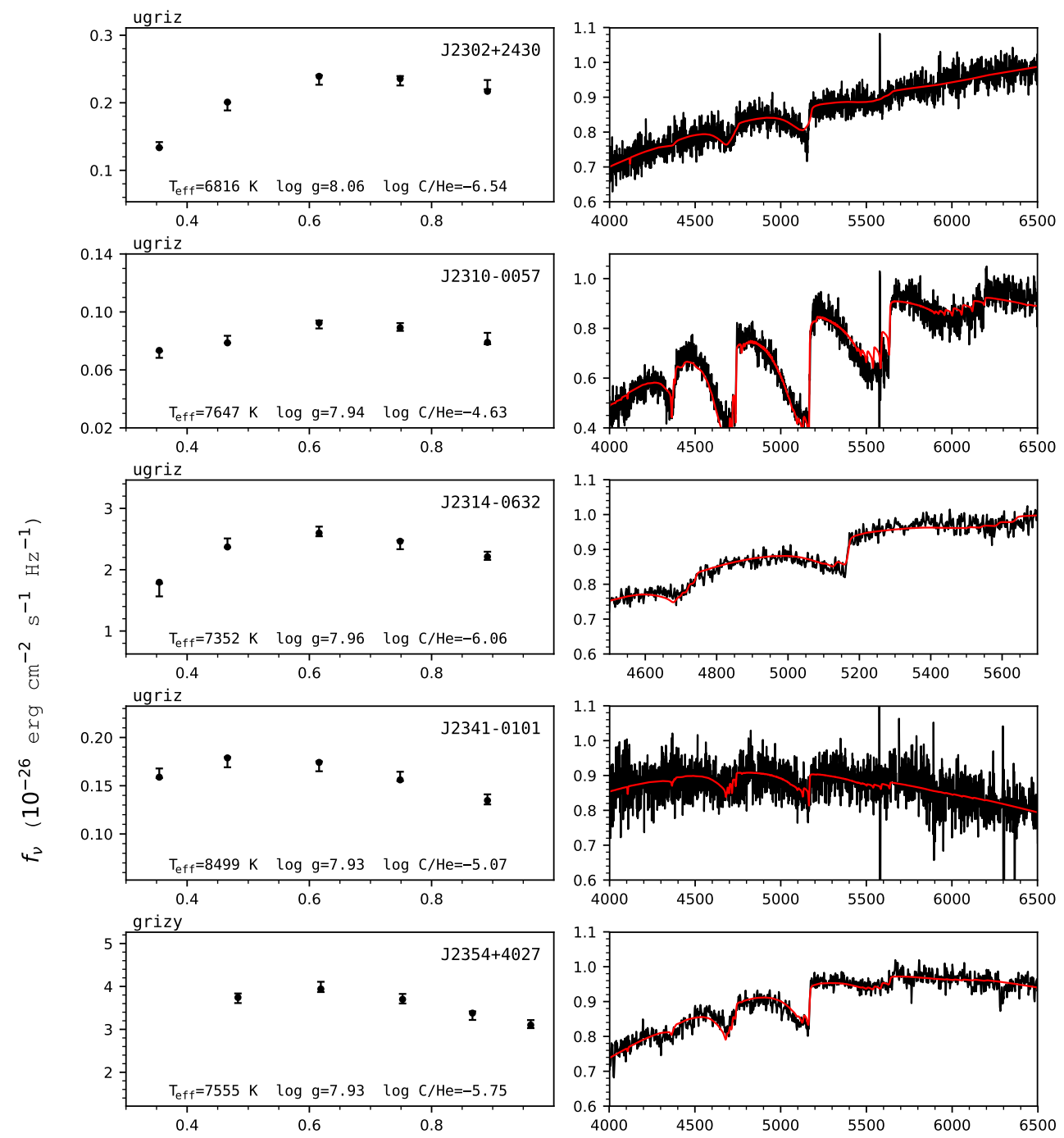

Figure 243. Fits to the DQ white dwarfs - continued. 